$$
\begin{array}{r}
M P I-P h / 95-104 \\
T U M-T 31-100 / 95 \\
\text { FERMILAB }-P U B-95 / 305-T \\
S L A C-P U B 7009
\end{array}
$$

\title{
WEAK DECAYS BEYOND LEADING LOGARITHMS
}

\author{
Gerhard Buchalla $^{3}$, Andrzej J. Buras ${ }^{1,2}$, Markus E. Lautenbacher ${ }^{1,4}$ * \\ ${ }^{1}$ Physik Department, Technische Universität München, \\ D-85748 Garching, Germany. \\ ${ }^{2}$ Max-Planck-Institut für Physik - Werner-Heisenberg-Institut, \\ Föhringer Ring 6, D-80805 München, Germany. \\ ${ }^{3}$ Theoretical Physics Department, \\ Fermi National Accelerator Laboratory, \\ P.O. Box 500, Batavia, IL 60510, USA. \\ 4 SLAC Theory Group, Stanford University, \\ P.O. Box 4349, Stanford, CA 94309, USA.
}

(November 1995)

to appear in Reviews of Modern Physics

\begin{abstract}
We review the present status of QCD corrections to weak decays beyond the leading logarithmic approximation including particle-antiparticle mixing and rare and CP violating decays. After presenting the basic formalism for these calculations we discuss in detail the effective hamiltonians for all decays for which the next-to-leading corrections are known. Subsequently, we present the phenomenological implications of these calculations. In particular we update the values of various parameters and we incorporate new information on $m_{t}$ in view of the recent top quark discovery. One of the central issues in our review are the theoretical uncertainties related to renormalization scale ambiguities which are substantially reduced by including next-to-leading order corrections. The impact of this theoretical improvement on the determination of the Cabibbo-Kobayashi-Maskawa matrix is then illustrated in various cases.
\end{abstract}

*email: buchalla@fnth20.fnal.gov, buras@feynman.t30.physik.tu-muenchen.de, lauten@feynman.t30.physik.tu-muenchen.de 


\section{Contents}

I Introduction $\quad 1$

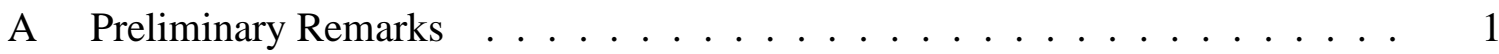

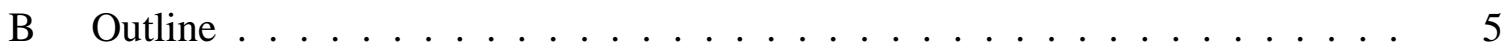

II Standard Electroweak Model $\quad 8$

A Particles and Interactions . . . . . . . . . . . . . . . . 8

B Standard Parametrization . . . . . . . . . . . . . . . . . . . 9

C Wolfenstein Parameterization Beyond Leading Order . . . . . . . . . . . . 10

D Unitarity Triangle Beyond Leading Order . . . . . . . . . . . . . . . . . . . 12

$\begin{array}{lll}\text { III Basic Formalism } & 14\end{array}$

A Renormalization of QCD . . . . . . . . . . . . . . . . 14

B Operator Product Expansion in Weak Decays - Preliminaries . . . . . . . . . 17

C OPE and Short Distance QCD Effects . . . . . . . . . . . . . . . . . . . . 20

D The Renormalization Group . . . . . . . . . . . . . . . . . . . . 26

1 Basic Concepts . . . . . . . . . . . . . . . . . 26

2 Threshold Effects in LLA . . . . . . . . . . . . . . . . . . . . . . . . . . . . . . . . . . . . .

3 Penguin Operators . . . . . . . . . . . . . . . . . . . . . 29

E Summary of Basic Formalism . . . . . . . . . . . . . . . . . 30

F Wilson Coefficients Beyond Leading Order . . . . . . . . . . . . . . . 31

1 The RG Formalism . . . . . . . . . . . . . . . . . . . 31

2 The Calculation of the Anomalous Dimensions . . . . . . . . . . . 35

3 Renormalization Scheme Dependence . . . . . . . . . . . . . 37

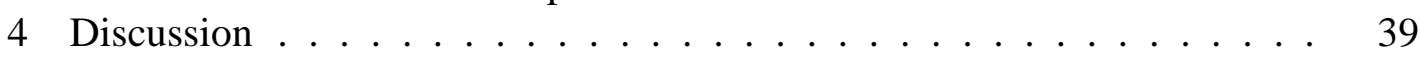

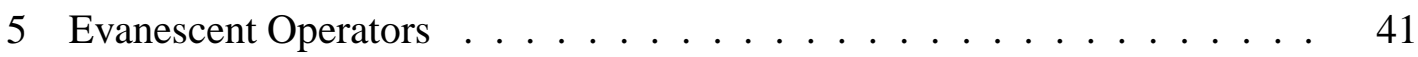

IV Guide to Effective Hamiltonians $\quad 44$

V The Effective $\Delta F=1$ Hamiltonian: Current-Current Operators 47

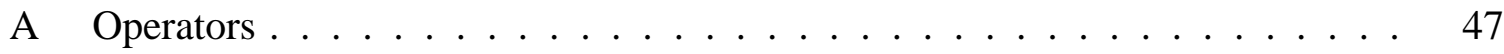

B Wilson Coefficients and RG Evolution .............. 47

VI The Effective $\Delta F=1$ Hamiltonian: Inclusion of QCD Penguin Operators 53

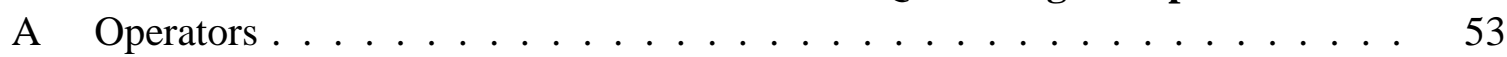

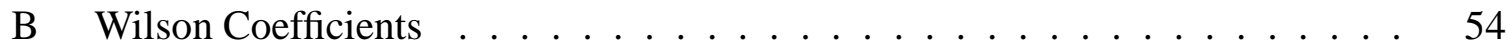

C Renormalization Group Evolution and Anomalous Dimension Matrices . . . . 56

D Quark Threshold Matching Matrix ................ 57

E Numerical Results for the $K \rightarrow \pi \pi$ Wilson Coefficients in Pure QCD . . . . . 57

F The $\Delta B=1$ Effective Hamiltonian in Pure QCD . . . . . . . . . . . . 59

G Numerical Results for the $\Delta B=1$ Wilson Coefficients in Pure QCD . . . . . 60 
VII The Effective $\Delta F=1$ Hamiltonian: Inclusion of Electroweak Penguin Operators 61

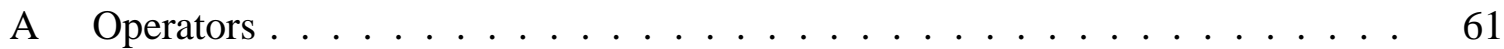

B Wilson Coefficients ...................... 62

C Renormalization Group Evolution and Anomalous Dimension Matrices . . . 63

D Quark Threshold Matching Matrix . . . . . . . . . . . . . . 66

E Numerical Results for the $K \rightarrow \pi \pi$ Wilson Coefficients . . . . . . . . . . 67

F The $\Delta B=1$ Effective Hamiltonian Including Electroweak Penguins $\ldots \ldots .68$

G Numerical Results for the $\Delta B=1$ Wilson Coefficients . . . . . . . . . 71

VIII The Effective Hamiltonian for $K_{L} \rightarrow \pi^{0} e^{+} e^{-} \quad \mathbf{7 3}$

A Operators ......................... 73

B Wilson Coefficients ....................... . 74

C Renormalization Group Evolution and Anomalous Dimension Matrices . . . . 75

D Quark Threshold Matching Matrix . . . . . . . . . . . . . . . 76

E Numerical Results for the $K_{L} \rightarrow \pi^{0} e^{+} e^{-}$Wilson Coefficients . . . . . . . 76

IX The Effective Hamiltonian for $B \rightarrow X_{s} \gamma$

A Operators ............................ 79

B Wilson Coefficients . . . . . . . . . . . . . . . . . . . . 79

C Renormalization Group Evolution and Anomalous Dimension Matrices . . . . 81

D Results for the Wilson Coefficients . . . . . . . . . . . . . . . 83

E Numerical Analysis . . . . . . . . . . . . . . . . . . . . . 83

X The Effective Hamiltonian for $B \rightarrow X_{s} e^{+} e^{-} \quad \mathbf{8 5}$

A Operators ........................... 85

B Wilson Coefficients ...................... 85

C Numerical Results . . . . . . . . . . . . . . . . . . . . . . . . . . 87

$\begin{array}{llr}\text { XI Effective Hamiltonians for Rare } K \text { - and } B \text {-Decays } & \mathbf{8 9}\end{array}$

A Overview .............................. 89

B The Decay $K^{+} \rightarrow \pi^{+} \nu \bar{\nu} \ldots \ldots \ldots \ldots \ldots \ldots \ldots \ldots \ldots \ldots \ldots \ldots$

1 The Next-to-Leading Order Effective Hamiltonian . . . . . . . . . . . 91

2 Z-Penguin and Box Contribution in the Top Sector . . . . . . . . . . 93

3 The $Z$-Penguin Contribution in the Charm Sector . . . . . . . . . . 94

4 The Box Contribution in the Charm Sector . . . . . . . . . . . . 96

5 Discussion . . . . . . . . . . . . . . . . . . . . 97

C The Decay $\left(K_{L} \rightarrow \mu^{+} \mu^{-}\right)_{S D} \ldots \ldots \ldots \ldots \ldots \ldots$

1 The Next-to-Leading Order Effective Hamiltonian . . . . . . . . . . . . 98

2 Discussion . . . . . . . . . . . . . . . . . . . . 99

D The Decays $K_{L} \rightarrow \pi^{0} \nu \bar{\nu}, B \rightarrow X_{s, d} \nu \bar{\nu}$ and $B_{s, d} \rightarrow l^{+} l^{-} \ldots \ldots \ldots \ldots$

$\begin{array}{lll}\text { XII The Effective Hamiltonian for } K^{0}-\bar{K}^{0} \text { Mixing } & 101\end{array}$

A General Structure . . . . . . . . . . . . . . . . . . . . 101

B The Top Contribution $-\eta_{2} \ldots \ldots \ldots \ldots \ldots \ldots \ldots \ldots$

C The Charm Contribution $-\eta_{1} \ldots \ldots \ldots \ldots \ldots$

D The Top-Charm Contribution $-\eta_{3} \ldots \ldots \ldots \ldots \ldots \ldots$ 
E Numerical Results . . . . . . . . . . . . . . . . . . . . . . . . . . . . . 114

1 General Remarks . . . . . . . . . . . . . . . . . . . . . . . . . . . . 114

2 Results for $\eta_{1}, \eta_{2}$ and $\eta_{3} \ldots \ldots \ldots \ldots \ldots \ldots$

$\begin{array}{ll}\text { XIII The Effective Hamiltonian for } B^{0}-\bar{B}^{0} \text { Mixing } & 118\end{array}$

A General Structure . . . . . . . . . . . . . . . . . . . . 118

B Numerical Results . . . . . . . . . . . . . . . . . . . . . . . . . . . . . . 119

$\begin{array}{ll}\text { XIV Penguin Box Expansion for FCNC Processes } & 121\end{array}$

XV Heavy Quark Effective Theory Beyond Leading Logs 124

A General Remarks . . . . . . . . . . . . . . . . . . . . . . . . . . . . . . . . . 124

B Basic Concepts . . . . . . . . . . . . . . . . . . . . . . . . . . . . . 124

C Heavy-Light Currents . . . . . . . . . . . . . . . . . . . . . . . 127

D The Pseudoscalar Decay Constant in the Static Limit . . . . . . . . . . . . . . 129

E $\Delta B=2$ Transitions in the Static Limit . . . . . . . . . . . . . . 130

XVI Comments on Input Parameters $\quad 136$

A CKM Element $\left|V_{c b}\right| \ldots \ldots \ldots \ldots \ldots \ldots \ldots \ldots \ldots$

B CKM Element Ratio $\left|V_{u b} / V_{c b}\right| \ldots \ldots \ldots \ldots \ldots \ldots \ldots \ldots$

C Top Quark Mass $m_{\mathrm{t}} \ldots \ldots \ldots \ldots \ldots \ldots \ldots$

$\begin{array}{ll}\text { XVII Inclusive B Decays } & 138\end{array}$

A General Remarks . . . . . . . . . . . . . . . . . . . . . . . . . 138

B b-Quark Decay Modes . . . . . . . . . . . . . . . . . . . . . . . . . . . 139

C The B Meson Semileptonic Branching Ratio . . . . . . . . . . . . . . . . . 142

XVIII $\varepsilon_{K}, B^{0}-\bar{B}^{0}$ Mixing and the Unitarity Triangle 145

A Basic Formula for $\varepsilon_{K} \ldots \ldots \ldots \ldots \ldots \ldots \ldots$

B Basic Formula for $B^{0}-\bar{B}^{0}$ Mixing . . . . . . . . . . . . . . . . . . . . 147

C $\sin (2 \beta)$ from $\varepsilon_{K}$ and $B^{0}-\bar{B}^{0}$ Mixing $\ldots \ldots \ldots \ldots \ldots \ldots$

D Phenomenological Analysis . . . . . . . . . . . . . . . . . . . 148

XIX $\varepsilon^{\prime} / \varepsilon$ Beyond Leading Logarithms 153

A Basic Formulae . . . . . . . . . . . . . . . . . . . . . . 153

B Hadronic Matrix Elements for $K \rightarrow \pi \pi \ldots \ldots \ldots \ldots \ldots \ldots \ldots$

C $\left\langle Q_{i}(\mu)\right\rangle_{2}$ for $(V-A) \otimes(V-A)$ Operators . . . . . . . . . . . 155

D $\left\langle Q_{i}(\mu)\right\rangle_{0}$ for $(V-A) \otimes(V-A)$ Operators $\ldots \ldots \ldots \ldots \ldots \ldots \ldots$

E $\left\langle Q_{i}(\mu)\right\rangle_{0,2}$ for $(V-A) \otimes(V+A)$ Operators . . . . . . . . . . . . 157

F The Four Dominant Contributions to $\varepsilon^{\prime} / \varepsilon \ldots \ldots \ldots \ldots \ldots$

G An Analytic Formula for $\varepsilon^{\prime} / \varepsilon \ldots \ldots \ldots \ldots$. . . . . . . . . . . 160

$\mathrm{H} \quad$ Numerical Results . . . . . . . . . . . . . . . . . . . . . . . . . . 162

$\begin{array}{llr}\text { XX } & K_{L}-K_{S} \text { Mass Difference and } \Delta I=1 / 2 \text { Rule } & \mathbf{1 6 7}\end{array}$

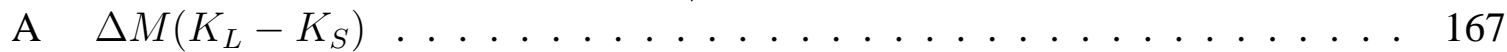

B The $\Delta I=1 / 2$ Rule $\ldots \ldots \ldots \ldots \ldots \ldots \ldots \ldots \ldots$ 
XXI The Decay $K_{L} \rightarrow \pi^{0} e^{+} e^{-} \quad 170$

A General Remarks . . . . . . . . . . . . . . . . . . . 170

B Analytic Formula for $B\left(K_{L} \rightarrow \pi^{0} e^{+} e^{-}\right)_{d i r} \ldots \ldots \ldots \ldots \ldots$. . . . . . 171

C Numerical Analysis . . . . . . . . . . . . . . . . . . 172

D The Indirectly CP Violating and CP Conserving Parts . . . . . . . . . . . . . 174

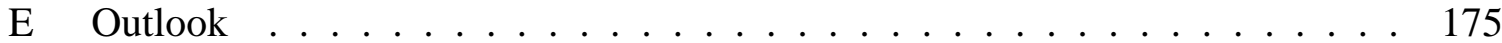

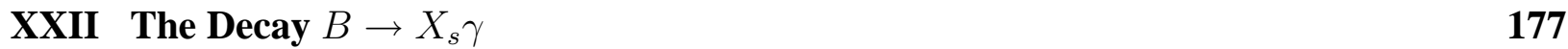

A General Remarks . . . . . . . . . . . . . . . . . . . . 177

B The Decay $B \rightarrow X_{s} \gamma$ in the Leading Log Approximation . . . . . . . . . . . 178

C Looking at $B \rightarrow X_{s} \gamma$ Beyond Leading Logarithms . . . . . . . . . . . . . 180

XXIII The Decay $B \rightarrow X_{s} e^{+} e^{-} \quad 182$

A General Remarks . . . . . . . . . . . . . . . . . . . . 182

B The Differential Decay Rate . . . . . . . . . . . . . . . . . . 183

C Numerical Analysis ........................ 184

XXIV The Decays $K^{+} \rightarrow \pi^{+} \nu \bar{\nu}$ and $K_{L} \rightarrow \pi^{0} \nu \bar{\nu} \quad 188$

A General Remarks on $K^{+} \rightarrow \pi^{+} \nu \bar{\nu} \ldots \ldots \ldots \ldots \ldots$. . . . . . . . 188

B Master Formulae for $K^{+} \rightarrow \pi^{+} \nu \bar{\nu} \ldots \ldots \ldots \ldots \ldots$. . . . . . . . 188

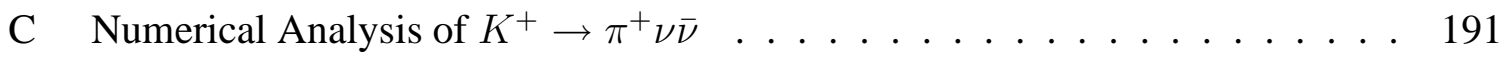

1 Renormalization Scale Uncertainties . . . . . . . . . . . . . . . 191

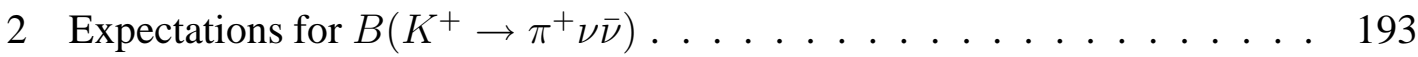

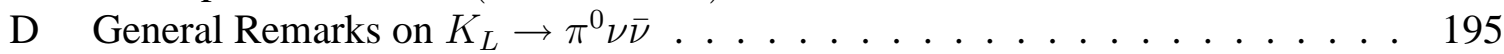

E Master Formulae for $K_{L} \rightarrow \pi^{0} \nu \bar{\nu} \ldots \ldots \ldots \ldots \ldots$

F $\quad$ Numerical Analysis of $K_{L} \rightarrow \pi^{0} \nu \bar{\nu} \quad \ldots \ldots \ldots$. . . . . . . . . . . . . 196

1 Renormalization Scale Uncertainties . . . . . . . . . . . . . . . . . 196

2 Expectations for $B\left(K_{L} \rightarrow \pi^{0} \nu \bar{\nu}\right) \ldots \ldots \ldots$. . . . . . . . . . . . . . . . . . 196

G Unitarity Triangle from $K \rightarrow \pi \nu \bar{\nu} \ldots \ldots \ldots$. . . . . . . . . . . . 197

$\mathrm{H} \quad \sin 2 \beta$ from $K \rightarrow \pi \nu \bar{\nu} \ldots \ldots \ldots \ldots \ldots \ldots$

XXV The Decays $K_{L} \rightarrow \mu^{+} \mu^{-}$and $K^{+} \rightarrow \pi^{+} \mu^{+} \mu^{-} \quad 200$

A General Remarks on $K_{L} \rightarrow \mu^{+} \mu^{-} \ldots \ldots \ldots \ldots$. . . . . . . . 200

B Master Formulae for $\left(K_{L} \rightarrow \mu^{+} \mu^{-}\right)_{\mathrm{SD}} \ldots \ldots \ldots \ldots \ldots \ldots$

C Numerical Analysis of $\left(K_{L} \rightarrow \mu^{+} \mu^{-}\right)_{S D} \ldots \ldots \ldots \ldots \ldots$. . . . . . . . . . . . . . . . 201

1 Renormalization Scale Uncertainties . . . . . . . . . . . . . . 201

2 Expectations for $B\left(K_{L} \rightarrow \mu^{+} \mu^{-}\right)_{S D} \ldots \ldots \ldots \ldots . \ldots \ldots 203$

D General Remarks on $K^{+} \rightarrow \pi^{+} \mu^{+} \mu^{-} \ldots \ldots \ldots \ldots$. . . . . . . . . . . . . . . 204

E Master Formulae for $\Delta_{L R} \ldots \ldots \ldots \ldots \ldots \ldots \ldots$

F Numerical Analysis of $\Delta_{L R} \ldots \ldots \ldots \ldots \ldots$

XXVI The Decays $B \rightarrow X \nu \bar{\nu}$ and $B \rightarrow \mu^{+} \mu^{-} \quad 208$

A General Remarks . . . . . . . . . . . . . . . . . . . 208

B The Decays $B \rightarrow X_{s} \nu \bar{\nu}$ and $B \rightarrow X_{d} \nu \bar{\nu} \ldots \ldots \ldots \ldots \ldots$

C The Decays $B_{s} \rightarrow \mu^{+} \mu^{-}$and $B_{d} \rightarrow \mu^{+} \mu^{-} \ldots \ldots \ldots \ldots$. . . . . . 209 
$\begin{array}{lll}\text { A } & \text { Compilation of Numerical Input Parameters } & 214\end{array}$

$\begin{array}{lr}\text { APPENDIXES } & 214\end{array}$ 


\section{INTRODUCTION}

\section{A. Preliminary Remarks}

Among the fundamental forces of nature the weak interactions clearly show the most complicated and diversified pattern from the point of view of our present day understanding represented by the Standard Model of particle physics. Although this theory of the strong and electroweak forces is capable of describing very successfully a huge amount of experimental information in a quantitative way and a great deal of phenomena at least qualitatively, there are many big question marks that remain. The most prominent among them like the problem of electroweak symmetry breaking and the origin of fermion masses and quark mixing are closely related to the part of the Standard Model describing weak interactions. Equally puzzling is the fact that whereas the discrete space-time symmetries $\mathrm{C}, \mathrm{P}, \mathrm{CP}$ and $\mathrm{T}$ are respected by strong and electromagnetic interactions, the weak force violates them all. Obviously, the weak interaction is the corner of the Standard Model that is least understood. The history of this field is full of surprises and still more of them might be expected in the future.

For these reasons big efforts have been and still are being undertaken in order to develop our theoretical understanding of weak interaction phenomena and to disentangle the basic mechanisms and parameters. An excellent laboratory for this enterprise is provided by the very rich phenomenology of weak meson decays.

The careful investigation of these decays is mandatory for further testing the Standard Model. Of particular importance is the determination of all Cabibbo-Kobayashi-Maskawa (CKM) parameters as a prerequisite for a decisive test of the consistency of the Standard Model ansatz including the unitarity of the CKM matrix and its compatibility with the quark masses. Many interesting issues within this context still remain unsettled. Let us just mention here the question of direct $\mathrm{CP}$ violation in non-leptonic $\mathrm{K}$ decays $\left(\varepsilon^{\prime} / \varepsilon\right)$, the yet completely unknown pattern of $\mathrm{CP}$ violation in the $\mathrm{B}$ system and the rare $\mathrm{K}$ and $\mathrm{B}$ decays, which are sensitive to the effects of virtual heavy particles, most notably the top quark, its mass and its weak couplings. Whether the CKM description of CP violation is correct, remains as an outstanding open question. It is clear that the need for a modification of the model is conceivable and that meson decay phenomena might provide a window for "new physics". However, independently of this possibility it is crucial to improve the theoretical predictions in the Standard Model itself, either to further establish its correctness, or to be able to make clear cut statements on its possible failure.

Now, for all attempts towards a theoretical understanding of these issues the obvious fact that the fundamental forces do not come in isolation is of crucial significance. Since hadrons are involved in the decays that are of interest here, QCD unavoidably gets into the game. In order to understand weak meson decays we have to understand the interplay of weak interactions with the strong forces.

To accomplish this task it is necessary to employ the field theoretical tools of the operator product expansion (OPE) (Wilson and Zimmermann, 1972) and the renormalization group (Stueckelberg and Petermann, 1953), (Gell-Mann and Low, 1954), (Ovsyannikov, 1956), (Symanzik, 1970), (Callan Jr, 1970), ('t Hooft, 1973), (Weinberg, 1973). The basic virtues of these two techniques may be characterized as follows. Consider the amplitude $A$ for some weak meson decay process. Using the OPE formalism this amplitude can be represented as (Witten, 1977) 


$$
A=\left\langle\mathcal{H}_{e f f}\right\rangle=\sum_{i} C_{i}\left(\mu, M_{W}\right)\left\langle Q_{i}(\mu)\right\rangle
$$

where it is factorized into the Wilson coefficient functions $C_{i}$ and the matrix elements of local operators $Q_{i}$. In this process the $\mathrm{W}$ boson and other fields with mass bigger than the factorization scale $\mu$ are "integrated out", that is removed from the theory as dynamical degrees of freedom. The effect of their existence is however implicitly taken into account in the Wilson coefficients. In a more intuitive interpretation one can view the expression $\sum C_{i} Q_{i}$ as an effective hamiltonian for the process considered, with $Q_{i}$ as the effective vertices and $C_{i}$ the corresponding coupling constants. Usually for weak decays only the operators of lowest dimension need to be taken into account. Contributions of higher dimensional operators are negligible since they are typically suppressed by powers of $p^{2} / M_{W}^{2}$, where $p$ is the momentum scale relevant for the decaying meson in question.

The essential point about the OPE is that it achieves a separation of the full problem into two distinct parts, the long-distance contributions contained in the operator matrix elements and the short-distance physics described by the Wilson coefficients. The renormalization scale $\mu$ separating the two regimes is typically chosen to be of the order $\mathcal{O}(\geq 1 \mathrm{GeV})$ for kaon decays and a few $\mathrm{GeV}$ for the decays of $\mathrm{D}$ and B mesons. The physical amplitude $A$ however cannot depend on $\mu$. The $\mu$ dependence of the Wilson coefficients has to cancel the $\mu$ dependence present in $\left\langle Q_{i}(\mu)\right\rangle$. In other words it is a matter of choice what exactly belongs to the matrix elements and what to the coefficient functions. This cancellation of $\mu$ dependence involves generally several terms in the expansion in (I.1).

The long-distance part in (I.1) deals with low energy strong interactions and therefore poses a very difficult problem. Many approaches, like lattice gauge theory, $1 / N$ - expansion, QCD- and hadronic sum rules or chiral perturbation theory, have been used in the past to obtain qualitative insight and some quantitative estimates of relevant hadronic matrix elements. In addition heavy quark effective theory (HQET) and heavy quark expansions (HQE) have been widely used for $B$ decays. Despite these efforts the problem is not yet solved satisfactorily.

In general in weak decays of mesons the hadronic matrix elements constitute the most important source of theoretical uncertainty. There are however a few special examples of semileptonic rare decays $\left(K^{+} \rightarrow \pi^{+} \nu \bar{\nu}, K_{L} \rightarrow \pi^{0} \nu \bar{\nu}, B \rightarrow X_{s} \nu \bar{\nu}\right)$ where the matrix elements needed can be extracted from well measured leading decays or calculated perturbatively or as in the case of $B_{s} \rightarrow \mu \bar{\mu}$ expressed fully in terms of meson decay constants. Thus practically the problem of long-distance QCD can be completely avoided. This makes these decay modes very attractive from a theoretical point of view, although due to very small branching ratios they are quite difficult to access experimentally today.

Contrary to the long-distance contributions the short-distance part can be analyzed systematically using well established field theoretical methods. Due to the asymptotic freedom property of QCD the strong interaction effects at short-distances are calculable in perturbation theory in the strong coupling $\alpha_{\mathrm{s}}(\mu)$. In fact $\alpha_{\mathrm{s}}(\mu)$ is small enough in the full range of relevant short distance scales of $\mathcal{O}\left(M_{W}\right)$ down to $\mathcal{O}(1 \mathrm{GeV})$ to serve as a reasonable expansion parameter. However the presence of large logarithms $\ln \left(M_{W} / \mu\right)$ multiplying $\alpha_{\mathrm{s}}(\mu)$ (where $\mu=\mathcal{O}(1 \mathrm{GeV})$ ) in the calculation of the coefficients $C_{i}\left(\mu, M_{W}\right)$ spoils the validity of the usual perturbation series. This is a characteristic feature of renormalizable quantum field theories when vastly different scales are present. It is therefore necessary to perform a renormalization group analysis which allows an efficient summation of logarithmic terms to all orders in perturbation theory. In this way the usual perturbation 
theory is replaced by the renormalization group improved perturbation theory in which the leading order (LO) corresponds to summing the leading logarithmic terms $\sim\left(\alpha_{\mathrm{s}} \ln \left(M_{W} / \mu\right)\right)^{n}$. Then at next-to-leading order (NLO), all terms of the form $\sim \alpha_{\mathrm{s}}\left(\alpha_{\mathrm{s}} \ln \left(M_{W} / \mu\right)\right)^{n}$ are summed in addition, and so on.

The evaluation of the short-distance coefficients in renormalization group improved perturbation theory is only a part of the entire problem, but one should stress that still it is indispensible to analyze this part systematically; the effective hamiltonians resulting from the short-distance analysis provide the necessary basis for any further computation of weak decay amplitudes. The longdistance matrix elements needed in addition can be treated separately and will hopefully be known with desirable accuracy one day.

The rather formal expression for the decay amplitudes given in (I.1) can always be cast in a form (Buchalla et al., 1991)

$$
A(M \rightarrow F)=\sum_{i} B_{i} V_{C K M}^{i} \eta_{Q C D}^{i} F_{i}\left(m_{t}, m_{c}\right)
$$

which is more useful for phenomenology. In writing (I.2) we have generalized (I.1) to include several CKM factors $V_{C K M}^{i}$. The functions $F_{i}\left(m_{t}, m_{c}\right)$ result from the evaluation of loop diagrams with internal top and charm exchanges and may also depend solely on $m_{\mathrm{t}}$ or $m_{\mathrm{c}}$. In certain cases $F_{i}$ are mass independent. The factors $\eta_{Q C D}^{i}$ summarize short distance QCD corrections which can be calculated by the formal methods mentioned above. Finally $B_{i}$ stand for nonperturbative factors related to the hadronic matrix elements of the contributing operators: the main theoretical uncertainty in the whole enterprise. A well known example of a $B_{i}$-factor is the renormalization group invariant parameter $B_{K}$ relevant for $K^{0}-\bar{K}^{0}$ mixing and the indirect CP violation in $K \rightarrow \pi \pi$. It is worth noting that the short-distance QCD contributions by themselves have already an important impact on weak decay processes. In non-leptonic K-decays, for example, they help to explain the famous $\Delta I=1 / 2$ rule and they generate penguin operators which are relevant for $\varepsilon^{\prime} / \varepsilon$. They suppress the semileptonic branching ratio in heavy quark decays and produce a significant enhancement of the weak radiative process $B \rightarrow X_{s} \gamma$.

Starting with the pioneering work of (Gaillard and Lee, 1974a) and (Altarelli and Maiani, 1974), who calculated the first leading logarithmic QCD effects in weak decays, considerable efforts have been devoted to the calculation of short-distance QCD corrections to weak meson decay processes. The analysis has been extended to a large variety of particular modes. Of great interest are especially processes sensitive to the virtual contribution of heavy quarks, like the top. A classic example of this type is the 1974 analysis of (Gaillard and Lee, 1974b) of $K^{0}-\bar{K}^{0}$ mixing and their estimate of the charm quark mass prior to its discovery, based on the dependence of the $\Delta S=2$ transition on virtual charm. This calculation constitutes the prototype application for present day analyses of virtual top contributions in $B^{0}-\bar{B}^{0}$ mixing, rare decays and $\mathrm{CP}$ violation, which are similar in spirit.

Until 1989 most of the calculations were done in LO, i.e. in the leading logarithmic approximation (Vainshtein et al., 1977), (Gilman and Wise, 1979), (Gilman and Wise, 1980), (Guberina and Peccei, 1980). An exception was the important work of (Altarelli et al., 1981) where the first NLO calculation in the theory of weak decays has been presented.

Today the effective hamiltonians for weak processes are available at the next-to-leading level for the most important and interesting cases due to a series of publications devoted to this enterprise beginning with the work of (Buras and Weisz, 1990). In table I we give a list of decays for which NLO QCD corrections are known at present. With the next-to-leading short-distance effects 
included, weak decays have in a sense now achieved the status that the conceptually similar field of deep inelastic lepton nucleon scattering had attained more then a decade ago (Buras, 1980).

TABLE I. Processes for which NLO QCD corrections have been calculated by now.

\begin{tabular}{|c|c|}
\hline Decay & Reference \\
\hline \multicolumn{2}{|c|}{ 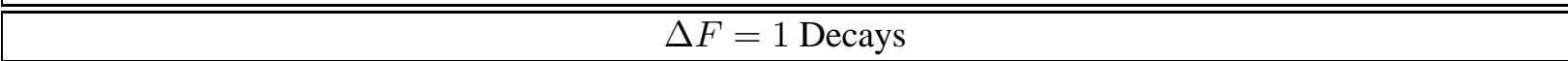 } \\
\hline current-current operators & (Altarelli et al., 1981), (Buras and Weisz, 1990) \\
\hline QCD penguin operators & $\begin{array}{l}\text { (Buras et al., 1993c), (Buras et al., 1993a), } \\
\text { (Ciuchini et al., 1994a) }\end{array}$ \\
\hline electroweak penguin operators & $\begin{array}{l}\text { (Buras et al., 1993c), (Buras et al., 1993a), } \\
\text { (Ciuchini et al., 1994a) }\end{array}$ \\
\hline magnetic penguin operators & (Misiak and Münz, 1995) \\
\hline$B(B \rightarrow X e \nu)$ & $\begin{array}{l}\text { (Altarelli et al., 1981), (Buchalla, 1993), } \\
\text { (Bagan et al., 1994), (Bagan et al., 1995b) }\end{array}$ \\
\hline Inclusive $\Delta S=1$ & (Jamin and Pich, 1994) \\
\hline \multicolumn{2}{|c|}{ Particle-Antiparticle Mixing } \\
\hline$\eta_{1}$ & (Herrlich and Nierste, 1994) \\
\hline$\eta_{2}, \eta_{B}$ & (Buras et al., 1990) \\
\hline$\eta_{3}$ & (Herrlich and Nierste, 1995a) \\
\hline \multicolumn{2}{|c|}{ Rare K- and B-Meson Decays } \\
\hline$K_{L}^{0} \rightarrow \pi^{0} \nu \bar{\nu}, B \rightarrow l^{+} l^{-}, B \rightarrow X_{\mathrm{s}} \nu \bar{\nu}$ & (Buchalla and Buras, 1993a) \\
\hline$K^{+} \rightarrow \pi^{+} \nu \bar{\nu}, K_{L} \rightarrow \mu^{+} \mu^{-}$ & (Buchalla and Buras, 1994a) \\
\hline$K^{+} \rightarrow \pi^{+} \mu \bar{\mu}$ & (Buchalla and Buras, 1994b) \\
\hline$K_{L} \rightarrow \pi^{0} e^{+} e^{-}$ & (Buras et al., 1994a) \\
\hline$B \rightarrow X_{s} e^{+} e^{-}$ & (Misiak, 1995), (Buras and Münz, 1995) \\
\hline
\end{tabular}

Let us recall why NLO calculations are important for weak decays and why it is worthwile to perform the very involved and complicated computations.

- The NLO is first of all necessary to test the validity of perturbation theory. In LO all the $\left(\alpha_{\mathrm{s}} \ln \left(M_{W} / \mu\right)\right)^{n}$ terms are summed, yielding a result of $\mathcal{O}(1)$; it is only at NLO where one obtains a truly perturbative $\mathcal{O}\left(\alpha_{\mathrm{s}}\right)$ correction relative to the LO and one can check whether it is small enough to justify the perturbative approach.

- Without going to NLO the scheme specific QCD scale $\Lambda_{\overline{M S}}$ extracted from various high energy processes cannot be used meaningfully in weak decays.

- Due to renormalization group (RG) invariance the physical amplitudes do not depend on the exact scales $\mu_{i}$ at which quark masses (top) are defined or heavy particles are integrated out. However in perturbation theory RG invariance is broken through the truncation of the series by terms of the neglected order. Numerically the resulting scale ambiguities, representing the theoretical uncertainty of the short-distance part, are a serious problem for the LO which can be reduced considerably by going to NLO. 
- The Wilson coefficients are renormalization scheme dependent quantities. The scheme dependence is first "felt" at NLO whereas the LO is completely insensitive to this important feature. In particular this issue is essential for a proper matching of the short distance contributions to the long distance matrix elements as obtained from lattice calculations.

- In some cases, particularly for $\varepsilon^{\prime} / \varepsilon, K_{L} \rightarrow \pi^{0} e^{+} e^{-}$and $B \rightarrow X_{s} e^{+} e^{-}$, the central issue of the top quark mass dependence is strictly speaking a NLO effect.

We would like to stress that short-distance QCD should be contrasted with an "intrinsically perturbative" theory like QED, where perturbation theory is almost the whole story since $\alpha_{Q E D}$ is exceedingly small. In QCD the coupling is much larger at interesting scales so that the conceptual questions like residual scale or scheme dependences, which are formally of the neglected higher order, become important numerically. Thus in this sense the question of higher order corrections is not only one of a quantitative improvement (of making precise predictions even more accurate, like in QED), but of a qualitative improvement as well.

We think that the time is appropriate to review the subject of QCD corrections to weak meson decays at the next-to-leading order level and to collect the most important results obtained in this field.

\section{B. Outline}

This review is divided into three parts, roughly speaking "basic concepts", "technicalities" and "phenomenological applications". The division is made especially for pedagogical reasons hoping to make the review as readable as possible to a wide audience of physicists.

In the first part we discuss the basic formalism necessary to obtain the effective hamiltonians for weak decays from the underlying full $S U(3) \otimes S U(2)_{L} \otimes U(1)_{Y}$ gauge theory of the Standard Model.

The second part constitutes a compendium of effective hamiltonians for all weak decays for which NLO corrections have been calculated in the literature and whose list is given in table I. We include also the discussion of the important decay $B \rightarrow X_{s} \gamma$ which is known only at the LO level.

The third part of our review then presents the phenomenological picture of weak decays beyond the leading logarithmic approximation using the results obtained in parts one and two.

We end our review of this exciting field with a brief summary of results and an outlook.

We are aware of the fact that some sections in this review are necessarily rather technical which is connected to the very nature of the subject of this review. We have however made efforts to present the material in a pedagogical fashion. Thus part one can be regarded as an elementary introduction to the formalism of QCD calculations which include renormalization group methods and the operator product expansion. Even if our compendium in part two looks rather technical at first sight, the guidelines to the effective hamiltonians presented in section IV should be helpful in following and using this important part of our review. In any case the phenomenological part three is almost self-contained and its material can be easily followed with the help of the guidelines in section IV without the necessity of fully understanding the details of NLO calculations. 



\section{Part One -}

\section{The Basic Formalism}

In this first part we will discuss the basic formalism behind radiative corrections to weak decays.

In section II we recall those ingredients of the standard $S U(3) \otimes S U(2) \otimes U(1)$ model, which play an important role in subsequent sections. In particular we recall the Cabibbo-KobayashiMaskawa matrix in two useful parametrizations and we briefly describe the unitarity triangle.

In section III we outline the basic formalism for the calculation of QCD effects in weak decays. Beginning with the idea of effective field theories we introduce subsequently the techniques of the operator product expansion and the renormalization group. These important concepts are illustrated explicitly using the simple, but phenomenologically relevant example of current-current operators, which allows to demonstrate the procedure in a transparent way. The central issue in this formalism is the computation of the Wilson coefficients $C_{i}$ of local operators in the $\mathrm{LO}$ and NLO approximation. This calculation involves the proper computation of $C_{i}$ at $\mu=\mathcal{O}\left(M_{\mathrm{W}}\right)$ and the renormalization group evolution down to low energy scales $\mu \ll M_{\mathrm{W}}$ relevant for the weak decays considered. The latter requires the evaluation of one-loop and two-loop anomalous dimensions of $Q_{i}$ or more generally the anomalous dimension matrices, which describe the mixing of these operators under renormalization. We outline the steps for a consistent calculation of the Wilson coefficients $C_{i}$ and formulate recipes for the determination of the anomalous dimensions of local operators. In section III F we give "master formulae" for the Wilson coefficients $C_{i}$, including NLO corrections. Since these formulae will be central for our review, we discuss their various properties in some detail. In particular we address the $\mu$ - and renormalization scheme dependences and we show on general grounds how these dependences are canceled by those present in the hadronic matrix elements. 


\section{STANDARD ELECTROWEAK MODEL}

\section{A. Particles and Interactions}

Throughout this review we will work in the context of the three generation model of quarks and leptons based on the gauge group $S U(3) \otimes S U(2)_{L} \otimes U(1)_{Y}$ spontaneously broken down to $S U(3) \otimes U(1)_{Q}$. Here $Y$ and $Q$ denote the weak hypercharge and the electric charge generators, respectively. $S U(3)$ stands for $Q C D$ which will be discussed in more detail in the following section. Here we would like to recall certain features of the electroweak part of the Standard Model which will be important for our considerations.

The left-handed leptons and quarks are put in $S U(2)_{L}$ doublets

$$
\begin{array}{ll}
\left(\begin{array}{c}
\nu_{e} \\
e^{-}
\end{array}\right)_{L} \quad\left(\begin{array}{c}
\nu_{\mu} \\
\mu^{-}
\end{array}\right)_{L} \quad\left(\begin{array}{c}
\nu_{\tau} \\
\tau^{-}
\end{array}\right)_{L} \\
\left(\begin{array}{c}
u \\
d^{\prime}
\end{array}\right)_{L} \quad\left(\begin{array}{c}
c \\
s^{\prime}
\end{array}\right)_{L} \quad\left(\begin{array}{c}
t \\
b^{\prime}
\end{array}\right)_{L}
\end{array}
$$

with the corresponding right-handed fields transforming as singlets under $S U(2)_{L}$. The primes are discussed below. The relevant electroweak charges $Q, Y$ and the third component of the weak isospin $T_{3}$ are collected in table II.

TABLE II. Electroweak charges $Q, Y$ and the third component of the weak isospin $T_{3}$ for quarks and

\begin{tabular}{|c|c|c|c|c|c|c|c|}
\hline & $\nu_{L}^{e}$ & $e_{L}^{-}$ & $e_{R}^{-}$ & $u_{L}$ & $d_{L}$ & $u_{R}$ & $d_{R}$ \\
\hline$Q$ & 0 & -1 & -1 & $2 / 3$ & $-1 / 3$ & $2 / 3$ & $-1 / 3$ \\
\hline$T_{3}$ & $1 / 2$ & $-1 / 2$ & 0 & $1 / 2$ & $-1 / 2$ & 0 & 0 \\
\hline$Y$ & -1 & -1 & -2 & $1 / 3$ & $1 / 3$ & $4 / 3$ & $-2 / 3$ \\
\hline
\end{tabular}
leptons in the Standard Model.

The electroweak interactions of quarks and leptons are mediated by the massive weak gauge bosons $W^{ \pm}$and $Z^{\circ}$ and by the photon $A$. These interactions are summarized by the Lagrangian

$$
\mathcal{L}_{i n t}=\mathcal{L}_{\mathrm{CC}}+\mathcal{L}_{\mathrm{NC}}
$$

where

$$
\mathcal{L}_{\mathrm{CC}}=\frac{g_{2}}{2 \sqrt{2}}\left(J_{\mu}^{+} W^{+\mu}+J_{\mu}^{-} W^{-\mu}\right)
$$

describes the charged current interactions and

$$
\mathcal{L}_{\mathrm{NC}}=e J_{\mu}^{e m} A^{\mu}+\frac{g_{2}}{2 \cos \Theta_{W}} J_{\mu}^{o} Z^{\mu}
$$

the neutral current interactions. Here $e$ is the QED coupling constant, $g_{2}$ is the $S U(2)_{L}$ coupling constant and $\Theta_{W}$ is the Weinberg angle. The currents are given as follows 


$$
\begin{gathered}
J_{\mu}^{+}=\left(\bar{u} d^{\prime}\right)_{V-A}+\left(\bar{c} s^{\prime}\right)_{V-A}+\left(\bar{t} b^{\prime}\right)_{V-A}+\left(\bar{\nu}_{e} e\right)_{V-A}+\left(\bar{\nu}_{\mu} \mu\right)_{V-A}+\left(\bar{\nu}_{\tau} \tau\right)_{V-A} \\
J_{\mu}^{e m}=\sum_{f} Q_{f} \bar{f} \gamma_{\mu} f \\
J_{\mu}^{o}=\sum_{f} \bar{f} \gamma_{\mu}\left(v_{f}-a_{f} \gamma_{5}\right) f \\
v_{f}=T_{3}^{f}-2 Q_{f} \sin ^{2} \Theta_{W} \quad a_{f}=T_{3}^{f}
\end{gathered}
$$

where $Q_{f}$ and $T_{3}^{f}$ denote the charge and the third component of the weak isospin of the left-handed fermion $f_{L}$.

In our discussion of weak decays an important role is played by the Fermi constant:

$$
\frac{G_{F}}{\sqrt{2}}=\frac{g_{2}^{2}}{8 M_{W}^{2}}
$$

which has the value

$$
G_{F}=1.16639 \cdot 10^{-5} \mathrm{GeV}^{-2}
$$

Other values of the relevant parameters will be collected in appendix A.

The interactions between the gauge bosons are standard and can be found in any textbook on gauge theories.

The primes in (II.2) indicate that the weak eigenstates $\left(d^{\prime}, s^{\prime}, b^{\prime}\right)$ are not equal to the corresponding mass eigenstates $(d, s, b)$, but are rather linear combinations of the latter. This is expressed through the relation

$$
\left(\begin{array}{c}
d^{\prime} \\
s^{\prime} \\
b^{\prime}
\end{array}\right)=\left(\begin{array}{lll}
V_{u d} & V_{u s} & V_{u b} \\
V_{c d} & V_{c s} & V_{c b} \\
V_{t d} & V_{t s} & V_{t b}
\end{array}\right)\left(\begin{array}{c}
d \\
s \\
b
\end{array}\right)
$$

where the unitary matrix connecting theses two sets of states is the Cabibbo-Kobayashi-Maskawa (CKM) matrix. Many parametrizations of this matrix have been proposed in the literature. We will use in this review two parametrizations: the standard parametrization recommended by the particle data group and the Wolfenstein parametrization.

\section{B. Standard Parametrization}

Let us introduce the notation $c_{i j}=\cos \theta_{i j}$ and $s_{i j}=\sin \theta_{i j}$ with $i$ and $j$ being generation labels $(i, j=1,2,3)$. The standard parametrization is then given as follows (Particle Data Group, 1994)

$$
V=\left(\begin{array}{ccc}
c_{12} c_{13} & s_{12} c_{13} & s_{13} e^{-i \delta} \\
-s_{12} c_{23}-c_{12} s_{23} s_{13} e^{i \delta} & c_{12} c_{23}-s_{12} s_{23} s_{13} e^{i \delta} & s_{23} c_{13} \\
s_{12} s_{23}-c_{12} c_{23} s_{13} e^{i \delta} & -s_{23} c_{12}-s_{12} c_{23} s_{13} e^{i \delta} & c_{23} c_{13}
\end{array}\right)
$$


where $\delta$ is the phase necessary for CP violation. $c_{i j}$ and $s_{i j}$ can all be chosen to be positive and $\delta$ may vary in the range $0 \leq \delta \leq 2 \pi$. However the measurements of $\mathrm{CP}$ violation in $\mathrm{K}$ decays force $\delta$ to be in the range $0<\delta<\pi$.

The extensive phenomenology of the last years has shown that $s_{13}$ and $s_{23}$ are small numbers: $\mathcal{O}\left(10^{-3}\right)$ and $\mathcal{O}\left(10^{-2}\right)$, respectively. Consequently to an excellent accuracy $c_{13}=c_{23}=1$ and the four independent parameters are given as follows

$$
s_{12}=\left|V_{u s}\right|, \quad s_{13}=\left|V_{u b}\right|, \quad s_{23}=\left|V_{c b}\right|, \quad \delta
$$

with the phase $\delta$ extracted from $\mathrm{CP}$ violating transitions or loop processes sensitive to $\left|V_{t d}\right|$. The latter fact is based on the observation that for $0 \leq \delta \leq \pi$, as required by the analysis of CP violation, there is a one-to-one correspondence between $\delta$ and $\left|V_{t d}\right|$ given by

$$
\left|V_{t d}\right|=\sqrt{a^{2}+b^{2}-2 a b \cos \delta}, \quad a=\left|V_{c d} V_{c b}\right|, \quad b=\left|V_{u d} V_{u b}\right|
$$

\section{Wolfenstein Parameterization Beyond Leading Order}

We will also use the Wolfenstein parametrization (Wolfenstein, 1983). It is an approximate parametrization of the CKM matrix in which each element is expanded as a power series in the small parameter $\lambda=\left|V_{u s}\right|=0.22$

$$
V=\left(\begin{array}{ccc}
1-\frac{\lambda^{2}}{2} & \lambda & A \lambda^{3}(\varrho-i \eta) \\
-\lambda & 1-\frac{\lambda^{2}}{2} & A \lambda^{2} \\
A \lambda^{3}(1-\varrho-i \eta) & -A \lambda^{2} & 1
\end{array}\right)+\mathcal{O}\left(\lambda^{4}\right)
$$

and the set (II.14) is replaced by

$$
\lambda, \quad A, \quad \varrho, \quad \eta \text {. }
$$

The Wolfenstein parameterization has several nice features. In particular it offers in conjunction with the unitarity triangle a very transparent geometrical representation of the structure of the CKM matrix and allows to derive several analytic results to be discussed below. This turns out to be very useful in the phenomenology of rare decays and of $\mathrm{CP}$ violation.

When using the Wolfenstein parametrization one should remember that it is an approximation and that in certain situations neglecting $\mathcal{O}\left(\lambda^{4}\right)$ terms may give wrong results. The question then arises how to find $\mathcal{O}\left(\lambda^{4}\right)$ and higher order terms ? The point is that since (II.16) is only an approximation the exact definiton of $\lambda$ is not unique by terms of the neglected order $\mathcal{O}\left(\lambda^{4}\right)$. This is the reason why in different papers in the literature different $\mathcal{O}\left(\lambda^{4}\right)$ terms can be found. They simply correspond to different definitons of the expansion parameter $\lambda$. Obviously the physics does not depend on this choice. Here it suffices to find an expansion in $\lambda$ which allows for simple relations between the parameters (II.14) and (II.17). This will also restore the unitarity of the CKM matrix which in the Wolfenstein parametrization as given in (II.16) is not satisfied exactly.

To this end we go back to (II.13) and we impose the relations (Buras et al., 1994b)

$$
s_{12}=\lambda \quad s_{23}=A \lambda^{2} \quad s_{13} e^{-i \delta}=A \lambda^{3}(\varrho-i \eta)
$$

to all orders in $\lambda$. In view of the comments made above this can certainly be done. It follows that 


$$
\varrho=\frac{s_{13}}{s_{12} s_{23}} \cos \delta \quad \eta=\frac{s_{13}}{s_{12} s_{23}} \sin \delta
$$

We observe that (II.18) and (II.19) represent simply the change of variables from (II.14) to (II.17). Making this change of variables in the standard parametrization (II.13) we find the CKM matrix as a function of $(\lambda, A, \varrho, \eta)$ which satisfies unitarity exactly! We also note that in view of $c_{13}=1-$ $\mathcal{O}\left(\lambda^{6}\right)$ the relations between $s_{i j}$ and $\left|V_{i j}\right|$ in (II.14) are satisfied to high accuracy. The relations in (II.19) have been first used in (Schmidtler and Schubert, 1992). However, the improved treatment of the unitarity triangle presented below goes beyond the analysis of these authors.

The procedure outlined above gives automatically the corrections to the Wolfenstein parametrization in (II.16). Indeed expressing (II.13) in terms of Wolfenstein parameters using (II.18) and then expanding in powers of $\lambda$ we recover the matrix in (II.16) and in addition find explicit corrections of $\mathcal{O}\left(\lambda^{4}\right)$ and higher order terms. $V_{u b}$ remains unchanged. The corrections to $V_{u s}$ and $V_{c b}$ appear only at $\mathcal{O}\left(\lambda^{7}\right)$ and $\mathcal{O}\left(\lambda^{8}\right)$, respectively. For many practical purposes the corrections to the real parts can also be neglected. The essential corrections to the imaginary parts are:

$$
\Delta V_{c d}=-i A^{2} \lambda^{5} \eta \quad \Delta V_{t s}=-i A \lambda^{4} \eta
$$

These two corrections have to be taken into account in the discussion of CP violation. On the other hand the imaginary part of $V_{c s}$ which in our expansion in $\lambda$ appears only at $\mathcal{O}\left(\lambda^{6}\right)$ can be fully neglected.

In order to improve the accuracy of the unitarity triangle discussed below we will also include the $\mathcal{O}\left(\lambda^{5}\right)$ correction to $V_{t d}$ which gives

$$
V_{t d}=A \lambda^{3}(1-\bar{\varrho}-i \bar{\eta})
$$

with

$$
\bar{\varrho}=\varrho\left(1-\frac{\lambda^{2}}{2}\right) \quad \bar{\eta}=\eta\left(1-\frac{\lambda^{2}}{2}\right) .
$$

In order to derive analytic results we need accurate explicit expressions for $\lambda_{i}=V_{i d} V_{i s}^{*}$ where $i=c, t$. We have

$$
\begin{gathered}
\operatorname{Im} \lambda_{t}=-\operatorname{Im} \lambda_{c}=\eta A^{2} \lambda^{5} \\
\operatorname{Re} \lambda_{c}=-\lambda\left(1-\frac{\lambda^{2}}{2}\right) \\
\operatorname{Re} \lambda_{t}=-\left(1-\frac{\lambda^{2}}{2}\right) A^{2} \lambda^{5}(1-\bar{\varrho})
\end{gathered}
$$

Expressions (II.23) and (II.24) represent to an accuracy of $0.2 \%$ the exact formulae obtained using (II.13). The expression (II.25) deviates by at most $2 \%$ from the exact formula in the full range of parameters considered. In order to keep the analytic expressions in the phenomenological applications in a transparent form we have dropped a small $\mathcal{O}\left(\lambda^{7}\right)$ term in deriving (II.25). After inserting the expressions (II.23)-(II.25) in exact formulae for quantities of interest, further expansion in $\lambda$ should not be made. 


\section{Unitarity Triangle Beyond Leading Order}

The unitarity of the CKM matrix provides us with several relations of which

$$
V_{u d} V_{u b}^{*}+V_{c d} V_{c b}^{*}+V_{t d} V_{t b}^{*}=0
$$

is the most useful one. In the complex plane the relation (II.26) can be represented as a triangle, the so-called "unitarity-triangle" (UT). Phenomenologically this triangle is very interesting as it involves simultaneously the elements $V_{u b}, V_{c b}$ and $V_{t d}$ which are under extensive discussion at present.

In the usual analyses of the unitarity triangle only terms $\mathcal{O}\left(\lambda^{3}\right)$ are kept in (II.26) (Buras and Harlander, 1992), (Nir, 1992), (Harris and Rosner, 1992), (Schmidtler and Schubert, 1992), (Dib et al., 1990), (Ali and London, 1995). It is however straightforward to include the next-to-leading $\mathcal{O}\left(\lambda^{5}\right)$ terms (Buras et al., 1994b). We note first that

$$
V_{c d} V_{c b}^{*}=-A \lambda^{3}+\mathcal{O}\left(\lambda^{7}\right) .
$$

Thus to an excellent accuracy $V_{c d} V_{c b}^{*}$ is real with $\left|V_{c d} V_{c b}^{*}\right|=A \lambda^{3}$. Keeping $\mathcal{O}\left(\lambda^{5}\right)$ corrections and rescaling all terms in (II.26) by $A \lambda^{3}$ we find

$$
\frac{1}{A \lambda^{3}} V_{u d} V_{u b}^{*}=\bar{\varrho}+i \bar{\eta} \quad, \quad \frac{1}{A \lambda^{3}} V_{t d} V_{t b}^{*}=1-(\bar{\varrho}+i \bar{\eta})
$$

with $\bar{\varrho}$ and $\bar{\eta}$ defined in (II.22). Thus we can represent (II.26) as the unitarity triangle in the complex $(\bar{\varrho}, \bar{\eta})$ plane. This is shown in fig. 1. The length of the side $C B$ which lies on the real axis equals unity when eq. (II.26) is rescaled by $V_{c d} V_{c b}^{*}$. We observe that beyond the leading order in $\lambda$ the point A does not correspond to $(\varrho, \eta)$ but to $(\bar{\varrho}, \bar{\eta})$. Clearly within $3 \%$ accuracy $\bar{\varrho}=\varrho$ and $\bar{\eta}=\eta$. Yet in the distant future the accuracy of experimental results and theoretical calculations may improve considerably so that the more accurate formulation given here will be appropriate.

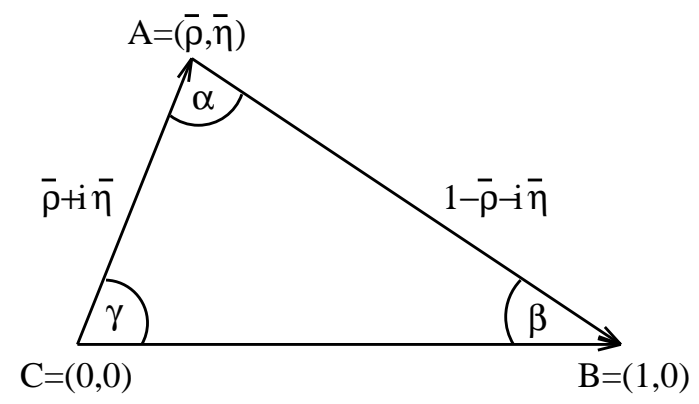

FIG. 1. Unitarity triangle in the complex $(\bar{\varrho}, \bar{\eta})$ plane.

Using simple trigonometry one can calculate $\sin \left(2 \phi_{i}\right), \phi_{i}=\alpha, \beta, \gamma$, in terms of $(\bar{\varrho}, \bar{\eta})$ with the result:

$$
\begin{gathered}
\sin (2 \alpha)=\frac{2 \bar{\eta}\left(\bar{\eta}^{2}+\bar{\varrho}^{2}-\bar{\varrho}\right)}{\left(\bar{\varrho}^{2}+\bar{\eta}^{2}\right)\left((1-\bar{\varrho})^{2}+\bar{\eta}^{2}\right)} \\
\sin (2 \beta)=\frac{2 \bar{\eta}(1-\bar{\varrho})}{(1-\bar{\varrho})^{2}+\bar{\eta}^{2}}
\end{gathered}
$$




$$
\sin (2 \gamma)=\frac{2 \bar{\varrho} \bar{\eta}}{\varrho^{2}+\bar{\eta}^{2}}=\frac{2 \varrho \eta}{\varrho^{2}+\eta^{2}}
$$

The lengths $C A$ and $B A$ in the rescaled triangle of fig. 1 to be denoted by $R_{b}$ and $R_{t}$, respectively, are given by

$$
\begin{gathered}
R_{b} \equiv \frac{\left|V_{u d} V_{u b}^{*}\right|}{\left|V_{c d} V_{c b}^{*}\right|}=\sqrt{\varrho^{2}+\bar{\eta}^{2}}=\left(1-\frac{\lambda^{2}}{2}\right) \frac{1}{\lambda}\left|\frac{V_{u b}}{V_{c b}}\right| \\
R_{t} \equiv \frac{\left|V_{t d} V_{t b}^{*}\right|}{\left|V_{c d} V_{c b}^{*}\right|}=\sqrt{(1-\bar{\varrho})^{2}+\bar{\eta}^{2}}=\frac{1}{\lambda}\left|\frac{V_{t d}}{V_{c b}}\right|
\end{gathered}
$$

The expressions for $R_{b}$ and $R_{t}$ given here in terms of $(\bar{\varrho}, \bar{\eta})$ are excellent approximations. Clearly $R_{b}$ and $R_{t}$ can also be determined by measuring two of the angles $\phi_{i}$ :

$$
\begin{aligned}
& R_{b}=\frac{\sin (\beta)}{\sin (\alpha)}=\frac{\sin (\alpha+\gamma)}{\sin (\alpha)}=\frac{\sin (\beta)}{\sin (\gamma+\beta)} \\
& R_{t}=\frac{\sin (\gamma)}{\sin (\alpha)}=\frac{\sin (\alpha+\beta)}{\sin (\alpha)}=\frac{\sin (\gamma)}{\sin (\gamma+\beta)}
\end{aligned}
$$




\section{BASIC FORMALISM}

\section{A. Renormalization of QCD}

As already emphasized in the introduction, the effects of QCD play an important role in the phenomenology of weak decays of hadrons. In fact in the theoretical analysis of these decays the investigation of QCD corrections is the most difficult and extensive part. In the present subsection we shall therefore briefly recall basic features of perturbative QCD and its renormalization. Thereby we will concentrate on those aspects, that will be needed for the present review. We will also take the opportunity to introduce for later reference the expressions for the running coupling, the running mass and the corresponding renormalization group functions.

The Lagrangian density of QCD reads

$$
\begin{aligned}
\mathcal{L}_{Q C D}= & -\frac{1}{4}\left(\partial_{\mu} A_{\nu}^{a}-\partial_{\nu} A_{\mu}^{a}\right)\left(\partial^{\mu} A^{a \nu}-\partial^{\nu} A^{a \mu}\right)-\frac{1}{2 \xi}\left(\partial^{\mu} A_{\mu}^{a}\right)^{2} \\
& +\bar{q}\left(i \not \partial-m_{q}\right) q+\chi^{a *} \partial^{\mu} \partial_{\mu} \chi^{a} \\
& -\frac{g}{2} f^{a b c}\left(\partial_{\mu} A_{\nu}^{a}-\partial_{\nu} A_{\mu}^{a}\right) A^{b \mu} A^{c \nu}-\frac{g^{2}}{4} f^{a b e} f^{c d e} A_{\mu}^{a} A_{\nu}^{b} A^{c \mu} A^{d \nu} \\
& +g \bar{q}_{i} T_{i j}^{a} \gamma^{\mu} q_{j} A_{\mu}^{a}+g f^{a b c}\left(\partial^{\mu} \chi^{a *}\right) \chi^{b} A_{\mu}^{c}
\end{aligned}
$$

Here $q=\left(q_{1}, q_{2}, q_{3}\right)$ is the color triplet of quark flavor $q, q=u, d, s, c, b, t . g$ is the QCD coupling, $A_{\mu}^{a}$ the gluon field, $\chi^{a}$ the ghost field and $\xi$ the gauge parameter. $T^{a}, f^{a b c}(a, b, c=1, \ldots, 8)$ are the generators and structure constants of $S U(3)$, respectively. From this Lagrangian one may read off the Feynman rules for QCD, e.g. $i g T_{i j}^{a} \gamma^{\mu}$ for the quark-gluon vertex.

In order to deal with divergences that appear in quantum (loop) corrections to Green functions, the theory has to be regularized to have an explicit parametrization of the singularities and subsequently renormalized to render the Green functions finite. For these purposes we will employ:

- Dimensional regularization (DR) by continuation to $D=4-2 \varepsilon$ space-time dimensions (Bollini and Giambiagi, 1972a), (Bollini and Giambiagi, 1972b), ('t Hooft and Veltman, 1972a), (Ashmore, 1972), (Cicuta and Montaldi, 1972).

- Subtraction of divergences in the minimal subtraction scheme $M S$ ('t Hooft, 1973) or the modified minimal subtraction scheme $(\overline{M S})$ (Bardeen et al., 1978).

To eliminate the divergences one has to renormalize the fields and parameters in the Lagrangian, in general through

$$
\begin{array}{lll}
A_{0 \mu}^{a}=Z_{3}^{1 / 2} A_{\mu}^{a} & q_{0}=Z_{q}^{1 / 2} q & \chi_{0}^{a}=\tilde{Z}_{3}^{1 / 2} \chi^{a} \\
g_{0}=Z_{g} g \mu^{\varepsilon} & \xi_{0}=Z_{3} \xi & m_{0}=Z_{m} m
\end{array}
$$

The index " 0 " indicates unrenormalized quantities. The factors $Z$ are the renormalization constants. The scale $\mu$ has been introduced to make $g$ dimensionless in $D=4-2 \varepsilon$ dimensions. Since we will not consider Green functions with external ghosts, we will not need the ghost field renormalization. We also do not need the gauge parameter renormalization if we are dealing with gauge independent quantities, as e.g. Wilson coefficient functions.

A straightforward way to implement renormalization is provided by the counterterm method. 
Thereby the parameters and fields in the original Lagrangian, which are to be considered as unrenormalized (bare) quantities, are reexpressed through renormalized ones by means of (III.2) from the very beginning. For instance, the quark kinetic term becomes

$$
\mathcal{L}_{F}=\bar{q}_{0} i \not \partial q_{0}-m_{0} \bar{q}_{0} q_{0} \equiv \bar{q} i \not \partial q-m \bar{q} q+\left(Z_{q}-1\right) \bar{q} i \not \partial q-\left(Z_{q} Z_{m}-1\right) m \bar{q} q
$$

The advantage then is, that only renormalized quantities are present in the Lagrangian. The counterterms $(\sim(Z-1))$, appearing in addition, can be formally treated as interaction terms that contribute to Green functions calculated in perturbation theory. The Feynman rule for the counterterms in (III.3), for example, reads ( $p$ is the quark momentum)

$$
i\left(Z_{q}-1\right) \not p-i\left(Z_{q} Z_{m}-1\right) m
$$

The constants $Z_{i}$ are then determined such that they cancel the divergences in the Green functions according to the chosen renormalization scheme. In an analogous way all renormalization constants can be fixed by considering the appropriate Green functions.

Of central importance for the study of perturbative QCD effects are the renormalization group equations, which govern the dependence of renormalized parameters and Green functions on the renormalization scale $\mu$. These differential equations are easily derived from the definition (III.2) by using the fact that bare quantities are $\mu$-independent. In this way one finds that the renormalized coupling $g(\mu)$ obeys (Gross, 1976)

$$
\frac{d}{d \ln \mu} g(\mu)=\beta(\varepsilon, g(\mu))
$$

where

$$
\beta(\varepsilon, g)=-\varepsilon g-g \frac{1}{Z_{g}} \frac{d Z_{g}}{d \ln \mu} \equiv-\varepsilon g+\beta(g)
$$

which defines the $\beta$ function. (III.5) is valid in arbitrary dimensions. In four dimensions $\beta(\varepsilon, g)$ reduces to $\beta(g)$. Similarly, the anomalous dimension of the mass, $\gamma_{m}$, defined through

$$
\frac{d m(\mu)}{d \ln \mu}=-\gamma_{m}(g) m(\mu)
$$

is given by

$$
\gamma_{m}(g)=\frac{1}{Z_{m}} \frac{d Z_{m}}{d \ln \mu}
$$

In the $M S(\overline{M S})$-scheme, where just the pole terms in $\varepsilon$ are present in the renormalization constants $Z_{i}$, these can be expanded as follows

$$
Z_{i}=1+\sum_{k=1}^{\infty} \frac{1}{\varepsilon^{k}} Z_{i, k}(g)
$$

Using (III.5), (III.6) one finds

$$
\frac{1}{Z_{i}} \frac{d Z_{i}}{d \ln \mu}=-2 g^{2} \frac{\partial Z_{i, 1}(g)}{\partial g^{2}}
$$


which allows a direct calculation of the renormalization group functions from the $1 / \varepsilon$-pole part of the renormalization constants. Along these lines one obtains at the two loop level, required for next-to-leading order calculations,

$$
\beta(g)=-\beta_{0} \frac{g^{3}}{16 \pi^{2}}-\beta_{1} \frac{g^{5}}{\left(16 \pi^{2}\right)^{2}}
$$

In terms of

$$
\alpha_{\mathrm{s}} \equiv \frac{g^{2}}{4 \pi}
$$

we have

$$
\frac{d \alpha_{\mathrm{s}}}{d \ln \mu}=-2 \beta_{0} \frac{\alpha_{\mathrm{s}}^{2}}{4 \pi}-2 \beta_{1} \frac{\alpha_{\mathrm{s}}^{3}}{(4 \pi)^{2}}
$$

Similarly, the two-loop expression for the quark mass anomalous dimension can be written as

$$
\gamma_{m}\left(\alpha_{\mathrm{s}}\right)=\gamma_{m 0} \frac{\alpha_{\mathrm{s}}}{4 \pi}+\gamma_{m 1}\left(\frac{\alpha_{\mathrm{s}}}{4 \pi}\right)^{2}
$$

We also give the $1 / \varepsilon$-pole part $Z_{q, 1}$ of the quark field renormalization constant $Z_{1}$ to $\mathcal{O}\left(\alpha_{\mathrm{s}}^{2}\right)$, which we will need later on

$$
Z_{q, 1}=a_{1} \frac{\alpha_{\mathrm{s}}}{4 \pi}+a_{2}\left(\frac{\alpha_{\mathrm{s}}}{4 \pi}\right)^{2}
$$

The coefficients in eqs. (III.13) - (III.15) read

$$
\begin{gathered}
\beta_{0}=\frac{11 N-2 f}{3} \quad \beta_{1}=\frac{34}{3} N^{2}-\frac{10}{3} N f-2 C_{F} f \quad C_{F}=\frac{N^{2}-1}{2 N} \\
\gamma_{m 0}=6 C_{F} \quad \gamma_{m 1}=C_{F}\left(3 C_{F}+\frac{97}{3} N-\frac{10}{3} f\right) \\
a_{1}=-C_{F} \quad a_{2}=C_{F}\left(\frac{3}{4} C_{F}-\frac{17}{4} N+\frac{1}{2} f\right)
\end{gathered}
$$

$N$ is the number of colors, $f$ the number of quark flavors. The coefficients are given in the $M S$ $(\overline{M S})$ scheme. However, $\beta_{0}, \beta_{1}, \gamma_{m 0}$ and $a_{1}$ are scheme independent. The expressions for $a_{1}$ and $a_{2}$ in (III.18) are valid in Feynman gauge, $\xi=1$.

At two-loop order the solution of the renormalization group equation (III.13) for $\alpha_{\mathrm{s}}(\mu)$ can always be written in the form

$$
\alpha_{\mathrm{s}}(\mu)=\frac{4 \pi}{\beta_{0} \ln \frac{\mu^{2}}{\Lambda^{2}}}\left[1-\frac{\beta_{1}}{\beta_{0}^{2}} \frac{\ln \ln \frac{\mu^{2}}{\Lambda^{2}}}{\ln \frac{\mu^{2}}{\Lambda^{2}}}\right]
$$

Eq. (III.19) gives the running coupling constant at NLO. $\alpha_{\mathrm{s}}(\mu)$ vanishes as $\mu / \Lambda \rightarrow \infty$ due to asymptotic freedom. We remark that, in accordance with the two-loop accuracy, (III.19) is valid 
up to terms of the order $\mathcal{O}\left(1 / \ln ^{3} \mu^{2} / \Lambda^{2}\right)$. For the purpose of counting orders in $1 / \ln \mu^{2} / \Lambda^{2}$ the double logarithmic expression $\ln \ln \mu^{2} / \Lambda^{2}$ may formally be viewed as a constant. Note that an additional term const. $/ \ln ^{2} \mu^{2} / \Lambda^{2}$, which is of the same order as the next-to-leading correction term in (III.19), can always be absorbed into a multiplicative redefinition of $\Lambda$. Hence the choice of the form (III.19) is possible without restriction, but one should keep in mind that the definition of $\Lambda$ is related to this particular choice. The introduction of the $\overline{M S}$ scheme and the corresponding definition of $\Lambda_{\overline{M S}}$ and its relation to $\Lambda_{M S}$ is discussed in section III F 4 .

Finally we write down the two-loop expression for the running quark mass in the $M S(\overline{M S})$ scheme, which results from integrating (III.7)

$$
m(\mu)=m(m)\left[\frac{\alpha_{\mathrm{s}}(\mu)}{\alpha_{\mathrm{s}}(m)}\right]^{\frac{\gamma_{m 0}}{2 \beta_{0}}}\left[1+\left(\frac{\gamma_{m 1}}{2 \beta_{0}}-\frac{\beta_{1} \gamma_{m 0}}{2 \beta_{0}^{2}}\right) \frac{\alpha_{\mathrm{s}}(\mu)-\alpha_{\mathrm{s}}(m)}{4 \pi}\right]
$$

\section{B. Operator Product Expansion in Weak Decays - Preliminaries}

Weak decays of hadrons are mediated through the weak interactions of their quark constituents, whose strong interactions, binding the constituents into hadrons, are characterized by a typical hadronic energy scale of the order of $1 \mathrm{GeV}$. Our goal is therefore to derive an effective low energy theory describing the weak interactions of quarks. The formal framework to achieve this is provided by the operator product expansion (OPE). In order to introduce the main ideas behind it, let us consider the simple example of the quark level transition $c \rightarrow s u \bar{d}$, which is relevant for Cabibbo-allowed decays of D mesons. Disregarding QCD effects for the moment, the tree-level W-exchange amplitude for $c \rightarrow s u \bar{d}$ is simply given by

$$
\begin{aligned}
A & =i \frac{G_{F}}{\sqrt{2}} V_{c s}^{*} V_{u d} \frac{M_{W}^{2}}{k^{2}-M_{W}^{2}}(\bar{s} c)_{V-A}(\bar{u} d)_{V-A} \\
& =-i \frac{G_{F}}{\sqrt{2}} V_{c s}^{*} V_{u d}(\bar{s} c)_{V-A}(\bar{u} d)_{V-A}+\mathcal{O}\left(\frac{k^{2}}{M_{W}^{2}}\right)
\end{aligned}
$$

where $(V-A)$ refers to the Lorentz structure $\gamma_{\mu}\left(1-\gamma_{5}\right)$.

Since $k$, the momentum transfer through the $\mathrm{W}$ propagator, is very small as compared to the W mass $M_{W}$, terms of the order $\mathcal{O}\left(k^{2} / M_{W}^{2}\right)$ can safely be neglected and the full amplitude $A$ can be approximated by the first term on the r.h.s. of (III.21). Now this term may obviously be also obtained from an effective hamiltonian defined by

$$
\mathcal{H}_{e f f}=\frac{G_{F}}{\sqrt{2}} V_{c s}^{*} V_{u d}(\bar{s} c)_{V-A}(\bar{u} d)_{V-A}+\ldots
$$

where the ellipsis denotes operators of higher dimensions, typically involving derivative terms, which can in principle be chosen so as to reproduce the terms of higher order in $k^{2} / M_{W}^{2}$ of the full amplitude in (III.21). This exercise already provides us with a simple example of an OPE. The product of two charged current operators is expanded into a series of local operators, whose contributions are weighted by effective coupling constants, the Wilson coefficients.

A more formal basis for this procedure may be given by considering the generating functional for Green functions in the path integral formalism. The part of the generating functional relevant for the present discussion is, up to an overall normalizing factor, given by 


$$
Z_{W} \sim \int\left[d W^{+}\right]\left[d W^{-}\right] \exp \left(i \int d^{4} x \mathcal{L}_{W}\right)
$$

where $\mathcal{L}_{W}$ is the Lagrangian density containing the kinetic terms of the $\mathrm{W}$ boson field and its interaction with charged currents

$$
\begin{array}{r}
\mathcal{L}_{W}=-\frac{1}{2}\left(\partial_{\mu} W_{\nu}^{+}-\partial_{\nu} W_{\mu}^{+}\right)\left(\partial^{\mu} W^{-\nu}-\partial^{\nu} W^{-\mu}\right)+M_{W}^{2} W_{\mu}^{+} W^{-\mu} \\
+\frac{g_{2}}{2 \sqrt{2}}\left(J_{\mu}^{+} W^{+\mu}+J_{\mu}^{-} W^{-\mu}\right) \\
J_{\mu}^{+}=V_{p n} \bar{p} \gamma_{\mu}\left(1-\gamma_{5}\right) n \quad p=(u, c, t) \quad n=(d, s, b) \quad J_{\mu}^{-}=\left(J_{\mu}^{+}\right)^{\dagger}
\end{array}
$$

Since we are not interested in Green functions with external W lines, we have not introduced external source terms for the $\mathrm{W}$ fields. In the present argument we will furthermore choose the unitary gauge for the $\mathrm{W}$ field for definiteness, however physical results do not depend on this choice.

Introducing the operator

$$
K_{\mu \nu}(x, y)=\delta^{(4)}(x-y)\left(g_{\mu \nu}\left(\partial^{2}+M_{W}^{2}\right)-\partial_{\mu} \partial_{\nu}\right)
$$

we may, after discarding a total derivative in the W kinetic term, rewrite (III.23) as

$$
\begin{gathered}
Z_{W} \sim \int\left[d W^{+}\right]\left[d W^{-}\right] \exp \left[i \int d^{4} x d^{4} y W_{\mu}^{+}(x) K^{\mu \nu}(x, y) W_{\nu}^{-}(y)\right. \\
\left.+i \frac{g_{2}}{2 \sqrt{2}} \int d^{4} x J_{\mu}^{+} W^{+\mu}+J_{\mu}^{-} W^{-\mu}\right]
\end{gathered}
$$

The inverse of $K_{\mu \nu}$, denoted by $\Delta_{\mu \nu}$, and defined through

$$
\int d^{4} y K_{\mu \nu}(x, y) \Delta^{\nu \lambda}(y, z)=g_{\mu}^{\lambda} \delta^{(4)}(x-z)
$$

is just the $\mathrm{W}$ propagator in the unitary gauge

$$
\begin{gathered}
\Delta_{\mu \nu}(x, y)=\int \frac{d^{4} k}{(2 \pi)^{4}} \Delta_{\mu \nu}(k) e^{-i k(x-y)} \\
\Delta_{\mu \nu}(k)=\frac{-1}{k^{2}-M_{W}^{2}}\left(g_{\mu \nu}-\frac{k_{\mu} k_{\nu}}{M_{W}^{2}}\right)
\end{gathered}
$$

Performing the gaussian functional integration over $W^{ \pm}(x)$ in (III.27) explicitly, this expression simplifies to

$$
Z_{W} \sim \exp \left[-i \int \frac{g_{2}^{2}}{8} J_{\mu}^{-}(x) \Delta^{\mu \nu}(x, y) J_{\nu}^{+}(y) d^{4} x d^{4} y\right]
$$

This result implies a nonlocal action functional for the quarks 


$$
\mathcal{S}_{n l}=\int d^{4} x \mathcal{L}_{k i n}-\frac{g_{2}^{2}}{8} \int d^{4} x d^{4} y J_{\mu}^{-}(x) \Delta^{\mu \nu}(x, y) J_{\nu}^{+}(y)
$$

where the first piece represents the quark kinetic terms and the second their charged current interactions.

We can now formally expand this second, nonlocal term in powers of $1 / M_{W}^{2}$ to yield a series of local interaction operators of dimensions that increase with the order in $1 / M_{W}^{2}$. To lowest order

$$
\Delta^{\mu \nu}(x, y) \approx \frac{g^{\mu \nu}}{M_{W}^{2}} \delta^{(4)}(x-y)
$$

and the second term in (III.32) becomes

$$
-\frac{g_{2}^{2}}{8 M_{W}^{2}} \int d^{4} x J_{\mu}^{-}(x) J^{+\mu}(x)
$$

corresponding to the usual effective charged current interaction Lagrangian

$$
\mathcal{L}_{\text {int }, e f f}=-\frac{G_{F}}{\sqrt{2}} J_{\mu}^{-} J^{+\mu}(x)=-\frac{G_{F}}{\sqrt{2}} V_{p n}^{*} V_{p^{\prime} n^{\prime}}(\bar{n} p)_{V-A}\left(\bar{p}^{\prime} n^{\prime}\right)_{V-A}
$$

which contains, among other terms, the leading contribution to (III.22).

The simple considerations we have presented so far already illustrate several of the basic aspects of the general approach.

- Formally, the procedure to approximate the interaction term in (III.32) by (III.34) is an example of a short-distance OPE. The product of the local operators $J_{\mu}^{-}(x)$ and $J_{\nu}^{+}(y)$, to be taken at short-distances due to the convolution with the massive, short-range $\mathrm{W}$ propagator $\Delta^{\mu \nu}(x, y)$ (compare (III.33)), is expanded into a series of composite local operators, of which the leading term is shown in (III.34).

- The dominant contributions in the short-distance expansion come from the operators of lowest dimension. In our case these are four-fermion operators of dimension six, whereas operators of higher dimensions can usually be neglected in weak decays.

- Note that, as far as the charged current weak interaction is concerned, no approximation is involved yet in the nonlocal interaction term in (III.32), except that we do not consider higher order weak corrections or processes with external W boson states. Correspondingly, the OPE series into which the nonlocal interaction is expanded, is equivalent to the original theory, when considered to all orders in $1 / M_{W}^{2}$. In other words, the full series will reproduce the complete Green functions for the charged current weak interactions of quarks. The truncation of the operator series then yields a systematic approximation scheme for low energy processes, neglecting contributions suppressed by powers of $k^{2} / M_{W}^{2}$. In this way one is able to construct low energy effective theories for weak decays.

- In going from the full to the effective theory the $\mathrm{W}$ boson is removed as an explicit, dynamical degree of freedom. This step is often refered to as "integrating out" the W boson, a terminology which is very obvious in the path integral language discussed above. Alternatively one could of course use the canonical operator formalism, where the W field instead of being intergrated out, gets "contracted out" through the application of Wick's theorem. 
- The effective local four-fermion interaction terms are a modern version of the classic Fermitheory of weak interactions.

- An intuitive interpretation of the OPE formalism discussed so far is, that from the point of view of low energy dynamics, the effects of a short-range exchange force mediated by a heavy boson approximately corresponds to a point interaction.

- The presentation we have given illustrates furthermore, that the approach of evaluating the relevant Green functions (or amplitudes) directly in order to construct the OPE, as in (III.21), actually gives the same result as the more formal technique employing path integrals. While the latter can give some useful insight into the general aspects of the method, the former is more convenient for practical calculations and we will make use of it throughout the discussion to follow.

- Up to now we have not talked about the strong interactions among quarks, which have of course to be taken into account. They are described by QCD and can at short-distances be calculated in perturbation theory, due to the property of asymptotic freedom of QCD. The corresponding gluon exchange contributions constitute quantum corrections to the simplified picture sketched above, which can in this sense be viewed as a classical approximation. We will describe the incorporation of QCD corrections and related additional features they imply for the OPE in the following section.

\section{OPE and Short Distance QCD Effects}

We will now take up the discussion of QCD quantum corrections at short-distances to the OPE for weak decays. A crucial point for this enterprise is the property of asymptotic freedom of QCD. This allows one to treat the short-distance corrections, that is the contribution of hard gluons at energies of the order $\mathcal{O}\left(M_{W}\right)$ down to hadronic scales $\geq 1 \mathrm{GeV}$, in perturbation theory. In the following, we will always restrict ourselves to the leading dimension six operators in the OPE and omit the negligible contributions of higher dimensional operators. Staying with our example of $c \rightarrow s u \bar{d}$ transitions, recall that we had for the amplitude without QCD

$$
A_{0}=-i \frac{G_{F}}{\sqrt{2}} V_{c s}^{*} V_{u d}\left(\bar{s}_{i} c_{i}\right)_{V-A}\left(\bar{u}_{j} d_{j}\right)_{V-A}
$$

where the summation over repeated color indices is understood. This result leads directly to the effective hamiltonian of (III.22) where the color indices have been suppressed. If we now include QCD effects, the effective hamiltonian, constructed to reproduce the low energy approximation of the exact theory, is generalized to

$$
\mathcal{H}_{e f f}=\frac{G_{F}}{\sqrt{2}} V_{c s}^{*} V_{u d}\left(C_{1} Q_{1}+C_{2} Q_{2}\right)
$$

where

$$
\begin{aligned}
Q_{1} & =\left(\bar{s}_{i} c_{j}\right)_{V-A}\left(\bar{u}_{j} d_{i}\right)_{V-A} \\
Q_{2} & =\left(\bar{s}_{i} c_{i}\right)_{V-A}\left(\bar{u}_{j} d_{j}\right)_{V-A}
\end{aligned}
$$

The essential features of this hamiltonian are: 
- In addition to the original operator $Q_{2}$ (with index 2 for historical reasons) a new operator $Q_{1}$ with the same flavor form but different color structure is generated. This is because a gluon linking the two color singlet weak current lines can "mix" the color indices due to the following relation for the color charges $T_{i j}^{a}$

$$
T_{i k}^{a} T_{j l}^{a}=-\frac{1}{2 N} \delta_{i k} \delta_{j l}+\frac{1}{2} \delta_{i l} \delta_{j k}
$$

- The Wilson coefficients $C_{1}$ and $C_{2}$, the coupling constants for the interaction terms $Q_{1}$ and $Q_{2}$, become calculable nontrivial functions of $\alpha_{\mathrm{s}}, M_{W}$ and the renormalization scale $\mu$. If QCD is neglected they have the trivial form $C_{1}=0, C_{2}=1$ and (III.37) reduces to (III.22).

In order to obtain the final result for the hamiltonian (III.37), we have to calculate the coefficients $C_{1,2}$. These are determined by the requirement that the amplitude $A$ in the full theory be reproduced by the corresponding amplitude in the effective theory (III.37), thus

$$
A=-i \frac{G_{F}}{\sqrt{2}} V_{c s}^{*} V_{u d}\left(C_{1}\left\langle Q_{1}\right\rangle+C_{2}\left\langle Q_{2}\right\rangle\right)
$$

If we calculate the amplitude $A$ and, to the same order in $\alpha_{\mathrm{s}}$, the matrix elements of operators $\left\langle Q_{1}\right\rangle$, $\left\langle Q_{2}\right\rangle$, we can obtain $C_{1}$ and $C_{2}$ via (III.41). This procedure is called matching the full theory onto the effective theory (III.37).

Here we use the term "amplitude" in the meaning of "amputated Green function". Correspondingly operator matrix elements are - within this perturbative context - amputated Green functions with operator insertion. In a diagrammatic language these amputated Green functions are given by Feynman graphs, but without gluonic self energy corrections in external legs, like e.g. in figs. 2 and 3 for the full and effective theory, respectively. In the present example penguin diagrams do not contribute due to the flavor structure of the $c \rightarrow s u \bar{d}$ transition.

Evaluating the current-current diagrams of fig. 2 (a)-(c), we find for the full amplitude $A$ to $\mathcal{O}\left(\alpha_{\mathrm{s}}\right)$

$$
A=-i \frac{G_{F}}{\sqrt{2}} V_{c s}^{*} V_{u d}\left[\left(1+2 C_{F} \frac{\alpha_{\mathrm{s}}}{4 \pi} \ln \frac{\mu^{2}}{-p^{2}}\right) S_{2}+\frac{3}{N} \frac{\alpha_{\mathrm{s}}}{4 \pi} \ln \frac{M_{W}^{2}}{-p^{2}} S_{2}-3 \frac{\alpha_{\mathrm{s}}}{4 \pi} \ln \frac{M_{W}^{2}}{-p^{2}} S_{1}\right]
$$

Here we have introduced the spinor amplitudes

$$
\begin{aligned}
& S_{1}=\left(\bar{s}_{i} c_{j}\right)_{V-A}\left(\bar{u}_{j} d_{i}\right)_{V-A} \\
& S_{2}=\left(\bar{s}_{i} c_{i}\right)_{V-A}\left(\bar{u}_{j} d_{j}\right)_{V-A}
\end{aligned}
$$

which are just the tree level matrix elements of $Q_{1}$ and $Q_{2}$. We have employed the Feynman gauge $(\xi=1)$ and taken all external quark lines massless and carrying the off-shell momentum $p$. Furthermore we have kept only logarithmic corrections $\sim \alpha_{\mathrm{s}} \cdot \log$ and discarded constant contributions of order $\mathcal{O}\left(\alpha_{\mathrm{s}}\right)$, which corresponds to the leading log approximation. The necessary renormalization of the quark fields in the $M S$-scheme is already incorporated into (III.42). It has removed a $1 / \varepsilon$ singularity in the first term of (III.42), which therefore carries an explicit $\mu$ dependence. 


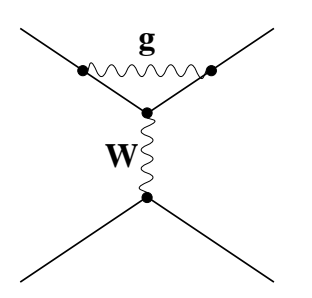

(a)

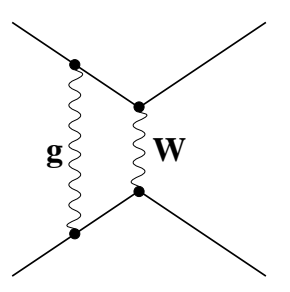

(b)

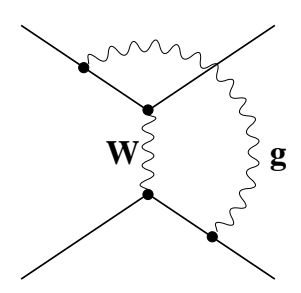

(c)

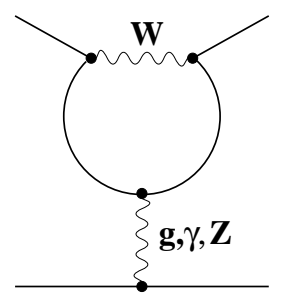

(d)

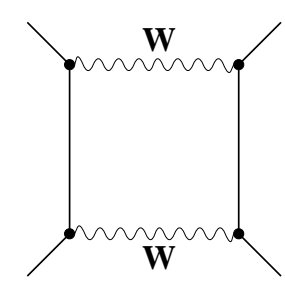

(e)

FIG. 2. One-loop current-current (a)-(c), penguin (d) and box (e) diagrams in the full theory. For pure QCD corrections as considered in this section and e.g. in VI the $\gamma$ - and $Z$-contributions in diagram (d) and the diagram (e) are absent. Possible left-right or up-down reflected diagrams are not shown.

Under the same conditions, the unrenormalized current-current matrix elements of the operators $Q_{1}$ and $Q_{2}$ are from fig. 3 (a)-(c) found to be

$$
\begin{aligned}
& \left\langle Q_{1}\right\rangle^{(0)}= \\
& \left(1+2 C_{F} \frac{\alpha_{\mathrm{s}}}{4 \pi}\left(\frac{1}{\varepsilon}+\ln \frac{\mu^{2}}{-p^{2}}\right)\right) S_{1}+\frac{3}{N} \frac{\alpha_{\mathrm{s}}}{4 \pi}\left(\frac{1}{\varepsilon}+\ln \frac{\mu^{2}}{-p^{2}}\right) S_{1}-3 \frac{\alpha_{\mathrm{s}}}{4 \pi}\left(\frac{1}{\varepsilon}+\ln \frac{\mu^{2}}{-p^{2}}\right) S_{2} \\
& \left\langle Q_{2}\right\rangle^{(0)}= \\
& \left(1+2 C_{F} \frac{\alpha_{\mathrm{s}}}{4 \pi}\left(\frac{1}{\varepsilon}+\ln \frac{\mu^{2}}{-p^{2}}\right)\right) S_{2}+\frac{3}{N} \frac{\alpha_{\mathrm{s}}}{4 \pi}\left(\frac{1}{\varepsilon}+\ln \frac{\mu^{2}}{-p^{2}}\right) S_{2}-3 \frac{\alpha_{\mathrm{s}}}{4 \pi}\left(\frac{1}{\varepsilon}+\ln \frac{\mu^{2}}{-p^{2}}\right) S_{1}
\end{aligned}
$$

Again, the divergences in the first terms are eliminated through field renormalization. However, in contrast to the full amplitude, the resulting expressions are still divergent. Therefore an additional multiplicative renormalization, refered to as operator renormalization, is necessary:

$$
Q_{i}^{(0)}=Z_{i j} Q_{j}
$$

Since (III.45) and (III.46) each involve both $S_{1}$ and $S_{2}$, the renormalization constant is in this case a $2 \times 2$ matrix $Z$. The relation between the unrenormalized $\left(\left\langle Q_{i}\right\rangle^{(0)}\right)$ and the renormalized amputated Green functions $\left(\left\langle Q_{i}\right\rangle\right)$ is then

$$
\left\langle Q_{i}\right\rangle^{(0)}=Z_{q}^{-2} Z_{i j}\left\langle Q_{j}\right\rangle
$$

From (III.45), (III.46) and (III.15) we read off ( $M S$-scheme)

$$
Z=1+\frac{\alpha_{\mathrm{s}}}{4 \pi} \frac{1}{\varepsilon}\left(\begin{array}{cc}
3 / N & -3 \\
-3 & 3 / N
\end{array}\right)
$$




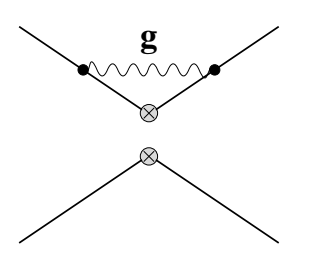

(a)

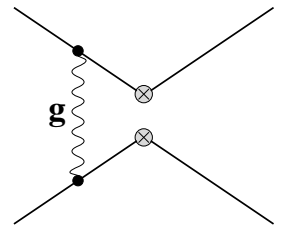

(b)

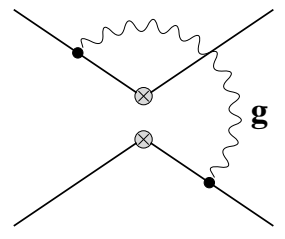

(c)

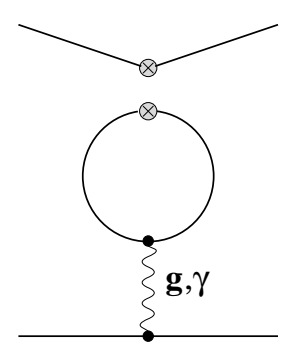

(d.1)

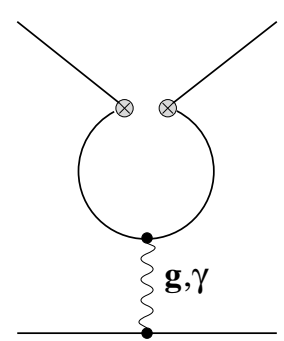

(d.2)

FIG. 3. One loop current-current (a)-(c) and penguin (d) diagrams contributing to the LO anomalous dimensions and matching conditions in the effective theory. The 4-vertex " $\otimes \otimes$ " denotes the insertion of a 4-fermion operator $Q_{i}$. For pure QCD corrections as considered in this section and e.g. in VI the contributions from $\gamma$ in diagrams (d.1) and (d.2) are absent. Again, possible left-right or up-down reflected diagrams are not shown.

It follows that the renormalized matrix elements $\left\langle Q_{i}\right\rangle$ are given by

$$
\begin{aligned}
& \left\langle Q_{1}\right\rangle=\left(1+2 C_{F} \frac{\alpha_{\mathrm{s}}}{4 \pi} \ln \frac{\mu^{2}}{-p^{2}}\right) S_{1}+\frac{3}{N} \frac{\alpha_{\mathrm{s}}}{4 \pi} \ln \frac{\mu^{2}}{-p^{2}} S_{1}-3 \frac{\alpha_{\mathrm{s}}}{4 \pi} \ln \frac{\mu^{2}}{-p^{2}} S_{2} \\
& \left\langle Q_{2}\right\rangle=\left(1+2 C_{F} \frac{\alpha_{\mathrm{s}}}{4 \pi} \ln \frac{\mu^{2}}{-p^{2}}\right) S_{2}+\frac{3}{N} \frac{\alpha_{\mathrm{s}}}{4 \pi} \ln \frac{\mu^{2}}{-p^{2}} S_{2}-3 \frac{\alpha_{\mathrm{s}}}{4 \pi} \ln \frac{\mu^{2}}{-p^{2}} S_{1}
\end{aligned}
$$

Inserting $\left\langle Q_{i}\right\rangle$ into (III.41) and comparing with (III.42) we derive

$$
C_{1}=-3 \frac{\alpha_{\mathrm{s}}}{4 \pi} \ln \frac{M_{W}^{2}}{\mu^{2}} \quad C_{2}=1+\frac{3}{N} \frac{\alpha_{\mathrm{s}}}{4 \pi} \ln \frac{M_{W}^{2}}{\mu^{2}}
$$

We would like to digress and add a comment on the renormalization of the interaction terms in the effective theory. The commonly used convention is to introduce via (III.48) the renormalization constants $Z_{i j}$, defined to absorb the divergences of the operator matrix elements. It is however instructive to view this renormalization in a slightly different, but of course equivalent way, corresponding to the standard counterterm method in perturbative renormalization. Consider, as usual, the hamiltonian of the effective theory as the starting point with fields and coupling constants as bare quantities, which are renormalized according to $(q=s, c, u, d)$

$$
\begin{gathered}
q^{(0)}=Z_{q}^{1 / 2} q \\
C_{i}^{(0)}=Z_{i j}^{c} C_{j}
\end{gathered}
$$


Then the hamiltonian (III.37) is essentially (omitting the factor $\frac{G_{F}}{\sqrt{2}} V_{c s}^{*} V_{u d}$ )

$$
C_{i}^{(0)} Q_{i}\left(q^{(0)}\right) \equiv Z_{q}^{2} Z_{i j}^{c} C_{j} Q_{i} \equiv C_{i} Q_{i}+\left(Z_{q}^{2} Z_{i j}^{c}-\delta_{i j}\right) C_{j} Q_{i}
$$

that is, it can be written in terms of renormalized couplings and fields $\left(C_{i} Q_{i}\right)$, plus counterterms. The argument $q^{(0)}$ in the first term in (III.55) indicates that the interaction term $Q_{i}$ is composed of bare fields. Calculating the amplitude with the hamiltonian (III.55), which includes the counterterms, we get the finite renormalized result

$$
Z_{q}^{2} Z_{i j}^{c} C_{j}\left\langle Q_{i}\right\rangle^{(0)}=C_{j}\left\langle Q_{j}\right\rangle
$$

Hence (compare (III.48))

$$
Z_{i j}^{c}=Z_{j i}^{-1}
$$

In short, it is sometimes useful to keep in mind that one can think of the "operator renormalization", which sounds like a new concept, in terms of the completely equivalent, but customary, renormalization of the coupling constants $C_{i}$, as in any field theory.

Now that we have presented in quite some detail the derivation of the Wilson coefficients in (III.52), we shall discuss and interpret the most important aspects of the short-distance expansion for weak decays, which can be studied very transparently on the explicit example we have given.

- First of all a further remark about the phenomenon of operator mixing that we encountered in our example. This occurs because gluonic corrections to the matrix element of the original operator $Q_{2}$ are not just proportional to $Q_{2}$ itself, but involve the additional structure $Q_{1}$ (and vice versa). Therefore, besides a $Q_{2}$-counterterm, a counterterm $\sim Q_{1}$ is needed to renormalize this matrix element - the operators in question are said to mix under renormalization. This however is nothing new in principle. It is just an algebraic generalization of the usual concepts. Indeed, if we introduce a different operator basis $Q_{ \pm}=\left(Q_{2} \pm Q_{1}\right) / 2$ (with coefficients $C_{ \pm}=C_{2} \pm C_{1}$ ) the renormalization becomes diagonal and matrix elements of $Q_{+}$and $Q_{-}$are renormalized multiplicatively. In this new basis the OPE reads

$$
A \equiv A_{+}+A_{-}=-i \frac{G_{F}}{\sqrt{2}} V_{c s}^{*} V_{u d}\left(C_{+}\left\langle Q_{+}\right\rangle+C_{-}\left\langle Q_{-}\right\rangle\right)
$$

where $\left(S_{ \pm}=\left(S_{2} \pm S_{1}\right) / 2\right)$

$$
A_{ \pm}=-i \frac{G_{F}}{\sqrt{2}} V_{c s}^{*} V_{u d}\left[\left(1+2 C_{F} \frac{\alpha_{\mathrm{s}}}{4 \pi} \ln \frac{\mu^{2}}{-p^{2}}\right) S_{ \pm}+\left(\frac{3}{N} \mp 3\right) \frac{\alpha_{\mathrm{s}}}{4 \pi} \ln \frac{M_{W}^{2}}{-p^{2}} S_{ \pm}\right]
$$

and

$$
\begin{gathered}
\left\langle Q_{ \pm}\right\rangle=\left(1+2 C_{F} \frac{\alpha_{\mathrm{s}}}{4 \pi} \ln \frac{\mu^{2}}{-p^{2}}\right) S_{ \pm}+\left(\frac{3}{N} \mp 3\right) \frac{\alpha_{\mathrm{s}}}{4 \pi} \ln \frac{\mu^{2}}{-p^{2}} S_{ \pm} \\
C_{ \pm}=1+\left(\frac{3}{N} \mp 3\right) \frac{\alpha_{\mathrm{s}}}{4 \pi} \ln \frac{M_{W}^{2}}{\mu^{2}}
\end{gathered}
$$


- In the calculation of the amplitude $A$ in (III.42) and of the matrix elements in (III.45) and (III.46) the off-shell momentum $p$ of the external quark legs represents an infrared regulator. The logarithmic infrared divergence of the gluon correction diagrams (figs. 2(a)-(c) and 3 (a)-(c)) as $p^{2} \rightarrow 0$ is evident from (III.42), (III.45) and (III.46). A similar observation can be made for the $M_{W}$ dependence of the full amplitude $A$. We see that (III.42) is logarithmically divergent in the limit $M_{W} \rightarrow \infty$. This behaviour is reflected in the ultraviolet divergences (persisting after field renormalization) of the matrix elements (III.45), (III.46) in the effective theory, whose local interaction terms correspond to the weak interactions in the infinite $M_{W}$ limit as they are just the leading contribution of the $1 / M_{W}$ operator product expansion. This also implies, that the characteristic logarithmic functional dependence of the leading $\mathcal{O}\left(\alpha_{\mathrm{s}}\right)$ corrections is closely related to the divergence structure of the effective theory, that is to the renormalization constants $Z_{i j}$.

- The most important feature of the OPE is that it provides a factorization of short-distance (coefficients) and long-distance (operator matrix elements) contributions. This is clearly exhibited in our example. The dependence of the amplitude (III.42) on $p^{2}$, representing the long-distance structure of $A$, is fully contained in the matrix elements of the local operators $Q_{i}$ (III.50), (III.51), whereas the Wilson coefficients $C_{i}$ in (III.52) are free from this dependence. Essentially, this factorization has the form (see (III.59) - (III.61))

$$
\left(1+\alpha_{\mathrm{s}} G \ln \frac{M_{W}^{2}}{-p^{2}}\right) \doteq\left(1+\alpha_{\mathrm{s}} G \ln \frac{M_{W}^{2}}{\mu^{2}}\right) \cdot\left(1+\alpha_{\mathrm{s}} G \ln \frac{\mu^{2}}{-p^{2}}\right)
$$

that is, amplitude $=$ coefficient function $\times$ operator matrix element. Hereby the logarithm on the 1.h.s. is split according to

$$
\ln \frac{M_{W}^{2}}{-p^{2}}=\ln \frac{M_{W}^{2}}{\mu^{2}}+\ln \frac{\mu^{2}}{-p^{2}}
$$

Since the logarithmic behaviour results from the integration over some virtual loop momentum, we may - roughly speaking - rewrite this as

$$
\int_{-p^{2}}^{M_{W}^{2}} \frac{d k^{2}}{k^{2}}=\int_{\mu^{2}}^{M_{W}^{2}} \frac{d k^{2}}{k^{2}}+\int_{-p^{2}}^{\mu^{2}} \frac{d k^{2}}{k^{2}}
$$

which illustrates that the coefficient contains the contributions from large virtual momenta of the loop correction from scales $\mu \approx 1 \mathrm{GeV}$ to $M_{W}$, whereas the low energy contributions are separated into the matrix elements.

Of course, the latter can not be calculated in perturbation theory for transitions between physical meson states. The point is, that we have calculated the OPE for unphysical offshell quark external states only to extract the Wilson coefficients, which we need to construct the effective hamiltonian (III.37). For this purpose the fact that we have considered an unphysical amplitude is irrelevant since the coefficient functions do not depend on the external states, but represent the short-distance structure of the theory. Once we have extracted the coefficients and written down the effective hamiltonian, the latter can be used at least in principle - to evaluate the physically interesting decay amplitudes by means of some nonperturbative approach. 
- In interpreting the role of the scale $\mu$ we may distinguish two different aspects. From the point of view of the effective theory $\mu$ is just a renormalization scale, introduced in the process of renormalizing the effective local interaction terms by the dimensional method. On the other hand, from the point of view of the full theory, $\mu$ acts as the scale at which the full contribution is separated into a low energy and a high energy part, as is evident from the above discussion. For this reason $\mu$ is sometimes also called the factorization scale.

- In our case the infrared structure of the amplitude is characterized by the off-shell momentum $p$. In general one could work with any other arbitrary momentum configuration, on-shell or off-shell, with or without external quark mass, with infrared divergences regulated by offshell momenta, quark masses, a fictitious gluon mass or by dimensional regularization. In the case of off-shell momenta the amplitude is furthermore dependent on the gauge parameter of the gluon field. All these things belong to the infrared or long-distance structure of the amplitude. Therefore the dependence on these choices is the same for the full amplitude and for the operator matrix elements and drops out in the coefficient functions. To check that this is really the case for a particular choice is of crucial importance for practical calculations. On the other hand one may use this freedom and choose the treatment of external lines according to convenience or taste. Sometimes it may however seem preferable to keep a slightly more inconvenient dependence on external masses and/or gluon gauge in order to have a useful check that this dependence does indeed cancel out for the Wilson coefficients one is calculating.

\section{The Renormalization Group}

\section{Basic Concepts}

So far we have computed the Wilson coefficient functions (III.61) in ordinary perturbation theory. This, however, is not sufficient for the problem at hand. The appropriate scale at which to normalize the hadronic matrix elements of local operators is a low energy scale - low compared to the weak scale $M_{W}$ - of a few $G e V$ typically. In our example of charm decay $\mu=\mathcal{O}\left(m_{c}\right)$. For such a low scale $\mu$ the logarithm $\ln \left(M_{W}^{2} / \mu^{2}\right)$ multiplying $\alpha_{\mathrm{s}}(\mu)$ in the expression (III.61) becomes large. Although $\alpha_{\mathrm{s}}(\mu)$ by itself is a valid expansion parameter down to scales of $\mathcal{O}(1 \mathrm{GeV})$, say, this is not longer true for the combination $\alpha_{\mathrm{s}}(\mu) \ln \left(M_{W}^{2} / \mu^{2}\right)$. In fact, for our example (III.61) the first order correction term amounts for $\mu=1 \mathrm{GeV}$ to $65-130 \%$ although $\alpha_{\mathrm{s}} / 4 \pi \approx 4 \%$. The reason for this breakdown of the naive perturbative expansion lies ultimately in the appearance of largely disparate scales $M_{W}$ and $\mu$ in the problem at hand.

This situation can be considerably improved by employing the method of the renormalization group (RG). The renormalization group is the group of transformations between different choices of the renormalization scale $\mu$. The renormalization group equations describe the change of renormalized quantities, Green functions and parameters, with $\mu$ in a differential form. As we shall illustrate below, solving these differential equations allows, in the leading logarithmic approximation (LLA), to sum up the terms $\left(\alpha_{\mathrm{s}} \ln \left(M_{W} / \mu\right)\right)^{n}$ to all orders $n(n=0, \ldots, \infty)$ in perturbation theory. This leads to the RG improved perturbation theory. Going one step beyond in this modified expansion, to the next-to-leading logarithmic approximation (NLLA), the summation is extended 
to all terms $\alpha_{\mathrm{s}}\left(\alpha_{\mathrm{s}} \ln \left(M_{W} / \mu\right)\right)^{n}$, and so on. In this context it is useful to consider $\alpha_{\mathrm{s}} \ln \left(M_{W} / \mu\right)$ with a large logarithm $\ln \left(M_{W} / \mu\right)$ as a quantity of order $\mathcal{O}(1)$

$$
\alpha_{\mathrm{S}} \ln \frac{M_{W}}{\mu}=\mathcal{O}(1) \quad \mu \ll M_{W}
$$

Therefore the series in powers of $\alpha_{\mathrm{s}} \ln \left(M_{W} / \mu\right)$ cannot be truncated. Summed to all orders it yields again a contribution of order $\mathcal{O}(1)$. Correspondingly the next-to-leading logs $\alpha_{\mathrm{s}}\left(\alpha_{\mathrm{s}} \ln \left(M_{W} / \mu\right)^{n}\right.$ represent an $\mathcal{O}\left(\alpha_{\mathrm{s}}\right)$ perturbative correction to the leading term.

The renormalization group equation for the Wilson coefficient functions follows from the fact, that the unrenormalized Wilson coefficients $\vec{C}^{(0)}=Z_{c} \vec{C}\left(\vec{C}^{T}=\left(C_{1}, C_{2}\right)\right)$ are $\mu$-independent. Defining the matrix of anomalous dimensions $\gamma$ by

$$
\gamma=Z^{-1} \frac{d}{d \ln \mu} Z
$$

and recalling that $Z_{c}^{T}=Z^{-1}$, we obtain the renormalization group equation

$$
\frac{d}{d \ln \mu} \vec{C}(\mu)=\gamma^{T}\left(\alpha_{\mathrm{s}}\right) \vec{C}(\mu)
$$

The solution of (III.67) may formally be written in terms of a $\mu$-evolution matrix $U$ as

$$
\vec{C}(\mu)=U\left(\mu, M_{W}\right) \vec{C}\left(M_{W}\right)
$$

From (III.49) and (III.66) we have to first order in $\alpha_{\mathrm{s}}$

$$
\gamma\left(\alpha_{\mathrm{s}}\right)=\frac{\alpha_{\mathrm{s}}}{4 \pi} \gamma^{(0)}=\frac{\alpha_{\mathrm{s}}}{4 \pi}\left(\begin{array}{cc}
-6 / N & 6 \\
6 & -6 / N
\end{array}\right)
$$

or in the diagonal basis

$$
\gamma_{ \pm}\left(\alpha_{\mathrm{s}}\right)=\frac{\alpha_{\mathrm{s}}}{4 \pi} \gamma_{ \pm}^{(0)} \quad \gamma_{ \pm}^{(0)}= \pm 6 \frac{N \mp 1}{N}
$$

Note that if we neglect QCD loop corrections completely, the couplings $\vec{C}$ are independent of $\mu$. The nontrivial $\mu$-dependence of $\vec{C}$ expressed in (III.67) is a genuine quantum effect. It implies an anomalous scaling behaviour for the dimensionless coefficients, i.e. one that is different from the classical theory. For this reason the factor $\gamma$ is called anomalous (scale) dimension (compare (III.67) with $\frac{d}{d \ln \mu} \mu^{n}=n \mu^{n}$ for an n-dimensional $\mu$-dependent term $\mu^{n}$ ).

Using (III.13) the RG equation (III.67) is easily solved with the result

$$
C_{ \pm}(\mu)=\left[\frac{\alpha_{\mathrm{s}}\left(M_{W}\right)}{\alpha_{\mathrm{s}}(\mu)}\right]^{\frac{\gamma_{ \pm}^{(0)}}{2 \beta_{0}}} C_{ \pm}\left(M_{W}\right)
$$

At a scale $\mu_{W}=M_{W}$ no large logarithms are present and $C_{ \pm}\left(M_{W}\right)$ can therefore be calculated in ordinary perturbation theory. From (III.61) we have to the order needed for the LLA

$$
C_{ \pm}\left(M_{W}\right)=1
$$


(III.71) and (III.72) give the final result for the coefficients in the leading log approximation of RG improved perturbation theory.

At this point one should emphasize, that the choice of the high energy matching scale $\mu_{W}=M_{W}$ is of course not unique. The only requirement is that the choice of $\mu_{W}$ must not introduce large logs $\ln \left(M_{W} / \mu_{W}\right)$ in order not to spoil the applicability of the usual perturbation theory. Therefore $\mu_{W}$ should be of order $\mathcal{O}\left(M_{W}\right)$. The logarithmic correction in (III.61) is then $\mathcal{O}\left(\alpha_{\mathrm{s}}\right)$ and is neglected in LLA. Then, still, $C_{ \pm}\left(\mu_{W}\right)=1$ and

$$
C_{ \pm}(\mu)=\left[\frac{\alpha_{\mathrm{s}}\left(\mu_{W}\right)}{\alpha_{\mathrm{s}}(\mu)}\right]^{\frac{\gamma_{ \pm}^{(0)}}{2 \beta_{0}}}=\left[\frac{\alpha_{\mathrm{s}}\left(M_{W}\right)}{\alpha_{\mathrm{s}}(\mu)}\right]^{\frac{\gamma_{ \pm}^{(0)}}{2 \beta_{0}}}\left(1+\mathcal{O}\left(\alpha_{\mathrm{s}}\right)\right)
$$

A change of $\mu_{W}$ around the value of $M_{W}$ causes an ambiguity of $\mathcal{O}\left(\alpha_{\mathrm{s}}\right)$ in the coefficient. This ambiguity represents a theoretical uncertainty in the determination of $C_{ \pm}(\mu)$. In order to reduce it, it is necessary to go beyond the leading order. At NLO the scale ambiguity is then reduced from $\mathcal{O}\left(\alpha_{\mathrm{s}}\right)$ to $\mathcal{O}\left(\alpha_{\mathrm{s}}^{2}\right)$. We will come back to this point below. Presently, we will set $\mu_{W}=M_{W}$, but it is important to keep the related uncertainty in mind.

Taking into account the leading order solution of the RG equation (III.13) for the coupling, which can be expressed in the form

$$
\alpha_{\mathrm{s}}(m)=\frac{\alpha_{\mathrm{s}}(\mu)}{1+\beta_{0} \frac{\alpha_{\mathrm{s}}(\mu)}{4 \pi} \ln \frac{m^{2}}{\mu^{2}}}
$$

we may rewrite (III.71) as

$$
C_{ \pm}(\mu)=\left(\frac{1}{1+\beta_{0} \frac{\alpha_{\mathrm{s}}(\mu)}{4 \pi} \ln \frac{M_{W}^{2}}{\mu^{2}}}\right)^{\frac{\gamma_{ \pm}^{(0)}}{2 \beta_{0}}}
$$

(III.75) contains the logarithmic corrections $\sim \alpha_{\mathrm{s}} \ln \left(M_{W}^{2} / \mu^{2}\right)$ to all orders in $\alpha_{\mathrm{s}}$. This shows very clearly that the leading log corrections have been summed up to all orders in perturbation theory by solving the RG equation. In particular, if we again expand (III.75) in powers of $\alpha_{\mathrm{s}}$, keeping the first term only we recover (III.61). This observation demonstrates, that the RG method allows to obtain solutions, which go beyond the conventional perturbation theory.

Before concluding this subsection, we would like to introduce still two generalizations of the approach developed so far, which will appear in the general discussion below.

\section{Threshold Effects in LLA}

First we may generalize the renormalization group evolution from $M_{W}$ down to $\mu \approx m_{c}$ to include the threshold effect of heavy quarks like $b$ or $t$ as follows

$$
\vec{C}(\mu)=U^{(f=4)}\left(\mu, \mu_{b}\right) U^{(f=5)}\left(\mu_{b}, \mu_{W}\right) \vec{C}\left(\mu_{W}\right)
$$

which is valid for the LLA. In our example of the $c \rightarrow s u \bar{d}$ transition the top quark gives no contribution at all. Being heavier (but comparable) in mass than the $\mathrm{W}$, it is simply removed from 
the theory along with the W-boson. In a first step the coefficients at the initial scale $\mu_{W} \approx M_{W}$ are evolved down to $\mu_{b} \approx m_{b}$ in an effective theory with five quark flavors $(f=5)$. Then, again in the spirit of the effective field theory technique, for scales below $\mu_{b}$ also the bottom quark is removed as an explicit degree of freedom from the effective theory, yielding a new effective theory with only four "active" quark flavors left. The matching corrections between both theories can be calculated in ordinary perturbation theory at the scale $\mu_{b}$, since due to $\mu_{b} \approx m_{b}$ no large logs can occur in this procedure. For the same reason matching corrections $\left(\mathcal{O}\left(\alpha_{\mathrm{s}}\right)\right)$ can be neglected in LLA and the coefficients at $\mu_{b}, \vec{C}\left(\mu_{b}\right)$, simply serve as the initial values for the RG evolution in the four quark theory down to $\mu \approx m_{c}$. In addition, continuity of the running coupling across the threshold $\mu_{b}$ is imposed by the requirement

$$
\alpha_{s, f=4}\left(\mu_{b}, \Lambda^{(4)}\right)=\alpha_{s, f=5}\left(\mu_{b}, \Lambda^{(5)}\right)
$$

which defines different QCD scales $\Lambda^{(f)}$ for each effective theory.

Neglecting the b-threshold, as we did before (III.68), one may just perform the full evolution from $\mu_{W}$ to $\mu$ in an effective four flavor theory. It turns out that in some cases the difference of these two approaches is even negligible.

We would like to add a comment on this effective field theory technique. At the first sight the idea to "remove by hand" heavy degrees of freedom may look somewhat artificial. However it appears quite natural when not viewed from the evolution from high towards low energies but vice versa (which actually corrsponds to the historical way). Suppose only the "light" quarks $u$, $d, s, c$ were known. Then in the attempt to formulate a theory of their weak interactions one would be lead to a generalized Fermi theory with (effective) four quark coupling constants to be determined somehow. Of course, we are in the lucky position to know the underlying theory in the form of the Standard Model. Therefore we can actually derive the coupling constants of the low energy effective theory from "first principles". This is exactly what is achieved technically by going through a series of effective theories, removing heavy degrees of freedom successively, by means of a step-by-step procedure.

\section{Penguin Operators}

A second, but very important issue is the generation of QCD penguin operators (Vainshtein et al., 1977). Consider for example the local operator $\left(\bar{s}_{i} u_{i}\right)_{V-A}\left(\bar{u}_{j} d_{j}\right)_{V-A}$, which is directly induced by $\mathrm{W}$-boson exchange. In this case, additional QCD correction diagrams, the penguin diagrams (d.1) and (d.2) with a gluon in fig. 3, contribute and as a consequence six operators are involved in the mixing under renormalization instead of two. These read

$$
\begin{array}{r}
Q_{1}=\left(\bar{s}_{i} u_{j}\right)_{V-A}\left(\bar{u}_{j} d_{i}\right)_{V-A} \\
Q_{2}=\left(\bar{s}_{i} u_{i}\right)_{V-A}\left(\bar{u}_{j} d_{j}\right)_{V-A} \\
Q_{3}=\left(\bar{s}_{i} d_{i}\right)_{V-A} \sum_{q}\left(\bar{q}_{j} q_{j}\right)_{V-A} \\
Q_{4}=\left(\bar{s}_{i} d_{j}\right)_{V-A} \sum_{q}\left(\bar{q}_{j} q_{i}\right)_{V-A} \\
Q_{5}=\left(\bar{s}_{i} d_{i}\right)_{V-A} \sum_{q}\left(\bar{q}_{j} q_{j}\right)_{V+A} \\
Q_{6}=\left(\bar{s}_{i} d_{j}\right)_{V-A} \sum_{q}\left(\bar{q}_{j} q_{i}\right)_{V+A}
\end{array}
$$

The sum over $q$ runs over all quark flavors that exist in the effective theory in question. The operators $Q_{1}$ and $Q_{2}$ are just the ones we have encountered in subsection IIIC, but with the cquark replaced by $u$. This modified flavor structure gives rise to the gluon penguin type diagrams 
shown in fig. 3 (d). Since the gluon coupling is of course flavor conserving, it is clear that penguins cannot be generated from the operator $(\bar{s} c)_{V-A}(\bar{u} d)_{V-A}$. The penguin graphs induce the new local interaction vertices $Q_{3}, \ldots, Q_{6}$, which have the same quantum numbers. Their structure is easily understood. The flavor content is determined by the $(\bar{s} d)_{V-A}$ current in the upper part and by a $\sum_{q}(\bar{q} q)_{V}$ vector current due to the gluon coupling in the lower. This vector structure is for convenience decomposed into a $(V-A)$ and a $(V+A)$ part. For each of these, two different color forms arise due to the color structure of the exchanged gluon (see (III.40)). Together this yields the four operators $Q_{3}, \ldots, Q_{6}$.

For all operators $Q_{1}, \ldots, Q_{6}$ all possible QCD corrections (that is all amputated Green functions with insertion of $Q_{i}$ ) of the current-current (fig. 3 (a)-(c)) as well as of the penguin type (fig. 3 (d.1) and (d.2)) have to be evaluated. In this process no new operators are generated, so that $Q_{1}, \ldots, Q_{6}$ form a complete set. They "close under renormalization". In analogy to the case of subsection III C the divergent parts of these Green functions determine, after field renormalization, the operator renormalization constants, which in the present case form a $6 \times 6$ matrix. The calculation of the corresponding anomalous dimension matrix and the renormalization group analysis then proceeds in the usual way. We will see that the inclusion of higher order electroweak interactions requires the introduction of still more operators.

\section{E. Summary of Basic Formalism}

We think that after this rather detailed discussion of the methods required for the short-distance calculations in weak decays, it is useful to give at this point a concise summary of the material covered so far. At the same time this may serve as an outline of the necessary procedure for practical calculations. Furthermore it will also provide a starting point for the extension of the formalism from the LLA considered until now to the NLLA to be presented in the next subsection.

Ultimately our goal is the evaluation of weak decay amplitudes involving hadrons in the framework of a low energy effective theory, of the form

$$
\left\langle\mathcal{H}_{e f f}\right\rangle=\frac{G_{F}}{\sqrt{2}} V_{C K M}\left\langle\vec{Q}^{T}(\mu)\right\rangle \vec{C}(\mu)
$$

The procedure for this calculation can be divided into the following three steps.

\section{Step 1: Perturbation Theory}

Calculation of Wilson coefficients $\vec{C}\left(\mu_{W}\right)$ at $\mu_{W} \approx M_{W}$ to the desired order in $\alpha_{\mathrm{s}}$. Since logarithms of the form $\ln \left(\mu_{W} / M_{W}\right)$ are not large, this can be performed in ordinary perturbation theory. It amounts to matching the full theory onto a five quark effective theory.

\section{Step 2: RG Improved Perturbation Theory}

- Calculation of the anomalous dimensions of the operators

- Solution of the renormalization group equation for $\vec{C}(\mu)$

- Evolution of the coefficients from $\mu_{W}$ down to the appropriate low energy scale $\mu$

$$
\vec{C}(\mu)=U\left(\mu, \mu_{W}\right) \vec{C}\left(\mu_{W}\right)
$$




\section{Step 3: Non-Perturbative Regime}

Calculation of hadronic matrix elements $\langle\vec{Q}(\mu)\rangle$, normalized at the appropriate low energy scale $\mu$, by means of some non-perturbative method.

Important issues in this procedure are:

- The OPE achieves a factorization of short- and long distance contributions.

- Correspondingly, in order to disentangle the short-distance from the long-distance part and to extract $\vec{C}\left(\mu_{W}\right)$ in actual calculations, a proper matching of the full onto the effective theory has to be performed.

- Similar comments apply to the matching of an effective theory with $f$ quark flavors to a theory with $(f-1)$ flavors during the RG evolution to lower scales.

- Furthermore, factorization implies, that the $\mu$-dependence and also the dependence on the renormalization scheme, which appears beyond the leading order, cancel between $C_{i}$ and $\left\langle Q_{i}\right\rangle$.

- Since the top quark is integrated out along with the W, the coefficients $\vec{C}\left(\mu_{W}\right)$ in general contain also the full dependence on the top quark mass $m_{t}$.

- A summation of large logs by means of the RG method is necessary. More specifically, in the $n$-th order of renormalization group improved perturbation theory the terms of the form

$$
\alpha_{\mathrm{s}}^{n}(\mu)\left(\alpha_{\mathrm{s}}(\mu) \ln \frac{M_{W}}{\mu}\right)^{k}
$$

are summed to all orders in $k(k=0,1,2, \ldots)$. This approach is justified as long as $\alpha_{\mathrm{s}}(\mu)$ is small enough, which requires that $\mu$ not be too low, typically not less than $1 \mathrm{GeV}$.

\section{F. Wilson Coefficients Beyond Leading Order}

\section{The RG Formalism}

We are now going to extend the renormalization group formalism for the coefficient functions to the next-to-leading order level. Subsequently we will discuss important aspects of the resulting formulae, in particular the scale- and scheme dependences and their cancellation.

To have something specific in mind, we may consider the calculation for the $\Delta S=1$ effective hamiltonian for nonleptonic decays, which without QCD effects and for low energy is given by

$$
\mathcal{H}_{\text {eff }}^{\Delta S=1}=\frac{G_{F}}{\sqrt{2}} V_{u s}^{*} V_{u d}(\bar{s} u)_{V-A}(\bar{u} d)_{V-A}
$$

At higher energies of course also the charm, bottom and top quark have to be taken into account. The Feynman diagrams contributing to $\mathcal{O}\left(\alpha_{\mathrm{s}}\right)$ corrections to this hamiltonian are shown in fig. 2 and 3. Including current-current- as well as penguin type corrections, the relevant operator basis consists of the six operators in (III.78).

On the one hand, this particular case is very important by itself since it provides the theoretical 
basis for a large variety of different decay modes. On the other hand we will at this stage keep the discussion fairly general, so that all important features of a general validity are exhibited. In addition, the central formulae of this subsection will be used at several places later on, if at times extended or modified to match the specific cases in question. In part two of this report we will give a more detailed discussion of the hamiltonians relevant for various decays. Here, we would rather like to concentrate on the presentation of the OPE and renormalization group formalism. The effective hamiltonian for nonleptonic decays may be written in general as

$$
\mathcal{H}_{\text {eff }}=\frac{G_{F}}{\sqrt{2}} \sum_{i} C_{i}(\mu) Q_{i}(\mu) \equiv \frac{G_{F}}{\sqrt{2}} \vec{Q}^{T}(\mu) \vec{C}(\mu)
$$

where the index $i$ runs over all contributing operators, in our example $Q_{1}, \ldots, Q_{6}$ of (III.78). It is straightforward to apply $\mathcal{H}_{\text {eff }}$ to D- and B-meson decays as well by changing the quark flavors appropriately. For the time being we omit CKM parameters, which can be reinserted later on. $\mu$ is some low energy scale of the order $\mathcal{O}(1 \mathrm{GeV}), \mathcal{O}\left(m_{c}\right)$ and $\mathcal{O}\left(m_{b}\right)$ for K-, D- and B-meson decays, respectively. The argument $\mu$ of the operators $Q_{i}(\mu)$ means, that their matrix elements are to be normalized at scale $\mu$.

The Wilson coefficient functions are given by

$$
\vec{C}(\mu)=U\left(\mu, \mu_{W}\right) \vec{C}\left(\mu_{W}\right)
$$

The coefficients at the scale $\mu_{W}=\mathcal{O}\left(M_{W}\right)$ can be evaluated in perturbation theory. The evolution matrix $U$ then includes the renormalization group improved perturbative contributions from the scale $\mu_{W}$ down to $\mu$.

In the first step we determine $\vec{C}\left(\mu_{W}\right)$ from a comparison of the amputated Green function with appropriate external lines in the full theory with the corresponding amplitude in the effective theory. At NLO we have to calculate to $\mathcal{O}\left(\alpha_{\mathrm{s}}\right)$, including non-logarithmic, constant terms. The full amplitude results from the current-current- and penguin type diagrams in fig. 2, is finite after field renormalization and can be written as

$$
A=\frac{G_{F}}{\sqrt{2}} \vec{S}^{T}\left(\vec{A}^{(0)}+\frac{\alpha_{\mathrm{s}}\left(\mu_{W}\right)}{4 \pi} \vec{A}^{(1)}\right)
$$

Here $\vec{S}$ denotes the tree level matrix elements of the operators $\vec{Q}$. In the effective theory (III.80) the current-current- and penguin corrections of fig. 3 have to be calculated for all the operators $Q_{i}$. In this case, besides the field renormalization, a renormalization of operators is necessary

$$
Z_{q}^{2}\langle\vec{Q}\rangle^{(0)}=Z\langle\vec{Q}\rangle
$$

where the matrix $\mathrm{Z}$ absorbes those divergences of the Green functions with operator $\vec{Q}$ insertion, that are not removed by the field renormalization. The renormalized matrix elements of the operators can then to $\mathcal{O}\left(\alpha_{\mathrm{s}}\right)$ be written as

$$
\left\langle\vec{Q}\left(\mu_{W}\right)\right\rangle=\left(1+\frac{\alpha_{\mathrm{s}}\left(\mu_{W}\right)}{4 \pi} r\right) \vec{S}
$$

and the amplitude in the effective theory to the same order becomes 


$$
A_{e f f}=\frac{G_{F}}{\sqrt{2}} \vec{S}^{T}\left(1+\frac{\alpha_{\mathrm{s}}\left(\mu_{W}\right)}{4 \pi} r^{T}\right) \vec{C}\left(\mu_{W}\right)
$$

Equating (III.82) and (III.85) we obtain

$$
\vec{C}\left(\mu_{W}\right)=\vec{A}^{(0)}+\frac{\alpha_{\mathrm{s}}\left(\mu_{W}\right)}{4 \pi}\left(\vec{A}^{(1)}-r^{T} \vec{A}^{(0)}\right)
$$

In general $\vec{A}^{(1)}$ in (III.82) involves logarithms $\ln \left(M_{W}^{2} /-p^{2}\right)$ where p denotes some global external momentum for the amplitudes in fig. 2. On the other hand, the matrix $r$ in (III.84), characterizing the radiative corrections to $\left\langle\vec{Q}\left(\mu_{W}\right)\right\rangle$, includes $\ln \left(-p^{2} / \mu_{W}^{2}\right)$. As we have seen in subsection III C, these logarithms combine to $\ln \left(M_{W}^{2} / \mu_{W}^{2}\right)$ in the Wilson coefficient (III.86). For $\mu_{W}=M_{W}$ this logarithm vanishes altogether. For $\mu_{W}=\mathcal{O}\left(M_{W}\right)$ the expression $\ln \left(M_{W}^{2} / \mu_{W}^{2}\right)$ is a "small logarithm" and the correction $\sim \alpha_{\mathrm{s}} \ln \left(M_{W}^{2} / \mu_{W}^{2}\right)$, which could be neglected in LLA, has to be kept in the perturbative calculation at NLO together with constant pieces of order $\mathcal{O}\left(\alpha_{\mathrm{s}}\right)$.

In the second step, the renormalization group equation for $\vec{C}$

$$
\frac{d}{d \ln \mu} \vec{C}(\mu)=\gamma^{T}(g) \vec{C}(\mu)
$$

has to be solved with boundary condition (III.86). The solution is written with the help of the U-matrix as in (III.81), where $U\left(\mu, \mu_{W}\right)$ obeys the same equation as $\vec{C}(\mu)$ in (III.87). The general solution is easily written down iteratively

$$
U(\mu, m)=1+\int_{g(m)}^{g(\mu)} d g_{1} \frac{\gamma^{T}\left(g_{1}\right)}{\beta\left(g_{1}\right)}+\int_{g(m)}^{g(\mu)} d g_{1} \int_{g(m)}^{g_{1}} d g_{2} \frac{\gamma^{T}\left(g_{1}\right)}{\beta\left(g_{1}\right)} \frac{\gamma^{T}\left(g_{2}\right)}{\beta\left(g_{2}\right)}+\ldots
$$

which using $d g / d \ln \mu=\beta(g)$ is readily seen to solve the renormalization group equation

$$
\frac{d}{d \ln \mu} U(\mu, m)=\gamma^{T}(g) U(\mu, m)
$$

The series in (III.88) can be more compactly expressed by introducing the notion of g-ordering

$$
U(\mu, m)=T_{g} \exp \int_{g(m)}^{g(\mu)} d g^{\prime} \frac{\gamma^{T}\left(g^{\prime}\right)}{\beta\left(g^{\prime}\right)}
$$

where in the case $g(\mu)>g(m)$ the g-ordering operator $T_{g}$ is defined through

$$
T_{g} f\left(g_{1}\right) \ldots f\left(g_{n}\right)=\sum_{p e r m} \Theta\left(g_{i_{1}}-g_{i_{2}}\right) \Theta\left(g_{i_{2}}-g_{i_{3}}\right) \ldots \Theta\left(g_{i_{n-1}}-g_{i_{n}}\right) f\left(g_{i_{1}}\right) \ldots f\left(g_{i_{n}}\right)
$$

and brings about an ordering of the factors $f\left(g_{i}\right)$ such that the coupling constants increase from right to left. The sum in (III.91) runs over all permutations $\left\{i_{1}, \ldots, i_{n}\right\}$ of $\{1,2, \ldots, n\}$. The $T_{g}$ ordering is necessary since in general the anomalous dimension matrices at different couplings do not commute beyond the leading order, $\left[\gamma\left(g_{1}\right), \gamma\left(g_{2}\right)\right] \neq 0$.

At next-to-leading order we have to keep the first two terms in the perturbative expansions for $\beta(g)$ (see (III.11)) and $\gamma(g)$

$$
\gamma\left(\alpha_{\mathrm{s}}\right)=\gamma^{(0)} \frac{\alpha_{\mathrm{s}}}{4 \pi}+\gamma^{(1)}\left(\frac{\alpha_{\mathrm{s}}}{4 \pi}\right)^{2}
$$


To this order the evolution matrix $U(\mu, m)$ is given by (Buras et al., 1992)

$$
U(\mu, m)=\left(1+\frac{\alpha_{\mathrm{s}}(\mu)}{4 \pi} J\right) U^{(0)}(\mu, m)\left(1-\frac{\alpha_{\mathrm{s}}(m)}{4 \pi} J\right)
$$

$U^{(0)}$ is the evolution matrix in leading logarithmic approximation and the matrix $J$ expresses the next-to-leading corrections to this evolution. We have

$$
U^{(0)}(\mu, m)=V\left(\left[\frac{\alpha_{\mathrm{S}}(m)}{\alpha_{\mathrm{S}}(\mu)}\right]^{\frac{\vec{\gamma}^{(0)}}{2 \beta_{0}}}\right)_{D} V^{-1}
$$

where $V$ diagonalizes $\gamma^{(0) T}$

$$
\gamma_{D}^{(0)}=V^{-1} \gamma^{(0) T} V
$$

and $\vec{\gamma}^{(0)}$ is the vector containing the diagonal elements of the diagonal matrix $\gamma_{D}^{(0)}$.

If we define

$$
G=V^{-1} \gamma^{(1) T} V
$$

and a matrix $H$ whose elements are

$$
H_{i j}=\delta_{i j} \gamma_{i}^{(0)} \frac{\beta_{1}}{2 \beta_{0}^{2}}-\frac{G_{i j}}{2 \beta_{0}+\gamma_{i}^{(0)}-\gamma_{j}^{(0)}}
$$

the matrix $J$ is given by

$$
J=V H V^{-1}
$$

The fact that (III.93) is indeed a solution of the RG equation (III.89) to the order considered is straightforwardly verified by differentiation with respect to $\ln \mu$. Combining now the initial values (III.86) with the evolution matrix (III.93) we obtain

$$
\vec{C}(\mu)=\left(1+\frac{\alpha_{\mathrm{s}}(\mu)}{4 \pi} J\right) U^{(0)}\left(\mu, \mu_{W}\right)\left(\vec{A}^{(0)}+\frac{\alpha_{\mathrm{s}}\left(\mu_{W}\right)}{4 \pi}\left[\vec{A}^{(1)}-\left(r^{T}+J\right) \vec{A}^{(0)}\right]\right)
$$

Using (III.99) we can calculate for example the coefficients at a scale $\mu=\mu_{b}=\mathcal{O}\left(m_{b}\right)$, working in an effective five flavor theory, $f=5$. If we have to evolve the coefficients to still lower values, we would like to formulate a new effective theory for $\mu<\mu_{b}$ where now also the b-quark is removed as an explicit degree of freedom. To calculate the coefficients in this new four flavor theory at the scale $\mu_{b}$, we have to determine the matching corrections at this scale.

We follow the same principles as in the case of integrating out the W-boson and require

$$
\left\langle\vec{Q}_{f}(m)\right\rangle^{T} \vec{C}_{f}(m)=\left\langle\vec{Q}_{f-1}(m)\right\rangle^{T} \vec{C}_{f-1}(m)
$$

in the general case of a change from an f-flavor to a (f-1)-flavor theory at a scale $m$. The "full amplitude" on the 1.h.s., which is now in an f-flavor effective theory, is expanded into matrix elements of the new (f-1)-flavor theory, multiplied by new Wilson coefficients $\vec{C}_{f-1}$. From (III.84), determining the matrix elements of operators to $\mathcal{O}\left(\alpha_{\mathrm{s}}\right)$, one finds 


$$
\left\langle\vec{Q}_{f}(m)\right\rangle=\left(1+\frac{\alpha_{\mathrm{s}}(m)}{4 \pi} \delta r\right)\left\langle\vec{Q}_{f-1}(m)\right\rangle
$$

where

$$
\delta r=r^{(f)}-r^{(f-1)}
$$

In (III.102) we have made explicit the dependence of the matrix $r$ on the number of quark flavors which enters in our example via the penguin contributions. From (III.100) and (III.101) we find

$$
\vec{C}_{f-1}(m)=M(m) \vec{C}_{f}(m)
$$

with

$$
M(m)=1+\frac{\alpha_{\mathrm{s}}(m)}{4 \pi} \delta r^{T}
$$

The general renormalization group matrix $U$ in (III.93), now evaluated for (f-1) flavors, can be used to evolve $\vec{C}_{f-1}(m)$ to lower values of the renormalization scale. It is clear that no large logarithms can appear in (III.104) and that therefore the matching corrections, expressed in the matrix $M(m)$ can be computed in usual perturbation theory. We note that this type of matching corrections enters in a nontrivial way for the first time at the NLO level. In the LLA $M \equiv 1$ and one can simply omit the b-flavor components in the penguin operators when crossing the bthreshold.

We also remark that the correction matrix $M$ introduces a small discontinuity of the coefficients, regarded as functions of $\mu$, at the matching scale $m$. This is however not surprising. In any case the $\vec{C}(\mu)$ are not physical quantities and their discontinuity precisely cancels the effect of removing the heavy quark flavor from the operators, which evidently is a "discontinuous" step. Hence, physical amplitudes are not affected and indeed the behaviour of $\vec{C}$ at the matching scale ensures that the same physical result will be obtained, whether we choose to calculate in the f-flavor- or in the (f-1)-flavor theory for scales around the matching scale $m$.

To conclude we will write down how the typical final result for the coefficient functions at $\mu \approx$ $1 \mathrm{GeV}$, appropriate for K-decays, looks like, if we combine all the contributions discussed above. Then we can write

$$
\vec{C}(\mu)=U_{3}\left(\mu, \mu_{c}\right) M\left(\mu_{c}\right) U_{4}\left(\mu_{c}, \mu_{b}\right) M\left(\mu_{b}\right) U_{5}\left(\mu_{b}, \mu_{W}\right) \vec{C}\left(\mu_{W}\right)
$$

where $U_{f}$ is the evolution matrix for $f$ active flavors. In the following discussion we will not always include the flavor thresholds when writing the expression for the RG evolution. It is clear, that they can be added in a straightforward fashion.

\section{The Calculation of the Anomalous Dimensions}

The matrix of anomalous dimensions is the most important ingredient for the renormalization group calculation of the Wilson coefficient functions. In the following we will summarize the essential steps of its calculation.

Recall that the evaluation of the amputated Green functions with insertion of the operators $\vec{Q}$ gives the relation 


$$
\langle\vec{Q}\rangle^{(0)}=Z_{q}^{-2} Z\langle\vec{Q}\rangle \equiv Z_{G F}\langle\vec{Q}\rangle
$$

$\langle\vec{Q}\rangle^{(0)},\langle\vec{Q}\rangle$ denote the unrenormalized and renormalized Green functions, respectively. $Z_{q}$ is the quark field renormalization constant and $Z$ is the renormalization constant matrix of the operators $\vec{Q}$.

The anomalous dimensions are given by

$$
\gamma(g)=Z^{-1} \frac{d}{d \ln \mu} Z
$$

In the $M S$ (or $\overline{M S}$ ) scheme the renormalization constants are chosen to absorb the pure pole divergences $1 / \varepsilon^{k}(D=4-2 \varepsilon)$, but no finite parts. $Z$ can then be expanded in inverse powers of $\varepsilon$ as follows

$$
Z=1+\sum_{k=1}^{\infty} \frac{1}{\varepsilon^{k}} Z_{k}(g)
$$

Using the expression for the $\beta$-function (III.6), valid for arbitrary $\varepsilon$ we derive the useful formula (Floratos et al., 1977)

$$
\gamma(g)=-2 g^{2} \frac{\partial Z_{1}(g)}{\partial g^{2}}=-2 \alpha_{\mathrm{s}} \frac{\partial Z_{1}\left(\alpha_{\mathrm{s}}\right)}{\partial \alpha_{\mathrm{s}}}
$$

Similarly to (III.108) we expand

$$
\begin{gathered}
Z_{q}=1+\sum_{k=1}^{\infty} \frac{1}{\varepsilon^{k}} Z_{q, k}(g) \\
Z_{G F}=1+\sum_{k=1}^{\infty} \frac{1}{\varepsilon^{k}} Z_{G F, k}(g)
\end{gathered}
$$

From the calculation of the unrenormalized Green functions (III.106) we immediately obtain $Z_{G F}$. What we need to compute $\gamma(g)$ is $Z_{1}(g)$ (III.109). From (III.106), (III.108), (III.110), (III.111) we find

$$
Z_{1}=2 Z_{q, 1}+Z_{G F, 1}
$$

At next-to-leading order we have from the $1 / \varepsilon$ poles of the unrenormalized Green functions

$$
Z_{G F, 1}=b_{1} \frac{\alpha_{\mathrm{s}}}{4 \pi}+b_{2}\left(\frac{\alpha_{\mathrm{s}}}{4 \pi}\right)^{2}
$$

The corresponding expression for the well known factor $Z_{q, 1}$ has been quoted in (III.15). Using (III.15), (III.109), (III.112), (III.113) we finally obtain for the one- and two-loop anomalous dimension matrices $\gamma^{(0)}$ and $\gamma^{(1)}$ in (III.92)

$$
\begin{aligned}
& \gamma_{i j}^{(0)}=-2\left[2 a_{1} \delta_{i j}+\left(b_{1}\right)_{i j}\right] \\
& \gamma_{i j}^{(1)}=-4\left[2 a_{2} \delta_{i j}+\left(b_{2}\right)_{i j}\right]
\end{aligned}
$$

(III.114) and (III.115) may be used as recipes to immediately extract the anomalous dimensions from the divergent parts of the unrenormalized Green functions. 


\section{Renormalization Scheme Dependence}

A further issue, which becomes important at next-to-leading order is the dependence of unphysical quantities, like the Wilson coefficients and the anomalous dimensions, on the choice of the renormalization scheme. This scheme dependence arises because the renormalization prescription involves an arbitrariness in the finite parts to be subtracted along with the ultraviolet singularities. Two different schemes are then related by a finite renormalization. Considering the quantities, which we encountered in subsection III F 1, the following of them are independent of the renormalization scheme

$$
\beta_{0}, \quad \beta_{1}, \quad \gamma^{(0)}, \quad \vec{A}^{(0)}, \quad \vec{A}^{(1)}, \quad r^{T}+J, \quad\langle\vec{Q}\rangle^{T} \vec{C}
$$

whereas

$$
r, \quad \gamma^{(1)}, \quad J, \quad \vec{C},\langle\vec{Q}\rangle
$$

are scheme dependent.

In the framework of dimensional regularization one example of how such a scheme dependence can occur is the treatment of $\gamma_{5}$ in $D$ dimensions. Possible choices are the "naive dimensional regularization" (NDR) scheme with $\gamma_{5}$ taken to be anticommuting or the 't-HooftVeltman (HV) scheme ('t Hooft and Veltman, 1972b), (Breitenlohner and Maison, 1977) with non-anticommuting $\gamma_{5}$. Another example is the use of operators in a color singlet or a non-singlet form, such as

$$
Q_{2}=\left(\bar{s}_{i} u_{i}\right)_{V-A}\left(\bar{u}_{j} d_{j}\right)_{V-A} \quad \text { or } \quad \tilde{Q}_{2}=\left(\bar{s}_{i} d_{j}\right)_{V-A}\left(\bar{u}_{j} u_{i}\right)_{V-A}
$$

where $i, j$ are color indices. In $D=4$ dimensions these operators are equivalent since they are related by a Fierz transformation. In the NDR scheme however these two choices yield different results for $r, \gamma^{(1)}$ and $J$ and thus constitute two different schemes, related by a nontrivial finite renormalization. On the other hand, both choices give the same $r, \gamma^{(1)}$ and $J$ if the HV scheme is employed.

Let us now discuss the question of renormalization scheme dependences in explicit terms in order to obtain an overview on how the scheme dependences arise, how various quantities transform under a change of the renormalization scheme and how the cancellation of scheme dependences is guaranteed for physically relevant quantities.

First of all, it is clear that the product

$$
\langle\vec{Q}(\mu)\rangle^{T} \vec{C}(\mu)
$$

representing the full amplitude, is independent of the renormalization scheme chosen. This is simply due to the fact, that it is precisely the factorization of the amplitude into Wilson coefficients and matrix elements of operators by means of the operator product expansion, which introduces the scheme dependence of $\vec{C}$ and $\langle\vec{Q}\rangle$. In other words, the scheme dependence of $\vec{C}$ and $\langle\vec{Q}\rangle$ represents the arbitrariness one has in splitting the full amplitude into coefficients and matrix elements and the scheme independence of the combined product (III.119) is manifest in the construction of the operator product expansion.

More explicitly, these quantities are in different schemes (primed and unprimed) related by 


$$
\langle\vec{Q}\rangle^{\prime}=\left(1+\frac{\alpha_{\mathrm{s}}}{4 \pi} s\right)\langle\vec{Q}\rangle \quad \vec{C}^{\prime}=\left(1-\frac{\alpha_{\mathrm{s}}}{4 \pi} s^{T}\right) \vec{C}
$$

where $s$ is a constant matrix. (III.120) represents a finite renormalization of $\vec{C}$ and $\langle\vec{Q}\rangle$. From (III.84) we immediately obtain

$$
r^{\prime}=r+s
$$

Furthermore from

$$
\langle\vec{Q}(\mu)\rangle^{T} \vec{C}(\mu) \equiv\langle\vec{Q}(\mu)\rangle^{T} U\left(\mu, M_{W}\right) \vec{C}\left(M_{W}\right)
$$

we have

$$
U^{\prime}\left(\mu, M_{W}\right)=\left(1-\frac{\alpha_{\mathrm{s}}(\mu)}{4 \pi} s^{T}\right) U\left(\mu, M_{W}\right)\left(1+\frac{\alpha_{\mathrm{s}}\left(M_{W}\right)}{4 \pi} s^{T}\right)
$$

A comparison with (III.93) yields

$$
J^{\prime}=J-s^{T}
$$

The renormalization constant matrix in the primed scheme, $Z^{\prime}$, follows from (III.120) and (III.106)

$$
Z^{\prime}=Z\left(1-\frac{\alpha_{\mathrm{s}}}{4 \pi} s\right)
$$

Recalling the definition of the matrix of anomalous dimensions, (III.107) and (III.92), we derive

$$
\gamma^{(0) \prime}=\gamma^{(0)} \quad \gamma^{(1) \prime}=\gamma^{(1)}+\left[s, \gamma^{(0)}\right]+2 \beta_{0} s
$$

With these general formulae at hand it is straightforward to clarify the cancellation of scheme dependences in all particular cases. Alternatively, they may be used to transform scheme dependent quantities from one scheme to another, if desired, or to check the compatibility of results obtained in different schemes.

In particular we immediately verify from (III.121) and (III.124) the scheme independence of the matrix $r^{T}+J$. This means that in the expression for $\vec{C}$ in (III.99) the factor on the right hand side of $U^{(0)}$, related to the "upper end" of the evolution, is independent of the renormalization scheme, as it must be. The same is true for $U^{(0)}$. On the other hand $\vec{C}$ still depends on the renormalization scheme through the matrix $J$ to the left of $U^{(0)}$. As is evident from (III.120), this dependence is compensated for by the corresponding scheme dependence of the matrix elements of operators so that a physically meaningful result for the decay amplitudes is obtained. To ensure a proper cancellation of the scheme dependence the matrix elements have to be evaluated in the same scheme (renormalization, $\gamma_{5}$, form of operators) as the coefficient functions, which is a nontrivial task for the necessary non-perturbative computations. In other words, beyond the leading order the matching between short- and long-distance contributions has to be performed properly not only with respect to the scale $\mu$, but also with respect to the renormalization scheme employed. 


\section{Discussion}

We will now specialize the presentation of the general formalism to the case of a single operator (that is without mixing). This situation is e.g. relevant for the operators $Q_{+}$and $Q_{-}$with four different quark flavors, which we encountered in section III C. The resulting simplifications are useful in order to display some more details of the structure of the calculation and to discuss the most salient features of the NLO analysis in a transparent way.

In the case where only one single operator contributes, the amplitude in the full theory (dynamical W-boson) may be written as (see (III.82))

$$
A=\frac{G_{F}}{\sqrt{2}}\left(1+\frac{\alpha_{\mathrm{s}}\left(\mu_{W}\right)}{4 \pi}\left[-\frac{\gamma^{(0)}}{2} \ln \frac{M_{W}^{2}}{-p^{2}}+\tilde{A}^{(1)}\right]\right) S
$$

where we have made the logarithmic dependence on the $\mathrm{W}$ mass explicit. In the effective theory the amplitude reads

$$
\begin{aligned}
A_{e f f} & =\frac{G_{F}}{\sqrt{2}} C\left(\mu_{W}\right)\left\langle Q\left(\mu_{W}\right)\right\rangle \\
& =\frac{G_{F}}{\sqrt{2}} C\left(\mu_{W}\right)\left(1+\frac{\alpha_{\mathrm{s}}\left(\mu_{W}\right)}{4 \pi}\left[\frac{\gamma^{(0)}}{2}\left(\ln \frac{-p^{2}}{\mu_{W}^{2}}+\gamma_{E}-\ln 4 \pi\right)+\tilde{r}\right]\right) S
\end{aligned}
$$

The divergent pole term $1 / \varepsilon$ has been subtracted minimally. A comparison of (III.127) and (III.128) yields the Wilson coefficient

$$
C\left(\mu_{W}\right)=1+\frac{\alpha_{\mathrm{s}}\left(\mu_{W}\right)}{4 \pi}\left[-\frac{\gamma^{(0)}}{2}\left(\ln \frac{M_{W}^{2}}{\mu_{W}^{2}}+\gamma_{E}-\ln 4 \pi\right)+B\right]
$$

where

$$
B=\tilde{A}^{(1)}-\tilde{r}
$$

In the leading log approximation we had simply $C\left(\mu_{W}\right)=1$. By contrast at NLO the $\mathcal{O}\left(\alpha_{\mathrm{s}}\right)$ correction has to be taken into account in addition. This correction term exhibits the following new features:

- The expression $\gamma_{E}-\ln 4 \pi$, which is characteristic to dimensional regularization appears. It is proportional to $\gamma^{(0)}$.

- A constant term $B$ is present. $B$ depends on the factorization scheme chosen.

- An explicit logarithmic dependence on the matching scale $\mu_{W}$ shows up.

We discuss these points one by one.

First, the term $\gamma_{E}-\ln 4 \pi$ is characteristic for the $M S$ scheme. It can be eliminated by going from the $M S$ - to the $\overline{M S}$ scheme. This issue is well known in the literature. We find it however useful to briefly repeat the definition of the $\overline{M S}$ scheme in the present context, since this is an important point for NLO analyses.

Consider the RG solution for the coefficient 


$$
\begin{aligned}
& C(\mu)= \\
& \left(1+\frac{\alpha_{\mathrm{s}}(\mu)}{4 \pi} J\right)\left[\frac{\alpha_{\mathrm{s}}\left(\mu_{W}\right)}{\alpha_{\mathrm{s}}(\mu)}\right]^{\frac{\gamma^{(0)}}{2 \beta_{0}}}\left(1+\frac{\alpha_{\mathrm{s}}\left(\mu_{W}\right)}{4 \pi}\left[-\frac{\gamma^{(0)}}{2}\left(\ln \frac{M_{W}^{2}}{\mu_{W}^{2}}+\gamma_{E}-\ln 4 \pi\right)+B-J\right]\right)
\end{aligned}
$$

This represents the solution for the $M S$ scheme. Therefore in (III.131) $\alpha_{\mathrm{s}}=\alpha_{s, M S}$. The redefinition of $\alpha_{s, M S}$ through

$$
\alpha_{s, M S}=\alpha_{s, \overline{M S}}\left(1+\beta_{0}\left(\gamma_{E}-\ln 4 \pi\right) \frac{\alpha_{s, \overline{M S}}}{4 \pi}\right)
$$

is a finite renormalization of the coupling, which defines the $\overline{M S}$ scheme. Since

$$
\left[\alpha_{s, M S}\left(\mu_{W}\right)\right]^{\frac{\gamma^{(0)}}{2 \beta_{0}}} \doteq\left[\alpha_{s, \overline{M S}}\left(\mu_{W}\right)\right]^{\frac{\gamma^{(0)}}{2 \beta_{0}}}\left(1+\frac{\gamma^{(0)}}{2}\left(\gamma_{E}-\ln 4 \pi\right) \frac{\alpha_{s, \overline{M S}}\left(\mu_{W}\right)}{4 \pi}\right)
$$

we see, that this transformation eliminates, to the order considered, the $\gamma_{E}-\ln 4 \pi$ term in (III.131). At the lower end of the evolution the same redefinition yields a factor

$$
1-\frac{\gamma^{(0)}}{2}\left(\gamma_{E}-\ln 4 \pi\right) \frac{\alpha_{s, \overline{M S}}(\mu)}{4 \pi}
$$

which removes the corresponding factor from the matrix element (see (III.128))

$$
\langle Q(\mu)\rangle_{M S} \equiv\left(1+\frac{\gamma^{(0)}}{2}\left(\gamma_{E}-\ln 4 \pi\right) \frac{\alpha_{s, \overline{M S}}(\mu)}{4 \pi}\right)\langle Q(\mu)\rangle_{\overline{M S}}
$$

At the next-to-leading log level we are working, the transformation (III.132) is equivalent to a redefinition of the scale $\Lambda$ according to

$$
\Lambda \frac{2}{M S}=4 \pi e^{-\gamma_{E}} \Lambda_{M S}^{2}
$$

as one can verify with the help of (III.19). In practice, one can just drop the $\left(\gamma_{E}-\ln 4 \pi\right)$ terms in (III.131). Then $\alpha_{\mathrm{s}}(\mu)$ and $\Lambda$ are to be taken in the $\overline{M S}$ scheme. Throughout the present report it is always understood that the transformation to $\overline{M S}$ has been performed. Then

$$
C(\mu)=\left(1+\frac{\alpha_{\mathrm{s}}(\mu)}{4 \pi} J\right)\left[\frac{\alpha_{\mathrm{s}}\left(\mu_{W}\right)}{\alpha_{\mathrm{s}}(\mu)}\right]^{\frac{\gamma^{(0)}}{2 \beta_{0}}}\left(1+\frac{\alpha_{\mathrm{s}}\left(\mu_{W}\right)}{4 \pi}\left[-\frac{\gamma^{(0)}}{2} \ln \frac{M_{W}^{2}}{\mu_{W}^{2}}+B-J\right]\right)
$$

Second, from the issue of the $M S-\overline{M S}$ transformation, or more generally an arbitrary redefinition of $\alpha_{\mathrm{s}}$ (or $\Lambda$ ) one should distinguish the renormalization scheme dependence due to the ambiguity in the renormalization of the operator. It suggests itself to use for the latter the term "factorization scheme dependence". This is the scheme dependence we have discussed in section IIIF3. A change in the factorization scheme transforms $\gamma^{(1)}, B$ and $J$ as

$$
\gamma^{(1)^{\prime}}=\gamma^{(1)}+2 \beta_{0} s \quad B^{\prime}=B-s \quad J^{\prime}=J-s
$$

where $s$ is a constant number. This follows from the formulae in section IIIF 3 and from the definition of $B$ in (III.130). Note that in the case of a single operator the relation between $\gamma^{(1)}$ and $J$ simplifies to 


$$
J=\frac{1}{2 \beta_{0}}\left(\frac{\beta_{1}}{\beta_{0}} \gamma^{(0)}-\gamma^{(1)}\right)
$$

Obviously the scheme dependence cancels in the difference $B-J$ in (III.137).

Third, due to the explicit $\mu_{W}$ dependence in the $\mathcal{O}\left(\alpha_{\mathrm{s}}\right)$ correction term the coefficient function is, to the order considered, independent of the precise value of the matching scale $\mu_{W}$, as it must be. Indeed

$$
\frac{d}{d \ln \mu_{W}} C(\mu)=\mathcal{O}\left(\alpha_{\mathrm{s}}^{2}\right)
$$

since

$$
\frac{d}{d \ln \mu_{W}} \alpha_{\mathrm{s}}\left(\mu_{W}\right)=-2 \beta_{0} \frac{\alpha_{\mathrm{s}}\left(\mu_{W}\right)^{2}}{4 \pi}+\mathcal{O}\left(\alpha_{\mathrm{s}}^{3}\right)
$$

In the same way one can also convince oneself that the coefficient function is independent of the heavy quark threshold scales, up to terms of the neglected order.

Of course the dependence on the low energy scale $\mu$ remains and has to be matched with the corresponding dependence of the operator matrix element.

All the points we have mentioned here apply in an analogous manner also to the case with operator mixing, only the algebra is then slightly more complicated.

We would like to stress once again that it is only at the NLO level, that these features enter the analysis in a nontrivial way, as should be evident from the presentation we have given above.

\section{Evanescent Operators}

Finally, we would like to mention the so called evanescent operators. These are operators which exist in $D \neq 4$ dimensions but vanish in $D=4$. It has been stressed in (Buras and Weisz, 1990) that a correct calculation of two-loop anomalous dimensions requires a proper treatment of these operators. This discussion has been extended in (Dugan and Grinstein, 1991) and further generalized in (Herrlich and Nierste, 1995b). In view of a rather technical nature of this aspect we refer the interested reader to the papers quoted above. 



\section{Part Two -}

\section{The Effective Hamiltonians}

The second part constitutes a compendium of effective hamiltonians for weak decays. We will deal with all decays for which NLO corrections have been calculated in the literature and whose list is given in table I. This includes a listing of the initial conditions $C_{i}\left(M_{\mathrm{W}}\right)$, a listing of all oneloop and two-loop anomalous dimension matrices and finally tables of numerical values of the relevant Wilson coefficients as functions of $\Lambda_{\overline{\mathrm{MS}}}, m_{\mathrm{t}}$ and the renormalization schemes considered. In certain cases we are able to give analytic formulae for $C_{i}$.

We will discuss all effective hamiltonians one by one. With the help of the master formulae and the procedure of section III it is easy to see similarities and differences between various cases. Our compendium includes also the $b \rightarrow s \gamma$ and $b \rightarrow s g$ transitions which although known only in the leading logarithmic approximation deserve special attention.

Finally, as a preparation for the third part we give a brief description of the "Penguin-Box Expansion" (PBE), which can be regarded as a version of OPE particularly suited for the study of the $m_{t}$ dependence in weak decays.

In addition we have also included a section on NLO QCD calculations in the context of HQET. This chapter lies somewhat outside our main line of presentation. Also a comprehensive discussion of HQET is clearly beyond the scope of the present paper. However, we would like to illustrate the application of the general formalism for short distance QCD corrections within this framework and summarize a few important NLO results that have been obtained in HQET. 


\section{GUIDE TO EFFECTIVE HAMILTONIANS}

In order to facilitate the presentation of effective hamiltonians in weak decays we give a complete compilation of the relevant operators below. Divided into six classes, these operators play a dominant role in the phenomenology of weak decays. The six classes are given as follows

Current-Current Operators (fig. 4 (a)):

$$
Q_{1}=\left(\bar{s}_{i} u_{j}\right)_{\mathrm{V}-\mathrm{A}}\left(\bar{u}_{j} d_{i}\right)_{\mathrm{V}-\mathrm{A}} \quad Q_{2}=(\bar{s} u)_{\mathrm{V}-\mathrm{A}}(\bar{u} d)_{\mathrm{V}-\mathrm{A}}
$$

QCD-Penguins Operators (fig. 4 (b)):

$$
\begin{aligned}
Q_{3} & =(\bar{s} d)_{\mathrm{V}-\mathrm{A}} \sum_{q}(\bar{q} q)_{\mathrm{V}-\mathrm{A}} & Q_{4} & =\left(\bar{s}_{i} d_{j}\right)_{\mathrm{V}-\mathrm{A}} \sum_{q}\left(\bar{q}_{j} q_{i}\right)_{\mathrm{V}-\mathrm{A}} \\
Q_{5} & =(\bar{s} d)_{\mathrm{V}-\mathrm{A}} \sum_{q}(\bar{q} q)_{\mathrm{V}+\mathrm{A}} & Q_{6} & =\left(\bar{s}_{i} d_{j}\right)_{\mathrm{V}-\mathrm{A}} \sum_{q}\left(\bar{q}_{j} q_{i}\right)_{\mathrm{V}+\mathrm{A}}
\end{aligned}
$$

Electroweak-Penguins Operators (fig. $4(\mathrm{c}))$ :

$$
\begin{aligned}
Q_{7} & =\frac{3}{2}(\bar{s} d)_{\mathrm{V}-\mathrm{A}} \sum_{q} e_{q}(\bar{q} q)_{\mathrm{V}+\mathrm{A}} & Q_{8} & =\frac{3}{2}\left(\bar{s}_{i} d_{j}\right)_{\mathrm{V}-\mathrm{A}} \sum_{q} e_{q}\left(\bar{q}_{j} q_{i}\right)_{\mathrm{V}+\mathrm{A}} \\
Q_{9} & =\frac{3}{2}(\bar{s} d)_{\mathrm{V}-\mathrm{A}} \sum_{q} e_{q}(\bar{q} q)_{\mathrm{V}-\mathrm{A}} & Q_{10} & =\frac{3}{2}\left(\bar{s}_{i} d_{j}\right)_{\mathrm{V}-\mathrm{A}} \sum_{q} e_{q}\left(\bar{q}_{j} q_{i}\right)_{\mathrm{V}-\mathrm{A}}
\end{aligned}
$$

Magnetic-Penguins Operators (fig. $4(\mathrm{~d}))$ :

$$
Q_{7 \gamma}=\frac{e}{8 \pi^{2}} m_{\mathrm{b}} \bar{s}_{i} \sigma^{\mu \nu}\left(1+\gamma_{5}\right) b_{i} F_{\mu \nu} \quad Q_{8 G}=\frac{g}{8 \pi^{2}} m_{\mathrm{b}} \bar{s}_{i} \sigma^{\mu \nu}\left(1+\gamma_{5}\right) T_{i j}^{a} b_{j} G_{\mu \nu}^{a}
$$

$\Delta S=2$ and $\Delta B=2$ Operators (fig. $4(\mathbf{e})$ ):

$$
Q(\Delta S=2)=(\bar{s} d)_{V-A}(\bar{s} d)_{V-A} \quad Q(\Delta B=2)=(\bar{b} d)_{V-A}(\bar{b} d)_{V-A}
$$

Semi-Leptonic Operators (fig. 4 (f)):

$$
\begin{array}{rl}
Q_{7 V}=(\bar{s} d)_{V-A}(\bar{e} e)_{V} & Q_{7 A}=(\bar{s} d)_{V-A}(\bar{e} e)_{A} \\
Q_{9 V}=(\bar{b} s)_{V-A}(\bar{e} e)_{V} & Q_{10 A}=(\bar{b} s)_{V-A}(\bar{e} e)_{A} \\
Q(\bar{\nu} \nu)=(\bar{s} d)_{V-A}(\bar{\nu} \nu)_{V-A} & Q(\bar{\mu} \mu)=(\bar{s} d)_{V-A}(\bar{\mu} \mu)_{V-A}
\end{array}
$$

where indices in color singlet currents have been suppressd for simplicity.

For illustrative purposes, typical diagrams in the full theory from which the operators (IV.1)(IV.10) originate are shown in fig. 4.

The operators listed above will enter our review in a systematic fashion. We begin in section $\mathrm{V}$ with the presentation of the effective hamiltonians involving the current-current operators $Q_{1}$ and $Q_{2}$ only. These effective hamiltonians are given in (V.4), (V.5) and (V.6) for $\Delta B=1, \Delta C=1$ and $\Delta S=1$ non-leptonic decays, respectively.

In section VI we will generalize the hamiltonians (V.4) and (V.6) to include the QCD-penguin operators $Q_{3}-Q_{6}$. The corresponding expressions are given in (VI.32) and (VI.1), respectively. This generalization does not affect the Wilson coefficients of $Q_{1}$ and $Q_{2}$. 

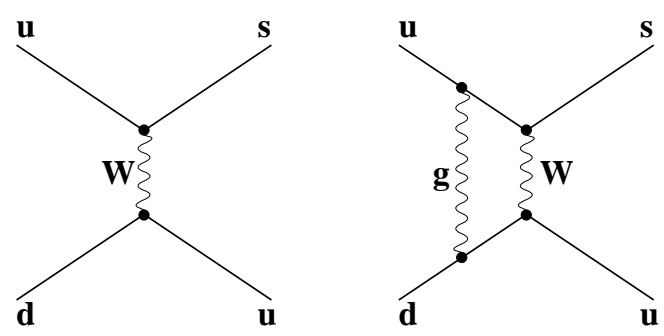

(a)
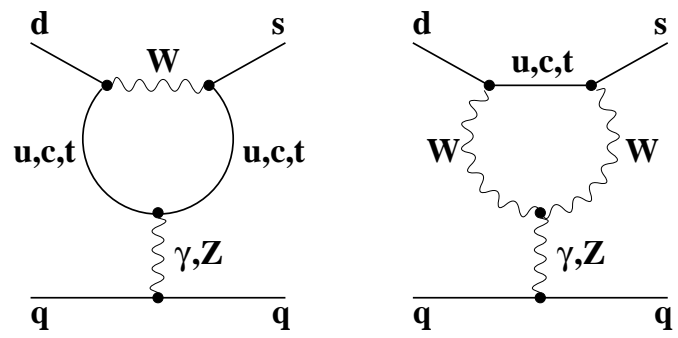

(c)

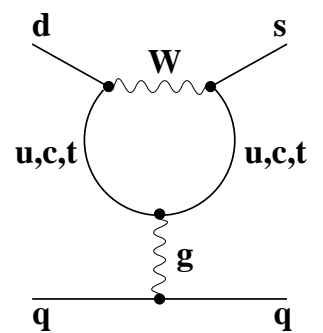

(b)

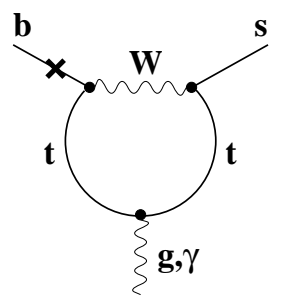

(d)

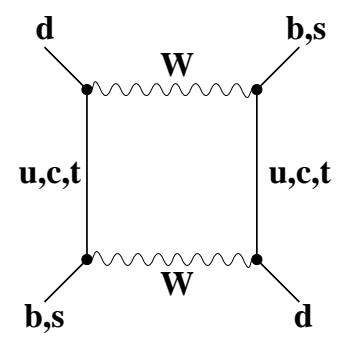

(e)

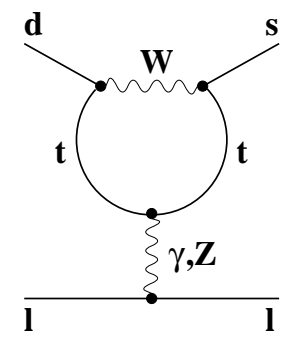

(f)

FIG. 4. Typical diagrams in the full theory from which the operators (IV.1)-(IV.10) originate. The cross in diagram (d) means a mass-insertion. It indicates that magnetic penguins originate from the mass-term on the external line in the usual QCD or QED penguin diagrams.

Next in section VII the $\Delta S=1$ and $\Delta B=1$ hamiltonians of section VI will be generalized to include the electroweak penguin operators $Q_{7}-Q_{10}$. These generalized hamiltonians are given in (VII.1) and (VII.37) for $\Delta S=1$ and $\Delta B=1$ non-leptonic decays, respectively. The inclusion of the electroweak penguin operators implies the inclusion of $Q E D$ effects. Consequently the coefficients of the operators $Q_{1}-Q_{6}$ given in this section will differ slightly from the ones presented in the previous sections.

In section VIII the effective hamiltonian for $K_{L} \rightarrow \pi^{0} e^{+} e^{-}$will be presented. It is given in (VIII.1). This hamiltonian can be considered as a generalization of the $\Delta S=1$ hamiltonian (VI.1) presented in section VI to include the semi-leptonic operators $Q_{7 V}$ and $Q_{7 A}$. This generalization does not modify the numerical values of the $\Delta S=1$ coefficients $C_{i}(i=1, \ldots, 6)$ given in section VI.

In section IX we will discuss the effective hamiltonian for $B \rightarrow X_{s} \gamma$. It is written down in (IX.1). This hamiltonian can be considered as a generalization of the $\Delta B=1$ hamiltonian (VI.32) to include the magnetic penguin operators $Q_{7 \gamma}$ and $Q_{8 G}$. This generalization does not modify the numerical values of the $\Delta B=1$ coefficients $C_{i}(i=1, \ldots, 6)$ from section VI. 
In section $\mathrm{X}$ we present the effective hamiltonian for $B \rightarrow X_{s} e^{+} e^{-}$. It is to be found in (X.1) and can be considered as the generalization of the $B \rightarrow X_{s} \gamma$ hamiltonian to include the semileptonic operators $Q_{9 V}$ and $Q_{10 A}$. The coefficients $C_{i}(i=1, \ldots, 6,7 \gamma, 8 G)$ given in section IX are not affected by this generalization.

In section XI the effective hamiltonians for $K^{+} \rightarrow \pi^{+} \nu \bar{\nu}, K_{L} \rightarrow \mu^{+} \mu^{-}, K_{L} \rightarrow \pi^{0} \nu \bar{\nu}(B \rightarrow$ $\left.X_{s, d} \nu \bar{\nu}\right)$ and $B \rightarrow l^{+} l^{-}$will be discussed. They are given in (XI.4), (XI.44), (XI.56) and (XI.57) respectively. Each of these hamiltonians involves only a single operator: $Q(\nu \bar{\nu})$ or $Q(\mu \bar{\mu})$ for $K^{+} \rightarrow \pi^{+} \nu \bar{\nu}\left(K_{L} \rightarrow \pi^{0} \nu \bar{\nu}\right)$ and $K_{L} \rightarrow \mu^{+} \mu^{-}$with analogous operators for $B \rightarrow X_{s, d} \nu \bar{\nu}$ and $B \rightarrow l^{+} l^{-}$.

Finally, sections XII and XIII present the effective hamiltonians for $\Delta S=2$ and $\Delta B=2$ transitions, respectively. These hamiltonians involve the operators $Q(\Delta S=2)$ and $Q(\Delta B=2)$ and can be found in (XII.1) and (XIII.1).

In table III we give the list of effective hamiltonians to be presented below, the equations in which they can be found and the list of operators entering different hamiltonians.

TABLE III. Compilation of various processes, equation no. of the corresponding effective hamiltonians and contributing operators.

\begin{tabular}{|l|l|l|}
\hline \hline \multicolumn{1}{|c|}{ Process } & \multicolumn{1}{c|}{ Cf. Equation } & \multicolumn{1}{c|}{ Contributing Operators } \\
\hline$\Delta F=1, F=B, C, S$ current-current & (V.4)-(V.6) & $Q_{1}, Q_{2}$ \\
$\Delta F=1$ pure QCD & (VI.1), (VI.32) & $Q_{1}, \ldots, Q_{6}$ \\
$\Delta F=1$ QCD and electroweak & (VII.1), (VII.37) & $Q_{1}, \ldots, Q_{10}$ \\
\hline$K_{L} \rightarrow \pi^{0} e^{+} e^{-}$ & (VIII.1) & $Q_{1}, \ldots, Q_{6}, Q_{7 V}, Q_{7 A}$ \\
\hline$B \rightarrow X_{s} \gamma$ & (IX.1) & $Q_{1}, \ldots, Q_{6}, Q_{7 \gamma}, Q_{8 G}$ \\
$B \rightarrow X_{s} e^{+} e^{-}$ & (X.1) & $Q_{1}, \ldots, Q_{6}, Q_{7 \gamma}, Q_{8 G}, Q_{9 V}, Q_{10 A}$ \\
\hline$K^{+} \rightarrow \pi^{+} \nu \bar{\nu},\left(K_{L} \rightarrow \mu^{+} \mu^{-}\right)_{S D}, K_{L} \rightarrow \pi^{0} \nu \bar{\nu}$, & (XI.4), (XI.44), (XI.56) & $Q(\bar{\nu} \nu), Q(\bar{\mu} \mu)$ \\
$B \rightarrow X_{s, d} \nu \bar{\nu}, B \rightarrow l^{+} l^{-}$ & \\
\hline$K^{0}-\bar{K}^{0}$ mixing & (XI.57) & $Q(\Delta S=2)$ \\
$B^{0}-\bar{B}^{0}$ mixing & (XII.1) & $Q(\Delta B=2)$ \\
\hline \hline
\end{tabular}




\section{THE EFFECTIVE $\Delta F=1$ HAMILTONIAN: CURRENT-CURRENT OPERATORS}

\section{A. Operators}

We begin our compendium by presenting the parts of effective hamiltonians involving the current-current operators only. These operators will be generally denoted by $Q_{1}$ and $Q_{2}$, although their flavour structure depends on the decay considered. To be specific we will consider

$$
\begin{aligned}
Q_{1}=\left(\bar{b}_{i} c_{j}\right)_{V-A}\left(\bar{u}_{j} d_{i}\right)_{V-A} & Q_{2}=\left(\bar{b}_{i} c_{i}\right)_{V-A}\left(\bar{u}_{j} d_{j}\right)_{V-A} \\
Q_{1}=\left(\bar{s}_{i} c_{j}\right)_{V-A}\left(\bar{u}_{j} d_{i}\right)_{V-A} & Q_{2}=\left(\bar{s}_{i} c_{i}\right)_{V-A}\left(\bar{u}_{j} d_{j}\right)_{V-A} \\
Q_{1}=\left(\bar{s}_{i} u_{j}\right)_{V-A}\left(\bar{u}_{j} d_{i}\right)_{V-A} & Q_{2}=\left(\bar{s}_{i} u_{i}\right)_{V-A}\left(\bar{u}_{j} d_{j}\right)_{V-A}
\end{aligned}
$$

for $\Delta B=1, \Delta C=1$ and $\Delta S=1$ decays respectively. Then the corresponding effective hamiltonians are given by

$$
\begin{array}{cc}
H_{e f f}(\Delta B=1)=\frac{G_{F}}{\sqrt{2}} V_{c b}^{*} V_{u d}\left[C_{1}(\mu) Q_{1}+C_{2}(\mu) Q_{2}\right] & \left(\mu=O\left(m_{b}\right)\right) \\
H_{e f f}(\Delta C=1)=\frac{G_{F}}{\sqrt{2}} V_{c s}^{*} V_{u d}\left[C_{1}(\mu) Q_{1}+C_{2}(\mu) Q_{2}\right] & \left(\mu=O\left(m_{c}\right)\right) \\
H_{\text {eff }}(\Delta S=1)=\frac{G_{F}}{\sqrt{2}} V_{u s}^{*} V_{u d}\left[C_{1}(\mu) Q_{1}+C_{2}(\mu) Q_{2}\right] \quad(\mu=O(1 \mathrm{GeV}))
\end{array}
$$

As we will see in subsequent sections these hamiltonians have to be generalized to include also penguin operators. This however will not change the Wilson coefficients $C_{1}(\mu)$ and $C_{2}(\mu)$ except for small $O(\alpha)$ corrections in a complete analysis which includes also electroweak penguin operators. For this reason it is useful to present the results for $C_{1,2}$ separately as they can be used in a large class of decays.

When analyzing $Q_{1}$ and $Q_{2}$ in isolation, it is useful to work with the operators $Q_{ \pm}$and their coefficients $z_{ \pm}$defined by

$$
Q_{ \pm}=\frac{1}{2}\left(Q_{2} \pm Q_{1}\right) \quad z_{ \pm}=C_{2} \pm C_{1}
$$

$Q_{+}$and $Q_{-}$do not mix under renormalization and the expression for $z_{ \pm}(\mu)$ is very simple.

\section{B. Wilson Coefficients and RG Evolution}

The initial conditions for $z_{ \pm}$at $\mu=M_{W}$ are obtained using the matching procedure between the full (fig. 2 (a)-(c)) and effective (fig. 3 (a)-(c)) theory summarized in section III F 1. Given the initial conditions for $z_{ \pm}$at scale $\mu=M_{W}$ 


$$
z_{ \pm}\left(M_{W}\right)=1+\frac{\alpha_{\mathrm{s}}\left(M_{W}\right)}{4 \pi} B_{ \pm}
$$

and using the NLO RG evolution formula (III.99) for the case without mixing one finds for the Wilson coefficients of $Q_{ \pm}$at some scale $\mu$

$$
z_{ \pm}(\mu)=\left[1+\frac{\alpha_{\mathrm{s}}(\mu)}{4 \pi} J_{ \pm}\right]\left[\frac{\alpha_{\mathrm{s}}\left(M_{W}\right)}{\alpha_{\mathrm{s}}(\mu)}\right]^{d_{ \pm}}\left[1+\frac{\alpha_{\mathrm{s}}\left(M_{W}\right)}{4 \pi}\left(B_{ \pm}-J_{ \pm}\right)\right]
$$

with

$$
J_{ \pm}=\frac{d_{ \pm}}{\beta_{0}} \beta_{1}-\frac{\gamma_{ \pm}^{(1)}}{2 \beta_{0}} \quad d_{ \pm}=\frac{\gamma_{ \pm}^{(0)}}{2 \beta_{0}}
$$

where the coefficients $\beta_{0}$ and $\beta_{1}$ of the QCD $\beta$-function are given in (III.16). Furthermore the LO and NLO expansion coefficients for the anomalous dimensions $\gamma_{ \pm}$of $Q_{ \pm}$in (V.10) and the coefficients $B_{ \pm}$in (V.8) are given by

$$
\begin{gathered}
\gamma_{ \pm}^{(0)}= \pm 12 \frac{N \mp 1}{2 N} \\
\gamma_{ \pm}^{(1)}=\frac{N \mp 1}{2 N}\left[-21 \pm \frac{57}{N} \mp \frac{19}{3} N \pm \frac{4}{3} f-2 \beta_{0} \kappa_{ \pm}\right] \\
B_{ \pm}=\frac{N \mp 1}{2 N}\left[ \pm 11+\kappa_{ \pm}\right]
\end{gathered}
$$

with $N$ being the number of colors. Here we have introduced the parameter $\kappa_{ \pm}$which conveniently distinguishes between various renormalization schemes

$$
\kappa_{ \pm}=\left\{\begin{array}{rl}
0 & \mathrm{NDR} \\
\mp 4 & \mathrm{HV}
\end{array} .\right.
$$

Thus, using $N=3$ in the following, $J_{ \pm}$in (V.10) can also be written as

$$
J_{ \pm}=\left(J_{ \pm}\right)_{\mathrm{NDR}}+\frac{3 \mp 1}{6} \kappa_{ \pm}=\left(J_{ \pm}\right)_{\mathrm{NDR}} \pm \frac{\gamma_{ \pm}^{(0)}}{12} \kappa_{ \pm}
$$

Setting $\gamma_{ \pm}^{(1)}, B_{ \pm}$and $\beta_{1}$ to zero gives the leading logarithmic approximation (Altarelli and Maiani, 1974), (Gaillard and Lee, 1974a).

The NLO calculations in the NDR scheme and in the HV scheme have been presented in (Buras and Weisz, 1990). In writing (V.12) we have incorporated the $-2 \gamma_{J}^{(1)}$ correction in the HV scheme resulting from the non-vanishing two-loop anomalous dimension of the weak current.

$$
\gamma_{J}^{(1)}= \begin{cases}0 & \text { NDR } \\ \frac{N^{2}-1}{N} 2 \beta_{0} & \mathrm{HV}\end{cases}
$$

The NLO corrections $\gamma_{ \pm}^{(1)}$ in the dimensional reduction scheme (DRED) have been first considered in (Altarelli et al., 1981) and later confirmed in (Buras and Weisz, 1990). Here one has $\kappa_{ \pm}=$ 
$\mp 6-N$. This value for $\kappa_{ \pm}$in DRED incorporates also a finite renormalization of $\alpha_{\mathrm{s}}$ in order to work in all schemes with the usual $\overline{M S}$ coupling.

As already discussed in section III F 3, the expression $\left(B_{ \pm}-J_{ \pm}\right)$is scheme independent. The scheme dependence of the Wilson coeffcients $z_{ \pm}(\mu)$ originates then entirely from the scheme dependence of $J_{ \pm}$at the lower end of the evolution which can be seen explicitly in (V.15).

In order to exhibit the $\mu$ dependence on the same footing as the scheme dependence, it is useful to rewrite (V.9) in the case of B-decays as follows:

$$
z_{ \pm}(\mu)=\left[1+\frac{\alpha_{\mathrm{s}}\left(m_{b}\right)}{4 \pi} \tilde{J}_{ \pm}(\mu)\right]\left[\frac{\alpha_{\mathrm{s}}\left(M_{W}\right)}{\alpha_{\mathrm{s}}\left(m_{b}\right)}\right]^{d_{ \pm}}\left[1+\frac{\alpha_{\mathrm{s}}\left(M_{W}\right)}{4 \pi}\left(B_{ \pm}-J_{ \pm}\right)\right]
$$

with

$$
\tilde{J}_{ \pm}(\mu)=\left(J_{ \pm}\right)_{N D R} \pm \frac{\gamma_{ \pm}^{(0)}}{12} \kappa_{ \pm}+\frac{\gamma_{ \pm}^{(0)}}{2} \ln \left(\frac{\mu^{2}}{m_{b}^{2}}\right)
$$

summarizing both the renormalization scheme dependence and the $\mu$-dependence. Note that in the first parenthesis in (V.17) we have set $\alpha_{\mathrm{s}}(\mu)=\alpha_{\mathrm{s}}\left(m_{b}\right)$ as the difference in the scales in this correction is still of higher order. We also note that a change of the renormalization scheme can be compensated by a change in $\mu$. From (V.18) we find generally

$$
\mu_{i}^{ \pm}=\mu_{N D R} \exp \left(\mp \frac{\kappa_{ \pm}^{(i)}}{12}\right)
$$

where $i$ denotes a given scheme. From (V.14) we then have

$$
\mu_{H V}=\mu_{N D R} \exp \left(\frac{1}{3}\right)
$$

Evidently the change in $\mu$ relating $\mathrm{HV}$ and $\mathrm{NDR}^{1}$ is the same for $z_{+}$and $z_{-}$and consequently for $C_{i}(\mu)$.

This discussion shows that a meaningful analysis of the $\mu$ dependence of $C_{i}(\mu)$ can only be made simultaneously with the analysis of the scheme dependence.

The coefficients $C_{i}(\mu)$ for B-decays can now be calculated using

$$
C_{1}(\mu)=\frac{z_{+}(\mu)-z_{-}(\mu)}{2} \quad C_{2}(\mu)=\frac{z_{+}(\mu)+z_{-}(\mu)}{2}
$$

To this end we set $f=5$ in the formulae above and use the two-loop $\alpha_{\mathrm{s}}(\mu)$ of eq. (III.19) with $\Lambda \equiv \Lambda \frac{(5)}{\mathrm{MS}}$. The actual numerical values used for $\alpha_{\mathrm{s}}\left(M_{Z}\right)$ or equivalently $\Lambda \frac{(5)}{\mathrm{MS}}$ are collected in appendix A together with other numerical input parameters.

In the case of D-decays and K-decays the relevant scales are $\mu=\mathcal{O}\left(m_{c}\right)$ and $\mu=\mathcal{O}(1 \mathrm{GeV})$, respectively. In order to calculate $C_{i}(\mu)$ for these cases one has to evolve these coefficients first

\footnotetext{
${ }^{1}$ The relation $\mu_{\mathrm{DRED}}^{ \pm}=\mu_{\mathrm{NDR}} \exp \left(\frac{2 \pm 1}{4}\right)$ between NDR and DRED is more involved. In any case $\mu_{\mathrm{HV}}$ and $\mu_{\mathrm{DRED}}^{ \pm}$are larger than $\mu_{\mathrm{NDR}}$.
} 
from $\mu=\mathcal{O}\left(m_{b}\right)$ further down to $\mu=\mathcal{O}\left(m_{c}\right)$ in an effective theory with $f=4$. Matching $\alpha_{\mathrm{s}}^{(5)}\left(m_{b}\right)=\alpha_{\mathrm{s}}^{(4)}\left(m_{b}\right)$ we find to a very good approximation $\Lambda_{\overline{\mathrm{MS}}}^{(4)}=(325 \pm 110) \mathrm{MeV}$. Unfortunately, the necessity to evolve $C_{i}(\mu)$ from $\mu=M_{W}$ down to $\mu=m_{c}$ in two different effective theories ( $f=5$ and $f=4$ ) and eventually in the case of K-decays with $f=3$ for $\mu<m_{c}$ makes the formulae for $C_{i}(\mu)$ in $\mathrm{D}$-decays and $\mathrm{K}$-decays rather complicated. They can be found in (Buras et al., 1993b). Fortunately all these complications can be avoided by a simple trick, which reproduces the results of (Buras et al., 1993b) to better than $1.5 \%$. In order to find $C_{i}(\mu)$ for $1 \mathrm{GeV} \leq \mu \leq 2 \mathrm{GeV}$ one can simply use the master formulae given above with $\Lambda \frac{(5)}{\mathrm{MS}}$ replaced by $\Lambda \frac{(4)}{\mathrm{MS}}$ and an "effective" number of active flavours $f=4.15$. The latter effective value for $f$ allows to obtain a very good agreement with (Buras et al., 1993b). This can be verified by comparing the results presented here with those in tables X and XII where no "tricks" have been used. The nice feature of this method is that the $\mu$ and renormalization scheme dependences of $C_{i}(\mu)$ can still be studied in simple terms.

The numerical coefficients $C_{i}(\mu)$ for $\mathrm{B}$-decays are shown in tables IV and $\mathrm{V}$ for different $\mu$ and $\Lambda \frac{(5)}{\mathrm{MS}}$. In addition to the results for the NDR and HV renormalization schemes we show the LO values $^{2}$. The corresponding results for $\mathrm{K}$-decays and D-decays are given in tables VI and VII.

TABLE IV. The coefficient $C_{1}(\mu)$ for B-decays.

\begin{tabular}{|c|c|c|c||r|r|r||r|r|r|}
\hline \hline & \multicolumn{3}{|c||}{$\Lambda \frac{(5)}{\mathrm{MS}}=140 \mathrm{MeV}$} & \multicolumn{3}{c||}{$\Lambda \frac{(5)}{\mathrm{MS}}=225 \mathrm{MeV}$} & \multicolumn{3}{c|}{$\Lambda \frac{(5)}{\mathrm{MS}}=310 \mathrm{MeV}$} \\
\hline$\mu[\mathrm{GeV}]$ & LO & NDR & \multicolumn{1}{c||}{ HV } & LO & NDR & HV & LO & NDR & HV \\
\hline \hline 4.0 & -0.274 & -0.175 & -0.211 & -0.310 & -0.197 & -0.239 & -0.341 & -0.216 & -0.264 \\
\hline 5.0 & -0.244 & -0.151 & -0.184 & -0.274 & -0.169 & -0.208 & -0.300 & -0.185 & -0.228 \\
\hline 6.0 & -0.221 & -0.133 & -0.164 & -0.248 & -0.148 & -0.184 & -0.269 & -0.161 & -0.201 \\
\hline 7.0 & -0.203 & -0.118 & -0.148 & -0.226 & -0.132 & -0.166 & -0.246 & -0.143 & -0.181 \\
\hline 8.0 & -0.188 & -0.106 & -0.135 & -0.209 & -0.118 & -0.151 & -0.226 & -0.128 & -0.164 \\
\hline \hline
\end{tabular}

TABLE V. The coefficient $C_{2}(\mu)$ for B-decays.

\begin{tabular}{|c|r|r|r||r|r|r||r|r|r|}
\hline \hline & \multicolumn{3}{|c||}{$\Lambda_{\overline{\mathrm{MS}}}^{(5)}=140 \mathrm{MeV}$} & \multicolumn{3}{c||}{$\Lambda_{\overline{\mathrm{MS}}}^{(5)}=225 \mathrm{MeV}$} & \multicolumn{3}{|c|}{$\Lambda_{\overline{\mathrm{MS}}}^{(5)}=310 \mathrm{MeV}$} \\
\hline$\mu[\mathrm{GeV}]$ & LO & NDR & HV & LO & NDR & HV & LO & NDR & HV \\
\hline \hline 4.0 & 1.121 & 1.074 & 1.092 & 1.141 & 1.086 & 1.107 & 1.158 & 1.096 & 1.120 \\
\hline 5.0 & 1.105 & 1.062 & 1.078 & 1.121 & 1.072 & 1.090 & 1.135 & 1.080 & 1.101 \\
\hline 6.0 & 1.093 & 1.054 & 1.069 & 1.107 & 1.062 & 1.079 & 1.118 & 1.068 & 1.087 \\
\hline 7.0 & 1.084 & 1.047 & 1.061 & 1.096 & 1.054 & 1.069 & 1.106 & 1.059 & 1.077 \\
\hline 8.0 & 1.077 & 1.042 & 1.055 & 1.087 & 1.047 & 1.062 & 1.096 & 1.052 & 1.069 \\
\hline \hline
\end{tabular}

\footnotetext{
${ }^{2}$ The results for the DRED scheme can be found in (Buras, 1995).
} 
From tables IV-IX we observe:

- The scheme dependence of the Wilson coefficients is sizable. This is in particular the case of $C_{1}$ which vanishes in the absence of QCD corrections.

- The differences between LO and NLO results in the case of $C_{1}$ are large showing the importance of next-to-leading corrections. In fact in the NDR scheme the corrections may be as large as $70 \%$. This comparison of LO and NLO coefficients can however be questioned because for the chosen values of $\Lambda_{\overline{\mathrm{MS}}}$ one has $\alpha_{\mathrm{s}}^{(L O)}\left(M_{Z}\right)=0.135 \pm 0.009$ to be compared with $\alpha_{\mathrm{s}}\left(M_{Z}\right)=0.117 \pm 0.007$ (Bethke, 1994), (Webber, 1994). Consequently the difference in LO and NLO results for $C_{i}$ originates partly in the change in the value of the QCD coupling.

- In view of the latter fact it is instructive to show also the LO results in which the next-toleading expression for $\alpha_{\mathrm{s}}$ is used. We give some examples in tables VIII and IX. Now the differences between LO and NLO results is considerably smaller although still as large as $30-40 \%$ in the case of $C_{1}$ and the NDR scheme.

- In any case the inclusion of NLO corrections in NDR and HV schemes weakens the impact of QCD on the Wilson coefficients of current-current operators. It is however important to keep in mind that such a behavior is specific to the scheme chosen and will in general be different in other schemes, reflecting the unphysical nature of the Wilson coefficient functions.

TABLE VI. The coefficient $C_{1}(\mu)$ for K-decays and D-decays.

\begin{tabular}{|c|c|c|c||r|r|r||r|r|r|}
\hline \hline & \multicolumn{3}{|c||}{$\Lambda \frac{(4)}{\mathrm{MS}}=215 \mathrm{MeV}$} & \multicolumn{3}{c||}{$\Lambda_{\overline{\mathrm{MS}}}^{(4)}=325 \mathrm{MeV}$} & \multicolumn{3}{c|}{$\Lambda_{\overline{\mathrm{MS}}}^{(4)}=435 \mathrm{MeV}$} \\
\hline$\mu[\mathrm{GeV}]$ & LO & NDR & \multicolumn{1}{c||}{ HV } & LO & NDR & HV & LO & NDR & HV \\
\hline \hline 1.00 & -0.602 & -0.410 & -0.491 & -0.742 & -0.510 & -0.631 & -0.899 & -0.632 & -0.825 \\
\hline 1.25 & -0.529 & -0.356 & -0.424 & -0.636 & -0.430 & -0.523 & -0.747 & -0.512 & -0.642 \\
\hline 1.50 & -0.478 & -0.319 & -0.379 & -0.565 & -0.378 & -0.457 & -0.653 & -0.439 & -0.543 \\
\hline 1.75 & -0.439 & -0.291 & -0.346 & -0.514 & -0.340 & -0.410 & -0.587 & -0.390 & -0.478 \\
\hline 2.00 & -0.409 & -0.269 & -0.320 & -0.475 & -0.311 & -0.375 & -0.537 & -0.353 & -0.431 \\
\hline \hline
\end{tabular}

We have made the whole discussion without invoking HQET (cf. section XV). It is sometimes stated in the literature that at $\mu=m_{b}$ in the case of B-decays one has to switch to HQET. In this case for $\mu<m_{b}$ the anomalous dimensions $\gamma_{ \pm}$differ from those given above. We should however stress that switching to HQET can be done at any $\mu<m_{b}$ provided the logarithms $\ln \left(m_{b} / \mu\right)$ in $\left\langle Q_{i}\right\rangle$ do not become too large. Similar comments apply to D-decays with respect to $\mu=m_{c}$. Of course the coefficients $C_{i}$ calculated in HQET for $\mu<m_{b}$ are different from the coefficients presented here. However the corresponding matrix elements $\left\langle Q_{i}\right\rangle$ in HQET are also different so that the physical amplitudes remain unchanged. 
TABLE VII. The coefficient $C_{2}(\mu)$ for K-decays and D-decays.

\begin{tabular}{|c|r|r|r||r|r|r||r|r|r|}
\hline \hline & \multicolumn{3}{|c||}{$\Lambda_{\overline{\mathrm{MS}}}^{(4)}=215 \mathrm{MeV}$} & \multicolumn{3}{c||}{$\Lambda_{\overline{\mathrm{MS}}}^{(4)}=325 \mathrm{MeV}$} & \multicolumn{3}{|c|}{$\Lambda_{\overline{\mathrm{MS}}}^{(4)}=435 \mathrm{MeV}$} \\
\hline$\mu[\mathrm{GeV}]$ & LO & NDR & HV & LO & NDR & HV & LO & NDR & HV \\
\hline \hline 1.00 & 1.323 & 1.208 & 1.259 & 1.422 & 1.275 & 1.358 & 1.539 & 1.363 & 1.506 \\
\hline 1.25 & 1.274 & 1.174 & 1.216 & 1.346 & 1.221 & 1.282 & 1.426 & 1.277 & 1.367 \\
\hline 1.50 & 1.241 & 1.152 & 1.187 & 1.298 & 1.188 & 1.237 & 1.358 & 1.228 & 1.296 \\
\hline 1.75 & 1.216 & 1.136 & 1.167 & 1.264 & 1.165 & 1.207 & 1.313 & 1.196 & 1.252 \\
\hline 2.00 & 1.198 & 1.123 & 1.152 & 1.239 & 1.148 & 1.185 & 1.279 & 1.174 & 1.221 \\
\hline \hline
\end{tabular}

TABLE VIII. $C_{1}^{\mathrm{LO}}$ and $C_{2}^{\mathrm{LO}}$ for B-decays with $\alpha_{\mathrm{s}}$ in NLO.

\begin{tabular}{|c|c|c||c|c||c|c|}
\hline \hline & \multicolumn{2}{|c||}{$\Lambda_{\overline{\mathrm{MS}}}^{(5)}=140 \mathrm{MeV}$} & \multicolumn{2}{c||}{$\Lambda_{\overline{\mathrm{MS}}}^{(5)}=225 \mathrm{MeV}$} & \multicolumn{2}{c|}{$\Lambda_{\overline{\mathrm{MS}}}^{(5)}=310 \mathrm{MeV}$} \\
\hline$\mu[\mathrm{GeV}]$ & $C_{1}$ & $C_{2}$ & $C_{1}$ & $C_{2}$ & $C_{1}$ & $C_{2}$ \\
\hline \hline 4.0 & -0.244 & 1.105 & -0.274 & 1.121 & -0.301 & 1.135 \\
\hline 5.0 & -0.217 & 1.091 & -0.243 & 1.105 & -0.265 & 1.116 \\
\hline 6.0 & -0.197 & 1.082 & -0.220 & 1.093 & -0.239 & 1.102 \\
\hline \hline
\end{tabular}

TABLE IX. $C_{1}^{\mathrm{LO}}$ and $C_{2}^{\mathrm{LO}}$ for K and D-decays with $\alpha_{\mathrm{s}}$ in NLO.

\begin{tabular}{|c|c|c||c|c||c|c|}
\hline \hline & \multicolumn{2}{|c||}{$\Lambda_{\overline{\mathrm{MS}}}^{(4)}=215 \mathrm{MeV}$} & \multicolumn{2}{c||}{$\Lambda_{\frac{(4)}{\mathrm{MS}}}=325 \mathrm{MeV}$} & \multicolumn{2}{c|}{$\Lambda_{\overline{\mathrm{MS}}}^{(4)}=435 \mathrm{MeV}$} \\
\hline$\mu[\mathrm{GeV}]$ & $C_{1}$ & $C_{2}$ & $C_{1}$ & $C_{2}$ & $C_{1}$ & $C_{2}$ \\
\hline \hline 1.0 & -0.524 & 1.271 & -0.664 & 1.366 & -0.851 & 1.502 \\
\hline 1.5 & -0.413 & 1.201 & -0.493 & 1.250 & -0.579 & 1.307 \\
\hline 2.0 & -0.354 & 1.165 & -0.412 & 1.200 & -0.469 & 1.235 \\
\hline \hline
\end{tabular}




\section{THE EFFECTIVE $\Delta F=1$ HAMILTONIAN: INCLUSION OF QCD PENGUIN OPERATORS}

In section $\mathrm{V}$ we have restricted ourselves to current-current operators when considering QCD corrections to the effective $\Delta F=1(F=B, C, S)$ hamiltonian for weak decays.

As already mentioned in section IIID 3 e.g. for the $\Delta S=1$ case the special flavour structure of $Q_{2}=(\bar{s} u)_{\mathrm{V}-\mathrm{A}}(\bar{u} d)_{\mathrm{V}-\mathrm{A}}$ allows not only for QCD corrections of the current-current type as in fig. 3 (a)-(c) from which the by now well known second current-current operator $Q_{1}$ is created. For a complete treatment of QCD corrections all possible ways of attaching a gluon to the initial weak $\Delta F=1$ transition operator $Q_{2}$ have to be taken into account. Therefore attaching gluons to $Q_{2}$ in the form of diagrams (d.1) and (d.2) in fig. 3, generates a completely new set of four-quark operators, the so-called QCD penguin operators, usually denoted as $Q_{3}, \ldots, Q_{6}{ }^{3}$. This procedure is often referred to as inserting $Q_{2}$ into type-1 and type-2 penguin diagrams.

The $\Delta S=1$ effective hamiltonian for $K \rightarrow \pi \pi$ at scales $\mu<m_{\mathrm{c}}$ then reads

$$
\mathcal{H}_{\text {eff }}(\Delta S=1)=\frac{G_{F}}{\sqrt{2}} V_{\text {us }}^{*} V_{\text {ud }} \sum_{i=1}^{6}\left(z_{i}(\mu)+\tau y_{i}(\mu)\right) Q_{i},
$$

with

$$
\tau=-\frac{V_{\mathrm{ts}}^{*} V_{\mathrm{td}}}{V_{\mathrm{us}}^{*} V_{\mathrm{ud}}}
$$

The set of four-quark operators $\vec{Q}(\mu)$ and Wilson coefficients $\vec{z}(\mu)$ and $\vec{y}(\mu)$ will be discussed one by one in the subsections below.

\section{A. Operators}

The basis of four-quark operators for the $\Delta S=1$ effective hamiltonian in (VI.1) is given in explicit form by

$$
\begin{aligned}
Q_{1} & =\left(\bar{s}_{i} u_{j}\right)_{\mathrm{V}-\mathrm{A}}\left(\bar{u}_{j} d_{i}\right)_{\mathrm{V}-\mathrm{A}}, \\
Q_{2} & =(\bar{s} u)_{\mathrm{V}-\mathrm{A}}(\bar{u} d)_{\mathrm{V}-\mathrm{A}}, \\
Q_{3} & =(\bar{s} d)_{\mathrm{V}-\mathrm{A}} \sum_{q}(\bar{q} q)_{\mathrm{V}-\mathrm{A}}, \\
Q_{4} & =\left(\bar{s}_{i} d_{j}\right)_{\mathrm{V}-\mathrm{A}} \sum_{q}\left(\bar{q}_{j} q_{i}\right)_{\mathrm{V}-\mathrm{A}}, \\
Q_{5} & =(\bar{s} d)_{\mathrm{V}-\mathrm{A}} \sum_{q}(\bar{q} q)_{\mathrm{V}+\mathrm{A}}, \\
Q_{6} & =\left(\bar{s}_{i} d_{j}\right)_{\mathrm{V}-\mathrm{A}} \sum_{q}\left(\bar{q}_{j} q_{i}\right)_{\mathrm{V}+\mathrm{A}} .
\end{aligned}
$$

\footnotetext{
${ }^{3}$ Obviously, whether or not it is possible to form a closed fermion loop as in a type- 1 insertion or to connect the two currents to yield a continuous fermion line as required for a type- 2 insertion strongly depends on the flavour structure of the operator considered. E.g. for $Q_{2}$ only the type-2 penguin diagram contributes. This feature can be exploited to obtain NLO anomalous dimension matrices in the NDR scheme without the necessity of calculating closed fermion loops with $\gamma_{5}$ (Buras et al., 1993c), (Buras et al., 1993a).
} 
As already mentioned, this basis closes under QCD renormalization.

For $\mu<m_{\mathrm{c}}$ the sums over active quark flavours in (VI.3) run over $u, d$ and $s$. However, when $m_{\mathrm{b}}>\mu>m_{\mathrm{c}}$ is considered also $q=c$ has to be included. Moreover, in this case two additional current-current operators have to be taken into account

$$
Q_{1}^{c}=\left(\bar{s}_{i} c_{j}\right)_{\mathrm{V}-\mathrm{A}}\left(\bar{c}_{j} d_{i}\right)_{\mathrm{V}-\mathrm{A}}, \quad Q_{2}^{c}=(\bar{s} c)_{\mathrm{V}-\mathrm{A}}(\bar{c} d)_{\mathrm{V}-\mathrm{A}} .
$$

and the effective hamiltonian takes the form

$$
\mathcal{H}_{\text {eff }}(\Delta S=1)=\frac{G_{F}}{\sqrt{2}} V_{\text {us }}^{*} V_{\text {ud }}\left[(1-\tau) \sum_{i=1}^{2} z_{i}(\mu)\left(Q_{i}-Q_{i}^{c}\right)+\tau \sum_{i=1}^{6} v_{i}(\mu) Q_{i}\right]
$$

\section{B. Wilson Coefficients}

For the Wilson coefficients $y_{i}(\mu)$ and $z_{i}(\mu)$ in eq. (VI.1) one has

$$
y_{i}(\mu)=v_{i}(\mu)-z_{i}(\mu)
$$

The coefficients $z_{i}$ and $v_{i}$ are the components of the six dimensional column vectors $\vec{v}(\mu)$ and $\vec{z}(\mu)$. Their RG evolution is given by

$$
\begin{gathered}
\vec{v}(\mu)=U_{3}\left(\mu, m_{\mathrm{c}}\right) M\left(m_{\mathrm{c}}\right) U_{4}\left(m_{\mathrm{c}}, m_{\mathrm{b}}\right) M\left(m_{\mathrm{b}}\right) U_{5}\left(m_{\mathrm{b}}, M_{\mathrm{W}}\right) \vec{C}\left(M_{\mathrm{W}}\right), \\
\vec{z}(\mu)=U_{3}\left(\mu, m_{\mathrm{c}}\right) \vec{z}\left(m_{\mathrm{c}}\right) .
\end{gathered}
$$

Here $U_{f}\left(m_{1}, m_{2}\right)$ denotes the full NLO evolution matrix for $f$ active flavours. $M\left(m_{i}\right)$ is the matching matrix at quark threshold $m_{i}$ given in eq. (III.104). These two matrices will be discussed in more detail in subsections VIC and VID, respectively.

The initial values $\vec{C}\left(M_{\mathrm{W}}\right)$ necessary for the RG evolution of $\vec{v}(\mu)$ in eq. (VI.7) can be found according to the procedure of matching the effective (fig. 3) onto the full theory (fig. 2) as summarized in section III F. For the NDR scheme one obtains (Buras et al., 1992)

$$
\begin{aligned}
& C_{1}\left(M_{\mathrm{W}}\right)=\frac{11}{2} \frac{\alpha_{\mathrm{s}}\left(M_{\mathrm{W}}\right)}{4 \pi}, \\
& C_{2}\left(M_{\mathrm{W}}\right)=1-\frac{11}{6} \frac{\alpha_{\mathrm{s}}\left(M_{\mathrm{W}}\right)}{4 \pi}, \\
& C_{3}\left(M_{\mathrm{W}}\right)=-\frac{\alpha_{\mathrm{s}}\left(M_{\mathrm{W}}\right)}{24 \pi} \widetilde{E}_{0}\left(x_{t}\right), \\
& C_{4}\left(M_{\mathrm{W}}\right)=\frac{\alpha_{\mathrm{s}}\left(M_{\mathrm{W}}\right)}{8 \pi} \widetilde{E}_{0}\left(x_{t}\right), \\
& C_{5}\left(M_{\mathrm{W}}\right)=-\frac{\alpha_{\mathrm{s}}\left(M_{\mathrm{W}}\right)}{24 \pi} \widetilde{E}_{0}\left(x_{t}\right), \\
& C_{6}\left(M_{\mathrm{W}}\right)=\frac{\alpha_{\mathrm{s}}\left(M_{\mathrm{W}}\right)}{8 \pi} \widetilde{E}_{0}\left(x_{t}\right),
\end{aligned}
$$

where 


$$
\begin{aligned}
E_{0}(x) & =-\frac{2}{3} \ln x+\frac{x\left(18-11 x-x^{2}\right)}{12(1-x)^{3}}+\frac{x^{2}\left(15-16 x+4 x^{2}\right)}{6(1-x)^{4}} \ln x, \\
\widetilde{E}_{0}\left(x_{t}\right) & =E_{0}\left(x_{t}\right)-\frac{2}{3}
\end{aligned}
$$

with

$$
x_{t}=\frac{m_{t}^{2}}{M_{\mathrm{W}}^{2}} .
$$

Here $E_{0}(x)$ results from the evaluation of the gluon penguin diagrams.

The initial values $\vec{C}\left(M_{\mathrm{W}}\right)$ in the $\mathrm{HV}$ scheme can be found in (Buras et al., 1992).

In order to calculate the initial conditions $\vec{z}\left(m_{\mathrm{c}}\right)$ for $z_{i}(\mu)$ in eq. (VI.8) one has to consider the difference $Q_{2}^{u}-Q_{2}^{c}$ of $Q_{2}$-type current-current operators as can be seen explicitly in (VI.5). Due to the GIM mechanism the coefficients $z_{i}(\mu)$ of penguin operators $Q_{i}, i \neq 1,2$ are zero in 5- and 4-flavour theories. The evolution for scales $\mu>m_{\mathrm{c}}$ involves then only the current-current operators $Q_{i}^{u}-Q_{i}^{c}, i=1,2$, with initial conditions at scale $\mu=M_{\mathrm{W}}$

$$
z_{1}\left(M_{\mathrm{W}}\right)=C_{1}\left(M_{\mathrm{W}}\right), \quad z_{2}\left(M_{\mathrm{W}}\right)=C_{2}\left(M_{\mathrm{W}}\right) .
$$

$Q_{1,2}^{u} \equiv Q_{1,2}$ and $Q_{1,2}^{c}$ do not mix with each other under renormalization. We then find

$$
\left(\begin{array}{c}
z_{1}\left(m_{\mathrm{c}}\right) \\
z_{2}\left(m_{\mathrm{c}}\right)
\end{array}\right)=U_{4}\left(m_{\mathrm{c}}, m_{\mathrm{b}}\right) M\left(m_{\mathrm{b}}\right) U_{5}\left(m_{\mathrm{b}}, M_{\mathrm{W}}\right)\left(\begin{array}{c}
z_{1}\left(M_{\mathrm{W}}\right) \\
z_{2}\left(M_{\mathrm{W}}\right)
\end{array}\right),
$$

where this time the evolution matrices $U_{4,5}$ contain only the $2 \times 2$ anomalous dimension submatrices describing the mixing between current-current operators. The matching matrix $M\left(m_{\mathrm{b}}\right)$ is then also only the corresponding $2 \times 2$ submatrix of the full $6 \times 6$ matrix in (VI.27). For the particular case of (VI.19) it simplifies to a unit matrix. When the charm quark is integrated out the operators $Q_{1,2}^{c}$ disappear from the effective hamiltonian and the coefficients $z_{i}(\mu), i \neq 1,2$ for penguin operators become non-zero. In order to calculate $z_{i}\left(m_{\mathrm{c}}\right)$ for penguin operators a proper matching between effective 4- and 3-quark theories, that is between (VI.5) and (VI.1), has to be made. For the 3-quark theory one obtains in the NDR scheme (Buras et al., 1993b)

$$
\vec{z}\left(m_{\mathrm{c}}\right)=\left(\begin{array}{c}
z_{1}\left(m_{\mathrm{c}}\right) \\
z_{2}\left(m_{\mathrm{c}}\right) \\
-\alpha_{\mathrm{s}} /(24 \pi) F_{\mathrm{s}}\left(m_{\mathrm{c}}\right) \\
\alpha_{\mathrm{s}} /(8 \pi) F_{\mathrm{s}}\left(m_{\mathrm{c}}\right) \\
-\alpha_{\mathrm{s}} /(24 \pi) F_{\mathrm{s}}\left(m_{\mathrm{c}}\right) \\
\alpha_{\mathrm{s}} /(8 \pi) F_{\mathrm{s}}\left(m_{\mathrm{c}}\right)
\end{array}\right)
$$

where

$$
F_{\mathrm{s}}\left(m_{\mathrm{c}}\right)=-\frac{2}{3} z_{2}\left(m_{\mathrm{c}}\right)
$$

In the HV scheme $z_{1,2}$ are modified and one has $F_{\mathrm{s}}\left(m_{\mathrm{c}}\right)=0$ or $z_{i}\left(m_{\mathrm{c}}\right)=0$ for $i \neq 1,2$. 


\section{Renormalization Group Evolution and Anomalous Dimension Matrices}

The general RG evolution matrix $U\left(m_{1}, m_{2}\right)$ from scale $m_{2}$ down to $m_{1}<m_{2}$ reads in pure QCD

$$
U\left(m_{1}, m_{2}\right) \equiv T_{g} \exp \int_{g\left(m_{2}\right)}^{g\left(m_{1}\right)} d g^{\prime} \frac{\gamma_{\mathrm{s}}^{T}\left(g^{\prime 2}\right)}{\beta\left(g^{\prime}\right)},
$$

with $\gamma_{\mathbf{s}}\left(g^{2}\right)$ being the full $6 \times 6$ QCD anomalous dimension matrix for $Q_{1}, \ldots, Q_{6}$.

For the case at hand it can be expanded in terms of $\alpha_{\mathrm{s}}$ as follows

$$
\gamma_{\mathrm{s}}\left(g^{2}\right)=\frac{\alpha_{\mathrm{s}}}{4 \pi} \gamma_{\mathrm{s}}^{(0)}+\frac{\alpha_{\mathrm{s}}^{2}}{(4 \pi)^{2}} \gamma_{\mathrm{s}}^{(1)}+\cdots
$$

Explicit expressions for $\gamma_{\mathrm{s}}^{(0)}$ and $\gamma_{\mathrm{s}}^{(1)}$ will be given below.

Using eq. (VI.23) the general QCD evolution matrix $U\left(m_{1}, m_{2}\right)$ of eq. (VI.22) can be written as in (III.93) (Buras et al., 1992).

$$
U\left(m_{1}, m_{2}\right)=\left(1+\frac{\alpha_{\mathrm{s}}\left(m_{1}\right)}{4 \pi} J\right) U^{(0)}\left(m_{1}, m_{2}\right)\left(1-\frac{\alpha_{\mathrm{s}}\left(m_{2}\right)}{4 \pi} J\right),
$$

where $U^{(0)}\left(m_{1}, m_{2}\right)$ denotes the evolution matrix in the leading logarithmic approximation and $J$ summarizes the next-to-leading correction to this evolution. Therefore, the full matrix $U\left(m_{1}, m_{2}\right)$ sums logarithms $\left(\alpha_{\mathrm{s}} t\right)^{n}$ and $\alpha_{\mathrm{s}}\left(\alpha_{\mathrm{s}} t\right)^{n}$ with $t=\ln \left(m_{2}^{2} / m_{1}^{2}\right)$. Explicit expressions for $U^{(0)}\left(m_{1}, m_{2}\right)$ and $J$ are given in eqs. (III.94)-(III.98).

The LO anomalous dimension matrix $\gamma_{\mathrm{s}}^{(0)}$ of eq. (VI.23) has the explicit form (Gaillard and Lee, 1974a), (Altarelli and Maiani, 1974), (Vainshtein et al., 1977), (Gilman and Wise, 1979), (Guberina and Peccei, 1980)

$$
\gamma_{\mathrm{s}}^{(0)}=\left(\begin{array}{cccccc}
\frac{-6}{N} & 6 & 0 & 0 & 0 & 0 \\
6 & \frac{-6}{N} & \frac{-2}{3 N} & \frac{2}{3} & \frac{-2}{3 N} & \frac{2}{3} \\
0 & 0 & \frac{-22}{3 N} & \frac{22}{3} & \frac{-4}{3 N} & \frac{4}{3} \\
0 & 0 & 6-\frac{2 f}{3 N} & \frac{-6}{N}+\frac{2 f}{3} & \frac{-2 f}{3 N} & \frac{2 f}{3} \\
0 & 0 & 0 & 0 & \frac{6}{N} & -6 \\
0 & 0 & \frac{-2 f}{3 N} & \frac{2 f}{3} & \frac{-2 f}{3 N} & \frac{-6\left(-1+N^{2}\right)}{N}+\frac{2 f}{3}
\end{array}\right)
$$

The NLO anomalous dimension matrix $\gamma_{\mathrm{s}}^{(1)}$ of eq. (VI.23) reads in the NDR scheme (Buras et al., 1992), (Ciuchini et al., 1994a) 


$$
\left.\gamma_{\mathrm{s}, \mathrm{NDR}}^{(1)}\right|_{N=3}=\left(\begin{array}{cccccc}
-\frac{21}{2}-\frac{2 f}{9} & \frac{7}{2}+\frac{2 f}{3} & \frac{79}{9} & -\frac{7}{3} & -\frac{65}{9} & -\frac{7}{3} \\
\frac{7}{2}+\frac{2 f}{3} & -\frac{21}{2}-\frac{2 f}{9} & -\frac{202}{243} & \frac{1354}{81} & -\frac{1192}{243} & \frac{904}{81} \\
0 & 0 & -\frac{5911}{486}+\frac{71 f}{9} & \frac{5983}{162}+\frac{f}{3} & -\frac{2384}{243}-\frac{71 f}{9} & \frac{1808}{81}-\frac{f}{3} \\
0 & 0 & \frac{379}{18}+\frac{56 f}{243} & -\frac{91}{6}+\frac{808 f}{81} & -\frac{130}{9}-\frac{502 f}{243} & -\frac{14}{3}+\frac{646 f}{81} \\
0 & 0 & \frac{-61 f}{9} & \frac{-11 f}{3} & \frac{71}{3}+\frac{61 f}{9} & -99+\frac{11 f}{3} \\
0 & 0 & \frac{-682 f}{243} & \frac{106 f}{81} & -\frac{225}{2}+\frac{1676 f}{243} & -\frac{1343}{6}+\frac{1348 f}{81}
\end{array}\right)
$$

In (VI.25) and (VI.26) $f$ denotes the number of active quark flavours at a certain scale $\mu$. The corresponding results for $\gamma_{\mathrm{s}}^{(1)}$ in the HV scheme can either be obtained by direct calculation or by using the relation (III.126). They can be found in (Buras et al., 1992), (Ciuchini et al., 1994a) where also the $N$ dependence of $\gamma_{\mathrm{s}}^{(1)}$ is given.

\section{Quark Threshold Matching Matrix}

As discussed in section IIIF 1 in general a matching matrix $M(m)$ has to be included in the $\mathrm{RG}$ evolution at NLO when going from a $f$-flavour effective theory to a $(f-1)$-flavour effective theory at quark threshold $\mu=m$ (Buras et al., 1992), (Buras et al., 1993b).

For the $\Delta S=1$ decay $K \rightarrow \pi \pi$ in pure QCD one has (Buras et al., 1992)

$$
M(m)=1+\frac{\alpha_{\mathrm{s}}(m)}{4 \pi} \delta r_{\mathrm{s}}^{T}
$$

At the quark thresholds $m=m_{b}$ and $m=m_{c}$ the matrix $\delta r_{\mathrm{s}}$ reads

$$
\delta r_{\mathrm{s}}^{T}=-\frac{5}{9} P(0,0,0,1,0,1)
$$

with

$$
P^{T}=\left(0,0,-\frac{1}{3}, 1,-\frac{1}{3}, 1\right)
$$

\section{E. Numerical Results for the $K \rightarrow \pi \pi$ Wilson Coeficients in Pure QCD}

Tables X-XII give the $\Delta S=1$ Wilson coefficients for $Q_{1}, \ldots, Q_{6}$ in pure QCD.

We observe a visible scheme dependence for all NLO Wilson coefficients. Notably we find $\left|y_{6}\right|$ to be smaller in the HV than in the NDR scheme.

In addition all coefficients, especially $z_{1}$ and $y_{3}, \ldots, y_{6}$, show a strong dependence on $\Lambda_{\overline{\mathrm{MS}}}$.

Next, at NLO the absolute values for $z_{1,2}$ and $y_{i}$ are suppressed relative to their LO results, except for $y_{5}$ in $\mathrm{HV}$ and $y_{4,6}$ in NDR for $\mu>m_{\mathrm{c}}$. The latter behaviour is related to the effect of the matching matrix $M\left(m_{\mathrm{c}}\right)$ absent for $\mu>m_{\mathrm{c}}$. 
TABLE X. $\Delta S=1$ Wilson coefficients at $\mu=1 \mathrm{GeV}$ for $m_{\mathrm{t}}=170 \mathrm{GeV} . y_{1}=y_{2} \equiv 0$.

\begin{tabular}{|c|c|c|c||c|c|c||c|c|c|}
\hline \hline & \multicolumn{3}{|c||}{$\Lambda_{\overline{\mathrm{MS}}}^{(4)}=215 \mathrm{MeV}$} & \multicolumn{3}{c||}{$\Lambda_{\overline{\mathrm{MS}}}^{(4)}=325 \mathrm{MeV}$} & \multicolumn{3}{c|}{$\Lambda_{\overline{\mathrm{MS}}}^{(4)}=435 \mathrm{MeV}$} \\
\hline Scheme & LO & NDR & HV & LO & NDR & HV & LO & NDR & HV \\
\hline$z_{1}$ & -0.602 & -0.407 & -0.491 & -0.743 & -0.506 & -0.636 & -0.901 & -0.622 & -0.836 \\
$z_{2}$ & 1.323 & 1.204 & 1.260 & 1.423 & 1.270 & 1.362 & 1.541 & 1.352 & 1.515 \\
\hline$z_{3}$ & 0.003 & 0.007 & 0.004 & 0.004 & 0.013 & 0.007 & 0.006 & 0.022 & 0.015 \\
$z_{4}$ & -0.008 & -0.022 & -0.010 & -0.012 & -0.034 & -0.016 & -0.016 & -0.058 & -0.029 \\
$z_{5}$ & 0.003 & 0.006 & 0.003 & 0.004 & 0.007 & 0.004 & 0.005 & 0.009 & 0.005 \\
$z_{6}$ & -0.009 & -0.021 & -0.009 & -0.013 & -0.034 & -0.014 & -0.018 & -0.058 & -0.025 \\
\hline$y_{3}$ & 0.029 & 0.023 & 0.026 & 0.036 & 0.031 & 0.036 & 0.045 & 0.040 & 0.048 \\
$y_{4}$ & -0.051 & -0.046 & -0.048 & -0.060 & -0.056 & -0.059 & -0.069 & -0.066 & -0.072 \\
$y_{5}$ & 0.012 & 0.004 & 0.013 & 0.013 & -0.001 & 0.016 & 0.014 & -0.013 & 0.020 \\
$y_{6}$ & -0.084 & -0.076 & -0.070 & -0.111 & -0.109 & -0.096 & -0.145 & -0.166 & -0.136 \\
\hline \hline
\end{tabular}

TABLE XI. $\Delta S=1$ Wilson coefficients at $\mu=m_{\mathrm{c}}=1.3 \mathrm{GeV}$ for $m_{\mathrm{t}}=170 \mathrm{GeV}$ and $f=3$ effective flavours. $\left|z_{3}\right|, \ldots,\left|z_{6}\right|$ are numerically irrelevant relative to $\left|z_{1,2}\right| . y_{1}=y_{2} \equiv 0$.

\begin{tabular}{|c|c|c|c||c|r|r||r|r|r|}
\hline \hline & \multicolumn{3}{|c||}{$\Lambda \frac{(4)}{\mathrm{MS}}=215 \mathrm{MeV}$} & \multicolumn{3}{c||}{$\Lambda_{\overline{\mathrm{MS}}}^{(4)}=325 \mathrm{MeV}$} & \multicolumn{3}{c|}{$\Lambda_{\overline{\mathrm{MS}}}^{(4)}=435 \mathrm{MeV}$} \\
\hline Scheme & LO & NDR & HV & LO & NDR & HV & LO & NDR & HV \\
\hline$z_{1}$ & -0.518 & -0.344 & -0.411 & -0.621 & -0.412 & -0.504 & -0.727 & -0.487 & -0.614 \\
$z_{2}$ & 1.266 & 1.166 & 1.207 & 1.336 & 1.208 & 1.269 & 1.411 & 1.258 & 1.346 \\
\hline$y_{3}$ & 0.026 & 0.021 & 0.024 & 0.032 & 0.027 & 0.031 & 0.039 & 0.035 & 0.040 \\
$y_{4}$ & -0.050 & -0.046 & -0.048 & -0.059 & -0.056 & -0.058 & -0.068 & -0.067 & -0.070 \\
$y_{5}$ & 0.013 & 0.007 & 0.013 & 0.015 & 0.005 & 0.016 & 0.016 & 0.001 & 0.018 \\
$y_{6}$ & -0.075 & -0.067 & -0.062 & -0.095 & -0.088 & -0.079 & -0.118 & -0.116 & -0.102 \\
\hline \hline
\end{tabular}

For $y_{3}, \ldots, y_{5}$ there is no visible $m_{\mathrm{t}}$ dependence in the range $m_{\mathrm{t}}=(170 \pm 15) \mathrm{GeV}$. For $\left|y_{6}\right|$ there is a relative variation of $\mathcal{O}( \pm 1.5 \%)$ for in/decreasing $m_{\mathrm{t}}$.

Finally, a comment on the Wilson coefficients in the HV Scheme as presented here is appropriate. As we have mentioned in section V B, the two-loop anomalous dimensions of the weak current in the HV scheme does not vanish. This peculiar feature of the HV scheme is also felt in $\gamma_{\mathrm{s}}^{(1)}$. The diagonal terms in $\gamma_{\mathrm{s}}^{(1)}$ aquire additional universal large $\mathcal{O}\left(N^{2}\right)$ terms $(44 / 3) N^{2}$ which are absent in the NDR scheme. These artificial terms can be removed by working with $\gamma_{\mathrm{s}}^{(1)}-2 \gamma_{J}^{(1)}$ instead of $\gamma_{\mathrm{s}}^{(1)}$. This procedure, adopted in this review and in (Buras et al., 1993b), corresponds effectively to a finite renormalization of operators which changes the coefficient of $\alpha_{\mathrm{s}} / 4 \pi$ in $C_{2}^{H V}\left(M_{\mathrm{W}}\right)$ from $-13 / 2$ to $-7 / 6$. The Rome group (Ciuchini et al., 1994a) has chosen not to make this additional finite renormalization and consequently their coefficients in the HV scheme differ from the HV coefficients presented here by a universal factor. They can be found by using

$$
C_{\text {Rome }}^{\mathrm{HV}}(\mu)=\left[1-\frac{\alpha_{\mathrm{s}}(\mu)}{4 \pi} 4 C_{F}\right] C^{\mathrm{HV}}(\mu)
$$


TABLE XII. $\Delta S=1$ Wilson coefficients at $\mu=2 \mathrm{GeV}$ for $m_{\mathrm{t}}=170 \mathrm{GeV}$. For $\mu>m_{\mathrm{c}}$ the GIM mechanism gives $z_{i} \equiv 0, i=3, \ldots, 6 . y_{1}=y_{2} \equiv 0$.

\begin{tabular}{|c|c|c|c||c|r|r||r|r|r|}
\hline \hline & \multicolumn{3}{|c||}{$\Lambda_{\overline{\mathrm{MS}}}^{(4)}=215 \mathrm{MeV}$} & \multicolumn{3}{c||}{$\Lambda_{\overline{\mathrm{MS}}}^{(4)}=325 \mathrm{MeV}$} & \multicolumn{3}{c|}{$\Lambda_{\overline{\mathrm{MS}}}^{(4)}=435 \mathrm{MeV}$} \\
\hline Scheme & LO & NDR & HV & LO & NDR & HV & LO & NDR & HV \\
\hline$z_{1}$ & -0.411 & -0.266 & -0.318 & -0.477 & -0.309 & -0.374 & -0.541 & -0.350 & -0.430 \\
$z_{2}$ & 1.199 & 1.121 & 1.151 & 1.240 & 1.145 & 1.185 & 1.282 & 1.170 & 1.220 \\
\hline$y_{3}$ & 0.019 & 0.019 & 0.018 & 0.023 & 0.023 & 0.022 & 0.027 & 0.027 & 0.026 \\
$y_{4}$ & -0.040 & -0.046 & -0.039 & -0.046 & -0.054 & -0.045 & -0.052 & -0.062 & -0.052 \\
$y_{5}$ & 0.011 & 0.010 & 0.011 & 0.012 & 0.010 & 0.013 & 0.013 & 0.010 & 0.015 \\
$y_{6}$ & -0.055 & -0.057 & -0.047 & -0.067 & -0.070 & -0.056 & -0.078 & -0.085 & -0.067 \\
\hline \hline
\end{tabular}

Clearly this difference is compensated by the corresponding difference in the hadronic matrix elements of the operators $Q_{i}$.

\section{F. The $\Delta B=1$ Effective Hamiltonian in Pure QCD}

An important application of the formalism developed in the previous subsections is for the case of $B$-meson decays. The LO calculation can be found e.g. in (Ponce, 1981), (Grinstein, 1989) where the importance of NLO calculations has already been pointed out. This section can be viewed as the generalization of Grinstein's analysis beyond the LO approximation. We will focus on the $\Delta B=1, \Delta C=0$ part of the effective hamiltonian which is of particular interest for the study of $\mathrm{CP}$ violation in decays to $\mathrm{CP}$ self-conjugate final states. The part of the hamiltonian inducing $\Delta B=1, \Delta C= \pm 1$ transitions involves no penguin operators and has already been discussed in $\mathrm{V}$.

At tree-level the effective hamiltonian of interest here is simply given by

$$
\mathcal{H}_{\text {eff }}(\Delta B=1)=\frac{G_{F}}{\sqrt{2}} \sum_{q=u, c} \sum_{q^{\prime}=d, s} V_{q b}^{*} V_{q q^{\prime}}(\bar{b} q)_{\mathrm{V}-\mathrm{A}}\left(\bar{q} q^{\prime}\right)_{\mathrm{V}-\mathrm{A}} .
$$

The cases $q^{\prime}=d$ and $q^{\prime}=s$ can be treated separately and have the same Wilson coefficients $C_{i}(\mu)$. Therefore we will restrict the discussion to $q^{\prime}=d$ in the following.

Using unitarity of the CKM matrix, $\xi_{u}+\xi_{c}+\xi_{t}=0$ with $\xi_{i}=V_{i b}^{*} V_{i d}$, and the fact that $Q_{1,2}^{u}$ and $Q_{1,2}^{c}$ have the same initial conditions at $\mu=M_{\mathrm{W}}$ one obtains for the effective $\Delta B=1$ hamiltonian at scales $\mu=\mathcal{O}\left(m_{\mathrm{b}}\right)$

$$
\begin{aligned}
\mathcal{H}_{\mathrm{eff}}(\Delta B=1)= & \frac{G_{F}}{\sqrt{2}}\left\{\xi_{c}\left[C_{1}(\mu) Q_{1}^{c}(\mu)+C_{2}(\mu) Q_{2}^{c}(\mu)\right]+\xi_{u}\left[C_{1}(\mu) Q_{1}^{u}(\mu)+C_{2}(\mu) Q_{2}^{u}(\mu)\right]\right. \\
& \left.-\xi_{t} \sum_{i=3}^{6} C_{i}(\mu) Q_{i}(\mu)\right\} .
\end{aligned}
$$

Here 


$$
\begin{aligned}
Q_{1}^{q} & =\left(\bar{b}_{i} q_{j}\right)_{\mathrm{V}-\mathrm{A}}\left(\bar{q}_{j} d_{i}\right)_{\mathrm{V}-\mathrm{A}}, \\
Q_{2}^{q} & =(\bar{b} q)_{\mathrm{V}-\mathrm{A}}(\bar{q} d)_{\mathrm{V}-\mathrm{A}}, \\
Q_{3} & =(\bar{b} d)_{\mathrm{V}-\mathrm{A}} \sum_{q}(\bar{q} q)_{\mathrm{V}-\mathrm{A}}, \\
Q_{4} & =\left(\bar{b}_{i} d_{j}\right)_{\mathrm{V}-\mathrm{A}} \sum_{q}\left(\bar{q}_{j} q_{i}\right)_{\mathrm{V}-\mathrm{A}}, \\
Q_{5} & =(\bar{b} d)_{\mathrm{V}-\mathrm{A}} \sum_{q}(\bar{q} q)_{\mathrm{V}+\mathrm{A}}, \\
Q_{6} & =\left(\bar{b}_{i} d_{j}\right)_{\mathrm{V}-\mathrm{A}} \sum_{q}\left(\bar{q}_{j} q_{i}\right)_{\mathrm{V}+\mathrm{A}},
\end{aligned}
$$

where the summation runs over $q=u, d, s, c, b$.

The corresponding $\Delta B=1$ Wilson coefficients at scale $\mu=\mathcal{O}\left(m_{\mathrm{b}}\right)$ are simply given by a truncated version of eq. (VI.7)

$$
\vec{C}\left(m_{\mathrm{b}}\right)=U_{5}\left(m_{\mathrm{b}}, M_{\mathrm{W}}\right) \vec{C}\left(M_{\mathrm{W}}\right) .
$$

Here $U_{5}$ is the $6 \times 6 \mathrm{RG}$ evolution matrix of eq. (VI.24) for $f=5$ active flavours. The initial conditions $\vec{C}\left(M_{\mathrm{W}}\right)$ are identical to those of (VI.9)-(VI.14) for the $\Delta S=1$ case.

\section{G. Numerical Results for the $\Delta B=1$ Wilson Coefficients in Pure QCD}

TABLE XIII. $\Delta B=1$ Wilson coefficients at $\mu=\bar{m}_{\mathrm{b}}\left(m_{\mathrm{b}}\right)=4.40 \mathrm{GeV}$ for $m_{\mathrm{t}}=170 \mathrm{GeV}$.

\begin{tabular}{|c|c|c|c||c|c|c||c|c|c|}
\hline \hline & \multicolumn{3}{|c||}{$\Lambda_{\overline{\mathrm{MS}}}^{(5)}=140 \mathrm{MeV}$} & \multicolumn{3}{c||}{$\Lambda_{\overline{\mathrm{MS}}}^{(5)}=225 \mathrm{MeV}$} & \multicolumn{3}{c|}{$\Lambda_{\overline{\mathrm{MS}}}^{(5)}=310 \mathrm{MeV}$} \\
\hline Scheme & LO & NDR & HV & LO & NDR & HV & LO & NDR & HV \\
\hline$C_{1}$ & -0.272 & -0.164 & -0.201 & -0.307 & -0.184 & -0.227 & -0.337 & -0.202 & -0.250 \\
$C_{2}$ & 1.120 & 1.068 & 1.087 & 1.139 & 1.078 & 1.101 & 1.155 & 1.087 & 1.113 \\
\hline$C_{3}$ & 0.012 & 0.012 & 0.011 & 0.013 & 0.013 & 0.012 & 0.015 & 0.015 & 0.014 \\
$C_{4}$ & -0.026 & -0.031 & -0.026 & -0.030 & -0.035 & -0.029 & -0.032 & -0.038 & -0.032 \\
$C_{5}$ & 0.008 & 0.008 & 0.008 & 0.009 & 0.009 & 0.009 & 0.009 & 0.009 & 0.010 \\
$C_{6}$ & -0.033 & -0.035 & -0.029 & -0.038 & -0.041 & -0.033 & -0.042 & -0.046 & -0.036 \\
\hline \hline
\end{tabular}

Table XIII lists the $\Delta B=1$ Wilson coefficients for $Q_{1}^{u, c}, Q_{2}^{u, c}, Q_{3}, \ldots, Q_{6}$ in pure QCD. $C_{1}, C_{4}$ and $C_{6}$ show a $\mathcal{O}(20 \%)$ scheme dependence while this dependence is much weaker for the rest of the coefficients.

Similarly to the $\Delta S=1$ case the numerical values for $\Delta B=1$ Wilson coefficients are sensitive to the value of $\Lambda_{\overline{\mathrm{MS}}}$ used to determine $\alpha_{\mathrm{s}}$ for the RG evolution. The sensitivity is however less pronounced than in the $\Delta S=1$ case due to the higher value $\mu=\bar{m}_{\mathrm{b}}\left(m_{\mathrm{b}}\right)$ of the renormalization scale.

Finally, one finds no visible $m_{\mathrm{t}}$ dependence in the range $m_{\mathrm{t}}=(170 \pm 15) \mathrm{GeV}$. 


\section{THE EFFECTIVE $\Delta F=1$ HAMILTONIAN: INCLUSION OF ELECTROWEAK PENGUIN OPERATORS}

Similarly to the creation of the penguin operators $Q_{3}, \ldots, Q_{6}$ through QCD corrections the inclusion of electroweak corrections, shown in figs. 2 (d) and (e), generates a set of new operators, the so-called electroweak penguin operators. For the $\Delta S=1$ decay $K \rightarrow \pi \pi$ they are usually denoted by $Q_{7}, \ldots, Q_{10}$.

This means that although now we will have to deal with technically more involved issues like an extended operator basis or the possibility of mixed QCD-QED contributions the underlying principles in performing the RG evolution will closely resemble those used in section VI for pure QCD. Obviously, the fundamental step has already been made when going from current-current operators only in section V, to the inclusion of QCD penguins in section VI. Hence, in this section we will wherever possible only point out the differences between the pure $6 \times 6 \mathrm{QCD}$ and the combined $10 \times 10$ QCD-QED case.

The full $\Delta S=1$ effective hamiltonian for $K \rightarrow \pi \pi$ at scales $\mu<m_{\mathrm{c}}$ reads including QCD and QED corrections ${ }^{4}$

$$
\mathcal{H}_{\mathrm{eff}}(\Delta S=1)=\frac{G_{F}}{\sqrt{2}} V_{\mathrm{us}}^{*} V_{\mathrm{ud}} \sum_{i=1}^{10}\left(z_{i}(\mu)+\tau y_{i}(\mu)\right) Q_{i}(\mu),
$$

with $\tau=-V_{\mathrm{ts}}^{*} V_{\mathrm{td}} /\left(V_{\mathrm{us}}^{*} V_{\mathrm{ud}}\right)$.

\section{A. Operators}

The basis of four-quark operators for the $\Delta S=1$ effective hamiltonian in (VII.1) is given by $Q_{1}, \ldots, Q_{6}$ of (VI.3) and the electroweak penguin operators

$$
\begin{aligned}
Q_{7} & =\frac{3}{2}(\bar{s} d)_{\mathrm{V}-\mathrm{A}} \sum_{q} e_{q}(\bar{q} q)_{\mathrm{V}+\mathrm{A}}, \\
Q_{8} & =\frac{3}{2}\left(\bar{s}_{i} d_{j}\right)_{\mathrm{V}-\mathrm{A}} \sum_{q} e_{q}\left(\bar{q}_{j} q_{i}\right)_{\mathrm{V}+\mathrm{A}}, \\
Q_{9} & =\frac{3}{2}(\bar{s} d)_{\mathrm{V}-\mathrm{A}} \sum_{q} e_{q}(\bar{q} q)_{\mathrm{V}-\mathrm{A}}, \\
Q_{10} & =\frac{3}{2}\left(\bar{s}_{i} d_{j}\right)_{\mathrm{V}-\mathrm{A}} \sum_{q} e_{q}\left(\bar{q}_{j} q_{i}\right)_{\mathrm{V}-\mathrm{A}} .
\end{aligned}
$$

Here, $e_{q}$ denotes the quark electric charge reflecting the electroweak origin of $Q_{7}, \ldots, Q_{10}$. The basis $Q_{1}, \ldots, Q_{10}$ closes under QCD and QED renormalization. Finally, for $m_{\mathrm{b}}>\mu>m_{\mathrm{c}}$ the operators $Q_{1}^{c}$ and $Q_{2}^{c}$ of eq. (VI.4) have to be included again similarly to the case of pure QCD.

\footnotetext{
${ }^{4}$ In principle also operators $Q_{11}=\frac{g_{\mathrm{s}}}{16 \pi^{2}} m_{\mathrm{s}} \bar{s} \sigma_{\mu \nu} T^{a} G_{a}^{\mu \nu}\left(1-\gamma_{5}\right) d$ and $Q_{12}=\frac{e e_{d}}{16 \pi^{2}} m_{\mathrm{s}} \bar{s} \sigma_{\mu \nu} F^{\mu \nu}\left(1-\gamma_{5}\right) d$ should be considered for $K \rightarrow \pi \pi$. However, as shown in (Bertolini et al., 1995a) their numerical contribution is negligible. Therefore $Q_{11}$ and $Q_{12}$ will not be included here for $K \rightarrow \pi \pi$.
} 


\section{B. Wilson Coefficients}

As far as formulae for Wilson coefficients are concerned the generalization of section VIB to the present case is to a large extent straightforward.

First, due to the extended operator basis $\vec{v}(\mu)$ and $\vec{z}(\mu)$ in eqs. (VI.7) and (VI.8) are now ten dimensional column vectors. Furthermore, the substitution

$$
U_{f}\left(m_{1}, m_{2}\right) \rightarrow U_{f}\left(m_{1}, m_{2}, \alpha\right)
$$

has to be made in the RG evolution equations (VI.7), (VI.8) and (VI.19). Here $U_{f}\left(m_{1}, m_{2}, \alpha\right)$ denotes the full $10 \times 10$ QCD- QED RG evolution matrix for $f$ active flavours. $U_{f}\left(m_{1}, m_{2}, \alpha\right)$ will still be discussed in more detail in subsection VII C.

The extended initial values $\vec{C}\left(M_{\mathrm{W}}\right)$ including now $\mathcal{O}(\alpha)$ corrections and additional entries for $Q_{7}, \ldots, Q_{10}$ can be obtained from the usual matching procedure between figs. 2 and 3. They read in the NDR scheme (Buras et al., 1993b)

$$
\begin{aligned}
& C_{1}\left(M_{\mathrm{W}}\right)=\frac{11}{2} \frac{\alpha_{\mathrm{s}}\left(M_{\mathrm{W}}\right)}{4 \pi} \\
& C_{2}\left(M_{\mathrm{W}}\right)=1-\frac{11}{6} \frac{\alpha_{\mathrm{s}}\left(M_{\mathrm{W}}\right)}{4 \pi}-\frac{35}{18} \frac{\alpha}{4 \pi} \\
& C_{3}\left(M_{\mathrm{W}}\right)=-\frac{\alpha_{\mathrm{s}}\left(M_{\mathrm{W}}\right)}{24 \pi} \widetilde{E}_{0}\left(x_{t}\right)+\frac{\alpha}{6 \pi} \frac{1}{\sin ^{2} \theta_{W}}\left[2 B_{0}\left(x_{t}\right)+C_{0}\left(x_{t}\right)\right] \\
& C_{4}\left(M_{\mathrm{W}}\right)=\frac{\alpha_{\mathrm{s}}\left(M_{\mathrm{W}}\right)}{8 \pi} \widetilde{E}_{0}\left(x_{t}\right) \\
& C_{5}\left(M_{\mathrm{W}}\right)=-\frac{\alpha_{\mathrm{s}}\left(M_{\mathrm{W}}\right)}{24 \pi} \widetilde{E}_{0}\left(x_{t}\right) \\
& C_{6}\left(M_{\mathrm{W}}\right)=\frac{\alpha_{\mathrm{s}}\left(M_{\mathrm{W}}\right)}{8 \pi} \widetilde{E}_{0}\left(x_{t}\right) \\
& C_{7}\left(M_{\mathrm{W}}\right)=\frac{\alpha}{6 \pi}\left[4 C_{0}\left(x_{t}\right)+\widetilde{D}_{0}\left(x_{t}\right)\right] \\
& C_{8}\left(M_{\mathrm{W}}\right)=0, \\
& C_{9}\left(M_{\mathrm{W}}\right)=\frac{\alpha}{6 \pi}\left[4 C_{0}\left(x_{t}\right)+\widetilde{D}_{0}\left(x_{t}\right)+\frac{1}{\sin ^{2} \theta_{W}}\left(10 B_{0}\left(x_{t}\right)-4 C_{0}\left(x_{t}\right)\right)\right], \\
& C_{10}\left(M_{\mathrm{W}}\right)=0,
\end{aligned}
$$

where

$$
\begin{aligned}
& B_{0}(x)=\frac{1}{4}\left[\frac{x}{1-x}+\frac{x \ln x}{(x-1)^{2}}\right] \\
& C_{0}(x)=\frac{x}{8}\left[\frac{x-6}{x-1}+\frac{3 x+2}{(x-1)^{2}} \ln x\right], \\
& D_{0}(x)=-\frac{4}{9} \ln x+\frac{-19 x^{3}+25 x^{2}}{36(x-1)^{3}}+\frac{x^{2}\left(5 x^{2}-2 x-6\right)}{18(x-1)^{4}} \ln x, \\
& \widetilde{D}_{0}\left(x_{t}\right)=D_{0}\left(x_{t}\right)-\frac{4}{9}
\end{aligned}
$$


$\widetilde{E}_{0}\left(x_{t}\right)$ and $x_{t}$ have already been defined in eqs. (VI.16) and (VI.17), respectively. Here $B_{0}(x)$ results from the evaluation of the box diagrams, $C_{0}(x)$ from the $Z^{0}$-penguin, $D_{0}(x)$ from the photon penguin and $E_{0}(x)$ in $\widetilde{E}_{0}\left(x_{t}\right)$ from the gluon penguin diagrams.

The initial values $\vec{C}\left(M_{\mathrm{W}}\right)$ in the HV scheme can be found in (Buras et al., 1993b).

Finally, the generalization of (VI.20) to the $Q_{1}, \ldots, Q_{10}$ basis reads (Buras et al., 1993b)

$$
\vec{z}\left(m_{\mathrm{c}}\right)=\left(\begin{array}{c}
z_{1}\left(m_{\mathrm{c}}\right) \\
z_{2}\left(m_{\mathrm{c}}\right) \\
-\alpha_{\mathrm{s}} /(24 \pi) F_{\mathrm{s}}\left(m_{\mathrm{c}}\right) \\
\alpha_{\mathrm{s}} /(8 \pi) F_{\mathrm{s}}\left(m_{\mathrm{c}}\right) \\
-\alpha_{\mathrm{s}} /(24 \pi) F_{\mathrm{s}}\left(m_{\mathrm{c}}\right) \\
\alpha_{\mathrm{s}} /(8 \pi) F_{\mathrm{s}}\left(m_{\mathrm{c}}\right) \\
\alpha /(6 \pi) F_{\mathrm{e}}\left(m_{\mathrm{c}}\right) \\
0 \\
\alpha /(6 \pi) F_{\mathrm{e}}\left(m_{\mathrm{c}}\right) \\
0
\end{array}\right),
$$

with $F_{\mathrm{s}}\left(m_{\mathrm{c}}\right)$ given by (VI.21) and

$$
F_{\mathrm{e}}\left(m_{\mathrm{c}}\right)=-\frac{4}{9}\left(3 z_{1}\left(m_{\mathrm{c}}\right)+z_{2}\left(m_{\mathrm{c}}\right)\right) .
$$

In the HV scheme, in addition to $z_{1,2}$ differing from their NDR values, one has $F_{\mathrm{s}}\left(m_{\mathrm{c}}\right)=$ $F_{\mathrm{e}}\left(m_{\mathrm{c}}\right)=0$ and, consequently, $z_{i}\left(m_{\mathrm{c}}\right)=0$ for $i \neq 1,2$.

\section{Renormalization Group Evolution and Anomalous Dimension Matrices}

Besides an extended operator basis the main difference between the pure QCD case of section $\mathrm{VI}$ and the present case consists in the additional presence of QED contributions to the RG evolution. This will make the actual formulae for the RG evolution matrices more involved, however the underlying concepts developed in sections $\mathrm{V}$ and VI remain the same.

Similarly to (VI.22) for pure QCD the general RG evolution matrix $U\left(m_{1}, m_{2}, \alpha\right)$ from scale $m_{2}$ down to $m_{1}<m_{2}$ can be written formally as ${ }^{5}$

$$
U\left(m_{1}, m_{2}, \alpha\right) \equiv T_{g} \exp \int_{g\left(m_{2}\right)}^{g\left(m_{1}\right)} d g^{\prime} \frac{\gamma^{T}\left(g^{\prime 2}, \alpha\right)}{\beta\left(g^{\prime}\right)},
$$

with $\gamma\left(g^{2}, \alpha\right)$ being now the full $10 \times 10$ anomalous dimension matrix including QCD and QED contributions.

For the case at hand $\gamma\left(g^{2}, \alpha\right)$ can be expanded in the following way

$$
\gamma\left(g^{2}, \alpha\right)=\gamma_{\mathrm{s}}\left(g^{2}\right)+\frac{\alpha}{4 \pi} \Gamma\left(g^{2}\right)+\ldots,
$$

\footnotetext{
${ }^{5}$ We neglect the running of the electromagnetic coupling $\alpha$, which is a very good approximation (Buchalla et al., 1990).
} 
with the pure $\alpha_{\mathrm{s}}$-expansion of $\gamma_{\mathrm{s}}\left(g^{2}\right)$ given in (VI.23). The term present due to QED corrections has the expansion

$$
\Gamma\left(g^{2}\right)=\gamma_{\mathrm{e}}^{(0)}+\frac{\alpha_{\mathrm{s}}}{4 \pi} \gamma_{\mathrm{se}}^{(1)}+\ldots
$$

Using (VII.20)-(VII.21) the general RG evolution matrix $U\left(m_{1}, m_{2}, \alpha\right)$ of eq. (VII.19) may then be decomposed as follows

$$
U\left(m_{1}, m_{2}, \alpha\right)=U\left(m_{1}, m_{2}\right)+\frac{\alpha}{4 \pi} R\left(m_{1}, m_{2}\right)
$$

Here $U\left(m_{1}, m_{2}\right)$ represents the pure QCD evolution already encountered in section VI but now generalized to an extended operator basis. $R\left(m_{1}, m_{2}\right)$ describes the additional evolution in the presence of the electromagnetic interaction. $U\left(m_{1}, m_{2}\right)$ sums the logarithms $\left(\alpha_{\mathrm{s}} t\right)^{n}$ and $\alpha_{\mathrm{s}}\left(\alpha_{\mathrm{s}} t\right)^{n}$ with $t=\ln \left(m_{2}^{2} / m_{1}^{2}\right)$, whereas $R\left(m_{1}, m_{2}\right)$ sums the logarithms $t\left(\alpha_{\mathrm{s}} t\right)^{n}$ and $\left(\alpha_{\mathrm{s}} t\right)^{n}$.

The formula for $U\left(m_{1}, m_{2}\right)$ has already been given in (VI.24). The leading order formula for $R\left(m_{1}, m_{2}\right)$ can be found in (Buchalla et al., 1990) except that there a different overall normalization (relative factor $-4 \pi$ in $R$ ) has been used. Here we give the general expression for $R\left(m_{1}, m_{2}\right)$ (Buras et al., 1993b)

$$
\begin{aligned}
R\left(m_{1}, m_{2}\right) & =\int_{g\left(m_{2}\right)}^{g\left(m_{1}\right)} d g^{\prime} \frac{U\left(m_{1}, m^{\prime}\right) \Gamma^{T}\left(g^{\prime}\right) U\left(m^{\prime}, m_{2}\right)}{\beta\left(g^{\prime}\right)} \\
& \equiv-\frac{2 \pi}{\beta_{0}} V\left(K^{(0)}\left(m_{1}, m_{2}\right)+\frac{1}{4 \pi} \sum_{i=1}^{3} K_{i}^{(1)}\left(m_{1}, m_{2}\right)\right) V^{-1},
\end{aligned}
$$

with $g^{\prime} \equiv g\left(m^{\prime}\right)$.

The matrix kernels in (VII.23) are defined by

$$
\begin{gathered}
\left(K^{(0)}\left(m_{1}, m_{2}\right)\right)_{i j}=\frac{M_{i j}^{(0)}}{a_{i}-a_{j}-1}\left[\left(\frac{\alpha_{\mathrm{s}}\left(m_{2}\right)}{\alpha_{\mathrm{s}}\left(m_{1}\right)}\right)^{a_{j}} \frac{1}{\alpha_{\mathrm{s}}\left(m_{1}\right)}-\left(\frac{\alpha_{\mathrm{s}}\left(m_{2}\right)}{\alpha_{\mathrm{s}}\left(m_{1}\right)}\right)^{a_{i}} \frac{1}{\alpha_{\mathrm{s}}\left(m_{2}\right)}\right] \\
\left(K_{1}^{(1)}\left(m_{1}, m_{2}\right)\right)_{i j}=\left\{\begin{array}{ll}
\frac{M_{i j}^{(1)}}{a_{i}-a_{j}}\left[\left(\frac{\alpha_{\mathrm{s}}\left(m_{2}\right)}{\alpha_{\mathrm{s}}\left(m_{1}\right)}\right)^{a_{j}}-\left(\frac{\alpha_{\mathrm{s}}\left(m_{2}\right)}{\alpha_{\mathrm{s}}\left(m_{1}\right)}\right)^{a_{i}}\right] & i \neq j \\
M_{i i}^{(1)}\left(\frac{\alpha_{\mathrm{s}}\left(m_{2}\right)}{\alpha_{\mathrm{s}}\left(m_{1}\right)}\right)^{a_{i}} \ln \frac{\alpha_{\mathrm{s}}\left(m_{1}\right)}{\alpha_{\mathrm{s}}\left(m_{2}\right)} & i=j
\end{array},\right. \\
K_{2}^{(1)}\left(m_{1}, m_{2}\right)=-\alpha_{\mathrm{s}}\left(m_{2}\right) K^{(0)}\left(m_{1}, m_{2}\right) H \\
K_{3}^{(1)}\left(m_{1}, m_{2}\right)=\alpha_{\mathrm{s}}\left(m_{1}\right) H K^{(0)}\left(m_{1}, m_{2}\right)
\end{gathered}
$$

with

$$
\begin{aligned}
& M^{(0)}=V^{-1} \gamma_{\mathrm{e}}^{(0) T} V \\
& M^{(1)}=V^{-1}\left(\gamma_{\mathrm{se}}^{(1) T}-\frac{\beta_{1}}{\beta_{0}} \gamma_{\mathrm{e}}^{(0) T}+\left[\gamma_{\mathrm{e}}^{(0) T}, J\right]\right) V .
\end{aligned}
$$

The matrix $H$ is defined in (III.97). 
After this formal description we now give explicit expressions for the $10 \times 10 \mathrm{LO}$ and NLO anomalous dimension matrices $\gamma_{\mathrm{s}}^{(0)}, \gamma_{\mathrm{e}}^{(0)}, \gamma_{\mathrm{s}}^{(1)}$ and $\gamma_{\mathrm{se}}^{(1)}$. The values quoted for the NLO matrices are in the NDR scheme (Buras et al., 1993c), (Buras et al., 1993a), (Ciuchini et al., 1994a). The corresponding results for $\gamma_{\mathrm{s}}^{(1)}$ and $\gamma_{\mathrm{se}}^{(1)}$ in the HV scheme can either be obtained by direct calculation or by using the QCD/QED version of eq. (III.126) given in (Buras et al., 1993a). They can be found in (Buras et al., 1993c), (Buras et al., 1993a) and (Ciuchini et al., 1993a), (Ciuchini et al., 1994a).

The $6 \times 6$ submatrices for $Q_{1}, \ldots, Q_{6}$ of the full LO and NLO $10 \times 10$ QCD matrices $\gamma_{\mathrm{s}}^{(0)}$ and $\gamma_{\mathrm{s}}^{(1)}$ are identical to the corresponding $6 \times 6$ matrices already given in eqs. (VI.25) and (VI.26), respectively. Next, $Q_{1}, \ldots, Q_{6}$ do not mix to $Q_{7}, \ldots, Q_{10}$ under QCD and hence

$$
\left[\gamma_{\mathrm{s}}^{(0)}\right]_{i j}=\left[\gamma_{\mathrm{s}}^{(1)}\right]_{i j}=0 \quad i=1, \ldots, 6 \quad j=7, \ldots, 10
$$

The remaining entries for rows 7-10 in $\gamma_{\mathrm{s}}^{(0)}$ (Bijnens and Wise, 1984) and $\gamma_{\mathrm{s}}^{(1)}$ (Buras et al., 1993c), (Ciuchini et al., 1994a) are given in tables XIV and XV, respectively. There $u$ and $d(f=u+d)$ denote the number of active up- and down-type quark flavours.

TABLE XIV. Rows $7-10$ of the LO anomalous dimension matrix $\gamma_{\mathrm{s}}^{(0)}$.

\begin{tabular}{|r|r|c|c|c|c|c|c|c|c|c|}
\hline \hline$(i, j)$ & 1 & 2 & 3 & 4 & 5 & 6 & 7 & 8 & 9 & 10 \\
\hline 7 & 0 & 0 & 0 & 0 & 0 & 0 & $\frac{6}{N}$ & -6 & 0 & 0 \\
8 & 0 & 0 & $\frac{-2(u-d / 2)}{3 N}$ & $\frac{2(u-d / 2)}{3}$ & $\frac{-2(u-d / 2)}{3 N}$ & $\frac{2(u-d / 2)}{3}$ & 0 & $\frac{-6\left(-1+N^{2}\right)}{N}$ & 0 & 0 \\
9 & 0 & 0 & $\frac{2}{3 N}$ & $-\frac{2}{3}$ & $\frac{2}{3 N}$ & $-\frac{2}{3}$ & 0 & 0 & $\frac{-6}{N}$ & 6 \\
10 & 0 & 0 & $\frac{-2(u-d / 2)}{3 N}$ & $\frac{2(u-d / 2)}{3}$ & $\frac{-2(u-d / 2)}{3 N}$ & $\frac{2(u-d / 2)}{3}$ & 0 & 0 & 6 & $\frac{-6}{N}$ \\
\hline \hline
\end{tabular}

TABLE XV. Rows 7-10 of the NLO anomalous dimension matrix $\gamma_{\mathrm{s}}^{(1)}$ for $N=3$ and NDR.

\begin{tabular}{rr|r|c|c|c|}
\hline \hline$(i, j)$ & 1 & 2 & 3 & 4 & 5 \\
\hline 7 & 0 & 0 & $\frac{-61(u-d / 2)}{9}$ & $\frac{-11(u-d / 2)}{3}$ & $\frac{83(u-d / 2)}{9}$ \\
8 & 0 & 0 & $\frac{-682(u-d / 2)}{243}$ & $\frac{106(u-d / 2)}{81}$ & $\frac{704(u-d / 2)}{243}$ \\
9 & 0 & 0 & $\frac{202}{243}+\frac{73(u-d / 2)}{9}$ & $-\frac{1354}{81}-\frac{(u-d / 2)}{3}$ & $\frac{1192}{243}-\frac{71(u-d / 2)}{9}$ \\
10 & 0 & 0 & $-\frac{79}{9}-\frac{106(u-d / 2)}{243}$ & $\frac{7}{3}+\frac{826(u-d / 2)}{81}$ & $\frac{65}{9}-\frac{502(u-d / 2)}{243}$ \\
\hline \hline
\end{tabular}

\begin{tabular}{|c|c|c|c|c|c|}
\hline$(i, j)$ & 6 & 7 & 8 & 9 & 10 \\
\hline 7 & $\frac{-11(u-d / 2)}{3}$ & $\frac{71}{3}-\frac{22 f}{9}$ & $-99+\frac{22 f}{3}$ & 0 & 0 \\
\hline 8 & $\frac{736(u-d / 2)}{81}$ & $-\frac{225}{2}+4 f$ & $-\frac{1343}{6}+\frac{68 f}{9}$ & 0 & 0 \\
\hline 9 & $-\frac{904}{81}-\frac{(u-d / 2)}{3}$ & 0 & 0 & $-\frac{21}{2}-\frac{2 f}{9}$ & $\frac{7}{2}+\frac{2 f}{3}$ \\
\hline 10 & $\frac{7}{3}+\frac{646(u-d / 2)}{81}$ & 0 & 0 & $\frac{7}{2}+\frac{2 f}{3}$ & $-\frac{21}{2}-\frac{2 f}{9}$ \\
\hline
\end{tabular}

The full $10 \times 10$ matrices $\gamma_{\mathrm{e}}^{(0)}$ (Lusignoli, 1989) and $\gamma_{\mathrm{se}}^{(1)}$ (Buras et al., 1993a), (Ciuchini et al., 1994a) can be found in tables XVI and XVII, respectively. 
TABLE XVI. The LO anomalous dimension matrix $\gamma_{\mathrm{e}}^{(0)}$.

\begin{tabular}{|r|c|c|c|c|c|c|c|c|c|c|}
\hline \hline$(i, j)$ & 1 & 2 & 3 & 4 & 5 & 6 & 7 & 8 & 9 & 10 \\
\hline 1 & $-\frac{8}{3}$ & 0 & 0 & 0 & 0 & 0 & $\frac{16 N}{27}$ & 0 & $\frac{16 N}{27}$ & 0 \\
2 & 0 & $-\frac{8}{3}$ & 0 & 0 & 0 & 0 & $\frac{16}{27}$ & 0 & $\frac{16}{27}$ & 0 \\
3 & 0 & 0 & 0 & 0 & 0 & 0 & $-\frac{16}{27}+\frac{16 N(u-d / 2)}{27}$ & 0 & $-\frac{88}{27}+\frac{16 N(u-d / 2)}{27}$ & 0 \\
4 & 0 & 0 & 0 & 0 & 0 & 0 & $\frac{-16 N+\frac{16(u-d / 2)}{27}}{27}$ & 0 & $\frac{-16 N}{27}+\frac{16(u-d / 2)}{27}$ & $-\frac{8}{3}$ \\
5 & 0 & 0 & 0 & 0 & 0 & 0 & $\frac{8}{3}+\frac{16 N(u-d / 2)}{27}$ & 0 & $\frac{16 N(u-d / 2)}{27}$ & 0 \\
6 & 0 & 0 & 0 & 0 & 0 & 0 & $\frac{16(u-d / 2)}{27}$ & $\frac{8}{3}$ & $\frac{16(u-d / 2)}{27}$ & 0 \\
7 & 0 & 0 & 0 & 0 & $\frac{4}{3}$ & 0 & $\frac{4}{3}+\frac{16 N(u+d / 4)}{27}$ & 0 & $\frac{16 N(u+d / 4)}{27}$ & 0 \\
8 & 0 & 0 & 0 & 0 & 0 & $\frac{4}{3}$ & $\frac{16(u+d / 4)}{27}$ & $\frac{4}{3}$ & $\frac{16(u+d / 4)}{27}$ & 0 \\
9 & 0 & 0 & $-\frac{4}{3}$ & 0 & 0 & 0 & $\frac{8}{27}+\frac{16 N(u+d / 4)}{27}$ & 0 & $-\frac{28}{27}+\frac{16 N(u+d / 4)}{27}$ & 0 \\
10 & 0 & 0 & 0 & $-\frac{4}{3}$ & 0 & 0 & $\frac{8 N}{27}+\frac{16(u+d / 4)}{27}$ & 0 & $\frac{8 N}{27}+\frac{16(u+d / 4)}{27}$ & $-\frac{4}{3}$ \\
\hline \hline
\end{tabular}

\section{Quark Threshold Matching Matrix}

Extending the matching matrix $M(m)$ of (VI.27) to the simultaneous presence of QCD and QED corrections yields

$$
M(m)=1+\frac{\alpha_{\mathrm{s}}(m)}{4 \pi} \delta r_{\mathrm{s}}^{T}+\frac{\alpha}{4 \pi} \delta r_{\mathrm{e}}^{T}
$$

At scale $\mu=m_{\mathrm{b}}$ the matrices $\delta r_{\mathrm{s}}$ and $\delta r_{\mathrm{e}}$ read

$$
\begin{gathered}
\delta r_{\mathrm{s}}^{T}=\frac{5}{18} P(0,0,0,-2,0,-2,0,1,0,1) \\
\delta r_{\mathrm{e}}^{T}=\frac{10}{81} \bar{P}(0,0,6,2,6,2,-3,-1,-3,-1)
\end{gathered}
$$

and at $\mu=m_{\mathrm{c}}$

$$
\begin{gathered}
\delta r_{\mathrm{s}}^{T}=-\frac{5}{9} P(0,0,0,1,0,1,0,1,0,1) \\
\delta r_{\mathrm{e}}^{T}=-\frac{40}{81} \bar{P}(0,0,3,1,3,1,3,1,3,1)
\end{gathered}
$$

with eq. (VI.29) generalized to

$$
\begin{aligned}
P^{T} & =\left(0,0,-\frac{1}{3}, 1,-\frac{1}{3}, 1,0,0,0,0\right), \\
\bar{P}^{T} & =(0,0,0,0,0,0,1,0,1,0) .
\end{aligned}
$$


TABLE XVII. The NLO anomalous dimension matrix $\gamma_{\mathrm{se}}^{(1)}$ for $N=3$ and NDR.

\begin{tabular}{|c|c|c|c|c|c|}
\hline$(i, j)$ & 1 & 2 & 3 & 4 & 5 \\
\hline 1 & $\frac{194}{9}$ & $-\frac{2}{3}$ & $-\frac{88}{243}$ & $\frac{88}{81}$ & $-\frac{88}{243}$ \\
\hline 2 & $\frac{25}{3}$ & $-\frac{49}{9}$ & $-\frac{556}{729}$ & $\frac{556}{243}$ & $-\frac{556}{729}$ \\
\hline 3 & 0 & 0 & $\frac{1690}{729}-\frac{136(u-d / 2)}{243}$ & $-\frac{1690}{243}+\frac{136(u-d / 2)}{81}$ & $\frac{232}{729}-\frac{136(u-d / 2)}{243}$ \\
\hline 4 & 0 & 0 & $-\frac{641}{243}-\frac{388 u}{729}+\frac{32 d}{729}$ & $-\frac{655}{81}+\frac{388 u}{243}-\frac{32 d}{243}$ & $\frac{88}{243}-\frac{388 u}{729}+\frac{32 d}{729}$ \\
\hline 5 & 0 & 0 & $\frac{-136(u-d / 2)}{243}$ & $\frac{136(u-d / 2)}{81}$ & $-2-\frac{136(u-d / 2)}{243}$ \\
\hline 6 & 0 & 0 & $\frac{-748 u}{729}+\frac{212 d}{729}$ & $\frac{748 u}{243}-\frac{212 d}{243}$ & $3-\frac{748 u}{729}+\frac{212 d}{729}$ \\
\hline 7 & 0 & 0 & $\frac{-136(u+d / 4)}{243}$ & $\frac{136(u+d / 4)}{81}$ & $-\frac{116}{9}-\frac{136(u+d / 4)}{243}$ \\
\hline 8 & 0 & 0 & $\frac{-748 u}{729}-\frac{106 d}{729}$ & $\frac{748 u}{243}+\frac{106 d}{243}$ & $-1-\frac{748 u}{729}-\frac{106 d}{729}$ \\
\hline 9 & 0 & 0 & $\frac{7012}{729}-\frac{136(u+d / 4)}{243}$ & $\frac{764}{243}+\frac{136(u+d / 4)}{81}$ & $-\frac{116}{729}-\frac{136(u+d / 4)}{243}$ \\
\hline 10 & 0 & 0 & $\frac{1333}{243}-\frac{388 u}{729}-\frac{16 d}{729}$ & $\frac{107}{81}+\frac{388 u}{243}+\frac{16 d}{243}$ & $-\frac{44}{243}-\frac{388 u}{729}-\frac{16 d}{729}$ \\
\hline
\end{tabular}

\begin{tabular}{|r|c|c|c|c|c|}
\hline \hline$(i, j)$ & 6 & 7 & 8 & 9 & 10 \\
\hline 1 & $\frac{88}{81}$ & $\frac{152}{27}$ & $\frac{40}{9}$ & $\frac{136}{27}$ & $\frac{56}{9}$ \\
2 & $\frac{556}{243}$ & $-\frac{484}{729}$ & $-\frac{124}{27}$ & $-\frac{3148}{729}$ & $\frac{172}{27}$ \\
3 & $-\frac{232}{243}+\frac{136(u-d / 2)}{81}$ & $\frac{3136}{729}+\frac{104(u-d / 2)}{27}$ & $\frac{64}{27}+\frac{88(u-d / 2)}{9}$ & $\frac{20272}{729}+\frac{184(u-d / 2)}{27}$ & $-\frac{112}{27}+\frac{8(u-d / 2)}{9}$ \\
4 & $-\frac{88}{81}+\frac{388 u}{243}-\frac{32 d}{243}$ & $-\frac{152}{27}+\frac{3140 u}{729}+\frac{656 d}{729}$ & $-\frac{40}{9}-\frac{100 u}{27}-\frac{16 d}{27}$ & $\frac{170}{27}+\frac{908 u}{729}+\frac{1232 d}{729}$ & $-\frac{14}{3}+\frac{148 u}{27}-\frac{80 d}{27}$ \\
5 & $6+\frac{136(u-d / 2)}{81}$ & $-\frac{232}{9}+\frac{104(u-d / 2)}{27}$ & $\frac{40}{3}+\frac{88(u-d / 2)}{9}$ & $\frac{184(u-d / 2)}{27}$ & $\frac{8(u-d / 2)}{9}$ \\
6 & $7+\frac{748 u}{243}-\frac{212 d}{243}$ & $-2-\frac{5212 u}{729}+\frac{4832 d}{729}$ & $\frac{182}{9}+\frac{188 u}{27}-\frac{160 d}{27}$ & $\frac{-2260 u}{729}+\frac{2816 d}{729}$ & $\frac{-140 u}{27}+\frac{64 d}{27}$ \\
7 & $\frac{20}{3}+\frac{136(u+d / 4)}{81}$ & $-\frac{134}{9}+\frac{104(u+d / 4)}{27}$ & $\frac{38}{3}+\frac{88(u+d / 4)}{9}$ & $\frac{184(u+d / 4)}{27}$ & $\frac{8(u+d / 4)}{9}$ \\
8 & $\frac{91}{9}+\frac{748 u}{243}+\frac{106 d}{243}$ & $2-\frac{5212 u}{729}-\frac{2416 d}{729}$ & $\frac{154}{9}+\frac{188 u}{27}+\frac{80 d}{27}$ & $\frac{-2260 u}{729}-\frac{1408 d}{729}$ & $\frac{-140 u}{27}-\frac{32 d}{27}$ \\
9 & $\frac{116}{243}+\frac{136(u+d / 4)}{81}$ & $-\frac{1568}{729}+\frac{104(u+d / 4)}{27}$ & $-\frac{32}{27}+\frac{88(u+d / 4)}{9}$ & $\frac{5578}{729}+\frac{184(u+d / 4)}{27}$ & $\frac{38}{27}+\frac{8(u+d / 4)}{9}$ \\
10 & $\frac{44}{81}+\frac{388 u}{243}+\frac{16 d}{243}$ & $\frac{76}{27}+\frac{3140 u}{729}-\frac{328 d}{729}$ & $\frac{20}{9}-\frac{100 u}{27}+\frac{8 d}{27}$ & $\frac{140}{27}+\frac{908 u}{729}-\frac{616 d}{729}$ & $-\frac{28}{9}+\frac{148 u}{27}+\frac{40 d}{27}$ \\
\hline \hline
\end{tabular}

\section{E. Numerical Results for the $K \rightarrow \pi \pi$ Wilson Coefficients}

Tables XVIII-XX give the $\Delta S=1$ Wilson coefficients for $Q_{1}, \ldots, Q_{10}$ in the mixed case of QCD and QED.

The coefficients for the current-current and QCD penguin operators $Q_{1}, \ldots, Q_{6}$ are only very weakly affected by the extension of the operator basis to the electroweak penguin operators $Q_{7}, \ldots, Q_{10}$. Therefore the discussion for $Q_{1}, \ldots, Q_{6}$ given in connection with tables X-XII for the case of pure QCD basically still holds and will not be repeated here.

For the remaining coefficients of $Q_{7}, \ldots, Q_{10}$ one finds a moderate scheme dependence for $y_{7}, y_{9}$ and $y_{10}$, but a $\mathcal{O}(9 \%)$ one for $y_{8}$. The notable feature of $\left|y_{6}\right|$ being larger in NDR than in HV still holds, but is now confronted with an exactly opposite dependence for the other important $\Delta S=1$ Wilson coefficient $y_{8}$ which is in addition enhanced over its $\mathrm{LO}$ value.

The particular dependence of $y_{6}$ and $y_{8}$ with respect to scheme, LO/NLO and $m_{\mathrm{t}}$ (see below) should be kept in mind for the later discussion of $\varepsilon^{\prime} / \varepsilon$ in section XIX. 
TABLE XVIII. $\Delta S=1$ Wilson coefficients at $\mu=1 \mathrm{GeV}$ for $m_{\mathrm{t}}=170 \mathrm{GeV} \cdot y_{1}=y_{2} \equiv 0$.

\begin{tabular}{|c|c|c|c||c|c|c||c|c|c|}
\hline \hline & \multicolumn{3}{|c||}{$\Lambda \frac{(4)}{\mathrm{MS}}=215 \mathrm{MeV}$} & \multicolumn{3}{c||}{$\Lambda_{\overline{\mathrm{MS}}}^{(4)}=325 \mathrm{MeV}$} & \multicolumn{3}{c|}{$\Lambda_{\mathrm{MS}}^{(4)}=435 \mathrm{MeV}$} \\
\hline Scheme & LO & NDR & HV & LO & NDR & HV & LO & NDR & HV \\
\hline$z_{1}$ & -0.607 & -0.409 & -0.494 & -0.748 & -0.509 & -0.640 & -0.907 & -0.625 & -0.841 \\
$z_{2}$ & 1.333 & 1.212 & 1.267 & 1.433 & 1.278 & 1.371 & 1.552 & 1.361 & 1.525 \\
\hline$z_{3}$ & 0.003 & 0.008 & 0.004 & 0.004 & 0.013 & 0.007 & 0.006 & 0.023 & 0.015 \\
$z_{4}$ & -0.008 & -0.022 & -0.010 & -0.012 & -0.035 & -0.017 & -0.017 & -0.058 & -0.029 \\
$z_{5}$ & 0.003 & 0.006 & 0.003 & 0.004 & 0.008 & 0.004 & 0.005 & 0.009 & 0.005 \\
$z_{6}$ & -0.009 & -0.022 & -0.009 & -0.013 & -0.035 & -0.014 & -0.018 & -0.059 & -0.025 \\
\hline$z_{7} / \alpha$ & 0.004 & 0.003 & -0.003 & 0.008 & 0.011 & -0.002 & 0.011 & 0.021 & -0.001 \\
$z_{8} / \alpha$ & 0 & 0.008 & 0.006 & 0.001 & 0.014 & 0.010 & 0.001 & 0.027 & 0.017 \\
$z_{9} / \alpha$ & 0.005 & 0.007 & 0 & 0.008 & 0.018 & 0.005 & 0.012 & 0.034 & 0.011 \\
$z_{10} / \alpha$ & 0 & -0.005 & -0.006 & -0.001 & -0.008 & -0.010 & -0.001 & -0.014 & -0.017 \\
\hline$y_{3}$ & 0.030 & 0.025 & 0.028 & 0.038 & 0.032 & 0.037 & 0.047 & 0.042 & 0.050 \\
$y_{4}$ & -0.052 & -0.048 & -0.050 & -0.061 & -0.058 & -0.061 & -0.071 & -0.068 & -0.074 \\
$y_{5}$ & 0.012 & 0.005 & 0.013 & 0.013 & -0.001 & 0.016 & 0.014 & -0.013 & 0.021 \\
$y_{6}$ & -0.085 & -0.078 & -0.071 & -0.113 & -0.111 & -0.097 & -0.148 & -0.169 & -0.139 \\
\hline$y_{7} / \alpha$ & 0.027 & -0.033 & -0.032 & 0.036 & -0.032 & -0.030 & 0.043 & -0.031 & -0.027 \\
$y_{8} / \alpha$ & 0.114 & 0.121 & 0.133 & 0.158 & 0.173 & 0.188 & 0.216 & 0.254 & 0.275 \\
$y_{9} / \alpha$ & -1.491 & -1.479 & -1.480 & -1.585 & -1.576 & -1.577 & -1.700 & -1.718 & -1.722 \\
$y_{10} / \alpha$ & 0.650 & 0.540 & 0.547 & 0.800 & 0.690 & 0.699 & 0.968 & 0.892 & 0.906 \\
\hline \hline
\end{tabular}

We also note that in the range of $m_{\mathrm{t}}$ considered here, $y_{7}$ is very small, $y_{9}$ is essentially unaffected by NLO QCD corrections and $y_{10}$ is suppressed for $\mu \geq m_{\mathrm{c}}$. It should also be stressed that $\left|y_{9}\right|$ and $\left|y_{10}\right|$ are substantially larger than $\left|y_{8}\right|$ although, as we will see in the analysis of $\varepsilon^{\prime} / \varepsilon$, the operator $Q_{8}$ is more important than $Q_{9}$ and $Q_{10}$ for this ratio.

Next, one infers from tables XVIII-XX that also in the mixed QCD/QED case the Wilson coefficients show a strong dependence on $\Lambda_{\overline{\mathrm{MS}}}$.

In contrast to the coefficients $y_{3}, \ldots, y_{6}$ for QCD penguins, $y_{7}, \ldots, y_{10}$ for the electroweak penguins show a sizeable $m_{\mathrm{t}}$ dependence in the range $m_{\mathrm{t}}=(170 \pm 15) \mathrm{GeV}$. With in/decreasing $m_{\mathrm{t}}$ there is a relative variation of $\mathcal{O}( \pm 19 \%)$ and $\mathcal{O}( \pm 10 \%)$ for the absolute values of $y_{8}$ and $y_{9,10}$, respectively. This is illustrated further in figs. 5 and 6 where the $m_{\mathrm{t}}$ dependence of these coefficients is shown explicitly. This strong $m_{\mathrm{t}}$-dependence originates in the $Z^{0}$-penguin diagrams. The $m_{\mathrm{t}}$-dependence of $y_{9}$ and $y_{10}$ can be conveniently parametrized by a linear function to an accuracy better than $0.5 \%$. Details of this $m_{\mathrm{t}}$-parametriziation can be found in table XXI.

Finally, in tables XVIII-XX one observes again the usual feature of decreasing Wilson coefficients with increasing scale $\mu$.

\section{F. The $\Delta B=1$ Effective Hamiltonian Including Electroweak Penguins}

Finally we present in this section the Wilson coefficient functions of the $\Delta B=1, \Delta C=0$ hamiltonian, including the effects of electroweak penguin contributions (Buras et al., 1993b). 
TABLE XIX. $\Delta S=1$ Wilson coefficients at $\mu=m_{\mathrm{c}}=1.3 \mathrm{GeV}$ for $m_{\mathrm{t}}=170 \mathrm{GeV}$ and $f=3$ effective flavours. $\left|z_{3}\right|, \ldots,\left|z_{10}\right|$ are numerically irrelevant relative to $\left|z_{1,2}\right| \cdot y_{1}=y_{2} \equiv 0$.

\begin{tabular}{|c|c|c|c||c|c|c||c|c|c|}
\hline \hline & \multicolumn{3}{|c||}{$\Lambda_{\overline{\mathrm{MS}}}^{(4)}=215 \mathrm{MeV}$} & \multicolumn{3}{c||}{$\Lambda_{\overline{\mathrm{MS}}}^{(4)}=325 \mathrm{MeV}$} & \multicolumn{3}{c|}{$\Lambda_{\overline{\mathrm{MS}}}^{(4)}=435 \mathrm{MeV}$} \\
\hline Scheme & LO & NDR & HV & LO & NDR & HV & LO & NDR & HV \\
\hline$z_{1}$ & -0.521 & -0.346 & -0.413 & -0.625 & -0.415 & -0.507 & -0.732 & -0.490 & -0.617 \\
$z_{2}$ & 1.275 & 1.172 & 1.214 & 1.345 & 1.216 & 1.276 & 1.420 & 1.265 & 1.354 \\
\hline$y_{3}$ & 0.027 & 0.023 & 0.025 & 0.034 & 0.029 & 0.033 & 0.041 & 0.036 & 0.042 \\
$y_{4}$ & -0.051 & -0.048 & -0.049 & -0.061 & -0.057 & -0.060 & -0.070 & -0.068 & -0.072 \\
$y_{5}$ & 0.013 & 0.007 & 0.014 & 0.015 & 0.005 & 0.016 & 0.017 & 0.001 & 0.018 \\
$y_{6}$ & -0.076 & -0.068 & -0.063 & -0.096 & -0.089 & -0.081 & -0.120 & -0.118 & -0.103 \\
\hline$y_{7} / \alpha$ & 0.030 & -0.031 & -0.031 & 0.039 & -0.030 & -0.028 & 0.048 & -0.029 & -0.026 \\
$y_{8} / \alpha$ & 0.092 & 0.103 & 0.112 & 0.121 & 0.136 & 0.145 & 0.155 & 0.179 & 0.189 \\
$y_{9} / \alpha$ & -1.428 & -1.423 & -1.423 & -1.490 & -1.479 & -1.479 & -1.559 & -1.548 & -1.549 \\
$y_{10} / \alpha$ & 0.558 & 0.451 & 0.457 & 0.668 & 0.547 & 0.553 & 0.781 & 0.656 & 0.664 \\
\hline \hline
\end{tabular}

TABLE XX. $\Delta S=1$ Wilson coefficients at $\mu=2 \mathrm{GeV}$ for $m_{\mathrm{t}}=170 \mathrm{GeV}$. For $\mu>m_{\mathrm{c}}$ the GIM mechanism gives $z_{i} \equiv 0, i=3, \ldots, 10 . y_{1}=y_{2} \equiv 0$.

\begin{tabular}{|c|c|c|c||c|c|c||c|c|c|}
\hline \hline & \multicolumn{3}{|c||}{$\Lambda \frac{(4)}{\mathrm{MS}}=215 \mathrm{MeV}$} & \multicolumn{3}{c||}{$\Lambda_{\overline{\mathrm{MS}}}^{(4)}=325 \mathrm{MeV}$} & \multicolumn{3}{c|}{$\Lambda_{\overline{\mathrm{MS}}}^{(4)}=435 \mathrm{MeV}$} \\
\hline Scheme & LO & NDR & HV & LO & NDR & HV & LO & NDR & HV \\
\hline$z_{1}$ & -0.413 & -0.268 & -0.320 & -0.480 & -0.310 & -0.376 & -0.544 & -0.352 & -0.432 \\
$z_{2}$ & 1.206 & 1.127 & 1.157 & 1.248 & 1.151 & 1.191 & 1.290 & 1.176 & 1.227 \\
\hline$y_{3}$ & 0.021 & 0.020 & 0.019 & 0.025 & 0.024 & 0.023 & 0.028 & 0.028 & 0.027 \\
$y_{4}$ & -0.041 & -0.046 & -0.040 & -0.047 & -0.055 & -0.046 & -0.053 & -0.063 & -0.053 \\
$y_{5}$ & 0.011 & 0.010 & 0.012 & 0.012 & 0.011 & 0.013 & 0.014 & 0.011 & 0.015 \\
$y_{6}$ & -0.056 & -0.058 & -0.047 & -0.068 & -0.071 & -0.057 & -0.079 & -0.086 & -0.068 \\
\hline$y_{7} / \alpha$ & 0.031 & -0.023 & -0.020 & 0.037 & -0.019 & -0.020 & 0.042 & -0.016 & -0.019 \\
$y_{8} / \alpha$ & 0.068 & 0.076 & 0.084 & 0.084 & 0.094 & 0.102 & 0.101 & 0.113 & 0.121 \\
$y_{9} / \alpha$ & -1.357 & -1.361 & -1.357 & -1.393 & -1.389 & -1.389 & -1.430 & -1.419 & -1.423 \\
$y_{10} / \alpha$ & 0.442 & 0.356 & 0.360 & 0.513 & 0.414 & 0.419 & 0.581 & 0.472 & 0.477 \\
\hline \hline
\end{tabular}

These effects play a role in certain penguin-induced B meson decays as discussed in (Fleischer, 1994a), (Fleischer, 1994b), (Deshpande et al., 1995), (Deshpande and He, 1995).

The generalization of the $\Delta B=1, \Delta C=0$ hamiltonian in pure QCD (VI.32) to incorporate also electroweak penguin operators is straightforward. One obtains

$$
\begin{aligned}
\mathcal{H}_{\mathrm{eff}}(\Delta B=1)= & \frac{G_{F}}{\sqrt{2}}\left\{\xi_{c}\left[C_{1}(\mu) Q_{1}^{c}(\mu)+C_{2}(\mu) Q_{2}^{c}(\mu)\right]+\xi_{u}\left[C_{1}(\mu) Q_{1}^{u}(\mu)+C_{2}(\mu) Q_{2}^{u}(\mu)\right]\right. \\
& \left.-\xi_{t} \sum_{i=3}^{10} C_{i}(\mu) Q_{i}(\mu)\right\}
\end{aligned}
$$


TABLE XXI. Coefficients in linear $m_{\mathrm{t}}$-parametriziation $y_{i} / \alpha=a+b \cdot\left(m_{\mathrm{t}} / 170 \mathrm{GeV}\right)$ of Wilson coefficients $y_{9} / \alpha$ and $y_{10} / \alpha$ at scale $\mu=m_{\mathrm{c}}$ for $\Lambda_{\overline{\mathrm{MS}}}^{(4)}=325 \mathrm{MeV}$.

\begin{tabular}{|c||c|c||c|c|}
\hline \hline \hline & & \multicolumn{2}{c||}{$y_{9} / \alpha$} & \multicolumn{2}{|c|}{$y_{10} / \alpha$} \\
\hline & $\mathrm{a}$ & $\mathrm{b}$ & $\mathrm{a}$ & $\mathrm{b}$ \\
\hline LO & 0.189 & -1.682 & -0.111 & 0.780 \\
NDR & 0.129 & -1.611 & -0.128 & 0.676 \\
HV & 0.129 & -1.611 & -0.121 & 0.676 \\
\hline \hline
\end{tabular}

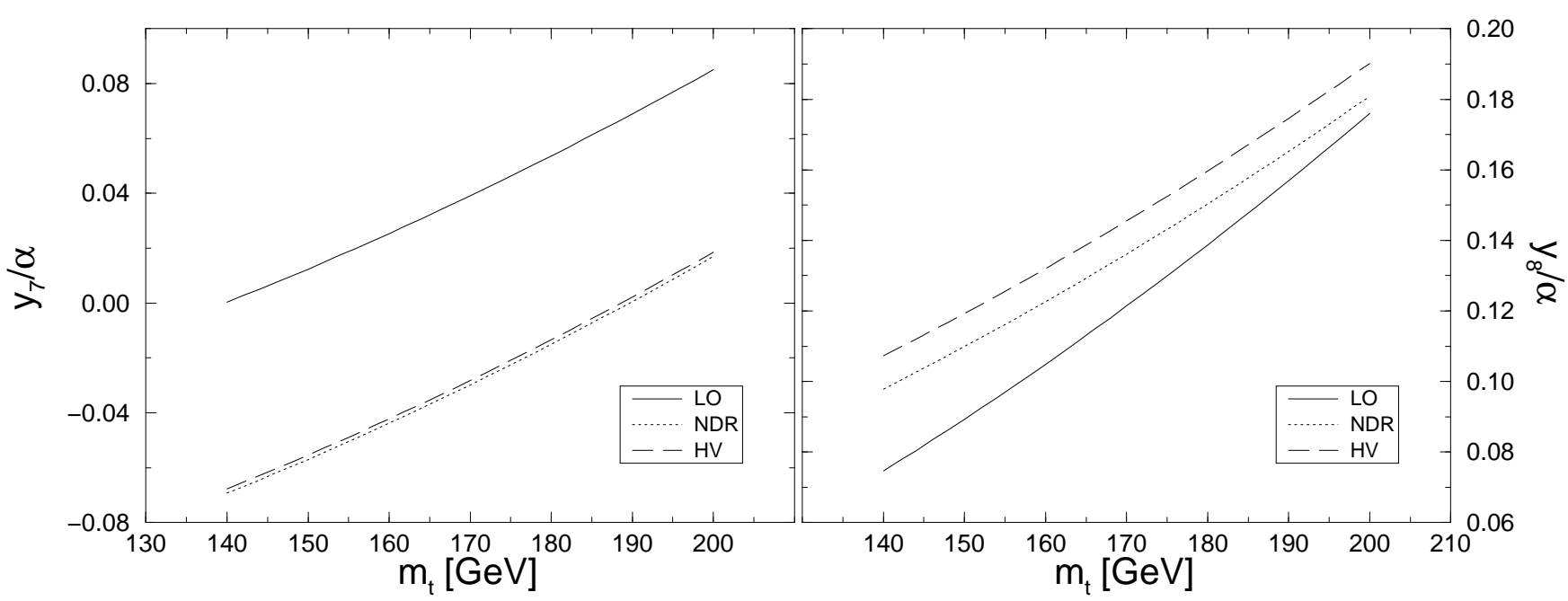

FIG. 5. Wilson coefficients $y_{7}\left(m_{\mathrm{c}}\right) / \alpha$ and $y_{8}\left(m_{\mathrm{c}}\right) / \alpha$ as functions of $m_{\mathrm{t}}$ for $\Lambda \frac{(4)}{\mathrm{MS}}=325 \mathrm{MeV}$.

where the operator basis now includes the electroweak penguin operators

$$
\begin{aligned}
Q_{7} & =\frac{3}{2}(\bar{b} d)_{\mathrm{V}-\mathrm{A}} \sum_{q} e_{q}(\bar{q} q)_{\mathrm{V}+\mathrm{A}}, \\
Q_{8} & =\frac{3}{2}\left(\bar{b}_{i} d_{j}\right)_{\mathrm{V}-\mathrm{A}} \sum_{q} e_{q}\left(\bar{q}_{j} q_{i}\right)_{\mathrm{V}+\mathrm{A}}, \\
Q_{9} & =\frac{3}{2}(\bar{b} d)_{\mathrm{V}-\mathrm{A}} \sum_{q} e_{q}(\bar{q} q)_{\mathrm{V}-\mathrm{A}}, \\
Q_{10} & =\frac{3}{2}\left(\bar{b}_{i} d_{j}\right)_{\mathrm{V}-\mathrm{A}} \sum_{q} e_{q}\left(\bar{q}_{j} q_{i}\right)_{\mathrm{V}-\mathrm{A}}
\end{aligned}
$$

in addition to (VI.33). The Wilson coefficients at $\mu=m_{b}$ read

$$
\vec{C}\left(m_{\mathrm{b}}\right)=U_{5}\left(m_{\mathrm{b}}, M_{\mathrm{W}}, \alpha\right) \vec{C}\left(M_{\mathrm{W}}\right) .
$$

where $U_{5}$ is the $10 \times 10$ evolution matrix of (VII.22) for $f=5$ flavors. The $\vec{C}\left(M_{\mathrm{W}}\right)$ are given in (VII.3) - (VII.12) in the NDR scheme. 


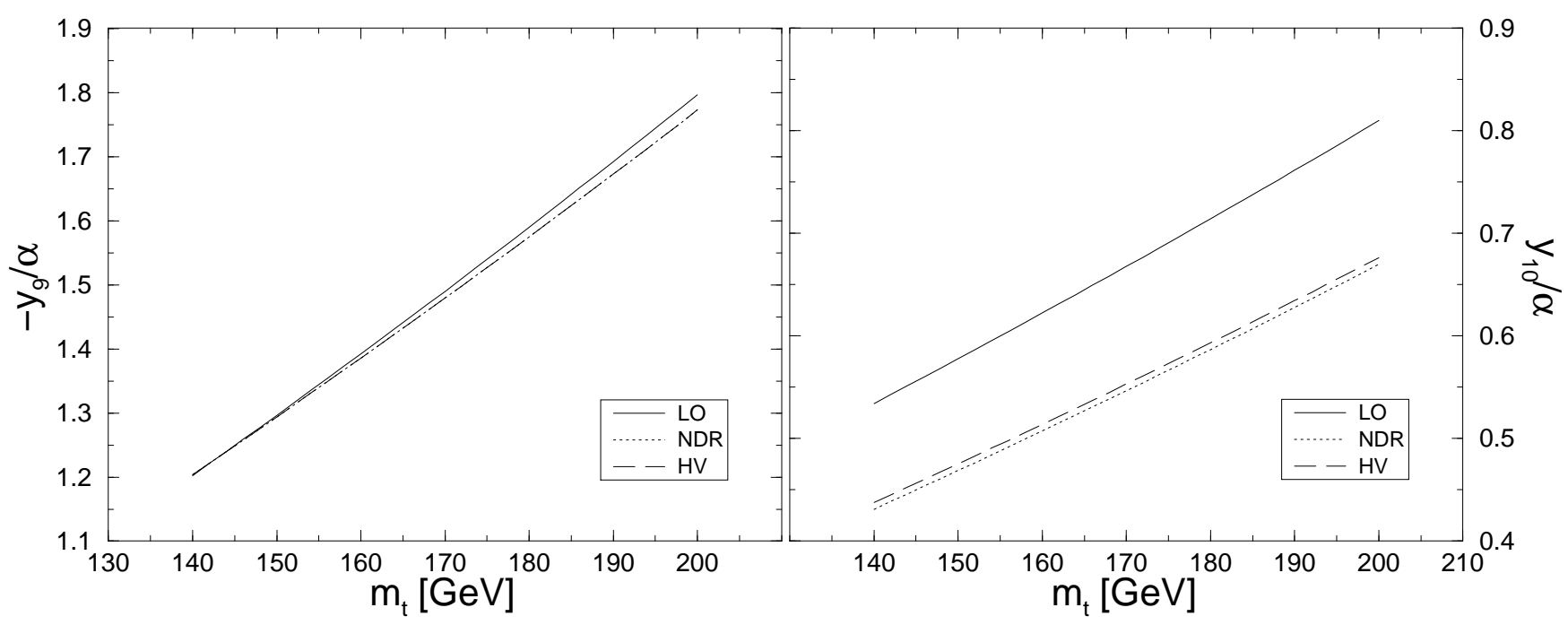

FIG. 6. Wilson coefficients $y_{9}\left(m_{\mathrm{c}}\right) / \alpha$ and $y_{10}\left(m_{\mathrm{c}}\right) / \alpha$ as a function of $m_{\mathrm{t}}$ for $\Lambda_{\overline{\mathrm{MS}}}^{(4)}=325 \mathrm{MeV}$.

G. Numerical Results for the $\Delta B=1$ Wilson Coefficients

TABLE XXII. $\Delta B=1$ Wilson coefficients at $\mu=\bar{m}_{\mathrm{b}}\left(m_{\mathrm{b}}\right)=4.40 \mathrm{GeV}$ for $m_{\mathrm{t}}=170 \mathrm{GeV}$.

\begin{tabular}{|c|c|c|c||c|c|c||c|c|c|}
\hline \hline & \multicolumn{3}{|c||}{$\Lambda_{\overline{\mathrm{MS}}}^{(5)}=140 \mathrm{MeV}$} & \multicolumn{3}{c||}{$\Lambda_{\overline{\mathrm{MS}}}^{(5)}=225 \mathrm{MeV}$} & \multicolumn{3}{c|}{$\Lambda_{\overline{\mathrm{MS}}}^{(5)}=310 \mathrm{MeV}$} \\
\hline Scheme & LO & NDR & HV & LO & NDR & HV & LO & NDR & HV \\
\hline$C_{1}$ & -0.273 & -0.165 & -0.202 & -0.308 & -0.185 & -0.228 & -0.339 & -0.203 & -0.251 \\
$C_{2}$ & 1.125 & 1.072 & 1.091 & 1.144 & 1.082 & 1.105 & 1.161 & 1.092 & 1.117 \\
\hline$C_{3}$ & 0.013 & 0.013 & 0.012 & 0.014 & 0.014 & 0.013 & 0.016 & 0.016 & 0.015 \\
$C_{4}$ & -0.027 & -0.031 & -0.026 & -0.030 & -0.035 & -0.029 & -0.033 & -0.039 & -0.033 \\
$C_{5}$ & 0.008 & 0.008 & 0.008 & 0.009 & 0.009 & 0.009 & 0.009 & 0.009 & 0.010 \\
$C_{6}$ & -0.033 & -0.036 & -0.029 & -0.038 & -0.041 & -0.033 & -0.043 & -0.046 & -0.037 \\
\hline$C_{7} / \alpha$ & 0.042 & -0.003 & 0.006 & 0.045 & -0.002 & 0.005 & 0.047 & -0.001 & 0.005 \\
$C_{8} / \alpha$ & 0.041 & 0.047 & 0.052 & 0.048 & 0.054 & 0.060 & 0.054 & 0.061 & 0.067 \\
$C_{9} / \alpha$ & -1.264 & -1.279 & -1.269 & -1.280 & -1.292 & -1.283 & -1.294 & -1.303 & -1.296 \\
$C_{10} / \alpha$ & 0.291 & 0.234 & 0.237 & 0.328 & 0.263 & 0.266 & 0.360 & 0.288 & 0.291 \\
\hline \hline
\end{tabular}

Table XXII lists the $\Delta B=1$ Wilson coefficients for $Q_{1}^{u, c}, Q_{2}^{u, c}, Q_{3}, \ldots, Q_{10}$ in the mixed case of QCD and QED.

Similarly to the $\Delta S=1$ case the coefficients for the current-current and QCD penguin operators $Q_{1}, \ldots, Q_{6}$ are only very weakly affected by the extension of the operator basis to the electroweak penguin operators $Q_{7}, \ldots, Q_{10}$. Therefore the discussion of $C_{1}, \ldots, C_{6}$ in connection with table XIII is also valid for the present case.

Here we therefore restrict our discussion to the coefficients $C_{7}, \ldots, C_{10}$ of the operators $Q_{7}, \ldots, Q_{10}$ in the extended basis. 
The coefficients $C_{7}, \ldots, C_{10}$ show a visible dependence on the scheme, $\Lambda_{\overline{\mathrm{MS}}}$ and LO/NLO. However, this dependence is less pronounced for the coefficient $C_{9}$ than it is for $C_{7,8,10}$. This is noteworthy since in $B$-meson decays $C_{9}$ usually resides in the dominant electroweak penguin contribution (Fleischer, 1994a), (Fleischer, 1994b), (Deshpande et al., 1995), (Deshpande and He, 1995).

In contrast to $C_{1}, \ldots, C_{6}$ the additional coefficients $C_{7}, \ldots, C_{10}$ show a non negligible $m_{\mathrm{t}}$ dependence in the range $m_{\mathrm{t}}=(170 \pm 15) \mathrm{GeV}$. With in/decreasing $m_{\mathrm{t}}$ there is similarly to the $\Delta S=1$ case a relative variation of $\mathcal{O}( \pm 19 \%)$ and $\mathcal{O}( \pm 10 \%)$ for the absolute values of $C_{8}$ and $C_{9,10}$, respectively.

Since the coefficients $C_{9}$ and $C_{10}$ play an important role in B decays we show in fig. 7 their $m_{\mathrm{t}}$ dependence explicitly. Again the $m_{\mathrm{t}}$-dependence can be parametrized by a linear function to an accuracy better than $0.5 \%$. Details of the $m_{\mathrm{t}}$-parametriziation are given in table XXIII.

TABLE XXIII. Coefficients in linear $m_{\mathrm{t}}$-parametriziation $C_{i} / \alpha=a+b \cdot\left(m_{\mathrm{t}} / 170 \mathrm{GeV}\right)$ of Wilson coefficients $C_{9} / \alpha$ and $C_{10} / \alpha$ at scale $\mu=m_{\mathrm{b}}=4.4 \mathrm{GeV}$ for $\Lambda \frac{(5)}{\mathrm{MS}}=225 \mathrm{MeV}$.

\begin{tabular}{|c||c|c||r|r|r|}
\hline \hline & & \multicolumn{1}{c||}{$C_{9} / \alpha$} & \multicolumn{2}{|c|}{$C_{10} / \alpha$} \\
\hline & $\mathrm{a}$ & $\mathrm{b}$ & $\mathrm{a}$ & $\mathrm{b}$ \\
\hline LO & 0.152 & -1.434 & -0.056 & 0.385 \\
NDR & 0.109 & -1.403 & -0.065 & 0.328 \\
$\mathrm{HV}$ & 0.117 & -1.403 & -0.062 & 0.328 \\
\hline \hline
\end{tabular}
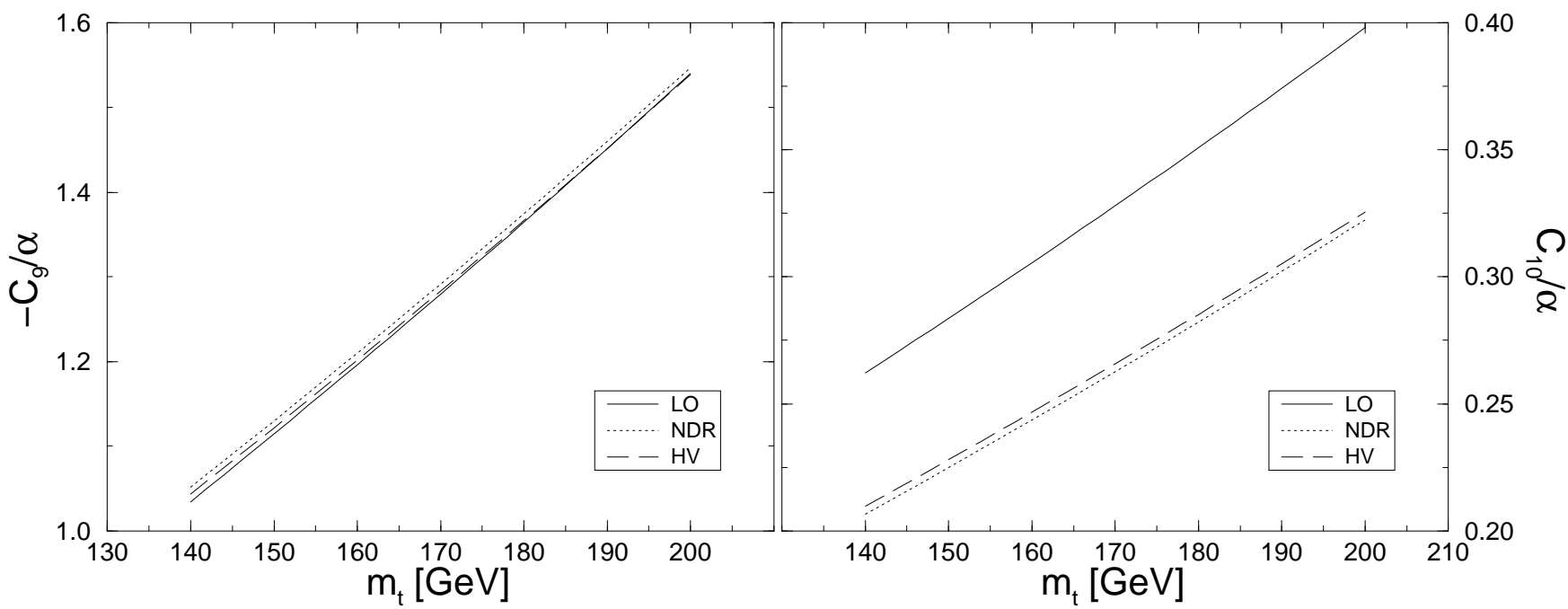

FIG. 7. Wilson coefficients $C_{9} / \alpha$ and $C_{10} / \alpha$ at $\mu=\bar{m}_{\mathrm{b}}\left(m_{\mathrm{b}}\right)=4.40 \mathrm{GeV}$ as a function of $m_{\mathrm{t}}$ for $\Lambda_{\overline{\mathrm{MS}}}^{(5)}=225 \mathrm{MeV}$. 


\section{THE EFFECTIVE HAMILTONIAN FOR $K_{L} \rightarrow \pi^{0} e^{+} e^{-}$}

The $\Delta S=1$ effective hamiltonian for $K_{L} \rightarrow \pi^{0} e^{+} e^{-}$at scales $\mu<m_{\mathrm{c}}$ is given by

$$
\mathcal{H}_{\mathrm{eff}}(\Delta S=1)=\frac{G_{F}}{\sqrt{2}} V_{\mathrm{us}}^{*} V_{\mathrm{ud}}\left[\sum_{i=1}^{6,7 V}\left(z_{i}(\mu)+\tau y_{i}(\mu)\right) Q_{i}(\mu)+\tau y_{7 A}\left(M_{\mathrm{W}}\right) Q_{7 A}\left(M_{\mathrm{W}}\right)\right]
$$

with

$$
\tau=-\frac{V_{\mathrm{ts}}^{*} V_{\mathrm{td}}}{V_{\mathrm{us}}^{*} V_{\mathrm{ud}}}
$$

\section{A. Operators}

In (VIII.1) $Q_{1,2}$ denote the $\Delta S=1$ current-current and $Q_{3}, \ldots, Q_{6}$ the QCD penguin operators of eq. (VI.3). For scales $\mu>m_{\mathrm{c}}$ again the current-current operators $Q_{1,2}^{c}$ of eq. (VI.4) have to be taken into account.

The new operators specific to the decay $K_{L} \rightarrow \pi^{0} e^{+} e^{-}$are

$$
\begin{aligned}
& Q_{7 V}=(\bar{s} d)_{\mathrm{V}-\mathrm{A}}(\bar{e} e)_{\mathrm{V}}, \\
& Q_{7 A}=(\bar{s} d)_{\mathrm{V}-\mathrm{A}}(\bar{e} e)_{\mathrm{A}} .
\end{aligned}
$$

They originate through the $\gamma$ - and $Z^{0}$-penguin and box diagrams of fig. 2 .

It is convenient to introduce the auxiliary operator

$$
Q_{7 V}^{\prime}=\left(\alpha / \alpha_{\mathrm{s}}\right)(\bar{s} d)_{\mathrm{V}-\mathrm{A}}(\bar{e} e)_{\mathrm{V}}
$$

and work for the renormalization group analysis in the basis $Q_{1}, \ldots, Q_{6}, Q_{7 V}^{\prime}$. The factor $\alpha / \alpha_{\mathrm{s}}$ in the definition of $Q_{7 V}^{\prime}$ implies that in this new basis the anomalous dimension matrix $\gamma$ will be a function of $\alpha_{\mathrm{s}}$ alone. At the end of the renormalization group analysis, this factor will be put back into the Wilson coefficient $C_{7 V}(\mu)$ of the operator $Q_{7 V}$ in eq. (VIII.3). There is no need to include a similar factor in $Q_{7 A}$ as this operator does not mix under renormalization with the remaining operators. Since $Q_{7 A}$ has no anomalous dimension its Wilson coeffcient is $\mu$-independent.

In principle one can think of including the electroweak four-quark penguin operators $Q_{7}, \ldots, Q_{10}$ of eq. (VII.2) in $\mathcal{H}_{\text {eff }}$ of (VIII.1). However, their Wilson coefficients and matrix elements for the decay $K_{L} \rightarrow \pi^{0} e^{+} e^{-}$are both of order $\mathcal{O}(\alpha)$ implying that these operators eventually would enter the amplitude $A\left(K_{L} \rightarrow \pi^{0} e^{+} e^{-}\right)$at $\mathcal{O}\left(\alpha^{2}\right)$. To the order considered here this contribution is thus negligible. This should be distinguished from the case of $K \rightarrow \pi \pi$ discussed in section VII. There, in spite of being suppressed by $\alpha / \alpha_{\mathrm{s}}$ relative to QCD penguin operators, the electroweak penguin operators have to be included in the analysis because of the additional enhancement factor $\operatorname{Re} A_{0} / \operatorname{Re} A_{2} \simeq 22$ present in the formula for $\varepsilon^{\prime} / \varepsilon$ (see section XIX). Such an enhancement factor is not present in the $K_{L} \rightarrow \pi^{0} e^{+} e^{-}$case and the electroweak penguin operators can be safely neglected.

Concerning the Wilson coefficients, the electroweak four-quark penguin operators would also affect through mixing under renormalization the coefficients $C_{3}, \ldots, C_{6}$ at $\mathcal{O}(\alpha)$ and $C_{7 V}$ at $\mathcal{O}\left(\alpha^{2}\right)$. 
Since the corresponding matrix elements are $\mathcal{O}(\alpha)$ and $\mathcal{O}(1)$, respectively, we again obtain a negligible $\mathcal{O}\left(\alpha^{2}\right)$ effect in $A\left(K_{L} \rightarrow \pi^{0} e^{+} e^{-}\right)$.

In summary, the electroweak four-quark penguin operators $Q_{7}, \ldots, Q_{10}$ can safely be neglected in the following discussion of $\mathcal{H}_{\text {eff }}(\Delta S=1)$ for $K_{L} \rightarrow \pi^{0} e^{+} e^{-}$.

We also neglect the "magnetic moment" operators.These operators, being of dimension five, do not influence the Wilson coefficients of the operators $Q_{1}, \ldots, Q_{6}, Q_{7 V}$ and $Q_{7 A}$. Since their contributions to $K_{L} \rightarrow \pi^{0} e^{+} e^{-}$are suppressed by an additional factor $m_{\mathrm{s}}$, they appear strictly speaking at higher order in chiral perturbation theory. On the other hand the magnetic moment type operators play a crucial role in $b \rightarrow s \gamma$ and $b \rightarrow d \gamma$ transitions as discussed in sections IX and XXII. They also have to be kept in the decay $B \rightarrow X_{s} e^{+} e^{-}$.

\section{B. Wilson Coefficients}

Eqs. (VI.6)-(VI.8) remain valid in the case of $K_{L} \rightarrow \pi^{0} e^{+} e^{-}$with $U_{f}\left(m_{1}, m_{2}\right)$ and $M\left(m_{i}\right)$ now denoting $7 \times 7$ matrices in the $Q_{1}, \ldots, Q_{6}, Q_{7 V}^{\prime}$ basis. The Wilson coefficients are given by seven-dimensional column vectors $\vec{z}(\mu)$ and $\vec{v}(\mu)$ having components $\left(z_{1}, \ldots, z_{6}, z_{7 V}^{\prime}\right)$ and $\left(v_{1}, \ldots, v_{6}, v_{7 V}^{\prime}\right)$, respectively. Here

$$
v_{7 V}^{\prime}(\mu)=\frac{\alpha_{\mathrm{s}}(\mu)}{\alpha} v_{7 V}(\mu), \quad z_{7 V}^{\prime}(\mu)=\frac{\alpha_{\mathrm{s}}(\mu)}{\alpha} z_{7 V}(\mu)
$$

are the rescaled Wilson coefficients of the auxiliary operator $Q_{7 V}^{\prime}$ used in the renormalization group evolution.

The initial conditions $C_{1}\left(M_{\mathrm{W}}\right), \ldots, C_{6}\left(M_{\mathrm{W}}\right), z_{1}\left(M_{\mathrm{W}}\right), z_{2}\left(M_{\mathrm{W}}\right)$ and $z_{1}\left(m_{\mathrm{c}}\right), \ldots, z_{6}\left(m_{\mathrm{c}}\right)$ for the four-quark operators $Q_{1}, \ldots, Q_{6}$ are readily obtained from eqs. (VI.9)-(VI.14), (VI.18) and (VI.20).

The corresponding initial conditions for the remaining operators $Q_{7 V}^{\prime}$ and $Q_{7 A}$ specific to $K_{L} \rightarrow \pi^{0} e^{+} e^{-}$are then given in the NDR scheme by

$$
C_{7 V}^{\prime}\left(M_{\mathrm{W}}\right)=\frac{\alpha_{\mathrm{s}}\left(M_{\mathrm{W}}\right)}{2 \pi}\left[\frac{C_{0}\left(x_{t}\right)-B_{0}\left(x_{t}\right)}{\sin ^{2} \theta_{W}}-\tilde{D}_{0}\left(x_{t}\right)-4 C_{0}\left(x_{t}\right)\right]
$$

and

$$
C_{7 A}\left(M_{\mathrm{W}}\right)=y_{7 A}\left(M_{\mathrm{W}}\right)=\frac{\alpha}{2 \pi} \frac{B_{0}\left(x_{t}\right)-C_{0}\left(x_{t}\right)}{\sin ^{2} \theta_{W}} .
$$

In order to find $z_{7 V}^{\prime}\left(m_{\mathrm{c}}\right)$ which results from the diagrams of fig. 3, we simply have to rescale the NDR result for $z_{7}\left(m_{\mathrm{c}}\right)$ in eq. (VII.17) by a factor of $-3 \alpha_{\mathrm{s}} / \alpha$. This yields

$$
z_{7 V}^{\prime}\left(m_{\mathrm{c}}\right)=-\frac{\alpha_{\mathrm{s}}\left(m_{\mathrm{c}}\right)}{2 \pi} F_{\mathrm{e}}\left(m_{\mathrm{c}}\right) .
$$




\section{Renormalization Group Evolution and Anomalous Dimension Matrices}

Working in the rescaled basis $Q_{1}, \ldots, Q_{6}, Q_{7 V}^{\prime}$ the anomalous dimension matrix $\gamma$ has per construction a pure $\mathcal{O}\left(\alpha_{\mathrm{s}}\right)$ expansion

$$
\gamma=\frac{\alpha_{\mathrm{s}}}{4 \pi} \gamma^{(0)}+\frac{\alpha_{\mathrm{s}}^{2}}{(4 \pi)^{2}} \gamma^{(1)}+\ldots
$$

where $\gamma^{(0)}$ and $\gamma^{(1)}$ are $7 \times 7$ matrices. The evolution matrices $U_{f}\left(m_{1}, m_{2}\right)$ in eqs. (VI.7) and (VI.8) are for the present case simply given by (VI.24) and (III.94)-(III.98).

The $6 \times 6$ submatrix of $\gamma^{(0)}$ involving the operators $Q_{1}, \ldots, Q_{6}$ has already been given in eq. (VI.25). Here we only give the remaining entries of $\gamma^{(0)}$ related to the additional presence of the operator $Q_{7 V}^{\prime}$

$$
\begin{aligned}
\gamma_{17}^{(0)} & =-\frac{16}{9} N & \gamma_{27}^{(0)} & =-\frac{16}{9} \\
\gamma_{37}^{(0)} & =-\frac{16}{9} N\left(u-\frac{d}{2}-\frac{1}{N}\right) & \gamma_{47}^{(0)} & =-\frac{16}{9}\left(u-\frac{d}{2}-N\right) \\
\gamma_{57}^{(0)} & =-\frac{16}{9} N\left(u-\frac{d}{2}\right) & \gamma_{67}^{(0)} & =-\frac{16}{9}\left(u-\frac{d}{2}\right) \\
\gamma_{77}^{(0)} & =-2 \beta_{0}=-\frac{22}{3} N+\frac{4}{3} f & \gamma_{7 i}^{(0)} & =0 \quad i=1, \ldots, 6
\end{aligned}
$$

where $N$ denotes the number of colours. These elements have been first calculated in (Gilman and Wise, 1980) except that $\gamma_{37}^{(0)}$ and $\gamma_{47}^{(0)}$ have been corrected in (Eeg and Picek, 1988), (Flynn and Randall, 1989a).

The $6 \times 6$ submatrix of $\gamma^{(1)}$ involving the operators $Q_{1}, \ldots, Q_{6}$ has already been presented as $\gamma_{\mathrm{s}}^{(1)}$ in eq. (VI.26) and the seventh column of $\gamma^{(1)}$ is given as follows in the NDR scheme (Buras et al., 1994a)

$$
\begin{aligned}
\gamma_{17}^{(1)} & =\frac{8}{3}\left(1-N^{2}\right), \\
\gamma_{27}^{(1)} & =\frac{200}{81}\left(N-\frac{1}{N}\right), \\
\gamma_{37}^{(1)} & =\frac{8}{3}\left(u-\frac{d}{2}\right)\left(1-N^{2}\right)+\frac{464}{81}\left(\frac{1}{N}-N\right), \\
\gamma_{47}^{(1)} & =\left(u \frac{280}{81}+d \frac{64}{81}\right)\left(\frac{1}{N}-N\right)+\frac{8}{3}\left(N^{2}-1\right), \\
\gamma_{57}^{(1)} & =\frac{8}{3}\left(u-\frac{d}{2}\right)\left(1-N^{2}\right), \\
\gamma_{67}^{(1)} & =\left(u \frac{440}{81}-d \frac{424}{81}\right)\left(N-\frac{1}{N}\right), \\
\gamma_{77}^{(1)} & =-2 \beta_{1}=-\frac{68}{3} N^{2}+\frac{20}{3} N f+4 C_{F} f \\
\gamma_{7 i}^{(1)} & =0 \quad i=1, \ldots, 6
\end{aligned}
$$

where $C_{F}=\left(N^{2}-1\right) /(2 N)$. The corresponding results in the HV scheme can be found in (Buras et al., 1994a). 


\section{Quark Threshold Matching Matrix}

For the case of $K_{L} \rightarrow \pi^{0} e^{+} e^{-}$the matching matrix $M(m)$ has in the basis $Q_{1}, \ldots, Q_{6}, Q_{7 V}^{\prime}$ the form

$$
M(m)=1+\frac{\alpha_{\mathrm{s}}(m)}{4 \pi} \delta r_{\mathrm{s}}^{T}
$$

where 1 and $\delta r_{\mathrm{s}}^{T}$ are $7 \times 7$ matrices and $m$ is the scale of the quark threshold.

The $6 \times 6$ submatrix of $M(m)$ involving $Q_{1}, \ldots, Q_{6}$ has been given in eq. (VI.28). The remaining entries of $\delta r_{\mathrm{s}}$ can be found from the matrix $\delta r_{\mathrm{e}}$ given in eqs. (VII.32) and (VII.34) by making a simple rescaling by $-3 \alpha_{\mathrm{s}} / \alpha$ as in the case of $z_{7}\left(m_{\mathrm{c}}\right)$.

In summary, for the quark threshold $m=m_{\mathrm{b}}$ the matrix $\delta r_{\mathrm{s}}$ reads

$$
\delta r_{\mathrm{s}}=\left(\begin{array}{ccccccc}
0 & 0 & 0 & 0 & 0 & 0 & 0 \\
0 & 0 & 0 & 0 & 0 & 0 & 0 \\
0 & 0 & 0 & 0 & 0 & 0 & -\frac{20}{9} \\
0 & 0 & \frac{5}{27} & -\frac{5}{9} & \frac{5}{27} & -\frac{5}{9} & -\frac{20}{27} \\
0 & 0 & 0 & 0 & 0 & 0 & -\frac{20}{9} \\
0 & 0 & \frac{5}{27} & -\frac{5}{9} & \frac{5}{27} & -\frac{5}{9} & -\frac{20}{27} \\
0 & 0 & 0 & 0 & 0 & 0 & 0
\end{array}\right) .
$$

For $m=m_{\mathrm{c}}$ the seventh column of $\delta r_{\mathrm{s}}$ in (VIII.14) has to be multiplied by -2 .

\section{E. Numerical Results for the $K_{L} \rightarrow \pi^{0} e^{+} e^{-}$Wilson Coefficients}

TABLE XXIV. $K_{\mathrm{L}} \rightarrow \pi^{0} e^{+} e^{-}$Wilson coefficients for $Q_{7 V, A}$ at $\mu=1 \mathrm{GeV}$ for $m_{\mathrm{t}}=170 \mathrm{GeV}$. The corresponding coefficients for $Q_{1}, \ldots, Q_{6}$ can be found in table X of section VI.

\begin{tabular}{|c|c|c|c||c|r|r||r|r|c|}
\hline \hline & \multicolumn{3}{|c||}{$\Lambda \frac{(4)}{\mathrm{MS}}=215 \mathrm{MeV}$} & \multicolumn{3}{c||}{$\Lambda \frac{(4)}{\mathrm{MS}}=325 \mathrm{MeV}$} & \multicolumn{3}{c|}{$\Lambda \frac{(4)}{\mathrm{MS}}=435 \mathrm{MeV}$} \\
\hline Scheme & LO & NDR & HV & LO & NDR & HV & LO & NDR & HV \\
\hline$z_{7 V} / \alpha$ & -0.014 & -0.015 & 0.005 & -0.024 & -0.046 & -0.003 & -0.035 & -0.084 & -0.011 \\
\hline$y_{7 V} / \alpha$ & 0.575 & 0.747 & 0.740 & 0.540 & 0.735 & 0.725 & 0.509 & 0.720 & 0.710 \\
$y_{7 A} / \alpha$ & -0.700 & -0.700 & -0.700 & -0.700 & -0.700 & -0.700 & -0.700 & -0.700 & -0.700 \\
\hline \hline
\end{tabular}

In the case of $K_{L} \rightarrow \pi^{0} e^{+} e^{-}$, due to $\gamma_{7 i}^{(0)}=\gamma_{7 i}^{(1)}=0, i=1, \ldots, 6$ in eq. (VIII.11) and (VIII.12), respectively, the RG evolution of $Q_{1}, \ldots, Q_{6}$ is completely unaffected by the additional presence of the operator $Q_{7 V}$. The $K_{L} \rightarrow \pi^{0} e^{+} e^{-}$Wilson coefficients $z_{i}$ and $y_{i}, i=1, \ldots, 6$ at scale $\mu=1 \mathrm{GeV}$ can therefore be found in table $\mathrm{X}$ of section VI.

The $K_{L} \rightarrow \pi^{0} e^{+} e^{-}$Wilson coefficients for the remaining operators $Q_{7 V}$ and $Q_{7 A}$ are given in table XXIV. Some insight in the analytic structure of $y_{7 V}$ will be gained by studying the analogous decay $B \rightarrow X_{s} e^{+} e^{-}$in section $\mathrm{X}$ and also in section XXI where the phenomenology of $K_{L} \rightarrow$ $\pi^{0} e^{+} e^{-}$will be presented. 
TABLE XXV. $K_{\mathrm{L}} \rightarrow \pi^{0} e^{+} e^{-}$Wilson coefficients $z_{7 V} / \alpha$ and $y_{7 V} / \alpha$ for $m_{\mathrm{t}}=170 \mathrm{GeV}$ and various values of $\mu$.

\begin{tabular}{|c|c|c|c||c|r|r||r|r|r|}
\hline \hline & \multicolumn{3}{|c||}{$\Lambda_{\overline{\mathrm{MS}}}^{(4)}=215 \mathrm{MeV}$} & \multicolumn{3}{c||}{$\Lambda_{\overline{\mathrm{MS}}}{ }^{(4)} 325 \mathrm{MeV}$} & \multicolumn{3}{c|}{$\Lambda_{\overline{\mathrm{MS}}}{ }^{(4)} 435 \mathrm{MeV}$} \\
\hline Scheme & LO & NDR & HV & LO & NDR & HV & LO & NDR & HV \\
\hline$\mu[\mathrm{GeV}]$ & \multicolumn{7}{|c||}{$z_{7 V} / \alpha$} \\
\hline 0.8 & -0.031 & -0.029 & 0.004 & -0.053 & -0.081 & -0.012 & -0.077 & -0.149 & -0.023 \\
1.0 & -0.014 & -0.015 & 0.005 & -0.024 & -0.046 & -0.003 & -0.035 & -0.084 & -0.011 \\
1.2 & -0.004 & -0.009 & 0.002 & -0.006 & -0.029 & 0 & -0.009 & -0.051 & -0.002 \\
\hline$\mu[\mathrm{GeV}]$ & \multicolumn{7}{|c||}{$y_{7 V} / \alpha$} \\
\hline 0.8 & 0.578 & 0.751 & 0.744 & 0.545 & 0.739 & 0.730 & 0.514 & 0.722 & 0.712 \\
1.0 & 0.575 & 0.747 & 0.740 & 0.540 & 0.735 & 0.725 & 0.509 & 0.720 & 0.710 \\
1.2 & 0.571 & 0.744 & 0.736 & 0.537 & 0.731 & 0.721 & 0.505 & 0.716 & 0.706 \\
\hline \hline
\end{tabular}

In table XXV we show the $\mu$-dependence of $z_{7 V} / \alpha$ and $y_{7 V} / \alpha$. We find a pronounced scheme and $\mu$-dependence for $z_{7 V}$. This signals that these dependences have to be carefully addressed in the calculation of the CP conserving part in the $K_{L} \rightarrow \pi^{0} e^{+} e^{-}$amplitude. On the other hand, the scheme and $\mu$-dependences for $y_{7 V}$ are below $\mathcal{O}(1.5 \%)$.

Similarly, $z_{7 V}$ shows a strong dependence on the choice of the QCD scale $\Lambda_{\overline{\mathrm{MS}}}$ while this dependence is small or absent for $y_{7 V}$ and $y_{7 A}$, respectively.

Finally, as seen from eq. (VIII.9) $z_{7 V}$ is independent of $m_{\mathrm{t}}$. However, with in/decreasing $m_{\mathrm{t}}$ in the range $m_{\mathrm{t}}=(170 \pm 15) \mathrm{GeV}$ there is a relative variation of $\mathcal{O}( \pm 3 \%)$ and $\mathcal{O}( \pm 14 \%)$ for the absolute values of $y_{7 V}$ and $y_{7 A}$, respectively. This is illustrated further in fig. 8 and table XXVI where the $m_{\mathrm{t}}$ dependence of these coefficients is shown explicitly. Accidentally for $m_{t} \approx 175 \mathrm{GeV}$ one finds $\left|y_{7 V}\right| \approx\left|y_{7 A}\right|$. Most importantly the impact of NLO corrections is to enhance the Wilson coefficient $y_{7 V}$ by roughly $25 \%$. As we will see in section XXI this implies an enhancement of the direct CP violation in $K_{L} \rightarrow \pi^{0} e^{+} e^{-}$.

TABLE XXVI. $K_{\mathrm{L}} \rightarrow \pi^{0} e^{+} e^{-}$Wilson coefficients $y_{7 V} / \alpha$ and $y_{7 A} / \alpha$ for $\mu=1.0 \mathrm{GeV}$ and various values of $m_{\mathrm{t}}$.

\begin{tabular}{|c|c|c|c|c|c|c|c|c|c|c|}
\hline \multirow[b]{3}{*}{$m_{\mathrm{t}}[\mathrm{GeV}]$} & \multicolumn{9}{|c|}{$y_{7 V} / \alpha$} & \multirow[t]{3}{*}{$y_{7 A} / \alpha$} \\
\hline & \multicolumn{3}{|c|}{$\Lambda_{\overline{\mathrm{MS}}}^{(4)}=215 \mathrm{MeV}$} & \multicolumn{3}{|c|}{$\Lambda_{\frac{(4)}{\mathrm{VS}}}^{(4)}=325 \mathrm{MeV}$} & \multicolumn{3}{|c|}{$\Lambda_{\overline{\mathrm{MS}}}^{(4)}=435 \mathrm{MeV}$} & \\
\hline & $\mathrm{LO}$ & NDR & HV & $\mathrm{LO}$ & NDR & $\mathrm{HV}$ & $\mathrm{LO}$ & NDR & $\mathrm{HV}$ & \\
\hline 150 & 0.546 & 0.719 & 0.711 & 0.512 & 0.706 & 0.697 & 0.481 & 0.692 & 0.681 & -0.576 \\
\hline 160 & 0.560 & 0.733 & 0.726 & 0.526 & 0.721 & 0.711 & 0.495 & 0.706 & 0.696 & -0.637 \\
\hline 170 & 0.575 & 0.747 & 0.740 & 0.540 & 0.735 & 0.725 & 0.509 & 0.720 & 0.710 & -0.700 \\
\hline 180 & 0.588 & 0.761 & 0.753 & 0.554 & 0.748 & 0.739 & 0.523 & 0.734 & 0.723 & -0.765 \\
\hline 190 & 0.601 & 0.774 & 0.766 & 0.567 & 0.761 & 0.752 & 0.536 & 0.747 & 0.736 & -0.833 \\
\hline 200 & 0.614 & 0.786 & 0.779 & 0.580 & 0.774 & 0.764 & 0.549 & 0.760 & 0.749 & -0.902 \\
\hline
\end{tabular}




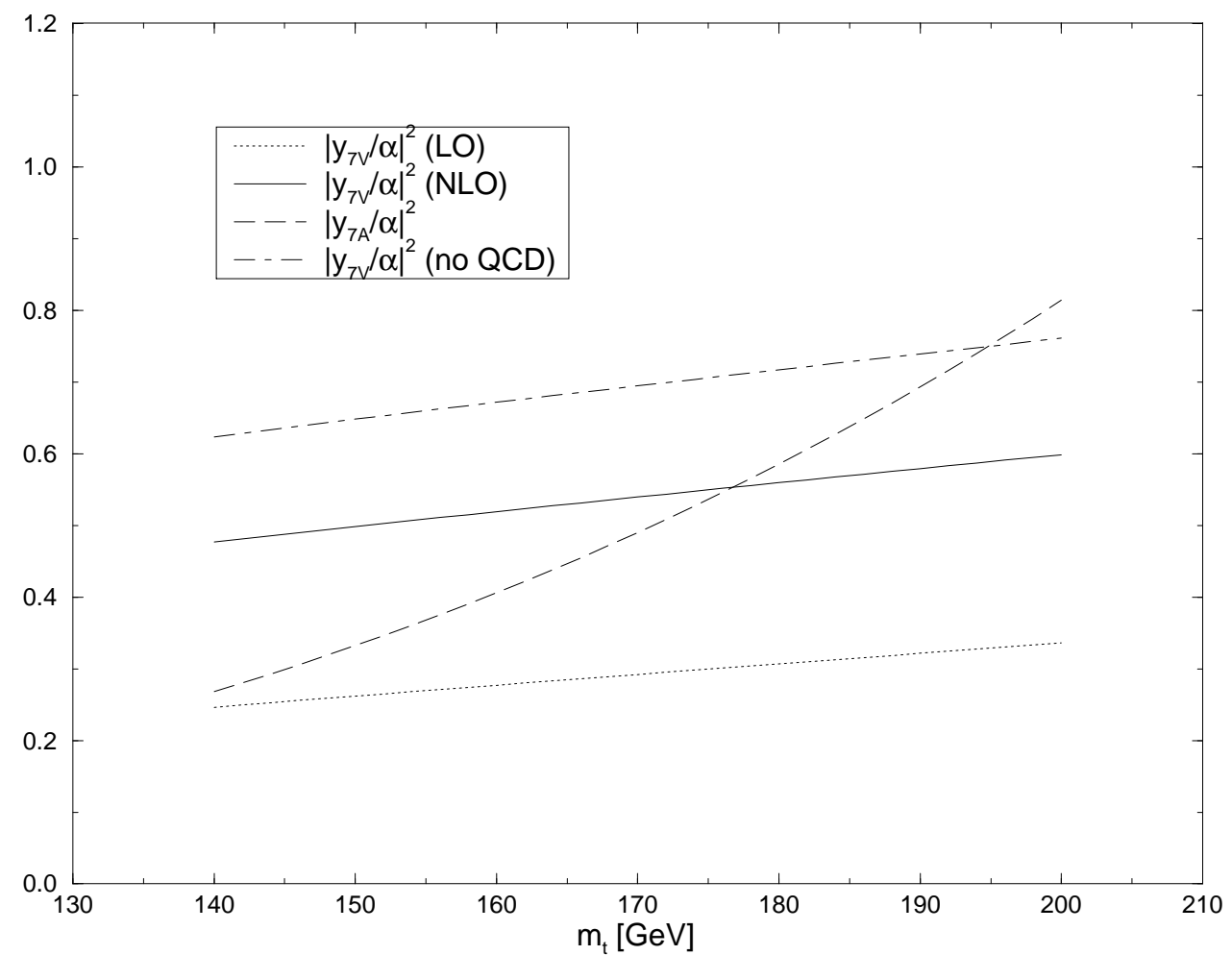

FIG. 8. Wilson coefficients $\left|y_{7 V} / \alpha\right|^{2}$ and $\left|y_{7 A} / \alpha\right|^{2}$ as a function of $m_{\mathrm{t}}$ for $\Lambda_{\overline{\mathrm{MS}}}^{(4)}=325 \mathrm{MeV}$ at scale $\mu=1.0 \mathrm{GeV}$. 


\section{THE EFFECTIVE HAMILTONIAN FOR $B \rightarrow X_{s} \gamma$}

The effective hamiltonian for $B \rightarrow X_{s} \gamma$ at scales $\mu=O\left(m_{\mathrm{b}}\right)$ is given by

$$
\mathcal{H}_{\mathrm{eff}}(b \rightarrow s \gamma)=-\frac{G_{F}}{\sqrt{2}} V_{t s}^{*} V_{t b}\left[\sum_{i=1}^{6} C_{i}(\mu) Q_{i}(\mu)+C_{7 \gamma}(\mu) Q_{7 \gamma}(\mu)+C_{8 G}(\mu) Q_{8 G}(\mu)\right]
$$

where in view of $\left|V_{u s}^{*} V_{u b} / V_{t s}^{*} V_{t b}\right|<0.02$ we have neglected the term proportional to $V_{u s}^{*} V_{u b}$.

\section{A. Operators}

The complete list of operators is given as follows

$$
\begin{aligned}
Q_{1} & =\left(\bar{s}_{i} c_{j}\right)_{V-A}\left(\bar{c}_{j} b_{i}\right)_{V-A} \\
Q_{2} & =(\bar{s} c)_{V-A}(\bar{c} b)_{V-A} \\
Q_{3} & =(\bar{s} b)_{V-A} \sum_{q}(\bar{q} q)_{V-A} \\
Q_{4} & =\left(\bar{s}_{i} b_{j}\right)_{V-A} \sum_{q}\left(\bar{q}_{j} q_{i}\right)_{V-A} \\
Q_{5} & =(\bar{s} b)_{V-A} \sum_{q}(\bar{q} q)_{V+A} \\
Q_{6} & =\left(\bar{s}_{i} b_{j}\right)_{V-A} \sum_{q}\left(\bar{q}_{j} q_{i}\right)_{V+A} \\
Q_{7 \gamma} & =\frac{e}{8 \pi^{2}} m_{\mathrm{b}} \bar{s}_{i} \sigma^{\mu \nu}\left(1+\gamma_{5}\right) b_{i} F_{\mu \nu} \\
Q_{8 G} & =\frac{g}{8 \pi^{2}} m_{\mathrm{b}} \bar{s}_{i} \sigma^{\mu \nu}\left(1+\gamma_{5}\right) T_{i j}^{a} b_{j} G_{\mu \nu}^{a}
\end{aligned}
$$

The current-current operators $Q_{1,2}$ and the QCD penguin operators $Q_{3}, \ldots, Q_{6}$ have been already contained in the usual $\Delta B=1$ hamiltonian presented in section VIF. The new operators $Q_{7 \gamma}$ and $Q_{8 G}$ specific for $b \rightarrow s \gamma$ and $b \rightarrow s g$ transitions carry the name of magnetic penguin operators. They originate from the mass-insertion on the external b-quark line in the QCD and QED penguin diagrams of fig. 4 (d), respectively. In view of $m_{\mathrm{s}} \ll m_{\mathrm{b}}$ we do not include the corresponding contributions from mass-insertions on the external s-quark line.

\section{B. Wilson Coefficients}

A very peculiar feature of the renormalization group analysis of the set of operators in (IX.2) is that the mixing under (infinite) renormalization between the set $Q_{1}, \ldots, Q_{6}$ and the operators $Q_{7 \gamma}, Q_{8 G}$ vanishes at the one-loop level. Consequently in order to calculate the coefficients $C_{7 \gamma}(\mu)$ and $C_{8 G}(\mu)$ in the leading logarithmic approximation, two-loop calculations of $\mathcal{O}\left(e g_{s}^{2}\right)$ and $\mathcal{O}\left(g_{s}^{3}\right)$ are necessary. The corresponding NLO analysis requires the evaluation of the mixing in question at the three-loop level. In view of this new feature it is useful to include additional couplings in the definition of $Q_{7 \gamma}$ and $Q_{8 G}$ as done in (IX.2). In this manner the entries in the anomalous dimension matrix representing the mixing between $Q_{1}, \ldots, Q_{6}$ and $Q_{7 \gamma}, Q_{8 G}$ at the two-loop level are $O\left(g_{s}^{2}\right)$ 
and enter the anomalous dimension matrix $\gamma_{s}^{(0)}$. Correspondingly the three-loop mixing between these two sets of operators enters the matrix $\gamma_{s}^{(1)}$.

The mixing under renormalization in the sector $Q_{7 \gamma}, Q_{8 G}$ proceeds in the usual manner and the corresponding entries in $\gamma_{s}^{(0)}$ and $\gamma_{s}^{(1)}$ result from one-loop and two-loop calculations, respectively.

At present, the coefficients $C_{7 \gamma}$ and $C_{8 G}$ are only known in the leading logarithmic approximation. Consequently we are in the position to give here only their values in this approximation. The work on NLO corrections to $C_{7 \gamma}$ and $C_{8 G}$ is in progress and we will summarize below what is already known about these corrections.

The peculiar feature of this decay mentioned above caused that the first fully correct calculation of the leading anomalous dimension matrix has been obtained only in 1993 (Ciuchini et al., 1993b), (Ciuchini et al., 1994c). It is instructive to clarify this right at the beginning. We follow here (Buras et al., 1994c).

The point is that the mixing between the sets $Q_{1}, \ldots, Q_{6}$ and $Q_{7 \gamma}, Q_{8 G}$ in $\gamma_{s}^{(0)}$ resulting from two-loop diagrams is generally regularization scheme dependent. This is certainly disturbing because the matrix $\gamma_{s}^{(0)}$, being the first term in the expansion for $\gamma_{s}$, is usually scheme independent. There is a simple way to circumvent this difficulty (Buras et al., 1994c).

As noticed in (Ciuchini et al., 1993b), (Ciuchini et al., 1994c) the regularization scheme dependence of $\gamma_{s}^{(0)}$ in the case of $b \rightarrow s \gamma$ and $b \rightarrow s g$ is signaled in the one-loop matrix elements of $Q_{1}, \ldots, Q_{6}$ for on-shell photons or gluons. They vanish in any 4-dimensional regularization scheme and in the HV scheme but some of them are non-zero in the NDR scheme. One has

$$
\left\langle Q_{i}\right\rangle_{\text {one-loop }}^{\gamma}=y_{i}\left\langle Q_{7 \gamma}\right\rangle_{\text {tree }}, \quad i=1, \ldots, 6
$$

and

$$
\left\langle Q_{i}\right\rangle_{\text {one-loop }}^{G}=z_{i}\left\langle Q_{8 G}\right\rangle_{\text {tree }}, \quad i=1, \ldots, 6 \text {. }
$$

In the HV scheme all the $y_{i}$ 's and $z_{i}$ 's vanish, while in the NDR scheme $\vec{y}=\left(0,0,0,0,-\frac{1}{3},-1\right)$ and $\vec{z}=(0,0,0,0,1,0)$. This regularization scheme dependence is canceled by a corresponding regularization scheme dependence in $\gamma_{s}^{(0)}$ as first demonstrated in (Ciuchini et al., 1993b), (Ciuchini et al., 1994c). It should be stressed that the numbers $y_{i}$ and $z_{i}$ come from divergent, i.e. purely short-distance parts of the one-loop integrals. So no reference to the spectator-model or to any other model for the matrix elements is necessary here.

In view of all this it is convenient in the leading order to introduce the so-called "effective coefficients" (Buras et al., 1994c) for the operators $Q_{7 \gamma}$ and $Q_{8 G}$ which are regularization scheme independent. They are given as follows:

$$
C_{7 \gamma}^{(0) e f f}(\mu)=C_{7 \gamma}^{(0)}(\mu)+\sum_{i=1}^{6} y_{i} C_{i}^{(0)}(\mu) .
$$

and

$$
C_{8 G}^{(0) e f f}(\mu)=C_{8 G}^{(0)}(\mu)+\sum_{i=1}^{6} z_{i} C_{i}^{(0)}(\mu)
$$

One can then introduce a scheme-independent vector

$$
\vec{C}^{(0) e f f}(\mu)=\left(C_{1}^{(0)}(\mu), \ldots, C_{6}^{(0)}(\mu), C_{7 \gamma}^{(0) e f f}(\mu), C_{8 G}^{(0) e f f}(\mu)\right)
$$


From the RGE for $\vec{C}^{(0)}(\mu)$ it is straightforward to derive the RGE for $\vec{C}^{(0) e f f}(\mu)$. It has the form

$$
\mu \frac{d}{d \mu} C_{i}^{(0) e f f}(\mu)=\frac{\alpha_{\mathrm{s}}}{4 \pi} \gamma_{j i}^{(0) e f f} C_{j}^{(0) e f f}(\mu)
$$

where

$$
\gamma_{j i}^{(0) e f f}=\left\{\begin{array}{cl}
\gamma_{j 7}^{(0)}+\sum_{k=1}^{6} y_{k} \gamma_{j k}^{(0)}-y_{j} \gamma_{77}^{(0)}-z_{j} \gamma_{87}^{(0)} & \mathrm{i}=7, \mathrm{j}=1, \ldots, 6 \\
\gamma_{j 8}^{(0)}+\sum_{k=1}^{6} z_{k} \gamma_{j k}^{(0)}-z_{j} \gamma_{88}^{(0)} & \mathrm{i}=8, \mathrm{j}=1, \ldots, 6 \\
\gamma_{j i}^{(0)} & \text { otherwise. }
\end{array}\right.
$$

The matrix $\gamma^{(0) e f f}$ is a scheme-independent quantity. It equals the matrix which one would directly obtain from two-loop diagrams in the HV scheme. In order to simplify the notation we will omit the label "eff" in the expressions for the elements of this effective one loop anomalous dimension matrix given below and keep it only in the Wilson coefficients of the operators $Q_{7 \gamma}$ and $Q_{8 G}$.

This discussion clarifies why it took so long to find the correct leading anomalous dimension matrix for the $b \rightarrow s \gamma$ decay (Ciuchini et al., 1993b), (Ciuchini et al., 1994c). All previous calculations (Grinstein et al., 1990), (Cella et al., 1990), (Misiak, 1993), (Adel and Yao, 1993), (Adel and Yao, 1994) of the leading order QCD corrections to $b \rightarrow s \gamma$ used the NDR scheme setting unfortunately $z_{i}$ and $y_{i}$ to zero, or not including all operators or making other mistakes. The discrepancy between the calculation of (Grigjanis et al., 1988) (DRED scheme) and (Grinstein et al., 1990) (NDR scheme) has been clarified by (Misiak, 1994).

\section{Renormalization Group Evolution and Anomalous Dimension Matrices}

The coefficients $C_{i}(\mu)$ in (IX.1) can be calculated by using

$$
\vec{C}(\mu)=U_{5}\left(\mu, M_{\mathrm{W}}\right) \vec{C}\left(M_{\mathrm{W}}\right)
$$

Here $U_{5}\left(\mu, M_{\mathrm{W}}\right)$ is the $8 \times 8$ evolution matrix which is given in general terms in (III.93) with $\gamma$ being this time an $8 \times 8$ anomalous dimension matrix. In the leading order $U_{5}\left(\mu, M_{\mathrm{W}}\right)$ is to be replaced by $U_{5}^{(0)}\left(\mu, M_{\mathrm{W}}\right)$ and the initial conditions by $\vec{C}^{(0)}\left(M_{\mathrm{W}}\right)$ given by (Grinstein et al., 1990)

$$
\begin{gathered}
C_{2}^{(0)}\left(M_{\mathrm{W}}\right)=1 \\
C_{7 \gamma}^{(0)}\left(M_{\mathrm{W}}\right)=\frac{3 x_{t}^{3}-2 x_{t}^{2}}{4\left(x_{t}-1\right)^{4}} \ln x_{t}+\frac{-8 x_{t}^{3}-5 x_{t}^{2}+7 x_{t}}{24\left(x_{t}-1\right)^{3}} \equiv-\frac{1}{2} D_{0}^{\prime}\left(x_{t}\right) \\
C_{8 G}^{(0)}\left(M_{\mathrm{W}}\right)=\frac{-3 x_{t}^{2}}{4\left(x_{t}-1\right)^{4}} \ln x_{t}+\frac{-x_{t}^{3}+5 x_{t}^{2}+2 x_{t}}{8\left(x_{t}-1\right)^{3}} \equiv-\frac{1}{2} E_{0}^{\prime}\left(x_{t}\right)
\end{gathered}
$$

with all remaining coefficients being zero at $\mu=M_{\mathrm{W}}$. The functions $D_{0}^{\prime}\left(x_{t}\right)$ and $E_{0}^{\prime}\left(x_{t}\right)$ are sometimes used in the literature. The $6 \times 6$ submatrix of $\gamma_{s}^{(0)}$ involving the operators $Q_{1}, \ldots, Q_{6}$ is given in (VI.25). Here we only give the remaining non-vanishing entries of $\gamma_{s}^{(0)}$ (Ciuchini et al., 1993b), (Ciuchini et al., 1994c). 
Denoting for simplicity $\gamma_{i j} \equiv\left(\gamma_{s}\right)_{i j}$, the elements $\gamma_{i 7}^{(0)}$ with $i=1, \ldots, 6$ are:

$$
\begin{aligned}
\gamma_{17}^{(0)}=0, & \gamma_{27}^{(0)}=\frac{104}{27} C_{F} \\
\gamma_{37}^{(0)}=-\frac{116}{27} C_{F} & \gamma_{47}^{(0)}=\left(\frac{104}{27} u-\frac{58}{27} d\right) C_{F} \\
\gamma_{57}^{(0)}=\frac{8}{3} C_{F} & \gamma_{67}^{(0)}=\left(\frac{50}{27} d-\frac{112}{27} u\right) C_{F}
\end{aligned}
$$

The elements $\gamma_{i 8}^{(0)}$ with $i=1, \ldots, 6$ are:

$$
\begin{aligned}
\gamma_{18}^{(0)}=3, & \gamma_{28}^{(0)}=\frac{11}{9} N-\frac{29}{9} \frac{1}{N} \\
\gamma_{38}^{(0)}=\frac{22}{9} N-\frac{58}{9} \frac{1}{N}+3 f & \gamma_{48}^{(0)}=6+\left(\frac{11}{9} N-\frac{29}{9} \frac{1}{N}\right) f \\
\gamma_{58}^{(0)}=-2 N+\frac{4}{N}-3 f & \gamma_{68}^{(0)}=-4-\left(\frac{16}{9} N-\frac{25}{9} \frac{1}{N}\right) f
\end{aligned}
$$

Finally the $2 \times 2$ one-loop anomalous dimension matrix in the sector $Q_{7 \gamma}, Q_{8 G}$ is given by (Grinstein et al., 1990)

$$
\begin{aligned}
\gamma_{77}^{(0)}=8 C_{F} & \gamma_{78}^{(0)}=0 \\
\gamma_{87}^{(0)}=-\frac{8}{3} C_{F} & \gamma_{88}^{(0)}=16 C_{F}-4 N
\end{aligned}
$$

As we discussed above, the first correct calculation of the two-loop mixing between $Q_{1}, \ldots, Q_{6}$ and $Q_{7 \gamma}, Q_{8 G}$ has been presented in (Ciuchini et al., 1993b), (Ciuchini et al., 1994c) and confirmed subsequently in (Cella et al., 1994a), (Cella et al., 1994b), (Misiak, 1995). In order to extend these calculations beyond the leading order one would have to calculate $\gamma_{s}^{(1)}$ (see (III.92)) and $O\left(\alpha_{\mathrm{s}}\right)$ corrections to the initial conditions in (IX.12) and (IX.13). We summarize below the present status of this NLO calculation.

The $6 \times 6$ two-loop submatrix of $\gamma_{s}^{(1)}$ involving the operators $Q_{1}, \ldots, Q_{6}$ is given in eq. (VI.26). The two-loop generalization of (IX.20) has been calculated only last year (Misiak and Münz, 1995). It is given for both NDR and HV schemes as follows

$$
\begin{aligned}
& \gamma_{77}^{(1)}=C_{F}\left(\frac{548}{9} N-16 C_{F}-\frac{56}{9} f\right) \\
& \gamma_{78}^{(1)}=0 \\
& \gamma_{87}^{(1)}=C_{F}\left(-\frac{404}{27} N+\frac{32}{3} C_{F}+\frac{56}{27} f\right) \\
& \gamma_{88}^{(1)}=-\frac{458}{9}-\frac{12}{N^{2}}+\frac{214}{9} N^{2}+\frac{56}{9} \frac{f}{N}-\frac{13}{9} f N
\end{aligned}
$$

The generalization of (IX.14)-(IX.19) to next-to-leading order requires three loop calculations which have not been done yet. The $O\left(\alpha_{\mathrm{s}}\right)$ corrections to $C_{7 \gamma}\left(M_{\mathrm{W}}\right)$ and $C_{8 G}\left(M_{\mathrm{W}}\right)$ have been considered in (Adel and Yao, 1993). 


\section{Results for the Wilson Coefficients}

The leading order results for the Wilson Coefficients of all operators entering the effective hamiltonian in (IX.1) can be written in an analytic form. They are (Buras et al., 1994c)

$$
\begin{aligned}
C_{j}^{(0)}(\mu) & =\sum_{i=1}^{8} k_{j i} \eta^{a_{i}} \quad(j=1, \ldots, 6) \\
C_{7 \gamma}^{(0) e f f}(\mu) & =\eta^{\frac{16}{23}} C_{7 \gamma}^{(0)}\left(M_{\mathrm{W}}\right)+\frac{8}{3}\left(\eta^{\frac{14}{23}}-\eta^{\frac{16}{23}}\right) C_{8 G}^{(0)}\left(M_{\mathrm{W}}\right)+C_{2}^{(0)}\left(M_{\mathrm{W}}\right) \sum_{i=1}^{8} h_{i} \eta^{a_{i}}, \\
C_{8 G}^{(0) e f f}(\mu) & =\eta^{\frac{14}{23}} C_{8 G}^{(0)}\left(M_{\mathrm{W}}\right)+C_{2}^{(0)}\left(M_{\mathrm{W}}\right) \sum_{i=1}^{8} \bar{h}_{i} \eta^{a_{i}},
\end{aligned}
$$

with

$$
\eta=\frac{\alpha_{\mathrm{s}}\left(M_{\mathrm{W}}\right)}{\alpha_{\mathrm{s}}(\mu)},
$$

\begin{tabular}{|c|c|c|c|c|c|c|c|c|}
\hline$i$ & 1 & 2 & 3 & 4 & 5 & 6 & 7 & 8 \\
\hline$a_{i}$ & $\frac{14}{23}$ & $\frac{16}{23}$ & $\frac{6}{23}$ & $-\frac{12}{23}$ & 0.4086 & -0.4230 & -0.8994 & 0.1456 \\
\hline$k_{1 i}$ & 0 & 0 & $\frac{1}{2}$ & $-\frac{1}{2}$ & 0 & 0 & 0 & 0 \\
\hline$k_{2 i}$ & 0 & 0 & $\frac{1}{2}$ & $\frac{1}{2}$ & 0 & 0 & 0 & 0 \\
\hline$k_{3 i}$ & 0 & 0 & $-\frac{1}{14}$ & $\frac{1}{6}$ & 0.0510 & -0.1403 & -0.0113 & 0.0054 \\
\hline$k_{4 i}$ & 0 & 0 & $-\frac{1}{14}$ & $-\frac{1}{6}$ & 0.0984 & 0.1214 & 0.0156 & 0.0026 \\
\hline$k_{5 i}$ & 0 & 0 & 0 & 0 & -0.0397 & 0.0117 & -0.0025 & 0.0304 \\
\hline$k_{6 i}$ & 0 & 0 & 0 & 0 & 0.0335 & 0.0239 & -0.0462 & -0.0112 \\
\hline$h_{i}$ & 2.2996 & -1.0880 & $-\frac{3}{7}$ & $-\frac{1}{14}$ & -0.6494 & -0.0380 & -0.0185 & -0.0057 \\
\hline $\bar{h}_{i}$ & 0.8623 & 0 & 0 & 0 & -0.9135 & 0.0873 & -0.0571 & 0.0209 \\
\hline
\end{tabular}

and $C_{7 \gamma}^{(0)}\left(M_{\mathrm{W}}\right)$ and $C_{8 G}^{(0)}\left(M_{\mathrm{W}}\right)$ given in (IX.12) and (IX.13), respectively. The numbers $a_{i}, k_{j i}, h_{i}$ and $\bar{h}_{i}$ are given in table XXVII.

TABLE XXVII.

\section{E. Numerical Analysis}

The decay $B \rightarrow X_{s} \gamma$ is the only decay in our review for which the complete NLO corrections are not available. In presenting the numerical values for the Wilson coefficients a few remarks on the choice of $\alpha_{\mathrm{s}}$ should therefore be made. In the leading order the leading order expression for $\alpha_{\mathrm{s}}$ should be used. The question then is what to use for $\Lambda_{\mathrm{QCD}}$ in this expression. In other decays for which NLO corrections were available this was not important because LO results were secondary. We have therefore simply inserted our standard $\Lambda_{\overline{\mathrm{MS}}}$ values into the LO formula for $\alpha_{\mathrm{s}}$. This procedure gives $\alpha_{\mathrm{s}}^{(5)}\left(M_{\mathrm{Z}}\right)=0.126,0.136,0.144$ for $\Lambda_{\overline{\mathrm{MS}}}^{(5)}=140 \mathrm{MeV}, 225 \mathrm{MeV}, 310 \mathrm{MeV}$, 
respectively. In view of these high values of $\alpha_{\mathrm{s}}^{(5)}\left(M_{\mathrm{Z}}\right)$ we will proceed here differently. Following (Buras et al., 1994c) we will use $\alpha_{\mathrm{s}}^{(5)}\left(M_{\mathrm{Z}}\right)=0.110,0.117,0.124$ as in our NLO calculations, but we will evolve $\alpha_{\mathrm{s}}(\mu)$ to $\mu \approx \mathcal{O}\left(m_{\mathrm{b}}\right)$ using the leading order expressions. In short, we will use

$$
\alpha_{\mathrm{s}}(\mu)=\frac{\alpha_{\mathrm{s}}\left(M_{\mathrm{Z}}\right)}{1-\beta_{0} \alpha_{\mathrm{s}}\left(M_{\mathrm{Z}}\right) / 2 \pi \ln \left(M_{\mathrm{Z}} / \mu\right)} .
$$

This discussion shows again the importance of the complete NLO calculation for this decay.

Before starting the discussion of the numerical values for the coefficients $C_{7 \gamma}^{(0) e f f}$ and $C_{8 G}^{(0) e f f}$, let us illustrate the relative numerical importance of the three terms in expression (IX.23) for $C_{7 \gamma}^{(0) e f f}$.

For instance, for $m_{\mathrm{t}}=170 \mathrm{GeV}, \mu=5 \mathrm{GeV}$ and $\alpha_{\mathrm{s}}^{(5)}\left(M_{\mathrm{Z}}\right)=0.117$ one obtains

$$
\begin{aligned}
C_{7 \gamma}^{(0) e f f}(\mu) & =0.698 C_{7 \gamma}^{(0)}\left(M_{\mathrm{W}}\right)+0.086 C_{8 G}^{(0)}\left(M_{\mathrm{W}}\right)-0.156 C_{2}^{(0)}\left(M_{\mathrm{W}}\right) \\
& =0.698(-0.193)+0.086(-0.096)-0.156=-0.299 .
\end{aligned}
$$

In the absence of QCD we would have $C_{7 \gamma}^{(0) e f f}(\mu)=C_{7 \gamma}^{(0)}\left(M_{\mathrm{W}}\right)$ (in that case one has $\eta=1$ ). Therefore, the dominant term in the above expression (the one proportional to $C_{2}^{(0)}\left(M_{\mathrm{W}}\right)$ ) is the additive QCD correction that causes the enormous QCD enhancement of the $b \rightarrow s \gamma$ rate (Bertolini et al., 1987), (Deshpande et al., 1987). It originates solely from the two-loop diagrams. On the other hand, the multiplicative QCD correction (the factor 0.698 above) tends to suppress the rate, but fails in the competition with the additive contributions.

In the case of $C_{8 G}^{(0) e f f}$ a similar enhancement is observed

$$
\begin{aligned}
C_{8 G}^{(0) e f f}(\mu) & =0.730 C_{8 G}^{(0)}\left(M_{\mathrm{W}}\right)-0.073 C_{2}^{(0)}\left(M_{\mathrm{W}}\right) \\
& =0.730(-0.096)-0.073=-0.143 .
\end{aligned}
$$

In table XXVIII we give the values of $C_{7 \gamma}^{(0) e f f}$ and $C_{8 G}^{(0) e f f}$ for different values of $\mu$ and $\alpha_{\mathrm{s}}^{(5)}\left(M_{\mathrm{Z}}\right)$. To this end (IX.26) has been used. A strong $\mu$-dependence of both coefficients is observed. We will return to this dependence in section XXII.

TABLE XXVIII. Wilson coefficients $C_{7 \gamma}^{(0) e f f}$ and $C_{8 G}^{(0) e f f}$ for $m_{\mathrm{t}}=170 \mathrm{GeV}$ and various values of $\alpha_{\mathrm{S}}^{(5)}\left(M_{\mathrm{Z}}\right)$ and $\mu$.

\begin{tabular}{|c||c|c||c|c||c|c|}
\hline \hline \multicolumn{1}{|c||}{} & \multicolumn{2}{c||}{$\alpha_{\mathrm{S}}^{(5)}\left(M_{\mathrm{Z}}\right)=0.110$} & \multicolumn{2}{c||}{$\alpha_{\mathrm{S}}^{(5)}\left(M_{\mathrm{Z}}\right)=0.117$} & \multicolumn{2}{c|}{$\alpha_{\mathrm{s}}^{(5)}\left(M_{\mathrm{Z}}\right)=0.124$} \\
\hline$\mu[\mathrm{GeV}]$ & $C_{7 \gamma}^{(0) e f f}$ & $C_{8 G}^{(0) e f f}$ & $C_{7 \gamma}^{(0) e f f}$ & $C_{8 G}^{(0) e f f}$ & $C_{7 \gamma}^{(0) e f f}$ & $C_{8 G}^{(0) e f f}$ \\
\hline 2.5 & -0.323 & -0.153 & -0.334 & -0.157 & -0.346 & -0.162 \\
5.0 & -0.291 & -0.140 & -0.299 & -0.143 & -0.307 & -0.147 \\
7.5 & -0.275 & -0.133 & -0.281 & -0.136 & -0.287 & -0.139 \\
10.0 & -0.263 & -0.129 & -0.268 & -0.131 & -0.274 & -0.133 \\
\hline \hline
\end{tabular}




\section{THE EFFECTIVE HAMILTONIAN FOR $B \rightarrow X_{s} e^{+} e^{-}$}

The effective hamiltonian for $B \rightarrow X_{s} e^{+} e^{-}$at scales $\mu=O\left(m_{\mathrm{b}}\right)$ is given by

$$
\begin{aligned}
\mathcal{H}_{\mathrm{eff}}\left(b \rightarrow s e^{+} e^{-}\right)= & \mathcal{H}_{\mathrm{eff}}(b \rightarrow s \gamma)- \\
& \frac{G_{F}}{\sqrt{2}} V_{t s}^{*} V_{t b}\left[C_{9 V}(\mu) Q_{9 V}(\mu)+C_{10 A}(\mu) Q_{10 A}(\mu)\right]
\end{aligned}
$$

where again we have neglected the term proportional to $V_{u s}^{*} V_{u b}$ and $\mathcal{H}_{\text {eff }}(b \rightarrow s \gamma)$ is given in (IX.1).

\section{A. Operators}

In addition to the operators relevant for $B \rightarrow X_{s} \gamma$, there are two new operators

$$
Q_{9 V}=(\bar{s} b)_{V-A}(\bar{e} e)_{V} \quad Q_{10 A}=(\bar{s} b)_{V-A}(\bar{e} e)_{A}
$$

where $V$ and $A$ refer to $\gamma_{\mu}$ and $\gamma_{\mu} \gamma_{5}$, respectively.

They originate in the $Z^{0}$ - and $\gamma$-penguin diagrams with external $\bar{e} e$ of fig. 4 (f) and from the corresponding box diagrams.

\section{B. Wilson Coefficients}

The coefficient $C_{10 A}(\mu)$ is given by

$$
C_{10 A}\left(M_{\mathrm{W}}\right)=\frac{\alpha}{2 \pi} \widetilde{C}_{10}\left(M_{\mathrm{W}}\right), \quad \widetilde{C}_{10}\left(M_{\mathrm{W}}\right)=-\frac{Y_{0}\left(x_{t}\right)}{\sin ^{2} \Theta_{W}}
$$

with $Y_{0}(x)$ given in (X.8). Since $Q_{10 A}$ does not renormalize under QCD, its coefficient does not depend on $\mu \approx \mathcal{O}\left(m_{\mathrm{b}}\right)$. The only renormalization scale dependence in (X.3) enters through the definition of the top quark mass. We will return to this issue in section XXIII C.

The coefficient $C_{9 V}(\mu)$ has been calculated over the last years with increasing precision by several groups (Grinstein et al., 1989), (Grigjanis et al., 1989), (Cella et al., 1991), (Misiak, 1993) culminating in two complete next-to-leading QCD calculations (Misiak, 1995), (Buras and Münz, 1995) which agree with each other.

In order to calculate the coefficient $C_{9 V}$ including next-to-leading order corrections we have to perform in principle a two-loop renormalization group analysis for the full set of operators contributing to (X.1). However, $Q_{10 A}$ is not renormalized and the dimension five operators $Q_{7 \gamma}$ and $Q_{8 G}$ have no impact on $C_{9 V}$. Consequently only a set of seven operators, $Q_{1}, \ldots, Q_{6}$ and $Q_{9 V}$, has to be considered. This is precisely the case of the decay $K_{L} \rightarrow \pi^{0} e^{+} e^{-}$discussed in (Buras et al., 1994a) and in section VIII, except for an appropriate change of quark flavours and the fact that now $\mu \approx \mathcal{O}\left(m_{\mathrm{b}}\right)$ instead of $\mu \approx \mathcal{O}(1 \mathrm{GeV})$. Since the NLO analysis of $K_{L} \rightarrow \pi^{0} e^{+} e^{-}$has already been presented in section VIII we will only give the final result for $C_{9 V}(\mu)$. Because of the one step evolution from $\mu=M_{\mathrm{W}}$ down to $\mu=m_{\mathrm{b}}$ without any thresholds in between it is possible to find an analytic formula for $C_{9 V}(\mu)$. Defining $\tilde{C}_{9}$ by 


$$
C_{9 V}(\mu)=\frac{\alpha}{2 \pi} \widetilde{C}_{9}(\mu)
$$

one finds (Buras and Münz, 1995) in the NDR scheme

$$
\widetilde{C}_{9}^{N D R}(\mu)=P_{0}^{N D R}+\frac{Y_{0}\left(x_{t}\right)}{\sin ^{2} \Theta_{W}}-4 Z_{0}\left(x_{t}\right)+P_{E} E_{0}\left(x_{t}\right)
$$

with

$$
\begin{aligned}
P_{0}^{N D R}= & \frac{\pi}{\alpha_{\mathrm{s}}\left(M_{\mathrm{W}}\right)}\left(-0.1875+\sum_{i=1}^{8} p_{i} \eta^{a_{i}+1}\right) \\
& +1.2468+\sum_{i=1}^{8} \eta^{a_{i}}\left[r_{i}^{N D R}+s_{i} \eta\right] \\
P_{E}= & 0.1405+\sum_{i=1}^{8} q_{i} \eta^{a_{i}+1} .
\end{aligned}
$$

The functions $Y_{0}(x)$ and $Z_{0}(x)$ are defined by

$$
Y_{0}(x)=C_{0}(x)-B_{0}(x) \quad Z_{0}(x)=C_{0}(x)+\frac{1}{4} D_{0}(x)
$$

\begin{tabular}{|c|c|c|c|c|c|c|c|c|}
\hline$i$ & 1 & 2 & 3 & 4 & 5 & 6 & 7 & 8 \\
\hline$p_{i}$ & 0 & 0 & $-\frac{80}{203}$ & $\frac{8}{33}$, & 0.0433 & 0.1384 & 0.1648 & -0.0073 \\
\hline$r_{i}^{N D R}$ & 0 & 0 & 0.8966 & -0.1960 & -0.2011 & 0.1328 & -0.0292 & -0.1858 \\
\hline$s_{i}$ & 0 & 0 & -0.2009 & -0.3579 & 0.0490 & -0.3616 & -0.3554 & 0.0072 \\
\hline$q_{i}$ & 0 & 0 & 0 & 0 & 0.0318 & 0.0918 & -0.2700 & 0.0059 \\
\hline$r_{i}^{H V}$ & 0 & 0 & -0.1193 & 0.1003 & -0.0473 & 0.2323 & -0.0133 & -0.1799 \\
\hline
\end{tabular}

with $B_{0}(x), C_{0}(x)$ and $D_{0}(x)$ given in (VII.13), (VII.14) and (VII.15), respectively. $E_{0}(x)$ is given in eq. (VI.15). The powers $a_{i}$ are the same as in table XXVII. The coefficients $p_{i}, r_{i}^{N D R}, s_{i}$, and $q_{i}$ can be found in table XXIX. $P_{E}$ is $\mathcal{O}\left(10^{-2}\right)$ and consequently the last term in (X.5) can be neglected. We keep it however in our numerical analysis. These results agree with (Misiak, 1995).

TABLE XXIX.

In the HV scheme only the coefficients $r_{i}$ are changed. They are given on the last line of table XXIX. Equivalently we can write

$$
P_{0}^{k}=P_{0}^{N D R}+\xi_{k} \frac{4}{9}\left(3 C_{1}^{(0)}+C_{2}^{(0)}-C_{3}^{(0)}-3 C_{4}^{(0)}\right)
$$

with

$$
\xi_{k}=\left\{\begin{array}{rl}
0 & k=\mathrm{NDR} \\
-1 & k=\mathrm{HV}
\end{array}\right.
$$

We note that 


$$
\begin{gathered}
\sum_{i=1}^{8} p_{i}=0.1875, \quad \sum_{i=1}^{8} q_{i}=-0.1405 \\
\sum_{i=1}^{8}\left(r_{i}^{k}+s_{i}\right)=-1.2468+\frac{4}{9}\left(1+\xi_{k}\right),
\end{gathered}
$$

In this way for $\eta=1$ one finds $P_{E}=0, P_{0}^{N D R}=4 / 9$ and $P_{0}^{H V}=0$ in accordance with the initial conditions at $\mu=M_{\mathrm{W}}$. Moreover, the second relation in (X.12) assures the correct large logarithm in $P_{0}^{N D R}$, i.e. $8 / 9 \ln \left(M_{\mathrm{W}} / \mu\right)$.

The special feature of $C_{9 V}(\mu)$ compared to the coefficients of the remaining operators contributing to $B \rightarrow X_{s} e^{+} e^{-}$is the large logarithm represented by $1 / \alpha_{\mathrm{s}}$ in $P_{0}$ in (X.6). Consequently the renormalization group improved perturbation theory for $C_{9 \mathrm{~V}}$ has the structure $\mathcal{O}\left(1 / \alpha_{\mathrm{s}}\right)+\mathcal{O}(1)+\mathcal{O}\left(\alpha_{\mathrm{s}}\right)+\ldots$, whereas the corresponding series for the remaining coefficients is $\mathcal{O}(1)+\mathcal{O}\left(\alpha_{\mathrm{s}}\right)+\ldots$. Therefore in order to find the next-to-leading $\mathcal{O}(1)$ term in the branching ratio for $B \rightarrow X_{s} e^{+} e^{-}$, the full two-loop renormalization group analysis has to be performed in order to find $C_{9 V}$, but the coefficients of the remaining operators should be taken in the leading logarithmic approximation. This is gratifying because the coefficient of the magnetic operator $Q_{7 \gamma}$ is known only in the leading logarithmic approximation.

\section{Numerical Results}

In our numerical analysis we will use the two-loop expression for $\alpha_{\mathrm{s}}$ and the parameters collected in the appendix. Our presentation follows closely the one given in (Buras and Münz, 1995).

In table XXX we show the constant $P_{0}$ in (X.6) for different $\mu$ and $\Lambda_{\overline{\mathrm{MS}}}$, in the leading order corresponding to the first term in (X.6) and for the NDR and HV schemes as given by (X.6) and (X.9), respectively. In table XXXI we show the corresponding values for $\widetilde{C}_{9}(\mu)$. To this end we set $m_{\mathrm{t}}=170 \mathrm{GeV}$.

TABLE XXX. The coefficient $P_{0}$ of $\widetilde{C}_{9}$ for various values of $\Lambda \frac{(5)}{\mathrm{MS}}$ and $\mu$.

\begin{tabular}{|c||c|r|r||r|r|r||r|r|r|}
\hline \hline \multicolumn{1}{|c||}{} & \multicolumn{3}{c||}{$\Lambda \frac{(5)}{\mathrm{MS}}=140 \mathrm{MeV}$} & \multicolumn{3}{c||}{$\Lambda \frac{(5)}{\mathrm{MS}}=225 \mathrm{MeV}$} & \multicolumn{3}{|c|}{$\Lambda \frac{(5)}{\mathrm{MS}}=310 \mathrm{MeV}$} \\
\hline$\mu[\mathrm{GeV}]$ & LO & NDR & HV & LO & NDR & HV & LO & NDR & HV \\
\hline 2.5 & 2.053 & 2.928 & 2.797 & 1.933 & 2.846 & 2.759 & 1.835 & 2.775 & 2.727 \\
5.0 & 1.852 & 2.625 & 2.404 & 1.788 & 2.591 & 2.395 & 1.736 & 2.562 & 2.388 \\
7.5 & 1.675 & 2.391 & 2.127 & 1.632 & 2.373 & 2.127 & 1.597 & 2.358 & 2.128 \\
10.0 & 1.526 & 2.204 & 1.912 & 1.494 & 2.194 & 1.917 & 1.469 & 2.185 & 1.921 \\
\hline \hline
\end{tabular}

We observe:

- The NLO corrections to $P_{0}$ enhance this constant relatively to the LO result by roughly $45 \%$ and $35 \%$ in the NDR and HV schemes, respectively. This enhancement is analogous to the one found in the case of $K_{L} \rightarrow \pi^{0} e^{+} e^{-}$. 
TABLE XXXI. Wilson coefficient $\widetilde{C}_{9}$ for $m_{\mathrm{t}}=170 \mathrm{GeV}$ and various values of $\Lambda \frac{(5)}{\mathrm{MS}}$ and $\mu$.

\begin{tabular}{|c||c|r|r||r|r|r||r|r|r|}
\hline \hline \multicolumn{1}{|c||}{} & \multicolumn{3}{c||}{$\Lambda \frac{(5)}{\mathrm{MS}}=140 \mathrm{MeV}$} & \multicolumn{3}{c||}{$\Lambda \frac{(5)}{\mathrm{MS}}=225 \mathrm{MeV}$} & \multicolumn{3}{c|}{$\Lambda \frac{(5)}{\mathrm{MS}}=310 \mathrm{MeV}$} \\
\hline$\mu[\mathrm{GeV}]$ & LO & NDR & \multicolumn{1}{c|}{ HV } & LO & NDR & HV & LO & NDR & HV \\
\hline 2.5 & 2.053 & 4.493 & 4.361 & 1.933 & 4.410 & 4.323 & 1.835 & 4.338 & 4.290 \\
5.0 & 1.852 & 4.191 & 3.970 & 1.788 & 4.156 & 3.961 & 1.736 & 4.127 & 3.954 \\
7.5 & 1.675 & 3.958 & 3.694 & 1.632 & 3.940 & 3.694 & 1.597 & 3.924 & 3.695 \\
10.0 & 1.526 & 3.772 & 3.480 & 1.494 & 3.761 & 3.485 & 1.469 & 3.752 & 3.488 \\
\hline \hline
\end{tabular}

- In calculating $P_{0}$ in the LO we have used $\alpha_{\mathrm{s}}(\mu)$ at one-loop level. Had we used the two-loop expression for $\alpha_{\mathrm{s}}(\mu)$ we would find for $\mu=5 \mathrm{GeV}$ and $\Lambda \frac{(5)}{\mathrm{MS}}=225 \mathrm{MeV}$ the value $P_{0}^{L O} \approx$ 1.98. Consequently the NLO corrections would have smaller impact. Ref. (Grinstein et al., 1989 ) including the next-to-leading term $4 / 9$ would find $P_{0}$ values roughly $20 \%$ smaller than $P_{0}^{N D R}$ given in table $\mathrm{XXX}$.

- It is tempting to compare $P_{0}$ in table XXX with that found in the absence of QCD corrections. In the limit $\alpha_{\mathrm{s}} \rightarrow 0$ we find $P_{0}^{N D R}=8 / 9 \ln \left(M_{\mathrm{W}} / \mu\right)+4 / 9$ and $P_{0}^{H V}=$ $8 / 9 \ln \left(M_{\mathrm{W}} / \mu\right)$ which for $\mu=5 \mathrm{GeV}$ give $P_{0}^{N D R}=2.91$ and $P_{0}^{H V}=2.46$. Comparing these values with table XXX we conclude that the QCD suppression of $P_{0}$ present in the leading order approximation is considerably weakened in the NDR treatment of $\gamma_{5}$ after the inclusion of NLO corrections. It is essentially removed for $\mu>5 \mathrm{GeV}$ in the HV scheme.

- The NLO corrections to $\widetilde{C}_{9}$ which include also the $m_{\mathrm{t}}$-dependent contributions are large as seen in table XXXI. The results in HV and NDR schemes are by more than a factor of two larger than the leading order result $\widetilde{C}_{9}=P_{0}^{L O}$ which consistently should not include $m_{\mathrm{t}^{-}}$ contributions. This demonstrates very clearly the necessity of NLO calculations which allow a consistent inclusion of the important $m_{\mathrm{t}}$-contributions. For the same set of parameters the authors of (Grinstein et al., 1989) would find $\widetilde{C}_{9}$ to be smaller than $\widetilde{C}_{9}^{N D R}$ by $10-15 \%$.

- The $\Lambda_{\overline{\mathrm{MS}}}$ dependence of $\widetilde{C}_{9}$ is rather weak. On the other hand its $\mu$ dependence is sizable ( $\sim 15 \%$ in the range of $\mu$ considered) although smaller than in the case of the coefficients $C_{7 \gamma}$ and $C_{8 G}$ given in table XXVIII. We also find that the $m_{\mathrm{t}}$ dependence of $\widetilde{C}_{9}$ is rather weak. Varying $m_{\mathrm{t}}$ between $150 \mathrm{GeV}$ and $190 \mathrm{GeV}$ changes $\widetilde{C}_{9}$ by at most $10 \%$. This weak $m_{\mathrm{t}}$ dependence of $\widetilde{C}_{9}$ originates in the partial cancelation of $m_{\mathrm{t}}$ dependences between $Y_{0}\left(x_{t}\right)$ and $Z_{0}\left(x_{t}\right)$ in (X.5) as already seen in the case of $K_{L} \rightarrow \pi^{0} e^{+} e^{-}$in fig. 8. Finally, the difference between $\widetilde{C}_{9}^{N D R}$ and $\widetilde{C}_{9}^{H V}$ is small and amounts to roughly $5 \%$.

- The dominant $m_{\mathrm{t}}$-dependence in this decay originates in the $m_{\mathrm{t}}$ dependence of $\tilde{C}_{10}\left(M_{\mathrm{W}}\right)$. In fact, as can be seen in section VIII $\tilde{C}_{10}\left(M_{\mathrm{W}}\right)=2 \pi y_{7 A} / \alpha$ with $y_{7 A}$ present in $K_{L} \rightarrow \pi^{0} e^{+} e^{-}$. The $m_{\mathrm{t}}$ dependence of $y_{7 A}$ is shown in fig. 8 . 


\section{EFFECTIVE HAMILTONIANS FOR RARE $K$ - AND $B$-DECAYS}

\section{A. Overview}

In the present section we will summarize the effective hamiltonians valid at next-to-leading logarithmic accuracy in QCD, which describe the semileptonic rare Flavour Changing Neutral Current (FCNC) transitions $K^{+} \rightarrow \pi^{+} \nu \bar{\nu},\left(K_{L} \rightarrow \mu^{+} \mu^{-}\right)_{S D}, K_{L} \rightarrow \pi^{0} \nu \bar{\nu}, B \rightarrow X_{s, d} \nu \bar{\nu}$ and $B \rightarrow l^{+} l^{-}$. These decay modes all are very similar in their structure and it is natural to discuss them together. On the other hand they differ from the decays $K \rightarrow \pi \pi, K \rightarrow \pi e^{+} e^{-}, B \rightarrow$ $X_{s} \gamma$ and $B \rightarrow X_{s} e^{+} e^{-}$discussed in previous sections. Before giving the detailed formulae, it will be useful to recall the most important general features of this class of processes first. In addition, characteristic differences between the specific modes will also become apparent from our presentation.

- Within the Standard Model all the decays listed above are loop-induced semileptonic FCNC processes determined by $Z^{0}$-penguin and box diagrams (fig. 2 (d) and (e)).

In particular, a distinguishing feature of the present class of decays is the absence of a photon penguin contribution. For the decay modes with neutrinos in the final state this is obvious, since the photon does not couple to neutrinos. For the mesons decaying into a charged lepton pair the photon penguin amplitude vanishes due to vector current conservation.

An important consequence is, that the decays considered here exhibit a hard GIM suppression, quadratic in (small) internal quark masses, which is a property of the $Z^{0}$-penguin and box graphs. By contrast, the GIM suppression resulting from photon penguin contributions is logarithmic. Decays where the photon penguin contributes are for example $K_{L} \rightarrow \pi^{0} e^{+} e^{-}$and $B \rightarrow X_{s} e^{+} e^{-}$. The differences in the basic structure of these processes, resulting from the different pattern of GIM suppression, are the reason why we have discussed $K_{L} \rightarrow \pi^{0} e^{+} e^{-}$and $B \rightarrow X_{s} e^{+} e^{-}$in a separate context.

- The investigation of low energy rare decay processes allows to probe, albeit indirectly, high energy scales of the theory. Of particular interest is the sensitivity to properties of the top quark, its mass $m_{t}$ and its CKM couplings $V_{t s}$ and $V_{t d}$.

- A particular and very important advantage of the processes under discussion is, that theoretically clean predictions can be obtained. The reasons for this are:

- The low energy hadronic matrix elements required are just the matrix elements of quark currents between hadron states, which can be extracted from the leading (nonrare) semileptonic decays. Other long-distance contributions are negligibly small. An exception is the decay $K_{L} \rightarrow \mu^{+} \mu^{-}$receiving important contributions from the two-photon intermediate state, which are difficult to calculate reliably. However, the short-distance part $\left(K_{L} \rightarrow \mu^{+} \mu^{-}\right)_{S D}$ alone, which we shall discuss here, is on the same footing as the other modes. The essential difficulty for phenomenological applications then is to separate the short-distance from the long-distance piece in the measured rate.

- According to the comments just made, the processes at hand are short-distance processes, calculable within a perturbative framework, possibly including renormalization group improvement. The necessary separation of the short-distance dynamics from the 
low energy matrix elements is achieved by means of an operator product expansion. The scale ambiguities, inherent to perturbative QCD, essentially constitute the only theoretical uncertainties present in the analysis. These uncertainties are well under control as they may be systematically reduced through calculations beyond leading order.

- The points made above emphasize, that the short-distance dominated loop-induced FCNC decays provide highly promising possibilities to investigate flavourdynamics at the quantum level. However, the very fact that these processes are based on higher order electroweak effects, which makes them interesting theoretically, at the same time implies, that the branching ratios will be very small and not easy to access experimentally.

The effective hamiltonians governing the decays $K^{+} \rightarrow \pi^{+} \nu \bar{\nu},\left(K_{L} \rightarrow \mu^{+} \mu^{-}\right)_{S D}, K_{L} \rightarrow \pi^{0} \nu \bar{\nu}$, $B \rightarrow X_{s, d} \nu \bar{\nu}, B \rightarrow l^{+} l^{-}$, resulting from the $Z^{0}$-penguin and box-type contributions, can all be written down in the following general form

$$
\mathcal{H}_{\text {eff }}=\frac{G_{F}}{\sqrt{2}} \frac{\alpha}{2 \pi \sin ^{2} \Theta_{W}}\left(\lambda_{c} F\left(x_{c}\right)+\lambda_{t} F\left(x_{t}\right)\right)\left(\bar{n} n^{\prime}\right)_{V-A}(\bar{r} r)_{V-A}
$$

where $n, n^{\prime}$ denote down-type quarks $\left(n, n^{\prime}=d, s, b\right.$ but $\left.n \neq n^{\prime}\right)$ and $r$ leptons, $r=l, \nu_{l}$ $(l=e, \mu, \tau)$. The $\lambda_{i}$ are products of CKM elements, in the general case $\lambda_{i}=V_{i n}^{*} V_{i n^{\prime}}$. Furthermore $x_{i}=m_{i}^{2} / M_{W}^{2}$.

The functions $F\left(x_{i}\right)$ describe the dependence on the internal up-type quark masses $m_{i}$ (and on lepton masses if necessary) and are understood to include QCD corrections. They are increasing functions of the quark masses, a property that is particularly important for the top contribution. Crucial features of the structure of the hamiltonian are furthermore determined by the hard GIM suppression characteristic for this class of decays. First we note that the dependence of the hamiltonian on the internal quarks comes in the form

$$
\sum_{i=u, c, t} \lambda_{i} F\left(x_{i}\right)=\lambda_{c}\left(F\left(x_{c}\right)-F\left(x_{u}\right)\right)+\lambda_{t}\left(F\left(x_{t}\right)-F\left(x_{u}\right)\right)
$$

where we have used the unitarity of the CKM matrix. Now, hard GIM suppression means that for $x \ll 1 F$ behaves quadratically in the quark masses. In the present case we have

$$
F(x) \sim x \ln x \quad \text { for } \quad x \ll 1
$$

The first important consequence is, that $F\left(x_{u}\right) \approx 0$ can be neglected. The hamiltonian acquires the form anticipated in (XI.1). It effectively consists of a charm and a top contribution. Therefore the relevant energy scales are $M_{W}$ or $m_{t}$ and, at least, $m_{c}$, which are large compared to $\Lambda_{Q C D}$. This fact indicates the short-distance nature of these processes.

A second consequence of (XI.3) is that $F\left(x_{c}\right) / F\left(x_{t}\right) \approx \mathcal{O}\left(10^{-3}\right) \ll 1$. Together with the weighting introduced by the CKM factors this relation determines the relative importance of the charm versus the top contribution in (XI.1). As seen in table XXXII a simple pattern emerges if one writes down the order of magnitude of $\lambda_{c}, \lambda_{t}$ in terms of powers of the Wolfenstein expansion parameter $\lambda$.

For the CP-violating decay $K_{L} \rightarrow \pi^{0} \nu \bar{\nu}$ and the B-decays the CKM factors $\lambda_{c}$ and $\lambda_{t}$ have the same order of magnitude. In view of $F\left(x_{c}\right) \ll F\left(x_{t}\right)$ the charm contribution is therefore 
TABLE XXXII. Order of magnitude of CKM parameters relevant for the various decays, expressed in powers of the Wolfenstein parameter $\lambda=0.22$. In the case of $K_{L} \rightarrow \pi^{0} \nu \bar{\nu}$, which is CP-violating, only the imaginary parts of $\lambda_{c, t}$ contribute.

\begin{tabular}{|c|c|c|c|c|}
\hline & $\begin{array}{c}K^{+} \rightarrow \pi^{+} \nu \bar{\nu} \\
\left(K_{L} \rightarrow \mu^{+} \mu^{-}\right)_{S D}\end{array}$ & $K_{L} \rightarrow \pi^{0} \nu \bar{\nu}$ & $\begin{array}{l}B \rightarrow X_{s} \nu \bar{\nu} \\
B_{s} \rightarrow l^{+} l^{-} \\
\end{array}$ & $\begin{array}{l}B \rightarrow X_{d} \nu \bar{\nu} \\
B_{d} \rightarrow l^{+} l^{-} \\
\end{array}$ \\
\hline$\lambda_{c}$ & $\overline{\nu \sim \lambda}$ & $\left(\overline{\operatorname{Im}} \lambda_{c} \sim \lambda^{5}\right)$ & 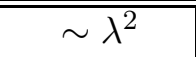 & $\bar{c} \sim \lambda^{3}$ \\
\hline$\lambda_{t}$ & $\sim \lambda^{5}$ & $\left(\operatorname{Im} \lambda_{t} \sim \lambda^{5}\right)$ & $\sim \lambda^{2}$ & $\sim \lambda^{3}$ \\
\hline
\end{tabular}

negligible and these decays are entirely determined by the top sector.

For $K^{+} \rightarrow \pi^{+} \nu \bar{\nu}$ and $\left(K_{L} \rightarrow \mu^{+} \mu^{-}\right)_{S D}$ on the other hand $\lambda_{t}$ is suppressed compared to $\lambda_{c}$ by a factor of order $\mathcal{O}\left(\lambda^{4}\right) \approx \mathcal{O}\left(10^{-3}\right)$, which roughly compensates for the $\mathcal{O}\left(10^{3}\right)$ enhancement of $F\left(x_{t}\right)$ over $F\left(x_{c}\right)$. Hence the top and charm contributions have the same order of magnitude and must both be taken into account.

In principle, as far as flavordynamics is concerned, the top and the charm sector have the same structure. The only difference comes from the quark masses. However, this difference has striking implications for the detailed formalism necessary to treat the strong interaction corrections. We have $m_{t} / M_{W}=\mathcal{O}(1)$ and $m_{c} / M_{W} \ll 1$. Correspondingly the QCD coupling $\alpha_{\mathrm{s}}$ is also somewhat smaller at $m_{t}$ than at $m_{c}$. For the charm contribution this implies that one can work to lowest order in the mass ratio $m_{c} / M_{W}$. On the other hand, for the same reason, logarithmic QCD corrections $\sim \alpha_{\mathrm{s}} \ln M_{W} / m_{c}$ are large and have to be resummed to all orders in perturbation theory by renormalization group methods. On the contrary, no large logarithms are present in the top sector, so that ordinary perturbation theory is applicable, but all orders in $m_{t} / M_{W}$ have to be taken into account. In fact we see that from the point of view of QCD corrections the charm and top contributions are quite "complementary" to each other, representing in a sense opposite limiting cases.

We are now ready to list the explicit expressions for the effective hamiltonians.

\section{B. The Decay $K^{+} \rightarrow \pi^{+} \nu \bar{\nu}$}

\section{The Next-to-Leading Order Effective Hamiltonian}

The final result for the effective hamiltonian inducing $K^{+} \rightarrow \pi^{+} \nu \bar{\nu}$ can be written as

$$
\mathcal{H}_{e f f}=\frac{G_{F}}{\sqrt{2}} \frac{\alpha}{2 \pi \sin ^{2} \Theta_{W}} \sum_{l=e, \mu, \tau}\left(V_{c s}^{*} V_{c d} X_{N L}^{l}+V_{t s}^{*} V_{t d} X\left(x_{t}\right)\right)(\bar{s} d)_{V-A}\left(\bar{\nu}_{l} \nu_{l}\right)_{V-A} .
$$

The index $l=e, \mu, \tau$ denotes the lepton flavor. The dependence on the charged lepton mass, resulting from the box-graph, is negligible for the top contribution. In the charm sector this is the case only for the electron and the muon, but not for the $\tau$-lepton.

The function $X(x)$, relevant for the top part, reads to $\mathcal{O}\left(\alpha_{\mathrm{s}}\right)$ and to all orders in $x=m^{2} / M_{W}^{2}$

$$
X(x)=X_{0}(x)+\frac{\alpha_{\mathrm{s}}}{4 \pi} X_{1}(x)
$$


with (Inami and Lim, 1981)

$$
X_{0}(x)=\frac{x}{8}\left[-\frac{2+x}{1-x}+\frac{3 x-6}{(1-x)^{2}} \ln x\right]
$$

and the QCD correction (Buchalla and Buras, 1993a)

$$
\begin{aligned}
X_{1}(x)= & -\frac{23 x+5 x^{2}-4 x^{3}}{3(1-x)^{2}}+\frac{x-11 x^{2}+x^{3}+x^{4}}{(1-x)^{3}} \ln x \\
& +\frac{8 x+4 x^{2}+x^{3}-x^{4}}{2(1-x)^{3}} \ln ^{2} x-\frac{4 x-x^{3}}{(1-x)^{2}} L_{2}(1-x) \\
& +8 x \frac{\partial X_{0}(x)}{\partial x} \ln x_{\mu}
\end{aligned}
$$

where $x_{\mu}=\mu^{2} / M_{W}^{2}$ with $\mu=\mathcal{O}\left(m_{t}\right)$ and

$$
L_{2}(1-x)=\int_{1}^{x} d t \frac{\ln t}{1-t}
$$

The $\mu$-dependence in the last term in (XI.7) cancels to the order considered the $\mu$-dependence of the leading term $X_{0}(x(\mu))$.

The expression corresponding to $X\left(x_{t}\right)$ in the charm sector is the function $X_{N L}^{l}$. It results from the $\mathrm{RG}$ calculation in NLLA and is given as follows:

$$
X_{N L}^{l}=C_{N L}-4 B_{N L}^{(1 / 2)}
$$

$C_{N L}$ and $B_{N L}^{(1 / 2)}$ correspond to the $Z^{0}$-penguin and the box-type contribution, respectively. One has (Buchalla and Buras, 1994a)

$$
\begin{aligned}
& C_{N L}=\frac{x(m)}{32} K_{c}^{\frac{24}{25}}\left[\left(\frac{48}{7} K_{+}+\frac{24}{11} K_{-}-\frac{696}{77} K_{33}\right)\left(\frac{4 \pi}{\alpha_{\mathrm{s}}(\mu)}+\frac{15212}{1875}\left(1-K_{c}^{-1}\right)\right)\right. \\
& +\left(1-\ln \frac{\mu^{2}}{m^{2}}\right)\left(16 K_{+}-8 K_{-}\right)-\frac{1176244}{13125} K_{+}-\frac{2302}{6875} K_{-}+\frac{3529184}{48125} K_{33} \\
& \left.+K\left(\frac{56248}{4375} K_{+}-\frac{81448}{6875} K_{-}+\frac{4563698}{144375} K_{33}\right)\right]
\end{aligned}
$$

where

$$
\begin{gathered}
K=\frac{\alpha_{\mathrm{s}}\left(M_{W}\right)}{\alpha_{\mathrm{s}}(\mu)} \quad K_{c}=\frac{\alpha_{\mathrm{s}}(\mu)}{\alpha_{\mathrm{s}}(m)} \\
K_{+}=K^{\frac{6}{25}} \quad K_{-}=K^{\frac{-12}{25}} \quad K_{33}=K^{\frac{-1}{25}} \\
B_{N L}^{(1 / 2)}=\frac{x(m)}{4} K_{c}^{\frac{24}{25}}\left[3\left(1-K_{2}\right)\left(\frac{4 \pi}{\alpha_{\mathrm{s}}(\mu)}+\frac{15212}{1875}\left(1-K_{c}^{-1}\right)\right)\right. \\
\left.-\ln \frac{\mu^{2}}{m^{2}}-\frac{r \ln r}{1-r}-\frac{305}{12}+\frac{15212}{625} K_{2}+\frac{15581}{7500} K K_{2}\right]
\end{gathered}
$$


Here $K_{2}=K^{-1 / 25}, m=m_{c}, r=m_{l}^{2} / m_{c}^{2}(\mu)$ and $m_{l}$ is the lepton mass. We will at times omit the index $l$ of $X_{N L}^{l}$. In (XI.10) - (XI.13) the scale is $\mu=\mathcal{O}\left(m_{c}\right)$. The two-loop expression for $\alpha_{\mathrm{s}}(\mu)$ is given in (III.19). Again - to the considered order - the explicit $\ln \left(\mu^{2} / m^{2}\right)$ terms in (XI.10) and (XI.13) cancel the $\mu$-dependence of the leading terms.

These formulae give the complete next-to-leading effective hamiltonian for $K^{+} \rightarrow \pi^{+} \nu \bar{\nu}$. The leading order expressions (Novikov et al., 1977), (Ellis and Hagelin, 1983), (Dib et al., 1991), (Buchalla et al., 1991) are obtained by replacing $X\left(x_{t}\right) \rightarrow X_{0}\left(x_{t}\right)$ and $X_{N L}^{l} \rightarrow X_{L}$ with $X_{L}$ found from (XI.10) and (XI.13) by retaining there only the $1 / \alpha_{\mathrm{s}}(\mu)$ terms. In LLA the one-loop expression should be used for $\alpha_{\mathrm{s}}$. This amounts to setting $\beta_{1}=0$ in (III.19). The numerical values for $X_{N L}$ for $\mu=m_{\mathrm{c}}$ and several values of $\Lambda \frac{(4)}{\mathrm{MS}}$ and $m_{\mathrm{c}}\left(m_{\mathrm{c}}\right)$ are given in table XXXIII. The $\mu$ dependence will be discussed in part three.

TABLE XXXIII. The functions $X_{N L}^{e}$ and $X_{N L}^{\tau}$ for various $\Lambda_{\overline{\mathrm{MS}}}^{(4)}$ and $m_{\mathrm{c}}$.

\begin{tabular}{|c|r|r|r|r|r|r|}
\hline \hline & \multicolumn{3}{|c|}{$X_{N L}^{e} / 10^{-4}$} & \multicolumn{3}{|c|}{$X_{N L}^{\tau} / 10^{-4}$} \\
\hline$\Lambda_{\overline{\mathrm{MS}}[\mathrm{MeV}] \backslash m_{\mathrm{c}}[\mathrm{GeV}]}$ & 1.25 & 1.30 & 1.35 & 1.25 & 1.30 & 1.35 \\
\hline 215 & 10.55 & 11.40 & 12.28 & 7.16 & 7.86 & 8.59 \\
325 & 9.71 & 10.55 & 11.41 & 6.32 & 7.01 & 7.72 \\
435 & 8.75 & 9.59 & 10.45 & 5.37 & 6.05 & 6.76 \\
\hline \hline
\end{tabular}

\section{Z-Penguin and Box Contribution in the Top Sector}

For completeness we give here in addition the expressions for the $Z^{0}$-penguin function $C(x)$ and the box function $B(x, 1 / 2)$ separately, which contribute to $X(x)$ in (XI.5) according to

$$
X(x)=C(x)-4 B(x, 1 / 2)
$$

The functions $C$ and $B$ depend on the gauge of the $W$-boson. In 't Hooft-Feynman-gauge ( $\xi=1$ ) they read

$$
C(x)=C_{0}(x)+\frac{\alpha_{\mathrm{s}}}{4 \pi} C_{1}(x)
$$

where (Inami and Lim, 1981)

$$
C_{0}(x)=\frac{x}{8}\left[\frac{6-x}{1-x}+\frac{3 x+2}{(1-x)^{2}} \ln x\right]
$$

and (Buchalla and Buras, 1993b)

$$
\begin{aligned}
C_{1}(x)= & \frac{29 x+7 x^{2}+4 x^{3}}{3(1-x)^{2}}-\frac{x-35 x^{2}-3 x^{3}-3 x^{4}}{3(1-x)^{3}} \ln x \\
& -\frac{20 x^{2}-x^{3}+x^{4}}{2(1-x)^{3}} \ln ^{2} x+\frac{4 x+x^{3}}{(1-x)^{2}} L_{2}(1-x) \\
& +8 x \frac{\partial C_{0}(x)}{\partial x} \ln x_{\mu}
\end{aligned}
$$


Similarly

$$
B(x, 1 / 2)=B_{0}(x)+\frac{\alpha_{\mathrm{s}}}{4 \pi} B_{1}(x, 1 / 2)
$$

with the one-loop function (Inami and Lim, 1981)

$$
B_{0}(x)=\frac{1}{4}\left[\frac{x}{1-x}+\frac{x}{(1-x)^{2}} \ln x\right]
$$

and (Buchalla and Buras, 1993a)

$$
\begin{aligned}
B_{1}(x, 1 / 2)= & \frac{13 x+3 x^{2}}{3(1-x)^{2}}-\frac{x-17 x^{2}}{3(1-x)^{3}} \ln x-\frac{x+3 x^{2}}{(1-x)^{3}} \ln ^{2} x+\frac{2 x}{(1-x)^{2}} L_{2}(1-x) \\
& +8 x \frac{\partial B_{0}(x)}{\partial x} \ln x_{\mu}
\end{aligned}
$$

The gauge dependence of $C$ and $B$ is canceled in the combination $X$ (XI.14). The second argument in $B(x, 1 / 2)$ indicates the weak isospin of the external leptons (the neutrinos in this case).

\section{The Z-Penguin Contribution in the Charm Sector}

In the next two paragraphs we would like to summarize the essential ingredients of the RG calculation for the charm sector leading to (XI.10) and (XI.13). In particular we present the operators involved, the initial values for the RG evolution of the Wilson coefficients and the required twoloop anomalous dimensions. We will first treat the $Z^{0}$-penguin contribution (XI.10) and discuss the box part (XI.13) subsequently. Further details can be found in (Buchalla and Buras, 1994a).

At renormalization scales of the order $\mathcal{O}\left(M_{W}\right)$ and after integrating out the $W$ - and $Z$-bosons the effective hamiltonian responsible for the $Z^{0}$-penguin contribution of the charm sector is given by

$$
\mathcal{H}_{e f f, c}^{(Z)}=\frac{G_{F}}{\sqrt{2}} \frac{\alpha}{2 \pi \sin ^{2} \Theta_{W}} \lambda_{c} \frac{\pi^{2}}{2 M_{W}^{2}}\left(v_{+} O_{+}+v_{-} O_{-}+v_{3} Q\right)
$$

where the operator basis is

$$
\begin{gathered}
O_{1}=-i \int d^{4} x T\left(\left(\bar{s}_{i} c_{j}\right)_{V-A}\left(\bar{c}_{j} d_{i}\right)_{V-A}\right)(x)\left(\left(\bar{c}_{k} c_{k}\right)_{V-A}(\bar{\nu} \nu)_{V-A}\right)(0)-\{c \rightarrow u\} \\
O_{2}=-i \int d^{4} x T\left(\left(\bar{s}_{i} c_{i}\right)_{V-A}\left(\bar{c}_{j} d_{j}\right)_{V-A}\right)(x)\left(\left(\bar{c}_{k} c_{k}\right)_{V-A}(\bar{\nu} \nu)_{V-A}\right)(0)-\{c \rightarrow u\} \\
O_{ \pm}=\frac{1}{2}\left(O_{2} \pm O_{1}\right) \\
Q=\frac{m^{2}}{g^{2}}(\bar{s} d)_{V-A}(\bar{\nu} \nu)_{V-A}
\end{gathered}
$$


The Wilson coefficients at $\mu=M_{W}$ are $\left(\vec{v}^{T} \equiv\left(v_{+}, v_{-}, v_{3}\right)\right)$

$$
\begin{gathered}
\vec{v}\left(M_{W}\right)=\vec{v}^{(0)}+\frac{\alpha_{\mathrm{s}}\left(M_{W}\right)}{4 \pi} \vec{v}^{(1)} \\
\vec{v}^{(0) T}=(1,1,0) \\
\vec{v}^{(1) T}=\left(B_{+}, B_{-}, B_{3}\right)
\end{gathered}
$$

where in the NDR scheme $\left(\overline{M S}\right.$, anticommuting $\gamma_{5}$ in $D \neq 4$ dimensions)

$$
B_{ \pm}= \pm 11 \frac{N \mp 1}{2 N} \quad B_{3}=16
$$

with $N$ denoting the number of colors.

In the basis of operators $\left\{O_{+}, O_{-}, Q\right\}$ the matrix of anomalous dimensions has the form

$$
\gamma=\left(\begin{array}{ccc}
\gamma_{+} & 0 & \gamma_{+3} \\
0 & \gamma_{-} & \gamma_{-3} \\
0 & 0 & \gamma_{33}
\end{array}\right)
$$

with the perturbative expansion

$$
\gamma\left(\alpha_{\mathrm{s}}\right)=\frac{\alpha_{\mathrm{s}}}{4 \pi} \gamma^{(0)}+\left(\frac{\alpha_{\mathrm{s}}}{4 \pi}\right)^{2} \gamma^{(1)}
$$

The nonvanishing entries of the anomalous dimension matrix read

$$
\begin{array}{ll}
\gamma_{33}^{(0)}=2\left(\gamma_{m 0}-\beta_{0}\right) & \gamma_{33}^{(1)}=2\left(\gamma_{m 1}-\beta_{1}\right) \\
\gamma_{ \pm}^{(0)}= \pm 6 \frac{N \mp 1}{N} & \gamma_{ \pm}^{(1)}=\frac{N \mp 1}{2 N}\left(-21 \pm \frac{57}{N} \mp \frac{19}{3} N \pm \frac{4}{3} f\right) \\
\gamma_{ \pm 3}^{(0)}= \pm 8(N \pm 1) & \gamma_{ \pm 3}^{(1)}=C_{F}( \pm 88 N-48)
\end{array}
$$

where $\gamma_{m 0}, \gamma_{m 1}, \beta_{0}, \beta_{1}$ can be found in (III.17) and (III.16), respectively. The expressions $\gamma^{(1)}$ refer to the NDR scheme, consistent with the scheme chosen for $\vec{v}\left(M_{W}\right)$. Following the general method for the solution of the RG equations explained in section III F 1, we can compute the Wilson coefficients $\vec{v}(\mu)$ at a scale $\mu=\mathcal{O}\left(m_{c}\right)$. It is convenient to work in an effective four-flavor theory $(f=4)$ in the full range of the RG evolution from $M_{W}$ down to $\mu$. The possible inclusion of a $b$-quark threshold would change the result for $X_{N L}$ by not more than $0.1 \%$ and can therefore be safely neglected.

After integrating out the charm quark at the scale $\mu=\mathcal{O}\left(m_{c}\right)$, the $Z^{0}$-penguin part of the charm contribution to the effective hamiltonian becomes

$$
\begin{gathered}
\mathcal{H}_{e f f, c}^{(Z)}=\frac{G_{F}}{\sqrt{2}} \frac{\alpha}{2 \pi \sin ^{2} \Theta_{W}} \lambda_{c} C_{N L}(\bar{s} d)_{V-A}(\bar{\nu} \nu)_{V-A} \\
C_{N L}=\frac{x(\mu)}{32}\left[\frac{1}{2}\left(1-\ln \frac{\mu^{2}}{m^{2}}\right)\left(\gamma_{+3}^{(0)} K_{+}+\gamma_{-3}^{(0)} K_{-}\right)+\frac{4 \pi}{\alpha_{\mathrm{s}}(\mu)} v_{3}(\mu)\right] .
\end{gathered}
$$

The explicit expression for $v_{3}(\mu)$ as obtained from solving the RG equation is given in (Buchalla and Buras, 1994a). Inserting this expression in (XI.34), expressing the charm quark mass $m(\mu)$ in terms of $m(m)$ and setting $N=3, f=4$, we finally end up with (XI.10). 


\section{The Box Contribution in the Charm Sector}

The RG analysis for the box contribution proceeds in analogy to the $Z^{0}$-penguin case. The box part is even somewhat simpler. When the $W$ boson is integrated out, the hamiltonian based on the box diagram reads

$$
\begin{gathered}
\mathcal{H}_{e f f, c}^{(B)}=-\frac{G_{F}}{\sqrt{2}} \frac{\alpha}{2 \pi \sin ^{2} \Theta_{W}} \lambda_{c}\left(-\frac{\pi^{2}}{M_{W}^{2}}\right)\left(c_{1} O+c_{2} Q\right) \\
O=-i \int d^{4} x T\left((\bar{s} c)_{V-A}(\bar{\nu} l)_{V-A}\right)(x)\left((\bar{l} \nu)_{V-A}(\bar{c} d)_{V-A}\right)(0)-\{c \rightarrow u\}
\end{gathered}
$$

with $Q$ alread given in (XI.25). The Wilson coefficients at $M_{W}$ in the NDR scheme are given by

$$
\vec{c}^{T}\left(M_{W}\right) \equiv\left(c_{1}\left(M_{W}\right), c_{2}\left(M_{W}\right)\right)=(1,0)+\frac{\alpha_{\mathrm{s}}\left(M_{W}\right)}{4 \pi}\left(0, B_{2}\right) \quad B_{2}=-36
$$

In the operator basis $\{O, Q\}$ the anomalous dimension matrix has the form

$$
\gamma=\left(\begin{array}{ll}
0 & \gamma_{12} \\
0 & \gamma_{22}
\end{array}\right)
$$

When expanded as

$$
\gamma=\frac{\alpha_{\mathrm{s}}}{4 \pi} \gamma^{(0)}+\left(\frac{\alpha_{\mathrm{s}}}{4 \pi}\right)^{2} \gamma^{(1)}
$$

the non-zero elements read (NDR scheme for $\gamma^{(1)}$ )

$$
\begin{array}{ll}
\gamma_{22}^{(0)}=2\left(\gamma_{m 0}-\beta_{0}\right) & \gamma_{22}^{(1)}=2\left(\gamma_{m 1}-\beta_{1}\right) \\
\gamma_{12}^{(0)}=-32 & \gamma_{12}^{(1)}=80 C_{F}
\end{array}
$$

Finally, after integrating out charm at $\mu=\mathcal{O}\left(m_{c}\right)$

$$
\begin{gathered}
\mathcal{H}_{e f f, c}^{(B)}=-\frac{G_{F}}{\sqrt{2}} \frac{\alpha}{2 \pi \sin ^{2} \Theta_{W}} \lambda_{c} 4 B_{N L}^{(1 / 2)}(\bar{s} d)_{V-A}\left(\bar{\nu}_{l} \nu_{l}\right)_{V-A} \\
B_{N L}^{(1 / 2)}=-\frac{x(\mu)}{64}\left[16\left(\ln \frac{\mu^{2}}{m^{2}}+\frac{5}{4}+\frac{r \ln r}{1-r}\right)+\frac{4 \pi}{\alpha_{\mathrm{s}}(\mu)} c_{2}(\mu)\right]
\end{gathered}
$$

(XI.41) is written here for one neutrino flavor. The index $(1 / 2)$ refers to the weak isospin of the final state leptons. From this result (XI.13) can be derived $(N=3, f=4)$. The explicit expression for $c_{2}(\mu)$ can be found in (Buchalla and Buras, 1994a).

Although Wilson coefficients and anomalous dimensions depend on the renormalization scheme, the final results in (XI.10) and (XI.13) are free from this dependence. The argument proceeds as in the general case presented in section III F 3. 


\section{Discussion}

It is instructive to consider furthermore the function $X(x)$ in the limiting case of small masses $(x \ll 1)$, keeping only terms linear in $x$ and including $\mathcal{O}\left(\alpha_{\mathrm{s}}\right)$ corrections:

$$
X(x) \doteq-\frac{3}{4} x \ln x-\frac{1}{4} x+\frac{\alpha_{\mathrm{s}}}{4 \pi}\left(-2 x \ln ^{2} x-7 x \ln x-\frac{23+2 \pi^{2}}{3} x\right)
$$

This simple and transparent expression can be regarded as a common limiting case of the top-and the charm contribution: On the one hand it follows from keeping only terms linear in $x$ in the top function (XI.5). On the other hand it can be obtained (up to the last term in (XI.43) which is $\mathcal{O}\left(\alpha_{\mathrm{s}} x\right)$ and goes beyond the NLLA) from expanding $X_{N L}$ (XI.9) (for $m_{l}=0$ ) to first order in $\alpha_{\mathrm{s}}$. This exercise provides one with a nice cross-check between the rather different looking functions $X_{N L}$ and $X\left(x_{t}\right)$ of the charm- and the top sector. Viewed the other way around, (XI.43) may serve to further illustrate the complementary character of the calculations necessary in each of the two sectors. $X\left(x_{t}\right)$ is the generalization of (XI.43) that includes all the higher order mass terms. $X_{N L}$ on the other hand generalizes (XI.43) to include all the leading logarithmic, $\mathcal{O}\left(x \alpha_{s}^{n} \ln ^{n+1} x\right)$, as well as the next-to-leading logarithmic $\mathcal{O}\left(x \alpha_{s}^{n} \ln ^{n} x\right)$ corrections, to all orders $n$ in $\alpha_{\mathrm{s}}$. Of these only the terms with $n=0$ and $n=1$ are contained in (XI.43).

Applying the approximation (XI.43) to the charm part directly, one can furthermore convince oneself, that the $\mathcal{O}\left(\alpha_{\mathrm{s}}\right)$ correction term would amount to more than $50 \%$ of the lowest order result. This observation illustrates very clearly the necessity to go beyond straightforward perturbation theory and to employ the RG summation technique. The importance of going still to next-to-leading order accuracy in the RG calculation is suggested by the relatively large size of the $\mathcal{O}\left(x \alpha_{\mathrm{s}} \ln x\right)$ term. Note also, that formally the non-logarithmic mass term $(-x / 4)$ in $(\mathrm{XI} .43)$ is a next-to-leading order effect in the framework of RG improved perturbation theory. The same is true for the dependence on the charged lepton mass, which can be taken into account consistently only in NLLA.

A crucial issue is the residual dependence of the functions $X_{N L}$ and $X\left(x_{t}\right)$ on the corresponding renormalization scales $\mu_{c}$ and $\mu_{t}$. Since the quark current operator in (XI.1) has no anomalous dimension, its matrix elements do not depend on the renormalization scale. The same must then hold for the coefficient functions $X_{N L}$ and $X\left(x_{t}\right)$. However, in practice this is only true up to terms of the neglected order in perturbation theory. The resulting scale ambiguities represent the theoretical uncertainties present in the calculation of the short-distance dominated processes under discussion. They can be systematically reduced by going to higher orders in the analysis. In table XXXIV we compare the order of the residual scale dependence in LLA and in NLLA for the top- and the charm contribution.

TABLE XXXIV. Residual scale ambiguity in the top and charm sector in LLA and NLLA.

\begin{tabular}{r|c|c|}
\hline \hline & Top Sector $\left(\mu_{t}=\mathcal{O}\left(m_{t}\right)\right)$ & Charm Sector $\left(\mu_{c}=\mathcal{O}\left(m_{c}\right)\right)$ \\
\hline LLA & $\mathcal{O}\left(\alpha_{\mathrm{s}}\right)$ & $\mathcal{O}\left(x_{c}\right)$ \\
\hline NLLA & $\mathcal{O}\left(\alpha_{s}^{2}\right)$ & $\mathcal{O}\left(\alpha_{\mathrm{s}} x_{c}\right)$ \\
\hline \hline
\end{tabular}

For numerical investigations we shall use $1 \mathrm{GeV} \leq \mu_{c} \leq 3 \mathrm{GeV}$ for the renormalization scale $\mu_{c}=\mathcal{O}\left(m_{c}\right)$ in the charm sector. Similarly, in the case of the top contribution we choose $\mu_{t}=$ 
$\mathcal{O}\left(m_{t}\right)$ in the range $100 \mathrm{GeV} \leq \mu_{t} \leq 300 \mathrm{GeV}$ for $m_{t}=170 \mathrm{GeV}$. Then, comparing LLA and NLLA, the theoretical uncertainty due to scale ambiguity is typically reduced from $\mathcal{O}(10 \%)$ to $\mathcal{O}(1 \%)$ in the top sector and from more than $50 \%$ to less than $20 \%$ in the charm sector. Here the quoted percentages refer to the total variation $\left(X_{\max }-X_{\min }\right) / X_{\text {central }}$ of the functions $X\left(x_{t}\right)$ or $X_{N L}$ within the range of scales considered. Phenomenological implications of this gain in accuracy will be discussed in section XXIV.

\section{The Decay $\left(K_{L} \rightarrow \mu^{+} \mu^{-}\right)_{S D}$}

\section{The Next-to-Leading Order Effective Hamiltonian}

The analysis of $\left(K_{L} \rightarrow \mu^{+} \mu^{-}\right)_{S D}$ proceeds in essentially the same manner as for $K^{+} \rightarrow \pi^{+} \nu \bar{\nu}$. The only difference is introduced through the reversed lepton line in the box contribution. In particular there is no lepton mass dependence, since only massless neutrinos appear as virtual leptons in the box diagram.

The effective hamiltonian in next-to-leading order can be written as follows:

$$
\mathcal{H}_{e f f}=-\frac{G_{F}}{\sqrt{2}} \frac{\alpha}{2 \pi \sin ^{2} \Theta_{W}}\left(V_{c s}^{*} V_{c d} Y_{N L}+V_{t s}^{*} V_{t d} Y\left(x_{t}\right)\right)(\bar{s} d)_{V-A}(\bar{\mu} \mu)_{V-A}+\text { h.c. }
$$

The function $Y(x)$ is given by

$$
Y(x)=Y_{0}(x)+\frac{\alpha_{\mathrm{s}}}{4 \pi} Y_{1}(x)
$$

where (Inami and Lim, 1981)

$$
Y_{0}(x)=\frac{x}{8}\left[\frac{4-x}{1-x}+\frac{3 x}{(1-x)^{2}} \ln x\right]
$$

and (Buchalla and Buras, 1993a)

$$
\begin{aligned}
Y_{1}(x)= & \frac{4 x+16 x^{2}+4 x^{3}}{3(1-x)^{2}}-\frac{4 x-10 x^{2}-x^{3}-x^{4}}{(1-x)^{3}} \ln x \\
& +\frac{2 x-14 x^{2}+x^{3}-x^{4}}{2(1-x)^{3}} \ln ^{2} x+\frac{2 x+x^{3}}{(1-x)^{2}} L_{2}(1-x) \\
& +8 x \frac{\partial Y_{0}(x)}{\partial x} \ln x_{\mu}
\end{aligned}
$$

The RG expression $Y_{N L}$ representing the charm contribution reads

$$
Y_{N L}=C_{N L}-B_{N L}^{(-1 / 2)}
$$

where $C_{N L}$ is the $Z^{0}$-penguin part given in (XI.10) and $B_{N L}^{(-1 / 2)}$ is the box contribution in the charm sector, relevant for the case of final state leptons with weak isospin $T_{3}=-1 / 2$. One has (Buchalla and Buras, 1994a) 


$$
\begin{aligned}
& B_{N L}^{(-1 / 2)}=\frac{x(m)}{4} K_{c}^{\frac{24}{25}}\left[3\left(1-K_{2}\right)\left(\frac{4 \pi}{\alpha_{\mathrm{s}}(\mu)}+\frac{15212}{1875}\left(1-K_{c}^{-1}\right)\right)\right. \\
& \left.-\ln \frac{\mu^{2}}{m^{2}}-\frac{329}{12}+\frac{15212}{625} K_{2}+\frac{30581}{7500} K K_{2}\right]
\end{aligned}
$$

Note the simple relation to $B_{N L}^{(1 / 2)}$ in (XI.13) (for $r=0$ )

$$
B_{N L}^{(-1 / 2)}-B_{N L}^{(1 / 2)}=\frac{x(m)}{2} K_{c}^{\frac{24}{25}}\left(K K_{2}-1\right)
$$

More details on the RG analysis in this case may be found in (Buchalla and Buras, 1994a).

TABLE XXXV. The function $Y_{N L}$ for various $\Lambda_{\mathrm{MS}}^{(4)}$ and $m_{\mathrm{c}}$.

\begin{tabular}{|c|r|r|r|}
\hline \hline & \multicolumn{3}{|c|}{$Y_{N L} / 10^{-4}$} \\
\hline$\Lambda \frac{(4)}{\mathrm{MS}}[\mathrm{MeV}] \backslash m_{\mathrm{c}}[\mathrm{GeV}]$ & 1.25 & 1.30 & 1.35 \\
\hline 215 & 3.09 & 3.31 & 3.53 \\
325 & 3.27 & 3.50 & 3.73 \\
435 & 3.40 & 3.64 & 3.89 \\
\hline \hline
\end{tabular}

\section{Discussion}

The gauge independent function $Y$ can be decomposed into the $Z^{0}$-penguin- and the box contribution

$$
Y(x)=C(x)-B(x,-1 / 2)
$$

In Feynman-gauge for the $W$ boson $C(x)$ is given in (XI.15). In the same gauge the box contribution reads

$$
B(x,-1 / 2)=B_{0}(x)+\frac{\alpha_{\mathrm{s}}}{4 \pi} B_{1}(x,-1 / 2)
$$

with $B_{0}(x)$ from (XI.19) and

$$
\begin{aligned}
B_{1}(x,-1 / 2)= & \frac{25 x-9 x^{2}}{3(1-x)^{2}}+\frac{11 x+5 x^{2}}{3(1-x)^{3}} \ln x-\frac{x+3 x^{2}}{(1-x)^{3}} \ln ^{2} x+\frac{2 x}{(1-x)^{2}} L_{2}(1-x) \\
& +8 x \frac{\partial B_{0}(x)}{\partial x} \ln x_{\mu}
\end{aligned}
$$

The equality $B(x, 1 / 2)=B(x,-1 / 2)$ at the one-loop level is a particular property of the Feynman-gauge. It is violated by $\mathcal{O}\left(\alpha_{\mathrm{s}}\right)$ corrections. There is however a very simple relation between $B_{1}(x, 1 / 2)$ and $B_{1}(x,-1 / 2)$

$$
B_{1}(x,-1 / 2)-B_{1}(x, 1 / 2)=16 B_{0}(x)
$$


We add a few comments on the most important differences between $Y_{N L}$ and $X_{N L}$. Expanding $Y_{N L}$ to first order in $\alpha_{\mathrm{s}}$ we find

$$
Y_{N L} \doteq \frac{1}{2} x+\frac{\alpha_{\mathrm{s}}}{4 \pi} x \ln ^{2} x+\mathcal{O}\left(\alpha_{\mathrm{s}} x\right)
$$

In contrast to $X_{N L}$ both the terms of $\mathcal{O}(x \ln x)$ and of $\mathcal{O}\left(\alpha_{\mathrm{s}} x \ln x\right)$ are absent in $Y_{N L}$. The cancellation of the leading $\mathcal{O}(x \ln x)$ terms between $Z^{0}$-penguin and box contribution implies that the non-leading $\mathcal{O}(x)$ term plays a much bigger role for $Y_{N L}$. A second consequence are the increased importance of QCD effects and the related larger sensitivity to $\mu_{c}$, resulting in a bigger theoretical uncertainty for $Y_{N L}$ than it happened to be the case for $X_{N L}$. In addition, whereas $X\left(x_{c}\right)$ is suppressed by $\sim 30 \%$ through QCD effects, the zeroth order expression for $Y$ is enhanced by as much as a factor of about 2.5. Nevertheless, QCD corrections included, $X_{N L}$ still exceeds $Y_{N L}$ by a factor of four, so that $Y_{N L}$ is less important for $\left(K_{L} \rightarrow \mu^{+} \mu^{-}\right)_{S D}$ than $X_{N L}$ is for $K^{+} \rightarrow \pi^{+} \nu \bar{\nu}$. Although the impact of the bigger uncertainties in $Y_{N L}$ is thus somewhat reduced in the complete result for $\left(K_{L} \rightarrow \mu^{+} \mu^{-}\right)_{S D}$, the remaining theoretical uncertainty due to scale ambiguity is still larger than for $K^{+} \rightarrow \pi^{+} \nu \bar{\nu}$. It will be investigated numerically in section XXV. The numerical values for $Y_{N L}$ for $\mu=m_{\mathrm{c}}$ and several values of $\Lambda_{\overline{\mathrm{MS}}}^{(4)}$ and $m_{\mathrm{c}}\left(m_{\mathrm{c}}\right)$ are given in table XXXV.

\section{The Decays $K_{L} \rightarrow \pi^{0} \nu \bar{\nu}, B \rightarrow X_{s, d} \nu \bar{\nu}$ and $B_{s, d} \rightarrow l^{+} l^{-}$}

After the above discussion it is easy to write down also the effective hamiltonians for $K_{L} \rightarrow$ $\pi^{0} \nu \bar{\nu}, B \rightarrow X_{s, d} \nu \bar{\nu}$ and $B_{s, d} \rightarrow l^{+} l^{-}$. As we have seen, only the top contribution is important in these cases and we can write

$$
\mathcal{H}_{e f f}=\frac{G_{F}}{\sqrt{2}} \frac{\alpha}{2 \pi \sin ^{2} \Theta_{W}} V_{t n}^{*} V_{t n^{\prime}} X\left(x_{t}\right)\left(\bar{n} n^{\prime}\right)_{V-A}(\bar{\nu} \nu)_{V-A}+\text { h.c. }
$$

for the decays $K_{L} \rightarrow \pi^{0} \nu \bar{\nu}, B \rightarrow X_{s} \nu \bar{\nu}$ and $B \rightarrow X_{d} \nu \bar{\nu}$, with $\left(\bar{n} n^{\prime}\right)=(\bar{s} d),(\bar{b} s),(\bar{b} d)$ respectively. Similarly

$$
\mathcal{H}_{\text {eff }}=-\frac{G_{F}}{\sqrt{2}} \frac{\alpha}{2 \pi \sin ^{2} \Theta_{W}} V_{t n}^{*} V_{t n^{\prime}} Y\left(x_{t}\right)\left(\bar{n} n^{\prime}\right)_{V-A}(\overline{l l})_{V-A}+\text { h.c. }
$$

for $B_{s} \rightarrow l^{+} l^{-}$and $B_{d} \rightarrow l^{+} l^{-}$, with $\left(\bar{n} n^{\prime}\right)=(\bar{b} s),(\bar{b} d)$. The functions $X, Y$ are given in (XI.5) and (XI.45). 


\section{THE EFFECTIVE HAMILTONIAN FOR $K^{0}-\bar{K}^{0}$ MIXING}

\section{A. General Structure}

The following chapter is devoted to the presentation of the effective hamiltonian for $\Delta S=2$ transitions. This hamiltonian incorporates the short-distance physics contributing to $K^{0}-\bar{K}^{0}$ mixing and is essential for the description of CP violation in the neutral K-meson system.

Being a FCNC process, $K^{0}-\bar{K}^{0}$ mixing can only occur at the loop level within the Standard Model. To lowest order it is induced through the box diagrams in fig. 4(e). Including QCD corrections the effective low energy hamiltonian, to be derived from these diagrams, can be written as follows $\left(\lambda_{i}=V_{i s}^{*} V_{i d}\right)$

$$
\begin{aligned}
\mathcal{H}_{e f f}^{\Delta S=2}= & \frac{G_{F}^{2}}{16 \pi^{2}} M_{W}^{2}\left[\lambda_{c}^{2} \eta_{1} S_{0}\left(x_{c}\right)+\lambda_{t}^{2} \eta_{2} S_{0}\left(x_{t}\right)+2 \lambda_{c} \lambda_{t} \eta_{3} S_{0}\left(x_{c}, x_{t}\right)\right] \times \\
& \times\left[\alpha_{\mathrm{s}}(\mu)\right]^{-2 / 9}\left[1+\frac{\alpha_{\mathrm{s}}(\mu)}{4 \pi} J_{3}\right] Q+\text { h.c. }
\end{aligned}
$$

This equation, together with (XII.31), (XII.10), (XII.68) for $\eta_{1}, \eta_{2}$ and $\eta_{3}$ respectively, represents the complete next-to-leading order short-distance hamiltonian for $\Delta S=2$ transitions. (XII.1) is valid for scales $\mu$ below the charm threshold $\mu_{c}=\mathcal{O}\left(m_{c}\right)$. In this case $\mathcal{H}_{\text {eff }}^{\Delta S=2}$ consists of a single four-quark operator

$$
Q=(\bar{s} d)_{V-A}(\bar{s} d)_{V-A}
$$

which is multiplied by the corresponding coefficient function. It is useful and customary to decompose this function into a charm-, a top- and a mixed charm-top contribution, as displayed in (XII.1). This form is obtained upon eliminating $\lambda_{u}$ by means of CKM matrix unitarity and setting $x_{u}=0$. The basic electroweak loop contributions without QCD correction are then expressed through the functions $S_{0}$, which read (Inami and Lim, 1981)

$$
\begin{gathered}
S_{0}\left(x_{c}\right) \doteq x_{c} \\
S_{0}\left(x_{t}\right)=\frac{4 x_{t}-11 x_{t}^{2}+x_{t}^{3}}{4\left(1-x_{t}\right)^{2}}-\frac{3 x_{t}^{3} \ln x_{t}}{2\left(1-x_{t}\right)^{3}} \\
S_{0}\left(x_{c}, x_{t}\right)=x_{c}\left[\ln \frac{x_{t}}{x_{c}}-\frac{3 x_{t}}{4\left(1-x_{t}\right)}-\frac{3 x_{t}^{2} \ln x_{t}}{4\left(1-x_{t}\right)^{2}}\right]
\end{gathered}
$$

Here again we keep only linear terms in $x_{c} \ll 1$, but of course all orders in $x_{t}$.

Short-distance QCD effects are described through the correction factors $\eta_{1}, \eta_{2}, \eta_{3}$ and the explicitly $\alpha_{\mathrm{s}}$-dependent terms in (XII.1). The discussion of these corrections will be the subject of the following sections.

Without QCD, i.e. in the limit $\alpha_{\mathrm{s}} \rightarrow 0$, one has $\eta_{i}\left[\alpha_{\mathrm{s}}\right]^{-2 / 9} \rightarrow 1$. In general, the complete coefficient function multiplying $Q$ in (XII.1) contains the QCD effects at high energies $\mu_{W}=\mathcal{O}\left(M_{W}\right), \mu_{t}=$ $\mathcal{O}\left(m_{t}\right)$ together with their RG evolution down to the scale $\mu=\mathcal{O}(1 \mathrm{GeV})$. A common ingredient for the three different contributions in (XII.1) is the anomalous dimension of the operator $Q$ and 
the corresponding evolution of its coefficient. The Fierz symmetric flavor structure of $Q$ implies that it acquires the same anomalous dimension as the Fierz symmetric operator $Q_{+}=\left(Q_{2}+Q_{1}\right) / 2$ (see section V), explicitly

$$
\begin{gathered}
\gamma=\frac{\alpha_{\mathrm{s}}}{4 \pi} \gamma^{(0)}+\left(\frac{\alpha_{\mathrm{s}}}{4 \pi}\right)^{2} \gamma^{(1)} \\
\gamma^{(0)}=6 \frac{N-1}{N} \quad \gamma^{(1)}=\frac{N-1}{2 N}\left[-21+\frac{57}{N}-\frac{19}{3} N+\frac{4}{3} f\right] \quad(\mathrm{NDR})
\end{gathered}
$$

The resulting evolution of the coefficient of $Q$ between general scales $\mu_{1}$ and $\mu$ then reads

$$
C_{Q}(\mu)=\left[1+\frac{\alpha_{\mathrm{s}}(\mu)-\alpha_{\mathrm{s}}\left(\mu_{1}\right)}{4 \pi} J_{f}\right]\left[\frac{\alpha_{\mathrm{s}}\left(\mu_{1}\right)}{\alpha_{\mathrm{s}}(\mu)}\right]^{d_{f}} C_{Q}\left(\mu_{1}\right)
$$

where

$$
d_{f}=\frac{\gamma^{(0)}}{2 \beta_{0}} \quad J_{f}=\frac{d_{f}}{\beta_{0}} \beta_{1}-\frac{\gamma^{(1)}}{2 \beta_{0}}
$$

depend on the number of active flavors $f$. At the lower end of the evolution $f=3$. The terms in (XII.8) depending on $\alpha_{\mathrm{s}}(\mu)$ are factored out explicitly in (XII.1) to exhibit the $\mu$-dependence of the coefficient function in the $f=3$ regime, which has to cancel the corresponding $\mu$-dependence of the hadronic matrix element of $Q$ between meson states in physical applications. A similar comment applies to the scheme dependence entering $J_{f}$ in (XII.9) through the scheme dependence of $\gamma^{(1)}$. Splitting off the $\mu$-dependence in (XII.1) is of course not unique. The way it is done belongs to the definition of the $\eta_{i}$-factors.

Let us finally compare the structure of (XII.1) with the effective hamiltonians for rare decays discussed in chapter XI. Common features of both types of processes include:

- Both are generated to lowest order via electroweak FCNC loop transitions involving heavy quarks.

- They contain a charm and a top contribution.

- The hamiltonian consists of a single dimension-6 operator.

Besides these similarities, however, there are also a few important differences, which have their root in the fact that the $\Delta S=2$ box diagrams involve two distinct quark lines as compared to the single quark line in semileptonic rare decays:

- The CKM parameter combinations $\lambda_{i}$ appear quadratically in (XII.1) instead of only linearly.

- (XII.1) in addition receives contributions from a mixed top-charm sector. This part in fact turns out to have the most involved structure of the three contributions.

- The operator $Q$ has a non-vanishing QCD anomalous dimension, resulting in a non-trivial scale and scheme dependence of the Wilson coefficient. 
- The hadronic matrix element of the four-quark operator $Q$ is a considerably more complicated object than the quark current matrix elements in semileptonic rare decays.

We will now present the complete next-to-leading order results for $\eta_{2}, \eta_{1}$ and $\eta_{3}$ in turn and discuss their most important theoretical features. The first leading log calculations of $\eta_{1}$ have been presented in (Vainshtein et al., 1976), (Novikov et al., 1977) and of $\eta_{2}$ in (Vysotskij, 1980). The complete leading log calculation inlcuding also $\eta_{3}$ has been first performed in (Gilman and Wise, 1983). Leading order calculations in the presence of a heavy top can be found in (Kaufman et al., 1989), (Flynn, 1990), (Datta et al., 1990) and (Datta et al., 1995).

\section{B. The Top Contribution $-\eta_{2}$}

The basic structure of the top quark sector in $\mathcal{H}_{e f f}^{\Delta S=2}$ is easy to understand. First the top quark is integrated out, along with the $W$, at a matching scale $\mu_{t}=\mathcal{O}\left(m_{t}\right)$, leaving a $m_{t}$-dependent coefficient normalized at $\mu_{t}$, multiplying the single operator $Q$. Subsequently the coefficient is simply renormalized down to scales $\mu=\mathcal{O}(1 \mathrm{GeV})$ by means of (XII.8). Including NLO corrections the resulting QCD factor $\eta_{2}$ from (XII.1) may be written (in $\overline{M S}$ ) as follows (Buras et al., 1990)

$$
\begin{aligned}
\eta_{2}= & {\left[\alpha_{\mathrm{s}}\left(m_{c}\right)\right]^{6 / 27}\left[\frac{\alpha_{\mathrm{s}}\left(m_{b}\right)}{\alpha_{\mathrm{s}}\left(m_{c}\right)}\right]^{6 / 25}\left[\frac{\alpha_{\mathrm{s}}\left(\mu_{t}\right)}{\alpha_{\mathrm{s}}\left(m_{b}\right)}\right]^{6 / 23} } \\
& \cdot\left[1+\frac{\alpha_{\mathrm{s}}\left(m_{c}\right)}{4 \pi}\left(J_{4}-J_{3}\right)+\frac{\alpha_{\mathrm{s}}\left(m_{b}\right)}{4 \pi}\left(J_{5}-J_{4}\right)\right. \\
& \left.+\frac{\alpha_{\mathrm{s}}\left(\mu_{t}\right)}{4 \pi}\left(\frac{S_{1}\left(x_{t}\right)}{S_{0}\left(x_{t}\right)}+B_{t}-J_{5}+\frac{\gamma^{(0)}}{2} \ln \frac{\mu_{t}^{2}}{M_{W}^{2}}+\gamma_{m 0} \frac{\partial \ln S_{0}\left(x_{t}\right)}{\partial \ln x_{t}} \ln \frac{\mu_{t}^{2}}{M_{W}^{2}}\right)\right]
\end{aligned}
$$

where $\gamma_{m 0}=6 C_{F}$,

$$
B_{t}=5 \frac{N-1}{2 N}+3 \frac{N^{2}-1}{2 N}
$$

and

$$
\begin{gathered}
S_{1}(x)=\frac{N-1}{2 N} S_{1}^{(8)}(x)+\frac{N^{2}-1}{2 N} S_{1}^{(1)}(x) \\
S_{1}^{(8)}(x)=-\frac{64-68 x-17 x^{2}+11 x^{3}}{4(1-x)^{2}}+\frac{32-68 x+32 x^{2}-28 x^{3}+3 x^{4}}{2(1-x)^{3}} \ln x \\
+\frac{x^{2}\left(4-7 x+7 x^{2}-2 x^{3}\right)}{2(1-x)^{4}} \ln ^{2} x+\frac{2 x\left(4-7 x-7 x^{2}+x^{3}\right)}{(1-x)^{3}} L_{2}(1-x) \\
+\frac{16}{x}\left(\frac{\pi^{2}}{6}-L_{2}(1-x)\right) \\
S_{1}^{(1)}(x)=-\frac{x\left(4-39 x+168 x^{2}+11 x^{3}\right)}{4(1-x)^{3}}-\frac{3 x\left(4-24 x+36 x^{2}+7 x^{3}+x^{4}\right)}{2(1-x)^{4}} \ln x \\
+\frac{3 x^{3}\left(13+4 x+x^{2}\right)}{2(1-x)^{4}} \ln ^{2} x-\frac{3 x^{3}(5+x)}{(1-x)^{3}} L_{2}(1-x)
\end{gathered}
$$


where the dilogarithm $L_{2}$ is defined in (XI.8).

In the expression (XII.10) we have taken into account the heavy quark thresholds at $m_{b}$ and $m_{c}$ in the RG evolution. As it must be, the dependence on the threshold scales is of the neglected order $\mathcal{O}\left(\alpha_{s}^{2}\right)$. In fact the threshold ambiguity is here of $\mathcal{O}\left(\alpha_{\mathrm{s}}^{2}\right)$ also in LLA since $\gamma^{(0)}$ is flavor independent. It turns out that this dependence is also very weak numerically and we therefore set $\mu_{c}=m_{c}$ and $\mu_{b}=m_{b}$. Furthermore it is a good approximation to neglect the b-threshold completely using an effective 4-flavor theory from $\mu_{t}$ down to $m_{c}$. This can be achieved by simply substituting $m_{b} \rightarrow \mu_{t}$ in (XII.10).

The leading order expression for $\eta_{2}$ is given by the first three factors on the r.h.s. of (XII.10). The fourth factor represents the next-to-leading order generalization. Let us discuss now the most interesting and important features of the NLO result for $\eta_{2}$ exhibited in (XII.10).

- $\eta_{2}$ is proportional to the initial value of the Wilson coefficient function at $\mu_{t}=M_{\mathrm{W}}$

$$
S(x)=S_{0}(x)+\frac{\alpha_{\mathrm{s}}}{4 \pi}\left(S_{1}(x)+B_{t} S_{0}(x)\right)
$$

which has to be extracted from the box graphs in fig. 4(e) and the corresponding gluon correction diagrams after a proper factorization of long- and short-distance contributions.

- $S(x)$ in (XII.15) is similar to the functions $X(x)$ and $Y(x)$ in sections XI B 1 and XIC 1 except that $S(x)$ is scheme dependent due to the renormalization that is required for the operator $Q$. This scheme dependence enters (XII.15) through the scheme dependent constant $B_{t}$, given in the NDR scheme in (XII.11). This scheme dependence is canceled in the combination $B_{t}-J_{5}$ by the two-loop anomalous dimension contained in $J_{5}$. Likewise the scheme dependence of $J_{f}$ cancels in the differences $\left(J_{f}-J_{f-1}\right)$ as is evident from the discussion of section III F 3 .

- A very important point is the dependence on the high energy matching scale $\mu_{t}$. This dependence enters the NLO $\alpha_{\mathrm{s}}\left(\mu_{t}\right)$-correction in (XII.10) in two distinct ways: First as a term proportional to $\gamma^{(0)}$ and, secondly, in conjunction with $\gamma_{m 0}$. The first of these terms cancels to $\mathcal{O}\left(\alpha_{\mathrm{s}}\right)$ the $\mu_{t}$-dependence present in the leading term $\left[\alpha_{\mathrm{s}}\left(\mu_{t}\right)\right]^{6 / 23}$. The second, on the other hand, leads to an $\mathcal{O}\left(\alpha_{\mathrm{s}}\right) \mu_{t}$-dependence of $\eta_{2}$ which is just the one needed to cancel the $\mu_{t}$-ambiguity of the leading function $S_{0}\left(x_{t}\left(\mu_{t}\right)\right)$ in the product $\eta_{2} S_{0}\left(x_{t}\right)$, such that in total physical results become independent of $\mu_{t}$ to $\mathcal{O}\left(\alpha_{\mathrm{s}}\right)$. From these observations it is obvious that one may interpret $\mu_{t}$ in the first case as the initial scale of the RG evolution and in the second case as the scale at which the top quark mass is defined. These two scales need not necessarily have the same value.

The important point is, that to leading logarithmic accuracy the $\mu_{t}$-dependence of both $\eta_{2}^{L O}\left(\mu_{t}\right)$ and $S_{0}\left(x_{t}\left(\mu_{t}\right)\right)$ remains uncompensated, leaving a disturbingly large uncertainty in the short-distance calculation.

- It is interesting to note that in the limit $m_{\mathrm{t}} \gg M_{\mathrm{W}}$ the dependence on $\mu_{t}$ enters $\eta_{2}$ as $\ln \mu_{t} / m_{\mathrm{t}}$, rather than $\ln \mu_{t} / M_{\mathrm{W}}$. This feature provides a formal justification for choosing $\mu_{t}=\mathcal{O}\left(m_{\mathrm{t}}\right)$ instead of $\mu_{t}=\mathcal{O}\left(M_{\mathrm{W}}\right)$. An explicit expression for the large $m_{\mathrm{t}}$ limit in the similar case of $\eta_{2 B}$ may be found in section XIII. 
- Although at NLO the product $\eta_{2} S_{0}\left(x_{t}\right)$ depends only very weakly on the precise value of $\mu_{t}$ as long as it is of $\mathcal{O}\left(m_{t}\right)$, the choice $\mu_{t}=m_{t}$ is again convenient: With this choice $\eta_{2}$ becomes almost independent of the top quark mass $m_{t}\left(m_{t}\right)$. By contrast, for $\mu_{t}=M_{W}$, say, $\eta_{2}$ would decrease with rising $m_{t}\left(m_{t}\right)$ in order to compensate for the increase of $S_{0}\left(x_{t}\left(M_{W}\right)\right)$ due to the use of a - for high $m_{t}$ - "unnaturally" low scale $M_{W}$.

- As mentioned above the dependence of the Wilson coefficient on the low energy scale $\mu$ and the remaining scheme dependence $\left(J_{3}\right)$ has been factored out explicitly in (XII.1). Therefore the QCD correction factor $\eta_{2}$ is by definition scale and scheme independent on the lower end of the RG evolution.

\section{The Charm Contribution $-\eta_{1}$}

The calculation of $\eta_{1}$ beyond leading logs has been presented in great detail in (Herrlich and Nierste, 1994), (Herrlich, 1994). Our task here will be to briefly describe the basic procedure and to summarize the main results.

In principle the charm contribution is similar in structure to the top contribution. However, since the quark mass $m_{c} \ll M_{W}$, the charm degrees of freedom can no longer be integrated out simultaneously with the $W$ boson, which would introduce large logarithmic corrections $\sim \alpha_{\mathrm{s}} \ln M_{W} / m_{c}$. To resum these logarithms one first constructs an effective theory at a scale $\mathcal{O}\left(M_{W}\right)$, where the $W$ boson is removed. The relevant operators are subsequently renormalized down to scales $\mu_{c}=\mathcal{O}\left(m_{c}\right)$, where the charm quark is then integrated out. After this step only the operator $Q$ (XII.2) remains and $\eta_{1}$ is finally obtained as discussed in section XII A.

Let us briefly outline this procedure for the case at hand. After integrating out $W$ the effective hamiltonian to first order in weak interactions, which is needed for the charm contribution, can be written as

$$
\mathcal{H}_{c}^{(1)}=\frac{G_{F}}{\sqrt{2}} \sum_{q, q^{\prime}=u, c} V_{q^{\prime} s}^{*} V_{q d}\left(C_{+} Q_{+}^{q^{\prime} q}+C_{-} Q_{-}^{q^{\prime} q}\right)
$$

where we have introduced the familiar $\Delta S=1$ four-quark operators in the multiplicatively renormalizable basis

$$
Q_{ \pm}^{q^{\prime} q}=\frac{1}{2}\left[\left(\bar{s}_{i} q_{i}^{\prime}\right)_{V-A}\left(\bar{q}_{j} d_{j}\right)_{V-A} \pm\left(\bar{s}_{i} q_{j}^{\prime}\right)_{V-A}\left(\bar{q}_{j} d_{i}\right)_{V-A}\right]
$$

We remark that no penguin operators appear in the present case due to GIM cancellation between charm quark and up quark contributions.

$\Delta S=2$ transitions occur to second order in the effective interaction (XII.16). The $\Delta S=2$ effective hamiltonian is therefore given by

$$
\mathcal{H}_{\text {eff }, c}^{\Delta S=2}=-\frac{i}{2} \int d^{4} x T\left(\mathcal{H}_{c}^{(1)}(x) \mathcal{H}_{c}^{(1)}(0)\right)
$$

Inserting (XII.16) into (XII.18), keeping only pieces that can contribute to the charm box diagrams and taking the GIM constraints into account, one obtains

$$
\mathcal{H}_{\text {eff }, c}^{\Delta S=2}=\frac{G_{F}^{2}}{2} \lambda_{c}^{2} \sum_{i, j=+,-} C_{i} C_{j} O_{i j}
$$


where

$$
O_{i j}=-\frac{i}{2} \int d^{4} x T\left[Q_{i}^{c c}(x) Q_{j}^{c c}(0)-Q_{i}^{u c}(x) Q_{j}^{c u}(0)-Q_{i}^{c u}(x) Q_{j}^{u c}(0)+Q_{i}^{u u}(x) Q_{j}^{u u}(0)\right]
$$

From the derivation of (XII.19) it is evident, that the Wilson coefficients of the bilocal operators $O_{i j}$ are simply given by the product $C_{i} C_{j}$ of the coefficients pertaining to the local operators $Q_{i}$, $Q_{j}$. The evolution of the $C_{i}$ from $M_{W}$ down to $\mu_{c}$ proceeds in the standard fashion and is described by equations of the type shown in (XII.8) with the appropriate anomalous dimensions inserted. In the following we list the required ingredients.

The Wilson coefficients at scale $\mu=M_{W}$ read

$$
\begin{aligned}
& C_{ \pm}\left(M_{W}\right)=1+\frac{\alpha_{\mathrm{s}}\left(M_{W}\right)}{4 \pi} B_{ \pm} \\
& B_{ \pm}= \pm 11 \frac{N \mp 1}{2 N} \quad(\mathrm{NDR})
\end{aligned}
$$

The two-loop anomalous dimensions are

$$
\begin{gathered}
\gamma_{ \pm}=\frac{\alpha_{\mathrm{s}}}{4 \pi} \gamma_{ \pm}^{(0)}+\left(\frac{\alpha_{\mathrm{s}}}{4 \pi}\right)^{2} \gamma_{ \pm}^{(1)} \\
\gamma_{ \pm}^{(0)}= \pm 6 \frac{N \mp 1}{N} \quad \gamma_{ \pm}^{(1)}=\frac{N \mp 1}{2 N}\left[-21 \pm \frac{57}{N} \mp \frac{19}{3} N \pm \frac{4}{3} f\right] \quad(\mathrm{NDR})
\end{gathered}
$$

For $i, j=+,-$ we introduce

$$
d_{i}^{(f)}=\frac{\gamma_{i}^{(0)}}{2 \beta_{0}} \quad J_{i}^{(f)}=\frac{d_{i}^{(f)}}{\beta_{0}} \beta_{1}-\frac{\gamma_{i}^{(1)}}{2 \beta_{0}}
$$

and

$$
d_{i j}^{(f)}=d_{i}^{(f)}+d_{j}^{(f)} \quad J_{i j}^{(f)}=J_{i}^{(f)}+J_{j}^{(f)}
$$

The essential step consists in matching (XII.19) onto an effective theory without charm, which will contain the single operator $Q=(\bar{s} d)_{V-A}(\bar{s} d)_{V-A}$. In NLO this matching has to be performed to $\mathcal{O}\left(\alpha_{\mathrm{s}}\right)$. At a normalization scale $\mu_{c}$ it reads explicitly, expressed in terms of operator matrix elements $(i, j=+,-)$

$$
\begin{gathered}
\left\langle O_{i j}\right\rangle=\frac{m_{c}^{2}\left(\mu_{c}\right)}{8 \pi^{2}}\left[\tau_{i j}+\frac{\alpha_{\mathrm{s}}\left(\mu_{c}\right)}{4 \pi}\left(\kappa_{i j} \ln \frac{\mu_{c}^{2}}{m_{c}^{2}}+\beta_{i j}\right)\right]\langle Q\rangle \\
\tau_{++}=\frac{N+3}{4} \quad \tau_{+-}=\tau_{-+}=-\frac{N-1}{4} \quad \tau_{--}=\frac{N-1}{4} \\
\kappa_{++}=3(N-1) \tau_{++} \quad \kappa_{+-}=\kappa_{-+}=3(N+1) \tau_{+-} \quad \kappa_{--}=3(N+3) \tau_{--}
\end{gathered}
$$


The $\beta_{i j}$ are scheme dependent. In the NDR scheme they are given by (Herrlich and Nierste, 1994)

$$
\begin{aligned}
\beta_{++} & =(1-N)\left(\frac{N^{2}-6}{12 N} \pi^{2}+3 \frac{-N^{2}+2 N+13}{4 N}\right) \\
\beta_{+-}= & \beta_{-+}=(1-N)\left(\frac{-N^{2}+2 N-2}{12 N} \pi^{2}+\frac{3 N^{2}+13}{4 N}\right) \\
\beta_{--} & =(1-N)\left(\frac{N^{2}-4 N+2}{12 N} \pi^{2}-\frac{3 N^{2}+10 N+13}{4 N}\right)
\end{aligned}
$$

Now, starting from (XII.19), evolving $C_{i}$ from $M_{W}$ down to $\mu_{c}$, integrating out charm at $\mu_{c}$ with the help of (XII.27), evolving the resulting coefficient according to (XII.8) and recalling the definition of $\eta_{1}$ in (XII.1), one finally obtains

$$
\begin{aligned}
\eta_{1}= & {\left[\alpha_{\mathrm{s}}\left(\mu_{c}\right)\right]^{d_{3}} \sum_{i, j=+,-}\left(\frac{\alpha_{\mathrm{s}}\left(m_{b}\right)}{\alpha_{\mathrm{s}}\left(\mu_{c}\right)}\right)^{d_{i j}^{(4)}}\left(\frac{\alpha_{\mathrm{s}}\left(M_{W}\right)}{\alpha_{\mathrm{s}}\left(m_{b}\right)}\right)^{d_{i j}^{(5)}} \times } \\
& \times\left[\tau_{i j}+\frac{\alpha_{\mathrm{s}}\left(\mu_{c}\right)}{4 \pi}\left(\kappa_{i j} \ln \frac{\mu_{c}^{2}}{m_{c}^{2}}+\tau_{i j}\left(J_{i j}^{(4)}-J_{3}\right)+\beta_{i j}\right)+\right. \\
& \left.+\tau_{i j}\left(\frac{\alpha_{\mathrm{s}}\left(m_{b}\right)}{4 \pi}\left(J_{i j}^{(5)}-J_{i j}^{(4)}\right)+\frac{\alpha_{\mathrm{s}}\left(M_{W}\right)}{4 \pi}\left(B_{i}+B_{j}-J_{i j}^{(5)}\right)\right)\right]
\end{aligned}
$$

We conclude this section with a discussion of a few important issues concerning the structure of this formula.

- (XII.31), as first obtained in (Herrlich and Nierste, 1994), represents the next-to-leading order generalization of the leading log expression for $\eta_{1}$ given in (Gilman and Wise, 1983). The latter follows as a special case of (XII.31) when the $\mathcal{O}\left(\alpha_{\mathrm{s}}\right)$ correction terms are put to zero.

- The expression (XII.31) is independent of the renormalization scheme up to terms of the neglected order $\mathcal{O}\left(\alpha_{s}^{2}\right)$. We have written $\eta_{1}$ in a form, in which this scheme independence becomes manifest: While the various $J$-terms, $B_{i}$ and $\beta_{i j}$ in (XII.31) all depend on the renormalization scheme when considered separately, the combinations $\tau_{i j}\left(J_{i j}^{(4)}-J_{3}\right)+\beta_{i j}$, $J_{i j}^{(5)}-J_{i j}^{(4)}$ and $B_{i}+B_{j}-J_{i j}^{(5)}$ are scheme invariant.

- The product $\eta_{1}\left(\mu_{c}\right) x_{c}\left(\mu_{c}\right)$ is independent of $\mu_{c}$ to the considered order,

$$
\frac{d}{d \ln \mu_{c}} \eta_{1}\left(\mu_{c}\right) x_{c}\left(\mu_{c}\right)=\mathcal{O}\left(\alpha_{s}^{2}\right)
$$

in accordance with the requirements of renormalization group invariance. The cancellation of the $\mu_{c}$-dependence to $\mathcal{O}\left(\alpha_{\mathrm{s}}\right)$ is related to the presence of an explicitly $\mu_{c}$-dependent term at NLO in (XII.31) and is guaranteed through the identity

$$
\kappa_{i j}=\tau_{i j}\left(\gamma_{m 0}+\frac{\gamma^{(0)}}{2}-\frac{\gamma_{i}^{(0)}+\gamma_{j}^{(0)}}{2}\right)
$$

which is easily verified using (III.17), (XII.7), (XII.24), (XII.28) and (XII.29). 
- Also the ambiguity in the scale $\mu_{W}$, at which $W$ is integrated out, is reduced from $\mathcal{O}\left(\alpha_{\mathrm{s}}\right)$ to $\mathcal{O}\left(\alpha_{s}^{2}\right)$ when going from leading to next-to-leading order. As mentioned above the dependence on the $b$-threshold scale $\mu_{b}$ is $\mathcal{O}\left(\alpha_{s}^{2}\right)$ in NLLA as well as in LLA. Numerically the dependence on $\mu_{b}$ is very small. Also the variation of the result with the high energy matching scale $\mu_{W}$ is considerably weaker than the residual dependence on $\mu_{c}$. Therefore we have set $\mu_{b}=m_{b}$ and $\mu_{W}=M_{W}$ in (XII.31). In numerical analyses we will take the dominant $\mu_{c}$-dependence as representative for the short-distance scale ambiguity of $\eta_{1}$. The generalization to the case $\mu_{W} \neq M_{W}$ is discussed in (Herrlich and Nierste, 1994). The more general case $\mu_{b} \neq m_{b}$ is trivially obtained by substituting $m_{b} \rightarrow \mu_{b}$ in (XII.31).

- Note that due to the GIM structure of $O_{i j}$ no mixing under infinite renormalization occurs between $O_{i j}$ and the local operator $Q$. This is related to the absence of the logarithm in the function $S_{0}\left(x_{c}\right)$ in (XII.3).

It is instructive to compare the results obtained for $\eta_{1}$ and $\eta_{2}$. Expanding (XII.31) to first order in $\alpha_{\mathrm{s}}$, in this way "switching off" the RG summations, we find

$$
\begin{aligned}
& {\left[\alpha_{\mathrm{s}}(\mu)\right]^{-2 / 9}\left(1+\frac{\alpha_{\mathrm{s}}(\mu)}{4 \pi} J_{3}\right) \eta_{1} \doteq} \\
& 1+\frac{\alpha_{\mathrm{s}}}{4 \pi}\left[\frac{\gamma^{(0)}}{2}\left(\ln \frac{\mu^{2}}{M_{W}^{2}}+\ln \frac{m^{2}}{M_{W}^{2}}-1+\frac{2}{9} \pi^{2}\right)+\gamma_{m 0}\left(\ln \frac{\mu^{2}}{m^{2}}+\frac{1}{3}\right)\right]
\end{aligned}
$$

where we have replaced $\mu_{c} \rightarrow \mu$ and $m_{c} \rightarrow m$. In deriving (XII.34) besides (XII.33) the following identities are useful

$$
\begin{gathered}
\sum_{i, j=+,-} \tau_{i j}=1 \quad \sum_{i, j=+,-} \tau_{i j} \frac{\gamma_{i}^{(0)}+\gamma_{j}^{(0)}}{2}=\gamma^{(0)} \\
\sum_{i, j=+,-} \tau_{i j}\left(B_{i}+B_{j}\right)=2 B_{+}
\end{gathered}
$$

The same result (XII.34) is obtained from $\eta_{2}$ as well, if we set $m_{c}=m_{b}=\mu_{t}=\mu, m_{t}=m$ in (XII.10) and expand for $m \ll M_{W}$. This exercise yields a useful cross-check between the calculations for $\eta_{1}$ and $\eta_{2}$. In addition it gives some further insight into the structure of the QCD corrections to $\Delta S=2$ box diagrams, establishing $\eta_{1}$ and $\eta_{2}$ as two different generalizations of the same asymptotic limit (XII.34).

\section{The Top-Charm Contribution $-\eta_{3}$}

To complete the description of the $K^{0}-\bar{K}^{0}$ effective hamiltonian we now turn to the mixed top-charm component, defined by the contribution $\sim \lambda_{c} \lambda_{t}$ in (XII.1), and the associated QCD correction factor $\eta_{3}$. The short distance QCD effects have been first obtained within the leading $\log$ approximation by (Gilman and Wise, 1983). The calculation of $\eta_{3}$ at next-to-leading order is due to the work of (Herrlich and Nierste, 1995a), (Nierste, 1995). As already mentioned, the renormalization group analysis necessary for $\eta_{3}$ is more involved than in the cases of $\eta_{1}$ and $\eta_{2}$. The characteristic differences will become clear from the following presentation. 
We begin by writing down the relevant $\Delta S=1$ hamiltonian, obtained after integrating out $W$ and top, which provides the basis for the construction of the $\Delta S=2$ effective hamiltonian we want to derive. It reads

$$
\mathcal{H}_{c t}^{(1)}=\frac{G_{F}}{\sqrt{2}}\left(\sum_{q, q^{\prime}=u, c} V_{q^{\prime} s}^{*} V_{q d} \sum_{i=1,2} C_{i} Q_{i}^{q^{\prime} q}-\lambda_{t} \sum_{i=3}^{6} C_{i} Q_{i}\right)
$$

with

$$
Q_{1}^{q^{\prime} q}=\left(\bar{s}_{i} q_{j}^{\prime}\right)_{V-A}\left(\bar{q}_{j} d_{i}\right)_{V-A} \quad Q_{2}^{q^{\prime} q}=\left(\bar{s}_{i} q_{i}^{\prime}\right)_{V-A}\left(\bar{q}_{j} d_{j}\right)_{V-A}
$$

and corresponds to the hamiltonian (VI.5), discussed in chapter VI, except that we have included the $\Delta C=1$ components $Q_{i}^{u c}, Q_{i}^{c u}$, which contribute in the analysis of $\eta_{3}$. By contrast to the simpler case of $\eta_{1}$ presented in the previous section, now also the penguin operators $Q_{i}, i=3, \ldots, 6$ (VI.3) have to be considered. Being proportional to $\lambda_{t}=V_{t s}^{*} V_{t d}$ they will contribute to the $\lambda_{c} \lambda_{t^{-}}$ part of (XII.1). We remark in this context that, on the other hand, the penguin contribution to the $\lambda_{t}^{2}$-sector is entirely negligible. Since only light quarks are involved in $Q_{3}, \ldots, Q_{6}$, the double penguin diagrams, which would contribute to the $\lambda_{t}^{2}$-piece of the $\Delta S=2$ amplitude, are suppressed by at least a factor of $m_{b}^{2} / m_{t}^{2}$ compared with the dominant top-exchange effects discussed in section XII B.

At second order in (XII.37) $\Delta S=2$ transitions are generated. Inserting (XII.37) in an expression similar to (XII.18), eliminating $\lambda_{u}$ by means of $\lambda_{u}=-\lambda_{c}-\lambda_{t}$ and collecting the terms proportional to $\lambda_{c} \lambda_{t}$, we obtain the top-charm component of the effective $\Delta S=2$ hamiltonian in the form

$$
\mathcal{H}_{\text {eff }, c t}^{\Delta S=2}=\frac{G_{F}^{2}}{2} \lambda_{c} \lambda_{t} \sum_{i= \pm}\left[\sum_{j=1}^{6} C_{i} C_{j} Q_{i j}+C_{7 i} Q_{7}\right]
$$

where

$$
Q_{i j}=-\frac{i}{2} \int d^{4} x T\left[2 Q_{i}^{u u}(x) Q_{j}^{u u}(0)-Q_{i}^{u c}(x) Q_{j}^{c u}(0)-Q_{i}^{c u}(x) Q_{j}^{u c}(0)\right]
$$

for $j=1,2$ and

$$
Q_{i j}=-\frac{i}{2} \int d^{4} x T\left[\left(Q_{i}^{u u}(x)-Q_{i}^{c c}(x)\right) Q_{j}(0)+Q_{j}(x)\left(Q_{i}^{u u}(0)-Q_{i}^{c c}(0)\right)\right]
$$

for $j=3, \ldots, 6$.

In defining these operators we have already omitted bilocal products with flavor structure like $(\bar{s} u \bar{u} d) \cdot(\bar{s} c \bar{c} d)$, which cannot contribute to $\Delta S=2$ box diagrams. Furthermore, for the factor entering the bilocal operators with index $i$ we have changed the basis from $Q_{1,2}^{q^{\prime} q}$ to $Q_{ \pm}^{q^{\prime} q}$ given in (XII.17). In addition local counterterms proportional to the $\Delta S=2$ operator

$$
Q_{7}=\frac{m_{c}^{2}}{g^{2}}(\bar{s} d)_{V-A}(\bar{s} d)_{V-A}
$$

have been added to (XII.39). These are necessary here because the bilocal operators can in general mix into $Q_{7}$ under infinite renormalization, a fact related to the logarithm present in the leading 
term $-x_{c} \ln x_{c}$ entering $S_{0}\left(x_{c}, x_{t}\right)$ in (XII.5). This behaviour is in contrast to the charm contribution, where the corresponding function $S_{0}\left(x_{c}\right)=x_{c}$ does not contain a logarithmic term and consequently no local $\Delta S=2$ counterterm is necessary in (XII.19). On the other hand the situation here is analogous to the case of the charm contribution to the effective hamiltonian for $K^{+} \rightarrow \pi^{+} \nu \bar{\nu}$ in section XI B which similarly behaves as $x_{c} \ln x_{c}$ in lowest order and correspondingly requires a counterterm, as displayed in (XI.21) and (XI.35).

After integrating out top and $W$ at the high energy matching scale $\mu_{W}=\mathcal{O}\left(M_{W}\right)$, the Wilson coefficients $C_{j}, j=1, \ldots 6$ of (XII.37) and (XII.39) are given in the NDR-scheme by (see section $\mathrm{VI})$

$$
\begin{aligned}
\vec{C}^{T}\left(\mu_{W}\right) & =(0,1,0,0,0,0)+\frac{\alpha_{s}\left(\mu_{W}\right)}{4 \pi}\left(6,-2,-\frac{2}{9}, \frac{2}{3},-\frac{2}{9}, \frac{2}{3}\right) \ln \frac{\mu_{W}}{M_{W}} \\
& +\frac{\alpha_{s}\left(\mu_{W}\right)}{4 \pi}\left(\frac{11}{2},-\frac{11}{6},-\frac{1}{6} \tilde{E}_{0}\left(x_{t}\right), \frac{1}{2} \tilde{E}_{0}\left(x_{t}\right),-\frac{1}{6} \tilde{E}_{0}\left(x_{t}\right), \frac{1}{2} \tilde{E}_{0}\left(x_{t}\right)\right)
\end{aligned}
$$

and $C_{ \pm}=C_{2} \pm C_{1} . \tilde{E}_{0}\left(x_{t}\right)$ can be found in (VI.16). The coefficient of $Q_{7}$ is obtained through matching the $\Delta S=2$ matrix element of the effective theory (XII.39) to the corresponding full theory matrix element, which is in the required approximation $\left(x_{c} \ll 1\right)$ given by (compare (XII.1))

$$
A_{\text {full }, c t}=\frac{G_{F}^{2}}{16 \pi^{2}} M_{W}^{2} 2 \lambda_{c} \lambda_{t} S_{0}\left(x_{c}, x_{t}\right)\langle Q\rangle
$$

At next-to-leading order this matching has to be done to one loop, including finite parts. Note that here the loop effect is due to electroweak interactions and QCD does not contribute explicitly in this step. The matching condition determines the sum $C_{7} \equiv C_{7+}+C_{7-}$, which in the NDR scheme and with the conventional definition of evanescent operators, (Buras and Weisz, 1990), see also (Herrlich and Nierste, 1995a), (Nierste, 1995), reads

$$
C_{7}\left(\mu_{W}\right)=\frac{\alpha_{s}\left(\mu_{W}\right)}{4 \pi}\left[-8 \ln \frac{\mu_{W}}{M_{W}}+4 \ln x_{t}-\frac{3 x_{t}}{1-x_{t}}-\frac{3 x_{t}^{2} \ln x_{t}}{\left(1-x_{t}\right)^{2}}+2\right]
$$

at next-to-leading order. In leading log approximation one simply would have $C_{7}\left(\mu_{W}\right)=0$.

The distribution of $C_{7}$ among $C_{7+}$ and $C_{7-}$ is arbitrary and has no impact on the physics. For example we may choose

$$
C_{7+}=C_{7} \quad C_{7-}=0
$$

Having determined the initial values of the Wilson coefficients

$$
\vec{C}^{( \pm) T} \equiv\left(C_{ \pm} C_{1}, \ldots, C_{ \pm} C_{6}, C_{7 \pm}\right)
$$

at a scale $\mu_{W}, \vec{C}^{( \pm)}\left(\mu_{W}\right)$, the next step consists in solving the RG equations to determine $\vec{C}^{( \pm)}\left(\mu_{c}\right)$ at the charm mass scale $\mu_{c}=\mathcal{O}\left(m_{c}\right)$. The renormalization group evolution of $\vec{C}^{( \pm)}$is given by

$$
\begin{gathered}
\frac{d}{d \ln \mu} \vec{C}^{( \pm)}(\mu)=\gamma_{c t}^{( \pm) T} \vec{C}^{( \pm)}(\mu) \\
\gamma_{c t}^{( \pm)}=\left(\begin{array}{cc}
\gamma_{s}+\gamma_{ \pm} \cdot 1 & \vec{\gamma}_{ \pm 7} \\
\overrightarrow{0}^{T} & \gamma_{77}
\end{array}\right)
\end{gathered}
$$


Here $\gamma_{s}$ is the standard $6 \times 6$ anomalous dimension matrix for the $\Delta S=1$ effective hamiltonian including QCD penguins from (VI.23), (VI.25) and (VI.26) (NDR-scheme). Similarly $\gamma_{ \pm}$are the anomalous dimensions of the current-current operators. They can be obtained as $\gamma_{ \pm}=\gamma_{s, 11} \pm \gamma_{s, 12}$ and are also given in section $\mathrm{V}$.

$\gamma_{77}$ represents the anomalous dimension of $Q_{7}$ (XII.42) and reads

$$
\gamma_{77}=\gamma_{+}+2 \gamma_{m}+2 \beta(g) / g=\frac{\alpha_{s}}{4 \pi} \gamma_{77}^{(0)}+\left(\frac{\alpha_{s}}{4 \pi}\right)^{2} \gamma_{77}^{(1)}
$$

For $N=3$ and in NDR

$$
\gamma_{77}^{(0)}=-2+\frac{4}{3} f \quad \gamma_{77}^{(1)}=\frac{175}{3}+\frac{152}{9} f
$$

Finally $\vec{\gamma}_{ \pm 7}$, the vector of anomalous dimensions expressing the mixing of the bilocal operators $Q_{ \pm i}(i=1, \ldots, 6)$ into $Q_{7}$, is given by

$$
\vec{\gamma}_{ \pm 7}=\frac{\alpha_{s}}{4 \pi} \vec{\gamma}_{ \pm 7}^{(0)}+\left(\frac{\alpha_{s}}{4 \pi}\right)^{2} \vec{\gamma}_{ \pm 7}^{(1)}
$$

where

$$
\begin{gathered}
\vec{\gamma}_{+7}^{(0) T}=(-16,-8,-32,-16,32,16) \\
\vec{\gamma}_{-7}^{(0) T}=(8,0,16,0,-16,0) \\
\vec{\gamma}_{+7}^{(1) T}=\left(-212,-28,-424,-56, \frac{1064}{3}, \frac{832}{3}\right) \\
\vec{\gamma}_{-7}^{(1) T}=\left(276,-92,552,-184,-\frac{1288}{3}, 0\right)
\end{gathered}
$$

The scheme-dependent numbers in $\vec{\gamma}_{ \pm 7}^{(1)}$ are given here in the NDR-scheme with the conventional treatment of evanescent operators as described in (Buras and Weisz, 1990), (Herrlich and Nierste, 1995a), (Nierste, 1995).

In order to solve the RG equation (XII.48) it is useful (Herrlich and Nierste, 1995a), (Nierste, $1995)$ to define the eight-dimensional vector $\left(\vec{C}^{T}=\left(C_{1}, \ldots, C_{6}\right)\right)$

$$
\vec{D}^{T}=\left(\vec{C}^{T}, C_{7+} / C_{+}, C_{7-} / C_{-}\right)
$$

which obeys

$$
\frac{d}{d \ln \mu} \vec{D}=\gamma_{c t}^{T} \vec{D}
$$

where

$$
\gamma_{c t}=\left(\begin{array}{ccc}
\gamma_{s} & \vec{\gamma}_{+7} & \vec{\gamma}_{-7} \\
\overrightarrow{0}^{T} & \gamma_{77}-\gamma_{+} & 0 \\
\overrightarrow{0}^{T} & 0 & \gamma_{77}-\gamma_{-}
\end{array}\right)
$$


The solution of (XII.58) proceeds in the standard fashion as described in section IIIF 1 and has the form

$$
\vec{D}\left(\mu_{c}\right)=U_{4}\left(\mu_{c}, \mu_{b}\right) M\left(\mu_{b}\right) U_{5}\left(\mu_{b}, \mu_{W}\right) \vec{D}\left(\mu_{W}\right)
$$

similarly to (III.105). The b-quark-threshold matching matrix $M\left(\mu_{b}\right)$ is an $8 \times 8$ matrix whose $6 \times 6$ submatrix $M_{i j}, i, j=1, \ldots, 6$ is identical to the matrix $M$ described in section VID. The remaining elements are $M_{77}=M_{88}=1$ and zero otherwise. From (XII.60) the Wilson coefficients $C_{i}\left(\mu_{c}\right)$ are obtained as

$$
C_{i}\left(\mu_{c}\right)=D_{i}\left(\mu_{c}\right) \quad i=1, \ldots, 6 \quad C_{7}\left(\mu_{c}\right)=C_{+}\left(\mu_{c}\right) D_{7}\left(\mu_{c}\right)+C_{-}\left(\mu_{c}\right) D_{8}\left(\mu_{c}\right)
$$

The final step in the calculation of $\eta_{3}$ consists in removing the charm degrees of freedom from the effective theory. Without charm the effective short-distance hamiltonian corresponding to (XII.39) can be written as

$$
\mathcal{H}_{e f f, c t}^{\Delta S=2}=\frac{G_{F}^{2}}{2} \lambda_{c} \lambda_{t} C_{c t} Q
$$

The matching condition is obtained by equating the matrix elements of (XII.39) and (XII.62), evaluated at a scale $\mu_{c}=\mathcal{O}\left(m_{c}\right)$. At next-to-leading order one needs the finite parts of the matrix elements of $Q_{i j}$, which can be written in the form

$$
\left\langle Q_{i j}\left(\mu_{c}\right)\right\rangle=\frac{m_{c}^{2}\left(\mu_{c}\right)}{8 \pi^{2}} r_{i j}\left(\mu_{c}\right)\langle Q\rangle
$$

where in the renormalization scheme described above after eq. (XII.56) the $r_{i j}$ are given by

$$
\begin{gathered}
r_{i j}\left(\mu_{c}\right)= \begin{cases}\left(4 \ln \left(\mu_{c} / m_{c}\right)-1\right) \tau_{i j} & j=1,2 \\
\left(8 \ln \left(\mu_{c} / m_{c}\right)-4\right) \tau_{i j} & j=3,4 \\
\left(-8 \ln \left(\mu_{c} / m_{c}\right)+4\right) \tau_{i j} & j=5,6\end{cases} \\
\tau_{ \pm 1}=\tau_{ \pm 3}=\tau_{ \pm 5}=(1 \pm 3) / 2 \\
\tau_{+j}=1 \quad \tau_{-j}=0 \quad j \text { even }
\end{gathered}
$$

Using (XII.63), the matching condition at $\mu_{c}$ between (XII.39) and (XII.62) implies

$$
C_{c t}\left(\mu_{c}\right)=\sum_{i= \pm} \sum_{j=1}^{6} C_{i}\left(\mu_{c}\right) C_{j}\left(\mu_{c}\right) \frac{m_{c}^{2}\left(\mu_{c}\right)}{8 \pi^{2}} r_{i j}\left(\mu_{c}\right)+C_{7}\left(\mu_{c}\right) \frac{m_{c}^{2}\left(\mu_{c}\right)}{4 \pi \alpha_{s}\left(\mu_{c}\right)}
$$

Evolving $C_{c t}$ from $\mu_{c}$ to $\mu<\mu_{c}$ in a three-flavor theory using (XII.8) and comparing (XII.62) with (XII.1), we obtain the final result

$$
\eta_{3}=\frac{x_{c}\left(\mu_{c}\right)}{S_{0}\left(x_{c}\left(\mu_{c}\right), x_{t}\left(\mu_{W}\right)\right)} \alpha_{s}\left(\mu_{c}\right)^{2 / 9}\left[\frac{\pi}{\alpha_{s}\left(\mu_{c}\right)} C_{7}\left(\mu_{c}\right)\left(1-\frac{\alpha_{s}\left(\mu_{c}\right)}{4 \pi} J_{3}\right)+\right.
$$




$$
\left.+\frac{1}{2} \sum_{i= \pm} \sum_{j=1}^{6} C_{i}\left(\mu_{c}\right) C_{j}\left(\mu_{c}\right) r_{i j}\left(\mu_{c}\right)\right]
$$

One may convince oneself, that $\eta_{3} S_{0}\left(x_{c}, x_{t}\right)$ is independent of the renormalization scales, in particular of $\mu_{c}$, up to terms of $\mathcal{O}\left(x_{c} \alpha_{s}^{2 / 9} \alpha_{s}\right)$.

Furthermore, using the formulae given in this section, it is easy to see from the explicit expression (XII.68), that $\eta_{3} \alpha_{s}^{-2 / 9} \rightarrow 1$ in the limit $\alpha_{s} \rightarrow 0$, as it should indeed be the case.

The next-to-leading order formula (XII.68) for $\eta_{3}$, first calculated in (Herrlich and Nierste, 1995a), (Nierste, 1995), provides the generalization of the leading log result obtained by (Gilman and Wise, 1983). It is instructive to compare (XII.68) with the leading order approximation, which can be written as

$$
\eta_{3}^{L O}=\alpha_{s}\left(\mu_{c}\right)^{2 / 9} \frac{-\pi C_{7}^{L O}\left(\mu_{c}\right)}{\alpha_{s}\left(\mu_{c}\right) \ln x_{c}}
$$

using the notation of (XII.68). $C_{7}^{L O}$ denotes the coefficient $C_{7}$, restricted to the leading logarithmic approximation. Formula (XII.69), derived here as a special case of (XII.68), is equivalent to the result obtained in (Gilman and Wise, 1983).

If penguin operators and the b-quark threshold in the RG evolution are neglected, it is possible to write down in closed form a relatively simple, explicit expression for $\eta_{3}$. Using a 4-flavor effective theory for the evolution from the $W$-scale down to the charm scale, we find in this approximation

$$
\begin{aligned}
\eta_{3}= & \frac{x_{c}\left(\mu_{c}\right)}{S_{0}\left(x_{c}\left(\mu_{c}\right), x_{t}\right)} \alpha_{s}\left(\mu_{c}\right)^{2 / 9} . \\
& \cdot\left[\frac{\pi}{\alpha_{s}\left(\mu_{c}\right)}\left(-\frac{18}{7} K_{++}-\frac{12}{11} K_{+-}+\frac{6}{29} K_{--}+\frac{7716}{2233} K_{7}\right)\left(1-\frac{\alpha_{s}\left(\mu_{c}\right)}{4 \pi} \frac{307}{162}\right)+\right. \\
& +\left(\ln \frac{\mu_{c}}{m_{c}}-\frac{1}{4}\right)\left(3 K_{++}-2 K_{+-}+K_{--}\right)+ \\
& +\frac{262497}{35000} K_{++}-\frac{123}{625} K_{+-}+\frac{1108657}{1305000} K_{--}-\frac{277133}{50750} K_{7}+ \\
& +K\left(-\frac{21093}{8750} K_{++}+\frac{13331}{13750} K_{+-}-\frac{10181}{18125} K_{--}-\frac{1731104}{2512125} K_{7}\right)+ \\
& \left.+\left(\ln x_{t}-\frac{3 x_{t}}{4\left(1-x_{t}\right)}-\frac{3 x_{t}^{2} \ln x_{t}}{4\left(1-x_{t}\right)^{2}}+\frac{1}{2}\right) K K_{7}\right]
\end{aligned}
$$

where

$$
\begin{gathered}
K_{++}=K^{12 / 25} \quad K_{+-}=K^{-6 / 25} \quad K_{--}=K^{-24 / 25} \\
K_{7}=K^{1 / 5} \quad K=\frac{\alpha_{s}\left(M_{W}\right)}{\alpha_{s}\left(\mu_{c}\right)}
\end{gathered}
$$

Here we have set $\mu_{W}=M_{W}$. (XII.70) represents the next-to-leading order generalization of an approximate formula for the leading $\log \eta_{3}$, also omitting gluon penguins, that has been first given in (Gilman and Wise, 1983). The analytical expression for $\eta_{3}$ in (XII.70) provides an excellent approximation, deviating generally by less than $1 \%$ from the full result. 


\section{E. Numerical Results}

\section{General Remarks}

After presenting the theoretical aspects of the short-distance QCD factors $\eta_{1}, \eta_{2}$ and $\eta_{3}$ in the previous sections, we shall now turn to a discussion of their numerical values. However, before considering explicit numbers, we would like to make a few general remarks.

First of all, it is important to recall that in the matrix element $\left\langle\bar{K}^{0}\left|\mathcal{H}_{\text {eff }}^{\Delta S=2}\right| K^{0}\right\rangle$ (see (XII.1)), only the complete products

$$
S_{0 i} \cdot \eta_{i}\left[\alpha_{\mathrm{s}}(\mu)\right]^{-2 / 9}\left[1+\frac{\alpha_{\mathrm{s}}(\mu)}{4 \pi} J_{3}\right]\left\langle\bar{K}^{0}|Q(\mu)| K^{0}\right\rangle \equiv C_{i}(\mu)\left\langle\bar{K}^{0}|Q(\mu)| K^{0}\right\rangle
$$

are physically relevant. Here $S_{0 i}$ denote the appropriate quark mass dependent functions $S_{0}$ for the three contributions $(i=1,2,3)$ in (XII.1). None of the factors in (XII.73) is physically meaningful by itself. In particular, there is some arbitrariness in splitting the product (XII.73) into the shortdistance part and the matrix element of $Q$ (XII.2) containing long distance contributions. This arbitrariness has of course no impact on the physical result. However, it is essential to employ a definition for the operator matrix element that is consistent with the short-distance QCD factor used.

Conventionally, the matrix element $\left\langle\bar{K}^{0}|Q| K^{0}\right\rangle$ is expressed in terms of the so-called bag parameter $B_{K}(\mu)$ defined through

$$
\left\langle\bar{K}^{0}|Q(\mu)| K^{0}\right\rangle \equiv \frac{8}{3} F_{K}^{2} m_{K}^{2} B_{K}(\mu)
$$

where $m_{K}$ is the kaon mass and $F_{K}=160 M e V$ is the kaon decay constant. In principle, one could just use the scale- and scheme dependent bag factor $B_{K}(\mu)$ along with the coefficient functions $C_{i}(\mu)$ as defined by (XII.73), evaluated at the same scale and in the same renormalization scheme. However, it has become customary to define the short-distance QCD correction factors $\eta_{i}$ by splitting off from the Wilson coefficient $C_{i}(\mu)$ the factor $\left[\alpha_{\mathrm{s}}(\mu)\right]^{-2 / 9}\left[1+\alpha_{\mathrm{s}}(\mu) /(4 \pi) J_{3}\right]$, which carries the dependence on the renormalization scheme and the scale $\mu$. This factor is then attributed to the matrix element of $Q$, formally cancelling its scale and scheme dependence. Accordingly one defines a renormalization scale and scheme invariant bag parameter $B_{K}$ (compare (XII.73), (XII.74))

$$
B_{K} \equiv\left[\alpha_{\mathrm{s}}(\mu)\right]^{-2 / 9}\left[1+\frac{\alpha_{\mathrm{s}}(\mu)}{4 \pi} J_{3}\right] B_{K}(\mu)
$$

If the $\eta_{i}$ as described in this report are employed to describe the short-distance QCD corrections, eq. (XII.75) is the consistent definition to be used for the kaon bag parameter.

Eventually the quantity $B_{K}(\mu)$ should be calculated within lattice QCD. At present, the analysis of (Sharpe, 1994), for example, gives a central value of $B_{K}(2 G e V)_{N D R}=0.616$, with some still sizable uncertainty. For a recent review see also (Soni, 1995). This result already incorporates the lattice-continuum theory matching and refers to the usual NDR scheme. It is clear that the NLO calculation of short-distance QCD effects is essential for consistency with this matching and for a proper treatment of the scheme dependence. Both require $\mathcal{O}\left(\alpha_{\mathrm{s}}\right)$ corrections, which go beyond 
the leading log approximation.

To convert to the scheme invariant parameter $B_{K}$ one uses (XII.75) with the NDR-scheme value for $J_{3}=307 / 162$ to obtain $B_{K}=0.84$. Note that the factor involving $J_{3}$ in (XII.75), which appears at $\mathrm{NLO}$, increases the r.h.s. of (XII.75) by $\approx 4.5 \%$. The leading factor $\alpha_{\mathrm{s}}^{-2 / 9}$ is about 1.31 . Of course, the fact that there is presently still a rather large uncertainty in the calculation of the hadronic matrix element is somewhat forgiving, regarding the precise definition of $B_{K}$. However, as the lattice calculations improve further and the errors decrease, the issue of a consistent definition of the $\eta_{i}$ and $B_{K}$ will become crucial and it is important to keep relation (XII.75) in mind.

Let us next add a side remark concerning the separation of the full amplitude into the formally RG invariant factors $\eta_{i}$ and $B_{K}$. This separation is essentially unique, up to trivial constant factors, if the evolution from the charm scale $\mu_{c}$ down to a "hadronic" scale $\mu<\mu_{c}$ is written in the resummed form as shown in (XII.8) and one requires that all factors depending on the scale $\mu$ are absorbed into the matrix element. On the other hand the hadronic scale $\mu=\mathcal{O}(1 G e V)$ is not really much different from the charm scale $\mu_{c}=\mathcal{O}\left(m_{c}\right)$, so that the logarithms $\ln \mu / \mu_{c}$ are not very large. Therefore one could argue that it is not necessary to resum those logarithms. In this case the first two factors on the r.h.s. of (XII.8) could be expanded to first order in $\alpha_{\mathrm{s}}$ and the amplitude (XII.73) would read

$$
C_{i}\left(\mu_{c}\right)\left(1+\frac{\alpha_{\mathrm{s}}}{\pi} \ln \frac{\mu}{\mu_{c}}\right)\left\langle\bar{K}^{0}|Q(\mu)| K^{0}\right\rangle
$$

From this expression it is obvious, that the separation of the physical amplitude into scheme invariant short-distance factors and a scheme invariant matrix element is in general not unique. This illustrates once more the ambiguity existing for theoretical concepts such as operator matrix elements or QCD correction factors, which only cancels in physical quantities.

For definiteness, we will stick to the RG improved form also for the evolution between $\mu_{c}$ and $\mu$ and the definitions for $\eta_{i}$ and $B_{K}$ that we have discussed in detail above.

\section{Results for $\eta_{1}, \eta_{2}$ and $\eta_{3}$}

We are now ready to quote numerical results for the short-distance QCD corrections $\eta_{i}$ at nextto-leading order and to compare them with the leading order approximation.

The factors $\eta_{1}$ and $\eta_{3}$ have been analyzed in detail in (Herrlich and Nierste, 1994) and (Nierste, 1995). Here we summarize briefly their main results. Using our central parameter values $m_{c}\left(m_{c}\right)=1.3 \mathrm{GeV}, \Lambda \frac{(4)}{\mathrm{MS}}=0.325 \mathrm{GeV}, m_{t}\left(m_{t}\right)=170 \mathrm{GeV}$ and fixing the scales as $\mu_{c}=m_{c}$, $\mu_{W}=M_{W}$ for $\eta_{1}, \mu_{W}=130 \mathrm{GeV}$ for $\eta_{3}$, one obtains at NLO

$$
\eta_{1}=1.38 \quad \eta_{3}=0.47
$$

This is to be compared with the $\mathrm{LO}$ values corresponding to the same input $\eta_{1}^{L O}=1.12, \eta_{3}^{L O}=$ 0.35 . We note that the next-to-leading order corrections are sizable, typically $20 \%-30 \%$, but still perturbative. The numbers above may be compared with the leading log values $\eta_{1}^{L O}=0.85$ and $\eta_{3}^{L O}=0.36$ that have been previously used in the literature, based on the choice $m_{c}=1.4 \mathrm{GeV}$, $\Lambda_{Q C D}=0.2 \mathrm{GeV}$ and $\mu_{W}=M_{W}$. The considerable difference between the two LO values for $\eta_{1}$ mainly reflects the large dependence of $\eta_{1}$ on $\Lambda_{Q C D}$.

In fact, when the QCD scale is allowed to vary within $\Lambda \frac{(4)}{\mathrm{MS}}=(0.325 \pm 0.110) \mathrm{GeV}$, the value for 
$\eta_{1}$ (NLO) changes by $\sim \pm 35 \%$. The leading order result $\eta_{1}^{L O}$ appears to be slightly less sensitive to $\Lambda_{Q C D}$. However, in this approximation the relation of $\Lambda_{Q C D}$ to $\Lambda_{\overline{M S}}^{(4)}$ is not well defined, which introduces an additional source of uncertainty when working to leading logarithmic accuracy.

The situation is much more favorable in the case of $\eta_{3}$, where the sensitivity to $\Lambda_{\frac{(4)}{\mathrm{MS}}}$ is quite small, $\sim \pm 3 \%$. Likewise the dependence on the charm quark mass is very small for both $\eta_{1}$ and $\eta_{3}$. Using $m_{c}\left(m_{c}\right)=(1.3 \pm 0.05) G e V$ and the central value for $\Lambda_{\overline{M S}}^{(4)}$ it is about $\pm 4 \%$ for $\eta_{1}$ and entirely negligible for $\eta_{3}$.

Finally, there are the purely theoretical uncertainties due to the renormalization scales. They are dominated by the ambiguity related to $\mu_{c}$. The products $S_{0}\left(x_{c}\left(\mu_{c}\right)\right) \cdot \eta_{1}\left(\mu_{c}\right)$ and $S_{0}\left(x_{c}\left(\mu_{c}\right), x_{t}\right)$. $\eta_{3}\left(\mu_{c}\right)$ are independent of $\mu_{c}$ up to terms of the neglected order in RG improved perturbation theory. In the case of $S_{0}\left(x_{c}\left(\mu_{c}\right)\right) \cdot \eta_{1}\left(\mu_{c}\right)\left(S_{0}\left(x_{c}\left(\mu_{c}\right), x_{t}\right) \cdot \eta_{3}\left(\mu_{c}\right)\right)$ the remaining sensitivity to $\mu_{c}$ amounts to typically $\pm 15 \%( \pm 7 \%)$ at NLO. These scale dependences are somewhat reduced compared to the leading order calculation, where the corresponding uncertainty is around $\pm 30 \%$ $( \pm 10 \%)$.

To summarize, sizable uncertainties are still associated with the number for the QCD factor $\eta_{1}$, whose central value is found to be $\eta_{1}=1.38$ (Herrlich and Nierste, 1994). On the other hand, the prediction for $\eta_{3}$ appears to be quite stable and can be reliably determined as $\eta_{3}=0.47 \pm$ 0.03 (Herrlich and Nierste, 1995a), (Nierste, 1995). One should emphasize however, that these conclusions have their firm basis only within the framework of a complete NLO analysis, as the one performed in (Herrlich and Nierste, 1994), (Nierste, 1995). Fortunately the quantity $\eta_{1}$, for which a high precision seems difficult to achieve, plays a less important role in the phenomenology of indirect $\mathrm{CP}$ violation.

Finally, we turn to a brief discussion of $\eta_{2}$ (Buras et al., 1990), representing the short-distance QCD effects of the top-quark contribution. For central parameter values, in particular $\Lambda \frac{(4)}{\mathrm{MS}}=$ $0.325 \mathrm{GeV}$ and $m_{t}\left(m_{t}\right)=170 \mathrm{GeV}$, and for $\mu_{t}=m_{t}\left(m_{t}\right)$ the numerical value is

$$
\eta_{2}=0.574
$$

Varying the QCD scale within $\Lambda_{\overline{\mathrm{MS}}}^{(4)}=(0.325 \pm 0.110) \mathrm{GeV}$ results in a $\pm 0.5 \%$ change in $\eta_{2}$. The dependence on $m_{t}\left(m_{t}\right)$ is even smaller, only $\pm 0.3 \%$ for $m_{t}\left(m_{t}\right)=(170 \pm 15) \mathrm{GeV}$. It is worthwhile to compare the NLO results with the leading log approximation. Using the same input as before yields a central value of $\eta_{2}^{L O}=0.612$, about $7 \%$ larger as the NLO result (XII.78). However, what is even more important than the difference in central values is the quite striking reduction of scale uncertainty when going from the leading log approximation to the full NLO treatment. Recall that the $\mu_{t}$-dependence in $\eta_{2}$ has to cancel the scale dependence of the function $S_{0}\left(x_{t}\left(\mu_{t}\right)\right)$. Allowing for a typical variation of the renormalization scale $\mu_{t}=\mathcal{O}\left(m_{t}\right)$ from $100 \mathrm{GeV}$ to $300 \mathrm{GeV}$ results in a sizable change in $S_{0}\left(x_{t}\left(\mu_{t}\right)\right) \eta_{2}^{L O}$ of $\pm 9 \%$. In fact, in leading order the $\mu_{t}$-dependence of $\eta_{2}$ has even the wrong sign, re-inforcing the scale dependence present in $S_{0}\left(x_{t}\left(\mu_{t}\right)\right)$ instead of reducing it. The large sensitivity to the unphysical parameter $\mu_{t}$ is essentially eliminated (to $\pm 0.4 \%$ ) for $\eta_{2} S_{0}\left(x_{t}\right)$ at NLO, a quite remarkable improvement of the theoretical accuracy. The situation here is similar to the case of the top-quark dominated rare $\mathrm{K}$ and $\mathrm{B}$ decays discussed in sections XI, XXIV and XXVI. For a further illustration of the reduction in scale uncertainty see the discussion of the analogous case of $\eta_{2 B}$ in section XIII B.

The dependence of $\eta_{2}$ on the charm and bottom threshold scales $\mu_{c}=\mathcal{O}\left(m_{c}\right)$ and $\mu_{b}=\mathcal{O}\left(m_{b}\right)$ is also extremely weak. Taking $1 \mathrm{GeV} \leq \mu_{c} \leq 3 \mathrm{GeV}$ and $3 \mathrm{GeV} \leq \mu_{b} \leq 9 \mathrm{GeV}$ results in a variation of $\eta_{2}$ by merely $\pm 0.26 \%$ and $\pm 0.06 \%$, respectively. 
In summary, the NLO result for $\eta_{2} S_{0}\left(x_{t}\right)$ is, by contrast to the leading logarithmic approximation, essentially free from theoretical uncertainties. Furthermore, $\eta_{2}$ is also rather insensitive to the input parameters $\Lambda_{\overline{\mathrm{MS}}}$ and $m_{t}$. The top contribution plays the dominant role for indirect CP violation in the neutral kaon system. The considerable improvement in the theoretical analysis of the short-distance QCD factor $\eta_{2}$ brought about by the next-to-leading order calculation is therefore particularly satisfying. 


\section{THE EFFECTIVE HAMILTONIAN FOR $B^{0}-\bar{B}^{0}$ MIXING}

\section{A. General Structure}

Due to the particular hierarchy of the CKM matrix elements only the top sector can contribute significantly to $B^{0}-\bar{B}^{0}$ mixing. The charm sector and the mixed top-charm contributions are entirely negligible here, in contrast to the $K^{0}-\bar{K}^{0}$ case, which considerably simplifies the analysis.

Refering to our earlier presentation of the top sector for $\Delta S=2$ transitions in section XII B we can immediately write down the effective $\Delta B=2$ hamiltonian. Performing the RG evolution only down to scales $\mu_{b}=\mathcal{O}\left(m_{b}\right)$ and making the necessary replacements $(s \rightarrow b)$ we get, in analogy to (XII.1) (Buras et al., 1990)

$$
\mathcal{H}_{e f f}^{\Delta B=2}=\frac{G_{F}^{2}}{16 \pi^{2}} M_{W}^{2}\left(V_{t b}^{*} V_{t d}\right)^{2} \eta_{2 B} S_{0}\left(x_{t}\right)\left[\alpha_{\mathrm{s}}\left(\mu_{b}\right)\right]^{-6 / 23}\left[1+\frac{\alpha_{\mathrm{s}}\left(\mu_{b}\right)}{4 \pi} J_{5}\right] Q+\text { h.c. }
$$

where here

$$
Q=(\bar{b} d)_{V-A}(\bar{b} d)_{V-A}
$$

and

$$
\begin{aligned}
& \eta_{2 B}=\left[\alpha_{\mathrm{s}}\left(\mu_{t}\right)\right]^{6 / 23} \times \\
& \times\left[1+\frac{\alpha_{\mathrm{s}}\left(\mu_{t}\right)}{4 \pi}\left(\frac{S_{1}\left(x_{t}\right)}{S_{0}\left(x_{t}\right)}+B_{t}-J_{5}+\frac{\gamma^{(0)}}{2} \ln \frac{\mu_{t}^{2}}{M_{W}^{2}}+\gamma_{m 0} \frac{\partial \ln S_{0}\left(x_{t}\right)}{\partial \ln x_{t}} \ln \frac{\mu_{t}^{2}}{M_{W}^{2}}\right)\right]
\end{aligned}
$$

The definitions of the various quantities in (XIII.3) can be found in section XII B. Several important aspects of $\eta_{2}$ in the kaon system have also been discussed in this section. Similar comments apply to the present case of $\eta_{2 B}$. Here we would still like to supplement this discussion by writing down the formula for $\eta_{2 B}$ in the limiting case $m_{\mathrm{t}} \gg M_{\mathrm{W}}$,

$$
\begin{aligned}
& \eta_{2 B}=\left[\alpha_{s}\left(\mu_{t}\right)\right]^{6 / 23} \times \\
& \times\left[1+\frac{\alpha_{s}\left(\mu_{t}\right)}{4 \pi}\left(\frac{\gamma^{(0)}}{2} \ln \frac{\mu_{t}^{2}}{m_{t}^{2}}+\gamma_{m 0} \ln \frac{\mu_{t}^{2}}{m_{t}^{2}}+11-\frac{20}{9} \pi^{2}+B_{t}-J_{5}+\mathcal{O}\left(\frac{M_{W}^{2}}{m_{t}^{2}}\right)\right)\right]
\end{aligned}
$$

This expression clarifies the structure of the RG evolution in the limit $m_{\mathrm{t}} \gg M_{\mathrm{W}}$. It also suggests that the renormalization scale is most naturally to be taken as $\mu_{t}=\mathcal{O}\left(m_{\mathrm{t}}\right)$ rather than $\mu_{t}=$ $\mathcal{O}\left(M_{\mathrm{W}}\right)$, both in the definition of the top quark mass and as the initial scale of the RG evolution. Formula (XIII.4) also holds, with obvious modifications, for the $\eta_{2}$ factor in the kaon system, which has been discussed in sec. XII B.

We finally mention that in the literature the $\mu_{b}$-dependent factors in (XIII.1) are sometimes not attributed to the matrix elements of $Q$, as implied by (XIII.1), but absorbed into the definition of the QCD correction factor

$$
\bar{\eta}_{2 B}=\eta_{2 B}\left[\alpha_{\mathrm{s}}\left(\mu_{b}\right)\right]^{-6 / 23}\left[1+\frac{\alpha_{\mathrm{s}}\left(\mu_{b}\right)}{4 \pi} J_{5}\right]
$$

Whichever definition is employed, it is important to remember this difference and to evaluate the hadronic matrix element consistently. Note that, in contrast to $\eta_{2 B}, \bar{\eta}_{2 B}$ is scale and scheme dependent. 


\section{B. Numerical Results}

The correction factor $\eta_{2 B}$ describes the short-distance QCD effects in the theoretical expression for $B^{0}-\bar{B}^{0}$ mixing. Due to the arbitrariness that exists in dividing the physical amplitude into short-distance contribution and hadronic matrix element, the short-distance QCD factor is strictly speaking an unphysical quantity and hence definition dependent. The $B$-factor, parametrizing the hadronic matrix element, has to match the convention used for $\eta_{2 B}$. With the definition of $\eta_{2 B}$ employed in this article and given explicitly in the previous section, the appropriate $B$-factor to be used is the so-called scheme independent bag-parameter $B_{B}$ as defined in eq. (XVIII.18), where $\mu=\mu_{b}=\mathcal{O}\left(m_{b}\right)$. We remark, that the factor $\eta_{2 B}$ is identical for $B_{d}-\bar{B}_{d}$ and $B_{s}-\bar{B}_{s}$ mixing. The effects of $S U(3)$ breaking enter only the hadronic matrix elements. This feature is a consequence of the factorization of short-distance and long-distance contributions inherent to the operator product expansion. For further comments see also the discussion of the analogous case of short-distance QCD factors in the neutral kaon system in section XII E 1.

In the following we summarize the main results of a numerical analysis of $\eta_{2 B}$. The factor $\eta_{2 B}$ is analogous to $\eta_{2}$ entering the top contribution to $K^{0}-\bar{K}^{0}$ mixing and both quantities share many important features.

The value of $\eta_{2 B}$ for $\Lambda_{\overline{\mathrm{MS}}}^{(4)}=0.325 \mathrm{GeV}, m_{\mathrm{t}}\left(m_{\mathrm{t}}\right)=170 \mathrm{GeV}$ and with $\mu_{t}$ set equal to $m_{\mathrm{t}}\left(m_{\mathrm{t}}\right)$ reads at NLO

$$
\eta_{2 B}=0.551
$$

This can be compared with $\eta_{2 B}^{L O}=0.580$, obtained, using the same input, in the leading logarithmic approximation. In the latter case the product $\eta_{2 B}^{L O}\left(\mu_{t}\right) \cdot S\left(x_{t}\left(\mu_{t}\right)\right)$ is, however, affected by a residual scale ambiguity of $\pm 9 \%$ (for $100 \mathrm{GeV} \leq \mu_{t} \leq 300 \mathrm{GeV}$ ). This uncertainty is reduced to the negligible amount of $\pm 0.3 \%$ in the complete NLO expression of $\eta_{2 B}\left(\mu_{t}\right) \cdot S\left(x_{t}\left(\mu_{t}\right)\right)$, corresponding to an increase in accuracy by a factor of 25 . The sensitivity to the unphysical scale $\mu_{t}$ in leading and next-to-leading order is illustrated in fig. 9.

In addition the number shown in (XIII.6) is also very stable against changes in the input parameters. Taking $\Lambda \frac{(4)}{\mathrm{MS}}=(0.325 \pm 0.110) \mathrm{GeV}$ and $m_{\mathrm{t}}\left(m_{\mathrm{t}}\right)=(170 \pm 15) \mathrm{GeV}$ results in a variation of $\eta_{2 B}$ by $\pm 1.3 \%$ and $\pm 0.3 \%$, respectively.

It is clear from this discussion, that the short-distance QCD effects in $B^{0}-\bar{B}^{0}$ mixing are very well under control, once NLO corrections have been properly included, and the remaining uncertainties are extremely small. The effective hamiltonian given in (XIII.1) therefore provides a solid foundation for the incorporation of non-perturbative effects, to be determined from lattice gauge theory, and for further phenomenological investigations related to $B^{0}-\bar{B}^{0}$ mixing phenomena. 


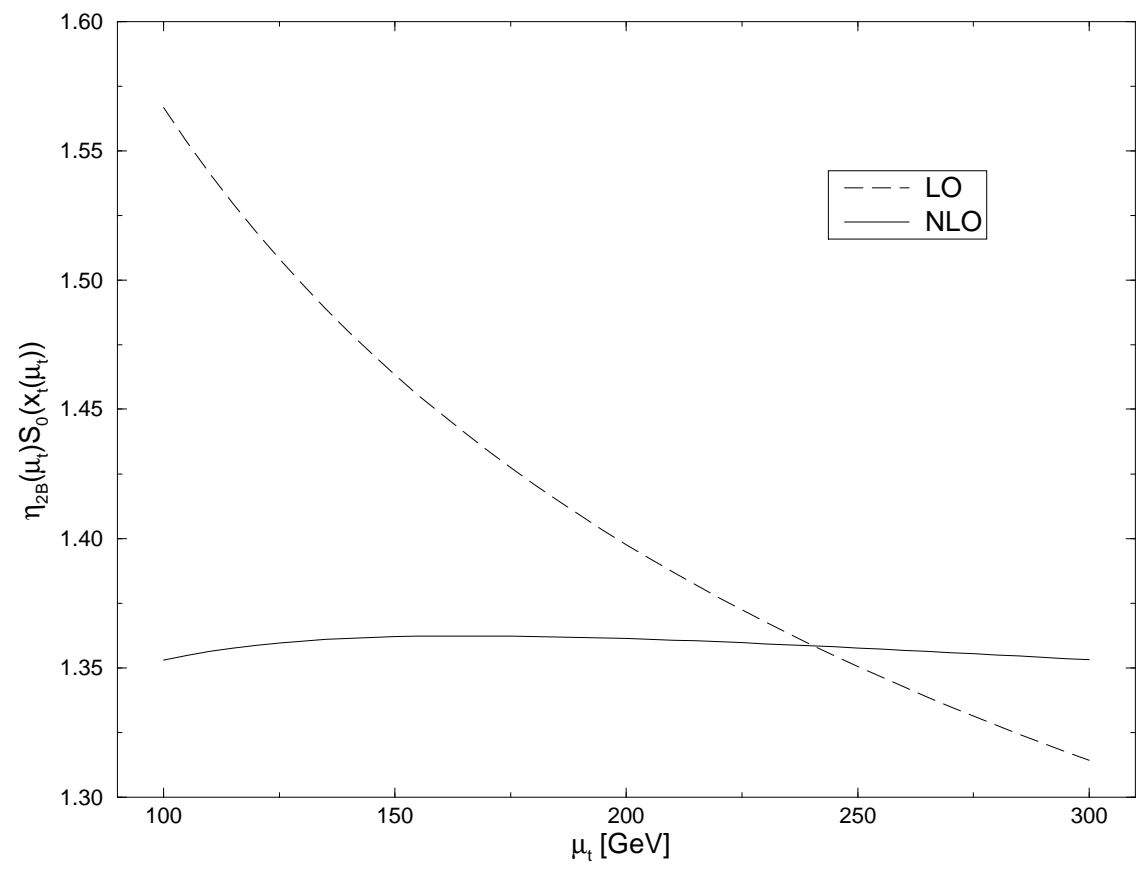

FIG. 9. Scale $\mu_{t}$ dependence of $\eta_{2 B}\left(\mu_{t}\right) S_{0}\left(x_{t}\left(\mu_{t}\right)\right)$ in LO and NLO. The quantity $\eta_{2 B}\left(\mu_{t}\right) S_{0}\left(x_{t}\left(\mu_{t}\right)\right)$ enters the theoretical expression for $\Delta m_{B}$, describing $B^{0}-\bar{B}^{0}$ mixing. It is independent of the precise value of the renormalization scale $\mu_{t}$ up to terms of the neglected order in $\alpha_{\mathrm{s}}$. The remaining sensitivity represents an unavoidable theoretical uncertainty. This ambiguity is shown here for the leading order (dashed) and the next-to-leading order calculation (solid). 


\section{PENGUIN BOX EXPANSION FOR FCNC PROCESSES}

An important virtue of OPE and RG is that with $m_{t}>M_{\mathrm{W}}$ the dependence of weak decays on the top quark mass is very elegantly isolated. It resides only in the initial conditions for the Wilson coefficients at scale $\mu \approx M_{\mathrm{W}}$ i.e. in $C_{i}\left(M_{\mathrm{W}}\right)$. A quick look at the initial conditions in the previous sections reveals the important fact that the leading $m_{t}$-dependence in all decays considered is represented universally by the $m_{t}$-dependent functions which result from exact calculations of the relevant penguin and box diagrams with internal top quark exchanges. These are the functions

$$
S_{0}\left(x_{t}\right), \quad B_{0}\left(x_{t}\right), \quad C_{0}\left(x_{t}\right), \quad D_{0}\left(x_{t}\right), \quad E_{0}\left(x_{t}\right), \quad D_{0}^{\prime}\left(x_{t}\right), \quad E_{0}^{\prime}\left(x_{t}\right)
$$

for which explicit expressions are given in (XII.4), eqs. (VII.13)-(VII.15), (VI.15), (IX.12) and (IX.13), respectively. In certain decays some of these functions do not appear because the corresponding penguin or box diagram does not contribute to the initial conditions. However, the function $C_{0}\left(x_{t}\right)$ resulting from the $Z^{0}$-penguin diagram enters all $\Delta F=1$ decays but $B \rightarrow X_{s} \gamma$. Having a quadratic dependence on $m_{t}$, this function is responsible for the dominant $m_{t}$-dependence of these decays. Since the non-leading $m_{t}$-dependence of $C_{0}\left(x_{t}\right)$ is gauge dependent, $C_{0}\left(x_{t}\right)$ is always accompanied by $B_{0}\left(x_{t}\right)$ or $D_{0}\left(x_{t}\right)$ in such a way that this dependence cancels. For this reason it is useful to replace the gauge dependent functions $B_{0}\left(x_{t}\right), C_{0}\left(x_{t}\right)$ and $D_{0}\left(x_{t}\right)$ by the gauge independent set (Buchalla et al., 1991)

$$
\begin{aligned}
X_{0}\left(x_{t}\right) & =C_{0}\left(x_{t}\right)-4 B_{0}\left(x_{t}\right) \\
Y_{0}\left(x_{t}\right) & =C_{0}\left(x_{t}\right)-B_{0}\left(x_{t}\right) \\
Z_{0}\left(x_{t}\right) & =C_{0}\left(x_{t}\right)+\frac{1}{4} D_{0}\left(x_{t}\right)
\end{aligned}
$$

as we have already done at various places in this review. The inclusion of NLO QCD corrections to $B^{0}-\bar{B}^{0}-, K^{0}-\bar{K}^{0}$-mixing and the rare $K$ - and $B$-decays of section XI requires the calculation of QCD corrections to penguin and box diagrams in the full theory. This results in the functions $\tilde{S}\left(x_{t}\right)=\eta_{2} S_{0}\left(x_{t}\right), X\left(x_{t}\right)$ and $Y\left(x_{t}\right)$, with the latter two given in (XI.5) and (XI.45), respectively. It turns out however that if the top quark mass is definded as $m_{t} \equiv \bar{m}_{t}\left(m_{t}\right)$ one has

$$
\tilde{S}\left(x_{t}\right)=\eta_{2} S_{0}\left(x_{t}\right), \quad X\left(x_{t}\right)=\eta_{X} X_{0}\left(x_{t}\right), \quad Y\left(x_{t}\right)=\eta_{Y} Y_{0}\left(x_{t}\right)
$$

with $\eta_{2}, \eta_{X}$ and $\eta_{Y}$ almost independent of $m_{t}$. Numerical values of $\eta_{X}$ and $\eta_{Y}$ are given in part three.

Consequently with this definition of $m_{t}$ the basic $m_{t}$-dependent functions listed in (XIV.1) and (XIV.2) represent the $m_{t}$-dependence of weak decays at the NLO level to a good approximation. It should be remarked that the QCD corrections to $D_{0}, E_{0}, D_{0}^{\prime}$ and $E_{0}^{\prime}$ have not been calculated yet. They would however be only required for still higher order corrections (NNLO) in the renormalization group improved perturbation theory as far as $D_{0}$ and $E_{0}$ are concerned. On the other hand, in the case of $D_{0}^{\prime}$ and $E_{0}^{\prime}$, which are relevant for the $b \rightarrow s \gamma$ decay, these corrections are necessary.

An inspection of the effective hamiltonians derived in the previous sections shows that for $B^{0}$ $\bar{B}^{0}$-mixing, $K^{0}-\bar{K}^{0}$-mixing and the rare decays of section XI the $m_{t}$ dependence of the effective hamiltonian is explicitly given in terms of the basic functions listed above. Due to the one step evolution from $\mu_{t}$ to $\mu_{b}$ we have also presented the explicit $m_{\mathrm{t}}$-dependence for $B \rightarrow X_{s} \gamma$ and $B \rightarrow X_{s} e^{+} e^{-}$decays. On the other hand in the case of $K \rightarrow \pi \pi$ and $K_{L} \rightarrow \pi^{0} e^{+} e^{-}$where 
the renormalization group evolution is very complicated the $m_{t}$ dependence of a given box or penguin diagram is distributed among various Wilson coefficient functions. In other words the $m_{t}$-dependence acquired at scale $\mu \approx \mathcal{O}\left(M_{\mathrm{W}}\right)$ is hidden in a complicated numerical evaluation of $U\left(\mu, M_{\mathrm{W}}\right)$.

For phenomenological applications it is more elegant and more convenient to have a formalism in which the final formulae for all amplitudes are given explicitly in terms of the basic $m_{t^{-}}$ dependent functions discussed above.

In (Buchalla et al., 1991) an approach has been presented which accomplishes this task. It gives the decay amplitudes as linear combinations of the basic, universal, process independent but $m_{t}$-dependent functions $F_{r}\left(x_{t}\right)$ of eq. (XIV.1) with corresponding coefficients $P_{r}$ characteristic for the decay under consideration. This approach termed "Penguin Box Expansion" (PBE) has the following general form

$$
\left.A(\text { decay })=P_{0} \text { (decay }\right)+\sum_{r} P_{r}(\text { decay }) F_{r}\left(x_{t}\right)
$$

where the sum runs over all possible functions contributing to a given amplitude. In (XIV.4) we have separated a $m_{t}$-independent term $P_{0}$ which summarizes contributions stemming from internal quarks other than the top, in particular the charm quark.

Many examples of PBE appear in this review. Several decays or transitions depend only on a single function out of the complete set (XIV.1). For completeness we give here the correspondence between various processes and the basic functions

$$
\begin{array}{ll}
B^{0}-\bar{B}^{0} \text {-mixing } & S_{0}\left(x_{t}\right) \\
K \rightarrow \pi \nu \bar{\nu}, B \rightarrow K \nu \bar{\nu}, B \rightarrow \pi \nu \bar{\nu} & X_{0}\left(x_{t}\right) \\
K \rightarrow \mu \bar{\mu}, B \rightarrow l \bar{l} & Y_{0}\left(x_{t}\right) \\
K_{L} \rightarrow \pi^{0} e^{+} e^{-} & Y_{0}\left(x_{t}\right), Z_{0}\left(x_{t}\right), E_{0}\left(x_{t}\right) \\
\varepsilon^{\prime} & X_{0}\left(x_{t}\right), Y_{0}\left(x_{t}\right), Z_{0}\left(x_{t}\right), E_{0}\left(x_{t}\right) \\
B \rightarrow X_{s} \gamma & D_{0}^{\prime}\left(x_{t}\right), E_{0}^{\prime}\left(x_{t}\right) \\
B \rightarrow X_{s} e^{+} e^{-} & Y_{0}\left(x_{t}\right), Z_{0}\left(x_{t}\right), E_{0}\left(x_{t}\right), D_{0}^{\prime}\left(x_{t}\right), E_{0}^{\prime}\left(x_{t}\right)
\end{array}
$$

In (Buchalla et al., 1991) an explicit transformation from OPE to PBE has been made. This transformation and the relation between these two expansions can be very clearly seen on the basis of

$$
A(P \rightarrow F)=\sum_{i, k}\left\langle F\left|O_{k}(\mu)\right| P\right\rangle U_{k j}\left(\mu, M_{\mathrm{W}}\right) C_{j}\left(M_{\mathrm{W}}\right)
$$

where $U_{k j}\left(\mu, M_{\mathrm{W}}\right)$ represents the renormalization group transformation from $M_{\mathrm{W}}$ down to $\mu$. As we have seen, OPE puts the last two factors in this formula together, mixing this way the physics around $M_{\mathrm{W}}$ with all physical contributions down to very low energy scales. The PBE is realized on the other hand by putting the first two factors together and rewriting $C_{j}\left(M_{\mathrm{W}}\right)$ in terms of the basic functions (XIV.1). This results in the expansion of eq. (XIV.4). Further technical details and the methods for the evaluation of the coefficients $P_{r}$ can be found in (Buchalla et al., 1991), where further virtues of PBE are discussed.

Finally, we give approximate formulae having power-like dependence on $x_{t}$ for the basic, gauge independent functions of $\mathrm{PBE}$ 


$$
\begin{array}{ll}
S_{0}\left(x_{t}\right)=0.784 \cdot x_{t}^{0.76} & X_{0}\left(x_{t}\right)=0.660 \cdot x_{t}^{0.575} \\
Y_{0}\left(x_{t}\right)=0.315 \cdot x_{t}^{0.78} & Z_{0}\left(x_{t}\right)=0.175 \cdot x_{t}^{0.93} \\
E_{0}\left(x_{t}\right)=0.564 \cdot x_{t}^{-0.51} & D_{0}^{\prime}\left(x_{t}\right)=0.244 \cdot x_{t}^{0.30} \\
E_{0}^{\prime}\left(x_{t}\right)=0.145 \cdot x_{t}^{0.19} &
\end{array}
$$

In the range $150 \mathrm{GeV} \leq m_{t} \leq 200 \mathrm{GeV}$ these approximations reproduce the exact expressions to an accuracy better than $1 \%$. 


\section{HEAVY QUARK EFFECTIVE THEORY BEYOND LEADING LOGS}

\section{A. General Remarks}

Since its advent in 1989 heavy quark effective theory (HQET) has developped into an elaborate and well-established formalism, providing a systematic framework for the treatment of hadrons containing a heavy quark. HQET represents a static approximation for the heavy quark, covariantly formulated in the language of an effective field theory. It allows to extract the dependence of hadronic matrix elements on the heavy quark mass and to exploit the simplifications that arise in QCD in the static limit.

There are several excellent reviews on this subject (Neubert, 1994c), (Georgi, 1991), (Grinstein, 1991), (Isgur and Wise, 1992), (Mannel, 1993) and we do not attempt here to cover the details of this extended field. However, we would like to emphasize the close parallels in the general formalism employed to calculate perturbative QCD effects for the effective weak hamiltonians we have been discussing in this review and in the context of HQET. In particular we will concentrate on results that have been obtained in HQET beyond the leading logarithmic approximation in QCD perturbation theory. Such calculations have been done mainly for bilinear current operators involving heavy quark fields, which have important applications in semileptonic decays of heavy hadrons. For the purpose of illustration we will focus on the simplest case of heavy-light currents as an important example. Furthermore, while existing reviews concentrate on semileptonic decays and current operators, we will also include results obtained for nonleptonic transitions and summarize the calculation of NLO QCD corrections to $B^{0}-\bar{B}^{0}$ mixing in HQET (Flynn et al., 1991), (Giménez, 1993). These latter papers generalize the leading-log results first obtained in (Voloshin and Shifman, 1987), (Politzer and Wise, 1988a), (Politzer and Wise, 1988b).

Throughout we will restrict ourselves to the leading order in HQET and not address the question of $1 / m$ corrections. For a discussion of this topic we refer the reader to the literature, in particular the above mentioned review articles.

\section{B. Basic Concepts}

Let us briefly recall the most important basic concepts underlying the idea of HQET. The Lagrangian describing a quark field $\Psi$ with mass $m$ and its QCD interactions with gluons reads

$$
\mathcal{L}=\bar{\Psi} i \not D \Psi-m \bar{\Psi} \Psi
$$

where $D_{\mu}=\partial_{\mu}-i g T^{a} A_{\mu}^{a}$ is the gauge-covariant derivative. If $\Psi$ is a heavy quark, i.e. its mass is large compared to the QCD scale, $\Lambda_{Q C D} / m \ll 1$, it acts approximately like a static color source and its QCD interactions simplify. A heavy quark inside a hadron moving with velocity $v$ has approximately the same velocity. Thus its momentum can be written as $p=m v+k$, where $k$ is a small residual momentum of the order of $\Lambda_{Q C D}$ and subject to changes of the same order through soft QCD interactions. To implement this approximation, the quark field $\Psi$ is decomposed into

$$
\Psi(x)=e^{-i m v \cdot x}\left(h_{v}(x)+H_{v}(x)\right)
$$

with $h_{v}$ and $H_{v}$ defined by 


$$
\begin{aligned}
& h_{v}(x)=e^{i m v \cdot x} \frac{1+\not x}{2} \Psi(x) \\
& H_{v}(x)=e^{i m v \cdot x} \frac{1-\not x}{2} \Psi(x)
\end{aligned}
$$

To be specific we consider here the case of a hadron containing a heavy quark, as opposed to a heavy antiquark. In order to describe a heavy antiquark, the definitions (XV.3) and (XV.4) are replaced by

$$
\begin{aligned}
& h_{v}^{(-)}(x)=e^{-i m v \cdot x} \frac{1-\not x}{2} \Psi(x) \\
& H_{v}^{(-)}(x)=e^{-i m v \cdot x} \frac{1+\not x}{2} \Psi(x)
\end{aligned}
$$

Consequently, for a heavy antiquark, one only needs to substitute $v \rightarrow-v$ in the expressions given below for the case of a heavy quark.

In the rest frame of the heavy quark $h_{v}$ and $H_{v}$ correspond to the upper and lower components of $\Psi$, respectively. In general, for $m \rightarrow \infty h_{v}$ and $H_{v}$ represent the "large" and "small" components of $\Psi$. In fact, the equations of motion of QCD imply that $H_{v}$ is suppressed by a factor $\Lambda_{Q C D} / m$ in comparison to $h_{v}$. The inclusion of an explicit exponential factor $\exp (-i m v \cdot x)$ in $(\mathrm{XV} .2)$ ensures that the momentum associated with the field $h_{v}$ is only a small residual momentum of order $\Lambda_{Q C D}$. Now an effective theory for $h_{v}$ is constructed by eliminating the small component field $H_{v}$ from explicitly appearing in the description of the heavy quark. On the classical level this can be done by using the equations of motion or, equivalently, by directly integrating out the $H_{v}$ degrees of freedom in the context of a path integral formulation (Mannel et al., 1992). The effective Lagrangian one obtains from (XV.1) along these lines is given by $\left(D_{\perp}^{\mu}=D^{\mu}-v^{\mu} v \cdot D\right)$

$$
\mathcal{L}_{e f f, t o t}=\bar{h}_{v} i v \cdot D h_{v}+\bar{h}_{v} i \not D_{\perp} \frac{1}{i v \cdot D+2 m-i \varepsilon} i \not D_{\perp} h_{v}
$$

The first term in (XV.7)

$$
\mathcal{L}_{e f f}=\bar{h}_{v}\left(i v^{\mu} \partial_{\mu}+g v^{\mu} T^{a} A_{\mu}^{a}\right) h_{v}
$$

represents the Lagrangian of HQET to lowest order in $1 / m$ and will be sufficient for our purposes. The second, nonlocal contribution in (XV.7) can be expanded into a series of local, higher dimension operators, carrying coefficients with increasing powers of $1 / \mathrm{m}$. To first order it yields the correction due to the residual heavy quark kinetic energy and the chromo-magnetic interaction term, coupling the heavy quark spin to the gluon field.

From (XV.8) one can obtain the Feynman rules of HQET, the propagator of the effective field $h_{v}$

$$
\frac{i}{v \cdot k} \frac{1+\not b}{2}
$$

and the $\bar{h}_{v}-h_{v}$-gluon vertex, $i g v^{\mu} T^{a}$. The explicit factor $(1+\not p) / 2$ in (XV.9) arises because the effective field $h_{v}$ is a constrained spinor, satisfying $\not h_{v} \equiv h_{v}$, as is obvious from (XV.3). The 
velocity $v_{\mu}$ is a constant in the effective theory and plays the role of a label for the effective field $h_{v}$. In principle, a different field $h_{v}$ has to be considered for every velocity $v$.

The Lagrangian in (XV.8) exhibits the crucial features of HQET: The quark-gluon coupling is independent of the quark's spin degrees of freedom and the Lagrangian is independent of the heavy quark flavor, since the heavy quark mass has been eliminated. This observation forms the basis for the spin-flavor symmetry of HQET (Isgur and Wise, 1989), (Isgur and Wise, 1990), which gives rise to important simplifications in the strong interactions of heavy quarks and allows to establish relations among the form factors of different heavy hadron matrix elements. The heavy quark symmetries are broken by $1 / m$-contributions as well as radiative corrections.

So far our discussion has been limited to the QCD interactions of the heavy quark. Weak interactions introduce new operators into the theory, which may be current operators, bilinear in quark fields, or four-quark operators, relevant for semileptonic and nonleptonic transitions, respectively. Such operators form the basic ingredients to be studied in weak decay phenomenology. They can as well be expanded in $1 / m$ and incorporated into the framework of HQET. For example a heavy-light current operator $\bar{q} \Gamma \Psi$ (evaluated at the origin, $x=0$ ), can be written (XV.2)

$$
\bar{q} \Gamma \Psi=\bar{q} \Gamma h_{v}+\mathcal{O}\left(\frac{1}{m}\right)
$$

to lowest order in HQET.

Up to now we have restricted our discussion to the classical level. In addition, of course, quantum radiative corrections have to be included. They will for example modify relations such as (XV.10). Technically their effects are taken into account by performing the appropriate matching calculations, relating operators in the effective theory to the corresponding operators in the full theory to the required order in renormalization group improved QCD perturbation theory. The procedure is very similar to the calculation of the usual effective hamiltonians for weak decays. The basic difference consists in the heavy degrees of freedom that are being integrated out in the matching process. In the general case of effective weak hamiltonians the heavy field to be removed as a dynamical variable is the $\mathrm{W}$ boson, whereas it is the lower component heavy quark field $H_{v}$ in the case of HQET. This similarity will become obvious from our presentation below.

At this point some comment might be in order concerning the relationship of the HQET formalism to the general weak effective hamiltonians discussed primarily in this review, in particular those relevant for b-physics.

The effective hamiltonians for $\Delta B=1,2$ nonleptonic transitions are the relevant hamiltonians for scales $\mu=\mathcal{O}\left(m_{b}\right)$, which are appropriate for B hadron decays, and their Wilson coefficients incorporate the QCD short distance dynamics between scales of $\mathcal{O}\left(M_{W}\right)$ and $\mathcal{O}\left(m_{b}\right)$. As already mentioned at the end of section $\mathrm{V}$ it is therefore not necessary to invoke HQET. The physics below $\mu=\mathcal{O}\left(m_{b}\right)$ is completely contained within the relevant hadronic matrix elements. On the other hand, HQET may be useful in certain cases, like e.g. $B^{0}-\bar{B}^{0}$ mixing, to gain additional insight into the structure of the hadronic matrix elements for scales below $m_{b}$, but still large compared to $\Lambda_{Q C D}$. These scales are still perturbative and the related contributions can be extracted analytically within HQET. In particular, this procedure makes the dependence of the matrix element on the heavy quark mass explicit, as we will see on examples below. Furthermore, this approach can be useful e.g. in connection with lattice calculations of hadronic matrix elements, which are easier to perform in the static limit for b-quarks, i.e. employing HQET (Sachrajda, 1992). The simplifications obtained are however at the expense of the approximation due to the expansion in $1 / m$. 
The most important application of HQET has been to the analysis of exclusive semileptonic transitions involving heavy quarks, where this formalism allows to exploit the consequences of heavy quark symmetry to relate formfactors and provides a basis for systematic corrections to the $m \rightarrow \infty$ limit. This area of weak decay phenomenology has been already reviewed in detail (Neubert, 1994c), (Georgi, 1991), (Grinstein, 1991), (Isgur and Wise, 1992), (Mannel, 1993) and will not be covered in the present article.

\section{Heavy-Light Currents}

As an example of a next-to-leading QCD calculation within the context of HQET, we will now discuss the case of a weak current, composed of one heavy and one light quark field, to leading order in the $1 / m$ expansion. For definitness we consider the axial vector heavy-light current, whose matrix elements determine the decay constants of pseudoscalar mesons containing a single heavy quark, like $f_{B}$ and $f_{D}$.

The axial vector current operator in the full theory is given by

$$
A=\bar{q} \gamma_{\mu} \gamma_{5} \Psi
$$

where $\Psi$ is the heavy and $q$ the light quark field. In HQET this operator can be expanded in the following way

$$
A=C_{1}(\mu) \tilde{A}_{1}+C_{2}(\mu) \tilde{A}_{2}+\mathcal{O}\left(\frac{1}{m}\right)
$$

where the operator basis in the effective theory reads

$$
\tilde{A}_{1}=\bar{q} \gamma_{\mu} \gamma_{5} h_{v} \quad \tilde{A}_{2}=\bar{q} v_{\mu} \gamma_{5} h_{v}
$$

with the heavy quark effective field $h_{v}$ defined in (XV.3). The use of the expansion (XV.12) is to make the dependence of the matrix elements of $A$ on the heavy quark mass $m$ explicit. The dependence on this mass is two-fold. First, there is a power dependence, which is manifest in the heavy quark expansion in powers of $1 / \mathrm{m}$. From this series only the lowest order term is shown in (XV.12). Second, there is a logarithmic dependence on $m$ due to QCD radiative corrections, which can be calculated in perturbation theory. This dependence is factorized into the coefficient functions $C_{1}, C_{2}$ in much the same way as the logarithmic dependence of nonleptonic weak decay amplitudes on the $\mathrm{W}$ boson mass is factorized into the Wilson coefficients of the usual weak hamiltonians. Since the dynamics of HQET is, by construction, independent of $m$, no further $m$ dependence is present in the matrix elements of the effective theory operators $\tilde{A}_{1,2}$, except for trivial factors of $m$ related to the normalization of meson states. Consequently the $m$ dependence of (XV.12) is determined explicitly.

We remark that in general the meson states in HQET to be used for the r.h.s. of (XV.12) differ from the meson states in the full theory to be used to sandwich the operator $A$ on the 1.h.s.. For the leading order in $1 / m$ we are working in this distinction is irrelevant, however.

An important point is that the operators $\tilde{A}_{1,2}$ in the effective theory have anomalous dimensions, although the operator $A$ in the full theory, being an axial vector current operator, does not. As a consequence matrix elements of $\tilde{A}_{1,2}$ will depend on the renormalization scale and scheme. This dependence is canceled however through a corresponding dependence of the coefficients 
so that the physical matrix elements of $A$ will be scale and scheme independent as they must be. The existence of anomalous dimensions for the effective theory operators merely reflects the logarithmic dependence on the heavy quark mass $m$ due to QCD effects. This dependence results in logarithmic divergences in the limit $m \rightarrow \infty$, corresponding to the effective theory, which require additional infinite renormalizations not present in full QCD. Obviously the situation is completely analogous to the case of constructing effective weak hamiltonians through integrating out the $\mathrm{W}$ boson, which we have described in detail in section III. In fact, the extraction of the coefficient functions by factorizing long and short distance contributions to quark level amplitudes and the renormalization group treatment follow exactly the same principles.

The Wilson coefficients at the high matching scale $\mu_{h}=\mathcal{O}(m)$, the initial condition to the RG evolution, can be calculated in ordinary perturbation theory with the result (NDR scheme)

$$
\begin{gathered}
C_{1}\left(\mu_{h}\right)=1+\frac{\alpha_{s}}{4 \pi}\left(\gamma_{h l}^{(0)} \ln \frac{\mu_{h}}{m}+B_{1}\right) \\
C_{2}\left(\mu_{h}\right)=\frac{\alpha_{s}}{4 \pi} B_{2}
\end{gathered}
$$

with

$$
B_{1}=-4 C_{F} \quad B_{2}=-2 C_{F}
$$

and $\gamma_{h l}^{(0)}$ given in (XV.18) below. $C_{F}$ is the QCD color factor $\left(N^{2}-1\right) /(2 N)$. We remark that the coefficient of the new operator $\tilde{A}_{2}$, generated at $\mathcal{O}\left(\alpha_{s}\right)$, is finite without requiring renormalization. As a consequence no explicit scale dependence appears in (XV.15) and $B_{2}$ is a scheme independent constant. For the same reason $\tilde{A}_{1}$ and $\tilde{A}_{2}$ do not mix under renormalization, but renormalize only multiplicatively. The anomalous dimension of the effective heavy quark currents is independent of the Dirac structure. It is the same for $\tilde{A}_{1}$ and $\tilde{A}_{2}$ and reads at two-loop order

$$
\gamma_{h l}=\gamma_{h l}^{(0)} \frac{\alpha_{s}}{4 \pi}+\gamma_{h l}^{(1)}\left(\frac{\alpha_{s}}{4 \pi}\right)^{2}
$$

where

$$
\begin{gathered}
\gamma_{h l}^{(0)}=-3 C_{F} \\
\gamma_{h l}^{(1)}=\left(-\frac{49}{6}+\frac{2}{3} \pi^{2}\right) N C_{F}+\left(\frac{5}{2}-\frac{8}{3} \pi^{2}\right) C_{F}^{2}+\frac{5}{3} C_{F} f= \\
=-\frac{254}{9}-\frac{56}{27} \pi^{2}+\frac{20}{9} f \quad(\mathrm{NDR})
\end{gathered}
$$

$N(f)$ denotes the number of colors (flavors). The anomalous dimension $\gamma_{h l}^{(0)}$ has been first calculated by (Voloshin and Shifman, 1987) and (Politzer and Wise, 1988a), (Politzer and Wise, 1988b). The generalization to next-to-leading order has been performed in (Ji and Musolf, 1991) and (Broadhurst and Grozin, 1991).

The RG equations are readily solved to obtain the coefficients at a lower but still perturbative scale $\mu$, where, formally, $\mu \ll \mu_{h}=\mathcal{O}(m)$. Using the results of section III F we have 


$$
\begin{gathered}
C_{1}(\mu)=\left(1+\frac{\alpha_{s}(\mu)}{4 \pi} J_{h l}\right)\left[\frac{\alpha_{s}\left(\mu_{h}\right)}{\alpha_{s}(\mu)}\right]^{d_{h l}}\left(1+\frac{\alpha_{s}\left(\mu_{h}\right)}{4 \pi}\left[\gamma_{h l}^{(0)} \ln \frac{\mu_{h}}{m}+B_{1}-J_{h l}\right]\right) \\
C_{2}(\mu)=\left[\frac{\alpha_{s}\left(\mu_{h}\right)}{\alpha_{s}(\mu)}\right]^{d_{h l}} \frac{\alpha_{s}\left(\mu_{h}\right)}{4 \pi} B_{2}
\end{gathered}
$$

with

$$
d_{h l}=\frac{\gamma_{h l}^{(0)}}{2 \beta_{0}} \quad J_{h l}=\frac{d_{h l}}{\beta_{0}} \beta_{1}-\frac{\gamma_{h l}^{(1)}}{2 \beta_{0}}
$$

We remark that the corresponding formulae for the vector current can be simply obtained from the above expressions by letting $\gamma_{5} \rightarrow 1$ and changing the sign of $B_{2}$.

In addition to the case of heavy-light currents considered here, the NLO corrections have also been calculated for flavor-conserving and flavor-changing heavy-heavy currents of the type $\bar{\Psi} \Gamma \Psi$ and $\bar{\Psi}_{1} \Gamma \Psi_{2}$ respectively, where $\Psi, \Psi_{1,2}$ are heavy quark fields $\left(\Gamma=\gamma_{\mu}, \gamma_{\mu} \gamma_{5}\right)$. In these cases the anomalous dimensions become velocity dependent. Additional complications arise in the analysis of flavor changing heavy-heavy currents due to the presence of two distinct heavy mass scales. For a detailed presentation see (Neubert, 1994c) and references cited therein.

\section{The Pseudoscalar Decay Constant in the Static Limit}

An important application of the results summarized in the last section is the calculation of the short distance QCD effects, from scales between $\mu_{h}=\mathcal{O}(m)$ and the low scale $\mu=\mathcal{O}(1 G e V)$, for the decay constants $f_{P}$ of pseudoscalar heavy mesons. Using only the leading term in the expansion (XV.12), omitting all $1 / m$ power corrections, corresponds to the static limit for $f_{P}$, which plays some role in lattice studies. As already mentioned we will restrict ourselves here to this limit. We should remark however, that non-negligible power corrections are known to exist for the realistic case of B or D meson decay constants (Sachrajda, 1992).

The decay constant $f_{P}$ is defined through

$$
\langle 0|A| P\rangle=-i f_{P} m_{P} v_{\mu}
$$

where the pseudoscalar meson state $|P\rangle$ is normalized in the conventional way $(\langle P \mid P\rangle=2 E V)$. The matrix elements of $\tilde{A}_{1,2}$ are related via heavy quark symmetry and are given by

$$
\left\langle 0\left|\tilde{A}_{1}\right| P\right\rangle=-\left\langle 0\left|\tilde{A}_{2}\right| P\right\rangle=-i \tilde{f}(\mu) \sqrt{m_{P}} v_{\mu}
$$

Apart from the explicit mass factor $\sqrt{m_{P}}$, which is merely due to the normalization of $|P\rangle$, these matrix elements are independent of the heavy quark mass. The "reduced" decay constant $\tilde{f}(\mu)$ is therefore $m$-independent. It does however depend on the renormalization scale and scheme chosen. The computation of $\tilde{f}(\mu)$ is a nonperturbative problem involving strong dynamics below scale $\mu$. Using (XV.12), (XV.20), (XV.21), (XV.23) and (XV.24) we obtain

$$
f_{P}=\frac{\tilde{f}(\mu)}{\sqrt{m_{P}}}\left(1+\frac{\alpha_{s}(\mu)}{4 \pi} J_{h l}\right)\left[\frac{\alpha_{s}\left(\mu_{h}\right)}{\alpha_{s}(\mu)}\right]^{d_{h l}}\left(1+\frac{\alpha_{s}\left(\mu_{h}\right)}{4 \pi}\left[\gamma_{h l}^{(0)} \ln \frac{\mu_{h}}{m}+B_{1}-J_{h l}-B_{2}\right]\right)
$$


The dependence of the coefficient function on the renormalization scheme through $J_{h l}$ in the second factor in (XV.25), and its dependence on $\mu$ cancel the corresponding dependences in the hadronic quantity $\tilde{f}(\mu)$ to the considered order in $\alpha_{s}$. The last factor in (XV.25) is scheme independent. Furthermore the cancellation of the dependence on $\mu_{h}$ to the required order can be seen explicitly. Note also the leading scaling behaviour $f_{P} \sim 1 / \sqrt{m_{P}}$, which is manifest in (XV.25).

Although $\tilde{f}(\mu)$ cannot be calculated without nonperturbative input, its independence of the heavy quark mass $m$ implies that $\tilde{f}$ will drop out in the ratio of $f_{B}$ over $f_{D}$, if charm is treated as a heavy quark. One thus obtains

$$
\begin{aligned}
\frac{f_{B}}{f_{D}}=\sqrt{\frac{m_{D}}{m_{B}}}\left[\frac{\alpha_{s}\left(\mu_{b}\right)}{\alpha_{s}\left(\mu_{c}\right)}\right]^{d_{h l}}(1 & +\frac{\alpha_{s}\left(\mu_{b}\right)-\alpha_{s}\left(\mu_{c}\right)}{4 \pi}\left(B_{1}-J_{h l}-B_{2}\right)+ \\
& \left.+\frac{\alpha_{s}\left(\mu_{b}\right)}{4 \pi} \gamma_{h l}^{(0)} \ln \frac{\mu_{b}}{m_{b}}-\frac{\alpha_{s}\left(\mu_{c}\right)}{4 \pi} \gamma_{h l}^{(0)} \ln \frac{\mu_{c}}{m_{c}}\right)
\end{aligned}
$$

The QCD factor on the right hand side of (XV.26) amounts to 1.14 for $m_{b}=4.8 G e V, m_{c}=$ $1.4 \mathrm{GeV}$ and $\Lambda_{\overline{M S}}=0.2 \mathrm{GeV}$ if we set $\mu_{i}=m_{i}, i=b, c$. If we allow for a variation of the renormalization scales as $2 / 3 \leq \mu_{i} / m_{i} \leq 2$, this factor lies within a range of 1.12 to 1.16 . This is to be compared with the leading log approximation, where the central value reads 1.12 with a variation from 1.10 to 1.15 . Note that due to cancellations in the ratio $f_{B} / f_{D}$ the scale ambiguity is not much larger in LLA than in NLLA. However the next-to-leading order QCD effects further enhance $f_{B} / f_{D}$ independently of the renormalization scheme.

\section{E. $\Delta B=2$ Transitions in the Static Limit}

In section XIII we have described the effective hamiltonian for $B^{0}-\bar{B}^{0}$ mixing. The calculation of the mixing amplitude requires in particular the evaluation of the matrix element $\left\langle\bar{B}^{0}|Q| B^{0}\right\rangle \equiv\langle Q\rangle$ of the operator

$$
Q=(\bar{b} d)_{V-A}(\bar{b} d)_{V-A}
$$

in addition to the short-distance Wilson coefficient. Coefficient function and operator matrix element are to be evaluated at a common renormalization scale, $\mu_{b}=\mathcal{O}\left(m_{b}\right)$, say. In contrast to the determination of the Wilson coefficient, the computation of the hadronic matrix element involves nonperturbative long-distance contributions. Ultimately this problem should be solved using lattice QCD. However, the b quark is rather heavy and it is therefore difficult to incorporate it as a fully dynamical field in the context of a lattice regularization approach. On the other hand QCD effects from scales below $\mu_{b}=\mathcal{O}\left(m_{b}\right)$ down to $\sim 1 G e V$ are still accessible to a perturbative treatment. HQET provides the tool to calculate these contributions. At the same time it allows one to extract the dependence of $\left\langle\bar{B}^{0}|Q| B^{0}\right\rangle$ on the bottom mass $m_{b}$ explicitly, albeit at the prize of the further approximation introduced by the expansion in inverse powers of $m_{b}$.

In a first step the operator $Q$ in (XV.27) is expressed as a linear combination of HQET operators by matching the "full" to the effective theory at a scale $\mu_{b}=\mathcal{O}\left(m_{b}\right)$

$$
\left\langle Q\left(\mu_{b}\right)\right\rangle=\left(1+\frac{\alpha_{s}\left(\mu_{b}\right)}{4 \pi}\left[\left(\tilde{\gamma}^{(0)}-\gamma^{(0)}\right) \ln \frac{\mu_{b}}{m_{b}}+\tilde{B}-B\right]\right)\left\langle\tilde{Q}\left(\mu_{b}\right)\right\rangle+\frac{\alpha_{s}\left(\mu_{b}\right)}{4 \pi} \tilde{B}_{s}\left\langle\tilde{Q}_{s}\left(\mu_{b}\right)\right\rangle
$$


Here

$$
\tilde{Q}=2(\bar{h} d)_{V-A}\left(\bar{h}^{(-)} d\right)_{V-A} \quad \tilde{Q}_{s}=2(\bar{h} d)_{S-P}\left(\bar{h}^{(-)} d\right)_{S-P}
$$

$\left((\bar{h} d)_{S-P} \equiv \bar{h}\left(1-\gamma_{5}\right) d\right)$ are the necessary operators in HQET relevant for the case of a $B^{0} \rightarrow \bar{B}^{0}$ transition. The field $\bar{h}$ creates a heavy quark, while $\bar{h}^{(-)}$annihilates a heavy antiquark. Since the effective theory field $\bar{h}\left(\bar{h}^{(-)}\right)$cannot, unlike the full theory field $\bar{b}$ in $Q$, at the same time annihilate (create) the heavy antiquark (heavy quark), explicit factors of two have to appear in (XV.29). Similarly to the case of the heavy-light current discussed in the previous section a new operator $\tilde{Q}_{s}$ with scalar-pseudoscalar structure is generated. Its coefficient is finite and hence no operator mixing under infinite renormalization occurs between $\tilde{Q}$ and $\tilde{Q}_{s}$.

In a second step, the matrix element $\left\langle\tilde{Q}\left(\mu_{b}\right)\right\rangle$ at the high scale $\mu_{b}$ has to be expressed through the matrix element of $\tilde{Q}$ evaluated at a lower scale $\mu \sim 1 \mathrm{GeV}$, which is relevant for a nonperturbative calculation, for example using lattice gauge theory. This relation can be obtained through the usual renormalization group evolution and reads in NLLA

$$
\left\langle\tilde{Q}\left(\mu_{b}\right)\right\rangle=\left[\frac{\alpha_{s}\left(\mu_{b}\right)}{\alpha_{s}(\mu)}\right]^{\tilde{d}}\left(1+\frac{\alpha_{s}(\mu)-\alpha_{s}\left(\mu_{b}\right)}{4 \pi} \tilde{J}\right)\langle\tilde{Q}(\mu)\rangle
$$

where

$$
\tilde{d}=\frac{\tilde{\gamma}^{(0)}}{2 \beta_{0}} \quad \tilde{J}=\frac{\tilde{d}}{\beta_{0}} \beta_{1}-\frac{\tilde{\gamma}^{(1)}}{2 \beta_{0}}
$$

with the beta-function coefficients $\beta_{0}$ and $\beta_{1}$ given in (III.16). The calculation of the one-loop anomalous dimension $\tilde{\gamma}^{(0)}$ of the HQET operator $\tilde{Q}$, required for the leading log approximation to (XV.30), has been first performed in (Voloshin and Shifman, 1987) and (Politzer and Wise, 1988a), (Politzer and Wise, 1988b). The computation of the two-loop anomalous dimension $\tilde{\gamma}^{(1)}$ is due to (Giménez, 1993). Finally, the next-to-leading order matching condition (XV.28) has been determined in (Flynn et al., 1991). In the following we summarize the results obtained in these papers.

The scheme dependent next-to-leading order quantities $B, \tilde{B}$ and $\tilde{\gamma}^{(1)}$ refer to the NDR scheme with anticommuting $\gamma_{5}$ and the usual subtraction of evanescent terms as defined in (Buras and Weisz, 1990). For $N=3$ colors we then have

$$
\begin{gathered}
\tilde{\gamma}^{(0)}=-8 \quad \gamma^{(0)}=4 \\
\tilde{B}-B=-14 \quad B=\frac{11}{3} \quad \tilde{B}_{s}=-8 \\
\tilde{\gamma}^{(1)}=-\frac{808}{9}-\frac{52}{27} \pi^{2}+\frac{64}{9} f
\end{gathered}
$$

where $f$ is the number of active flavors.

At this point we would like to make the following comments.

- The logarithmic term in (XV.28) reflects the $\mathcal{O}\left(\alpha_{s}\right)$ scale dependence of the matrix elements of $Q$ and $\tilde{Q}$. Accordingly its coefficient is given by the difference in the one-loop anomalous dimensions of these operators, $\gamma^{(0)}$ and $\tilde{\gamma}^{(0)}$. 
- The one-loop anomalous dimension of the effective theory operator $\tilde{Q}, \tilde{\gamma}^{(0)}$, is exactly twice as large as the one-loop anomalous dimension of the heavy-light current discussed in section XV C (see eq. (XV.18). Therefore the scale dependence of $\langle\tilde{Q}\rangle$ below $\mu_{b}$ is entirely contained in the scale dependence of the decay constant squared $\tilde{f}^{2}(\mu)$. This implies the well known result that in leading log approximation the parameter $B_{B}$ has no perturbative scale dependence in the static theory below $\mu_{b}$. As the result of (Giménez, 1993) for $\tilde{\gamma}^{(1)}$ shows, this somewhat accidental cancellation is not valid beyond the one-loop level.

- The matching condition (XV.28) contains besides the logarithm a scheme dependent constant term in the relation between $\langle Q\rangle$ and $\langle\tilde{Q}\rangle$. We have written this coefficient in the form $\tilde{B}-B$ in order to make the cancellation of scheme dependences, to be discussed below, more transparent. Here $B$ is identical to $B_{+}$introduced in (V.8) and characterizes the scheme dependence of $\langle Q\rangle$ (see also sections XII and XIII).

- The quantity $\tilde{\gamma}^{(1)}$ has been originally calculated in (Giménez, 1993) using dimensional reduction (DRED) instead of NDR as renormalization scheme. However, $\tilde{B}$ turns out to be the same in DRED and NDR, implying that also $\tilde{\gamma}^{(1)}$ coincides in these schemes (Giménez, 1993).

Finally we may put together (XV.28) and (XV.30), omitting for the moment the scheme independent, constant correction due to $\tilde{Q}_{s}$, to obtain

$$
\left\langle Q\left(\mu_{b}\right)\right\rangle=\left[\frac{\alpha_{s}\left(\mu_{b}\right)}{\alpha_{s}(\mu)}\right]^{\tilde{d}}\left(1+\frac{\alpha_{s}\left(\mu_{b}\right)}{4 \pi}\left[\left(\tilde{\gamma}^{(0)}-\gamma^{(0)}\right) \ln \frac{\mu_{b}}{m_{b}}+\tilde{B}-B-\tilde{J}\right]+\frac{\alpha_{s}(\mu)}{4 \pi} \tilde{J}\right)\langle\tilde{Q}(\mu)\rangle
$$

This relation serves to express the $B^{0}-\bar{B}^{0}$ matrix element of the operator $Q$, evaluated at a scale $\mu_{b}=\mathcal{O}\left(m_{b}\right)$, which is the relevant scale for the effective hamiltonian of section XIII, in terms of the static theory matrix element $\langle\tilde{Q}(\mu)\rangle$ normalized at a low scale $\mu \sim 1 \mathrm{GeV}$. The latter is more readily accessible to a nonperturbative lattice calculation than the full theory matrix element $\left\langle Q\left(\mu_{b}\right)\right\rangle$. Note that (XV.35) as it stands is valid in the continuum theory. In order to use lattice results one still has to perform an $\mathcal{O}\left(\alpha_{s}\right)$ matching of $\tilde{Q}$ to its lattice counterpart. This step however does not require any further renormalization group improvement since by means of (XV.35) $\tilde{Q}$ is already normalized at the appropriate low scale $\mu$. The continuum - lattice theory matching was determined in (Flynn et al., 1991) and is also discussed in (Giménez, 1993).

Of course, the right hand side in (XV.35) gives only the leading contribution in the $1 / m$ expansion of the full matrix element $\left\langle Q\left(\mu_{b}\right)\right\rangle$ (apart from $\tilde{Q}_{s}$ ). Going beyond this approximation would require the consideration of several new operators, which appear at the next order in $1 / \mathrm{m}$. These contributions have been studied in (Kilian and Mannel, 1993) in the leading logarithmic approximation. On the other hand (XV.35), while restricted to the static limit, includes and resums all leading and next-to-leading logarithmic corrections between the scales $\mu_{b}=\mathcal{O}\left(m_{b}\right)$ and $\mu \sim 1 G e V$ in the relation among $Q$ and $\tilde{Q}$. It is interesting to consider the scale and scheme dependences present in (XV.35). The dependence on $\mu$ in the first factor on the r.h.s. of (XV.35) is canceled by the $\mu$-dependence of $\langle\tilde{Q}(\mu)\rangle$. The dependence on $\mu_{b}$ of this factor is canceled by the explicit $\ln \mu_{b}$ term proportional to $\tilde{\gamma}^{(0)}$. Hence the only scale dependence remaining on the r.h.s., to the considered order $\mathcal{O}\left(\alpha_{s}\right)$, is the one $\sim \alpha_{s}\left(\mu_{b}\right) \gamma^{(0)} \ln \mu_{b}$. This is precisely the scale 
dependence of the full theory matrix element on the 1.h.s., which is required to cancel the corresponding dependence of the Wilson coefficient. Similarly the term $\sim \alpha_{s}\left(\mu_{b}\right) B$ represents the correct scheme dependence of $\left\langle Q\left(\mu_{b}\right)\right\rangle$, while the scheme dependence of $\alpha_{s}(\mu) \tilde{J}$ cancels with the scheme dependence of $\langle\tilde{Q}(\mu)\rangle$ and the difference $\tilde{B}-\tilde{J}$ is scheme independent by itself. This discussion demonstrates explicitly that the transition from full QCD to HQET can be made at an arbitrary scale $\mu_{b}=\mathcal{O}\left(m_{b}\right)$, as we have already emphasized above.

Finally we would like to remark that since the logarithm $\ln \mu_{b} / \mu$ is not really very large in the present case, one might take the attitude of neglecting higher order resummations of logarithmic terms altogether and restricting oneself to the $\mathcal{O}\left(\alpha_{s}\right)$ corrections alone. Then (XV.28) would be already the final result, as it was used in (Flynn et al., 1991). This approximation is fully consistent from a theoretical point of view. Yet it is useful to have the more complete expression (XV.35) at hand. Of course, as indicated above, the finite $\mathcal{O}\left(\alpha_{s}\right)$ correction due to the matrix element of $\tilde{Q}_{s}$ in (XV.28) must still be added to the r.h.s. of (XV.35). However, to complete the NLO renormalization group calculation also the leading logarithmic corrections related to the operator $\tilde{Q}_{s}$ should then be resummed. To our knowledge this part of the analysis has not yet been performed in the literature so far. 



\section{Part Three -}

\section{The Phenomenology of Weak Decays}

The third part of our review presents the phenomenological picture of weak decays beyond the leading logarithmic approximation.

There is essentially a one-to-one correspondence between the sections in the second and in the third part of this review. Part three uses heavily the results derived in part two. In spite of this, the third part is meant to be essentially self-contained and can be followed without difficulties by those readers who only scanned the material of the second part and read section II.

The phenomenological part of our review is organized as follows. We begin with a few comments on the input parameters in section XVI. Next, as an application of the NLO corrections in the current-current sector, we summarize the present status of the tree level inclusive B-decays, in particular the theoretical status of the semi-leptonic branching ratio. The issue of exclusive two-body non-leptonic decays and the question of factorization will not be discussed here. The numerical values of the related factors $a_{i}$ for various renormalization schemes can be found in (Buras, 1995).

The main part of the phenomenology begins in section XVIII where we update the "standard" analysis of the unitarity triangle based on the indirect CP violation in $K \rightarrow \pi \pi$ (the parameter $\varepsilon_{K}$ ) and the $B_{d}^{0}-\bar{B}_{d}^{0}$ mixing described by $x_{d}$. We incorporate in this analysis the most recent values of $m_{t}, V_{u b} / V_{c b}, V_{c b}, B_{K}$ and $F_{B}$. In addition to the analysis of the unitarity triangle we determine several quantities of interest. These results will be used frequently in subsequent sections.

In section XIX we present $\varepsilon^{\prime} / \epsilon$ beyond leading logarithms, summarizing and updating the extensive analysis presented in (Buras et al., 1993b). $\varepsilon^{\prime}$ measures the size of the direct CP violation in $K \rightarrow \pi \pi$ and its accurate estimate is an important but very difficult task. In section XX we discuss briefly the $K_{L}-K_{S}$ mass difference and the $\Delta I=1 / 2$ rule. Next, in section XXI we present an update for $K_{L} \rightarrow \pi^{0} e^{+} e^{-}$.

Next in sections XXII and XXIII we consider $B \rightarrow X_{s} \gamma$ and $B \rightarrow X_{s} e^{+} e^{-}$, respectively. $B \rightarrow X_{s} \gamma$ is known only in the LO approximation. However, in view of its importance we summarize the leading order formulae and show the standard model prediction compared with the CLEO II findings. We also summarize the present status of NLO calculations for this decay. The NLO calculations for $B \rightarrow X_{s} e^{+} e^{-}$have been completed and we give a brief account of these results.

Sections XXIV-XXVI discuss $K \rightarrow \pi \nu \bar{\nu}, K_{L} \rightarrow \mu^{+} \mu^{-}$and rare B-decays $\left(B \rightarrow X_{s} \nu \bar{\nu}\right.$, $B \rightarrow l^{+} l^{-}$). Except for $K_{L} \rightarrow \mu^{+} \mu^{-}$, all these decays have only very small hadronic uncertainties and the dominant theoretical errors are related to various renormalization scale ambiguities. We demonstrate that these uncertainties are considerably reduced by including NLO corrections, which will improve the determination of the CKM matrix in forthcoming experiments. Using the results of section XVIII, we also give updated standard model predictions for these decays. 


\section{COMMENTS ON INPUT PARAMETERS}

The phenomenology of weak decays depends sensitively on a number of input parameters. We have collected the numerical values of these parameters in appendix A. To this end we have frequently used the values quoted by (Particle Data Group, 1994). The basis for our choice of the numerical values for various non-perturbative parameters, such as $B_{K}$ or $F_{B}$, will be given in the course of our presentation. In certain cases, like the B-meson life-times and the size of the $B_{d}^{0}-\bar{B}_{d}^{0}$ mixing, for which the experimental world averages change constantly we have chosen values, which are in the ball park of those presented at various conferences and workshops during the summer of 1995. Here we would like to comment briefly on three important parameters: $\left|V_{c b}\right|$, $\left|V_{u b} / V_{c b}\right|$ and $m_{\mathrm{t}}$. The importance of these parameters lies in the fact that many branching ratios and also the $\mathrm{CP}$ violation in the Standard Model depend sensitively on them.

\section{A. CKM Element $\left|V_{c b}\right|$}

During the last two years there has been a considerable progress made by experimentalists (Patterson, 1995) and theorists in the extraction of $\left|V_{c b}\right|$ from the exclusive and inclusive B-decays. In these investigations the HQET in the case of exclusive decays and the Heavy Quark Expansions for inclusive decays played a considerable role. In particular we would like to mention the important papers (Neubert, 1994a), (Shifman et al., 1995) and (Ball et al., 1995a) on the basis of which one is entitled to use:

$$
\left|V_{c b}\right|=0.040 \pm 0.003 \quad \Rightarrow \quad A=0.82 \pm 0.06
$$

This should be compared with an error of \pm 0.006 for $\left|V_{c b}\right|$ quoted still in 1993 . The corresponding reduction of the error in $A$ by a factor of 2 has important consequences for the phenomenology of weak decays.

\section{B. CKM Element Ratio $\left|V_{u b} / V_{c b}\right|$}

Here the situation is much worse and the value

$$
\left|\frac{V_{u b}}{V_{c b}}\right|=0.08 \pm 0.02
$$

quoted by (Particle Data Group, 1994) appears to be still valid. There is a hope that the error could be reduced by a factor of 2 to 4 in the coming years both due to the theory (Ball et al., 1995a) and the recent CLEO measurements of the exclusive semileptonic decays $B \rightarrow(\pi, \varrho) l \nu_{l}$ (Thorndike, 1995).

\section{Top Quark Mass $m_{\mathrm{t}}$}

Next it is important to stress that the discovery of the top quark (Abe et al., 1994a), (Abe et al., 1994b), (Abe et al., 1994c), (Abachi et al., 1995) and its mass measurement had an important impact on the field of rare decays and $\mathrm{CP}$ violation reducing considerably one potential uncertainty. 
It is however important to keep in mind that the parameter $m_{\mathrm{t}}$, the top quark mass, used in weak decays is not equal to the one used in the electroweak precision studies at LEP or SLD. In the latter investigations the so-called pole mass is used, whereas in all the NLO calculations listed in table I and used in this review, $m_{\mathrm{t}}$ refers to the running current top quark mass normalized at $\mu=m_{\mathrm{t}}$ : $\bar{m}_{\mathrm{t}}\left(m_{\mathrm{t}}\right)$. One has

$$
m_{\mathrm{t}}^{(\text {pole })}=\bar{m}_{\mathrm{t}}\left(m_{\mathrm{t}}\right)\left[1+\frac{4}{3} \frac{\alpha_{\mathrm{s}}\left(m_{\mathrm{t}}\right)}{\pi}\right]
$$

so that for $m_{\mathrm{t}}=\mathcal{O}(170 \mathrm{GeV}), \bar{m}_{\mathrm{t}}\left(m_{\mathrm{t}}\right)$ is typically by $8 \mathrm{GeV}$ smaller than $m_{\mathrm{t}}^{(\text {Pole) }}$. This difference will matter in a few years.

In principle any definition $\bar{m}_{\mathrm{t}}\left(\mu_{t}\right)$ with $\mu_{t}=\mathcal{O}\left(m_{\mathrm{t}}\right)$ could be used. In the leading order this arbitrariness in the choice of $\mu_{t}$ introduces a potential theoretical uncertainty in those branching ratios which depend sensitively on the top quark mass. The inclusion of NLO corrections reduces this uncertainty considerably so that the resulting branching ratios remain essentially independent of the choice of $\mu_{t}$. We have discussed this point already in previous sections. Numerical examples will be given in this part below. The choice $\mu_{t}=m_{\mathrm{t}}$ turns out to be convenient and will be adopted in what follows.

Using the $m_{\mathrm{t}}^{(\text {pole) }}$ quoted by CDF (Abe et al., 1994a), (Abe et al., 1994b), (Abe et al., 1994c) together with the relation (XVI.3) we find roughly

$$
m_{\mathrm{t}} \equiv \bar{m}_{\mathrm{t}}\left(m_{\mathrm{t}}\right)=(170 \pm 15) \mathrm{GeV}
$$

which we will use in our phenomenological applications. In principle an error of $\pm 11 \mathrm{GeV}$ could be used but we prefer to be conservative. 


\section{INCLUSIVE B DECAYS}

\section{A. General Remarks}

Inclusive decays of $B$ mesons constitute an important testing ground for our understanding of strong interaction dynamics in its interplay with the weak forces. At the same time inclusive semileptonic modes provide useful information on $\left|V_{c b}\right|$.

Due to quark-hadron duality inclusive decays of heavy mesons can, in general, be calculated more reliably than corresponding exclusive modes. During recent years a systematic formulation for the treatment of inclusive heavy meson decays has been developed. It is based on operator product and heavy quark expansion, which are applied to the $B$ meson inclusive width, expressed as the absorptive part of the $B$ forward scattering amplitude

$$
\Gamma(B \rightarrow X)=\frac{1}{2 m_{B}} \operatorname{Im}\left(i \int d^{4} x\left\langle B\left|T \mathcal{H}_{e f f}^{(X)}(x) \mathcal{H}_{e f f}^{(X)}(0)\right| B\right\rangle\right)
$$

Here $\mathcal{H}_{\text {eff }}^{(X)}$ is the part of the complete $\Delta B=1$ effective hamiltonian that contributes to the particular inclusive final state $X$ under consideration. E.g. for inclusive semileptonic decays

$$
\mathcal{H}_{e f f, \Delta B=1}^{(S L)}=\frac{G_{F}}{\sqrt{2}} V_{c b}(\bar{c} b)_{V-A} \sum_{l=e, \mu, \tau}\left(\bar{l} \nu_{l}\right)_{V-A}+\text { h.c. }
$$

For nonleptonic modes the relevant expression is the $\Delta B=1$ short distance effective hamiltonian given in (VI.32). It has been shown in (Chay et al., 1990), (Bjorken et al., 1992), (Bigi et al., 1992), (Bigi et al., 1993), (Manohar and Wise, 1994), (Blok et al., 1994), (Falk et al., 1994), (Mannel, 1994), (Bigi et al., 1994a), that the leading term in a systematic expansion of (XVII.1) in $1 / m_{b}$ is determined by the decay width of a free b-quark calculated in the parton picture. Furthermore, the nonperturbative corrections to this perturbative result start at order $\left(\Lambda / m_{b}\right)^{2}$, where $\Lambda$ is a hadronic scale $\sim 1 \mathrm{GeV}$, and are quite small in the case of $\mathrm{B}$ decays. In the light of this formulation it becomes apparent that the perturbative, partonic description of heavy hadron decay is thus promoted from the status of a model calculation to the leading contribution in a systematic expansion based on QCD. We will still comment on the $\left(\Lambda / m_{b}\right)^{2}$ corrections below. In the following we will however concentrate on the leading quark level analysis of inclusive $B$ decays. As we shall see, the treatment of short-distance QCD effects at the next-to-leading order level - at least for the dominant modes - is of crucial importance for a proper understanding of these processes. The calculation of b-quark decay starts from the effective $\Delta B=1$ hamiltonian containing the relevant four-fermion operators multiplied by Wilson coefficients. To obtain the decay rate, the matrix elements (squared) of these operators have to be calculated perturbatively to the required order in $\alpha_{\mathrm{s}}$. While in LLA a zeroth order evaluation is sufficient, $\mathcal{O}\left(\alpha_{\mathrm{s}}\right)$ virtual gluon effects (along with real gluon bremsstrahlung contributions for the proper cancellation of infrared divergences in the inclusive rate) have to be taken into account at NLO. In this way the renormalization scale and scheme dependence present in the coefficient functions is canceled to the considered order $\left(\mathcal{O}\left(\alpha_{\mathrm{s}}\right)\right)$ in the decay rate. Thus, by contrast to low energy decays, in the case of inclusive heavy quark decay, a physical final result can be obtained within perturbation theory alone.

Our goal will be in particular to review the present status of the theoretical prediction for the $B$ meson semileptonic branching ratio $B_{S L}$. This quantity has received some attention in recent years 
since theoretical calculations (Altarelli and Petrarca, 1991), (Tanimoto, 1992), (Palmer and Stech, 1993), (Bigi et al., 1994b), (Falk et al., 1995) tended to yield values around $12.5-13.5 \%$, above the experimental figure $B_{S L}=(10.4 \pm 0.4) \%$ (Particle Data Group, 1994). However, these earlier analyses have not been complete in regard to the inclusion of final state mass effects and NLO QCD corrections in the nonleptonic widths. More precisely, these calculations took into account mass effects appropriate for the leading order in QCD along with NLO QCD corrections obtained for massless final state quarks. Recently the most important of these - so far lacking - mass effects have been properly included in the NLO QCD calculation through the work of (Bagan et al., 1994), (Bagan et al., 1995a), (Bagan et al., 1995b). These $\mathcal{O}\left(\alpha_{\mathrm{s}}\right)$ mass effects tend to decrease $B_{S L}$ and, according to the analysis of these authors essentially bring it, within theoretical uncertainties, into agreement with the experimental number. Before further discussing these issues, it is appropriate to start with a short overview summarizing the possible b-quark decay modes and classifying their relative importance.

\section{B. b-Quark Decay Modes}

First of all, a b-quark can decay semileptonically to the final states $c l \bar{\nu}_{l}$ and $u l \bar{\nu}_{l}$ with $l=e, \mu$, $\tau$.

In the case of nonleptonic final states we may distinguish three classes: Decays induced through current-current operators alone (class I), decays induced by both current-current and penguin operators (class II) and pure penguin transitions (class III). We have

\begin{tabular}{|c|llrr|}
\hline Class & \multicolumn{4}{|c|}{ Final State } \\
\hline I & $c \bar{u} d$, & $c \bar{u} s ;$ & $u \bar{c} s$, & $u \bar{c} d$ \\
II & $c \bar{c} s$, & $c \bar{c} d ;$ & $u \bar{u} d$, & $u \bar{u} s$ \\
III & $d \bar{d} d$, & $d \bar{d} s ;$ & $s \bar{s} d$, & $s \bar{s} s$ \\
\hline
\end{tabular}

Clearly there is a rich structure of possible decay modes even at the quark level and a complete treatment would be quite complicated. However, not all of these final states are equally important. In order to perform the analysis of b-quark decay, in particular in view of the calculation of $B_{S L}$, it is useful to identify the most important channels and to introduce appropriate approximations in dealing with less prominent decays. To organize the procedure, we make the following observations:

- The dominant, i.e. CKM allowed and tree-level induced, decays are $b \rightarrow c l \nu, b \rightarrow c \bar{u} d$ and $b \rightarrow c \bar{c} s$. For these a complete NLO calculation including final state mass effects is necessary.

- The channels $c \bar{u} s, c \bar{c} d, u \bar{c} d, u \bar{u} s$ may be incorporated with excellent accuracy into the modes $c \bar{u} d, c \bar{c} s, u \bar{c} s, u \bar{u} d$, respectively, using the approximate CKM unitarity in the first two generations. The error introduced thereby through the $s-d$ mass difference is entirely negligible.

- Penguin transitions are generally suppressed by the smallness of their Wilson coefficient functions, which are typically of the order of a few percent. For this reason, one may neglect the pure penguin decays of class III altogether as their decay rates involve penguin coefficients squared. 
- Furthermore we may neglect the penguin contributions to the CKM suppressed $b \rightarrow u$ transitions of class II.

- In addition one may treat the remaining smaller effects, namely $b \rightarrow u$ transitions and the interference of penguins with the leading current-current contribution in $b \rightarrow c \bar{c} s$ within the leading log approximation.

- Finally, rare, flavor-changing neutral current b-decay modes are negligible in the present context as well.

Next we will write down expressions for the relevant decay rate contributions we have discussed. For the dominant modes $b \rightarrow c l \nu, b \rightarrow c \bar{u} d$ and $b \rightarrow c \bar{c} s$ (without penguin effects) one has at next-to-leading order:

$$
\begin{aligned}
& \Gamma(b \rightarrow c l \nu)=\Gamma_{0} P\left(x_{c}, x_{l}, 0\right)\left[1+\frac{2 \alpha_{\mathrm{s}}(\mu)}{3 \pi} g\left(x_{c}, x_{l}, 0\right)\right] \\
& \Gamma(b \rightarrow c \bar{u} d)= \Gamma_{0} P\left(x_{c}, 0,0\right)\left[2 L_{+}^{2}+L_{-}^{2}+\frac{\alpha_{\mathrm{s}}\left(M_{W}\right)-\alpha_{\mathrm{s}}(\mu)}{2 \pi}\left(2 L_{+}^{2} R_{+}+L_{-}^{2} R_{-}\right)\right. \\
&+ \frac{2 \alpha_{\mathrm{s}}(\mu)}{3 \pi}\left(\frac{3}{4}\left(L_{+}-L_{-}\right)^{2} g_{11}\left(x_{c}\right)+\frac{3}{4}\left(L_{+}+L_{-}\right)^{2} g_{22}\left(x_{c}\right)\right. \\
&\left.\left.+\frac{1}{2}\left(L_{+}^{2}-L_{-}^{2}\right)\left(g_{12}\left(x_{c}\right)-12 \ln \frac{\mu}{m_{b}}\right)\right)\right] \\
& \Gamma(b \rightarrow c \bar{c} s)= \Gamma_{0} P\left(x_{c}, x_{c}, x_{s}\right)\left[2 L_{+}^{2}+L_{-}^{2}+\frac{\alpha_{\mathrm{s}}\left(M_{W}\right)-\alpha_{\mathrm{s}}(\mu)}{2 \pi}\left(2 L_{+}^{2} R_{+}+L_{-}^{2} R_{-}\right)\right. \\
&+ \frac{2 \alpha_{\mathrm{s}}(\mu)}{3 \pi}\left(\frac{3}{4}\left(L_{+}-L_{-}\right)^{2} h_{11}\left(x_{c}\right)+\frac{3}{4}\left(L_{+}+L_{-}\right)^{2} h_{22}\left(x_{c}\right)\right. \\
&\left.\left.+\frac{1}{2}\left(L_{+}^{2}-L_{-}^{2}\right)\left(h_{12}\left(x_{c}\right)-12 \ln \frac{\mu}{m_{b}}\right)\right)\right]
\end{aligned}
$$

Eq. (XVII.5) neglects small strange quark mass effects in the NLO terms, which have however been included in the numerical analysis in (Bagan et al., 1995b). In the equations above $\Gamma_{0}=$ $G_{F}^{2} m_{b}^{5}\left|V_{c b}\right|^{2} /\left(192 \pi^{3}\right)$ and $P\left(x_{1}, x_{2}, x_{3}\right)$ is the leading order phase space factor given for arbitrary masses $x_{i}=m_{i} / m_{b}$ by

$$
\begin{gathered}
P\left(x_{1}, x_{2}, x_{3}\right)=12 \int_{\left(x_{2}+x_{3}\right)^{2}}^{\left(1-x_{1}\right)^{2}} \frac{d s}{s}\left(s-x_{2}^{2}-x_{3}^{2}\right)\left(1+x_{1}^{2}-s\right) w\left(s, x_{2}^{2}, x_{3}^{2}\right) w\left(s, x_{1}^{2}, 1\right) \\
w(a, b, c)=\left(a^{2}+b^{2}+c^{2}-2 a b-2 a c-2 b c\right)^{1 / 2}
\end{gathered}
$$

$P$ is a completely symmetric function of its arguments.

Furthermore 


$$
L_{ \pm}=L_{ \pm}(\mu)=\left[\frac{\alpha_{\mathrm{s}}\left(M_{W}\right)}{\alpha_{\mathrm{s}}(\mu)}\right]^{d_{ \pm}}
$$

with $d_{+}=6 / 23, d_{-}=-12 / 23$ (see (V.10)) and $\mu=\mathcal{O}\left(m_{b}\right)$. The scheme independent $R_{ \pm}$come from the NLO renormalization group evolution and are given by $R_{ \pm}=B_{ \pm}-J_{ \pm}$(see (V.9)). For $f=5$ flavors $R_{+}=6473 / 3174, R_{-}=-9371 / 1587$. Note that the leading dependence of $L_{ \pm}$on the renormalization scale $\mu$ is canceled to $\mathcal{O}\left(\alpha_{\mathrm{s}}\right)$ by the explicit $\mu$-dependence in the $\alpha_{\mathrm{s}}$-correction terms. Virtual gluon and bremsstrahlung corrections to the matrix elements of four fermion operators are contained in the mass dependent functions $g, g_{i j}$ and $h_{i j}$.

The function $g\left(x_{1}, x_{2}, x_{3}\right)$ is available for arbitrary $x_{1}, x_{2}, x_{3}$ from (Hokim and Pham, 1983), (Hokim and Pham, 1984). The special case $g\left(x_{1}, 0,0\right)$ has been analysed also in (Cabibbo and Maiani, 1978). Analytical expressions have been given in (Nir, 1989) for $g\left(x_{1}, 0,0\right)$ and in (Bagan et al., 1994) for $g\left(0, x_{2}, 0\right)$. The functions $g_{11}(x), g_{12}(x)$ and $g_{22}(x)$ are calculated analytically in (Bagan et al., 1994). Furthermore, as discussed in (Bagan et al., 1994), $h_{11}(x)$ and $h_{22}(x)$ can be obtained from the work of (Hokim and Pham, 1983), (Hokim and Pham, 1984). Finally, $h_{12}(x)$ has been determined in (Bagan et al., 1995b). For the full mass dependence of these functions we refer the reader to the cited literature. Here we quote the results obtained in the massless limit. These have been computed in (Altarelli et al., 1981), (Buchalla, 1993) for $g_{i j}, h_{i j}\left(g_{i j}(0)=h_{i j}(0)\right.$ )

$$
g_{11}(0)=g_{22}(0)=\frac{31}{4}-\pi^{2} \quad g_{12}(0)=g_{11}(0)-\frac{19}{2}
$$

Furthermore

$$
g(0,0,0)=\frac{25}{4}-\pi^{2}
$$

In table XXXVI we have listed some typical numbers extracted from (Bagan et al., 1995a), (Bagan et al., 1995b) illustrating the impact of charm mass effects (for $x_{c}=0.3$ ) in the NLO correction terms by giving the enhancment factor of the NLO over the LO results. There are of course various ambiguities involved in this comparison. The numbers in table XXXVI are therefore merely intended to show the general trend. Note the sizable enhancement through NLO mass effects in the nonleptonic channels, in particular $b \rightarrow c \bar{c} s$. A large QCD enhancement in the latter case has also been reported in (Voloshin, 1995).

TABLE XXXVI. Typical values for the ratio of NLO to LO results for dominant b-decay channels with (I) and without (II) including finite charm mass effects in the NLO correction terms. The leading order final state mass effects (through the function $P$ ) are taken into account in all cases.

\begin{tabular}{c|cc|c|c|c|}
\hline \hline & $b \rightarrow c e \nu$ & $b \rightarrow c \tau \nu$ & $b \rightarrow c \bar{u} d$ & $b \rightarrow c \bar{c} s$ \\
\hline $\mathrm{I}$ & 0.85 & 0.88 & 1.06 & 1.32 \\
\hline $\mathrm{II}$ & 0.79 & 0.80 & 1.01 & 1.02 \\
\hline \hline
\end{tabular}

To complete the presentation of $\mathrm{b}$ decay modes we next write down expressions for the CKM suppressed channels $b \rightarrow u l \nu, b \rightarrow u \bar{c} s$ and $b \rightarrow u \bar{u} d$ (without penguins) as well as the contribution to the $b \rightarrow c \bar{c} s$ rate due to interference of the leading, current-current type transitions with penguin operators. Restricting ourselves to the LLA for these small contributions we obtain 


$$
\begin{gathered}
\Gamma\left(b \rightarrow u \sum_{l} l \nu\right)=\Gamma_{0}\left|\frac{V_{u b}}{V_{c b}}\right|^{2} \sum_{l} P\left(0, x_{l}, 0\right) \\
\Gamma(b \rightarrow u \bar{c} s)=\Gamma_{0}\left|\frac{V_{u b}}{V_{c b}}\right|^{2} P\left(0, x_{c}, x_{s}\right)\left[2 L_{+}^{2}+L_{-}^{2}\right] \\
\Gamma(b \rightarrow u \bar{u} d)=\Gamma_{0}\left|\frac{V_{u b}}{V_{c b}}\right|^{2}\left[2 L_{+}^{2}+L_{-}^{2}\right] \\
\Delta \Gamma_{\text {penguin }}(b \rightarrow c \bar{c} s)=6 \Gamma_{0} P\left(x_{c}, x_{c}, x_{s}\right)\left[c_{1}\left(c_{3}+\frac{1}{3} c_{4}+F\left(c_{5}+\frac{1}{3} c_{6}\right)\right)\right. \\
\left.+c_{2}\left(\frac{1}{3} c_{3}+c_{4}+F\left(\frac{1}{3} c_{5}+c_{6}\right)\right)\right]
\end{gathered}
$$

where $c_{1}, \ldots, c_{6}$ are the leading order Wilson coefficients and

$$
F=\frac{6 x_{c}^{2}}{P\left(x_{c}, x_{c}, x_{s}\right)} \int_{\left(x_{c}+x_{s}\right)^{2}}^{\left(1-x_{c}\right)^{2}} \frac{d s}{s^{2}}\left(s+x_{s}^{2}-x_{c}^{2}\right)\left(1+s-x_{c}^{2}\right) w\left(s, x_{c}^{2}, x_{s}^{2}\right) w\left(1, s, x_{c}^{2}\right)
$$

Numerically we have for $\left|V_{u b} / V_{c b}\right|=0.1$

$$
\begin{gathered}
\Gamma\left(b \rightarrow u \sum_{l} l \nu\right) \approx 0.024 \Gamma_{0} \quad \Gamma(b \rightarrow u \bar{c} s) \approx 0.017 \Gamma_{0} \\
\Gamma(b \rightarrow u \bar{u} d) \approx 0.034 \Gamma_{0} \quad \Delta \Gamma_{\text {penguin }}(b \rightarrow c \bar{c} s) \approx-0.041 \Gamma_{0}
\end{gathered}
$$

Note that the contribution due to the interference with penguin transitions in $b \rightarrow c \bar{c} s$ is negative. Hence, in addition to being small the effects in (XVII.16) and (XVII.17) tend to cancel each other in the total nonleptonic width.

Finally one may also incorporate nonperturbative corrections. These have been derived in (Bigi et al., 1992) and are also discussed in (Bagan et al., 1994). As mentioned above, nonperturbative effects are suppressed by two powers of the heavy b-quark mass and amount typically to a few percent. For details we refer the reader to the cited articles.

\section{The B Meson Semileptonic Branching Ratio}

An important application of the results described in the previous section is the theoretical prediction for the inclusive semileptonic branching ratio of $B$ mesons

$$
B_{S L}=\frac{\Gamma(B \rightarrow X e \nu)}{\Gamma_{t o t}(B)}
$$

On the parton level $\Gamma(B \rightarrow X e \nu) \simeq \Gamma(b \rightarrow c e \nu)$ and 


$$
\Gamma_{\text {tot }}(B) \simeq \sum_{l=e, \mu, \tau} \Gamma(b \rightarrow c l \nu)+\Gamma(b \rightarrow c \bar{u} d)+\Gamma(b \rightarrow c \bar{c} s)+\Delta \Gamma_{\text {penguin }}(b \rightarrow c \bar{c} s)+\Gamma(b \rightarrow u)
$$

(XVII.19)

Here we have applied the approximations discussed above. $\Gamma(b \rightarrow u)$ summarizes the $b \rightarrow u$ transitions.

Based on a similar treatment of the partonic rates, including in particular next-to-leading QCD corrections for the dominant channels and also incorporating nonperturbative corrections, the authors of (Bagan et al., 1995a), (Bagan et al., 1995b) have carried out an analysis of $B_{S L}$ and estimated the theoretical uncertainties. They obtain (Bagan et al., 1995b)

$$
B_{S L}=(12.0 \pm 1.4) \% \quad \text { and } \quad B_{S L}=(11.2 \pm 1.7) \%
$$

using pole and $\overline{M S}$ masses, respectively. The error is dominated in both cases by the renormalization scale uncertainty $\left(m_{\mathrm{b}} / 2<\mu<2 m_{\mathrm{b}}\right)$. Note also the sizable scheme ambiguity.

Within existing uncertainties, the theoretical prediction does not disagree significantly with the experimental value $B_{S L, \text { exp }}=(10.4 \pm 0.4) \%$ (Particle Data Group, 1994), although it seems to lie still somewhat on the high side.

It is amusing to note, that the naive mode counting estimate for $B_{S L}$, neglecting QCD and final state mass effects completely, yields $B_{S L}=1 / 9=11.1 \%$ in (almost) "perfect agreement" with experiment. Including the final state masses, still neglecting QCD, enhances this number to $B_{S L}=15.8 \%$. Incorporating in addition QCD effects at the leading log level increases the hadronic modes, thus leading to a decrease in $B_{S L}$, resulting typically in $B_{S L}=14.7 \%$. A substantial further decrease is finally brought about through the NLO QCD corrections, which both further enhance hadronic channels, in particular $b \rightarrow c \bar{c} s$, and simultaneously reduce $b \rightarrow c e \nu$. As pointed out in (Bagan et al., 1995a), (Bagan et al., 1995b) and illustrated in table XXXVI final state mass effects in the NLO correction terms play a nonnegligile role for this enhancement of hadronic decays. The nonperturbative effects also lead to a slight decrease of $B_{S L}$.

In short, leading final state mass effects and QCD corrections, acting in opposite directions on $B_{S L}$, tend to cancel each other, resulting in a number for $B_{S L}$ not too different from the simple modecounting guess.

We finally mention that, besides a calculation of $B_{S L}$, the partonic treatment of heavy meson decay has further important applications, such as the determination of $\left|V_{c b}\right|$ from inclusive semileptonic $B$ decay, $B \rightarrow X_{c} e \nu$. Analyses of this type have been presented in (Luke and Savage, 1994), (Bigi and Uraltsev, 1994), (Ball and Nierste, 1994), (Shifman et al., 1995).

Exact results beyond the presently known NLO accuracy seem extremely difficult to obtain, even for relatively simple quantities like the semileptonic b-quark decay rate. There exist however calculations in the literature devoted to the investigation of these higher order perturbative effects. Due to the severe technical difficulties, those calculations require additional assumptions. For instance, in an interesting study (Ball et al., 1995a) have investigated the effects of the running of $\alpha_{\mathrm{s}}$ on the semileptonic b-quark decay rate to all orders in perturbation theory. This calculation is equivalent to a resummation of all terms of the form $\alpha_{\mathrm{s}}\left(\beta_{0} \alpha_{\mathrm{s}}\right)^{n}$, which are related to one-gluon exchange diagrams containing an arbitrary number $n$ of fermion bubbles. The work of (Ball et al., 1995a) applies the renormalon techniques developped in (Beneke and Braun, 1995), (Ball et al., 1995b) and generalizes the $\mathcal{O}\left(\beta_{0} \alpha_{s}^{2}\right)$ results computed in (Luke et al., 1995). The underlying idea is similar in spirit to the BLM approach (Brodsky et al., 1983). An important application of the 
result is the extraction of $\left|V_{c b}\right|$ (Ball et al., 1995a). The formalism has also been used to study higher order QCD corrections to the $\tau$ lepton hadronic width (Ball et al., 1995b). Irrespective of the ultimate reliability of the approximation, these investigations are useful from a conceptual point of view as they help to illustrate important features of the higher order behavior of the perturbative expansion.

In principle the discussion we have given for b-decays may of course, with appropriate modifications, be applied to the case of charm as well. However here the nonperturbative corrections to the parton picture, which scale like $1 / m_{Q}^{2}$ with the heavy quark mass $m_{Q}$, are by an order of magnitude larger than for B mesons and accurate theoretical predictions are much more difficult to obtain (Blok and Shifman, 1993). 


\section{XVIII. $\varepsilon_{K}, B^{0}-\bar{B}^{0}$ MIXING AND THE UNITARITY TRIANGLE}

\section{A. Basic Formula for $\varepsilon_{K}$}

The indirect $\mathrm{CP}$ violation in $K \rightarrow \pi \pi$ is described by the well known parameter $\varepsilon_{K}$. The general formula for $\varepsilon_{K}$ is given as follows

$$
\varepsilon_{K}=\frac{\exp (i \pi / 4)}{\sqrt{2} \Delta M_{K}}\left(\operatorname{Im} M_{12}+2 \xi \operatorname{Re} M_{12}\right)
$$

where

$$
\xi=\frac{\operatorname{Im} A_{0}}{\operatorname{Re} A_{0}}
$$

with $A_{0} \equiv A\left(K \rightarrow(\pi \pi)_{I=0}\right)$ and $\Delta M_{K}$ being the $K_{L}-K_{S}$ mass difference. The off-diagonal element $M_{12}$ in the neutral $K$-meson mass matrix represents the $K^{0}-\bar{K}^{0}$ mixing. It is given by

$$
2 m_{K} M_{12}^{*}=\left\langle\bar{K}^{0}\left|\mathcal{H}_{\mathrm{eff}}(\Delta S=2)\right| K^{0}\right\rangle
$$

where $\mathcal{H}_{\text {eff }}(\Delta S=2)$ is the effective hamiltonian of eq. (XII.1). Defining the renormalization group invariant parameter $B_{K}$ by

$$
\begin{aligned}
& B_{K}=B_{K}(\mu)\left[\alpha_{\mathrm{s}}^{(3)}(\mu)\right]^{-2 / 9}\left[1+\frac{\alpha_{\mathrm{s}}^{(3)}(\mu)}{4 \pi} J_{3}\right] \\
& \left\langle\bar{K}^{0}\left|(\bar{s} d)_{V-A}(\bar{s} d)_{V-A}\right| K^{0}\right\rangle \equiv \frac{8}{3} B_{K}(\mu) F_{K}^{2} m_{K}^{2}
\end{aligned}
$$

and using (XII.1) we find

$$
M_{12}=\frac{G_{F}^{2}}{12 \pi^{2}} F_{K}^{2} B_{K} m_{K} M_{\mathrm{W}}^{2}\left[\lambda_{c}^{* 2} \eta_{1} S_{0}\left(x_{c}\right)+\lambda_{t}^{* 2} \eta_{2} S_{0}\left(x_{t}\right)+2 \lambda_{c}^{*} \lambda_{t}^{*} \eta_{3} S_{0}\left(x_{c}, x_{t}\right)\right]
$$

where the functions $S_{0}\left(x_{i}\right)$ and $S_{0}\left(x_{i}, x_{j}\right)$ are those of eq. (XII.3)-(XII.5). $F_{K}$ is the $K$-meson decay constant and $m_{K}$ the $K$-meson mass. The coefficient $J_{3}$ is given in (XII.9) and the QCD factors $\eta_{i}$ have been discussed in section XII. Their numerical values are

$$
\eta_{1}=1.38 \quad \eta_{2}=0.57 \quad \eta_{3}=0.47
$$

The last term in (XVIII.1) constitutes at most a $2 \%$ correction to $\varepsilon_{K}$ and consequently can be neglected in view of other uncertainties, in particular those connected with $B_{K}$. Inserting (XVIII.6) into (XVIII.1) we find

$$
\varepsilon_{K}=C_{\varepsilon} B_{K} \operatorname{Im} \lambda_{t}\left\{\operatorname{Re} \lambda_{c}\left[\eta_{1} S_{0}\left(x_{c}\right)-\eta_{3} S_{0}\left(x_{c}, x_{t}\right)\right]-\operatorname{Re} \lambda_{t} \eta_{2} S_{0}\left(x_{t}\right)\right\} \exp (i \pi / 4)
$$

where we have used the unitarity relation $\operatorname{Im} \lambda_{c}^{*}=\operatorname{Im} \lambda_{t}$ and we have neglected $\operatorname{Re} \lambda_{t} / \operatorname{Re} \lambda_{c}=$ $\mathcal{O}\left(\lambda^{4}\right)$ in evaluating $\operatorname{Im}\left(\lambda_{c}^{*} \lambda_{t}^{*}\right)$. The numerical constant $C_{\varepsilon}$ is given by 


$$
C_{\varepsilon}=\frac{G_{F}^{2} F_{K}^{2} m_{K} M_{\mathrm{W}}^{2}}{6 \sqrt{2} \pi^{2} \Delta M_{K}}=3.78 \cdot 10^{4} .
$$

Using the standard parametrization of (II.13) to evaluate $\operatorname{Im} \lambda_{i}$ and $\operatorname{Re} \lambda_{i}$, setting the values for $s_{12}$, $s_{13}, s_{23}$ and $m_{\mathrm{t}}$ in accordance with appendix A and taking a value for $B_{K}$ (see below) one can determine the phase $\delta$ by comparing (XVIII.8) with the experimental value for $\varepsilon_{K}$.

Once $\delta$ has been determined in this manner one can find the corresponding point $(\bar{\varrho}, \bar{\eta})$ by using (II.19) and (II.22). Actually for a given set $\left(s_{12}, s_{13}, s_{23}, m_{\mathrm{t}}, B_{K}\right)$ there are two solutions for $\delta$ and consequently two solutions for $(\bar{\varrho}, \bar{\eta})$. In order to see this clearly it is useful to use the Wolfenstein parametrization in which $\operatorname{Im} \lambda_{t}, \operatorname{Re} \lambda_{c}$ and $\operatorname{Re} \lambda_{t}$ are given to a very good approximation by (II.23)(II.25). We then find that (XVIII.8) and the experimental value for $\varepsilon_{K}$ specify a hyperbola in the $(\bar{\varrho}, \bar{\eta})$ plane given by

$$
\bar{\eta}\left\{(1-\bar{\varrho}) A^{2} \eta_{2} S_{0}\left(x_{t}\right)+P_{0}(\varepsilon)\right\} A^{2} B_{K}=0.226 .
$$

(XVIII.10)

where

$$
P_{0}(\varepsilon)=\left[\eta_{3} S_{0}\left(x_{c}, x_{t}\right)-\eta_{1} x_{c}\right] \frac{1}{\lambda^{4}} .
$$

The hyperbola (XVIII.10) intersects the circle given by (II.32) in two points which correspond to the two solutions for $\delta$ mentioned earlier.

The position of the hyperbola (XVIII.10) in the $(\bar{\varrho}, \bar{\eta})$ plane depends on $m_{\mathrm{t}},\left|V_{c b}\right|=A \lambda^{2}$ and $B_{K}$. With decreasing $m_{\mathrm{t}},\left|V_{c b}\right|$ and $B_{K}$ the $\varepsilon_{K}$-hyperbola moves away from the origin of the $(\bar{\varrho}, \bar{\eta})$ plane. When the hyperbola and the circle (II.32) touch each other lower bounds consistent with $\varepsilon_{K}^{\exp }$ for $m_{\mathrm{t}},\left|V_{c b}\right|,\left|V_{u b} / V_{c b}\right|$ and $B_{K}$ can be found. The lower bound on $m_{\mathrm{t}}$ is discussed in (Buras, 1993). Corresponding results for $\left|V_{u b} / V_{c b}\right|$ and $B_{K}$ are shown in fig. 11 and 12 , respectively. They will be discussed below.

Moreover approximate analytic expressions for these bounds can be derived. One has

$$
\begin{aligned}
\left(m_{\mathrm{t}}\right)_{\min } & =M_{\mathrm{W}}\left[\frac{1}{2 A^{2}}\left(\frac{1}{A^{2} B_{K} R_{b}}-1.4\right)\right]^{0.658} \\
\left|V_{u b} / V_{c b}\right|_{\min } & =\frac{\lambda}{1-\lambda^{2} / 2}\left[A^{2} B_{K}\left(2 x_{t}^{0.76} A^{2}+1.4\right)\right]^{-1} \\
\left(B_{K}\right)_{\min } & =\left[A^{2} R_{b}\left(2 x_{t}^{0.76} A^{2}+1.4\right)\right]^{-1}
\end{aligned}
$$

Concerning the parameter $B_{K}$, the analyses of (Sharpe, 1994), (Ishizuka, 1993) ( $B_{K}=0.83 \pm$ 0.03) using the lattice method and of (Bijnens and Prades, 1995) using a somewhat modified form of the $1 / N$ approach of (Bardeen et al., 1988), (Gerrard, 1990) give results in the ball park of the $1 / N$ result $B_{K}=0.70 \pm 0.10$ obtained some time ago in (Bardeen et al., 1988), (Gérard, 1990). In particular the analysis of (Bijnens and Prades, 1995) seems to have explained the difference between these values for $B_{K}$ and the lower values obtained using the QCD Hadronic Duality approach (Pich and de Rafael, 1985), (Prades et al., 1991) $\left(B_{K}=0.39 \pm 0.10\right)$ or using SU(3) symmetry and PCAC $\left(B_{K}=1 / 3\right)$ (Donoghue et al., 1982). These higher values of $B_{K}$ are also found in the most recent lattice analysis (Crisafulli et al., 1995) $\left(B_{K}=0.86 \pm 0.15\right)$ and in the lattice calculations of Bernard and Soni $\left(B_{K}=0.78 \pm 0.11\right)$ and the JLQCD group ( $B_{K}=$ $0.67 \pm 0.07$ ) with the quoted values obtained on the basis on the review by (Soni, 1995). In our numerical analysis we will use

$$
B_{K}=0.75 \pm 0.15 .
$$




\section{B. Basic Formula for $B^{0}-\bar{B}^{0}$ Mixing}

The $B^{0}-\bar{B}^{0}$ mixing is usually described by

$$
x_{d, s} \equiv \frac{(\Delta M)_{B_{d, s}}}{\Gamma_{B_{d, s}}}=\frac{2\left|M_{12}\right|_{B_{d, s}}}{\Gamma_{B_{d, s}}}
$$

where $(\Delta M)_{B_{d, s}}$ is the mass difference between the mass eigenstates in the $B_{d}^{0}-\bar{B}_{d}^{0}$ system and the $B_{s}^{0}-\bar{B}_{s}^{0}$ system, respectively, and $\Gamma_{B_{d, s}}=1 / \tau_{B_{d, s}}$ with $\tau_{B_{d, s}}$ being the corresponding lifetimes. The off-diagonal term $M_{12}$ in (XVIII.16) is given by

$$
2 m_{B}\left|M_{12}\right|=\left|\left\langle\bar{B}^{0}\left|\mathcal{H}_{\text {eff }}(\Delta B=2)\right| B^{0}\right\rangle\right|
$$

where $\mathcal{H}_{\text {eff }}(\Delta B=2)$ is the effective hamiltonian of (XIII.1). Defining the renormalization group invariant parameter $B_{B}$ by

$$
\begin{gathered}
B_{B}=B_{B}(\mu)\left[\alpha_{\mathrm{s}}^{(5)}(\mu)\right]^{-6 / 23}\left[1+\frac{\alpha_{\mathrm{s}}^{(5)}(\mu)}{4 \pi} J_{5}\right] \\
\left\langle\bar{B}^{0}\left|(\bar{b} d)_{V-A}(\bar{b} d)_{V-A}\right| B^{0}\right\rangle \equiv \frac{8}{3} B_{B}(\mu) F_{B}^{2} m_{B}^{2}
\end{gathered}
$$

and using (XIII.1) we find

$$
x_{d, s}=\tau_{B_{d, s}} \frac{G_{F}^{2}}{6 \pi^{2}} \eta_{B} m_{B_{d, s}}\left(B_{B_{d, s}} F_{B_{d, s}}^{2}\right) M_{\mathrm{W}}^{2} S_{0}\left(x_{t}\right)\left|V_{t(d, s)}\right|^{2}
$$

with the QCD factor $\eta_{B}$ discussed in section XIII and given by $\eta_{B}=0.55$.

The measurement of $B_{d}^{0}-\bar{B}_{d}^{0}$ mixing allows then to determine $\left|V_{t d}\right|$ or $R_{t}$ of (II.33)

$$
\left|V_{t d}\right|=A \lambda^{3} R_{t} \quad R_{t}=1.52 \frac{R_{0}}{\sqrt{S_{0}\left(x_{t}\right)}}
$$

where

$$
R_{0}=\left[\frac{0.040}{\left|V_{c b}\right|}\right]\left[\frac{200 \mathrm{MeV}}{\sqrt{B_{B_{d}}} F_{B_{d}}}\right]\left[\frac{x_{d}}{0.75}\right]^{0.5}\left[\frac{1.6 p s}{\tau_{B}}\right]^{0.5}\left[\frac{0.55}{\eta_{B}}\right]^{0.5} .
$$

which gives setting $\eta_{B}=0.55$

$$
\left|V_{t d}\right|=8.56 \cdot 10^{-3}\left[\frac{170 \mathrm{GeV}}{\bar{m}_{t}\left(m_{t}\right)}\right]^{0.76}\left[\frac{200 \mathrm{MeV}}{\sqrt{B_{B_{d}}} F_{B_{d}}}\right]\left[\frac{x_{d}}{0.75}\right]^{0.5}\left[\frac{1.6 p s}{\tau_{B}}\right]^{0.5} .
$$

There is a vast literature on the lattice calculations of $F_{B}$. The most recent results are somewhat lower than quoted a few years ago. Based on a review by (Sachrajda, 1994), the recent extensive study by (Duncan et al., 1995) and the analyses in (Bernard et al., 1994), (Draper and McNeile, 1994) we conclude that $F_{B_{d}}=(180 \pm 40) \mathrm{MeV}$. This together with the earlier result of the European Collaboration (Abada et al., 1992) for $B_{B}$, gives $F_{B_{d}} \sqrt{B_{B_{d}}}=194 \pm 45 \mathrm{MeV}$. A 
reduction of the error in this important quantity is desirable. These results for $F_{B}$ are compatible with the results obtained using QCD sum rules (e.g. (Bagan et al., 1992), (Neubert, 1992)). An interesting upper bound $F_{B_{d}}<195 \mathrm{MeV}$ using QCD dispersion relations has also recently been obtained (Boyd et al., 1995). In our numerical analysis we will use

$$
\sqrt{B_{B_{d}}} F_{B_{d}}=(200 \pm 40) \mathrm{MeV}
$$

The accuracy of the determination of $R_{t}$ can be considerably improved by measuring simultaneously the $B_{s}^{0}-\bar{B}_{s}^{0}$ mixing described by $x_{s}$. We have

$$
R_{t}=\frac{1}{\sqrt{R_{d s}}} \sqrt{\frac{x_{d}}{x_{s}}} \frac{1}{\lambda} \sqrt{1-\lambda^{2}(1-2 \varrho)} \quad R_{d s}=\frac{\tau_{B_{d}}}{\tau_{B_{s}}} \frac{m_{B_{d}}}{m_{B_{s}}}\left[\frac{F_{B_{d}} \sqrt{B_{B_{d}}}}{F_{B_{s}} \sqrt{B_{B_{s}}}}\right]^{2} .
$$

Note that $m_{\mathrm{t}}$ and $\left|V_{c b}\right|$ have been eliminated in this way and that $R_{d s}$ depends only on $S U(3)$ flavour breaking effects which contain much smaller theoretical uncertainties than the hadronic matrix elements in $x_{d}$ and $x_{s}$ separately. Provided $x_{d} / x_{s}$ has been accurately measured a determination of $R_{t}$ within $\pm 10 \%$ should be possible. Indeed the most recent lattice results (Duncan et al., 1995), (Baxter et al., 1994) give $F_{B_{s}} / F_{B_{d}}=1.22 \pm 0.04$. A similar result $F_{B_{s}} / F_{B_{d}}=1.16 \pm 0.05$ has been obtained using QCD sum rules (Narison, 1994). It would be useful to know $B_{B_{s}} / B_{B_{d}}$ with a similar precision. For $B_{B_{s}}=B_{B_{d}}$ we find using the lattice result $R_{d s}=0.66 \pm 0.07$.

\section{C. $\sin (2 \beta)$ from $\varepsilon_{K}$ and $B^{0}-\bar{B}^{0}$ Mixing}

Combining (XVIII.10) and (XVIII.20) one can derive an analytic formula for $\sin (2 \beta)$ (Buras et al., 1994b)

$$
\sin (2 \beta)=\frac{1}{1.16 A^{2} \eta_{2} R_{0}^{2}}\left[\frac{0.226}{A^{2} B_{K}}-\bar{\eta} P_{0}(\varepsilon)\right]
$$

$P_{0}(\varepsilon)$ is weakly dependent on $m_{\mathrm{t}}$ and for $155 \mathrm{GeV} \leq m_{\mathrm{t}} \leq 185 \mathrm{GeV}$ one has $P_{0}(\varepsilon) \approx 0.31 \pm 0.02$. As $\bar{\eta} \leq 0.45$ for $\left|V_{u b} / V_{c b}\right| \leq 0.1$ the first term in parenthesis is generally by a factor of 2-3 larger than the second term. Since this dominant term is independent of $m_{\mathrm{t}}$, the values for $\sin (2 \beta)$ extracted from $\varepsilon_{K}$ and $B^{0}-\bar{B}^{0}$ mixing show only a weak dependence on $m_{\mathrm{t}}$ as stressed in particular in (Rosner, 1992).

Since in addition $A^{2} R_{0}^{2}$ is independent of $\left|V_{c b}\right|$, the dominant uncertainty in this determination of $\sin (2 \beta)$ resides in $A^{2} B_{K}$ in the first term in the parenthesis and in $F_{B_{d}} \sqrt{B_{B_{d}}}$ contained in $R_{0}^{2}$.

\section{Phenomenological Analysis}

We will now combine the analyses of $\varepsilon_{K}$ and of $B_{d}^{0}-\bar{B}_{d}^{0}$ mixing to obtain allowed ranges for several quantities of interest. We consider two sets of input parameters, which are collected in the appendix. The first set represents the present situation. The second set can be considered as a "future vision" in which the errors on various input parameters have been decreased. It is plausible that such errors will be achieved at the end of this decade, although one cannot guarantee that the central values will remain. In table XXXVII we show the results for $\delta, \operatorname{Im} \lambda_{t}, \sin 2 \alpha, \sin 2 \beta, \sin \gamma$, 
$\left|V_{t d}\right|$ and $x_{s}$. They correspond to the two sets of parameters in question, with and without the constraint from $B_{d}^{0}-\bar{B}_{d}^{0}$ mixing. The results for $\operatorname{Im} \lambda_{t}$ and $\left|V_{t d}\right|$ will play an important role in the phenomenology of rare decays and $\mathrm{CP}$ violation. For completeness we also show the expectations for $\sin 2 \alpha, \sin 2 \beta$ and $\sin \gamma$ which enter various $\mathrm{CP}$ asymmetries in B-decays. As already discussed in detail in (Buras et al., 1994b), $\sin 2 \alpha$ cannot be predicted accurately this way. On the other hand $\sin 2 \beta$ and $\sin \gamma$ are more constrained and the resulting ranges for these quantities indicate that large $\mathrm{CP}$ asymmetries should be observed in a variety of B-decays.

TABLE XXXVII. Predictions for various quantities using present and future input parameter ranges given in appendix A. $\operatorname{Im} \lambda_{t}$ and $\left|V_{t d}\right|$ are given in units of $10^{-4}$ and $10^{-3}$, respectively. $\delta$ is in degrees.

\begin{tabular}{|c|r|r|r|r|}
\hline \hline & \multicolumn{2}{|c|}{ no $x_{d}$ constraint } & \multicolumn{2}{c|}{ with $x_{d}$ constraint } \\
\hline & Present & Future & \multicolumn{1}{c|}{ Present } & \multicolumn{1}{c|}{ Future } \\
\hline$\delta$ & $37.7-160.0$ & $57.4-144.9$ & $37.7-140.2$ & $58.5-93.3$ \\
$\operatorname{Im} \lambda_{t}$ & $0.64-1.75$ & $0.82-1.50$ & $0.87-1.75$ & $1.12-1.50$ \\
$\left|V_{t d}\right|$ & $6.7-13.5$ & $7.7-12.1$ & $6.7-11.9$ & $7.8-9.3$ \\
$x_{s}$ & - & - & $11.1-47.0$ & $19.6-29.6$ \\
$\sin 2 \alpha$ & $-0.86-1.00$ & $-0.323-1.00$ & $-0.86-1.00$ & $-0.30-0.73$ \\
$\sin 2 \beta$ & $0.21-0.80$ & $0.34-0.73$ & $0.34-0.80$ & $0.57-0.73$ \\
$\sin \gamma$ & $0.34-1.00$ & $0.58-1.00$ & $0.61-1.00$ & $0.85-1.00$ \\
\hline \hline
\end{tabular}

In fig. 10 we show $\operatorname{Im} \lambda_{t}$ as a function of $m_{\mathrm{t}}$. In fig. 11 the lower bound on $\left|V_{u b} / V_{c b}\right|$ resulting from the $\varepsilon_{K}$-constraint is shown as a function of $\left|V_{c b}\right|$ for various values of $B_{K}$. To this end we have set $m_{\mathrm{t}}=185 \mathrm{GeV}$. For lower values of $m_{\mathrm{t}}$ the lower bound on $\left|V_{u b} / V_{c b}\right|$ is stronger. A similar analysis has been made by (Herrlich and Nierste, 1995a). The latter work and the plot in fig. 11 demonstrate clearly the impact of the $\varepsilon_{K}$ constraint on the allowed values of $\left|V_{u b} / V_{c b}\right|$ and $\left|V_{c b}\right|$. Simultaneously small values of $\left|V_{u b} / V_{c b}\right|$ and $\left|V_{c b}\right|$, although still consistent with treelevel decays, are not allowed by the size of the indirect $\mathrm{CP}$ violation observed in $K \rightarrow \pi \pi$. Another representation of this behaviour is shown in fig. 12 where we plot the minimal value of $B_{K}$ consistent with the experimental value of $\varepsilon_{K}$ as a function of $V_{c b}$ for different $\left|V_{u b} / V_{c b}\right|$ and $m_{\mathrm{t}}<185 \mathrm{GeV}$.

Finally in fig. 13 we show the allowed ranges in the $(\bar{\rho}, \bar{\eta})$ plane obtained using the information from $V_{c b},\left|V_{u b} / V_{c b}\right|, \varepsilon_{K}$ and $B_{d}^{0}-\bar{B}_{d}^{0}$ mixing. In this plot we also show the impact of a future measurement of $B_{s}^{0}-\bar{B}_{s}^{0}$ mixing with $x_{s}=10,15,25,40$, which by means of the formula (XVIII.25) gives an important measurement of the side $R_{t}$ of the unitarity triangle. Whereas at present a broad range in the $(\bar{\rho}, \bar{\eta})$ plane is allowed, the situation might change in the future allowing only the values $0 \leq \bar{\rho} \leq 0.2$ and $0.30 \leq \bar{\eta} \leq 0.40$. This results in smaller ranges for various quantities of interest as explicitly seen in table XXXVII.

Other analyses of the unitarity triangle can be found in (Peccei and Wang, 1995), (Ciuchini et al., 1995), (Herrlich and Nierste, 1995a), (Ali and London, 1995). 


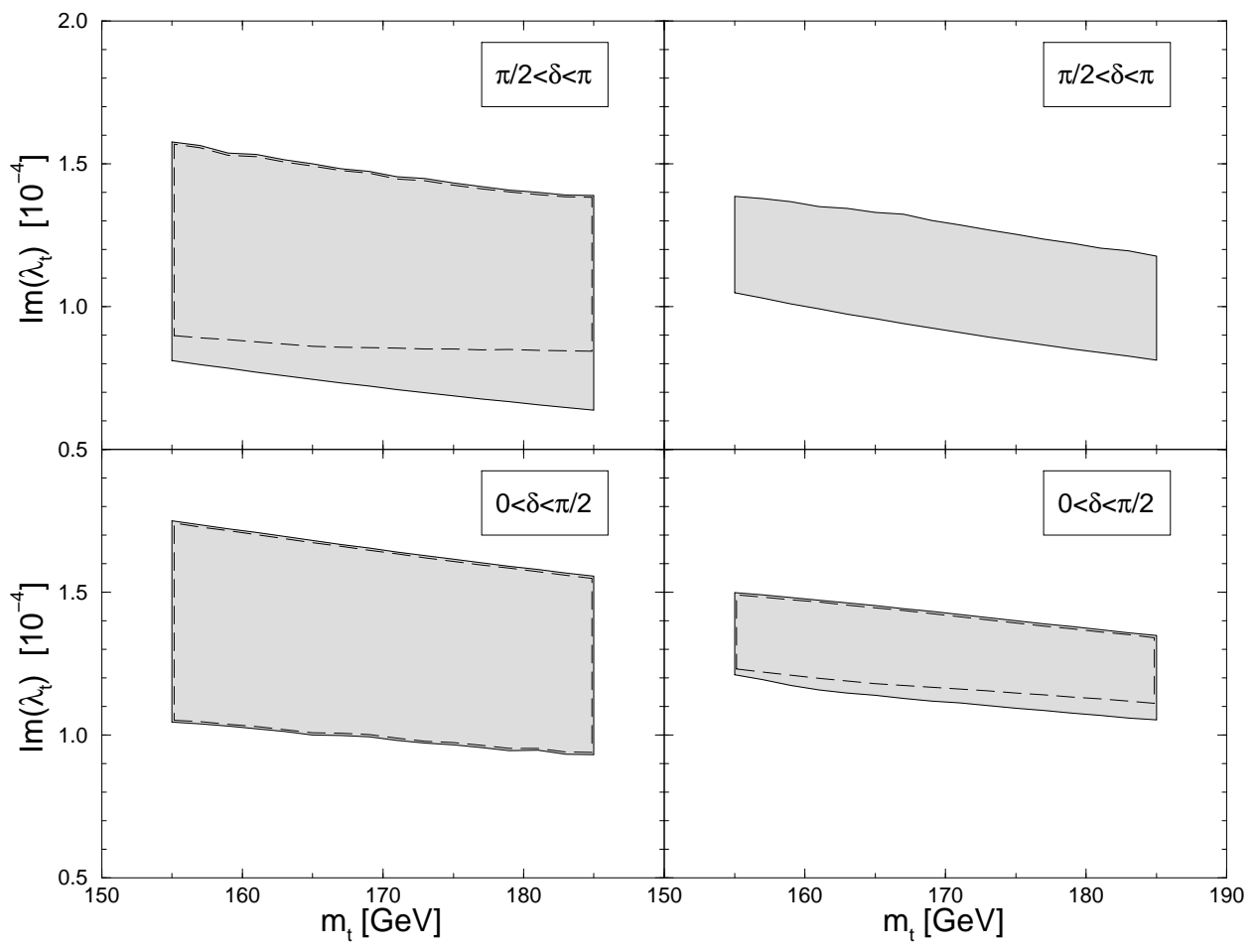

FIG. 10. Present (left) and future (right) allowed ranges for $\operatorname{Im}\left(\lambda_{t}\right)$. The ranges have been obtained by fitting $\varepsilon_{K}$ in (XVIII.8) to the experimental value. Input parameter ranges are given in appendix A. The impact of the additional constraint coming from $x_{d}$ is illustrated by the dashed lines. With the $x_{d}$ constraint imposed the solution $\pi / 2<\delta<\pi$ is completely eliminated for the future scenario. 


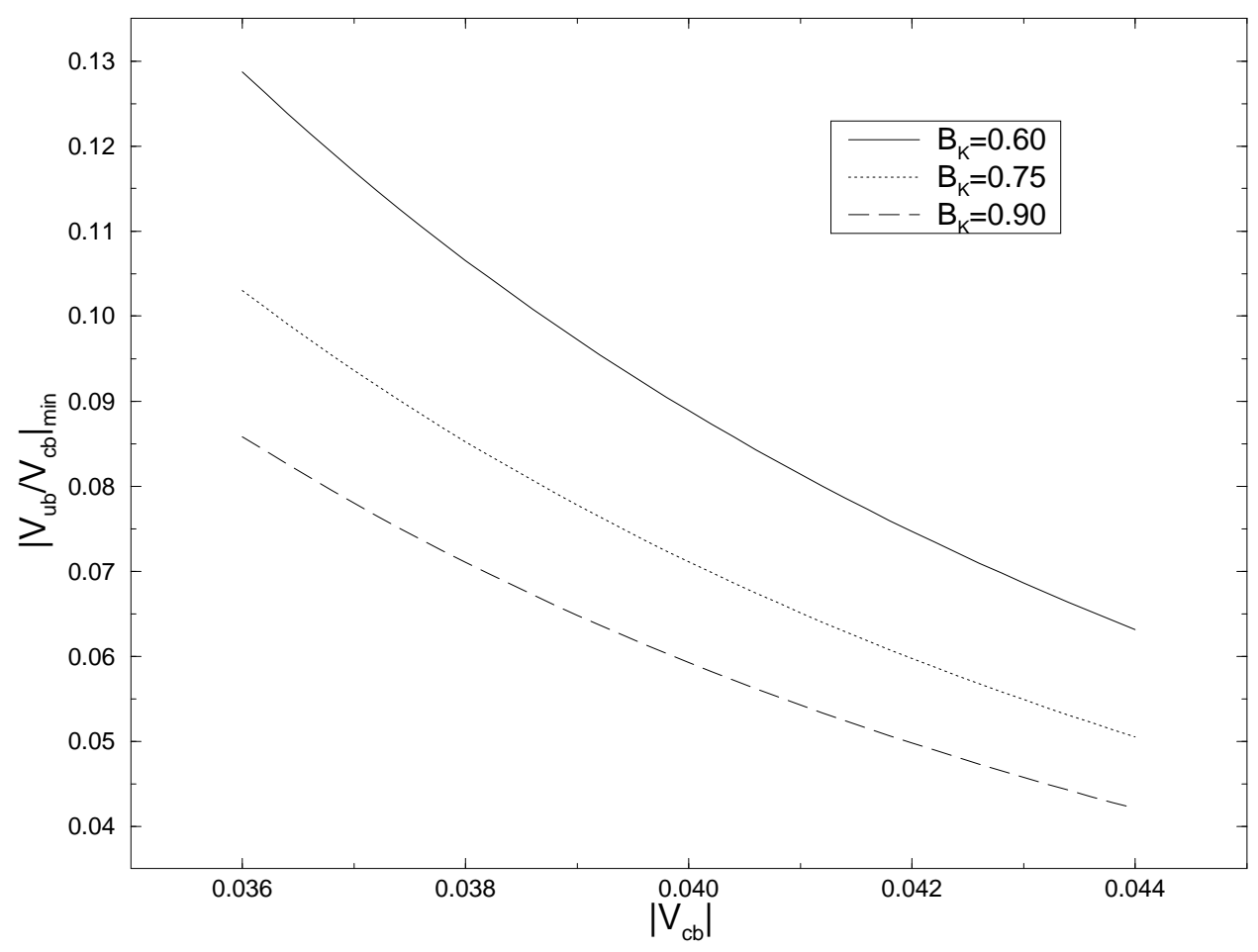

FIG. 11. $\left|V_{u b} / V_{c b}\right|_{\min }$ for $m_{\mathrm{t}} \leq 185 \mathrm{GeV}$ and various choices of $B_{K}$.

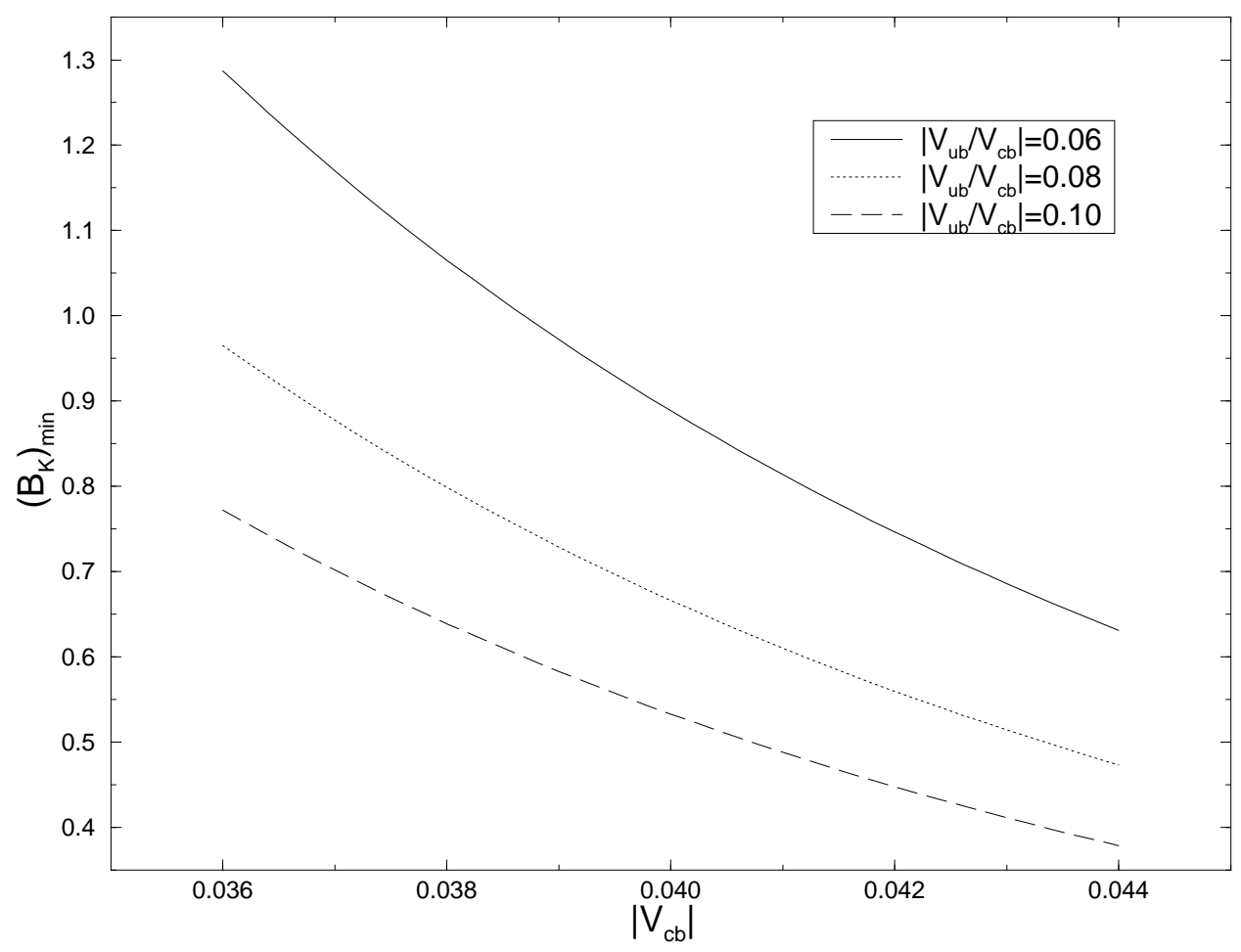

FIG. 12. $\left(B_{K}\right)_{\min }$ of eq. (XVIII.14) for $m_{\mathrm{t}} \leq 185 \mathrm{GeV}$ and various choices of $\left|V_{u b} / V_{c b}\right|$. 


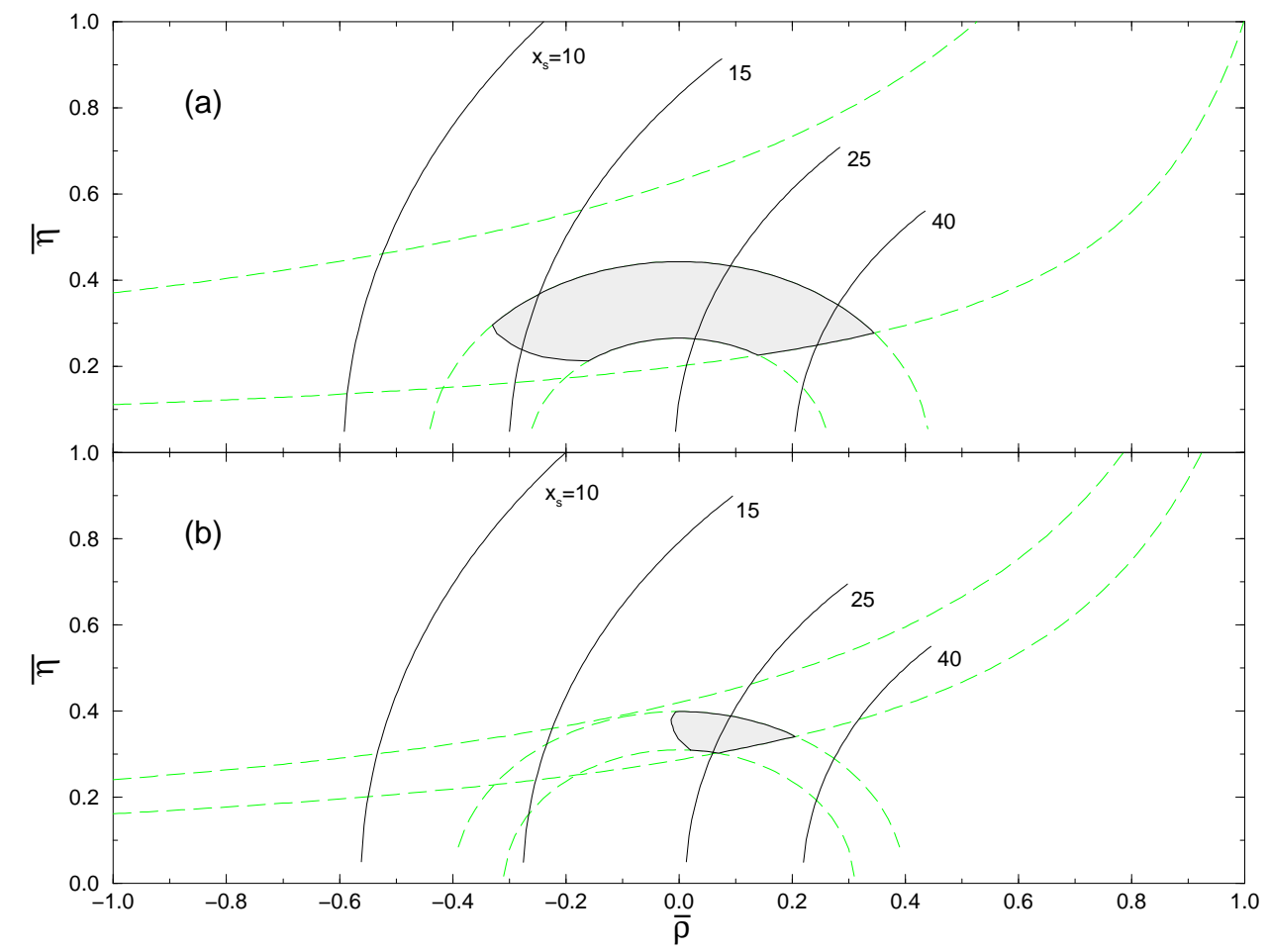

FIG. 13. Present (a) and future (b) allowed ranges for the upper corner A of the UT using data from $K^{0}-\bar{K}^{0}$-, $B^{0}-\bar{B}^{0}$-mixing and tree-level $B$-decays. Input parameter ranges are given in appendix A. The solid lines correspond to $\left(R_{t}\right)_{\max }$ from eq.(XVIII.25) using $R_{d s}=0.66$ and $x_{s} \geq 10,15,25$ and 40, respectively. 


\section{XIX. $\varepsilon^{\prime} / \varepsilon$ BEYOND LEADING LOGARITHMS}

\section{A. Basic Formulae}

The direct CP violation in $K \rightarrow \pi \pi$ is described by $\varepsilon^{\prime}$. The parameter $\varepsilon^{\prime}$ is given in terms of the amplitudes $A_{0} \equiv A\left(K \rightarrow(\pi \pi)_{I=0}\right)$ and $A_{2} \equiv A\left(K \rightarrow(\pi \pi)_{I=2}\right)$ as follows

$$
\varepsilon^{\prime}=-\frac{\omega}{\sqrt{2}} \xi(1-\Omega) \exp (i \Phi)
$$

where

$$
\xi=\frac{\operatorname{Im} A_{0}}{\operatorname{Re} A_{0}}, \quad \omega=\frac{\operatorname{Re} A_{2}}{\operatorname{Re} A_{0}}, \quad \Omega=\frac{1}{\omega} \frac{\operatorname{Im} A_{2}}{\operatorname{Im} A_{0}}
$$

and $\Phi=\pi / 2+\delta_{2}-\delta_{0} \approx \pi / 4$

When using (XIX.1) and (XIX.2) in phenomenological applications one usually takes $\operatorname{Re} A_{0}$ and $\omega$ from experiment, i.e.

$$
\operatorname{Re} A_{0}=3.33 \cdot 10^{-7} \mathrm{GeV} \quad \operatorname{Re} A_{2}=1.50 \cdot 10^{-8} \mathrm{GeV} \quad \omega=0.045
$$

where the last relation reflects the so-called $\Delta I=1 / 2$ rule. The main reason for this strategy is the unpleasant fact that until today nobody succeded in fully explaining this rule which to a large extent is believed to originate in the long-distance QCD contributions. We will be more specific about this in the next section. On the other hand the imaginary parts of the amplitudes in (XIX.2) being related to $\mathrm{CP}$ violation and the top quark physics should be dominated by short-distance contributions. Therefore $\operatorname{Im} A_{0}$ and $\operatorname{Im} A_{2}$ are usually calculated using the effective hamiltonian given in (VII.1). Using this hamiltonian and the experimental values for $\varepsilon, \operatorname{Re} A_{0}$ and $\omega$ the ratio $\varepsilon^{\prime} / \varepsilon$ can be written as follows

$$
\varepsilon^{\prime} / \varepsilon=\operatorname{Im} \lambda_{t}\left[P^{(1 / 2)}-P^{(3 / 2)}\right]
$$

where

$$
\begin{aligned}
& P^{(1 / 2)}=\sum P_{i}^{(1 / 2)}=r \sum y_{i}\left\langle Q_{i}\right\rangle_{0}\left(1-\Omega_{\eta+\eta^{\prime}}\right) \\
& P^{(3 / 2)}=\sum P_{i}^{(3 / 2)}=\frac{r}{\omega} \sum y_{i}\left\langle Q_{i}\right\rangle_{2}
\end{aligned}
$$

with

$$
r=\frac{G_{F} \omega}{2|\varepsilon| \operatorname{Re} A_{0}} .
$$

Here the hadronic matrix element shorthand notation is

$$
\left\langle Q_{i}\right\rangle_{I} \equiv\left\langle(\pi \pi)_{I}\left|Q_{i}\right| K\right\rangle
$$

and the sum in (XIX.5) and (XIX.6) runs over all contributing operators. This means for $\mu>m_{\mathrm{c}}$ also contributions from operators $Q_{1,2}^{c}$ to $P^{(1 / 2)}$ and $P^{(3 / 2)}$ have to be taken into account. These are necessary for $P^{(1 / 2)}$ and $P^{(3 / 2)}$ to be independent of the renormalization scale $\mu$. Next, 


$$
\Omega_{\eta+\eta^{\prime}}=\frac{1}{\omega} \frac{\left(\operatorname{Im} A_{2}\right)_{\text {I.B. }}}{\operatorname{Im} A_{0}}
$$

represents the contribution stemming from isospin breaking in the quark masses $\left(m_{u} \neq m_{d}\right)$. For $\Omega_{\eta+\eta^{\prime}}$ we will take

$$
\Omega_{\eta+\eta^{\prime}}=0.25 \pm 0.05
$$

which is in the ball park of the values obtained in the $1 / N_{c}$ approach (Buras and Gérard, 1987) and in chiral perturbation theory (Donoghue et al., 1986), (Lusignoli, 1989). $\Omega_{\eta+\eta^{\prime}}$ is independent of $m_{\mathrm{t}}$.

The numerical values of the Wilson coefficients $y_{i}$ have been already given in section VIIE. We therefore turn now our attention to the hadronic matrix elements (XIX.8) which constitute the main source of uncertainty in the calculation of $\varepsilon^{\prime} / \varepsilon$.

\section{B. Hadronic Matrix Elements for $K \rightarrow \pi \pi$}

The hadronic matrix elements $\left\langle Q_{i}\right\rangle_{I}$ depend generally on the renormalization scale $\mu$ and on the scheme used to renormalize the operators $Q_{i}$. These two dependences are canceled by those present in the Wilson coefficients $C_{i}(\mu)$ so that the resulting physical amplitudes do not depend on $\mu$ and on the renormalization scheme of the operators. Unfortunately the accuracy of the present non-perturbative methods used to evalutate $\left\langle Q_{i}\right\rangle_{I}$, like lattice methods or $1 / N_{c}$ expansion, is not sufficient to obtain the required $\mu$ and scheme dependences of $\left\langle Q_{i}\right\rangle_{I}$. A review of the existing methods and their comparison can be found in (Buras et al., 1993b), (Ciuchini et al., 1995). In view of this situation it has been suggested (Buras et al., 1993b) to determine as many matrix elements $\left\langle Q_{i}\right\rangle_{I}$ as possible from the leading CP conserving $K \rightarrow \pi \pi$ decays, for which the experimental data are summarized in (XIX.3). To this end it turned out to be very convenient to determine $\left\langle Q_{i}\right\rangle_{I}$ at a scale $\mu=m_{\mathrm{c}}$. Using the renormalization group evolution one can then find $\left\langle Q_{i}\right\rangle_{I}$ at any other scale $\mu \neq m_{\mathrm{c}}$. The details of this procedure can be found in (Buras et al., 1993b). Here we simply summarize the results of this work.

We first express the matrix elements $\left\langle Q_{i}\right\rangle_{I}$ in terms of the non-perturbative parameters $B_{i}^{(1 / 2)}$ and $B_{i}^{(3 / 2)}$ for $\left\langle Q_{i}\right\rangle_{0}$ and $\left\langle Q_{i}\right\rangle_{2}$, respectively. For $\mu \leq m_{\mathrm{c}}$ we have (Buras et al., 1993b)

$$
\begin{aligned}
\left\langle Q_{1}\right\rangle_{0} & =-\frac{1}{9} X B_{1}^{(1 / 2)} \\
\left\langle Q_{2}\right\rangle_{0} & =\frac{5}{9} X B_{2}^{(1 / 2)} \\
\left\langle Q_{3}\right\rangle_{0} & =\frac{1}{3} X B_{3}^{(1 / 2)} \\
\left\langle Q_{4}\right\rangle_{0} & =\left\langle Q_{3}\right\rangle_{0}+\left\langle Q_{2}\right\rangle_{0}-\left\langle Q_{1}\right\rangle_{0} \\
\left\langle Q_{5}\right\rangle_{0} & =\frac{1}{3} B_{5}^{(1 / 2)}\left\langle\overline{Q_{6}}\right\rangle_{0}, \\
\left\langle Q_{6}\right\rangle_{0} & =-4 \sqrt{\frac{3}{2}}\left[\frac{m_{\mathrm{K}}^{2}}{m_{\mathrm{s}}(\mu)+m_{\mathrm{d}}(\mu)}\right]^{2} \frac{F_{\pi}}{\kappa} B_{6}^{(1 / 2)}, \\
\left\langle Q_{7}\right\rangle_{0} & =-\left[\frac{1}{6}\left\langle\overline{Q_{6}}\right\rangle_{0}(\kappa+1)-\frac{X}{2}\right] B_{7}^{(1 / 2)},
\end{aligned}
$$




$$
\begin{aligned}
\left\langle Q_{8}\right\rangle_{0} & =-\left[\frac{1}{2}\left\langle\overline{Q_{6}}\right\rangle_{0}(\kappa+1)-\frac{X}{6}\right] B_{8}^{(1 / 2)}, \\
\left\langle Q_{9}\right\rangle_{0}=\frac{3}{2}\left\langle Q_{1}\right\rangle_{0}-\frac{1}{2}\left\langle Q_{3}\right\rangle_{0} & \\
\left\langle Q_{10}\right\rangle_{0}=\left\langle Q_{2}\right\rangle_{0}+\frac{1}{2}\left\langle Q_{1}\right\rangle_{0}-\frac{1}{2}\left\langle Q_{3}\right\rangle_{0} & \\
\left\langle Q_{1}\right\rangle_{2} & =\left\langle Q_{2}\right\rangle_{2}=\frac{4 \sqrt{2}}{9} X B_{1}^{(3 / 2)} \\
\left\langle Q_{i}\right\rangle_{2} & =0, \quad i=3, \ldots, 6 \\
\left\langle Q_{7}\right\rangle_{2} & =-\left[\frac{\kappa}{6 \sqrt{2}}\left\langle\overline{Q_{6}}\right\rangle_{0}+\frac{X}{\sqrt{2}}\right] B_{7}^{(3 / 2)}, \\
\left\langle Q_{8}\right\rangle_{2} & =-\left[\frac{\kappa}{2 \sqrt{2}}\left\langle\overline{Q_{6}}\right\rangle_{0}+\frac{\sqrt{2}}{6} X\right] B_{8}^{(3 / 2)} \\
\left\langle Q_{9}\right\rangle_{2} & =\left\langle Q_{10}\right\rangle_{2}=\frac{3}{2}\left\langle Q_{1}\right\rangle_{2},
\end{aligned}
$$

where

$$
\begin{gathered}
\kappa=\frac{\Lambda_{\chi}^{2}}{m_{\mathrm{K}}^{2}-m_{\pi}^{2}}=\frac{F_{\pi}}{F_{\mathrm{K}}-F_{\pi}}, \\
X=\sqrt{\frac{3}{2}} F_{\pi}\left(m_{\mathrm{K}}^{2}-m_{\pi}^{2}\right),
\end{gathered}
$$

and

$$
\left\langle\overline{Q_{6}}\right\rangle_{0}=\frac{\left\langle Q_{6}\right\rangle_{0}}{B_{6}^{(1 / 2)}} .
$$

The actual numerical values used for $m_{\mathrm{K}}, m_{\pi}, F_{\mathrm{K}}, F_{\pi}$ are collected in appendix A.

In the vacuum insertion method $B_{i}=1$ independent of $\mu$. In QCD, however, the hadronic parameters $B_{i}$ generally depend on the renormalizations scale $\mu$ and the renormalization scheme considered.

\section{C. $\left\langle Q_{i}(\mu)\right\rangle_{2}$ for $(V-A) \otimes(V-A)$ Operators}

The matrix elements $\left\langle Q_{1}\right\rangle_{2},\left\langle Q_{2}\right\rangle_{2},\left\langle Q_{9}\right\rangle_{2}$ and $\left\langle Q_{10}\right\rangle_{2}$ can to a very good approximation be determined from $\operatorname{Re} A_{2}$ in (XIX.3) as functions of $\Lambda_{\overline{\mathrm{MS}}}, \mu$ and the renormalization scheme considered. To this end it is useful to set $\alpha=0$, as the $\mathcal{O}(\alpha)$ effects in CP conserving amplitudes, such as the contributions of electroweak penguins, are very small. One then finds

$$
\left\langle Q_{1}(\mu)\right\rangle_{2}=\left\langle Q_{2}(\mu)\right\rangle_{2}=\frac{10^{6} \mathrm{GeV}^{2}}{1.77} \frac{\operatorname{Re} A_{2}}{z_{+}(\mu)}=\frac{8.47 \cdot 10^{-3} \mathrm{GeV}^{3}}{z_{+}(\mu)}
$$

and comparing with (XIX.21) 


$$
B_{1}^{(3 / 2)}(\mu)=\frac{0.363}{z_{+}(\mu)}
$$

with $z_{+}=z_{1}+z_{2}$. Since $z_{+}(\mu)$ depends on the scale $\mu$ and the renormalization scheme used, (XIX.30) gives automatically the scheme and $\mu$ dependence of $B_{1}^{(3 / 2)}$ and of the related matrix elements $\left\langle Q_{1}\right\rangle_{2},\left\langle Q_{2}\right\rangle_{2},\left\langle Q_{9}\right\rangle_{2}$ and $\left\langle Q_{10}\right\rangle_{2}$. The impact of $\mathcal{O}(\alpha)$ corrections on this result has been analysed in (Buras et al., 1993b). It amounts only to a few percent as expected. These corrections are of course included in the numerical analysis presented in this reference and here as well. Using $\mu=m_{\mathrm{c}}=1.3 \mathrm{GeV}, \Lambda \frac{(4)}{\mathrm{MS}}=325 \mathrm{MeV}$ and $z_{+}\left(m_{\mathrm{c}}\right)$ of table XIX we find according to (XIX.30)

$$
B_{1, N D R}^{(3 / 2)}\left(m_{\mathrm{c}}\right)=0.453 \quad B_{1, H V}^{(3 / 2)}\left(m_{\mathrm{c}}\right)=0.472 .
$$

The following comments should be made:

- $B_{1}^{(3 / 2)}(\mu)$ decreases with increasing $\mu$.

- The extracted value for $B_{1}^{(3 / 2)}$ is by more than a factor of two smaller than the vacuum insertion estimate.

- It is compatible with the $1 / N_{c}$ value $B_{1}^{(3 / 2)}(1 \mathrm{GeV}) \approx 0.55$ (Bardeen et al., 1987a) and somewhat smaller than the lattice result $B_{1}^{(3 / 2)}(2 \mathrm{GeV}) \approx 0.6$ (Ciuchini et al., 1995).

\section{D. $\left\langle Q_{i}(\mu)\right\rangle_{0}$ for $(V-A) \otimes(V-A)$ Operators}

The determination of $\left\langle Q_{i}(\mu)\right\rangle_{0}$ matrix elements is more involved because several operators may contribute to $\operatorname{Re} A_{0}$. The main idea of (Buras et al., 1993b) is then to set $\mu=m_{\mathrm{c}}$, as at this scale only $Q_{1}$ and $Q_{2}$ operators contribute to $\operatorname{Re} A_{0}$ in the $\mathrm{HV}$ scheme. One then finds $\left\langle Q_{1}\left(m_{\mathrm{c}}\right)\right\rangle_{0}$ as a function of $\left\langle Q_{2}\left(m_{\mathrm{c}}\right)\right\rangle_{0}$

$$
\left\langle Q_{1}\left(m_{\mathrm{c}}\right)\right\rangle_{0}=\frac{10^{6} \mathrm{GeV}^{2}}{1.77} \frac{\operatorname{Re} A_{0}}{z_{1}\left(m_{\mathrm{c}}\right)}-\frac{z_{2}\left(m_{\mathrm{c}}\right)}{z_{1}\left(m_{\mathrm{c}}\right)}\left\langle Q_{2}\left(m_{\mathrm{c}}\right)\right\rangle_{0}
$$

where the reference in $\left\langle Q_{1,2}\left(m_{\mathrm{c}}\right)\right\rangle_{0}$ to the HV scheme has been suppressed for convenience. Using next the relations (XIX.14), (XIX.19) and (XIX.20) one is able to obtain $\left\langle Q_{4}\left(m_{\mathrm{c}}\right)\right\rangle_{0},\left\langle Q_{9}\left(m_{\mathrm{c}}\right)\right\rangle_{0}$ and $\left\langle Q_{10}\left(m_{\mathrm{c}}\right)\right\rangle_{0}$ as functions of $\left\langle Q_{2}\left(m_{\mathrm{c}}\right)\right\rangle_{0}$ and $\left\langle Q_{3}\left(m_{\mathrm{c}}\right)\right\rangle_{0}$. Because $\left\langle Q_{3}\left(m_{\mathrm{c}}\right)\right\rangle_{0}$ is colour suppressed it is less essential for this analysis than $\left\langle Q_{2}\left(m_{\mathrm{c}}\right)\right\rangle_{0}$. Moreover its Wilson coefficient is small and similarly to $\left\langle Q_{9}\left(m_{\mathrm{c}}\right)\right\rangle_{0}$ and $\left\langle Q_{10}\left(m_{\mathrm{c}}\right)\right\rangle_{0}$ also $\left\langle Q_{3}\left(m_{\mathrm{c}}\right)\right\rangle_{0}$ has only a small impact on $\varepsilon^{\prime} / \varepsilon$. On the other hand the coefficient $y_{4}$ is substantial and consequently $\left\langle Q_{4}\left(m_{\mathrm{c}}\right)\right\rangle_{0}$ plays a considerable role in the analysis of $\varepsilon^{\prime} / \varepsilon$. The matrix element $\left\langle Q_{3}\left(m_{\mathrm{c}}\right)\right\rangle_{0}$ has then an indirect impact on $\varepsilon^{\prime} / \varepsilon$ through relation (XIX.14). For numerical evaluation, $\left\langle Q_{3}\left(m_{\mathrm{c}}\right)\right\rangle_{0}$ of (XIX.13) with $B_{3}^{(1 / 2)}=1$ can be used keeping in mind that this may introduce a small uncertainty in the final analysis. This uncertainty has been investigated in (Buras et al., 1993b).

Once the matrix elements in question have been determined as functions of $\left\langle Q_{2}\left(m_{\mathrm{c}}\right)\right\rangle_{0}$ in the HV scheme, they can be found by a finite renormalization in any other scheme. Details can be found in (Buras et al., 1993b). 
If one in addition makes the very plausible assumption valid in all known non-perturbative approaches that $\left\langle Q_{-}\left(m_{\mathrm{c}}\right)\right\rangle_{0} \geq\left\langle Q_{+}\left(m_{\mathrm{c}}\right)\right\rangle_{0} \geq 0$ the experimental value of $\operatorname{Re} A_{0}$ in (XIX.3) together with (XIX.32) and table XIX implies for $\Lambda \frac{(4)}{\mathrm{MS}}=325 \mathrm{MeV}$

$$
B_{2, L O}^{(1 / 2)}\left(m_{\mathrm{c}}\right)=5.7 \pm 1.1 \quad B_{2, N D R}^{(1 / 2)}\left(m_{\mathrm{c}}\right)=6.6 \pm 1.0 \quad B_{2, H V}^{(1 / 2)}\left(m_{\mathrm{c}}\right)=6.2 \pm 1.0
$$

The extraction of $B_{1}^{(1 / 2)}\left(m_{\mathrm{c}}\right)$ and of an analogous parameter $B_{4}^{(1 / 2)}\left(m_{\mathrm{c}}\right)$ are presented in detail in (Buras et al., 1993b). $B_{1}^{(1 / 2)}\left(m_{\mathrm{c}}\right)$ depends very sensitively on $B_{2}^{(1 / 2)}\left(m_{\mathrm{c}}\right)$ and its central value is as high as $15 . B_{4}^{(1 / 2)}\left(m_{\mathrm{c}}\right)$ is less sensitive and typically by $(10-15) \%$ lower than $B_{2}^{(1 / 2)}\left(m_{\mathrm{c}}\right)$. In any case this analysis shows very large departures from the results of the vacuum insertion method.

\section{E. $\left\langle Q_{i}(\mu)\right\rangle_{0,2}$ for $(V-A) \otimes(V+A)$ Operators}

The matrix elements of the $(V-A) \otimes(V+A)$ operators $Q_{5}-Q_{8}$ cannot be constrained by $\mathrm{CP}$ conserving data and one has to rely on existing non-perturbative methods to calculate them. Fortunately, there are some indications that the existing non-perturbative estimates of $\left\langle Q_{i}(\mu)\right\rangle_{0,2}$, $i=5, \ldots, 8$ are more reliable than the corresponding calculations for $(V-A) \otimes(V-A)$ operators.

First of all, the parameters $B_{5,6}^{(1 / 2)}$ (Kilcup, 1991), (Sharpe, 1991) and $B_{7,8}^{(3 / 2)}$ (Franco et al., 1989), (Kilcup, 1991), (Sharpe, 1991), (Bernard and Soni, 1991) calculated in the lattice approach

$$
B_{5,6}^{(1 / 2)}=1.0 \pm 0.2 \quad B_{7,8}^{(3 / 2)}=1.0 \pm 0.2
$$

agree well with the vacuum insertion values $\left(B_{i}=1\right)$ and in the case of $B_{6}^{(1 / 2)}$ and $B_{8}^{(3 / 2)}$ with the $1 / N_{c}$ approach $\left(B_{6}^{(1 / 2)}=B_{8}^{(3 / 2)}=1\right.$ ) (Bardeen et al., 1987b), (Buras and Gérard, 1987).

We note next that with fixed values for $B_{5,6}^{(1 / 2)}$ and $B_{7,8}^{(3 / 2)}$ the $\mu$-dependence of $\left\langle Q_{5,6}\right\rangle_{0}$ and $\left\langle Q_{7,8}\right\rangle_{2}$ is governed by the $\mu$ dependence of $m_{\mathrm{s}}(\mu)$. For $\left\langle Q_{6}\right\rangle_{0}$ and $\left\langle Q_{8}\right\rangle_{2}$ this property has been first found in the $1 / N_{c}$ approach (Buras and Gérard, 1987): in the large- $N_{c}$ limit the anomalous dimensions of $Q_{6}$ and $Q_{8}$ are simply twice the anomalous dimension of the mass operator leading to $\sim 1 / m_{\mathrm{s}}^{2}(\mu)$ for the corresponding matrix elements. Another support comes from a renormalization study in (Buras et al., 1993b). In this analysis the $B_{i}$-factors in (XIX.34) have been set to unity at $\mu=m_{\mathrm{c}}$. Subsequently the evolution of the matrix elements in the range $1 \mathrm{GeV} \leq \mu \leq 4 \mathrm{GeV}$ has been calculated showing that for the NDR scheme $B_{5,6}^{(1 / 2)}$ and $B_{7,8}^{(3 / 2)}$ were $\mu$ independent within an accuracy of (2-3) \%. The $\mu$ dependence in the HV scheme has been found to be stronger but still below $10 \%$.

Concerning $B_{7,8}^{(1 / 2)}$ one can simply set $B_{7,8}^{(1 / 2)}=1$ as the matrix elementes $\left\langle Q_{7,8}\right\rangle_{0}$ play only a minor role in the $\varepsilon^{\prime} / \varepsilon$ analysis.

In summary, our treatment of $\left\langle Q_{i}\right\rangle_{0,2}, i=5, \ldots 8$ follows the one used in (Buras et al., 1993b). We will set

$$
B_{7,8}^{(1 / 2)}\left(m_{\mathrm{c}}\right)=1 \quad B_{5}^{(1 / 2)}\left(m_{\mathrm{c}}\right)=B_{6}^{(1 / 2)}\left(m_{\mathrm{c}}\right) \quad B_{7}^{(3 / 2)}\left(m_{\mathrm{c}}\right)=B_{8}^{(3 / 2)}\left(m_{\mathrm{c}}\right)
$$

and we will treat $B_{6}^{(1 / 2)}\left(m_{\mathrm{c}}\right)$ and $B_{8}^{(3 / 2)}\left(m_{\mathrm{c}}\right)$ as free parameters in the neighbourhood of the values given in (XIX.34). Then the main uncertainty in the values of $\left\langle Q_{i}\right\rangle_{0,2}, i=5, \ldots 8$ results from the value of the strange quark mass $m_{\mathrm{s}}\left(m_{\mathrm{c}}\right)$. The present estimates give 


$$
m_{\mathrm{s}}\left(m_{\mathrm{c}}\right)=(170 \pm 20) \mathrm{MeV}
$$

with the lower values coming from recent lattice calculations (Allton et al., 1994) and the higher ones from QCD sum rules (Jamin and Münz, 1995), (Chetyrkin et al., 1995).

\section{F. The Four Dominant Contributions to $\varepsilon^{\prime} / \varepsilon$}

$P^{(1 / 2)}$ and $P^{(3 / 2)}$ in (XIX.4) can be written as linear combinations of two independent hadronic parameters $B_{6}^{(1 / 2)}$ and $B_{8}^{(3 / 2)}$ (Buras et al., 1993b). This $B_{i}$-expansion reads

$$
\begin{aligned}
& P^{(1 / 2)}=a_{0}^{(1 / 2)}+\left[\frac{178 \mathrm{MeV}}{m_{\mathrm{s}}\left(m_{\mathrm{c}}\right)+m_{\mathrm{d}}\left(m_{\mathrm{c}}\right)}\right]^{2} a_{6}^{(1 / 2)} B_{6}^{(1 / 2)} \\
& P^{(3 / 2)}=a_{0}^{(3 / 2)}+\left[\frac{178 \mathrm{MeV}}{m_{\mathrm{s}}\left(m_{\mathrm{c}}\right)+m_{\mathrm{d}}\left(m_{\mathrm{c}}\right)}\right]^{2} a_{8}^{(3 / 2)} B_{8}^{(3 / 2)} .
\end{aligned}
$$

Here $a_{0}^{(1 / 2)}$ and $a_{0}^{(3 / 2)}$ effectively summarize all dependences other than $B_{6}^{(1 / 2)}$ and $B_{8}^{(3 / 2)}$, especially $B_{2}^{(1 / 2)}$ in the case of $a_{0}^{(1 / 2)}$. Note that in contrast to (Buras et al., 1993b) we have absorbed the dependence on $B_{2}^{(1 / 2)}$ into $a_{0}^{(1 / 2)}$ and we have exhibited the dependence on $m_{\mathrm{s}}$ which was not shown explicitly there. The residual $m_{\mathrm{s}}$ dependence present in $a_{0}^{(1 / 2)}$ and $a_{0}^{(3 / 2)}$ is negligible. Setting $\mu=m_{\mathrm{c}}$, and using the strategy for hadronic matrix elements outlined above one finds the coefficients $a_{i}^{(1 / 2)}$ and $a_{i}^{(3 / 2)}$ as functions of $\Lambda_{\overline{\mathrm{MS}}}, m_{\mathrm{t}}$ and the renormalization scheme considered. These dependences are given in tables XXXVIII and XXXIX. We should however stress that $P^{(1 / 2)}$ and $P^{(3 / 2)}$ are independent of $\mu$ and the renormalization scheme considered.

TABLE XXXVIII. $B_{i}$-expansion coefficients for $P^{(1 / 2)}$.

\begin{tabular}{|c|c||c|r||r|r||r|r|}
\hline \hline & \multicolumn{1}{|c||}{} & \multicolumn{2}{c||}{ LO } & \multicolumn{2}{c||}{ NDR } & \multicolumn{2}{c|}{ HV } \\
\hline$\Lambda_{\mathrm{MS}}^{(4)}[\mathrm{MeV}]$ & $m_{\mathrm{t}}[\mathrm{GeV}]$ & $a_{0}^{(1 / 2)}$ & $a_{6}^{(1 / 2)}$ & $a_{0}^{(1 / 2)}$ & $a_{6}^{(1 / 2)}$ & $a_{0}^{(1 / 2)}$ & $a_{6}^{(1 / 2)}$ \\
\hline \multirow{3}{*}{215} & 155 & -2.138 & 5.110 & -2.251 & 4.676 & -2.215 & 4.159 \\
& 170 & -2.070 & 5.138 & -2.187 & 4.698 & -2.150 & 4.181 \\
& 185 & -1.996 & 5.162 & -2.117 & 4.716 & -2.081 & 4.200 \\
\hline \multirow{3}{*}{325} & 155 & -2.231 & 6.540 & -2.414 & 6.255 & -2.362 & 5.389 \\
& 170 & -2.161 & 6.576 & -2.350 & 6.282 & -2.298 & 5.416 \\
& 185 & -2.085 & 6.606 & -2.281 & 6.306 & -2.229 & 5.439 \\
\hline \multirow{3}{*}{435} & 155 & -2.288 & 8.171 & -2.549 & 8.417 & -2.473 & 6.972 \\
& 170 & -2.212 & 8.214 & -2.482 & 8.451 & -2.406 & 7.005 \\
& 185 & -2.130 & 8.251 & -2.409 & 8.480 & -2.333 & 7.035 \\
\hline \hline
\end{tabular}

Inspecting (XIX.37), (XIX.38) and tables XXXVIII, XXXIX we identify the following four contributions which govern the ratio $\varepsilon^{\prime} / \varepsilon$ at scales $\mu=\mathcal{O}\left(m_{\mathrm{c}}\right)$ :

i. The contribution of $(V-A) \otimes(V-A)$ operators to $P^{(1 / 2)}$ is dominantly represented by $a_{0}^{(1 / 2)}$. This term is to a large extent fixed by the experimental value of $A_{0}$ and consequently 
TABLE XXXIX. $B_{i}$-expansion coefficients for $P^{(3 / 2)}$.

\begin{tabular}{|c|c||c|r||r|r||r|r|}
\hline \hline & \multicolumn{1}{|c||}{} & \multicolumn{3}{|c||}{ LO } & \multicolumn{2}{c||}{ NDR } & \multicolumn{2}{c|}{ HV } \\
\hline$\Lambda \frac{(4)}{\mathrm{MS}}[\mathrm{MeV}]$ & $m_{\mathrm{t}}[\mathrm{GeV}]$ & $a_{0}^{(3 / 2)}$ & $a_{8}^{(3 / 2)}$ & $a_{0}^{(3 / 2)}$ & $a_{8}^{(3 / 2)}$ & $a_{0}^{(3 / 2)}$ & $a_{8}^{(3 / 2)}$ \\
\hline \multirow{3}{*}{215} & 155 & -0.797 & 1.961 & -0.819 & 1.887 & -0.838 & 2.114 \\
& 170 & -0.880 & 2.602 & -0.900 & 2.438 & -0.919 & 2.666 \\
& 185 & -0.965 & 3.296 & -0.983 & 3.036 & -1.002 & 3.263 \\
\hline \multirow{3}{*}{325} & 155 & -0.788 & 2.645 & -0.814 & 2.639 & -0.837 & 2.894 \\
& 170 & -0.870 & 3.422 & -0.895 & 3.305 & -0.917 & 3.560 \\
& 185 & -0.956 & 4.264 & -0.978 & 4.027 & -1.000 & 4.281 \\
\hline \multirow{3}{*}{435} & 155 & -0.779 & 3.425 & -0.809 & 3.622 & -0.835 & 3.899 \\
& 170 & -0.861 & 4.360 & -0.889 & 4.435 & -0.915 & 4.712 \\
& 185 & -0.947 & 5.372 & -0.971 & 5.316 & -0.998 & 5.593 \\
\hline \hline
\end{tabular}

is only very weakly dependent on $\Lambda_{\overline{\mathrm{MS}}}$ and the renormalization scheme considered. The weak dependence on $m_{\mathrm{t}}$ results from small contributions of electroweak penguin operators. Taking $\Lambda_{\mathrm{MS}}^{(4)}=325 \mathrm{MeV}, \mu=m_{\mathrm{c}}$ and $m_{\mathrm{t}}=170 \mathrm{GeV}$ we have $a_{0}^{(1 / 2)} \approx-2.3$ for both schemes considered. We observe that the contribution of $(V-A) \otimes(V-A)$ operators, in particular $Q_{4}$, to $\varepsilon^{\prime} / \varepsilon$ is negative.

ii. The contribution of $(V-A) \otimes(V+A)$ QCD penguin operators to $P^{(1 / 2)}$ is given by the second term in (XIX.37). This contribution is large and positive. The coefficient $a_{6}^{(1 / 2)}$ depends sensitively on $\Lambda_{\overline{\mathrm{MS}}}$ which results from the strong dependence of $y_{6}$ on the QCD scale. The dependence on $m_{\mathrm{t}}$ is very weak on the other hand. Taking $\Lambda \frac{(4)}{\mathrm{MS}}=325 \mathrm{MeV}$, $m_{\mathrm{s}}\left(m_{\mathrm{c}}\right)=170 \mathrm{MeV}$ and $m_{\mathrm{t}}=170 \mathrm{GeV}$ and setting as an example $B_{6}^{(1 / 2)}=1$ in the NDR and $\mathrm{HV}$ schemes we find a positve contribution to $\varepsilon^{\prime} / \varepsilon$ amounting to 6.3 and 5.4 in the NDR and HV scheme, respectively.

iii. The contribution of the $(V-A) \otimes(V-A)$ electroweak penguin operators $Q_{9}$ and $Q_{10}$ to $P^{(3 / 2)}$ is represented by $a_{0}^{(3 / 2)}$. As in the case of the contribution $\mathrm{i}$, the matrix elements contributing to $a_{0}^{(3 / 2)}$ are fixed by the CP conserving data, this time by the amplitude $A_{2}$. Consequently, the scheme and the $\Lambda_{\overline{\mathrm{MS}}}$ dependence of $a_{0}^{(3 / 2)}$ is very weak. The sizeable $m_{\mathrm{t}}$ dependence of $a_{0}^{(3 / 2)}$ results from the $m_{\mathrm{t}}$ dependence of $y_{9}+y_{10} \cdot a_{0}^{(3 / 2)}$ contributes positively to $\varepsilon^{\prime} / \varepsilon$. For $m_{\mathrm{t}}=170 \mathrm{GeV}$ this contribution is roughly 0.9 for both renormalization schemes and the full range of $\Lambda_{\overline{\mathrm{MS}}}$ considered.

iv. The contribution of the $(V-A) \otimes(V+A)$ electroweak penguin operators $Q_{7}$ and $Q_{8}$ to $P^{(3 / 2)}$ is represented by the second term in (XIX.38). This contribution depends sensitively on $m_{\mathrm{t}}$ and $\Lambda_{\overline{\mathrm{MS}}}$ as could be expected on the basis of $y_{7}$ and $y_{8}$. Taking again $B_{8}^{(3 / 2)}=1$ in both renormalization schemes we find for the central values of $\Lambda_{\overline{\mathrm{MS}}}^{(4)}, m_{\mathrm{t}}$ and $m_{\mathrm{c}}$ a negative contribution to $\varepsilon^{\prime} / \varepsilon$ equal to -3.9 and -3.6 for the NDR and HV scheme, respecetively.

Before analysing $\varepsilon^{\prime} / \varepsilon$ numerically in more detail, let us just set $\operatorname{Im} \lambda_{t}=1.3 \cdot 10^{-4}$ and $B_{6}^{(1 / 2)}=$ 
$B_{8}^{(3 / 2)}=1$ in both schemes. Then for the central values of the remaining parameters one obtains $\varepsilon^{\prime} / \varepsilon=2.0 \cdot 10^{-4}$ and $\varepsilon^{\prime} / \varepsilon=0.6 \cdot 10^{-4}$ for the NDR and HV scheme, respectively. This strong scheme dependence can only be compensated for by having $B_{6}^{(1 / 2)}$ and $B_{8}^{(3 / 2)}$ different in the two schemes considered. As we will see below the strong cancellations between various contributions at $m_{\mathrm{t}} \approx 170 \mathrm{GeV}$ make the prediction for $\varepsilon^{\prime} / \varepsilon$ rather uncertain. One should also stress that the formulation presented here does not exhibit analytically the $m_{\mathrm{t}}$ dependence. As the coefficients $a_{0}^{(3 / 2)}$ and $a_{8}^{(3 / 2)}$ depend very sensitively on $m_{\mathrm{t}}$ it is useful to display this dependence in an analytic form.

\section{G. An Analytic Formula for $\varepsilon^{\prime} / \varepsilon$}

As shown in (Buras and Lautenbacher, 1993) it is possible to cast the above discussion into an analytic formula which exhibits the $m_{\mathrm{t}}$ dependence together with the dependence on $m_{\mathrm{s}}, B_{6}^{(1 / 2)}$ and $B_{8}^{(3 / 2)}$. Such an analytic formula should be useful for those phenomenologists and experimentalists who are not interested in getting involved with the technicalities discussed in preceding sections.

In order to find an analytic expression for $\varepsilon^{\prime} / \varepsilon$ which exactly reproduces the results discussed above one uses the PBE presented in section XIV. The resulting analytic expression for $\varepsilon^{\prime} / \varepsilon$ is then given as follows

$$
\varepsilon^{\prime} / \varepsilon=\operatorname{Im} \lambda_{t} F\left(x_{t}\right)
$$

where

$$
F\left(x_{t}\right)=P_{0}+P_{X} X_{0}\left(x_{t}\right)+P_{Y} Y_{0}\left(x_{t}\right)+P_{Z} Z_{0}\left(x_{t}\right)+P_{E} E_{0}\left(x_{t}\right)
$$

with the $m_{\mathrm{t}}$ dependent functions listed in section XIV. The coefficients $P_{i}$ are given in terms of $B_{6}^{(1 / 2)} \equiv B_{6}^{(1 / 2)}\left(m_{\mathrm{c}}\right), B_{8}^{(3 / 2)} \equiv B_{8}^{(3 / 2)}\left(m_{\mathrm{c}}\right)$ and $m_{\mathrm{s}}\left(m_{\mathrm{c}}\right)$ as follows

$$
P_{i}=r_{i}^{(0)}+\left[\frac{178 \mathrm{MeV}}{m_{\mathrm{s}}\left(m_{\mathrm{c}}\right)+m_{\mathrm{d}}\left(m_{\mathrm{c}}\right)}\right]^{2}\left(r_{i}^{(6)} B_{6}^{(1 / 2)}+r_{i}^{(8)} B_{8}^{(3 / 2)}\right) .
$$

The $P_{i}$ are $\mu$ and renormalization scheme independent. They depend however on $\Lambda_{\overline{\mathrm{MS}}}$. In table XL we give the numerical values of $r_{i}^{(0)}, r_{i}^{(6)}$ and $r_{i}^{(8)}$ for different values of $\Lambda_{\overline{\mathrm{MS}}}$ at $\mu=m_{\mathrm{c}}$ in the NDR renormalization scheme. Analogous results in the HV scheme are given in table XLI. The coefficients $r_{i}^{(0)}, r_{i}^{(6)}$ and $r_{i}^{(8)}$ do not depend on $m_{\mathrm{s}}\left(m_{\mathrm{c}}\right)$ as this dependence has been factored out. $r_{i}^{(0)}$ does, however, depend on the particular choice for the parameter $B_{2}^{(1 / 2)}$ in the parametrization of the matrix element $\left\langle Q_{2}\right\rangle_{0}$. The values given in the tables correspond to the central values in (XIX.33). Variation of $B_{2}^{(1 / 2)}$ in the full allowed range introduces an uncertainty of at most $18 \%$ in the $r_{i}^{(0)}$ column of the tables. Since the parameters $r_{i}^{(0)}$ give only subdominant contributions to $\varepsilon^{\prime} / \varepsilon$ keeping $B_{2}^{(1 / 2)}$ and $r_{i}^{(0)}$ at their central values is a very good approximation.

For different scales $\mu$ the numerical values in the tables change without modifying the values of the $P_{i}$ 's as it should be. To this end also $B_{6}^{(1 / 2)}$ and $B_{8}^{(3 / 2)}$ have to be modified as they depend albeit weakly on $\mu$. 
Concerning the scheme dependence we note that whereas $r_{0}$ coefficients are scheme dependent, the coefficients $r_{i}, i=X, Y, Z, E$ do not show any scheme dependence. This is related to the fact that the $m_{\mathrm{t}}$ dependence in $\varepsilon^{\prime} / \varepsilon$ enters first at the NLO level and consequently all coefficients $r_{i}$ in front of the $m_{\mathrm{t}}$ dependent functions must be scheme independent. That this turns out to be indeed the case is a nice check of our calculations.

Consequently when changing the renormalization scheme one is only obliged to change appropriately $B_{6}^{(1 / 2)}$ and $B_{8}^{(3 / 2)}$ in the formula for $P_{0}$ in order to obtain a scheme independence of $\varepsilon^{\prime} / \varepsilon$. In calculating $P_{i}$ where $i \neq 0, B_{6}^{(1 / 2)}$ and $B_{8}^{(3 / 2)}$ can in fact remain unchanged, because their variation in this part corresponds to higher order contributions to $\varepsilon^{\prime} / \varepsilon$ which would have to be taken into account in the next order of perturbation theory.

For similar reasons the NLO analysis of $\varepsilon^{\prime} / \varepsilon$ is still insensitive to the precise definition of $m_{\mathrm{t}}$. In view of the fact that the NLO calculations of $\operatorname{Im} \lambda_{t}$ have been done with $m_{\mathrm{t}}=\bar{m}_{t}\left(m_{\mathrm{t}}\right)$ we will also use this definition in calculating $F\left(x_{t}\right)$.

TABLE XL. $\Delta S=1$ PBE coefficients for various $\Lambda_{\overline{\mathrm{MS}}}$ in the NDR scheme.

\begin{tabular}{|c||c|c|c||c|c|c||c|c|c|}
\hline \hline \multicolumn{1}{|c||}{} & \multicolumn{3}{c||}{$\Lambda_{\overline{\mathrm{MS}}}^{(4)}=215 \mathrm{MeV}$} & \multicolumn{3}{c||}{$\Lambda_{\overline{\mathrm{MS}}}^{(4)}=325 \mathrm{MeV}$} & \multicolumn{3}{c|}{$\Lambda_{\overline{\mathrm{MS}}}^{(4)}=435 \mathrm{MeV}$} \\
\hline$i$ & $r_{i}^{(0)}$ & $r_{i}^{(6)}$ & $r_{i}^{(8)}$ & $r_{i}^{(0)}$ & $r_{i}^{(6)}$ & $r_{i}^{(8)}$ & $r_{i}^{(0)}$ & $r_{i}^{(6)}$ & $r_{i}^{(8)}$ \\
\hline 0 & -2.644 & 4.784 & 0.876 & -2.749 & 6.376 & 0.689 & -2.845 & 8.547 & 0.436 \\
$X$ & 0.555 & 0.008 & 0 & 0.521 & 0.012 & 0 & 0.495 & 0.017 & 0 \\
$Y$ & 0.422 & 0.037 & 0 & 0.385 & 0.046 & 0 & 0.356 & 0.057 & 0 \\
$Z$ & 0.074 & -0.007 & -4.798 & 0.149 & -0.009 & -5.789 & 0.237 & -0.011 & -7.064 \\
$E$ & 0.209 & -0.591 & 0.205 & 0.181 & -0.727 & 0.265 & 0.152 & -0.892 & 0.342 \\
\hline \hline
\end{tabular}

TABLE XLI. $\Delta S=1 \mathrm{PBE}$ coefficients for various $\Lambda_{\overline{\mathrm{MS}}}$ in the HV scheme.

\begin{tabular}{|c||c|c|c||c|c|c||c|c|c|}
\hline \hline & \multicolumn{3}{c||}{$\Lambda_{\overline{\mathrm{MS}}}^{(4)}=215 \mathrm{MeV}$} & \multicolumn{3}{c||}{$\Lambda_{\overline{\mathrm{MS}}}^{(4)}=325 \mathrm{MeV}$} & \multicolumn{3}{c|}{$\Lambda_{\overline{\mathrm{MS}}}^{(4)}=435 \mathrm{MeV}$} \\
\hline$i$ & $r_{i}^{(0)}$ & $r_{i}^{(6)}$ & $r_{i}^{(8)}$ & $r_{i}^{(0)}$ & $r_{i}^{(6)}$ & $r_{i}^{(8)}$ & $r_{i}^{(0)}$ & $r_{i}^{(6)}$ & $r_{i}^{(8)}$ \\
\hline 0 & -2.631 & 4.291 & 0.668 & -2.735 & 5.548 & 0.457 & -2.830 & 7.163 & 0.185 \\
$X$ & 0.555 & 0.008 & 0 & 0.521 & 0.012 & 0 & 0.495 & 0.017 & 0 \\
$Y$ & 0.422 & 0.037 & 0 & 0.385 & 0.046 & 0 & 0.356 & 0.057 & 0 \\
$Z$ & 0.074 & -0.007 & -4.798 & 0.149 & -0.009 & -5.789 & 0.237 & -0.011 & -7.064 \\
$E$ & 0.209 & -0.591 & 0.205 & 0.181 & -0.727 & 0.265 & 0.152 & -0.892 & 0.342 \\
\hline \hline
\end{tabular}

The inspection of tables XL and XLI shows that the terms involving $r_{0}^{(6)}$ and $r_{Z}^{(8)}$ dominate the ratio $\varepsilon^{\prime} / \varepsilon$. The function $Z_{0}\left(x_{t}\right)$ representing a gauge invariant combination of $Z^{0}$ - and $\gamma$-penguins grows rapidly with $m_{\mathrm{t}}$ and due to $r_{Z}^{(8)}<0$ these contributions suppress $\varepsilon^{\prime} / \varepsilon$ strongly for large $m_{\mathrm{t}}$ (Flynn and Randall, 1989b), (Buchalla et al., 1990). These two dominant terms $r_{0}^{(6)}$ and $r_{Z}^{(8)}$ correspond essentially to the second terms in (XIX.37) and (XIX.38), respectively. The first term in (XIX.37) corresponds roughly to $r_{0}^{(0)}$ given here, while the first term in (XIX.38) is represented 
to a large extent by the positve contributions of $X_{0}\left(x_{t}\right)$ and $Y_{0}\left(x_{t}\right)$. The last term in (XIX.40) representing the residual $m_{\mathrm{t}}$ dependence of QCD penguins plays only a minor role in the full analysis of $\varepsilon^{\prime} / \varepsilon$.

\section{H. Numerical Results}

Let us define two effective B-factors:

$$
\left(B_{i}^{(j)}\left(m_{c}\right)\right)_{e f f}=\left[\frac{178 \mathrm{MeV}}{\bar{m}_{s}\left(m_{c}\right)+\bar{m}_{d}\left(m_{c}\right)}\right]^{2} B_{i}^{(j)}\left(m_{c}\right)
$$

In fig. 14 we show $\varepsilon^{\prime} / \varepsilon$ for $m_{\mathrm{t}}=170 \mathrm{GeV}$ as a function of $\Lambda_{\overline{\mathrm{MS}}}$ for different choices of the effective $B_{i}$ factors. We show here only the results in the NDR scheme. As discussed above $\varepsilon^{\prime} / \varepsilon$ is generally lower in the HV scheme, if the same values for $B_{6}^{(1 / 2)}$ and $B_{8}^{(3 / 2)}$ are used in both schemes. In view of the fact that the differences between NDR and HV schemes are smaller than the uncertainties in $B_{6}^{(1 / 2)}$ and $B_{8}^{(3 / 2)}$ we think it is sufficient to present only the results in the NDR scheme here. The results in the HV scheme can be found in (Buras et al., 1993b), (Ciuchini et al., 1995).

Fig. 14 shows strong dependence of $\varepsilon^{\prime} / \varepsilon$ on $\Lambda_{\overline{\mathrm{MS}}}$. However the main uncertainty originates in the poor knowledge of $\left(B_{i}\right)_{e f f}$. In case a) in which the QCD-penguin contributions dominate, $\varepsilon^{\prime} / \varepsilon$ can reach values as high as $1 \cdot 10^{-3}$. However, in case c) the electroweak penguin contributions are large enough to cancel essentially the QCD-penguin contributions completely. Consequently in this case $\left|\varepsilon^{\prime} / \varepsilon\right|<2 \cdot 10^{-5}$ and the standard model prediction of $\varepsilon^{\prime} / \varepsilon$ cannot be distinguished from a superweak theory. As shown in fig. 15 higher values of $\varepsilon^{\prime} / \varepsilon$ can be obtained for $m_{\mathrm{t}}=155 \mathrm{GeV}$ although still $\varepsilon^{\prime} / \varepsilon<13 \cdot 10^{-4}$.

For $m_{\mathrm{t}}=185 \mathrm{GeV}$ the values of $\varepsilon^{\prime} / \varepsilon$ are correspondingly smaller and in case c) small negative values are found for $\varepsilon^{\prime} / \varepsilon$. In figs. 14-16 the dark grey regions refer to the future ranges for $\operatorname{Im} \lambda_{t}$. Of course one should hope that also the knowledge of $\left(B_{i}\right)_{e f f}$ and of $\Lambda \frac{(4)}{\mathrm{MS}}$ will be improved in the future so that a firmer prediction for $\varepsilon^{\prime} / \varepsilon$ can be obtained.

Finally, fig. 17 shows the interrelated influence of $m_{\mathrm{t}}$ and the two most important hadronic matrix elements for penguin operators on the theoretical prediction of $\varepsilon^{\prime} / \varepsilon$. For a dominant QCD penguin matrix element $<Q_{6}>_{0} \varepsilon^{\prime} / \varepsilon$ stays positive for all $m_{\mathrm{t}}$ values considered. $\varepsilon^{\prime} / \varepsilon \approx 0$ becomes possible for equally weighted matrix elements $<Q_{6}>_{0}$ and $<Q_{8}>_{2}$ around $m_{\mathrm{t}}=$ $205 \mathrm{GeV}$. A dominant electroweak pengiun matrix element $<Q_{8}>_{2}$ shifts the point $\varepsilon^{\prime} / \varepsilon \approx 0$ to $m_{\mathrm{t}} \approx 165 \mathrm{GeV}$ and even allows for a negative $\varepsilon^{\prime} / \varepsilon$ for higher values of $m_{\mathrm{t}}$. The key issue to understand this behaviour of $\varepsilon^{\prime} / \varepsilon$ is the observation that the $Q_{6}$ contribution to $\varepsilon^{\prime} / \varepsilon$ is positive and only weakly $m_{\mathrm{t}}$ dependent. On the other hand the contribution coming from $Q_{8}$ is negative and shows a strong $m_{\mathrm{t}}$ dependence.

The results in fig. 14-17 use only the $\varepsilon_{K}$ constraint. In order to complete our analysis we want to impose also the $x_{d}$-constraint and vary $m_{s}\left(m_{c}\right), B_{6}^{(1 / 2)}$ and $B_{8}^{(3 / 2)}$ in the full ranges given in (XIX.34) and (XIX.36).

This gives for the "present" scenario

$$
-2.1 \cdot 10^{-4} \leq \varepsilon^{\prime} / \varepsilon \leq 13.2 \cdot 10^{-4}
$$

to be compared with 


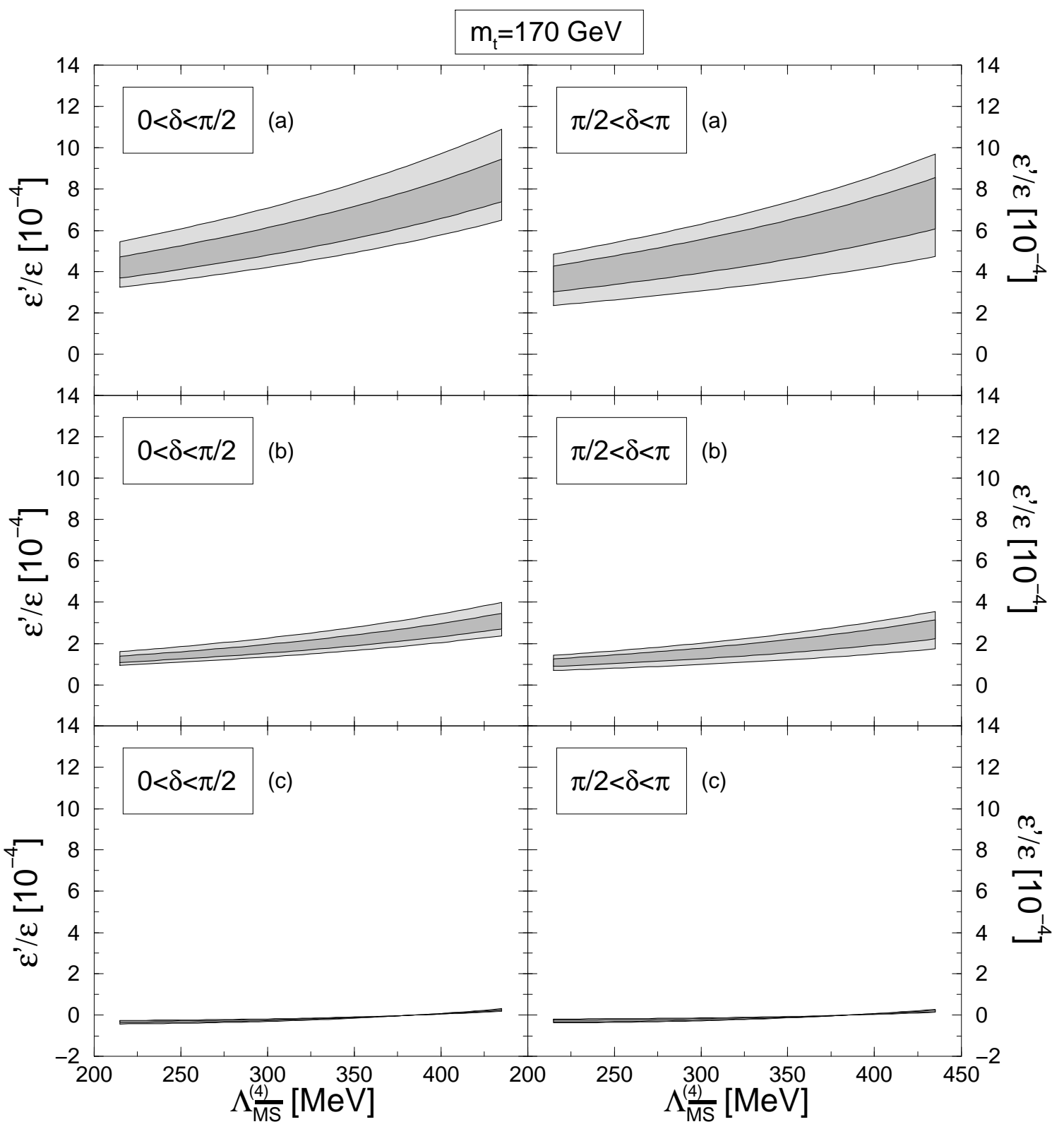

FIG. 14. The ranges of $\varepsilon^{\prime} / \varepsilon$ in the NDR scheme as a function of $\Lambda \frac{(4)}{\mathrm{MS}}$ for $m_{\mathrm{t}}=170 \mathrm{GeV}$ and present (light grey) and future (dark grey) parameter ranges given in appendix A. The three pairs of $\varepsilon^{\prime} / \varepsilon$ plots correspond to hadronic parameter sets (a) $\left(B_{6}^{(1 / 2)}\left(m_{\mathrm{c}}\right)\right)_{\mathrm{eff}}=1.5,\left(B_{8}^{(3 / 2)}\left(m_{\mathrm{c}}\right)\right)_{\mathrm{eff}}=1.0$, (b) $\left(B_{6}^{(1 / 2)}\left(m_{\mathrm{c}}\right)\right)_{\mathrm{eff}}=1.0,\left(B_{8}^{(3 / 2)}\left(m_{\mathrm{c}}\right)\right)_{\mathrm{eff}}=1.0$, and $(\mathrm{c})\left(B_{6}^{(1 / 2)}\left(m_{\mathrm{c}}\right)\right)_{\mathrm{eff}}=1.0,\left(B_{8}^{(3 / 2)}\left(m_{\mathrm{c}}\right)\right)_{\mathrm{eff}}=1.5$, respectively.

$$
-1.1 \cdot 10^{-4} \leq \varepsilon^{\prime} / \varepsilon \leq 10.4 \cdot 10^{-4}
$$

in the case of the "future" scenario. In both cases the $x_{d}$-constraint has essentially no impact on the predicted range for $\varepsilon^{\prime} / \varepsilon$.

Finally, extending the "future" scenario to $m_{\mathrm{s}}\left(m_{\mathrm{c}}\right)=(170 \pm 10) \mathrm{MeV}, \Lambda \frac{(4)}{\mathrm{MS}}=(325 \pm 50) \mathrm{MeV}$ and $B_{6}^{(1 / 2)}, B_{8}^{(3 / 2)}=1.0 \pm 0.1$ would give 


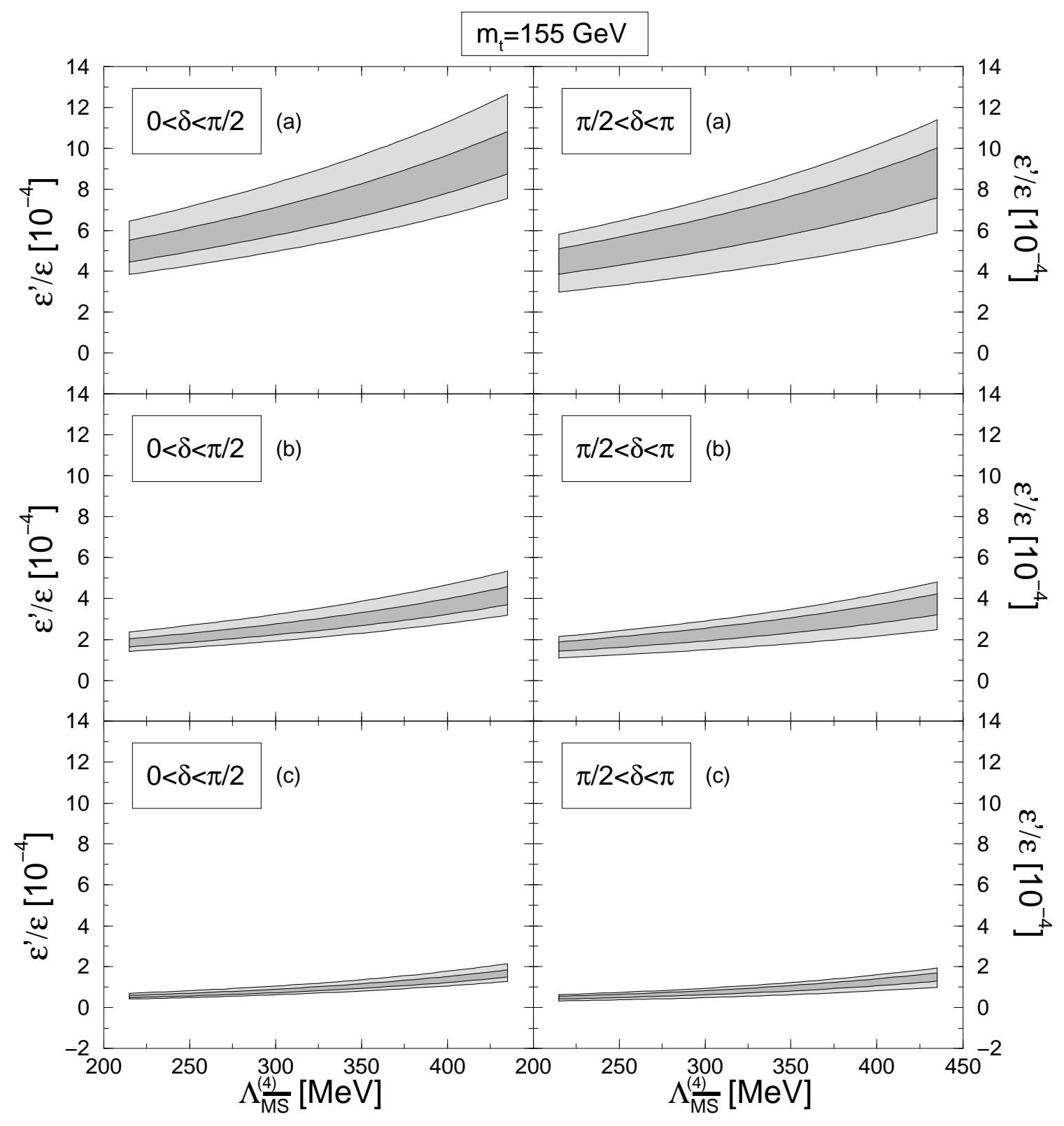

FIG. 15. Same as fig. 14 but for $m_{\mathrm{t}}=155 \mathrm{GeV}$.

$$
0.3 \cdot 10^{-4} \leq \varepsilon^{\prime} / \varepsilon \leq 5.4 \cdot 10^{-4}
$$

again with no impact from imposing the $x_{d}$-constraint.

Allowing for the additional variation $B_{2, N D R}^{(1 / 2)}\left(m_{\mathrm{c}}\right)=6.6 \pm 1.0$ extends ranges (XIX.43)(XIX.45) to $-2.5 \cdot 10^{-4} \leq \varepsilon^{\prime} / \varepsilon \leq 13.7 \cdot 10^{-4},-1.5 \cdot 10^{-4} \leq \varepsilon^{\prime} / \varepsilon \leq 10.8 \cdot 10^{-4}$ and $0.1 \cdot 10^{-4} \leq$ $\varepsilon^{\prime} / \varepsilon \leq 5.8 \cdot 10^{-4}$, respectively.

An analysis of the Rome group (Ciuchini et al., 1995) gives $\operatorname{Re}\left(\varepsilon^{\prime} / \varepsilon\right)=(3.1 \pm 2.5) \cdot 10^{-4}$ which is compatible with our results. Similar results are found with hadronic matrix elements calculated in the chiral quark model (Bertolini et al., 1995a), (Bertolini et al., 1995b).

The difference in the range for $\varepsilon^{\prime} / \varepsilon$ presentend here by us and the Rome group is related to the 


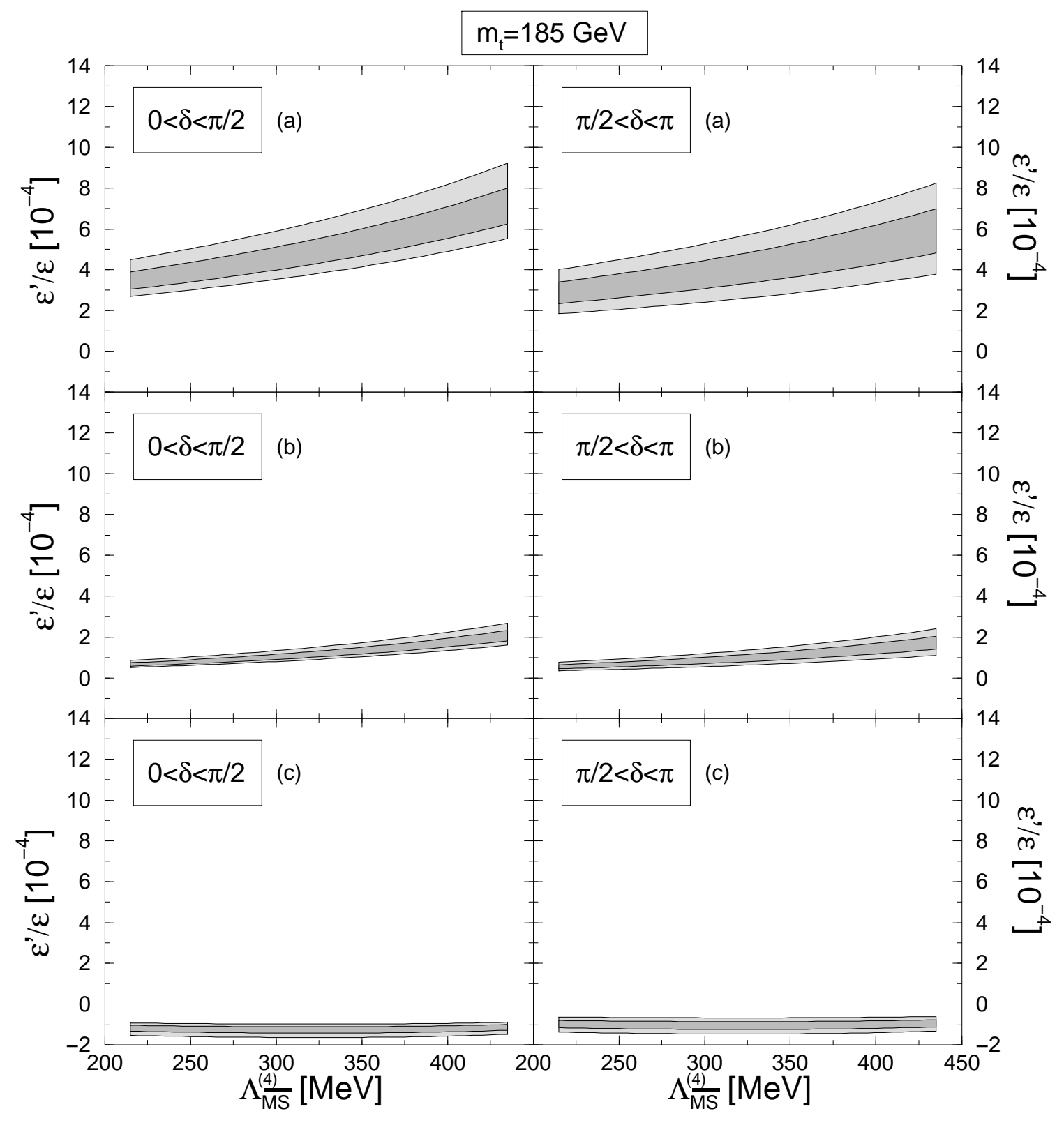

FIG. 16. Same as fig. 14 but for $m_{\mathrm{t}}=185 \mathrm{GeV}$.

different treatment of theoretical and experimental errors. Whereas we simply scan all parameters within one standard deviation, (Ciuchini et al., 1995) use Gaussian distributions in treating the experimental errors. Consequently our procedure is more conservative. We agree however with these authors that values for $\varepsilon^{\prime} / \varepsilon$ above $1 \cdot 10^{-3}$ although not excluded are very improbable. This should be contrasted with the work of the Dortmund group (Fröhlich et al., 1991), (Heinrich et al., 1992) which finds values for $\varepsilon^{\prime} / \varepsilon$ in the ball park of $(2-3) \cdot 10^{-3}$. We do not know any consistent framework for hadronic matrix elements which would give such high values within the Standard Model.

The experimental situation on $\operatorname{Re}\left(\varepsilon^{\prime} / \varepsilon\right)$ is unclear at present. While the result of the NA31 


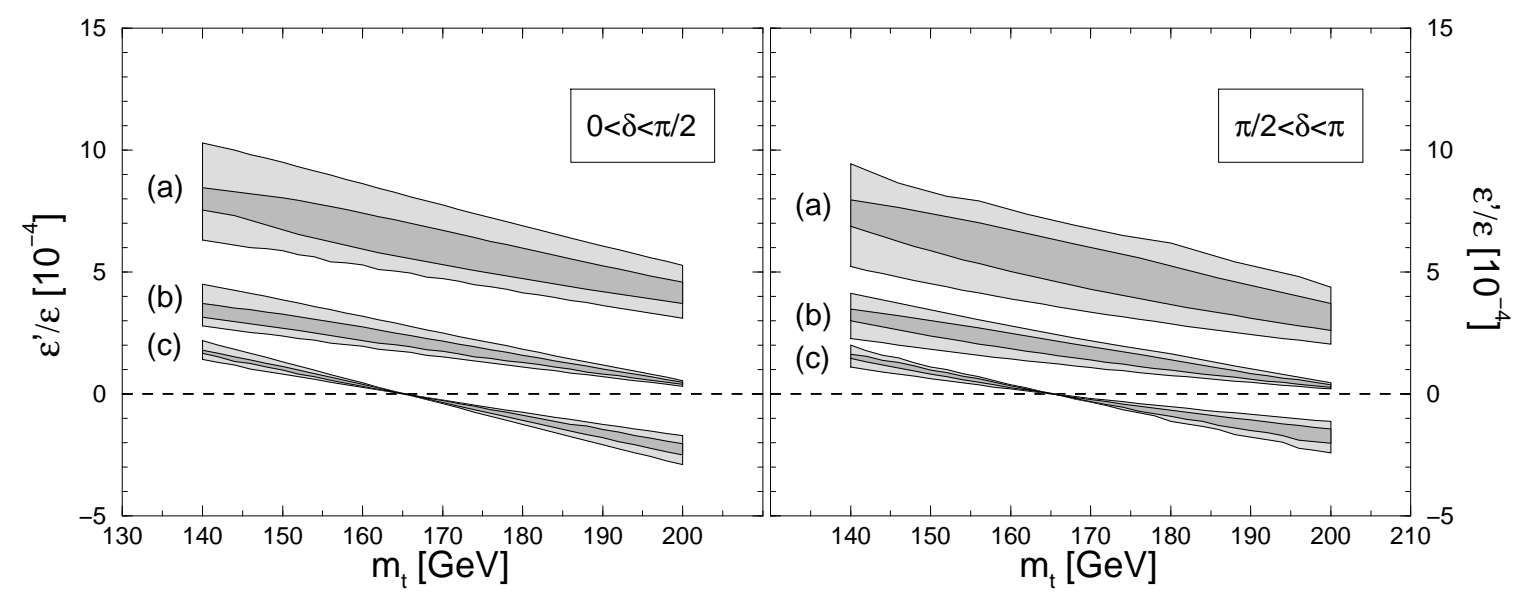

FIG. 17. The ranges of $\varepsilon^{\prime} / \varepsilon$ in the NDR scheme as a function of $m_{\mathrm{t}}$ for $\Lambda_{\frac{(4)}{\mathrm{MS}}}^{(4)} 325 \mathrm{MeV}$ and present (light grey) and future (dark grey) parameter ranges given in appendix A. The three bands correspond to hadronic parameter sets (a) $\left(B_{6}^{(1 / 2)}\left(m_{\mathrm{c}}\right)\right)_{\mathrm{eff}}=1.5,\left(B_{8}^{(3 / 2)}\left(m_{\mathrm{c}}\right)\right)_{\mathrm{eff}}=1.0$, (b) $\left(B_{6}^{(1 / 2)}\left(m_{\mathrm{c}}\right)\right)_{\mathrm{eff}}=1.0$, $\left(B_{8}^{(3 / 2)}\left(m_{\mathrm{c}}\right)\right)_{\mathrm{eff}}=1.0$, and (c) $\left(B_{6}^{(1 / 2)}\left(m_{\mathrm{c}}\right)\right)_{\mathrm{eff}}=1.0,\left(B_{8}^{(3 / 2)}\left(m_{\mathrm{c}}\right)\right)_{\mathrm{eff}}=1.5$, respectively.

collaboration at CERN with $\operatorname{Re}\left(\varepsilon^{\prime} / \varepsilon\right)=(23 \pm 7) \cdot 10^{-4}$ (Barr et al., 1993) clearly indicates direct $\mathrm{CP}$ violation, the value of E731 at Fermilab, $\operatorname{Re}\left(\varepsilon^{\prime} / \varepsilon\right)=(7.4 \pm 5.9) \cdot 10^{-4}$ (Gibbons et al., 1993), is compatible with superweak theories (Wolfenstein, 1964) in which $\varepsilon^{\prime} / \varepsilon=0$. The E731 result is in the ball park of the theoretical estimates. The NA31 value appears a bit high compared to the range given in (XIX.43) above.

Hopefully, in about three years the experimental situation concerning $\varepsilon^{\prime} / \varepsilon$ will be clarified through the improved measurements by the two collaborations at the $10^{-4}$ level and by experiments at the $\Phi$ factory in Frascati. One should also hope that the theoretical situation of $\varepsilon^{\prime} / \varepsilon$ will improve by then to confront the new data. 


\section{XX. $K_{L}-K_{S}$ MASS DIFFERENCE AND $\Delta I=1 / 2$ RULE} by

It is probably a good moment to make a few comments on the $K_{L}-K_{S}$ mass difference given

$$
\Delta M=M\left(K_{L}\right)-M\left(K_{S}\right)=3.51 \cdot 10^{-15} \mathrm{GeV}
$$

and the approximate $\Delta I=1 / 2$ rule in $K \rightarrow \pi \pi$ decays. As we have already briefly mentioned in the beginning of section XIX A, this empirical rule manifests itself in the dominance of $\Delta I=1 / 2$ over $\Delta I=3 / 2$ decay amplitudes. It can be expressed as

$$
\frac{\operatorname{Re} A_{0}}{\operatorname{Re} A_{2}}=22.2
$$

using the notation of section XIX A.

$$
\text { A. } \Delta M\left(K_{L}-K_{S}\right)
$$

The $K_{L}-K_{S}$ mass difference can be written as

$$
\Delta M=2 \operatorname{Re} M_{12}+(\Delta M)_{L D}
$$

with $M_{12}$ given in (XVIII.6) and $(\Delta M)_{L D}$ representing long distance contributions, corresponding for instance to the exchange of intermediate light pseudoscalar mesons $\left(\pi^{0}, \eta\right)$. The first term in (XX.3), the so-called short distance contribution, is dominated by the first term in (XVIII.6) so that

$$
(\Delta M)_{S D}=\frac{G_{F}^{2}}{6 \pi^{2}} F_{K}^{2} B_{K} m_{K} M_{W}^{2}\left[\lambda_{c}^{2} \eta_{1} \frac{m_{c}^{2}}{M_{W}^{2}}+\Delta_{t o p}\right]
$$

where $\Delta_{\text {top }}$ represents the two top dependent terms in (XVIII.6). In writing (XX.4) we are neglecting the tiny imaginary part in $\lambda_{c}=V_{c s}^{*} V_{c d}$. A very extensive numerical analysis of (XX.4) has been presented by (Herrlich and Nierste, 1994), who calculated the NLO corrections to $\eta_{1}$ and also to $\eta_{3}$ (Herrlich and Nierste, 1995a) which enters $\Delta_{t o p}$. The NLO calculation of the short distance contributions improves the matching to the non-perturbative matrix element parametrized by $B_{K}$ and clarifies the proper definition of $B_{K}$ to be used along with the QCD factors $\eta_{i}$. In addition the NLO study reveals an enhancement of $\eta_{1}$ over its LO estimate by about $20 \%$. Although sizable, this enhancement can still be considered being perturbative, as required by the consistency of the calculation. This increase in $\eta_{1}$, reinforced by updates in input parameters $\left(\Lambda_{\overline{\mathrm{MS}}}\right)$, brings $(\Delta M)_{S D}$ closer to the experimental value in (XX.1). With $\Lambda \frac{(4)}{\mathrm{MS}}=325 \mathrm{MeV}$ and $m_{\mathrm{c}}=1.3 \mathrm{GeV}$, giving $\eta_{1}^{N L O}=1.38$, one finds that typically $70 \%$ of $\Delta M$ can be described by the short distance component. The exact value is still somewhat uncertain because $\eta_{1}$ is rather sensitive to $\Lambda_{\overline{\mathrm{MS}}}$. Further uncertainties are introduced by the error in $B_{K}$ and due to the renormalization scale ambiguity, which is still quite pronounced even at NLO. Yet the result is certainly more reliable than previous LO estimates. Using the old value $\eta_{1}^{L O}=0.85$, corresponding to $m_{\mathrm{c}}=1.4 \mathrm{GeV}$ and $\Lambda_{Q C D}=200 \mathrm{MeV},(\Delta M)_{S D} / \Delta M$ would be below $50 \%$, suggesting a dominance of long distance contributions in $\Delta M$. As discussed in (Herrlich and Nierste, 1994), such a situation would 
be "unnatural" since the long distance component is formally suppressed by $\Lambda_{Q C D}^{2} / m_{c}^{2}$. Hence the short distance dominance indicated by the NLO analysis is also gratifying in this respect.

The long distance contributions, to which one can attribute the remaining $\sim 30 \%$ in $\Delta M$ not explained by the short distance part, are nicely discussed in (Bijnens et al., 1991).

In summary, the observed $K_{L}-K_{S}$ mass difference can be roughly described within the standard model after the NLO corrections have been taken into account. The remaining theoretical uncertainties in the dominant part in (XX.4) and the uncertainties in $(\Delta M)_{L D}$ do not allow however to use $\Delta M$ as a constraint on the CKM parameters.

\section{B. The $\Delta I=1 / 2$ Rule}

Using the effective hamiltonian in (VII.1) and keeping only the dominant terms one has

$$
\frac{\operatorname{Re} A_{0}}{\operatorname{Re} A_{2}} \approx \frac{z_{1}(\mu)\left\langle Q_{1}(\mu)\right\rangle_{0}+z_{2}(\mu)\left\langle Q_{2}(\mu)\right\rangle_{0}+z_{6}(\mu)\left\langle Q_{6}(\mu)\right\rangle_{0}}{z_{1}(\mu)\left\langle Q_{1}(\mu)\right\rangle_{2}+z_{2}(\mu)\left\langle Q_{2}(\mu)\right\rangle_{2}}
$$

where $\left\langle Q_{i}\right\rangle_{0,2}$ are defined in (XIX.8). The coefficients $z_{i}(\mu)$ can be found in table XVIII. For the hadronic matrix elements we use the formulae (XIX.11), (XIX.12), (XIX.16) and (XIX.21), which have been discussed in section XIX B. We find then, separating current-current and penguin contributions

$$
\begin{gathered}
\frac{\operatorname{Re} A_{0}}{\operatorname{Re} A_{2}}=R_{c}+R_{p} \\
R_{c}=\frac{5 z_{2}(\mu) B_{2}^{(1 / 2)}-z_{1}(\mu) B_{1}^{(1 / 2)}}{4 \sqrt{2} z_{+}(\mu) B_{1}^{(3 / 2)}} z_{+}=z_{1}+z_{2} \\
R_{p}=-11.9 \frac{z_{6}(\mu)}{z_{+}(\mu)} \frac{B_{6}^{(1 / 2)}}{B_{1}^{(3 / 2)}}\left[\frac{178 \mathrm{MeV}}{m_{s}(\mu)+m_{d}(\mu)}\right]^{2}
\end{gathered}
$$

The factor 11.9 expresses the enhancement of the matrix elements of the penguin operator $Q_{6}$ over $\left\langle Q_{1,2}\right\rangle$ first pointed out in (Vainshtein et al., 1977). It is instructive to calculate $R_{c}$ and $R_{p}$ using the vacuum insertion estimate for which $B_{1}^{(1 / 2)}=B_{2}^{(1 / 2)}=B_{1}^{(3 / 2)}=B_{6}^{(1 / 2)}=1$. Without QCD effects one finds then $R_{c}=0.9$ and $R_{p}=0$ in complete disagreement with the data. In table XLII we show the values of $R_{c}$ and $R_{p}$ at $\mu=1 \mathrm{GeV}$ using the results of table XVIII. We have set $m_{s}+m_{d}=178 \mathrm{MeV}$.

The inclusion of QCD effects enhances both $R_{c}$ and $R_{p}$ (Gaillard and Lee, 1974a), (Altarelli and Maiani, 1974), however even for the highest values of $\Lambda_{\overline{\mathrm{MS}}}^{(4)}$ the ratio $\operatorname{Re} A_{0} / \operatorname{Re} A_{2}$ is by at least a factor of 8 smaller than the experimental value in (XX.2). Moreover a considerable scheme dependence is observed. Lowering $\mu$ would improve the situation, but for $\mu<1 \mathrm{GeV}$ the perturbative calculations of $z_{i}(\mu)$ can no longer be trusted. Similarly lowering $m_{s}$ down to $100 \mathrm{MeV}$ would increase the penguin contribution. In view of the most recent estimates in (XIX.36) such a low value of $m_{s}$ seems to be excluded however. We conclude therefore, as already known since many years, that the vacuum insertion estimate fails completely in explaining the $\Delta I=1 / 2$ rule. 
TABLE XLII. The quantities $R_{c}$ and $R_{p}$ contributing to $\operatorname{Re} A_{0} / \operatorname{Re} A_{2}$ as described in the text, calculated using the vacuum insertion estimate for the hadronic matrix elements. The Wilson coefficient functions are evaluated for various $\Lambda_{\overline{\mathrm{MS}}}^{(4)}$ in leading logarithmic approximation as well as in next-to-leading order in two different schemes (NDR and HV).

\begin{tabular}{|c|r|r|r|r|r|r|r|r|r|}
\hline \hline & \multicolumn{3}{|c|}{$\Lambda \frac{(4)}{\mathrm{MS}}=215 \mathrm{MeV}$} & \multicolumn{3}{|c|}{$\Lambda_{\overline{\mathrm{MS}}}^{(4)}=325 \mathrm{MeV}$} & \multicolumn{3}{|c|}{$\Lambda_{\overline{\mathrm{MS}}}^{(4)}=435 \mathrm{MeV}$} \\
\hline Scheme & LO & NDR & HV & LO & NDR & HV & LO & NDR & HV \\
\hline$R_{c}$ & 1.8 & 1.4 & 1.6 & 2.0 & 1.6 & 1.8 & 2.4 & 1.8 & 2.2 \\
\hline$R_{p}$ & 0.1 & 0.3 & 0.1 & 0.2 & 0.5 & 0.2 & 0.3 & 1.0 & 0.4 \\
\hline \hline
\end{tabular}

As we have discussed in section XIX the vacuum insertion estimate $B_{6}^{(1 / 2)}=1$ is supported by the $1 / N$ expansion approach and by lattice calculations. Consequently the only solution to the $\Delta I=1 / 2$ rule problem appears to be a change in the values of the remaining $B_{i}$ factors. For instance repeating the above calculation with $B_{1}^{(3 / 2)}=0.48, B_{2}^{(1 / 2)}=5$ and $B_{1}^{(1 / 2)}=10$ would give in the NDR scheme $R_{c} \approx 20, R_{p} \approx 2$ and $\operatorname{Re} A_{0} / \operatorname{Re} A_{2} \approx 22$ in accordance with the experimental value.

There have been several attempts to explain the $\Delta I=1 / 2$ rule, which basically use the effective hamiltonian in (VII.1) but employ different methods for the hadronic matrix elements. In particular we would like to mention the $1 / N$ approach (Bardeen et al., 1987a), the work of (Pich and de Rafael, 1991) based on an effective action for four-quark operators, the diquark approach in (Neubert and Stech, 1991), QCD sum rules (Jamin and Pich, 1994), the chiral perturbation calculations in (Kambor et al., 1990), (Kambor et al., 1991) and very recently an analysis (Antonelli et al., 1995) in the framework of the chiral quark model (Cohen and Manohar, 1984).

With these methods values for $\operatorname{Re} A_{0} / \operatorname{Re} A_{2}$ in the range 15-20 can be obtained. It is beyond the scope of this review to discuss the weak and strong points of each method, although at least one of us believes that the "meson evolution" picture advocated in (Bardeen et al., 1987a) represents the main bulk of the physics behind the number 22. In view of the uncertainties present in these approaches, we have not used them in our analysis of $\varepsilon^{\prime} / \varepsilon$, but have constrained the hadronic matrix elements so that they satisfy the $\Delta I=1 / 2$ rule exactly. 


\section{THE DECAY $K_{L} \rightarrow \pi^{0} e^{+} e^{-}$}

\section{A. General Remarks}

Let us next move on to discuss the rare decay $K_{L} \rightarrow \pi^{0} e^{+} e^{-}$. Whereas in $K \rightarrow \pi \pi$ decays the $\mathrm{CP}$ violating contribution is only a tiny part of the full amplitude and the direct $\mathrm{CP}$ violation as we have just seen is expected to be at least by three orders of magnitude smaller than the indirect CP violation, the corresponding hierarchies are very different for $K_{L} \rightarrow \pi^{0} e^{+} e^{-}$. At lowest order in electroweak interactions (one-loop photon penguin, $Z^{0}$-penguin and $\mathrm{W}$-box diagrams), this decay takes place only if CP symmetry is violated. The CP conserving contribution to the amplitude comes from a two photon exchange, which although of higher order in $\alpha$ could in principle be sizable. Extensive studies of several groups indicate however that the CP conserving part is likely to be smaller than the $\mathrm{CP}$ violating contributions. We will be more specific about this at the end of this section.

The CP violating part can again be divided into a direct and an indirect one. The latter is given by the $K_{S} \rightarrow \pi^{0} e^{+} e^{-}$amplitude times the $\mathrm{CP}$ violating parameter $\varepsilon_{K}$. The amplitude $A\left(K_{S} \rightarrow\right.$ $\left.\pi^{0} e^{+} e^{-}\right)$can be written as

$$
A\left(K_{S} \rightarrow \pi^{0} e^{+} e^{-}\right)=\left\langle\pi^{0} e^{+} e^{-}\left|\mathcal{H}_{e f f}\right| K_{S}\right\rangle
$$

where $\mathcal{H}_{\text {eff }}$ can be found in (VIII.1) with the operators $Q_{1}, \ldots, Q_{6}$ defined in (VI.3), the operators $Q_{7 V}$ and $Q_{7 A}$ given by

$$
Q_{7 V}=(\bar{s} d)_{V-A}(\bar{e} e)_{V} \quad Q_{7 A}=(\bar{s} d)_{V-A}(\bar{e} e)_{A}
$$

and the Wilson coefficients $z_{i}$ and $y_{i}$ calculated in section VIII.

Let us next note that the coefficients of $Q_{7 V}$ and $Q_{7 A}$ are $\mathcal{O}(\alpha)$, but their matrix elements $\left\langle\pi^{0} e^{+} e^{-}\left|Q_{7 V, A}\right| K_{S}\right\rangle$ are $\mathcal{O}(1)$. In the case of $Q_{i}(i=1, \ldots, 6)$ the situation is reversed: the Wilson coefficients are $\mathcal{O}(1)$, but the matrix elements $\left\langle\pi^{0} e^{+} e^{-}\left|Q_{i}\right| K_{S}\right\rangle$ are $\mathcal{O}(\alpha)$. Consequently at $\mathcal{O}(\alpha)$ all operators contribute to $A\left(K_{S} \rightarrow \pi^{0} e^{+} e^{-}\right)$. However because $K_{S} \rightarrow \pi^{0} e^{+} e^{-}$is CP conserving, the coefficients $y_{i}$ multiplied by $\tau=\mathcal{O}\left(\lambda^{4}\right)$ can be fully neglected and the operator $Q_{7 A}$ drops out in this approximation. Now whereas $\left\langle\pi^{0} e^{+} e^{-}\left|Q_{7 V}\right| K_{S}\right\rangle$ can be trivially calculated, this is not the case for $\left\langle\pi^{0} e^{+} e^{-}\left|Q_{i}\right| K_{S}\right\rangle$ with $i=1, \ldots, 6$ which can only be evaluated using non-perturbative methods. Moreover it is clear from the short-distance analysis of section VIII that the inclusion of $Q_{i}$ in the estimate of $A\left(K_{S} \rightarrow \pi^{0} e^{+} e^{-}\right)$cannot be avoided. Indeed, whereas $\left\langle\pi^{0} e^{+} e^{-}\left|Q_{7 V}\right| K_{S}\right\rangle$ is independent of $\mu$ and the renormalization scheme, the coefficient $z_{7 V}$ shows very strong scheme and $\mu$-dependences. They can only be canceled by the contributions from the four-quark operators $Q_{i}$. All this demonstrates that the estimate of the indirect CP violation in $K_{L} \rightarrow \pi^{0} e^{+} e^{-}$cannot be done very reliably at present. Some estimates in the framework of chiral perturbation theory will be discussed below. On the other hand, a much better assessment of the importance of indirect CP violation in $K_{L} \rightarrow \pi^{0} e^{+} e^{-}$will become possible after a measurement of $B\left(K_{S} \rightarrow \pi^{0} e^{+} e^{-}\right)$. Fortunately the directly CP violating contribution can be fully calculated as a function of $m_{t}, \mathrm{CKM}$ parameters and the QCD coupling constant $\alpha_{\mathrm{s}}$. There are practically no theoretical uncertainties related to hadronic matrix elements because $\left\langle\pi^{0}\left|(\bar{s} d)_{V-A}\right| K_{L}\right\rangle$ can be extracted using isospin symmetry from the well measured decay $K^{+} \rightarrow \pi^{0} e^{+} \nu$. In what follows, we will concentrate on this contribution. 


\section{B. Analytic Formula for $B\left(K_{L} \rightarrow \pi^{0} e^{+} e^{-}\right)_{d i r}$}

The directly $\mathrm{CP}$ violating contribution is governed by the coefficients $y_{i}$ and consequently only the penguin operators $Q_{3}, \ldots, Q_{6}, Q_{7 V}$ and $Q_{7 A}$ have to be considered. Since $y_{i}=\mathcal{O}\left(\alpha_{\mathrm{s}}\right)$ for $i=3, \ldots, 6$, the contribution of QCD penguins to $B\left(K_{L} \rightarrow \pi^{0} e^{+} e^{-}\right)_{d i r}$ is really $\mathcal{O}\left(\alpha \alpha_{\mathrm{s}}\right)$ to be compared with the $\mathcal{O}(\alpha)$ contributions of $Q_{7 V}$ and $Q_{7 A}$. In deriving the final formula we will therefore neglect the contributions of the operators $Q_{3}, \ldots, Q_{6}$, i.e. we will assume that

$$
\sum_{i=3}^{6} y_{i}(\mu)\left\langle\pi^{0} e^{+} e^{-}\left|Q_{i}\right| K_{L}\right\rangle \ll y_{7 V}(\mu)\left\langle\pi^{0} e^{+} e^{-}\left|Q_{7 V}\right| K_{L}\right\rangle
$$

This assumption is supported by the corresponding relation for the quark-level matrix elements

$$
\sum_{i=3}^{6} y_{i}(\mu)\left\langle d e^{+} e^{-}\left|Q_{i}\right| s\right\rangle \ll y_{7 V}(\mu)\left\langle d e^{+} e^{-}\left|Q_{7 V}\right| s\right\rangle
$$

that can be easily verified perturbatively.

The neglect of the QCD penguin operators is compatible with the scheme and $\mu$-independence of the resulting branching ratio. Indeed $y_{7 A}$ does not depend on $\mu$ and the renormalization scheme at all and the corresponding dependences in $y_{7 V}$ are at the level of $\pm 1 \%$ as discussed in section VIII E. Introducing the numerical constant

$$
\kappa_{e}=\frac{1}{V_{u s}^{2}} \frac{\tau\left(K_{L}\right)}{\tau\left(K^{+}\right)}\left(\frac{\alpha}{2 \pi}\right)^{2} B\left(K^{+} \rightarrow \pi^{0} e^{+} \nu\right)=6.3 \cdot 10^{-6}
$$

one then finds

$$
B\left(K_{L} \rightarrow \pi^{0} e^{+} e^{-}\right)_{d i r}=\kappa_{e}\left(\operatorname{Im} \lambda_{t}\right)^{2}\left(\tilde{y}_{7 V}^{2}+\tilde{y}_{7 A}^{2}\right)
$$

where

$$
y_{i}=\frac{\alpha}{2 \pi} \tilde{y}_{i}
$$

Using next the method of the penguin-box expansion (section XIV) we can write similarly to (X.5) and $(\mathrm{X} .3)$

$$
\begin{gathered}
\tilde{y}_{7 V}=P_{0}+\frac{Y_{0}\left(x_{t}\right)}{\sin ^{2} \Theta_{W}}-4 Z_{0}\left(x_{t}\right)+P_{E} E_{0}\left(x_{t}\right) \\
\tilde{y}_{7 A}=-\frac{1}{\sin ^{2} \Theta_{W}} Y_{0}\left(x_{t}\right)
\end{gathered}
$$

with $Y_{0}, Z_{0}$ and $E_{0}$ given in (XI.46), (XIV.2) and (VI.15). $P_{E}$ is $\mathcal{O}\left(10^{-2}\right)$ and consequently the last term in (XXI.8) can be neglected. $P_{0}$ is given for different values of $\mu$ and $\Lambda_{\overline{M S}}$ in table XLIII. There we also show the leading order results and the case without QCD corrections.

The analytic expressions in (XXI.8) and (XXI.9) are useful as they display not only the explicit $m_{t}$-dependence, but also isolate the impact of leading and next-to-leading QCD effects. These effects modify only the constants $P_{0}$ and $P_{E}$. As anticipated from the results of section VIII E, $P_{0}$ 
TABLE XLIII. PBE coefficient $P_{0}$ of $y_{7 V}$ for various values of $\Lambda_{\mathrm{MS}}^{(4)}$ and $\mu$. In the absence of QCD $P_{0}=8 / 9 \ln \left(M_{W} / m_{c}\right)=3.664$ holds universally.

\begin{tabular}{|c|c||c|r|r|}
\hline \hline \multicolumn{2}{|c||}{} & \multicolumn{3}{|c|}{$P_{0}$} \\
\hline$\Lambda \frac{(4)}{\mathrm{MS}}[\mathrm{MeV}]$ & $\mu[\mathrm{GeV}]$ & LO & NDR & HV \\
\multirow{2}{*}{215} & 0.8 & 2.073 & 3.159 & 3.110 \\
& 1.0 & 2.048 & 3.133 & 3.084 \\
& 1.2 & 2.027 & 3.112 & 3.063 \\
325 & 0.8 & 1.863 & 3.080 & 3.024 \\
& 1.0 & 1.834 & 3.053 & 2.996 \\
& 1.2 & 1.811 & 3.028 & 2.970 \\
\hline \multirow{2}{*}{435} & 0.8 & 1.672 & 2.976 & 2.914 \\
& 1.0 & 1.640 & 2.965 & 2.899 \\
& 1.2 & 1.613 & 2.939 & 2.872 \\
\hline \hline
\end{tabular}

is strongly enhanced relatively to the LO result. This enhancement amounts roughly to a factor of $1.6 \pm 0.1$. Partially this enhancement is however due to the fact that for $\Lambda_{L O}=\Lambda_{\overline{M S}}$ the QCD coupling constant in the leading order is $20-30 \%$ larger than its next-to-leading order value. Calculating $P_{0}$ in LO but with the full $\alpha_{\mathrm{s}}$ of (III.19) we have found that the enhancement then amounts to a factor of $1.33 \pm 0.06$. In any case the inclusion of NLO QCD effects and a meaningful use of $\Lambda_{\overline{M S}}$ show that the next-to-leading order effects weaken the QCD suppression of $y_{7 V}$. As seen in table XLIII, the suppression of $P_{0}$ by QCD corrections amounts to about $15 \%$ in the complete next-to-leading order calculation.

\section{Numerical Analysis}

In fig. 8 of section VIII $E$ we have shown $\left|y_{7 V} / \alpha\right|^{2}$ and $\left|y_{7 A} / \alpha\right|^{2}$ as functions of $m_{t}$ together with the leading order result for $\left|y_{7 V} / \alpha\right|^{2}$ and the case without QCD corrections. From there it is obvious that the dominant $m_{t}$-dependence of $B\left(K_{L} \rightarrow \pi^{0} e^{+} e^{-}\right)_{\text {dir }}$ originates from the coefficient of the operator $Q_{7 A}$. Another noteworthy feature was that accidentally for $m_{t} \approx 175 \mathrm{GeV}$ one finds $y_{7 V} \approx y_{7 A}$.

In fig. 18 the ratio $B\left(K_{L} \rightarrow \pi^{0} e^{+} e^{-}\right)_{d i r} /\left(\operatorname{Im} \lambda_{t}\right)^{2}$ is shown as a function of $m_{t}$. The enhancement of the directly $\mathrm{CP}$ violating contribution through NLO corrections relatively to the LO estimate is clearly visible on this plot. As we will see below, due to large uncertainties present in $\operatorname{Im} \lambda_{t}$ this enhancement cannot yet be fully appreciated phenomenologically.

The very weak dependence on $\Lambda_{\overline{M S}}$ should be contrasted with the very strong dependence found in the case of $\varepsilon^{\prime} / \varepsilon$. Therefore, provided the other two contributions to $K_{L} \rightarrow \pi^{0} e^{+} e^{-}$can be shown to be small or can be reliably calculated one day, the measurement of $B\left(K_{L} \rightarrow \pi^{0} e^{+} e^{-}\right)$ should offer a good determination of $\operatorname{Im} \lambda_{t}$.

Next we would like to comment on the possible uncertainties due to the definition of $m_{t}$. At the level of accuracy at which we work we cannot fully address this question yet. In order to be able to do it, one needs to know the perturbative QCD corrections to $Y_{0}\left(x_{t}\right)$ and $Z_{0}\left(x_{t}\right)$ and for 


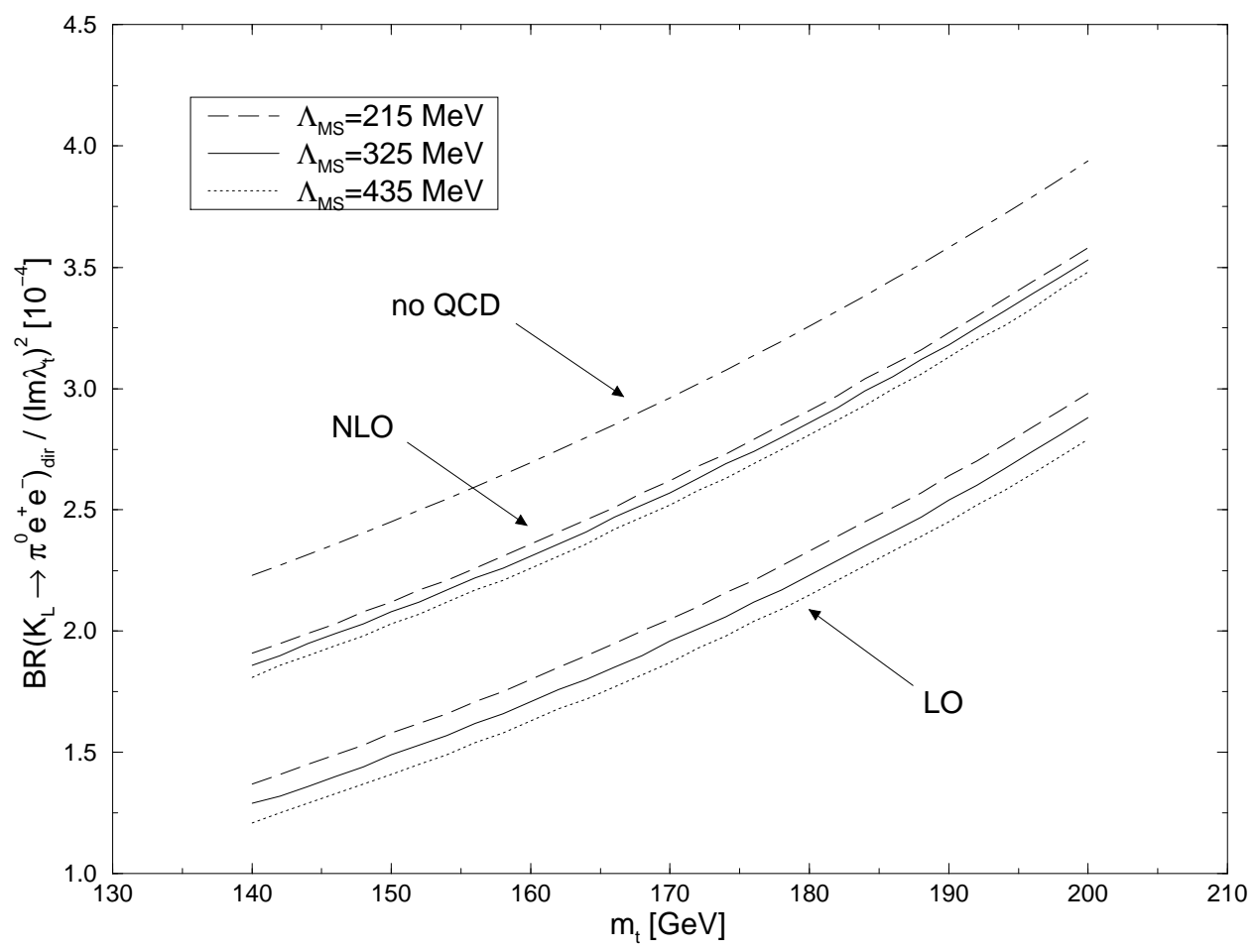

FIG. 18. $B\left(K_{L} \rightarrow \pi^{0} e^{+} e^{-}\right)_{\mathrm{dir}} /\left(\operatorname{Im} \lambda_{t}\right)^{2}$ as a function of $m_{\mathrm{t}}$ for various values of $\Lambda_{\frac{(4)}{\mathrm{MS}}}$ at scale $\mu=1.0 \mathrm{GeV}$.

consistency an additional order in the renormalization group improved calculation of $P_{0}$. Since the $m_{t}$-dependence of $y_{7 V}$ is rather moderate, the main concern in this issue is the coefficient $y_{7} \mathrm{~A}$ whose $m_{t}$-dependence is fully given by $Y\left(x_{t}\right)$. Fortunately the QCD corrected function $Y\left(x_{t}\right)$ is known from the analysis of $K_{L} \rightarrow \mu^{+} \mu^{-}$and can be directly used here. As we will discuss in section XXV, for $m_{t}=m_{t}\left(m_{t}\right)$ the QCD corrections to $Y_{0}\left(x_{t}\right)$ are around $2 \%$. On this basis we believe that if $m_{t}=m_{t}\left(m_{t}\right)$ is chosen, the additional QCD corrections to $B\left(K_{L} \rightarrow \pi^{0} e^{+} e^{-}\right)_{d i r}$ should be small.

Finally we give the predictions for the present and future sets of input parameters as described in apppendix A. It should be emphasized that the uncertainties in these predictions result entirely from the CKM parameters. This situation will improve considerably in the era of dedicated Bphysics experiments in the next decade, allowing a precise prediction for $B\left(K_{L} \rightarrow \pi^{0} e^{+} e^{-}\right)_{\mathrm{dir}}$.

We find

$$
\begin{aligned}
& B\left(K_{L} \rightarrow \pi^{0} e^{+} e^{-}\right)_{\operatorname{dir}}=\left\{\begin{array}{l}
(4.26 \pm 3.03) \cdot 10^{-12} \text { no } x_{d} \text { constraint } \\
(4.48 \pm 2.77) \cdot 10^{-12} \text { with } x_{d} \text { constraint }
\end{array}\right. \\
& B\left(K_{L} \rightarrow \pi^{0} e^{+} e^{-}\right)_{\operatorname{dir}}=\left\{\begin{array}{l}
(3.71 \pm 1.61) \cdot 10^{-12} \text { no } x_{d} \text { constraint } \\
(4.32 \pm 0.96) \cdot 10^{-12} \text { with } x_{d} \text { constraint }
\end{array}\right.
\end{aligned}
$$

These results are compatible with those found in (Buras et al., 1994a), (Donoghue and Gabbiani, 1995), (Köhler and Paschos, 1995) with differences originating in various choices of CKM parameters. 


\section{The Indirectly CP Violating and CP Conserving Parts}

Now we want to compare the results obtained for the direct $\mathrm{CP}$ violating part with the estimates made for the indirect CP-violating contribution and the CP-conserving one. The most recent discussions have been presented in (Cohen et al., 1993), (Heiliger and Seghal, 1993), (Donoghue and Gabbiani, 1995), (Köhler and Paschos, 1995) where references to earlier papers can be found.

The indirect $\mathrm{CP}$ violating amplitude is given by the $K_{S} \rightarrow \pi^{0} e^{+} e^{-}$amplitude times the CP parameter $\varepsilon_{K}$. Once $B\left(K_{S} \rightarrow \pi^{0} e^{+} e^{-}\right)$has been accurately measured, it will be possible to calculate this contribution precisely. Using chiral perturbation theory it is however possible to get an estimate by relating $K_{S} \rightarrow \pi^{0} e^{+} e^{-}$to the $K^{+} \rightarrow \pi^{+} e^{+} e^{-}$transition (Ecker et al., 1987), (Ecker et al., 1988). To this end one can write

$$
B\left(K_{L} \rightarrow \pi^{0} e^{+} e^{-}\right)_{\text {indir }}=B\left(K^{+} \rightarrow \pi^{+} e^{+} e^{-}\right) \frac{\tau\left(K_{L}\right)}{\tau\left(K^{+}\right)}\left|\varepsilon_{K}\right|^{2} r^{2}
$$

where

$$
r^{2}=\frac{\Gamma\left(K_{S} \rightarrow \pi^{0} e^{+} e^{-}\right)}{\Gamma\left(K^{+} \rightarrow \pi^{+} e^{+} e^{-}\right)}
$$

With $B\left(K^{+} \rightarrow \pi^{+} e^{+} e^{-}\right)=(2.74 \pm 0.23) \cdot 10^{-7}$ (Alliegro et al., 1992) and the most recent chiral perturbation theory estimate $|r| \leq 0.5$ (Ecker et al., 1988), (Bruno and Prades, 1993) one has

$$
B\left(K_{L} \rightarrow \pi^{0} e^{+} e^{-}\right)_{\text {indir }}=(5.9 \pm 0.5) \cdot 10^{-12} r^{2} \leq 1.6 \cdot 10^{-12},
$$

i.e. a branching ratio more than a factor of 2 below the direct $\mathrm{CP}$ violating contribution.

Yet as emphasized recently in (Donoghue and Gabbiani, 1995) and also in (Heiliger and Seghal, 1993) the knowledge of $r$ is very uncertain at present. In particular the estimate in (XXI.14) is based on a relation between two non-perturbative parameters, which is rather ad hoc and certainly not a consequence of chiral symmetry. As shown in (Donoghue and Gabbiani, 1995) a small deviation from this relation increases $r$ to values above unity so that $B\left(K_{L} \rightarrow \pi^{0} e^{+} e^{-}\right)_{\text {indir }}$ could be comparable or even large than $B\left(K_{L} \rightarrow \pi^{0} e^{+} e^{-}\right)_{\text {dir. }}$. It appears then that this enormous uncertainty in the indirectly $\mathrm{CP}$ violating part can only be removed by measuring the rate of $K_{S} \rightarrow \pi^{0} e^{+} e^{-}$.

It should also be stressed, that in reality the $\mathrm{CP}$ indirect amplitude may interfere with the vector part of the CP direct amplitude. The full CP violating amplitude can then be written following (Dib et al., 1989a), (Dib et al., 1989b) as follows

$$
B\left(K_{L} \rightarrow \pi^{0} e^{+} e^{-}\right)_{C P}=\left|2.43 \cdot 10^{-6} r e^{i \pi / 4}-i \sqrt{\kappa_{e}} \operatorname{Im} \lambda_{t} \tilde{y}_{7 V}\right|^{2}+\kappa_{e}\left(\operatorname{Im} \lambda_{t}\right)^{2} \tilde{y}_{7 A}^{2}
$$

As an example we show in fig. $19 B\left(K_{L} \rightarrow \pi^{0} e^{+} e^{-}\right)_{\mathrm{CP}}$ for $m_{\mathrm{t}}=170 \mathrm{GeV}, \Lambda \frac{(4)}{\mathrm{MS}}=325 \mathrm{MeV}$ and $\operatorname{Im} \lambda_{t}=1.3 \cdot 10^{-4}$ as a function of $r$. We observe that whereas for $0 \leq r \leq 1$ the dependence of $B\left(K_{L} \rightarrow \pi^{0} e^{+} e^{-}\right)_{\mathrm{CP}}$ on $r$ is moderate, it is rather strong otherwise and already for $r<-0.6$ values as high as $10^{-11}$ are found.

The estimate of the CP conserving contribution is also difficult. We refer the reader to (Cohen et al., 1993), (Heiliger and Seghal, 1993) and (Donoghue and Gabbiani, 1995) where further references to an extensive literature on this subject can be found. The measurement of the branching ratio 


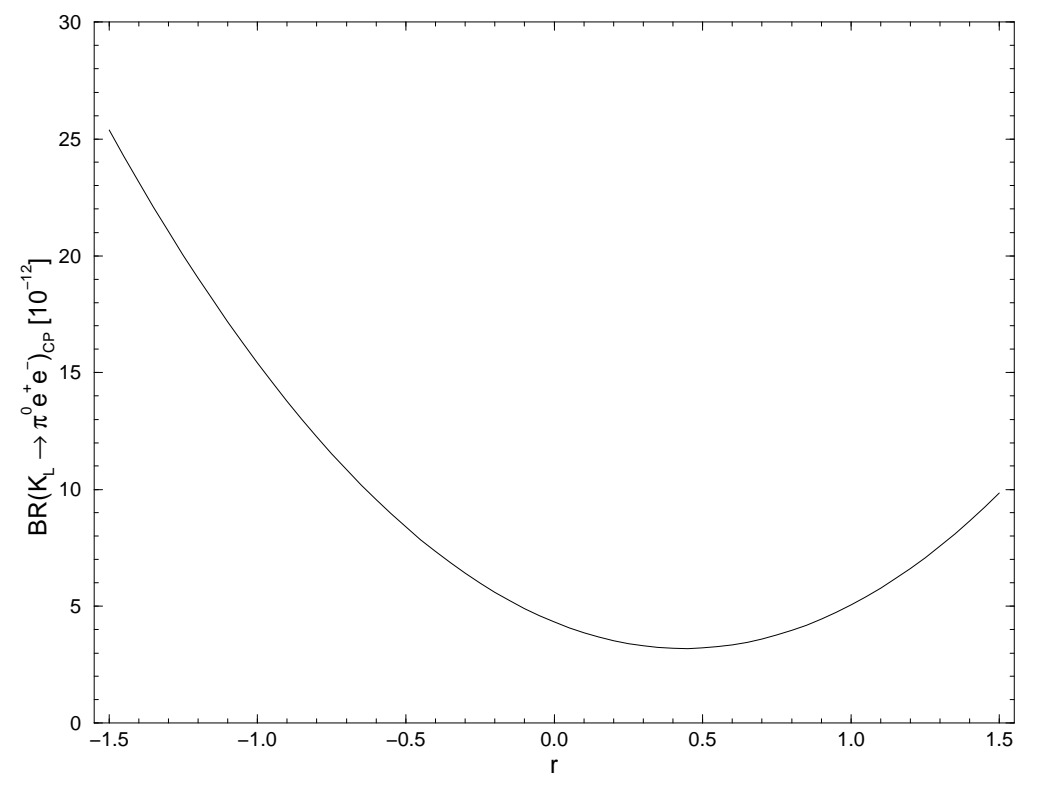

FIG. 19. $B\left(K_{L} \rightarrow \pi^{0} e^{+} e^{-}\right)_{\mathrm{CP}}$ for $m_{\mathrm{t}}=170 \mathrm{GeV}, \Lambda \frac{(4)}{\mathrm{MS}}=325 \mathrm{MeV}$ and $\operatorname{Im} \lambda_{t}=1.3 \cdot 10^{-4}$ as a function of $r$.

$$
B\left(K_{L} \rightarrow \pi^{0} \gamma \gamma\right) \leq \begin{cases}(1.7 \pm 0.3) \cdot 10^{-6} & \text { (Barr et al., 1992) } \\ (2.0 \pm 1.0) \cdot 10^{-6} & \text { (Papadimitriou et al., 1991) }\end{cases}
$$

and of the shape of the $\gamma \gamma$ mass spectrum plays an important role in this estimate. The most recent analyses give

$$
B\left(K_{L} \rightarrow \pi^{0} e^{+} e^{-}\right)_{c o n s} \approx \begin{cases}(0.3-1.8) \cdot 10^{-12} & \text { (Cohen } \text { et al. }, 1993) \\ 4.0 \cdot 10^{-12} & \text { (Heiliger and Seghal, 1993) } \\ (5 \pm 5) \cdot 10^{-12} & \text { (Donoghue and Gabbiani, 1995) }\end{cases}
$$

i.e. not necesarily below the $\mathrm{CP}$ violating contribution. An improved estimate of this component is certainly desirable. It should be noted that there is no interference in the rate between the CP conserving and $\mathrm{CP}$ violating contributions so that the results in fig. 19 and (XXI.17) can simply be added.

\section{E. Outlook}

The results discussed above indicate that within the Standard Model $B\left(K_{L} \rightarrow \pi^{0} e^{+} e^{-}\right)$could be as high as $1 \cdot 10^{-11}$. Moreover the direct $\mathrm{CP}$ violating contribution is found to be important and could even be dominant. Unfortunately the large uncertainties in the remaining two contributions will probably not allow an easy identification of the direct $\mathrm{CP}$ violation by measuring the branching ratio only. The future measurements of $B\left(K_{S} \rightarrow \pi^{0} e^{+} e^{-}\right)$and improvements in the estimate of the $\mathrm{CP}$ conserving part may of course change this unsatisfactory situation. Alternatively the measurements of the electron energy asymmetry (Heiliger and Seghal, 1993), (Donoghue and Gabbiani, 1995) and the study of the time evolution of $K^{0} \rightarrow \pi^{0} e^{+} e^{-}$(Littenberg, 1989b), (Donoghue and 
Gabbiani, 1995), (Köhler and Paschos, 1995) could allow for a refined study of CP violation in this decay.

The present experimental bounds

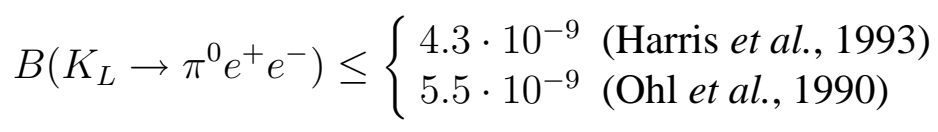

are still by three orders of magnitude away from the theoretical expectations. Yet the prospects of getting the required sensitivity of order $10^{-11}-10^{-12}$ in five years are encouraging (Littenberg and Valencia, 1993), (Winstein and Wolfenstein, 1993), (Ritchie and Wojcicki, 1993). 


\section{THE DECAY $B \rightarrow X_{s} \gamma$}

\section{A. General Remarks}

The $B \rightarrow X_{s} \gamma$ decay is known to be extremely sensitive to the structure of fundamental interactions at the electroweak scale. As any FCNC process, it does not arise at the tree level in the Standard Model. The one-loop W-exchange diagrams that generate this decay at the lowest order in the Standard Model are small enough to be comparable to possible nonstandard contributions (charged scalar exchanges, SUSY one loop diagrams, $W_{R}$ exchanges in the L-R symmetric models, etc.).

The $B \rightarrow X_{s} \gamma$ decay is particularly interesting because its rate is of order $G_{F}^{2} \alpha$, while most of the other FCNC processes involving leptons or photons are of order $G_{F}^{2} \alpha^{2}$. The long-range strong interactions are expected to play a minor role in the inclusive $B \rightarrow X_{s} \gamma$ decay. This is because the mass of the $b$-quark is much larger than the QCD scale $\Lambda$. Moreover, the only relevant intermediate hadronic states $\psi X_{s}$ are expected to give very small contributions, as long as we assume no interference between short- and long-distance terms in the inclusive rate. Therefore, it has become quite common to use the following approximate equality to estimate the $B \rightarrow X_{s} \gamma$ rate:

$$
\frac{\Gamma\left(B \rightarrow X_{s} \gamma\right)}{\Gamma\left(B \rightarrow X_{c} e \bar{\nu}_{e}\right)} \simeq \frac{\Gamma(b \rightarrow s \gamma)}{\Gamma\left(b \rightarrow c e \bar{\nu}_{e}\right)} \equiv R\left(m_{\mathrm{t}}, \alpha_{\mathrm{s}}, \xi\right)
$$

where the quantities on the r.h.s are calculated in the spectator model corrected for short-distance QCD effects. The normalization to the semileptonic rate is usually introduced in order to cancel the uncertainties due to the Cabibbo-Kobayashi-Maskawa (CKM) matrix elements and factors of $m_{\mathrm{b}}^{5}$ in the r.h.s. of eq. (XXII.1). Additional support for the approximation given above comes from the heavy quark expansions. Indeed the spectator model has been shown to correspond to the leading order approximation of an expansion in $1 / m_{\mathrm{b}}$. The first corrections appear at the $\mathcal{O}\left(1 / m_{\mathrm{b}}^{2}\right)$ level. The latter terms have been studied by several authors (Chay et al., 1990), (Bjorken et al., 1992), (Bigi et al., 1992), (Bigi et al., 1993), (Manohar and Wise, 1994), (Blok et al., 1994), (Falk et al., 1994), (Mannel, 1994), (Bigi et al., 1994a) with the result that they affect $B\left(B \rightarrow X_{s} \gamma\right)$ and $B\left(B \rightarrow X_{c} e \bar{\nu}_{e}\right)$ by only a few percent.

As indicated above, the ratio $R$ depends only on $m_{\mathrm{t}}$ and $\alpha_{\mathrm{s}}$ in the Standard Model. In extensions of the Standard Model, additional parameters are present. They have been commonly denoted by $\xi$. The main point to be stressed here is that $R$ is a calculable function of its parameters in the framework of a renormalization group improved perturbation theory. Consequently, the decay in question is particularly suited for tests of the Standard Model and its extensions.

One of the main difficulties in analyzing the inclusive $B \rightarrow X_{s} \gamma$ decay is calculating the shortdistance QCD effects due to hard gluon exchanges between the quark lines of the leading one-loop electroweak diagrams. These effects are known (Bertolini et al., 1987), (Deshpande et al., 1987), (Grinstein et al., 1990), (Grigjanis et al., 1988), (Grigjanis et al., 1992), (Misiak, 1991) to enhance the $B \rightarrow X_{s} \gamma$ rate in the Standard Model by a factor of 2-3, depending on the top quark mass. So the $B \rightarrow X_{s} \gamma$ decay appears to be the only known short distance process in the Standard Model that is dominated by two-loop contributions.

The $B \rightarrow X_{s} \gamma$ decay has already been measured. In 1993 CLEO reported (Ammar et al., 1993) the following branching ratio for the exclusive $B \rightarrow K^{*} \gamma$ decay 


$$
B\left(B \rightarrow K^{*} \gamma\right)=(4.5 \pm 1.5 \pm 0.9) \times 10^{-5} .
$$

In 1994 a first measurement of the inclusive rate has been presented (Alam et al., 1995)

$$
B\left(B \rightarrow X_{s} \gamma\right)=(2.32 \pm 0.57 \pm 0.35) \times 10^{-4}
$$

where the first error is statistical and the second is systematic.

As we will see below these experimental findings are in the ball park of the Standard Model expectations based on the leading logarithmic approximation.

In fact a complete leading order analysis of $B\left(B \rightarrow X_{s} \gamma\right)$ in the Standard Model has been presented almost a year before the CLEO result giving (Buras et al., 1994c)

$$
B\left(B \rightarrow X_{s} \gamma\right)_{T H}=(2.8 \pm 0.8) \times 10^{-4} .
$$

where the error is dominated by the uncertainty in the choice of the renormalization scale $m_{\mathrm{b}} / 2<\mu<2 m_{\mathrm{b}}$ as first stressed by Ali and Greub (Ali and Greub, 1993) and confirmed in (Buras et al., 1994c). Since $B \rightarrow X_{s} \gamma$ is dominated by QCD effects, it is not surprising that this scale-uncertainty in the leading order is particularly large. Such an uncertainty, inherent in any finite order of perturbation theory can be reduced by including next-to-leading order corrections. Unfortunately, it will take some time before the $\mu$-dependences present in $B \rightarrow X_{s} \gamma$ can be reduced in the same manner as it was done for the other decays (Buras et al., 1990), (Buchalla and Buras, 1993a), (Buchalla and Buras, 1994a), (Herrlich and Nierste, 1994). As we already stated in section IX B, a full next-to-leading order computation of $B \rightarrow X_{s} \gamma$ would require calculation of three-loop mixings between the operators $Q_{1}, \ldots, Q_{6}$ and the magnetic penguin operators $Q_{7 \gamma}, Q_{8 G}$. Moreover, certain two-loop matrix elements of the relevant operators should be calculated in the spectator model. A formal analysis at the next-to-leading level (Buras et al., 1994c) is however very encouraging and shows that the $\mu$-dependence can be considerably reduced once all the necessary calculations have been performed. We will return to this issue below.

\section{B. The Decay $B \rightarrow X_{s} \gamma$ in the Leading Log Approximation}

The leading logarithmic calculations (Grinstein et al., 1990), (Misiak, 1993), (Ali and Greub, 1993), (Ciuchini et al., 1994c), (Cella et al., 1994a), (Misiak, 1995), (Buras et al., 1994c) can be summarized in a compact form, as follows:

$$
R=\frac{\Gamma(b \rightarrow s \gamma)}{\Gamma\left(b \rightarrow c e \bar{\nu}_{e}\right)}=\frac{\left|V_{t s}^{*} V_{t b}\right|^{2}}{\left|V_{c b}\right|^{2}} \frac{6 \alpha}{\pi f(z)}\left|C_{7 \gamma}^{(0) e f f}(\mu)\right|^{2}
$$

where $C_{7 \gamma}^{(0) e f f}(\mu)$ is the effective coefficient given in (IX.23) and table XXVIII, $z=\frac{m_{\mathrm{c}}}{m_{\mathrm{b}}}$, and

$$
f(z)=1-8 z^{2}+8 z^{6}-z^{8}-24 z^{4} \ln z
$$

is the phase space factor in the semileptonic b-decay. Note, that at this stage one should not include the $\mathcal{O}\left(\alpha_{\mathrm{s}}\right)$ corrections to $\Gamma(b \rightarrow c e \bar{\nu})$ since they are part of the next-to-leading effects. For the same reason we do not include the $\mathcal{O}\left(\alpha_{\mathrm{s}}\right)$ QCD corrections to the matrix element of the operator $Q_{7 \gamma}$ (the QCD bremsstrahlung $b \rightarrow s \gamma+g$ and the virtual corrections to $b \rightarrow s \gamma$ ) which 
are known (Ali and Greub, 1991a), (Ali and Greub, 1991b), (Pott, 1995) and will be a part of a future NLO analysis.

Formula (XXII.5) and the expression (IX.23) for $C_{7 \gamma}^{(0) e f f}(\mu)$ summarize the complete leading logarithmic (LO) approximation for the $B \rightarrow X_{s} \gamma$ rate in the Standard Model. Their important property is that they are exactly the same in many interesting extensions of the Standard Model, such as the Two-Higgs-Doublet Model (2HDM) (Grinstein et al., 1990), (Hewett, 1993), (Barger et al., 1993), (Hayashi et al., 1993), (Buras et al., 1994c) or the Minimal Supersymmetric Standard Model (MSSM) (Bertolini et al., 1991a), (Barbieri and Giudice, 1993), (Borzumati, 1994). The only quantities that change are the coefficients $C_{2}^{(0)}\left(M_{\mathrm{W}}\right), C_{7 \gamma}^{(0)}\left(M_{\mathrm{W}}\right)$ and $C_{8 G}^{(0)}\left(M_{\mathrm{W}}\right)$. On the other hand in a general $S U(2)_{L} \times S U(2)_{R} \times U(1)$ model additional modifications are necessary, because new operators enter (Cho and Misiak, 1994).

A critical analysis of theoretical and experimental uncertainties present in the prediction for $B\left(B \rightarrow X_{s} \gamma\right)$ based on the above formulae has been made (Buras et al., 1994c). Here we just briefly list the main findings:

- First of all, eq. (XXII.5) is based on the spectator model. As we have mentioned above the heavy quark expansion gives a strong support for this model in inclusive B-decays. On a conservative side one can assume the error due to the use of the spectator model in $B \rightarrow X_{s} \gamma$ to amount to at most $\pm 10 \%$.

- The uncertainty coming from the ratio $z=\frac{m_{\mathrm{c}}}{m_{\mathrm{b}}}$ in the phase-space factor $f(z)$ for the semileptonic decay is estimated to be around $6 \%$.

- The error due to the ratio of the CKM parameters in eq. (XXII.5) is small. Assuming unitarity of the $3 \times 3 \mathrm{CKM}$ matrix and imposing the constraints from the CP-violating parameter $\epsilon_{\mathrm{K}}$ and $B^{0}-\bar{B}^{0}$ mixing one finds

$$
\frac{\left|V_{t s}^{*} V_{t b}\right|^{2}}{\left|V_{c b}\right|^{2}}=0.95 \pm 0.03
$$

- There exists an uncertainty due to the determination of $\alpha_{\mathrm{s}}$. This uncertainty is not small because of the importance of QCD corrections in the considered decay. For instance the difference between the ratios $R$ of eq. (XXII.5) obtained with help of $\alpha \overline{M S}\left(M_{\mathrm{Z}}\right)=0.11$ and 0.13 , respectively, is roughly $20 \%$.

- The dominant uncertainty in eq. (XXII.5) comes from the unknown next-to-leading order contributions. This uncertainty is best signaled by the strong $\mu$-dependence of the leading order expression (XXII.5), which is shown by the solid line in fig. 20, for the case $m_{\mathrm{t}}=$ $170 \mathrm{GeV}$.

One can see that when $\mu$ is varied by a factor of 2 in both directions around $m_{\mathrm{b}} \simeq 5 \mathrm{GeV}$, the ratio (XXII.5) changes by around $\pm 25 \%$, i.e. the ratios $R$ obtained for $\mu=2.5 \mathrm{GeV}$ and $\mu=10 \mathrm{GeV}$ differ by a factor of 1.6 (Ali and Greub, 1993).

The dashed lines in fig. 20 show the expected $\mu$-dependence of the ratio (XXII.5) once a complete next-to-leading calculation is performed. The $\mu$-dependence is then much weaker, but until one performs the calculation explicitly one cannot say which of the dashed curves is the proper one. The way the dashed lines are obtained is described in (Buras et al., 1994c). 


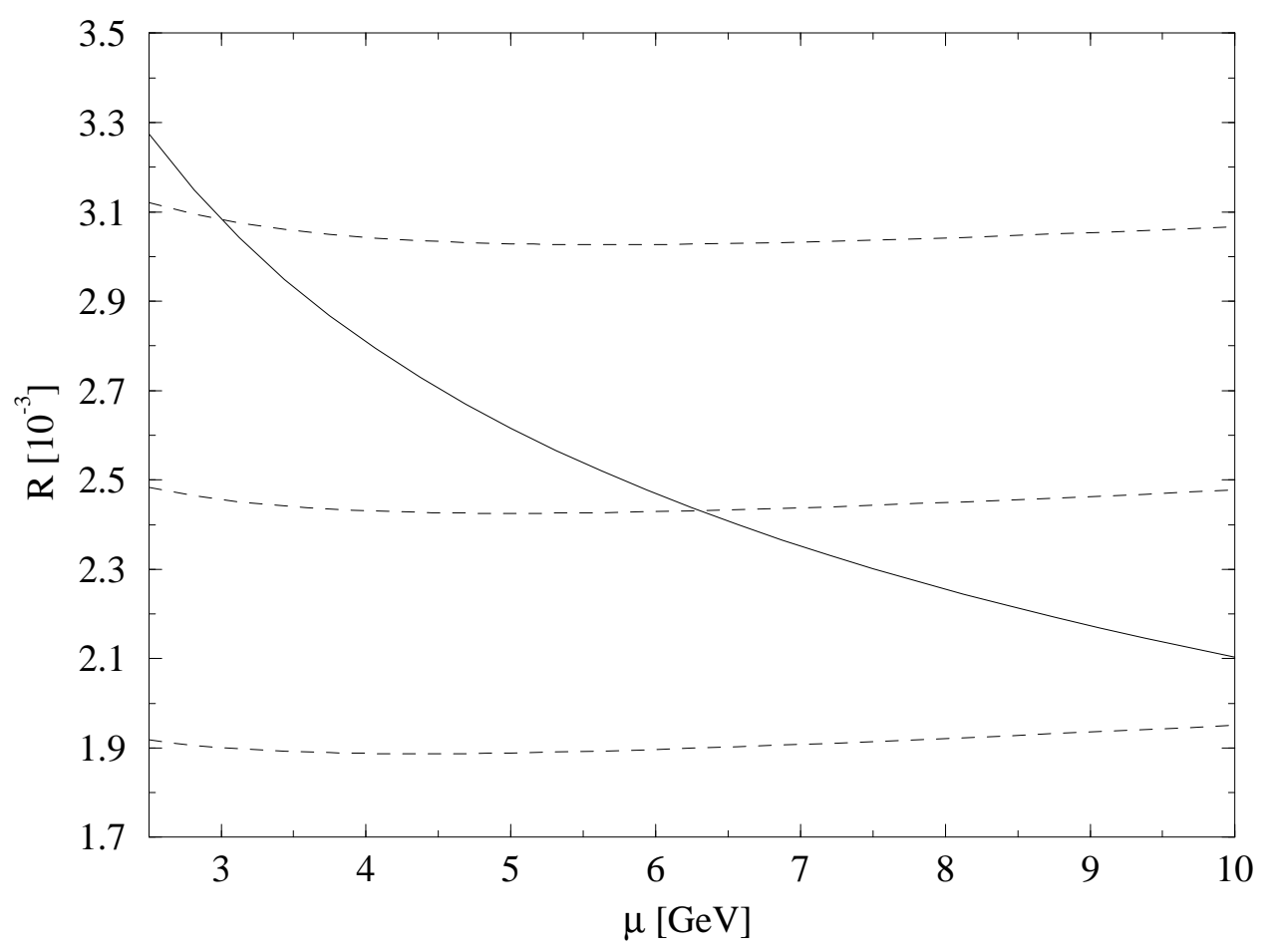

FIG. 20. $\mu$-dependence of the theoretical prediction for the ratio $R$ for $m_{\mathrm{t}}=170 \mathrm{GeV}$ and $\Lambda \frac{(5)}{\mathrm{MS}}=225 \mathrm{MeV}$. The solid line corresponds to the leading order prediction. The dashed lines describe possible next-to-leading results.

- Finally, there exists a $\pm 2.4 \%$ error in determining $B\left(B \rightarrow X_{s} \gamma\right)$ from eq. (XXII.1), which is due to the error in the experimental measurement of $B\left(B \rightarrow X_{c} e \bar{\nu}_{e}\right)=(10.43 \pm 0.24) \%$ (Particle Data Group, 1994).

- The uncertainty due to the value of $m_{\mathrm{t}}$ is small as is shown explicitly below.

Fig. 21 based on (Buras et al., 1994c) presents the Standard Model prediction for the inclusive $B \rightarrow X_{s} \gamma$ branching ratio including the errors listed above as a function of $m_{\mathrm{t}}$ together with the CLEO result.

We stress that the theoretical curves have been obtained prior to the experimental result. Since the theoretical error is dominated by scale ambiguities a complete NLO analysis is very desirable.

\section{Looking at $B \rightarrow X_{s} \gamma$ Beyond Leading Logarithms}

In this section we describe briefly a complete next-to-leading calculation of $B \rightarrow X_{s} \gamma$ in general terms. This section collects the most important findings of section 4 of (Buras et al., 1994c).

Let us first enumerate what has been already calculated in the literature and which calculations are still required in order to complete the next-to-leading calculation of $B\left(B \rightarrow X_{s} \gamma\right)$.

The present status is as follows: 


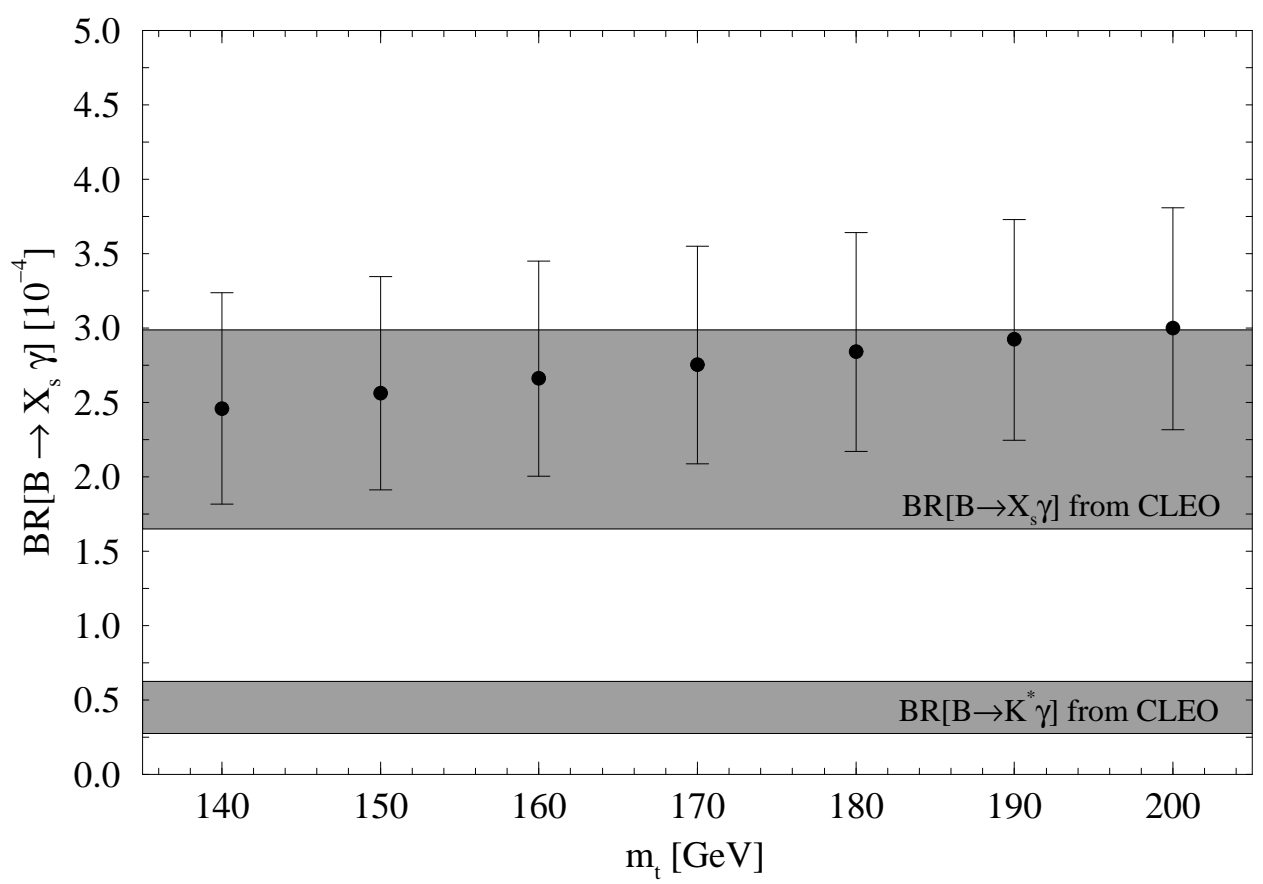

FIG. 21. Predictions for $B \rightarrow X_{s} \gamma$ in the SM as a function of the top quark mass with the theoretical uncertainties taken into account.

- The $6 \times 6$ submatrix of $\gamma^{(1)}$ describing the two-loop mixing of $\left(Q_{1}, \ldots, Q_{6}\right)$ and the corresponding $\mathcal{O}\left(\alpha_{\mathrm{s}}\right)$ corrections in $\vec{C}\left(M_{\mathrm{W}}\right)$ have been already calculated. They are given in section VI.

- The two-loop mixing in the $\left(Q_{7 \gamma}, Q_{8 G}\right)$ sector of $\gamma^{(1)}$ is known (Misiak and Münz, 1995) and given in section IX C.

- The $\mathcal{O}\left(\alpha_{\mathrm{s}}\right)$ corrections to the matrix element of the operators $Q_{7 \gamma}$ and $Q_{8 G}$ have been calculated (Ali and Greub, 1991a), (Ali and Greub, 1991b). They have been recently confirmed by (Pott, 1995) who also presents the results for the matrix elements of the remaining operators.

The remaining ingredients of a next-to-leading analysis of $B\left(B \rightarrow X_{s} \gamma\right)$ are:

- The three-loop mixing between the sectors $\left(Q_{1}, \ldots, Q_{6}\right)$ and $\left(Q_{7 \gamma}, Q_{8 G}\right)$ which, with our normalizations, contributes to $\gamma^{(1)}$.

- The $\mathcal{O}\left(\alpha_{\mathrm{s}}\right)$ corrections to $C_{7 \gamma}\left(M_{\mathrm{W}}\right)$ and $C_{8 G}\left(M_{\mathrm{W}}\right)$ in (IX.12) and (IX.13). This requires evaluation of two-loop penguin diagrams with internal $\mathrm{W}$ and top quark masses and a proper matching with the effective five-quark theory. An attempt to calculate the necessary twoloop Standard Model diagrams has been made in (Adel and Yao, 1994).

- The finite parts of the effective theory two-loop diagrams with the insertions of the fourquark operators . 
All these calculations are very involved, and the necessary three-loop calculation is a truly formidable task! Yet, as stressed in (Buras et al., 1994c) all these calculations have to be done if we want to reduce the theoretical uncertainties in $b \rightarrow s \gamma$ to around $10 \%$.

As demonstrated formally in (Buras et al., 1994c) the cancellation of the dominant $\mu$ dependence in the leading order can be achieved by calculating the relevant two-loop matrix element of the dominant four-quark operator $Q_{2}$. This matrix element is however renormalizationscheme dependent and moreover mixing with other operators takes place. This scheme dependence can only be canceled by calculating $\gamma^{(1)}$ in the same renormalization scheme. This point has been extensively discussed in this review and we will not repeat this discussion here. However, it is clear from these remarks, that in order to address the $\mu$-dependence and the renormalizationscheme dependence as well as their cancellations, it is necessary to perform a complete next-toleading order analysis of $\vec{C}(\mu)$ and of the corresponding matrix elements.

In this context we would like to comment on an analysis of (Ciuchini et al., 1994b) in which the known two-loop mixing of $Q_{1}, \ldots, Q_{6}$ has been added to the leading order analysis of $B \rightarrow$ $X_{s} \gamma$. Strong renormalization scheme dependence of the resulting branching ratio has been found, giving the branching ratio $(1.7 \pm 0.2) \times 10^{-4}$ and $(2.3 \pm 0.2) \times 10^{-4}$ at $\mu=5 \mathrm{GeV}$ for $\mathrm{HV}$ and NDR schemes, respectively. It has also been observed that whereas in the HV scheme the $\mu$ dependence has been weakened, it remained still strong in the NDR scheme. In our opinion this partial cancellation of the $\mu$-dependence in the HV scheme is rather accidental and has nothing to do with the cancellation of the $\mu$-dependence discussed above. The latter requires the evaluation of finite parts in two-loop matrix elements of the four-quark operators $Q_{1}, \ldots, Q_{6}$. On the other hand the strong scheme dependence in the partial NLO analysis presented in (Ciuchini et al., 1994b) demonstrates very clearly the need for a full analysis. In view of this discussion we think that the decrease of the branching ratio for $B \rightarrow X_{s} \gamma$ relative to the LO prediction, found in (Ciuchini et al., 1994b), and given by $B\left(B \rightarrow X_{s} \gamma\right)=(1.9 \pm 0.2 \pm 0.5) \cdot 10^{-4}$ is still premature and one should wait until the full NLO analysis has been done.

\section{THE DECAY $B \rightarrow X_{s} e^{+} e^{-}$}

\section{A. General Remarks}

The rare decay $B \rightarrow X_{s} e^{+} e^{-}$has been the subject of many theoretical studies in the framework of the Standard Model and its extensions such as the two Higgs doublet models and models involving supersymmetry (Hou et al., 1987), (Grinstein et al., 1989), (Jaus and Wyler, 1990), (Bertolini et al., 1991b), (Ali et al., 1991), (Deshpande et al., 1993), (Ali et al., 1995), (Greub et al., 1995). In particular the strong dependence of $B \rightarrow X_{s} e^{+} e^{-}$on $m_{\mathrm{t}}$ has been stressed in (Hou et al., 1987). It is clear that once $B \rightarrow X_{s} e^{+} e^{-}$has been observed, it will offer a useful test of the Standard Model and its extensions. To this end the relevant branching ratio, the dilepton invariant mass distribution and other distributions of interest should be calculated with sufficient precision. In particular the QCD effects should be properly taken into account.

The central element in any analysis of $B \rightarrow X_{s} e^{+} e^{-}$is the effective hamiltonian for this decay given in section $X$ where a detailed analysis of the Wilson coefficients has been presented. However, the actual calculation of $B \rightarrow X_{s} e^{+} e^{-}$involves not only the evaluation of Wilson coefficients of the relevant local operators but also the calculation of the corresponding matrix elements of these operators relevant for $B \rightarrow X_{s} e^{+} e^{-}$. The latter part of the analysis can be done in the 
spectator model, which, as indicated by the heavy quark expansion should offer a good approximation to QCD for B-decays. One can also include the non-perturbative $\mathcal{O}\left(1 / m_{\mathrm{b}}^{2}\right)$ corrections to the spectator model which enhance the rate for $B \rightarrow X_{s} e^{+} e^{-}$by roughly $10 \%$ (Falk et al., 1994). A realistic phenomenological analysis should also include the long-distance contributions which are mainly due to the $J / \psi$ and $\psi^{\prime}$ resonances (Lim et al., 1989), (Deshpande et al., 1989), (O’Donnell and Tung, 1991). Since in this review we are mainly interested in the next-to-leading short-distance QCD effects we will not include these complications in what follows. This section closely follows (Buras and Münz, 1995) execpt that the numerical results in figs. 22-24 have been slightly changed in accordance with the input parameters of appendix A.

We stress again that in a consistent NLO analysis of the decay $B \rightarrow X_{s} e^{+} e^{-}$, one should on one hand calculate the Wilson coefficient of the operator $Q_{9 V}=(\bar{s} b)_{V-A}(\bar{e} e)_{V}$ including leading and next-to-leading logarithms, but on the other hand only leading logarithms should be kept in the remaining Wilson coefficients. Only then a scheme independent amplitude can be obtained. As already discussed in section X, this special treatment of $Q_{9}$ is related to the fact that strictly speaking in the leading logarithmic approximation only this operator contributes to $B \rightarrow X_{s} e^{+} e^{-}$. The contributions of the usual current-current operators, QCD penguin operators, magnetic penguin operators and of $Q_{10 A}=(\bar{s} b)_{V-A}(\bar{e} e)_{A}$ enter only at the NLO level and to be consistent only the leading contributions to the corresponding Wilson coefficients should be included.

\section{B. The Differential Decay Rate}

Introducing

$$
\hat{s}=\frac{\left(p_{e^{+}}+p_{e^{-}}\right)^{2}}{m_{\mathrm{b}}^{2}}, \quad z=\frac{m_{\mathrm{c}}}{m_{\mathrm{b}}}
$$

and calculating the one-loop matrix elements of $Q_{i}$ using the spectator model in the NDR scheme one finds (Misiak, 1995), (Buras and Münz, 1995)

$$
\begin{aligned}
& R(\hat{s}) \equiv \frac{d / d \hat{s} \Gamma\left(b \rightarrow s e^{+} e^{-}\right)}{\Gamma(b \rightarrow c e \bar{\nu})}=\frac{\alpha^{2}}{4 \pi^{2}}\left|\frac{V_{t s}}{V_{c b}}\right|^{2} \frac{(1-\hat{s})^{2}}{f(z) \kappa(z)} \times \\
& {\left[(1+2 \hat{s})\left(\left|\widetilde{C}_{9}^{e f f}\right|^{2}+\left|\widetilde{C}_{10}\right|^{2}\right)+4\left(1+\frac{2}{\hat{s}}\right)\left|C_{7 \gamma}^{(0) e f f}\right|^{2}+12 C_{7 \gamma}^{(0) e f f} \operatorname{Re} \widetilde{C}_{9}^{\text {eff }}\right]}
\end{aligned}
$$

where

$$
\begin{aligned}
\widetilde{C}_{9}^{e f f}= & \widetilde{C}_{9}^{N D R} \tilde{\eta}(\hat{s})+h(z, \hat{s})\left(3 C_{1}^{(0)}+C_{2}^{(0)}+3 C_{3}^{(0)}+C_{4}^{(0)}+3 C_{5}^{(0)}+C_{6}^{(0)}\right)- \\
& \frac{1}{2} h(1, \hat{s})\left(4 C_{3}^{(0)}+4 C_{4}^{(0)}+3 C_{5}^{(0)}+C_{6}^{(0)}\right)- \\
& \frac{1}{2} h(0, \hat{s})\left(C_{3}^{(0)}+3 C_{4}^{(0)}\right)+\frac{2}{9}\left(3 C_{3}^{(0)}+C_{4}^{(0)}+3 C_{5}^{(0)}+C_{6}^{(0)}\right) .
\end{aligned}
$$

The general expression (XXIII.2) with $\kappa(z)=1$ has been first presented by (Grinstein et al., 1989) who in their approximate leading order renormalization group analysis kept only the operators $Q_{1}, Q_{2}, Q_{7 \gamma}, Q_{9 V}, Q_{10 A}$.

The various entries in (XXIII.2) are given as follows 


$$
\begin{aligned}
h(z, \hat{s})= & -\frac{8}{9} \ln \frac{m_{\mathrm{b}}}{\mu}-\frac{8}{9} \ln z+\frac{8}{27}+\frac{4}{9} x- \\
& \frac{2}{9}(2+x)|1-x|^{1 / 2} \begin{cases}\left(\ln \left|\frac{\sqrt{1-x}+1}{\sqrt{1-x}-1}\right|-i \pi\right), & \text { for } x \equiv 4 z^{2} / \hat{s}<1 \\
2 \arctan \frac{1}{\sqrt{x-1}}, & \text { for } x \equiv 4 z^{2} / \hat{s}>1,\end{cases} \\
h(0, \hat{s})= & \frac{8}{27}-\frac{8}{9} \ln \frac{m_{\mathrm{b}}}{\mu}-\frac{4}{9} \ln \hat{s}+\frac{4}{9} i \pi . \\
f(z)= & 1-8 z^{2}+8 z^{6}-z^{8}-24 z^{4} \ln z, \\
\kappa(z)= & 1-\frac{2 \alpha_{\mathrm{s}}(\mu)}{3 \pi}\left[\left(\pi^{2}-\frac{31}{4}\right)(1-z)^{2}+\frac{3}{2}\right] \\
\tilde{\eta}(\hat{s})= & 1+\frac{\alpha_{\mathrm{s}}(\mu)}{\pi} \omega(\hat{s})
\end{aligned}
$$

with

$$
\begin{aligned}
\omega(\hat{s})= & -\frac{2}{9} \pi^{2}-\frac{4}{3} \operatorname{Li}_{2}(s)-\frac{2}{3} \ln s \ln (1-s)-\frac{5+4 s}{3(1+2 s)} \ln (1-s)- \\
& \frac{2 s(1+s)(1-2 s)}{3(1-s)^{2}(1+2 s)} \ln s+\frac{5+9 s-6 s^{2}}{6(1-s)(1+2 s)} .
\end{aligned}
$$

Here $f(z)$ is the phase-space factor for $b \rightarrow c e \bar{\nu} . \kappa(z)$ is the corresponding single gluon QCD correction (Cabibbo and Maiani, 1978) in the approximation of (Kim and Martin, 1989). $\tilde{\eta}$ on the other hand represents single gluon corrections to the matrix element of $Q_{9}$ with $m_{\mathrm{s}}=0$ (Jezabek and Kühn, 1989), (Misiak, 1995). For consistency reasons this correction should only multiply the leading logarithmic term in $\tilde{C}_{9}^{\mathrm{NDR}}$.

In the HV scheme the one-loop matrix elements are different and one finds an additional explicit contribution to (XXIII.3) given by (Buras and Münz, 1995)

$$
-\xi^{H V} \frac{4}{9}\left(3 C_{1}^{(0)}+C_{2}^{(0)}-C_{3}^{(0)}-3 C_{4}^{(0)}\right) .
$$

However $\widetilde{C}_{9}^{N D R}$ has to be replaced by $\widetilde{C}_{9}^{H V}$ given in (X.5) and (X.9) and consequently $\widetilde{C}_{9}^{e f f}$ is the same in both schemes.

The first term in the function $h(z, \hat{s})$ in (XXIII.4) represents the leading $\mu$-dependence in the matrix elements. It is canceled by the $\mu$-dependence present in the leading logarithm in $\tilde{C}_{9}$. This is precisely the type of cancellation of the $\mu$-dependence which one would like to achieve in the case of $B \rightarrow X_{s} \gamma$. The $\mu$-dependence present in the coefficients of the other operators can only be canceled by going to still higher order in the renormalization group improved perturbation theory. To this end the matrix elements of four-quark operators should be evaluated at two-loop level. Also certain unknown three-loop anomalous dimensions should be included in the evaluation of $C_{7 \gamma}^{e f f}$ and $C_{9 V}$. Certainly this is beyond the scope of this review and we will only investigate the left-over $\mu$-dependence below.

\section{Numerical Analysis}

A detailed numerical analysis of the formulae above has been presented in (Buras and Münz, 1995). We give here a brief account of this work. We set first $\left|V_{t s} / V_{c b}\right|=1$ which in view of 
(XXII.7) is a good approximation. We keep in mind that for $\hat{s} \approx m_{\psi}^{2} / m_{\mathrm{b}}^{2}, \hat{s} \approx m_{\psi^{\prime}}^{2} / m_{\mathrm{b}}^{2}$ etc. the spectator model cannot be the full story and additional long-distance contributions discussed in (Lim et al., 1989), (Deshpande et al., 1989), (O'Donnell and Tung, 1991) have to be taken into account in a phenomenological analysis. Similarly we do not include $1 / \mathrm{m}_{\mathrm{b}}^{2}$ corrections calculated in (Falk et al., 1994) which typically enhance the differential rate by about $10 \%$.

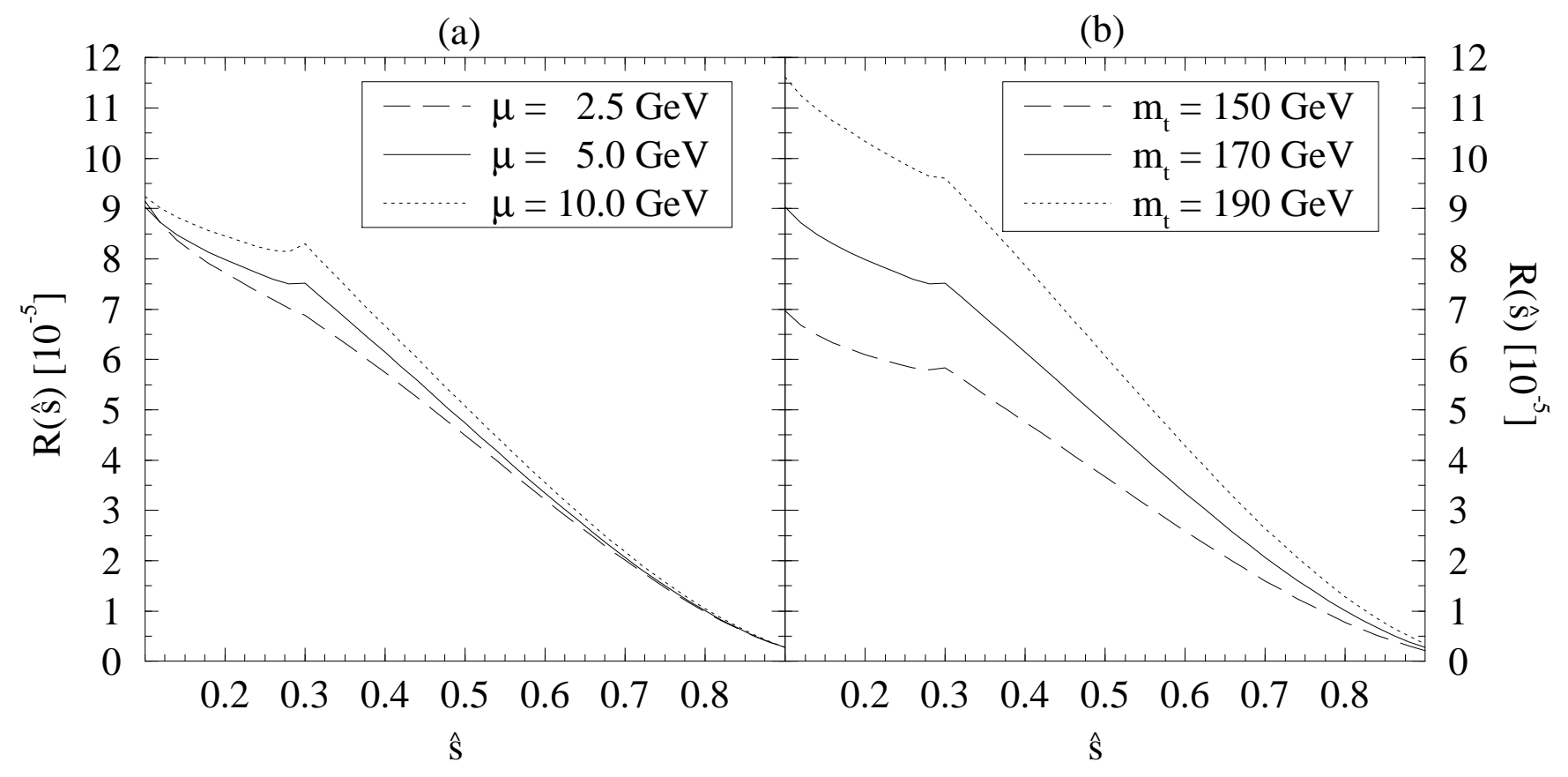

FIG. 22. (a) $R(\hat{s})$ for $m_{\mathrm{t}}=170 \mathrm{GeV}, \Lambda \frac{(5)}{\mathrm{MS}}=225 \mathrm{MeV}$ and differents values of $\mu$.

(b) $R(\hat{s})$ for $\mu=5 \mathrm{GeV}, \Lambda \frac{(5)}{\mathrm{MS}}=225 \mathrm{MeV}$ and various values of $m_{\mathrm{t}}$.

In fig. 22 (a) we show $R(\hat{s})$ for $m_{\mathrm{t}}=170 \mathrm{GeV}, \Lambda_{\overline{\mathrm{MS}}}=225 \mathrm{MeV}$ and different values of $\mu$. In fig. 22 (b) we set $\mu=5 \mathrm{GeV}$ and vary $m_{\mathrm{t}}$ from $150 \mathrm{GeV}$ to $190 \mathrm{GeV}$. The remaining $\mu$ dependence is rather weak and amounts to at most $\pm 8 \%$ in the full range of parameters considered. The $m_{\mathrm{t}}$ dependence of $R(\hat{s})$ is sizeable. Varying $m_{\mathrm{t}}$ between $150 \mathrm{GeV}$ and $190 \mathrm{GeV}$ changes $R(\hat{s})$ by typically $60-65 \%$ which in this range of $m_{\mathrm{t}}$ corresponds to $R(\hat{s}) \sim m_{\mathrm{t}}^{2}$. It is easy to verify that this strong $m_{\mathrm{t}}$ dependence originates in the coefficient $\widetilde{C}_{10}$ given in (X.3) as already stressed by several authors in the past (Hou et al., 1987), (Grinstein et al., 1989), (Bertolini et al., 1991b), (Deshpande et al., 1993), (Greub et al., 1995), (Ali et al., 1995), (Ali et al., 1991), (Jaus and Wyler, 1990).

We do not show the $\Lambda_{\overline{\mathrm{MS}}}$ dependence as it is very weak. Typically, changing $\Lambda_{\overline{\mathrm{MS}}}$ from $140 \mathrm{MeV}$ to $310 \mathrm{MeV}$ decreases $R(\hat{s})$ by about $5 \%$.

$R(\hat{s})$ is governed by three coefficients, $\widetilde{C}_{9}^{\text {eff }}, \widetilde{C}_{10}$ and $C_{7 \gamma}^{(0) e f f}$. The importance of various contributions has been investigated in (Buras and Münz, 1995). To this end one sets $\Lambda \frac{(5)}{\mathrm{MS}}=$ $225 \mathrm{GeV}, m_{\mathrm{t}}=170 \mathrm{GeV}$ and $\mu=5 \mathrm{GeV}$. In fig. 23 we show $R(\hat{s})$ keeping only $\widetilde{C}_{9}^{e f f}, \widetilde{C}_{10}$, $C_{7 \gamma}^{(0) e f f}$ and the $C_{7 \gamma}^{(0) e f f}-\widetilde{C}_{9}^{\text {eff }}$ interference term, respectively. Denoting these contributions by $R_{9}$, $R_{10}, R_{7}$ and $R_{7 / 9}$ we observe that the term $R_{7}$ plays only a minor role in $R(\hat{s})$. On the other hand the presence of $C_{7 \gamma}^{(0) e f f}$ cannot be ignored because the interference term $R_{7 / 9}$ is significant. 


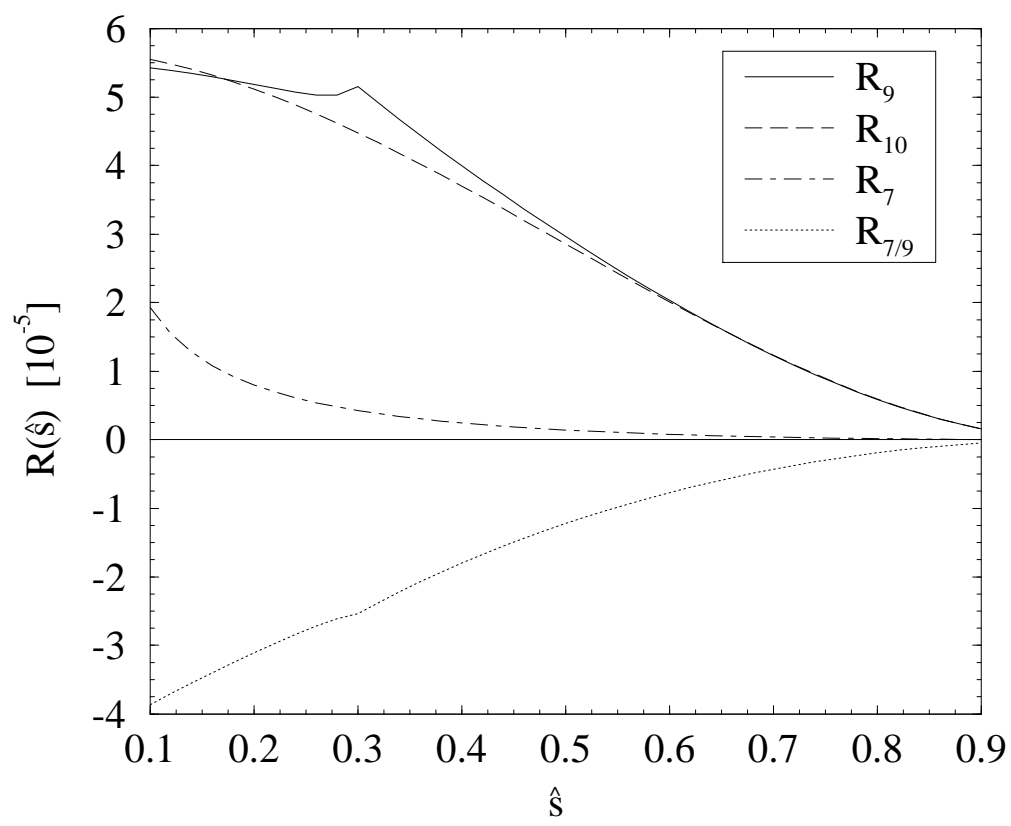

FIG. 23. Comparison of the four different contributions to $R(\hat{s})$ according to eq. (XXIII.2).

In fact the presence of this large interference term could be used to measure experimentally the relative sign of $C_{7 \gamma}^{(0) e f f}$ and $\operatorname{Re} \widetilde{C}_{9}^{e f f}$ (Grinstein et al., 1989), (Jaus and Wyler, 1990), (Ali et al., 1991), (Greub et al., 1995), (Ali et al., 1995) which as seen in fig. 23 is negative in the Standard Model. However, the most important contributions are $R_{9}$ and $R_{10}$ in the full range of $\hat{s}$ considered. For $m_{\mathrm{t}} \approx 170 \mathrm{GeV}$ these two contributions are roughly of the same size. Due to a strong $m_{\mathrm{t}}$ dependence of $R_{10}$, this contribution dominates for higher values of $m_{\mathrm{t}}$ and is less important than $R_{9}$ for $m_{\mathrm{t}}<170 \mathrm{GeV}$.

Next, in fig. 24 we show $R(\hat{s})$ for $\mu=5 \mathrm{GeV}, m_{\mathrm{t}}=170 \mathrm{GeV}$ and $\Lambda_{\overline{\mathrm{MS}}}=225 \mathrm{MeV}$ compared to the case of no QCD corrections and to the results (Grinstein et al., 1989) would obtain for our set of parameters using their approximate leading order formulae.

The discussion of the definition of $m_{\mathrm{t}}$ used here is identical to the one in the case of $K_{L} \rightarrow$ $\pi^{0} e^{+} e^{-}$and will not be repeated here. On the basis of the arguments given there we believe that if $m_{\mathrm{t}}=\bar{m}_{\mathrm{t}}\left(m_{\mathrm{t}}\right)$ is chosen, the additional short-distance QCD corrections to $B\left(B \rightarrow X_{s} e^{+} e^{-}\right)$ should be small.

Our discussion has been restricted to $B\left(B \rightarrow X_{s} \gamma\right)$. Also the photon spectrum has been the subject of several papers. We just refer to the most recent articles (Neubert, 1994b), (Shifman et al., 1994), (Dikeman et al., 1995), (Kapustin and Ligeti, 1995), (Kapustin et al., 1995), (Ali and Greub, 1995), (Pott, 1995) where further references can be found. 


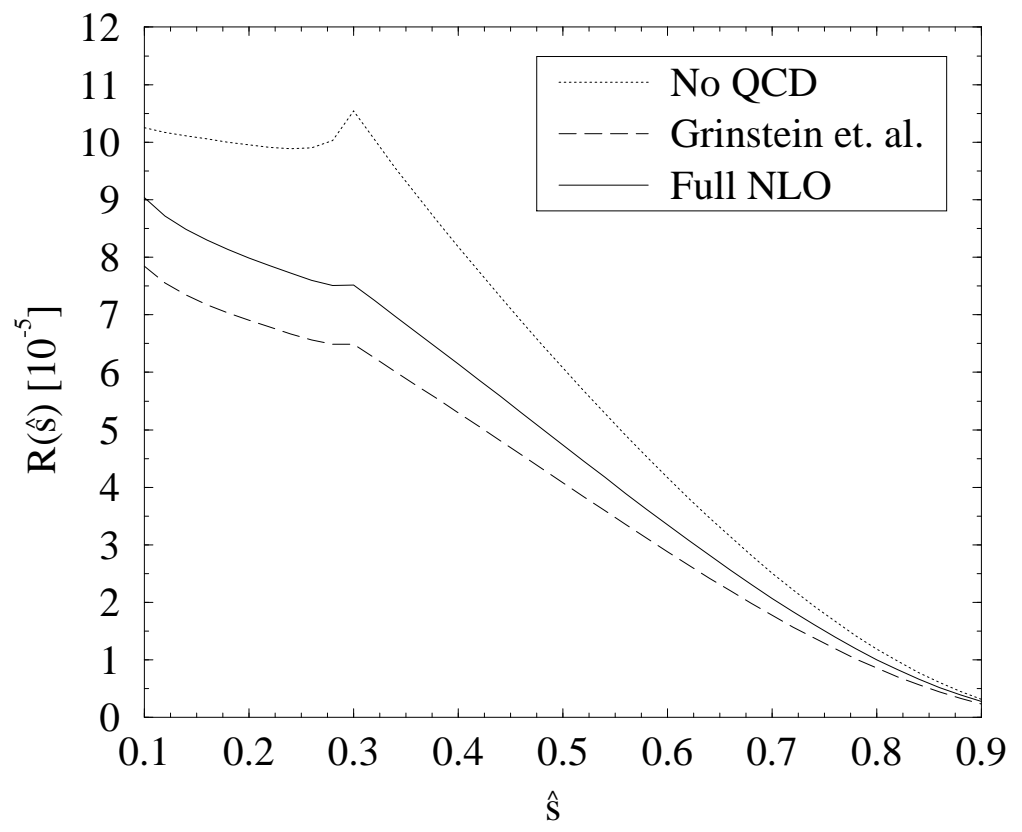

FIG. 24. $R(\hat{s})$ for $m_{\mathrm{t}}=170 \mathrm{GeV}, \Lambda \frac{(5)}{\mathrm{MS}}=225 \mathrm{MeV}$ and $\mu=5 \mathrm{GeV}$. 


\section{THE DECAYS $K^{+} \rightarrow \pi^{+} \nu \bar{\nu}$ AND $K_{L} \rightarrow \pi^{0} \nu \bar{\nu}$}

\section{A. General Remarks on $K^{+} \rightarrow \pi^{+} \nu \bar{\nu}$}

The rare decay $K^{+} \rightarrow \pi^{+} \nu \bar{\nu}$ is one of the theoretically cleanest decays. As such it is very well suited for the determination of CKM parameters, in particular of the element $V_{t d} . K^{+} \rightarrow \pi^{+} \nu \bar{\nu}$ is $\mathrm{CP}$ conserving and receives contributions from both internal top and charm exchanges. The inclusion of next-to-leading QCD corrections incorporated in the effective hamiltonian in (XI.4) and discussed in detail in section XI B reduces considerably the theoretical uncertainties due to the choice of the renormalization scales present in the leading order expressions. We will illustrate this below. Since in addition the relevant hadronic matrix element of the weak current $(\bar{s} d)_{V-A}$ can be measured in the leading decay $K^{+} \rightarrow \pi^{0} e^{+} \nu$, the resulting theoretical expression for $B\left(K^{+} \rightarrow \pi^{+} \nu \bar{\nu}\right)$ is only a function of the CKM parameters, the QCD scale $\Lambda_{\overline{M S}}$ and the quark masses $m_{t}$ and $m_{c}$. The long-distance contributions to $K^{+} \rightarrow \pi^{+} \nu \bar{\nu}$ have been found to be very small: a few percent of the charm contribution to the amplitude at most, which is safely negligible (Rein and Sehgal, 1989), (Hagelin and Littenberg, 1989) and (Lu and Wise, 1994).

Conventionally the branching fraction $B\left(K^{+} \rightarrow \pi^{+} \nu \bar{\nu}\right)$ is related to the experimentally well known quantity $B\left(K^{+} \rightarrow \pi^{0} e^{+} \nu\right)$ using isospin symmetry. Corrections to this approximation have recently been studied in (Marciano and Parsa, 1995). The breaking of isospin is due to quark mass effects and electroweak radiative corrections. In the case of $K^{+} \rightarrow \pi^{+} \nu \bar{\nu}$ these effects result in a decrease of the branching ratio by $10 \%$. The corresponding corrections in $K_{L} \rightarrow \pi^{0} \nu \bar{\nu}$ lead to a $5.6 \%$ reduction of $B\left(K_{L} \rightarrow \pi^{0} \nu \bar{\nu}\right)$. We have checked the analysis of (Marciano and Parsa, 1995) and agree with their findings. Once calculated, the inclusion of these effects is straightforward as they only amount to an overall factor for the branching ratio and do not affect the short-distance structure of $K \rightarrow \pi \nu \bar{\nu}$. We shall neglect the isospin violating corrections in the following chapters, where the focus is primarily on the short-distance physics. The effects are however incorporated in the final prediction quoted in our summary table in section XXVII.

In the following we shall concentrate on a discussion of $K^{+} \rightarrow \pi^{+} \nu \bar{\nu}$ within the framework of the standard model. The impact of various scenarios of new physics on this decay has been considered for instance in (Bigi and Gabbiani, 1991).

\section{B. Master Formulae for $K^{+} \rightarrow \pi^{+} \nu \bar{\nu}$}

Using the effective hamiltonian (XI.4) and summing over the three neutrino flavors one finds

$$
\begin{gathered}
B\left(K^{+} \rightarrow \pi^{+} \nu \bar{\nu}\right)=\kappa_{+} \cdot\left[\left(\frac{\operatorname{Im} \lambda_{t}}{\lambda^{5}} X\left(x_{t}\right)\right)^{2}+\left(\frac{\operatorname{Re} \lambda_{c}}{\lambda} P_{0}(X)+\frac{\operatorname{Re} \lambda_{t}}{\lambda^{5}} X\left(x_{t}\right)\right)^{2}\right] \\
\kappa_{+}=\frac{3 \alpha^{2} B\left(K^{+} \rightarrow \pi^{0} e^{+} \nu\right)}{2 \pi^{2} \sin ^{4} \Theta_{W}} \lambda^{8}=4.57 \cdot 10^{-11}
\end{gathered}
$$

where we have used

$$
\alpha=\frac{1}{129} \quad \sin ^{2} \Theta_{W}=0.23 \quad B\left(K^{+} \rightarrow \pi^{0} e^{+} \nu\right)=4.82 \cdot 10^{-2}
$$


Here $\lambda_{i}=V_{i s}^{*} V_{i d}$ with $\lambda_{c}$ being real to a very high accuracy. The function $X$ of (XI.5) can also be written as

$$
X(x)=\eta_{X} \cdot X_{0}(x) \quad \eta_{X}=0.985
$$

where $\eta_{X}$ summarizes the NLO corrections discussed in section XIB. With $m_{t} \equiv m_{t}\left(m_{t}\right)$ the QCD factor $\eta_{X}$ is practically independent of $m_{t}$ and $\Lambda_{\overline{M S}}$. Next

$$
P_{0}(X)=\frac{1}{\lambda^{4}}\left[\frac{2}{3} X_{N L}^{e}+\frac{1}{3} X_{N L}^{\tau}\right]
$$

with the numerical values for $X_{N L}^{l}$ given in table XXXIII. The corresponding values for $P_{0}(X)$ as a function of $\Lambda_{\overline{M S}}$ and $m_{c} \equiv m_{c}\left(m_{c}\right)$ are collected in table XLIV. We remark that a negligibly small term $\sim\left(X_{N L}^{e}-X_{N L}^{\tau}\right)^{2}(\sim 0.2 \%$ effect on the branching ratio) has been discarded in formula (XXIV.1).

TABLE XLIV. The function $P_{0}(X)$ for various $\Lambda_{\mathrm{MS}}^{(4)}$ and $m_{c}$.

\begin{tabular}{|l|c|c|c|}
\hline \hline & \multicolumn{3}{|c|}{$P_{0}(X)$} \\
\hline$\Lambda \frac{(4)}{\mathrm{MS}} \backslash m_{c}$ & $1.25 \mathrm{GeV}$ & $1.30 \mathrm{GeV}$ & $1.35 \mathrm{GeV}$ \\
\hline $215 \mathrm{MeV}$ & 0.402 & 0.436 & 0.472 \\
$325 \mathrm{MeV}$ & 0.366 & 0.400 & 0.435 \\
$435 \mathrm{MeV}$ & 0.325 & 0.359 & 0.393 \\
\hline \hline
\end{tabular}

Using the improved Wolfenstein parametrization and the approximate formulae (II.23) - (II.25) we can next write

$$
B\left(K^{+} \rightarrow \pi^{+} \nu \bar{\nu}\right)=4.57 \cdot 10^{-11} A^{4} X^{2}\left(x_{t}\right) \frac{1}{\sigma}\left[(\sigma \bar{\eta})^{2}+\left(\varrho_{0}-\bar{\varrho}\right)^{2}\right]
$$

where

$$
\sigma=\left(\frac{1}{1-\frac{\lambda^{2}}{2}}\right)^{2}
$$

The measured value of $\mathrm{B}\left(K^{+} \rightarrow \pi^{+} \nu \bar{\nu}\right)$ then determines an ellipse in the $(\bar{\varrho}, \bar{\eta})$ plane centered at $\left(\varrho_{0}, 0\right)$ with (Buras et al., 1994b)

$$
\varrho_{0}=1+\frac{P_{0}(X)}{A^{2} X\left(x_{t}\right)}
$$

and having the squared axes

$$
\bar{\varrho}_{1}^{2}=r_{0}^{2} \quad \bar{\eta}_{1}^{2}=\left(\frac{r_{0}}{\sigma}\right)^{2}
$$

where 


$$
r_{0}^{2}=\frac{1}{A^{4} X^{2}\left(x_{t}\right)}\left[\frac{\sigma \cdot B R\left(K^{+} \rightarrow \pi^{+} \nu \bar{\nu}\right)}{4.57 \cdot 10^{-11}}\right]
$$

The departure of $\varrho_{0}$ from unity measures the relative importance of the internal charm contributions.

The ellipse defined by $r_{0}, \varrho_{0}$ and $\sigma$ given above intersects with the circle (II.32). This allows to determine $\bar{\varrho}$ and $\bar{\eta}$ with

$$
\bar{\varrho}=\frac{1}{1-\sigma^{2}}\left(\varrho_{0}-\sqrt{\sigma^{2} \varrho_{0}^{2}+\left(1-\sigma^{2}\right)\left(r_{0}^{2}-\sigma^{2} R_{b}^{2}\right)}\right) \quad \bar{\eta}=\sqrt{R_{b}^{2}-\bar{\varrho}^{2}}
$$

and consequently

$$
R_{t}^{2}=1+R_{b}^{2}-2 \bar{\varrho}
$$

where $\bar{\eta}$ is assumed to be positive.

In the leading order of the Wolfenstein parametrization

$$
\sigma \rightarrow 1 \quad \bar{\eta} \rightarrow \eta \quad \bar{\varrho} \rightarrow \varrho
$$

and $B\left(K^{+} \rightarrow \pi^{+} \nu \bar{\nu}\right)$ determines a circle in the $(\varrho, \eta)$ plane centered at $\left(\varrho_{0}, 0\right)$ and having the radius $r_{0}$ of (XXIV.10) with $\sigma=1$. Formulae (XXIV.11) and (XXIV.12) then simplify to (Buchalla and Buras, 1994a)

$$
R_{t}^{2}=1+R_{b}^{2}+\frac{r_{0}^{2}-R_{b}^{2}}{\varrho_{0}}-\varrho_{0} \quad \varrho=\frac{1}{2}\left(\varrho_{0}+\frac{R_{b}^{2}-r_{0}^{2}}{\varrho_{0}}\right)
$$

Given $\bar{\varrho}$ and $\bar{\eta}$ one can determine $V_{t d}$ :

$$
V_{t d}=A \lambda^{3}(1-\bar{\varrho}-i \bar{\eta}) \quad\left|V_{t d}\right|=A \lambda^{3} R_{t}
$$

Before proceeding to the numerical analysis a few remarks are in order:

- The determination of $\left|V_{t d}\right|$ and of the unitarity triangle requires the knowledge of $V_{c b}$ (or $A$ ) and of $\left|V_{u b} / V_{c b}\right|$. Both values are subject to theoretical uncertainties present in the existing analyses of tree level decays. Whereas the dependence on $\left|V_{u b} / V_{c b}\right|$ is rather weak, the very strong dependence of $B\left(K^{+} \rightarrow \pi^{+} \nu \bar{\nu}\right)$ on $A$ or $V_{c b}$ makes a precise prediction for this branching ratio difficult at present. We will return to this below.

- The dependence of $B\left(K^{+} \rightarrow \pi^{+} \nu \bar{\nu}\right)$ on $m_{t}$ is also strong. However $m_{t}$ should be known already in this decade within $\pm 5 \%$ and consequently the uncertainty in $m_{t}$ will soon be less serious for $B\left(K^{+} \rightarrow \pi^{+} \nu \bar{\nu}\right)$ than the corresponding uncertainty in $V_{c b}$.

- Once $\varrho$ and $\eta$ are known precisely from CP asymmetries in B decays, some of the uncertainties present in (XXIV.6) related to $\left|V_{u b} / V_{c b}\right|$ (but not to $V_{c b}$ ) will be removed.

- A very clean determination of $\sin 2 \beta$ without essentially any dependence on $m_{t}$ and $V_{c b}$ can be made by combining $B\left(K^{+} \rightarrow \pi^{+} \nu \bar{\nu}\right)$ with $B\left(K_{L} \rightarrow \pi^{0} \nu \bar{\nu}\right)$ discussed below. We will present an analysis of this type in section XXIV H. 


\section{Numerical Analysis of $K^{+} \rightarrow \pi^{+} \nu \bar{\nu}$ \\ 1. Renormalization Scale Uncertainties}

We will now investigate the uncertainties in $X\left(x_{t}\right), X_{N L}, B\left(K^{+} \rightarrow \pi^{+} \nu \bar{\nu}\right),\left|V_{t d}\right|$ and in the determination of the unitarity triangle related to the choice of the renormalization scales $\mu_{t}$ and $\mu_{c}$ (see section XIB). To this end we will fix the remaining parameters as follows

$$
\begin{array}{cc}
m_{c} \equiv m_{c}\left(m_{c}\right)=1.3 \mathrm{GeV} & m_{t} \equiv m_{t}\left(m_{t}\right)=170 \mathrm{GeV} \\
V_{c b}=0.040 & \left|V_{u b} / V_{c b}\right|=0.08
\end{array}
$$

In the case of $B\left(K^{+} \rightarrow \pi^{+} \nu \bar{\nu}\right)$ we need the values of both $\varrho$ and $\bar{\eta}$. Therefore in this case we will work with

$$
\bar{\varrho}=0 \quad \bar{\eta}=0.36
$$

rather than with $\left|V_{u b} / V_{c b}\right|$. Finally we will set $\Lambda \frac{(4)}{M S}=0.325 \mathrm{GeV}$ and $\Lambda \frac{(5)}{M S}=0.225 \mathrm{GeV}$ for the charm part and top part, respectively. We then vary the scales $\mu_{c}$ and $\mu_{t}$, entering $m_{c}\left(\mu_{c}\right)$ and $m_{t}\left(\mu_{t}\right)$ respectively, in the ranges

$$
1 \mathrm{GeV} \leq \mu_{c} \leq 3 \mathrm{GeV} \quad 100 \mathrm{GeV} \leq \mu_{t} \leq 300 \mathrm{GeV}
$$

In fig. 25 we show the charm function $X_{N L}$ (for $m_{l}=0$ ) compared to the leading-log result $X_{L}$ and the case without QCD as functions of $\mu_{c}$. We observe the following features:

- The residual slope of $X_{N L}$ is considerably reduced in comparison to $X_{L}$, which exhibits a quite substantial dependence on the unphysical scale $\mu_{c}$. The variation of $X$ (defined as $\left.(X(1 \mathrm{GeV})-X(3 \mathrm{GeV})) / X\left(m_{c}\right)\right)$ is $24.5 \%$ in NLLA compared to $56.6 \%$ in LLA.

- The suppression of the uncorrected function through QCD effects is somewhat less pronounced in NLLA.

- The next-to-leading effects amount to a $\sim 10 \%$ correction relative to $X_{L}$ at $\mu=m_{c}$. However the size of this correction strongly depends on $\mu$ due to the scale ambiguity of the leading order result. This means that the question of how large the next-to-leading effects compared to the LLA really are cannot be answered uniquely. Therefore the relevant result is actually the reduction of the $\mu$-dependence in NLLA .

In fig. 26 we show the analogous results for the top function $X\left(x_{t}\right)$ as a function of $\mu_{t}$. We observe:

- Due to $\mu_{t} \gg \mu_{c}$ the scale dependences in the top function are substantially smaller than in the case of charm. Note in particular how the yet appreciable scale dependence of $X_{0}$ gets flattened out almost perfectly when the $\mathcal{O}\left(\alpha_{\mathrm{s}}\right)$ effects are taken into account. The total variation of $X\left(x_{t}\right)$ with $100 \mathrm{GeV} \leq \mu_{t} \leq 300 \mathrm{GeV}$ is around $1 \%$ in NLLA compared to $10 \%$ in LLA.

- As already stated above after (XXIV.4), with the choice $\mu_{t}=m_{t}$ the NLO correction is very small. It is substantially larger for $\mu_{t}$ very different from $m_{t}$. 


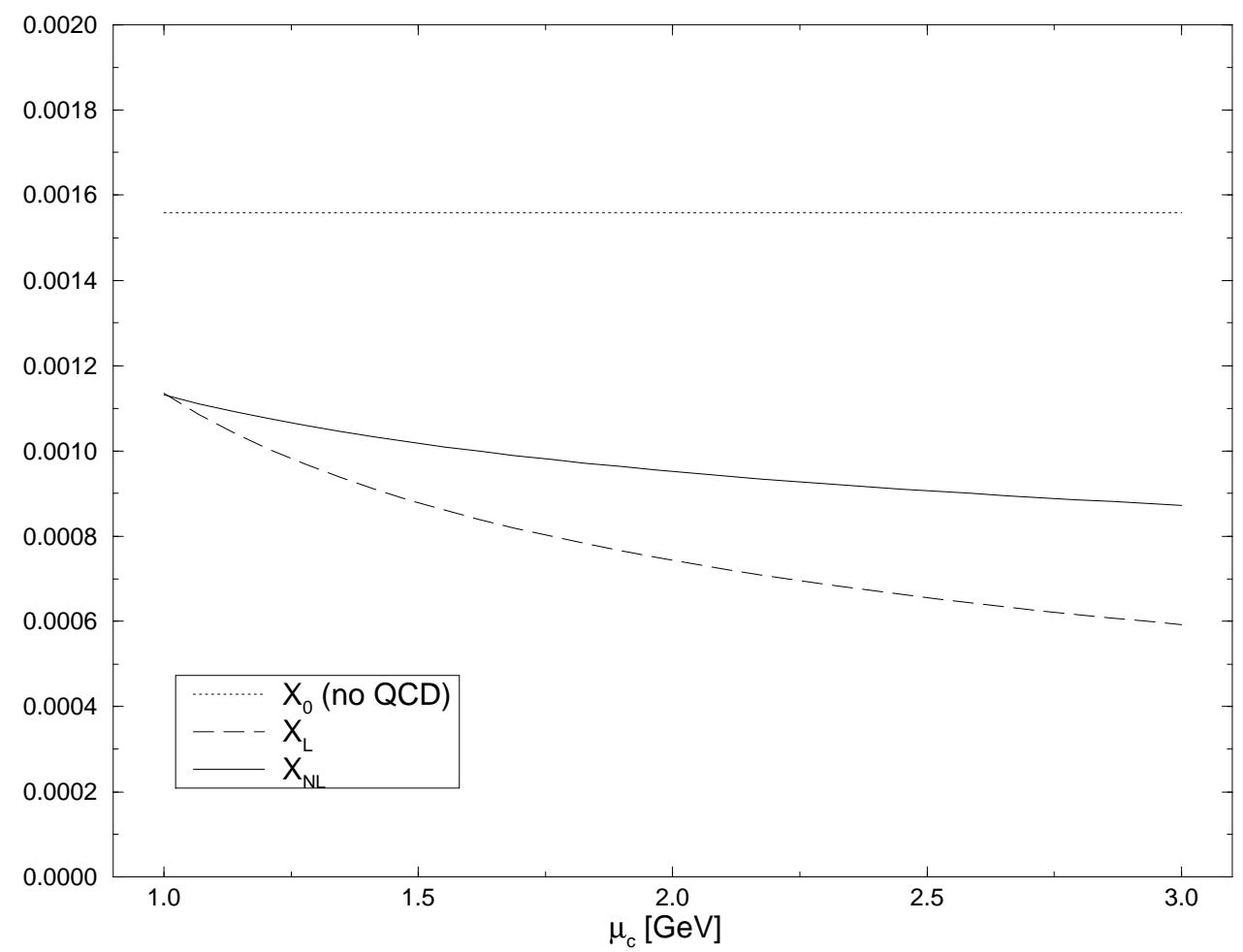

FIG. 25. Charm quark function $X_{N L}$ (for $m_{l}=0$ ) compared to the leading-log result $X_{L}$ and the case without QCD as functions of $\mu_{c}$.

Using (XXIV.1) and varying $\mu_{c, t}$ in the ranges (XXIV.19) we find that for the above choice of the remaining parameters the uncertainty in $B\left(K^{+} \rightarrow \pi^{+} \nu \bar{\nu}\right)$

$$
0.76 \cdot 10^{-10} \leq B\left(K^{+} \rightarrow \pi^{+} \nu \bar{\nu}\right) \leq 1.20 \cdot 10^{-10}
$$

present in the leading order is reduced to

$$
0.88 \cdot 10^{-10} \leq B\left(K^{+} \rightarrow \pi^{+} \nu \bar{\nu}\right) \leq 1.02 \cdot 10^{-10}
$$

after including NLO corrections. Similarly we obtain

$$
\begin{gathered}
8.24 \cdot 10^{-3} \leq\left|V_{t d}\right| \leq 10.97 \cdot 10^{-3} \quad \text { LLA } \\
9.23 \cdot 10^{-3} \leq\left|V_{t d}\right| \leq 10.10 \cdot 10^{-3} \quad \mathrm{NLLA}
\end{gathered}
$$

where we have set $B\left(K^{+} \rightarrow \pi^{+} \nu \bar{\nu}\right)=1 \cdot 10^{-10}$. We observe that including the full next-toleading corrections reduces the uncertainty in the determination of $\left|V_{t d}\right|$ from $\pm 14 \%$ (LLA) to $\pm 4.6 \%$ (NLLA) in the present example. The main bulk of this theoretical error stems from the charm sector. Indeed, keeping $\mu_{c}=m_{c}$ fixed and varying only $\mu_{t}$, the uncertainties in the determination of $\left|V_{t d}\right|$ would shrink to $\pm 4.7 \%$ (LLA) and $\pm 0.6 \%$ (NLLA). Similar comments apply to $B\left(K^{+} \rightarrow \pi^{+} \nu \bar{\nu}\right)$ where, as seen in (XXIV.20) and (XXIV.21), the theoretical uncertainty due to $\mu_{c, t}$ is reduced from $\pm 22 \%$ (LLA) to $\pm 7 \%$ (NLLA). 


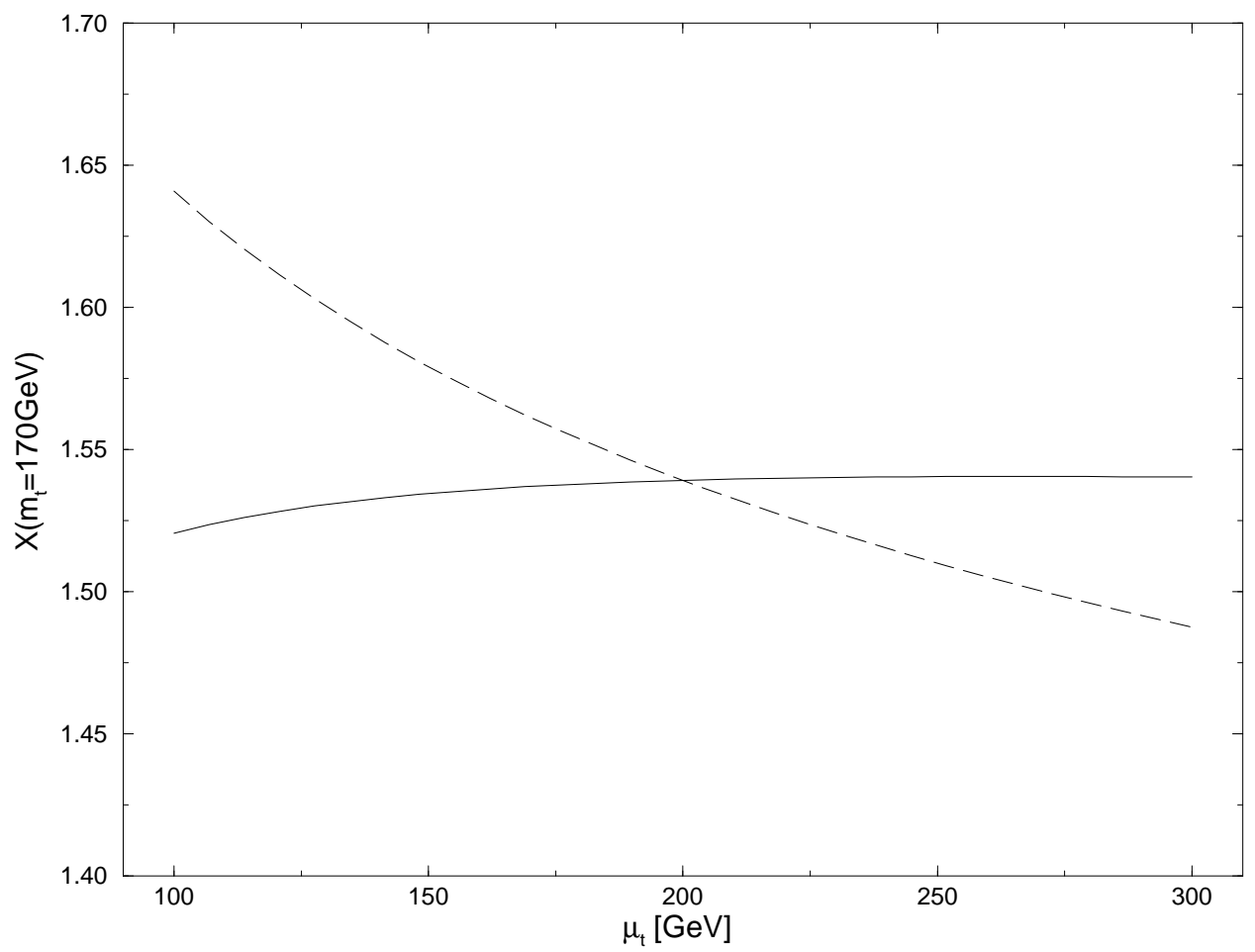

FIG. 26. Top quark function $X\left(x_{t}\right)$ as a function of $\mu_{t}$ for fixed $m_{\mathrm{t}}\left(m_{\mathrm{t}}\right)=170 \mathrm{GeV}$ with (solid curve) and without (dashed curve) $\mathcal{O}\left(\alpha_{\mathrm{s}}\right)$ corrections.

Finally in fig. 27 we show the position of the point $(\bar{\varrho}, \bar{\eta})$ which determines the unitarity triangle. To this end we have fixed all parameters as stated above except for $R_{b}$, for which we have chosen three representative numbers, $R_{b}=0.25,0.36,0.47$. The full and the reduced ranges represent LLA and NLLA respectively. The impact of the inclusion of NLO corrections on the accuracy of determining the unitarity triangle is clearly visible.

\section{Expectations for $B\left(K^{+} \rightarrow \pi^{+} \nu \bar{\nu}\right)$}

The purely theoretical uncertainties discussed so far should be distinguished from the uncertainties coming from the input parameters such as $m_{t}, V_{c b},\left|V_{u b} / V_{c b}\right|$ etc.. As we will see the latter uncertainties are still rather large to date. Consequently the progress achieved by the NLO calculations (Buchalla and Buras, 1994a) cannot yet be fully exploited phenomenologically at present. However the determination of the relevant parameters should improve in the future. Once the precision in the input parameters will have attained the desired level, the gain in accuracy of the theoretical prediction for $K^{+} \rightarrow \pi^{+} \nu \bar{\nu}$ in NLLA by a factor of more than 3 compared to the LLA will become very important.

Using our standard set of input parameters specified in appendix A and the constraints implied by the analysis of $\varepsilon_{K}$ and $B_{d}-\bar{B}_{d}$ mixing as described in section XVIII, we find for the $K^{+} \rightarrow$ $\pi^{+} \nu \bar{\nu}$ branching fraction the range

$$
0.6 \cdot 10^{-10} \leq B\left(K^{+} \rightarrow \pi^{+} \nu \bar{\nu}\right) \leq 1.5 \cdot 10^{-10}
$$




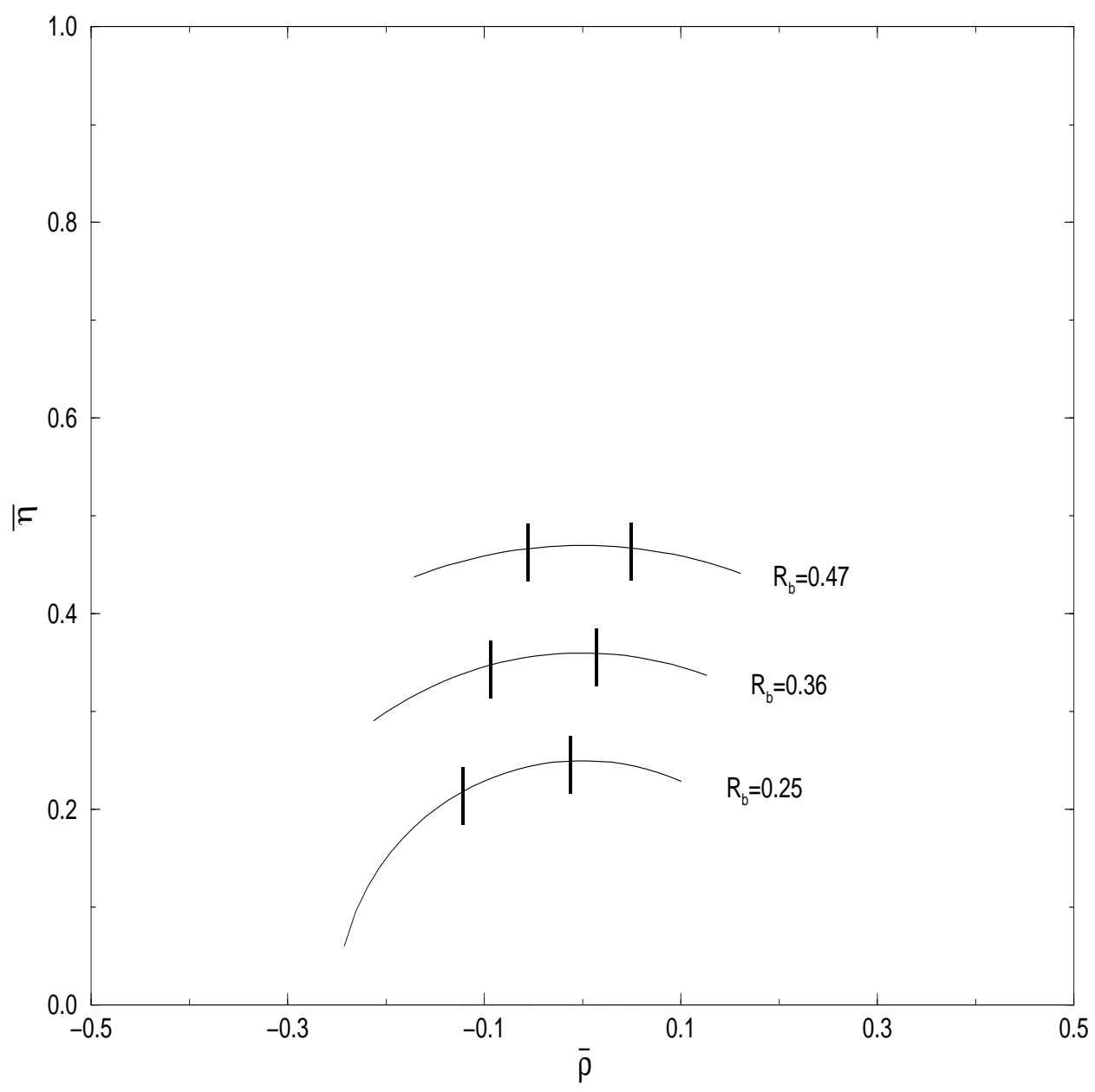

FIG. 27. The theoretical uncertainties in the determination of the unitarity triangle (UT) in the $(\bar{\varrho}, \bar{\eta})$ plane from $B\left(K^{+} \rightarrow \pi^{+} \nu \bar{\nu}\right)$. With fixed input parameters the vertex of the UT has to lie on a circle around the origin with radius $R_{b}$. A variation of the scales $\mu_{c}, \mu_{t}$ within $1 \mathrm{GeV} \leq \mu_{c} \leq 3 \mathrm{GeV}$ and $100 \mathrm{GeV} \leq \mu_{t} \leq 300 \mathrm{GeV}$ then yields the indicated ranges in LLA (full) and NLLA (reduced). We show the cases $R_{b}=0.25,0.36,0.47$.

Eq. (XXIV.24) represents the current standard model expectation for $B\left(K^{+} \rightarrow \pi^{+} \nu \bar{\nu}\right.$ ) (neglecting small isospin breaking corrections). To obtain this estimate we have allowed for a variation of the parameters $m_{t},\left|V_{c b}\right|,\left|V_{u b} / V_{c b}\right|, B_{K}, F_{B}^{2} B_{B}, x_{d}$ within their uncertainties as summarized in appendix $\mathrm{A}$. The uncertainties in $m_{c}$ and $\Lambda_{\overline{M S}}$, on the other hand, are small in comparison and have been neglected in this context. The above range would be reduced to

$$
0.8 \cdot 10^{-10} \leq B\left(K^{+} \rightarrow \pi^{+} \nu \bar{\nu}\right) \leq 1.0 \cdot 10^{-10}
$$

if the uncertainties in the input parameters could be decreased as assumed by our "future" scenario in appendix A.

It should be remarked that the $x_{d}$-constraint, excluding a part of the second quadrant for the CKM phase $\delta$, plays an essentail role in obtaining the upper bounds given above, without essentially any effect on the lower bounds. Without the $x_{d}$-constraint the upper bounds in (XXIV.24) and (XXIV.25) are relaxed to $2.3 \cdot 10^{-10}$ and $1.6 \cdot 10^{-10}$, respectively. 


\section{General Remarks on $K_{L} \rightarrow \pi^{0} \nu \bar{\nu}$}

The rare decay $K_{L} \rightarrow \pi^{0} \nu \bar{\nu}$ is even cleaner than $K^{+} \rightarrow \pi^{+} \nu \bar{\nu}$. It proceeds almost entirely through direct CP violation (Littenberg, 1989a) and is completely dominated by short-distance loop diagrams with top quark exchanges. In fact the $m_{t}$-dependence of $B\left(K_{L} \rightarrow \pi^{0} \nu \bar{\nu}\right)$ is again described by $X\left(x_{t}\right)$. Since the charm contribution can be fully neglected also the theoretical uncertainties present in $K^{+} \rightarrow \pi^{+} \nu \bar{\nu}$ due to $m_{c}, \mu_{c}$ and $\Lambda_{\overline{M S}}$ are absent here. For this reason $K_{L} \rightarrow \pi^{0} \nu \bar{\nu}$ is very well suited for the determination of CKM parameters, in particular the Wolfenstein parameter $\eta$.

\section{E. Master Formulae for $K_{L} \rightarrow \pi^{0} \nu \bar{\nu}$}

Using the effective hamiltonian (XI.56) and summing over three neutrino flavors one finds

$$
\begin{gathered}
B\left(K_{L} \rightarrow \pi^{0} \nu \bar{\nu}\right)=\kappa_{L} \cdot\left(\frac{\operatorname{Im} \lambda_{t}}{\lambda^{5}} X\left(x_{t}\right)\right)^{2} \\
\kappa_{L}=\kappa_{+} \frac{\tau\left(K_{L}\right)}{\tau\left(K^{+}\right)}=1.91 \cdot 10^{-10}
\end{gathered}
$$

with $\kappa_{+}$given in (XXIV.2). Using the Wolfenstein parametrization we can rewrite (XXIV.26) as

$$
B\left(K_{L} \rightarrow \pi^{0} \nu \bar{\nu}\right)=1.91 \cdot 10^{-10} \eta^{2} A^{4} X^{2}\left(x_{t}\right)
$$

or

$$
B\left(K_{L} \rightarrow \pi^{0} \nu \bar{\nu}\right)=3.48 \cdot 10^{-5} \eta^{2}\left|V_{c b}\right|^{4} X^{2}\left(x_{t}\right)
$$

A few remarks are in order:

- The determination of $\eta$ using $B\left(K_{L} \rightarrow \pi^{0} \nu \bar{\nu}\right)$ requires the knowledge of $V_{c b}$ and $m_{t}$. The very strong dependence on $V_{c b}$ or $A$ makes a precise prediction for this branching ratio difficult at present.

- It has been pointed out (Buras, 1994) that the strong dependence of $B\left(K_{L} \rightarrow \pi^{0} \nu \bar{\nu}\right)$ on $V_{c b}$, together with the clean nature of this decay, can be used to determine this element without any hadronic uncertainties. To this end $\eta$ and $m_{t}$ have to be known with sufficient precision in addition to $B\left(K_{L} \rightarrow \pi^{0} \nu \bar{\nu}\right) . \eta$ should be measured accurately in CP asymmetries in $B$ decays and the value of $m_{t}$ known to better than $\pm 5 \mathrm{GeV}$ from TEVATRON and future LHC experiments. Inverting (XXIV.29) and using a very accurate approximation for $X\left(x_{t}\right)$ (valid for $\left.m_{\mathrm{t}}=\bar{m}_{\mathrm{t}}\left(m_{\mathrm{t}}\right)\right)$ as given by (XXIV.4) and (XIV.6)

$$
X\left(x_{t}\right)=0.65 \cdot x_{t}^{0.575}
$$

one finds 


$$
V_{c b}=39.3 \cdot 10^{-3} \sqrt{\frac{0.39}{\eta}}\left[\frac{170 \mathrm{GeV}}{m_{t}}\right]^{0.575}\left[\frac{B\left(K_{L} \rightarrow \pi^{0} \nu \bar{\nu}\right)}{3 \cdot 10^{-11}}\right]^{1 / 4}
$$

We note that the weak dependence of $V_{c b}$ on $B\left(K_{L} \rightarrow \pi^{0} \nu \bar{\nu}\right)$ allows to achieve a high precision for this CKM element even when $B\left(K_{L} \rightarrow \pi^{0} \nu \bar{\nu}\right)$ is known with only relatively moderate accuracy, e.g. 10-15\%. Needless to say that any measurement of $B\left(K_{L} \rightarrow \pi^{0} \nu \bar{\nu}\right)$ is extremely challenging. A numerical analysis of (XXIV.31) can be found in (Buras, 1994).

\section{F. Numerical Analysis of $K_{L} \rightarrow \pi^{0} \nu \bar{\nu}$}

\section{Renormalization Scale Uncertainties}

The scale ambiguities present in the function $X\left(x_{t}\right)$ have already been discussed in connection with $K^{+} \rightarrow \pi^{+} \nu \bar{\nu}$. After the inclusion of NLO corrections they are so small that they can be neglected for all practical purposes. Effectively they could also be taken into account by introducing an additional error $\Delta m_{t} \leq \pm 1 \mathrm{GeV}$. At the level of $B\left(K_{L} \rightarrow \pi^{0} \nu \bar{\nu}\right)$ the ambiguity in the choice of $\mu_{t}$ is reduced from $\pm 10 \%$ (LLA) down to $\pm 1 \%$ (NLLA), which considerably increases the predictive power of the theory. Varying $\mu_{t}$ according to (XXIV.19) and using the input parameters of section XXIV $\mathrm{C}$ we find that the uncertainty in $B\left(K_{L} \rightarrow \pi^{0} \nu \bar{\nu}\right)$

$$
2.68 \cdot 10^{-11} \leq B\left(K_{L} \rightarrow \pi^{0} \nu \bar{\nu}\right) \leq 3.26 \cdot 10^{-11}
$$

present in the leading order is reduced to

$$
2.80 \cdot 10^{-11} \leq B\left(K_{L} \rightarrow \pi^{0} \nu \bar{\nu}\right) \leq 2.88 \cdot 10^{-11}
$$

after including NLO corrections. This means that the theoretical uncertainty in the determination of $\eta$ amounts to only $\pm 0.7 \%$ in NLLA which is safely negligible. The reduction of the scale ambiguity for $B\left(K_{L} \rightarrow \pi^{0} \nu \bar{\nu}\right)$ is further illustrated in fig. 28 .

\section{Expectations for $B\left(K_{L} \rightarrow \pi^{0} \nu \bar{\nu}\right)$}

From an analysis of $B\left(K_{L} \rightarrow \pi^{0} \nu \bar{\nu}\right)$ similar to the one described for $K^{+} \rightarrow \pi^{+} \nu \bar{\nu}$ in section XXIV C 2 we obtain the standard model expectation

$$
1.1 \cdot 10^{-11} \leq B\left(K_{L} \rightarrow \pi^{0} \nu \bar{\nu}\right) \leq 5.0 \cdot 10^{-11}
$$

corresponding to present day errors in the relevant input parameters. This would change into

$$
2.2 \cdot 10^{-11} \leq B\left(K_{L} \rightarrow \pi^{0} \nu \bar{\nu}\right) \leq 3.6 \cdot 10^{-11}
$$

if the parameter uncertainties would decrease as anticipated by our "future" scenario defined in appendix A. 


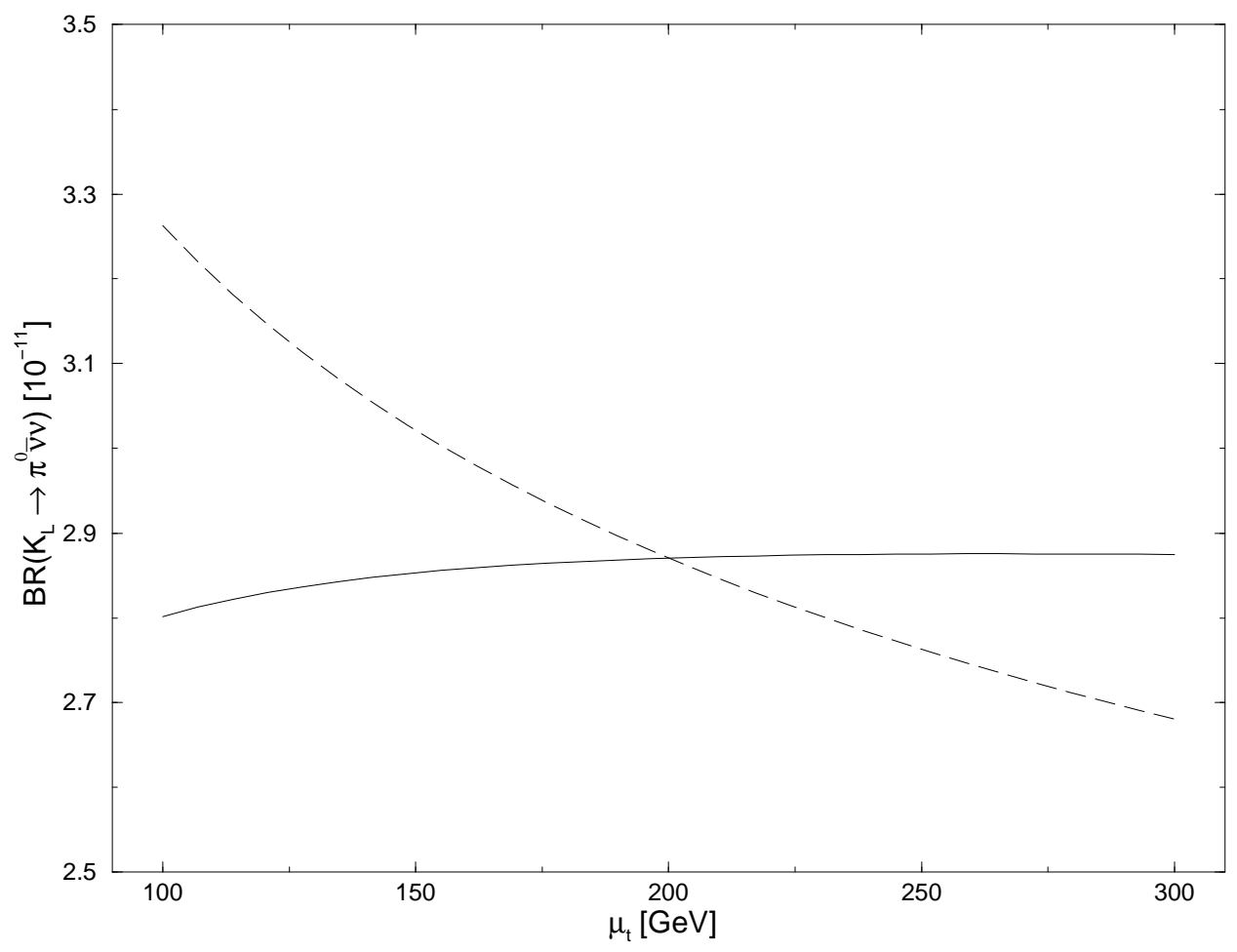

FIG. 28. The $\mu_{t}$-dependence of $B\left(K_{L} \rightarrow \pi^{0} \nu \bar{\nu}\right) / 10^{-11}$ with (solid curve) and without (dashed curve) $\mathcal{O}\left(\alpha_{\mathrm{s}}\right)$ corrections for $m_{\mathrm{t}}\left(m_{\mathrm{t}}\right)=170 \mathrm{GeV},\left|V_{c b}\right|=0.04$ and $\bar{\eta}=0.36$.

\section{G. Unitarity Triangle from $K \rightarrow \pi \nu \bar{\nu}$}

The measurement of $B\left(K^{+} \rightarrow \pi^{+} \nu \bar{\nu}\right)$ and $B\left(K_{L} \rightarrow \pi^{0} \nu \bar{\nu}\right)$ can determine the unitarity triangle completely provided $m_{t}$ and $V_{c b}$ are known. Using these two branching ratios simultaneously allows to eliminate $\left|V_{u b} / V_{c b}\right|$ from the analysis which removes considerable uncertainty. Indeed it is evident from (XXIV.1) and (XXIV.26) that, given $B\left(K^{+} \rightarrow \pi^{+} \nu \bar{\nu}\right)$ and $B\left(K_{L} \rightarrow \pi^{0} \nu \bar{\nu}\right)$, one can extract both $\operatorname{Im} \lambda_{t}$ and $\operatorname{Re} \lambda_{t}$. We get

$$
\operatorname{Im} \lambda_{t}=\lambda^{5} \frac{\sqrt{B_{2}}}{X\left(x_{t}\right)} \quad \operatorname{Re} \lambda_{t}=-\lambda^{5} \frac{\frac{\operatorname{Re} \lambda_{c}}{\lambda} P_{0}(X)+\sqrt{B_{1}-B_{2}}}{X\left(x_{t}\right)}
$$

where we have defined the "reduced" branching ratios

$$
B_{1}=\frac{B\left(K^{+} \rightarrow \pi^{+} \nu \bar{\nu}\right)}{4.57 \cdot 10^{-11}} \quad B_{2}=\frac{B\left(K_{L} \rightarrow \pi^{0} \nu \bar{\nu}\right)}{1.91 \cdot 10^{-10}}
$$

Using next the expressions for $\operatorname{Im} \lambda_{t}, \operatorname{Re} \lambda_{t}$ and $\operatorname{Re} \lambda_{c}$ given in (II.23) - (II.25) we find

$$
\bar{\varrho}=1+\frac{P_{0}(X)-\sqrt{\sigma\left(B_{1}-B_{2}\right)}}{A^{2} X\left(x_{t}\right)} \quad \bar{\eta}=\frac{\sqrt{B_{2}}}{\sqrt{\sigma} A^{2} X\left(x_{t}\right)}
$$

with $\sigma$ defined in (XXIV.7). An exact treatment of the CKM matrix shows that the formulae (XXIV.38) are rather precise (Buchalla and Buras, 1994c). The error in $\bar{\eta}$ is below $0.1 \%$ and $\bar{\varrho}$ may deviate from the exact expression by at most $\Delta \bar{\varrho}=0.02$ with essentially negligible error for 
$0 \leq \bar{\varrho} \leq 0.25$

As an illustrative example, let us consider the following scenario. We assume that the branching ratios are known to within $\pm 10 \%$

$$
B\left(K^{+} \rightarrow \pi^{+} \nu \bar{\nu}\right)=(1.0 \pm 0.1) \cdot 10^{-10} \quad B\left(K_{L} \rightarrow \pi^{0} \nu \bar{\nu}\right)=(2.5 \pm 0.25) \cdot 10^{-11}
$$

Next we take $\left(m_{i} \equiv m_{i}\left(m_{i}\right)\right)$

$$
m_{t}=(170 \pm 5) \mathrm{GeV} \quad m_{c}=(1.30 \pm 0.05) \mathrm{GeV} \quad V_{c b}=0.040 \pm 0.001
$$

where the quoted errors are quite reasonable if one keeps in mind that it will take at least ten years to achieve the accuracy assumed in (XXIV.39). Finally, we use

$$
\Lambda \frac{(4)}{\mathrm{MS}}=(200-350) \mathrm{MeV} \quad \mu_{c}=(1-3) \mathrm{GeV}
$$

where $\mu_{c}$ is the renormalization scale present in the analysis of the charm contribution. Its variation gives an indication of the theoretical uncertainty involved in the calculation. In comparison to this error we neglect the effect of varying $\mu_{W}=\mathcal{O}\left(M_{W}\right)$, the high energy matching scale at which the $\mathrm{W}$ boson is integrated out, as well as the very small scale dependence of the top quark contribution. As reference parameters we use the central values in (XXIV.39) and (XXIV.40) and $\Lambda \frac{(4)}{\mathrm{MS}}=300 \mathrm{MeV}, \mu_{c}=m_{c}$. The results that would be obtained in such a scenario for $\bar{\eta},\left|V_{t d}\right|$ and $\bar{\varrho}$ are collected in table XLV.

\begin{tabular}{|c|c|c|c|c|c|c|}
\hline & & $\Delta(B R)$ & $\overline{\Delta\left(m_{t}, V_{c b}\right)}$ & $\Delta\left(m_{c}, \Lambda_{\frac{\mathrm{MS}}{(4)}}\right)$ & $\Delta\left(\mu_{c}\right)$ & $\Delta_{\text {total }}$ \\
\hline $\bar{\eta}$ & 0.33 & \pm 0.02 & \pm 0.03 & \pm 0.00 & \pm 0.00 & \pm 0.05 \\
\hline$\left|V_{t d}\right| / 10^{-3}$ & 9.3 & \pm 0.6 & \pm 0.6 & \pm 0.5 & \pm 0.4 & \pm 2.1 \\
\hline $\bar{\varrho}$ & 0.00 & \pm 0.08 & \pm 0.09 & \pm 0.06 & \pm 0.04 & \pm 0.27 \\
\hline
\end{tabular}

TABLE XLV. $\bar{\eta},\left|V_{t d}\right|$ and $\bar{\varrho}$ determined from $K^{+} \rightarrow \pi^{+} \nu \bar{\nu}$ and $K_{L} \rightarrow \pi^{0} \nu \bar{\nu}$ for the scenario described in the text together with the uncertainties related to various parameters.

There we have also displayed separately the associated, symmetrized errors $(\Delta)$ coming from the uncertainties in the branching ratios, $m_{t}$ and $V_{c b}, m_{c}$ and $\Lambda \frac{(4)}{\mathrm{MS}}, \mu_{c}$, as well as the total uncertainty.

We observe that respectable determinations of $\bar{\eta}$ and $\left|V_{t d}\right|$ can be obtained. On the other hand the determination of $\bar{\varrho}$ is rather poor. We also note that a sizable part of the total uncertainty results in each case from the strong dependence of both branching ratios on $m_{t}$ and $V_{c b}$. There is however one important quantity for which the strong dependence of $B\left(K^{+} \rightarrow \pi^{+} \nu \bar{\nu}\right)$ and $B\left(K_{L} \rightarrow \pi^{0} \nu \bar{\nu}\right)$ on $m_{t}$ and $V_{c b}$ does not matter at all.

$$
\text { H. } \sin 2 \beta \text { from } K \rightarrow \pi \nu \bar{\nu}
$$

Using (XXIV.38) one finds (Buchalla and Buras, 1994c) 


$$
r_{s}=r_{s}\left(B_{1}, B_{2}\right) \equiv \frac{1-\bar{\varrho}}{\bar{\eta}}=\cot \beta \quad \sin 2 \beta=\frac{2 r_{s}}{1+r_{s}^{2}}
$$

with

$$
r_{s}\left(B_{1}, B_{2}\right)=\sqrt{\sigma} \frac{\sqrt{\sigma\left(B_{1}-B_{2}\right)}-P_{0}(X)}{\sqrt{B_{2}}}
$$

Thus within the approximation of (XXIV.38) $\sin 2 \beta$ is independent of $V_{c b}$ (or $A$ ) and $m_{t}$. An exact treatment of the CKM matrix confirms this finding to a high accuracy. The dependence on $V_{c b}$ and $m_{t}$ enters only at order $\mathcal{O}\left(\lambda^{2}\right)$ and as a numerical analysis shows this dependence can be fully neglected.

It should be stressed that $\sin 2 \beta$ determined this way depends only on two measurable branching ratios and on the function $P_{0}(X)$ which is completely calculable in perturbation theory. Consequently this determination is free from any hadronic uncertainties and its accuracy can be estimated with a high degree of confidence. To this end we use the input given in (XXIV.39) - (XXIV.41) to find

$$
\sin 2 \beta=0.60 \pm 0.06 \pm 0.03 \pm 0.02
$$

where the first error comes from $B\left(K^{+} \rightarrow \pi^{+} \nu \bar{\nu}\right)$ and $B\left(K_{L} \rightarrow \pi^{0} \nu \bar{\nu}\right)$, the second from $m_{c}$ and $\Lambda_{\overline{M S}}$ and the last one from the uncertainty due to $\mu_{c}$. We note that the largest partial uncertainty results from the branching ratios themselves. It can be probably reduced with time as is the case with the \pm 0.03 uncertainty related to $\Lambda_{\overline{M S}}$ and $m_{c}$. Note that the theoretical uncertainty represented by $\Delta\left(\mu_{c}\right)$, which ultimately limits the accuracy of the analysis, is small. This reflects the clean nature of the $K \rightarrow \pi \nu \bar{\nu}$ decays. However the small uncertainty of \pm 0.02 is only achieved by including next-to-leading order QCD corrections. In the leading logarithmic approximation the corresponding error would amount to \pm 0.05 , larger than the one coming from $m_{c}$ and $\Lambda_{\overline{M S}}$.

The accuracy to which $\sin 2 \beta$ can be obtained from $K \rightarrow \pi \nu \bar{\nu}$ is, in our example, comparable to the one expected in determining $\sin 2 \beta$ from $\mathrm{CP}$ asymmetries in $\mathrm{B}$ decays prior to LHC experiments. In this case $\sin 2 \beta$ is determined best by measuring the time integrated $\mathrm{CP}$ violating asymmetry in $B_{d}^{0} \rightarrow \psi K_{S}$ which is given by

$$
\begin{aligned}
A_{C P}\left(\psi K_{S}\right) & =\frac{\int_{0}^{\infty}\left[\Gamma\left(B \rightarrow \psi K_{S}\right)-\Gamma\left(\bar{B} \rightarrow \psi K_{S}\right)\right] d t}{\int_{0}^{\infty}\left[\Gamma\left(B \rightarrow \psi K_{S}\right)+\Gamma\left(\bar{B} \rightarrow \psi K_{S}\right)\right] d t} \\
& =-\sin 2 \beta \frac{x_{d}}{1+x_{d}^{2}}
\end{aligned}
$$

where $x_{d}=\Delta m / \Gamma$ gives the size of $B_{d}^{0}-\bar{B}_{d}^{0}$ mixing. Combining (XXIV.42) and (XXIV.45) we obtain an interesting connection between rare $\mathrm{K}$ decays and $\mathrm{B}$ physics

$$
\frac{2 r_{s}\left(B_{1}, B_{2}\right)}{1+r_{s}^{2}\left(B_{1}, B_{2}\right)}=-A_{C P}\left(\psi K_{S}\right) \frac{1+x_{d}^{2}}{x_{d}}
$$

which must be satisfied in the Standard Model. We stress that except for $P_{0}(X)$ given in table XLIV all quantities in (XXIV.46) can be directly measured in experiment and that this relationship is essentially independent of $m_{t}$ and $V_{c b}$. 


\section{THE DECAYS $K_{L} \rightarrow \mu^{+} \mu^{-}$AND $K^{+} \rightarrow \pi^{+} \mu^{+} \mu^{-}$}

\section{A. General Remarks on $K_{L} \rightarrow \mu^{+} \mu^{-}$}

The rare decay $K_{L} \rightarrow \mu^{+} \mu^{-}$is CP conserving and in addition to its short-distance part receives important contributions from the two-photon intermediate state, which are difficult to calculate reliably (Geng and Ng, 1990), (Bélanger and Geng, 1991), (Ko, 1992).

This latter fact is rather unfortunate because the short-distance part is, similarly to $K^{+} \rightarrow$ $\pi^{+} \nu \bar{\nu}$, free of hadronic uncertainties and if extracted from the data would give a useful determination of the Wolfenstein parameter $\varrho$. The separation of the short-distance from the long-distance piece in the measured rate is very difficult however.

In spite of all this we will present here the analysis of the short-distance contribution because on one hand it may turn out to be useful one day for $K_{L} \rightarrow \mu^{+} \mu^{-}$and on the other hand it also plays an important role in a parity violating asymmetry, which can be measured in $K^{+} \rightarrow \pi^{+} \mu^{+} \mu^{-}$. We will discuss this latter topic later on in this section.

The analysis of $\left(K_{L} \rightarrow \mu^{+} \mu^{-}\right)_{S D}$ proceeds in essentially the same manner as for $K^{+} \rightarrow \pi^{+} \nu \bar{\nu}$. The only difference enters through the lepton line in the box contribution. This change introduces two new functions $Y_{N L}$ and $Y\left(x_{t}\right)$ for the charm and top contributions respectively (section XIC), which will be discussed in detail below.

\section{B. Master Formulae for $\left(K_{L} \rightarrow \mu^{+} \mu^{-}\right)_{\mathrm{SD}}$}

Using the effective hamiltonian (XI.44) and relating $\left\langle 0\left|(\bar{s} d)_{V-A}\right| K_{L}\right\rangle$ to $B\left(K^{+} \rightarrow \mu^{+} \nu\right)$ we find

$$
\begin{gathered}
B\left(K_{L} \rightarrow \mu^{+} \mu^{-}\right)_{S D}=\kappa_{\mu}\left[\frac{\operatorname{Re} \lambda_{c}}{\lambda} P_{0}(Y)+\frac{\operatorname{Re} \lambda_{t}}{\lambda^{5}} Y\left(x_{t}\right)\right]^{2} \\
\kappa_{\mu}=\frac{\alpha^{2} B\left(K^{+} \rightarrow \mu^{+} \nu\right)}{\pi^{2} \sin ^{4} \Theta_{W}} \frac{\tau\left(K_{L}\right)}{\tau\left(K^{+}\right)} \lambda^{8}=1.68 \cdot 10^{-9}
\end{gathered}
$$

where we have used

$$
\alpha=\frac{1}{129} \quad \sin ^{2} \Theta_{W}=0.23 \quad B\left(K^{+} \rightarrow \mu^{+} \nu\right)=0.635
$$

The function $Y(x)$ of (XI.45) can also be written as

$$
Y(x)=\eta_{Y} \cdot Y_{0}(x) \quad \eta_{Y}=1.026 \pm 0.006
$$

where $\eta_{Y}$ summarizes the NLO corrections discussed in section XIC. With $m_{t} \equiv m_{t}\left(m_{t}\right)$ this QCD factor depends only very weakly on $m_{t}$. The range in (XXV.4) corresponds to $150 \mathrm{GeV} \leq$ $m_{t} \leq 190 \mathrm{GeV}$. The dependence on $\Lambda_{\overline{M S}}$ can be neglected. Next

$$
P_{0}(Y)=\frac{Y_{N L}}{\lambda^{4}}
$$


TABLE XLVI. The function $P_{0}(Y)$ for various $\Lambda \frac{(4)}{\mathrm{MS}}$ and $m_{c}$.

\begin{tabular}{|c|c|c|c|}
\hline \hline & & $P_{0}(Y)$ & \\
\hline$\Lambda_{\overline{\mathrm{MS}}}^{(4)} / m_{c}$ & $1.25 \mathrm{GeV}$ & $1.30 \mathrm{GeV}$ & $1.35 \mathrm{GeV}$ \\
\hline $215 \mathrm{MeV}$ & 0.132 & 0.141 & 0.151 \\
$325 \mathrm{MeV}$ & 0.140 & 0.149 & 0.159 \\
$435 \mathrm{MeV}$ & 0.145 & 0.156 & 0.166 \\
\hline \hline
\end{tabular}

with $Y_{N L}$ calculated in section XIC. Values for $P_{0}(Y)$ as a function of $\Lambda_{\overline{M S}}$ and $m_{c} \equiv m_{c}\left(m_{c}\right)$ are collected in table XLVI.

Using the improved Wolfenstein parametrization and the approximate formulae (II.23) - (II.25) we can next write

$$
B\left(K_{L} \rightarrow \mu^{+} \mu^{-}\right)_{S D}=1.68 \cdot 10^{-9} A^{4} Y^{2}\left(x_{t}\right) \frac{1}{\sigma}\left(\bar{\varrho}_{0}-\bar{\varrho}\right)^{2}
$$

with

$$
\bar{\varrho}_{0}=1+\frac{P_{0}(Y)}{A^{2} Y\left(x_{t}\right)} \quad \sigma=\left(\frac{1}{1-\frac{\lambda^{2}}{2}}\right)^{2}
$$

The "experimental" value of $B\left(K_{L} \rightarrow \mu^{+} \mu^{-}\right)_{S D}$ determines the value of $\bar{\varrho}$ given by

$$
\bar{\varrho}=\bar{\varrho}_{0}-\bar{r}_{0} \quad \bar{r}_{0}^{2}=\frac{1}{A^{4} Y^{2}\left(x_{t}\right)}\left[\frac{\sigma B\left(K_{L} \rightarrow \mu^{+} \mu^{-}\right)_{S D}}{1.68 \cdot 10^{-9}}\right]
$$

Similarly to $r_{0}$ in the case of $K^{+} \rightarrow \pi^{+} \nu \bar{\nu}$, the value of $\bar{r}_{0}$ is fully determined by the top contribution which has only a very weak renormalization scale ambiguity after the inclusion of $\mathcal{O}\left(\alpha_{\mathrm{s}}\right)$ corrections. The main scale ambiguity resides in $\bar{\varrho}_{0}$ whose departure from unity measures the relative importance of the charm contribution.

\section{Numerical Analysis of $\left(K_{L} \rightarrow \mu^{+} \mu^{-}\right)_{S D}$}

\section{Renormalization Scale Uncertainties}

We will now investigate the uncertainties in $Y\left(x_{t}\right), Y_{N L}, B\left(K_{L} \rightarrow \mu^{+} \mu^{-}\right)_{S D}$ and $\varrho$ related to the dependence of these quantities on the choice of the renormalization scales $\mu_{t}$ and $\mu_{c}$. To this end we proceed as in section XXIV C 1 . We fix all the remaining parameters as given in (XXIV.16) and (XXIV.17) and we vary $\mu_{c}$ and $\mu_{t}$ within the ranges stated in (XXIV.19).

Fig. 29 shows the charm function $Y_{N L}$ compared to the leading-log result $Y_{L}$ and the case without QCD as a function of $\mu_{c}$. We note the following points:

- The residual slope of $Y_{N L}$ is considerably smaller than in $Y_{L}$ although still sizable. The variation of $Y$ with $\mu$ defined as $(Y(1 \mathrm{GeV})-Y(3 \mathrm{GeV})) / Y\left(m_{c}\right)$ is $53 \%$ in NLLA compared to $92 \%$ in LLA. 


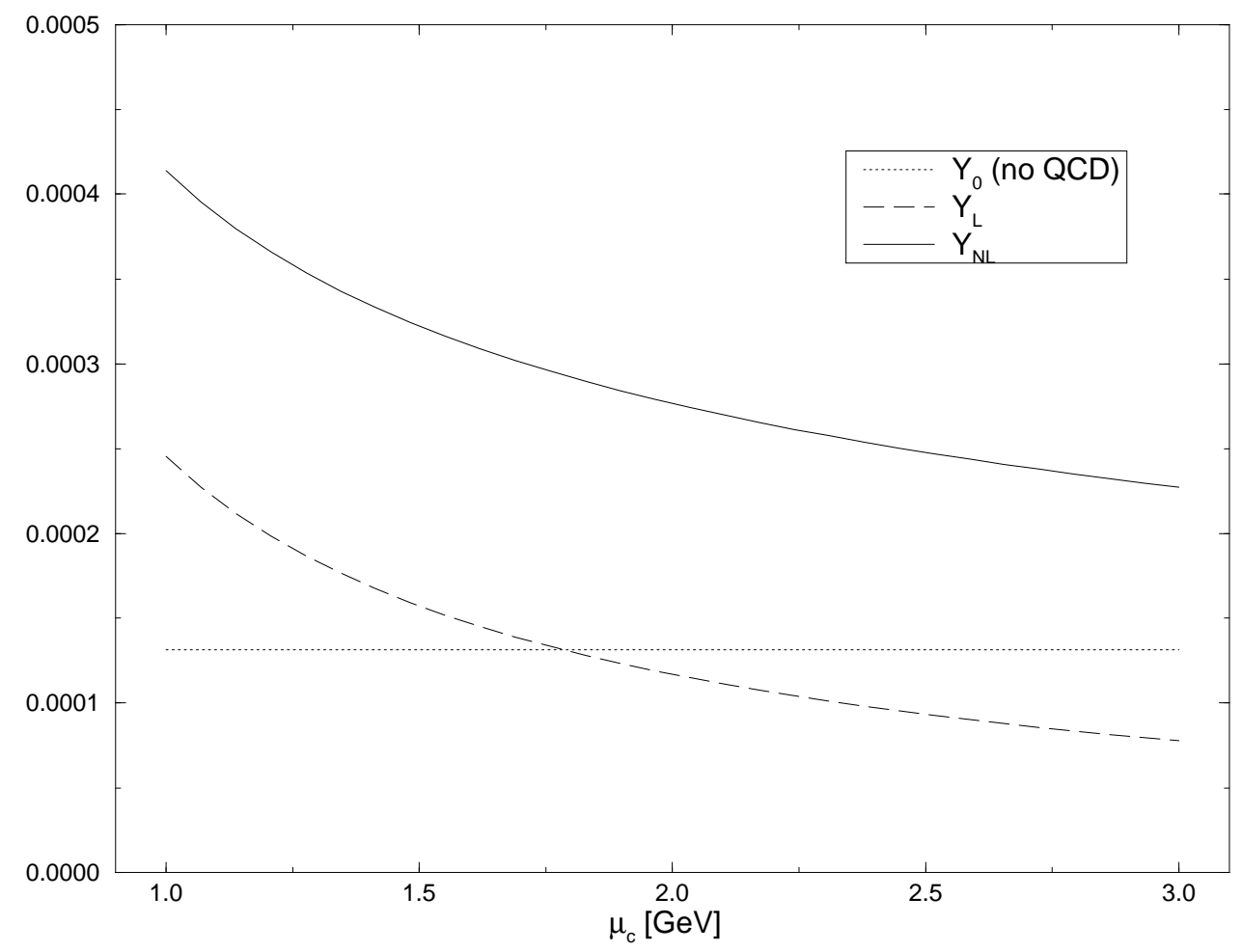

FIG. 29. Charm quark function $Y_{N L}$ compared to the leading-log result $Y_{L}$ and the case without QCD as functions of $\mu_{c}$.

- There is a strong enhancement of $Y_{0}$ through QCD corrections in contrast to the suppression found in the case of $X_{0}$.

In fig. 30 we show the analogous results for $Y\left(x_{t}\right)$ as a function of $\mu_{t}$. The observed features are similar to the ones found in the case of $X\left(x_{t}\right)$ :

- Considerable reduction of the scale uncertainties in NLLA relative to the LLA with a tiny residual uncertainty after the inclusion of NLO corrections.

- Small NLO correction for the choice $\mu_{t}=m_{t}$ as summarized by $\eta_{Y}$ in (XXV.4).

Using (XXV.1) and varying $\mu_{c, t}$ in the ranges (XXIV.19) we find that for our choice of input parameters the uncertainty in $B\left(K_{L} \rightarrow \mu^{+} \mu^{-}\right)_{S D}$

$$
0.816 \cdot 10^{-9} \leq B\left(K_{L} \rightarrow \mu^{+} \mu^{-}\right)_{S D} \leq 1.33 \cdot 10^{-9}
$$

present in the leading order is reduced to

$$
1.02 \cdot 10^{-9} \leq B\left(K_{L} \rightarrow \mu^{+} \mu^{-}\right)_{S D} \leq 1.25 \cdot 10^{-9}
$$

after including NLO corrections. Here we have assumed $\bar{\varrho}=0$.

Similarly we find

$$
-0.117 \leq \bar{\varrho} \leq 0.165 \quad \text { LLA }
$$




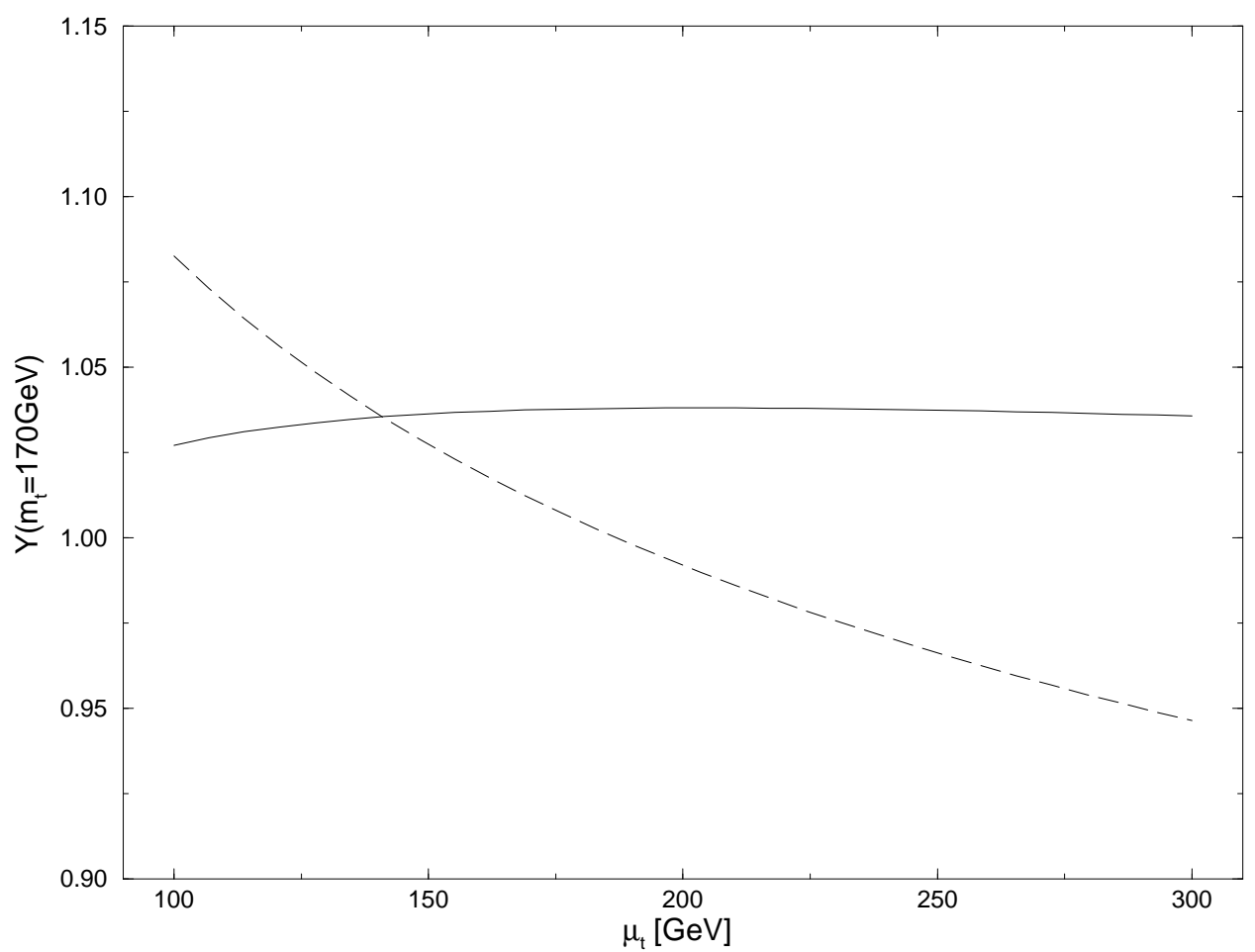

FIG. 30. Top quark function $Y\left(x_{t}\right)$ as a function of $\mu_{t}$ for fixed $m_{\mathrm{t}}\left(m_{\mathrm{t}}\right)=170 \mathrm{GeV}$ with (solid curve) and without (dashed curve) $\mathcal{O}\left(\alpha_{\mathrm{s}}\right)$ corrections.

$$
0.011 \leq \bar{\varrho} \leq 0.134 \quad \text { NLLA }
$$

where we have set $B\left(K_{L} \rightarrow \mu^{+} \mu^{-}\right)_{S D}=1 \cdot 10^{-9}$. We observe again a considerable reduction of the theoretical error when the NLO effects are included in the analyses. Also in this case the remaining ambiguity is largely dominated by the uncertainty in the charm sector.

\section{Expectations for $B\left(K_{L} \rightarrow \mu^{+} \mu^{-}\right)_{S D}$}

We finally quote the standard model expectation for the short-distance contribution to the $K_{L} \rightarrow \mu^{+} \mu^{-}$branching ratio. Using the analysis of $\varepsilon_{K}$ and the constraint implied by $B_{d}-\bar{B}_{d}$ mixing in analogy to the case of $K^{+} \rightarrow \pi^{+} \nu \bar{\nu}$ described in section XXIV C 2, we find

$$
0.6 \cdot 10^{-9} \leq B\left(K_{L} \rightarrow \mu^{+} \mu^{-}\right)_{S D} \leq 2.0 \cdot 10^{-9}
$$

and

$$
0.9 \cdot 10^{-9} \leq B\left(K_{L} \rightarrow \mu^{+} \mu^{-}\right)_{S D} \leq 1.2 \cdot 10^{-9}
$$

for present parameter uncertainties and our "future" scenario, respectively. The relevant sets of input parameters and their errors are collected in appendix A. Removing the $x_{d}$ constraint would increase the upper bounds in (XXV.13) and (XXV.14) to $3.5 \cdot 10^{-9}$ and $2.2 \cdot 10^{-9}$, respectively. 


\section{General Remarks on $K^{+} \rightarrow \pi^{+} \mu^{+} \mu^{-}$}

Obviously, the short distance effective hamiltonian in (XI.44) also gives rise to an amplitude for the transition $K^{+} \rightarrow \pi^{+} \mu^{+} \mu^{-}$. This amplitude, however, is by three orders of magnitude smaller than the dominant contribution to $K^{+} \rightarrow \pi^{+} \mu^{+} \mu^{-}$given by the one-photon exchange diagram (Ecker et al., 1987) and is therefore negligible in the total decay rate. On the other hand the coupling to the muon pair is purely vector-like for the one-photon amplitude, whereas it contains an axial vector part in the case of the SD contribution mediated by $Z^{0}$-penguin and W-box diagrams. Thus, as was pointed out by (Savage and Wise, 1990) and discussed in detail in (Lu et al., 1992), the interference of the one-photon and the SD contribution, which is odd under parity, generates a parity violating longitudinal muon polarization asymmetry

$$
\Delta_{L R}=\frac{\Gamma_{R}-\Gamma_{L}}{\Gamma_{R}+\Gamma_{L}}
$$

in the decay $K^{+} \rightarrow \pi^{+} \mu^{+} \mu^{-}$. Here $\Gamma_{R}\left(\Gamma_{L}\right)$ denotes the rate of producing a right- (left-) handed $\mu^{+}$, that is a $\mu^{+}$with spin along (opposite to) its three-momentum direction. In this way a measurement of the asymmetry $\Delta_{L R}$ could probe the phenomenologically interesting short distance physics, which is not visible in the total rate.

The $K^{+} \rightarrow \pi^{+} \gamma^{*}$ vertex is described by a form factor $f(s)$ ( $s$ being the invariant mass squared of the muon pair), that determines the one-photon amplitude and hence the total rate of $K^{+} \rightarrow$ $\pi^{+} \mu^{+} \mu^{-}$, but also enters the asymmetry $\Delta_{L R}$. This formfactor has been analyzed in detail in (Ecker et al., 1987) within the framework of chiral perturbation theory. The imaginary part $\operatorname{Im} f(s)$ turns out to be much smaller than $\operatorname{Re} f(s)$ and can safely be neglected in the calculation of $\Delta_{L R}$. For this reason $f(s) \approx \operatorname{Re} f(s)$, which depends on a constant not fixed by chiral perturbation theory, may also be directly extracted from experimental data on $K^{+} \rightarrow \pi^{+} e^{+} e^{-}$(Alliegro et al., 1992), sensitive to $|f(s)|$. We follow (Lu et al., 1992) in adopting this procedure.

The dominance of $\operatorname{Re} f(s)$ further implies that $\Delta_{L R}$ actually measures the real part of the short distance amplitude. As emphasized in (Bélanger et al., 1993), $\Delta_{L R}$ is therefore closely related to the short distance part of $K_{L} \rightarrow \mu^{+} \mu^{-}$and could possibly yield useful information on this contribution, which is difficult to extract from experimental results on $K_{L} \rightarrow \mu^{+} \mu^{-}$. Like $\left(K_{L} \rightarrow \mu^{+} \mu^{-}\right)_{S D}, \Delta_{L R}$ is in particular a measure of the Wolfenstein parameter $\varrho$.

The authors of (Lu et al., 1992) have also considered potential long distance contributions to $\Delta_{L R}$ originating from two-photon exchange amplitudes. Unfortunately these are very difficult to calculate in a reliable manner. The discussion in (Lu et al., 1992) indicates however, that they are likely to be much smaller than the short distance contributions considered above. We will focus here on the short distance part, keeping in mind the uncertainty due to possible non-negligible long distance corrections.

One should stress that the short distance part by itself, although calculable in a well defined perturbative framework, is not completely free from theoretical uncertainty. The natural context to discuss this issue is a next-to-leading order analysis, which for $\Delta_{L R}$ has been presented in (Buchalla and Buras, 1994b), generalizing the previous leading log calculations (Savage and Wise, 1990), (Lu et al., 1992), (Bélanger et al., 1993). We will summarize the results of (Buchalla and Buras, 1994b) below.

We finally mention that other asymmetries in $K^{+} \rightarrow \pi^{+} \mu^{+} \mu^{-}$, which are odd under time reversal and are also sensitive to short distance contributions, have been discussed in the literature 
(Savage and Wise, 1990), (Lu et al., 1992), (Agrawal et al., 1991), (Agrawal et al., 1992). They involve both the $\mu^{+}$and $\mu^{-}$polarizations and are considerably more difficult to measure than $\Delta_{L R}$. Possibilities for measuring the polarization of muons from $K^{+} \rightarrow \pi^{+} \mu^{+} \mu^{-}$in future experiments, based on studying the angular distribution of $e^{ \pm}$from muon decay, are described in (Kuno, 1992).

\section{E. Master Formulae for $\Delta_{L R}$}

The absolute value of the asymmetry $\Delta_{L R}$ can be written as

$$
\left|\Delta_{L R}\right|=r \cdot|\operatorname{Re} \xi|
$$

The factor $r$ arises from phase space integrations. It depends only on the particle masses $m_{K}, m_{\pi}$ and $m_{\mu}$, on the form factors of the matrix element $\left\langle\pi^{+}\left|(\bar{s} d)_{V-A}\right| K^{+}\right\rangle$, as well as on the form factor of the $K^{+} \rightarrow \pi^{+} \gamma^{*}$ transition, relevant for the one-photon amplitude. In addition $r$ depends on a possible cut which may be imposed on $\theta$, the angle between the three-momenta of the $\mu^{-}$and the pion in the rest frame of the $\mu^{+} \mu^{-}$pair. Without any cuts one has $r=2.3$ (Lu et al., 1992). If $\cos \theta$ is restricted to lie in the region $-0.5 \leq \cos \theta \leq 1.0$, this factor is increased to $r=4.1$. As discussed in (Lu et al., 1992), such a cut in $\cos \theta$ could be useful since it enhances $\Delta_{L R}$ by $80 \%$ with only a $22 \%$ decrease in the total number of events.

$\operatorname{Re} \xi$ is a function containing the information on the short distance physics. It depends on CKM parameters, the QCD scale $\Lambda_{\overline{M S}}$, the quark masses $m_{t}$ and $m_{c}$ and is given by

$$
\begin{aligned}
& \operatorname{Re} \xi=\kappa \cdot\left[\frac{\operatorname{Re} \lambda_{c}}{\lambda} P_{0}(Y)+\frac{\operatorname{Re} \lambda_{t}}{\lambda^{5}} Y\left(x_{t}\right)\right] \\
& \kappa=\frac{\lambda^{4}}{2 \pi \sin ^{2} \Theta_{W}\left(1-\frac{\lambda^{2}}{2}\right)}=1.66 \cdot 10^{-3}
\end{aligned}
$$

Here $\lambda=\left|V_{u s}\right|=0.22, \sin ^{2} \Theta_{W}=0.23, x_{t}=m_{t}^{2} / M_{W}^{2}, \lambda_{i}=V_{i s}^{*} V_{i d}$ and

$$
P_{0}(Y)=\frac{Y_{N L}}{\lambda^{4}}
$$

The functions $Y_{N L}$ and $Y\left(x_{t}\right)$ represent the charm and the top contribution, respectively. They are to next-to-leading logarithmic accuracy given in (XI.48) and (XI.45) and have already been discussed in chapter XI C and in the previous sections on the phenomenology of $\left(K_{L} \rightarrow \mu^{+} \mu^{-}\right)_{S D}$. Numerical values for $P_{0}(Y)$ can be found in table XLVI. From (XXV.16) and (XXV.17) we can obtain $\operatorname{Re} \lambda_{t}$ expressed as a function of $\left|\Delta_{L R}\right|$

$$
\operatorname{Re} \lambda_{t}=-\lambda^{5} \frac{\left|\Delta_{L R}\right| / r \kappa-\left(1-\frac{\lambda^{2}}{2}\right) P_{0}(Y)}{Y\left(x_{t}\right)}
$$

Since $\operatorname{Re} \lambda_{t}$ is related to the Wolfenstein parameter $\bar{\varrho}$ (see section II), one may use (XXV.20) to extract $\varrho$ from a given value of $\left|\Delta_{L R}\right|$. 


\section{F. Numerical Analysis of $\Delta_{L R}$}

To illustrate the phenomenological implications of the next-to-leading order calculation, let us consider the following scenario. We assume a typical value for $\Delta_{L R}$, allowing for an uncertainty of $\pm 10 \%$

$$
\Delta_{L R}=(6.0 \pm 0.6) \cdot 10^{-3}
$$

Here a cut on $\cos \theta,-0.5 \leq \cos \theta \leq 1.0$, is understood. Next we take $\left(m_{i} \equiv \bar{m}_{i}\left(m_{i}\right)\right)$

$$
\begin{gathered}
m_{t}=(170 \pm 5) \mathrm{GeV} \quad m_{c}=(1.30 \pm 0.05) \mathrm{GeV} \quad V_{c b}=0.040 \pm 0.001 \\
\Lambda_{\frac{\mathrm{MS}}{(4)}}=(300 \pm 50) \mathrm{MeV}
\end{gathered}
$$

Table XLVII shows the central value of $\bar{\varrho}$ that is extracted from $\Delta_{L R}$ in our example together with the uncertainties associated to the relevant input. Combined errors due to a simultaneous variation of several parameters can be obtained to a good approximation by simply adding the errors in table XLVII.

\begin{tabular}{|c|c|c|c|c|c|c|}
\hline & & $\Delta\left(\Delta_{L R}\right)$ & $\overline{\Delta\left(m_{t}\right)}$ & $\overline{\Delta\left(V_{c b}\right)}$ & $\overline{\Delta\left(m_{c}\right)}$ & $\overline{\Delta\left(\Lambda_{\overline{M S}}\right)}$ \\
\hline $\bar{Q} \bar{\varrho}||$ & -0.06 & \pm 0.13 & \pm 0.05 & \pm 0.06 & \pm 0.01 & \pm 0.00 \\
\hline
\end{tabular}

TABLE XLVII. $\varrho$ determined from $\Delta_{L R}$ for the scenario described in the text together with the uncertainties related to various input parameters.

These errors should be compared with the purely theoretical uncertainty of the short distance calculation, estimated by a variation of the renormalization scales $\mu_{c}$ and $\mu_{t}$. Varying these scales as given in (XXIV.19) and keeping all other parameters at their central values we find

$$
\begin{aligned}
& -0.15 \leq \bar{\varrho} \leq-0.03 \quad(\text { NLLA }) \\
& -0.31 \leq \bar{\varrho} \leq 0.02 \quad(\mathrm{LLA})
\end{aligned}
$$

We observe that at NLO the scale ambiguity is reduced by almost a factor of 3 compared to the leading log approximation. However, even in the NLLA the remaining uncertainty is still sizable, though moderate in comparison with the errors in table XLVII. Note that the remaining error in (XXV.24) is almost completely due to the charm sector, since the scale uncertainty in the top contribution is practically eliminated at NLO.

We remark that for definiteness we have incorporated the numerically important piece $x_{c} / 2$ in the leading $\log$ expression for the charm function $Y$, although this is strictly speaking a next-toleading order term. This procedure corresponds to a central value of $\bar{\varrho}=-0.12$ in LLA. Omitting the $x_{c} / 2$ term and employing the strict leading log result shifts this value to $\bar{\varrho}=-0.20$. Within NLLA this ambiguity is avoided in a natural way.

Finally we give the Standard Model expectation for $\Delta_{L R}$, based on the short distance contribution 
in (XXV.16), for the Wolfenstein parameter $\varrho$ in the range $-0.25 \leq \varrho \leq 0.25, V_{c b}=0.040 \pm 0.004$ and $m_{t}=(170 \pm 20) \mathrm{GeV}$. Including the uncertainties due to $m_{c}, \Lambda_{\overline{M S}}, \mu_{c}$ and $\mu_{t}$ and imposing the cut $-0.5 \leq \cos \theta \leq 1$, we find

$$
3.0 \cdot 10^{-3} \leq\left|\Delta_{L R}\right| \leq 9.6 \cdot 10^{-3}
$$

employing next-to-leading order formulae. Anticipating improvements in $V_{c b}, m_{t}$ and $\varrho$ we also consider a future scenario in which $\varrho=0.00 \pm 0.02, V_{c b}=0.040 \pm 0.001$ and $m_{t}=(170 \pm$ 5) $\mathrm{GeV}$. The very precise determination of $\varrho$ used here should be achieved through measuring CP asymmetries in B decays in the LHC era (Buras, 1994). Then (XXV.26) reduces to

$$
4.8 \cdot 10^{-3} \leq\left|\Delta_{L R}\right| \leq 6.6 \cdot 10^{-3}
$$

One should mention that although the top contribution dominates the short distance prediction for $\left|\Delta_{L R}\right|$, the charm part is still important and should not be neglected, as done in (Bélanger et al., 1993). It is easy to convince oneself that the charm sector contributes to $\bar{\varrho}$ the sizable amount $\Delta \bar{\varrho}_{\text {charm }} \approx 0.2$. Furthermore, as we have shown above, the charm part is the dominant source of theoretical uncertainty in the short distance calculation of $\Delta_{L R}$.

To summarize, we have seen that the scale ambiguity in the perturbative short distance contribution to $\Delta_{L R}$ can be considerably reduced by incorporating next-to-leading order QCD corrections. The corresponding theoretical error in the determination of $\bar{\varrho}$ from an anticipated measurement of $\left|\Delta_{L R}\right|$ is then decreased by a factor of 3 , in a typical example. Unfortunately the remaining scale uncertainty is quite visible even at NLO. In addition there are further uncertainties due to various input parameters and due to possible long distance effects. Together this implies that the accuracy to which $\varrho$ can be extracted from $\Delta_{L R}$ appears to be limited and $\Delta_{L R}$ can not fully compete with the "gold-plated" $K \rightarrow \pi \nu \bar{\nu}$ decay modes. Still, a measurement of $\Delta_{L R}$ might give interesting constraints on SM parameters, $\varrho$ in particular, and we feel it is worthwhile to further pursue this interesting additional possibility. 


\section{THE DECAYS $B \rightarrow X \nu \bar{\nu}$ AND $B \rightarrow \mu^{+} \mu^{-}$}

\section{A. General Remarks}

The rare decays $B \rightarrow X_{s} \nu \bar{\nu}, B \rightarrow X_{d} \nu \bar{\nu}$ and $B_{s} \rightarrow \mu^{+} \mu^{-}, B_{d} \rightarrow \mu^{+} \mu^{-}$are fully dominated by internal top quark contributions. The relevant effective hamiltonians are given in (XI.56) and (XI.57) respectively. Only the top functions $X\left(x_{t}\right)$ and $Y\left(x_{t}\right)$ enter these expressions and the uncertainties due to $m_{c}$ and $\Lambda_{\overline{M S}}$ affecting $K^{+} \rightarrow \pi^{+} \nu \bar{\nu}$ and $K_{L} \rightarrow \mu^{+} \mu^{-}$are absent here. Consequently these two decays are theoretically very clean. In particular the residual renormalization scale dependence of the relevant branching ratios, though sizable in leading order, can essentially be neglected after the inclusion of next-to-leading order corrections. On the other hand a measurement of these rare $\mathrm{B}$ decays, in particular of $B \rightarrow X_{s} \nu \bar{\nu}$ and $B \rightarrow X_{d} \nu \bar{\nu}$, is experimentally very challenging. In addition, as we will see below, $B\left(B_{s} \rightarrow \mu^{+} \mu^{-}\right)$and $B\left(B_{d} \rightarrow \mu^{+} \mu^{-}\right)$is subject to the uncertainties in the values of the B meson decay constants $F_{B_{s}}$ and $F_{B_{d}}$, which hopefully will be removed one day.

\section{B. The Decays $B \rightarrow X_{s} \nu \bar{\nu}$ and $B \rightarrow X_{d} \nu \bar{\nu}$}

The branching fraction for $B \rightarrow X_{s} \nu \bar{\nu}$ is given by

$$
\frac{B\left(B \rightarrow X_{s} \nu \bar{\nu}\right)}{B\left(B \rightarrow X_{c} e \bar{\nu}\right)}=\frac{3 \alpha^{2}}{4 \pi^{2} \sin ^{4} \Theta_{W}} \frac{\left|V_{t s}\right|^{2}}{\left|V_{c b}\right|^{2}} \frac{X^{2}\left(x_{t}\right)}{f(z)} \frac{\bar{\eta}}{\kappa(z)}
$$

Here $f(z), z=m_{c} / m_{b}$ is the phase-space factor for $B \rightarrow X_{c} e \bar{\nu}$ defined already in (XXII.6) and $\kappa(z)$ is the corresponding QCD correction (Cabibbo and Maiani, 1978) given in (XXIII.7). The factor $\bar{\eta}$ represents the QCD correction to the matrix element of the $b \rightarrow s \nu \bar{\nu}$ transition due to virtual and bremsstrahlung contributions and is given by the well known expression

$$
\bar{\eta}=\kappa(0)=1+\frac{2 \alpha_{s}\left(m_{b}\right)}{3 \pi}\left(\frac{25}{4}-\pi^{2}\right) \approx 0.83
$$

For the numerical analysis we will use $\Lambda_{Q C D}^{(5)}=225 \mathrm{MeV}$, (XXIV.3), $\left|V_{t s}\right|=\left|V_{c b}\right|, m_{t}\left(m_{t}\right)=$ $170 \mathrm{GeV}, B\left(B \rightarrow X_{c} e \bar{\nu}\right)=0.104, f(z)=0.49$ and $\kappa(z)=0.88$, keeping in mind the QCD uncertainties in $B \rightarrow X_{c} e \bar{\nu}$ discussed in section XVII.

Varying $\mu_{t}$ as in (XXIV.19) we find that the ambiguity

$$
3.82 \cdot 10^{-5} \leq B\left(B \rightarrow X_{s} \nu \bar{\nu}\right) \leq 4.65 \cdot 10^{-5}
$$

present in the leading order is reduced to

$$
3.99 \cdot 10^{-5} \leq B\left(B \rightarrow X_{s} \nu \bar{\nu}\right) \leq 4.09 \cdot 10^{-5}
$$

after the inclusion of QCD corrections (Buchalla and Buras, 1993a).

It should be noted that $B\left(B \rightarrow X_{s} \nu \bar{\nu}\right)$ as given in (XXVI.1) is in view of $\left|V_{t s} / V_{c b}\right|^{2} \approx 0.95 \pm$ 0.03 essentially independent of the CKM parameters and the main uncertainty resides in the value of $m_{\mathrm{t}}$. Setting all parameters as given above and in appendix A, and using (XXIV.30) we have 


$$
B\left(B \rightarrow X_{s} \nu \bar{\nu}\right)=4.1 \cdot 10^{-5} \frac{\left|V_{t s}\right|^{2}}{\left|V_{c b}\right|^{2}}\left[\frac{m_{\mathrm{t}}\left(m_{\mathrm{t}}\right)}{170 \mathrm{GeV}}\right]^{2.30} .
$$

In view of a new interest in this decay (Grossman et al., 1995) we quote the Standard Model expectation for $B\left(B \rightarrow X_{s} \nu \bar{\nu}\right)$ based on the input parameters collected in the appendix A. We find

$$
3.1 \cdot 10^{-5} \leq B\left(B \rightarrow X_{s} \nu \bar{\nu}\right) \leq 4.9 \cdot 10^{-5}
$$

for the "present day" uncertainties in the input parameters and

$$
3.6 \cdot 10^{-5} \leq B\left(B \rightarrow X_{s} \nu \bar{\nu}\right) \leq 4.2 \cdot 10^{-5}
$$

for our "future" scenario.

In the case of $B \rightarrow X_{d} \nu \bar{\nu}$ one has to replace $V_{t s}$ by $V_{t d}$ which results in a decrease of the branching ratio by roughly an order of magnitude.

\section{The Decays $B_{s} \rightarrow \mu^{+} \mu^{-}$and $B_{d} \rightarrow \mu^{+} \mu^{-}$}

The branching ratio for $B_{s} \rightarrow l^{+} l^{-}$is given by (Buchalla and Buras, 1993a)

$$
B\left(B_{s} \rightarrow l^{+} l^{-}\right)=\tau\left(B_{s}\right) \frac{G_{F}^{2}}{\pi}\left(\frac{\alpha}{4 \pi \sin ^{2} \Theta_{W}}\right)^{2} F_{B_{s}}^{2} m_{l}^{2} m_{B_{s}} \sqrt{1-4 \frac{m_{l}^{2}}{m_{B_{s}}^{2}}}\left|V_{t b}^{*} V_{t s}\right|^{2} Y^{2}\left(x_{t}\right)
$$

where $B_{s}$ denotes the flavor eigenstate $(\bar{b} s)$ and $F_{B_{s}}$ is the corresponding decay constant (normalized as $F_{\pi}=131 \mathrm{MeV}$ ). Using (XXIV.3), (XXV.4) and (XIV.6) we find in the case of $B_{s} \rightarrow \mu^{+} \mu^{-}$

$$
B\left(B_{s} \rightarrow \mu^{+} \mu^{-}\right)=4.18 \cdot 10^{-9}\left[\frac{\tau\left(B_{s}\right)}{1.6 p s}\right]\left[\frac{F_{B_{s}}}{230 \mathrm{MeV}}\right]^{2}\left[\frac{\left|V_{t s}\right|}{0.040}\right]^{2}\left[\frac{m_{t}\left(m_{t}\right)}{170 \mathrm{GeV}}\right]^{3.12}
$$

which approximates the next-to-leading order result.

Taking the central values for $\tau\left(B_{s}\right), F_{B_{s}},\left|V_{t s}\right|$ and $m_{t}\left(m_{t}\right)$ and varying $\mu_{t}$ as in (XXIV.19) we find that the uncertainty

$$
3.44 \cdot 10^{-9} \leq B\left(B_{s} \rightarrow \mu^{+} \mu^{-}\right) \leq 4.50 \cdot 10^{-9}
$$

present in the leading order is reduced to

$$
4.05 \cdot 10^{-9} \leq B\left(B_{s} \rightarrow \mu^{+} \mu^{-}\right) \leq 4.14 \cdot 10^{-9}
$$

when the QCD corrections are included. This feature is once more illustrated in fig. 31.

Finally, we quote the standard model expectation for $B\left(B_{s} \rightarrow \mu^{+} \mu^{-}\right)$based on the input parameters collected in the Appendix. We find

$$
1.7 \cdot 10^{-9} \leq B\left(B_{s} \rightarrow \mu^{+} \mu^{-}\right) \leq 8.4 \cdot 10^{-9}
$$

using present day uncertainties in the parameters and $F_{B_{s}}=230 \pm 40 \mathrm{MeV}$. With reduced errors for the input quantities, corresponding to our second scenario as defined in Appendix A, and taking $F_{B_{s}}=230 \pm 10 \mathrm{MeV}$ this range would shrink to 


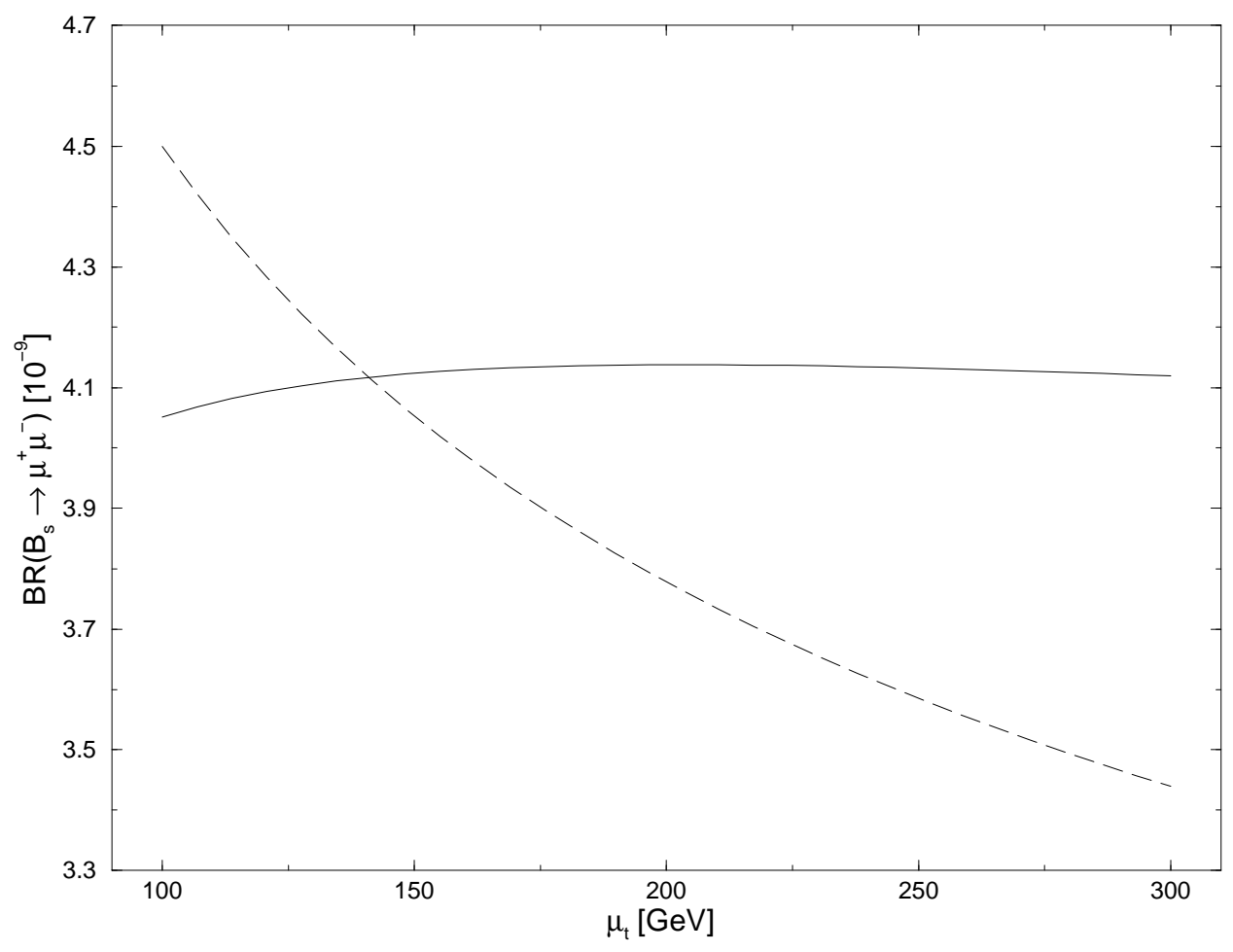

FIG. 31. The $\mu_{t}$-dependence of $B\left(B_{s} \rightarrow \mu^{+} \mu^{-}\right)\left[10^{-9}\right]$ with (solid curve) and without (dashed curve) $\mathcal{O}\left(\alpha_{\mathrm{s}}\right)$ corrections for fixed parameter values as described in the text.

$$
3.1 \cdot 10^{-9} \leq B\left(B_{s} \rightarrow \mu^{+} \mu^{-}\right) \leq 5.0 \cdot 10^{-9}
$$

For the case of $B_{d} \rightarrow \mu^{+} \mu^{-}$similar formulae hold with obvious replacements of labels $(s \rightarrow d)$. Provided the decay constants $F_{B_{s}}$ and $F_{B_{d}}$ will have been calculated reliably by non-perturbative methods or measured in leading leptonic decays one day, the rare processes $B_{s} \rightarrow \mu^{+} \mu^{-}$and $B_{d} \rightarrow \mu^{+} \mu^{-}$should offer clean determinations of $\left|V_{t s}\right|$ and $\left|V_{t d}\right|$. The accuracy of the related analysis will profit considerably from the reduction of theoretical ambiguity achieved through the inclusion of short-distance QCD effects. In particular $B\left(B_{s} \rightarrow \mu^{+} \mu^{-}\right)$, which is expected to be $\mathcal{O}\left(4 \cdot 10^{-9}\right)$, should be attainable at hadronic machines such as HERA-B, Tevatron and LHC. 


\section{SUMMARY}

In this review we have described in detail the present status of higher order QCD corrections to weak decays of hadrons. We have emphasized that during the last years considerable progress has been made in this field through the calculation of the next-to-leading QCD corrections to essentially all of the most interesting and important processes. This effort reduced considerably the theoretical uncertainties in the relevant formulae and thereby improves the determination of the CKM parameters to be achieved in future experiments. We have illustrated this with several examples.

In this review we have concentrated on weak decays in the Standard Model. The structure of weak decays in extensions of the Standard Model will generally be modified. Although we do not expect substantial effects due to "new physics" in tree level decays, the picture of loop induced processes, such as rare and $\mathrm{CP}$ violating decays, may turn out to be different from the one presented here. The basic structure of QCD calculations will remain valid, however. In certain extensions of the Standard Model, in which no new local operators occur, only the initial conditions to the renormalization group evolution will have to be modified. In more complicated extensions additional operators can be present and in addition to the change of the initial conditions, also the evolution matrix will have to be generalized.

Yet in order to be able to decide whether modifications of the standard theory are required by the data, it is essential that the theoretical calculations within the Standard Model itself reach the necessary precision. As far as the short distance contributions are concerned, we think that in most cases such a precision has been already achieved.

Important exceptions are the $b \rightarrow s \gamma$ and $b \rightarrow s g$ transitions for which the complete NLO corrections are not yet available. On the other hand the status of long distance contributions represented by the hadronic matrix elements of local operators or equivalently by various $B_{i}$ parameters, is much less satisfactory. This is in particular the case of non-leptonic decays, where the progress is very slow. Yet without these difficult non-perturbative calculations it is impossible to give reliable theoretical predictions for non-leptonic decays even if the Wilson coefficients of the relevant operators have been calculated with high precision. Moreover these coefficients have unphysical renormalization scale and renormalization scheme dependences which can only be canceled by the corresponding dependences in the hadronic matrix elements. All efforts should be made to improve the status of non-perturbative calculations.

The next ten years should be very exciting for the field of weak decays. The experimental efforts in several laboratories will provide many new results for the rare and $\mathrm{CP}$ violating decays which will offer new tests of the Standard Model and possibly signal some "new physics". As we have stressed in this review the NLO calculations presented here will play undoubtedly an important role in these investigations. Let us just imagine that $B_{s}^{0}-\bar{B}_{s}^{0}$ mixing and the branching ratios for $K^{+} \rightarrow \pi^{+} \nu \bar{\nu}, K_{L} \rightarrow \pi^{0} \nu \bar{\nu}, B \rightarrow X_{s} \nu \bar{\nu}$ and $B_{s} \rightarrow \mu^{+} \mu^{-}$have been measured to an acceptable accuracy. Having in addition at our disposal accurate values of $\left|V_{u b} / V_{c b}\right|,\left|V_{c b}\right|, m_{t}$, $F_{B}, B_{B}$ and $B_{K}$ as well as respectable results for the angles $(\alpha, \beta, \gamma)$ from the $\mathrm{CP}$ asymmetries in $\mathrm{B}-$ decays, we could really get a great insight into the physics of quark mixing and $\mathrm{CP}$ violation. One should hope that this progress on the experimental side will be paralleled by the progress in the calculations of hadronic matrix elements as well as by the calculations of QCD corrections in potential extensions of the Standard Model.

We would like to end our review with a summary of theoretical predictions and present exper- 
imental results for the rare and CP violating decays discussed by us. This summary is given in table XLVIII.

TABLE XLVIII. Summary of theoretical predictions and experimental results for the rare and CP violating processes discussed in this review. The entry "input" indicates that the corresponding measurement is used to determine or to constrain CKM parameters needed for the calculation of other decays. For $B\left(K_{L} \rightarrow \mu^{+} \mu^{-}\right)$the theoretical value refers only to the short-distance contribution. In the case of $B\left(K_{L} \rightarrow \pi^{0} e^{+} e^{-}\right)$the SM prediction corresponds to the contribution from direct CP violation. The SM predictions for $K^{+} \rightarrow \pi^{+} \nu \bar{\nu}$ and $K_{L} \rightarrow \pi^{0} \nu \bar{\nu}$ include the isospin breaking corrections considered in (Marciano and Parsa, 1995).

\begin{tabular}{|c|c|c|l|}
\hline \hline Quantity & SM Prediction & Experiment & \multicolumn{1}{c|}{ Exp. Reference } \\
\hline \hline \multicolumn{5}{|c|}{ K-Decays } \\
\hline$\varepsilon_{K} \mid$ & input & $(2.266 \pm 0.023) \cdot 10^{-3}$ & (Particle Data Group, 1994) \\
\hline$\varepsilon^{\prime} / \varepsilon$ & $(5.6 \pm 7.7) \cdot 10^{-4}$ & $(15 \pm 8) \cdot 10^{-4}$ & (Particle Data Group, 1994) \\
\hline$B\left(K_{L} \rightarrow \pi^{0} e^{+} e^{-}\right)$ & $(4.5 \pm 2.8) \cdot 10^{-12}[\mathrm{CP}$ dir $]$ & $<4.3 \cdot 10^{-9}$ & (Harris et al., 1993) $)$ \\
\hline$B\left(K^{+} \rightarrow \pi^{+} \nu \bar{\nu}\right)$ & $(1.0 \pm 0.4) \cdot 10^{-10}$ & $<2.4 \cdot 10^{-9}$ & (Adler et al., 1995) \\
\hline$B\left(K_{L} \rightarrow \pi^{0} \nu \bar{\nu}\right)$ & $(2.9 \pm 1.9) \cdot 10^{-11}$ & $<5.8 \cdot 10^{-5}$ & (Weaver et al., 1994) \\
\hline$B\left(K_{L} \rightarrow \mu^{+} \mu^{-}\right)$ & $(1.3 \pm 0.7) \cdot 10^{-9}[\mathrm{SD}]$ & $(7.4 \pm 0.4) \cdot 10^{-9}$ & (Particle Data Group, 1994) \\
\hline $\mid \Delta_{L R}\left(K^{+} \rightarrow \pi^{+} \mu^{+} \mu^{-}\right)$ & $(6 \pm 3) \cdot 10^{-3}$ & - & - \\
\hline \multicolumn{5}{|c|}{ B-Decays } & \\
\hline$x_{d}$ & input & $0.75 \pm 0.06$ & (Browder and Honscheid, 1995) \\
\hline$B\left(B \rightarrow X_{s} \gamma\right)$ & $(2.8 \pm 0.8) \cdot 10^{-4}$ & $(2.32 \pm 0.67) \cdot 10^{-4}$ & (Alam et al., 1995) \\
\hline$B\left(B \rightarrow X_{s} \nu \bar{\nu}\right)$ & $(4.0 \pm 0.9) \cdot 10^{-5}$ & $<3.9 \cdot 10^{-4}$ & (Grossman et al., 1995) \\
\hline$B\left(B_{s} \rightarrow \tau^{+} \tau^{-}\right)$ & $(1.1 \pm 0.7) \cdot 10^{-6}$ & - & - \\
\hline$B\left(B_{s} \rightarrow \mu^{+} \mu^{-}\right)$ & $(5.1 \pm 3.3) \cdot 10^{-9}$ & $<8.4 \cdot 10^{-6}$ & (Kroll et al., 1995) \\
\hline$B\left(B_{s} \rightarrow e^{+} e^{-}\right)$ & $(1.2 \pm 0.8) \cdot 10^{-13}$ & - & - \\
\hline$B\left(B_{d} \rightarrow \mu^{+} \mu^{-}\right)$ & $\sim 10^{-10}$ & $<1.6 \cdot 10^{-6}$ & (Kroll et al., 1995) \\
\hline$B\left(B_{d} \rightarrow e^{+} e^{-}\right)$ & $\sim 10^{-14}$ & $<5.9 \cdot 10^{-6}$ & (Ammar et al., 1994) \\
\hline \hline
\end{tabular}

Let us hope that the next ten years will bring a further reduction of uncertainties in the theoretical predictions and will provide us with accurate measurements of various branching ratios for which, as seen in table XLVIII, only upper bounds are available at present. 


\section{ACKNOWLEDGMENTS}

We would like to thank M. Jamin for extensive discussions and numerical checks in sections VII and XIX, M. Münz for providing the updated figures in sections XXII and XXIII, and S. Herrlich and U. Nierste for valuable comments on section XII.

Thanks are also due to B. Bardeen, M. Beneke, G. Burdman, I. Dunietz, A. El-Khadra, B. Gough, C. Greub, C. Hill, B. Kayser, W. Kilian, A. Kronfeld, A. Lenz, L. Littenberg, M. Misiak, T. Onogi, S. Parke, J. Simone and B. Winstein for useful discussions. M.E.L. acknowledges support by the Deutsche Forschungsgemeinschaft (DFG) and the hospitality of the SLAC theory group during parts of this work.

This work has been supported by the German Bundesministerium für Bildung und Forschung under contract 06 TM 743, the CEC Science project SC1-CT91-0729 and DFG contracts La 924/11 and ESMEX Li 519/2-1.

Fermilab is operated by Universities Research Association, Inc., under contract DE-AC0276CHO3000 with the United States Department of Energy. 


\section{APPENDIX A: COMPILATION OF NUMERICAL INPUT PARAMETERS}

Below we give for the convenience of the reader a compilation of input parameters that were used in the numerical parts of this review.

Running quark masses:

$$
\begin{array}{ll}
\bar{m}_{\mathrm{d}}\left(m_{\mathrm{c}}\right)=8 \mathrm{MeV} & \bar{m}_{\mathrm{s}}\left(m_{\mathrm{c}}\right)=(170 \pm 20) \mathrm{MeV} \\
\bar{m}_{\mathrm{c}}\left(m_{\mathrm{c}}\right)=1.3 \mathrm{GeV} & \\
\bar{m}_{\mathrm{b}}\left(m_{\mathrm{b}}\right)=4.4 \mathrm{GeV} & m_{\mathrm{b}}^{(\text {pole })}=4.8 \mathrm{GeV}
\end{array}
$$

Scalar meson masses and decay constants:

$$
\begin{array}{ll}
m_{\pi}=135 \mathrm{MeV} & F_{\pi}=131 \mathrm{MeV} \\
m_{\mathrm{K}}=498 \mathrm{MeV} & F_{\mathrm{K}}=160 \mathrm{MeV} \\
m_{B_{d}}=5.28 \mathrm{GeV} & \tau\left(B_{d}\right)=1.6 \cdot 10^{-12} \mathrm{~s} \\
m_{B_{s}}=5.38 \mathrm{GeV} & \tau\left(B_{s}\right)=1.6 \cdot 10^{-12} \mathrm{~s}
\end{array}
$$

\section{QCD and electroweak parameters:}

$$
\begin{aligned}
& \alpha_{\mathrm{s}}\left(M_{\mathrm{Z}}\right)=0.117 \pm 0.007 \\
& \Lambda \frac{(5)}{\mathrm{MS}}=(225 \pm 85) \mathrm{MeV} \\
& \alpha=1 / 129 \\
& M_{\mathrm{W}}=80.2 \mathrm{GeV} \\
& \sin \theta_{\mathrm{W}}=0.23
\end{aligned}
$$

\section{CKM elements:}

$$
\left|V_{\mathrm{us}}\right|=0.22 \quad\left|V_{\mathrm{ud}}\right|=0.975
$$

$K$-decays, $K^{0}-\bar{K}^{0}$ and $B^{0}-\bar{B}^{0}$ mixing:

$$
\begin{array}{lll}
\tau\left(K_{\mathrm{L}}\right)=5.17 \cdot 10^{-8} \mathrm{~s} & \tau\left(K^{+}\right)=1.237 \cdot 10^{-8} \mathrm{~s} \\
B R\left(K^{+} \rightarrow \pi^{0} e^{+} \nu\right)=0.0482 & & \\
\left|\varepsilon_{\mathrm{K}}\right|=(2.266 \pm 0.023) \cdot 10^{-3} & \Delta M_{\mathrm{K}}=3.51 \cdot 10^{-15} \mathrm{GeV} \\
\operatorname{Re} A_{0}=3.33 \cdot 10^{-7} \mathrm{GeV} & \operatorname{Re} A_{2}=1.50 \cdot 10^{-8} \mathrm{GeV} \\
\Omega_{\eta \eta^{\prime}}=0.25 & & \\
\eta_{1}=1.38 & \eta_{2}=0.57 \\
\eta_{3}=0.47 & \eta_{B}=0.55
\end{array}
$$

The values for $\operatorname{Re} A_{0,2}$ have been obtained from PDG using isospin analysis.

Hadronic matrix element parameters for $K \rightarrow \pi \pi$ :

$$
\begin{aligned}
& B_{2, L O}^{(1 / 2)}\left(m_{\mathrm{c}}\right)=5.7 \pm 1.1 \quad B_{2, N D R}^{(1 / 2)}\left(m_{\mathrm{c}}\right)=6.6 \pm 1.0 \quad \text { for } \Lambda_{\overline{\mathrm{MS}}}^{(4)}=325 \mathrm{MeV} \\
& B_{2, H V}^{(1 / 2)}\left(m_{\mathrm{c}}\right)=6.2 \pm 1.0 \\
& B_{3}^{(1 / 2)}=B_{5}^{(1 / 2)}=B_{6}^{(1 / 2)}=B_{7}^{(1 / 2)}=B_{8}^{(1 / 2)}=B_{7}^{(3 / 2)}=B_{8}^{(3 / 2)}=1 \quad \text { (central values) }
\end{aligned}
$$


In numerical investigations we have for illustrative purposes sometimes used actual present as well as estimated future errors for various input parameters. In the table below this is indicated by labels "present" and "future".

\begin{tabular}{|c|c|c|c|}
\hline Quantity & Central & Present & Future \\
\hline$\left|V_{\mathrm{cb}}\right|$ & 0.040 & \pm 0.003 & \pm 0.001 \\
$\left|V_{\mathrm{ub}} / V_{\mathrm{cb}}\right|$ & 0.08 & \pm 0.02 & \pm 0.01 \\
$B_{\mathrm{K}}$ & 0.75 & \pm 0.15 & \pm 0.05 \\
$\sqrt{B_{d}} F_{B_{d}}$ & $200 \mathrm{MeV}$ & $\pm 40 \mathrm{MeV}$ & $\pm 10 \mathrm{MeV}$ \\
$x_{d}$ & 0.75 & \pm 0.06 & \pm 0.03 \\
$m_{\mathrm{t}}$ & $170 \mathrm{GeV}$ & $\pm 15 \mathrm{GeV}$ & $\pm 5 \mathrm{GeV}$ \\
\hline
\end{tabular}




\section{REFERENCES}

Abachi, S. et al., 1995, Phys. Rev. Lett. 74, 2632.

Abada, A. et al., 1992, Nucl. Phys. B376, 172.

Abe, F. et al., 1994a, Phys. Rev. D50, 2966.

Abe, F. et al., 1994b, Phys. Rev. Lett 73, 225.

Abe, F. et al., 1994c, Phys. Rev. D51, 4623.

Adel, K. and Y. P. Yao, 1993, Mod. Phys. Lett. A8, 1679.

Adel, K. and Y. P. Yao, 1994, Phys. Rev. D49, 4945.

Adler, S. et al., 1995, BNL preprint, BNL-62327; hep-ex/9510006.

Agrawal, P., J. Ng, G. Bélanger, and C. Geng, 1991, Phys. Rev. Lett. 67, 537.

Agrawal, P., J. Ng, G. Bélanger, and C. Geng, 1992, Phys. Rev. D45, 2383.

Alam, M. S. et al., 1995, Phys. Rev. Lett. 74, 2885.

Ali, A. and C. Greub, 1991a, Z. Phys. C49, 431.

Ali, A. and C. Greub, 1991b, Phys. Lett. B259, 182.

Ali, A. and C. Greub, 1993, Z. Phys. C60, 433.

Ali, A. and C. Greub, 1995, Phys. Lett. B361, 146.

Ali, A. and D. London, 1995, DESY preprint DESY-95-148; hep-ph/9508272.

Ali, A., T. Mannel, and T. Morozumi, 1991, Phys. Lett. B273, 505.

Ali, A., G. F. Giudice, and T. Mannel, 1995, Z. Phys. C67, 417.

Alliegro, C. et al., 1992, Phys. Rev. Lett. 68, 278.

Allton, C. R., M. Ciuchini, M. Crisafulli, V. Lubicz, and G. Martinelli, 1994, Nucl. Phys. B431, 667.

Altarelli, G. and L. Maiani, 1974, Phys. Lett. B52, 351.

Altarelli, G. and S. Petrarca, 1991, Phys. Lett. B261, 303.

Altarelli, G., G. Curci, G. Martinelli, and S. Petrarca, 1981, Nucl. Phys. B187, 461.

Ammar, R. et al., 1993, Phys. Rev. Lett. 71, 674.

Ammar, R. et al., 1994, Phys. Rev. D49, 5701.

Antonelli, V., S. Bertolini, M. Fabrichesi, and E. I. Lashin, 1995, SISSA preprint, SISSA 102/95/EP.

Ashmore, J. F., 1972, Lett. Nuovo Cim. 4, 289.

Bagan, E., P. Ball, V. Braun, and H. G. Dosch, 1992, Phys. Lett. B278, 457.

Bagan, E., P. Ball, V. Braun, and P. Gosdzinsky, 1994, Nucl. Phys. B432, 3.

Bagan, E., P. Ball, V. Braun, and P. Gosdzinsky, 1995a, Phys. Lett. B342, 362.

Bagan, E., P. Ball, B. Fiol, and P. Gosdzinsky, 1995b, Phys. Lett. B351, 546.

Ball, P. and U. Nierste, 1994, Phys. Rev. D50, 5841.

Ball, P., M. Beneke, and V. M. Braun, 1995a, Phys. Rev. D52, 3929.

Ball, P., M. Beneke, and V. M. Braun, 1995b, Nucl. Phys. D452, 563.

Barbieri, R. and G. F. Giudice, 1993, Phys. Lett. B309, 86.

Bardeen, W. A., A. J. Buras, D. W. Duke, and T. Muta, 1978, Phys. Rev. D18, 3998.

Bardeen, W. A., A. J. Buras, and J.-M. Gérard, 1987a, Phys. Lett. B192, 138.

Bardeen, W. A., A. J. Buras, and J.-M. Gérard, 1987b, Nucl. Phys. B293, 787.

Bardeen, W. A., A. J. Buras, and J.-M. Gérard, 1988, Phys. Lett. B211, 343.

Barger, V., M. S. Berger, and R. J. N. Phillips, 1993, Phys. Rev. Lett. 70, 1368.

Barr, G. D. et al., 1992, Phys. Lett. B284, 440.

Barr, G. D. et al., 1993, Phys. Lett. B317, 233. 
Baxter, R. M. et al., 1994, Phys. Rev. D49, 1594.

Bélanger, G. and C. Q. Geng, 1991, Phys. Rev. D43, 140.

Bélanger, G., C. Geng, and P. Turcotte, 1993, Nucl. Phys. B390, 253.

Beneke, M. and V. Braun, 1995, Phys. Lett. B348, 513.

Bernard, C. and A. Soni, 1991, Nucl. Phys. (Proc. Suppl.) 9, 155.

Bernard, C., J. Labrenz, and A. Soni, 1994, Phys. Rev. D49, 2536.

Bertolini, S., F. Borzumati, and A. Masiero, 1987, Phys. Rev. Lett. 59, 180.

Bertolini, S., F. Borzumati, A. Masiero, and G. Ridolfi, 1991a, Nucl. Phys. B353, 591.

Bertolini, S., F. Borzumati, A. Masiero, and G. Ridolfi, 1991b, Nucl. Phys. B353, 591.

Bertolini, S., J. Eeg, and M. Fabbrichesi, 1995a, Nucl. Phys. B449, 197.

Bertolini, S., J. O. Eeg, and M. Fabrichesi, 1995b, SISSA preprint, SISSA 103/95/EP.

Bethke, S., 1994, talk presented at the QCD '94 Conference, Montpellier, France, July 7-13.

Bigi, I. I. and F. Gabbiani, 1991, Nucl. Phys. B367, 3.

Bigi, I. I. and N. Uraltsev, 1994, Z. Phys. . C62, 623.

Bigi, I. I., N. G. Uraltsev, and A. I. Vainshtein, 1992, Phys. Lett. B293, 430; erratum ibid. Phys. Lett. B297 (1993) 477.

Bigi, I. I. et al., 1993, Phys. Rev. Lett. 71, 496.

Bigi, I. I., B. Blok, M. Shifman, N. G. Uraltsev, and A. I. Vainshtein, 1994a, in B-Decays (2nd

Edition), editor S. L. Stone, World Scientific, Singapore, (1994) p. 132; (1992), p. 610.

Bigi, I. I., B. Blok, M. Shifman, and A. Vainshtein, 1994b, Phys. Lett. B323, 408.

Bijnens, J. and J. Prades, 1995, Nucl. Phys. B444, 523.

Bijnens, J. and M. B. Wise, 1984, Phys. Lett. B137, 245.

Bijnens, J., J.-M. Gérard, and G. Klein, 1991, Phys. Lett. B257, 191.

Bjorken, J. D., I. Dunietz, and J. Taron, 1992, Nucl. Phys. B371, 111.

Blok, B. and M. Shifman, 1993, talk presented at the 3rd Workshop on the Tau-Charm Factory, Marbella, Spain, 1-6 June, 1993, hep-ph/9311331.

Blok, B., L. Koyrakh, M. Shifman, and A. I. Vainshtein, 1994, Phys. Rev. D49, 3356.

Bollini, C. G. and J. J. Giambiagi, 1972a, Phys. Lett. B40, 566.

Bollini, C. G. and J. J. Giambiagi, 1972b, Nuovo Cim. 12B, 20.

Borzumati, F., 1994, Z. Phys. C63, 291.

Boyd, C., B. Grinstein, and R. Lebed, 1995, Phys. Rev. Lett. 74, 4603.

Breitenlohner, P. and D. Maison, 1977, Commun. Math. Phys. 52, 11, 39, 55.

Broadhurst, D. and A. Grozin, 1991, Phys. Lett. B267, 105.

Brodsky, S. J., G. P. Lepage, and P. B. Mackenzie, 1983, Phys. Rev. D28, 228.

Browder, T. E. and K. Honscheid, 1995, Prog. Part. Nucl. Phys. 35, 81.

Bruno, C. and J. Prades, 1993, Z. Phys. C57, 585.

Buchalla, G. and A. J. Buras, 1993a, Nucl. Phys. B400, 225.

Buchalla, G. and A. J. Buras, 1993b, Nucl. Phys. B398, 285.

Buchalla, G. and A. J. Buras, 1994a, Nucl. Phys. B412, 106.

Buchalla, G. and A. J. Buras, 1994b, Phys. Lett. B336, 263.

Buchalla, G. and A. J. Buras, 1994c, Phys. Lett. B333, 221.

Buchalla, G., A. J. Buras, and M. K. Harlander, 1990, Nucl. Phys. B337, 313.

Buchalla, G., A. J. Buras, and M. K. Harlander, 1991, Nucl. Phys. B349, 1.

Buchalla, G., 1993, Nucl. Phys. B391, 501.

Buras, A. J. and J.-M. Gérard, 1987, Phys. Lett. B192, 156. 
Buras, A. J. and M. K. Harlander, 1992, in Heavy Flavours, editors A. J. Buras and M. Lindner, World Scientific, Singapore, (1992), p. 58.

Buras, A. J. and M. E. Lautenbacher, 1993, Phys. Lett. B318, 212.

Buras, A. J. and M. Münz, 1995, Phys. Rev. D52, 186.

Buras, A. J. and P. H. Weisz, 1990, Nucl. Phys. B333, 66.

Buras, A. J., M. Jamin, and P. H. Weisz, 1990, Nucl. Phys. B347, 491.

Buras, A. J., M. Jamin, M. E. Lautenbacher, and P. H. Weisz, 1992, Nucl. Phys. B370, 69; addendum ibid. Nucl. Phys. $B 375$ (1992) 501.

Buras, A. J., M. Jamin, and M. E. Lautenbacher, 1993a, Nucl. Phys. B400, 75.

Buras, A. J., M. Jamin, and M. E. Lautenbacher, 1993b, Nucl. Phys. B408, 209.

Buras, A. J., M. Jamin, M. E. Lautenbacher, and P. H. Weisz, 1993c, Nucl. Phys. B400, 37.

Buras, A. J., M. E. Lautenbacher, M. Misiak, and M. Münz, 1994a, Nucl. Phys. B423, 349.

Buras, A. J., M. E. Lautenbacher, and G. Ostermaier, 1994b, Phys. Rev. D50, 3433.

Buras, A. J., M. Misiak, M. Münz, and S. Pokorski, 1994c, Nucl. Phys. B424, 374.

Buras, A. J., 1980, Rev. Mod. Phys. 52, 199.

Buras, A. J., 1993, Phys. Lett. B317, 449.

Buras, A. J., 1994, Phys. Lett. B333, 476.

Buras, A. J., 1995, Nucl. Phys. B434, 606.

Cabibbo, N. and L. Maiani, 1978, Phys. Lett. B79, 109.

Callan Jr, C. G., 1970, Phys. Rev. D2, 1541.

Cella, G., G. Curci, G. Ricciardi, and A. Viceré, 1990, Phys. Lett. B248, 181.

Cella, G., G. Ricciardi, and A. Viceré, 1991, Phys. Lett. B258, 212.

Cella, G., G. Curci, G. Ricciardi, and A. Viceré, 1994a, Phys. Lett. B325, 227.

Cella, G., G. Curci, G. Ricciardi, and A. Viceré, 1994b, Nucl. Phys. B431, 417.

Chay, J., H. Georgi, and B. Grinstein, 1990, Phys. Lett. B247, 399.

Chetyrkin, K. G., C. A. Dominguez, D. Pirjol, and K. Schilcher, 1995, Phys. Rev. D51, 5090.

Cho, P. and M. Misiak, 1994, Phys. Rev. D49, 5894.

Cicuta, G. M. and E. Montaldi, 1972, Lett. Nuovo Cim. 4, 329.

Ciuchini, M., E. Franco, G. Martinelli, and L. Reina, 1993a, Phys. Lett. B301, 263.

Ciuchini, M., E. Franco, G. Martinelli, L. Reina, and L. Silvestrini, 1993b, Phys. Lett. B316, 127.

Ciuchini, M., E. Franco, G. Martinelli, and L. Reina, 1994a, Nucl. Phys. B415, 403.

Ciuchini, M., E. Franco, G. Martinelli, L. Reina, and L. Silvestrini, 1994b, Phys. Lett. B334, 137.

Ciuchini, M., E. Franco, L. Reina, and L. Silvestrini, 1994c, Nucl. Phys. B421, 41.

Ciuchini, M., E. Franco, G. Martinelli, L. Reina, and L. Silvestrini, 1995, Z. Phys. C68, 239.

Cohen, A. G. and A. Manohar, 1984, Phys. Lett. B143, 481.

Cohen, A. G., G. Ecker, and A. Pich, 1993, Phys. Lett. B304, 347.

Crisafulli, M. et al., 1995, preprint, hep-lat/9509029.

Datta, A., J. Fröhlich, and E. A. Paschos, 1990, Z. Phys. C46, 63.

Datta, A., E. A. Paschos, J.-M. Schwarz, and M. N. S. Roy, 1995, University of Dortmund preprint, DO-TH 95/12.

Deshpande, N. G. and X.-G. He, 1995, Phys. Rev. Lett. 74, 26; erratum ibid. Phys. Rev. Lett. 74 (1995) 4099.

Deshpande, N. G., P. Lo, J. Trampetić, G. Eilam, and P. Singer, 1987, Phys. Rev. Lett. 59, 183. 
Deshpande, N. G., J. Trampetić, and K. Panose, 1989, Phys. Rev. D39, 1461.

Deshpande, N. G., K. Panose, and J. Trampetić, 1993, Phys. Lett. B308, 322.

Deshpande, N. G., X.-G. He, and J. Trampetić, 1995, Phys. Lett. B345, 547.

Dib, C. O., I. Dunietz, and F. J. Gilman, 1989a, Phys. Lett. B218, 487.

Dib, C. O., I. Dunietz, and F. J. Gilman, 1989b, Phys. Rev. D39, 2639.

Dib, C. O., I. Dunietz, F. J. Gilman, and Y. Nir, 1990, Phys. Rev. D 41, 1522.

Dib, C. O., I. Dunietz, and F. J. Gilman, 1991, Mod. Phys. Lett. A6, 3573.

Dikeman, R. D., M. Shifman, and N. G. Uraltsev, 1995, University of Minnesota preprint TPI-MINN-95/9-T; hep-ph/9505397.

Donoghue, J. F. and F. Gabbiani, 1995, Phys. Rev. D51, 2187.

Donoghue, J. F., E. Golowich, and B. R. Holstein, 1982, Phys. Lett. B119, 412.

Donoghue, J. F., E. Golowich, B. R. Holstein, and J. Trampetić, 1986, Phys. Lett. B179, 361.

Draper, T. and C. McNeile, 1994, Nucl. Phys. B (Proc. Suppl.) 34, 453.

Dugan, M. J. and B. Grinstein, 1991, Phys. Lett. B256, 239.

Duncan, A., E. Eichten, J. Flynn, B. Hill, G. Hockney, and H. Thacker, 1995, Phys. Rev. D51, 5101.

Ecker, G., A. Pich, and E. de Rafael, 1987, Nucl. Phys. B291, 692.

Ecker, G., A. Pich, and E. de Rafael, 1988, Nucl. Phys. B303, 665.

Eeg, J. O. and I. Picek, 1988, Phys. Lett. B214, 651.

Ellis, J. and J. S. Hagelin, 1983, Nucl. Phys. B217, 189.

Falk, A., M. Luke, and M. J. Savage, 1994, Phys. Rev. D49, 3367.

Falk, A., M. Wise, and I. Dunietz, 1995, Phys. Rev. D51, 1183.

Fleischer, R., 1994a, Z. Phys. C62, 81.

Fleischer, R., 1994b, Phys. Lett. B332, 419.

Floratos, E. G., D. A. Ross, and C. T. Sachrajda, 1977, Nucl. Phys. B129, 66.

Flynn, J. M. and L. Randall, 1989a, Nucl. Phys. B326, 31.

Flynn, J. M. and L. Randall, 1989b, Phys. Lett. B224, 221; erratum ibid. Phys. Lett. B235 (1990) 412.

Flynn, J., O. Hernández, and B. Hill, 1991, Phys. Rev. D43, 3709.

Flynn, J. M., 1990, Mod. Phys. Lett. A5, 877.

Franco, E., L. Maiani, G. Martinelli, and A. Morelli, 1989, Nucl. Phys. B317, 63.

Fröhlich, J., J. Heinrich, E. A. Paschos, and J.-M. Schwarz, 1991, University of Dortmund preprint, DO-TH 02/91.

Gaillard, M. K. and B. W. Lee, 1974a, Phys. Rev. Lett. 33, 108.

Gaillard, M. K. and B. W. Lee, 1974b, Phys. Rev. D10, 897.

Gell-Mann, M. and F. E. Low, 1954, Phys. Rev. 95, 1300.

Geng, C. Q. and J. N. Ng, 1990, Phys. Rev. D41, 2351.

Georgi, H., 1991, in Proceedings of TASI-91, editors R.K. Ellis et al., World Scientific, Singapore, (1991), p589.

Gérard, J.-M., 1990, Acta Physica Polonica B21, 257.

Gibbons, L. K. et al., 1993, Phys. Rev. Lett. 70, 1203.

Gilman, F. J. and M. B. Wise, 1979, Phys. Rev. D20, 2392.

Gilman, F. J. and M. B. Wise, 1980, Phys. Rev. D21, 3150.

Gilman, F. J. and M. B. Wise, 1983, Phys. Rev. D27, 1128.

Giménez, V., 1993, Nucl. Phys. B401, 116. 
Greub, C., A. Ioannissian, and D. Wyler, 1995, Phys. Lett. B346, 149.

Grigjanis, R., P. J. O’Donnell, M. Sutherland, and H. Navelet, 1988, Phys. Lett. B213, 355.

Grigjanis, R., P. J. O’Donnell, M. Sutherland, and H. Navelet, 1989, Phys. Lett. B223, 239.

Grigjanis, R., P. O’Donnell, M. Sutherland, and H. Navelet, 1992, Phys. Lett. B286, 413E.

Grinstein, B., M. J. Savage, and M. B. Wise, 1989, Nucl. Phys. B319, 271.

Grinstein, B., R. Springer, and M. B. Wise, 1990, Nucl. Phys. B339, 269.

Grinstein, B., 1989, Phys. Lett. B229, 280.

Grinstein, B., 1991, in High Energy Phenomenology, editors R. Huerta and M.A. Péres, World Scientific, Singapore, (1991), pi61.

Gross, D., 1976, in Methods In Field Theory (Les Houches 1975, Proceedings), editors R. Balian and J. Zinn-Justin, North-Holland, Amsterdam, (1976), p. 141.

Grossman, Y., Z. Ligeti, and E. Nardi, 1995, CALTEC preprint, CALT-68-2022; hepph/9510378.

Guberina, B. and R. D. Peccei, 1980, Nucl. Phys. B163, 289.

Hagelin, J. S. and L. S. Littenberg, 1989, Prog. Part. Nucl. Phys. 23, 1.

Harris, G. R. and J. L. Rosner, 1992, Phys. Rev. D45, 946.

Harris, D. A. et al., 1993, Phys. Rev. Lett. 71, 3918.

Hayashi, T., M. Matsuda, and M. Tanimoto, 1993, Prog. Theo. Phys. 89, 1047.

Heiliger, P. and L. Seghal, 1993, Phys. Rev. D47, 4920.

Heinrich, J., E. A. Paschos, J.-M. Schwarz, and Y. L. Wu, 1992, Phys. Lett. B279, 140.

Herrlich, S. and U. Nierste, 1994, Nucl. Phys. B419, 292.

Herrlich, S. and U. Nierste, 1995a, Phys. Rev. D52, 6505.

Herrlich, S. and U. Nierste, 1995b, Nucl. Phys. B455, 39.

Herrlich, S., 1994, Ph.D. dissertation, Munich Technical University (unpublished).

Hewett, J. L., 1993, Phys. Rev. Lett. 70, 1045.

Hokim, Q. and X. Pham, 1983, Phys. Lett. B122, 297.

Hokim, Q. and X. Pham, 1984, Ann. Phys. 155, 202.

Hou, W. S., R. I. Willey, and A. Soni, 1987, Phys. Rev. Lett. 58, 1608.

Inami, T. and C. S. Lim, 1981, Progr. Theor. Phys. 65, 297.

Isgur, N. and M. Wise, 1989, Phys. Lett. B232, 113.

Isgur, N. and M. Wise, 1990, Phys. Lett. B237, 527.

Isgur, N. and M. Wise, 1992, in Heavy Flavours, editors A. J. Buras and M. Lindner, World Scientific, Singapore, (1992), p. 234.

Ishizuka, N., 1993, Phys. Rev. Lett. 71, 24.

Jamin, M. and M. Münz, 1995, Z. Phys. C66, 633.

Jamin, M. and A. Pich, 1994, Nucl. Phys. B425, 15.

Jaus, W. and D. Wyler, 1990, Phys. Rev. D41, 3405.

Jezabek, M. and J. H. Kühn, 1989, Nucl. Phys. B320, 20.

Ji, X. and M. Musolf, 1991, Phys. Lett. B257, 409.

Kambor, J., J. Missimer, and D. Wyler, 1990, Nucl. Phys. B346, 17.

Kambor, J., J. Missimer, and D. Wyler, 1991, Phys. Lett. B261, 496.

Kapustin, A. and Z. Ligeti, 1995, Phys. Lett. B355, 318.

Kapustin, A., Z. Ligeti, and H. D. Politzer, 1995, Phys. Lett. B357, 653.

Kaufman, W. A., H. Steger, and Y. P. Yao, 1989, Mod. Phys. Lett. A3, 1479.

Kilcup, G. W., 1991, Nucl. Phys. B (Proc. Suppl.) 20, 417. 
Kilian, W. and T. Mannel, 1993, Phys. Lett. B301, 382.

Kim, C. S. and A. D. Martin, 1989, Phys. Lett. B225, 186.

Ko, P., 1992, Phys. Rev. D45, 174.

Köhler, G. O. and E. A. Paschos, 1995, Phys. Rev. D52, 175.

Kroll, J. et al., 1995, Fermilab preprint, FERMILAB-CONF-95/229-E.

Kuno, Y., 1992, KEK-preprint 92-190, published in Proc. 10th Int. Symp. on High Energy Spin Physics, Nagoya, Japan (1992), p. 769.

Lim, C. S., T. Morozumi, and A. I. Sanda, 1989, Phys. Lett. B218, 343.

Littenberg, L. and G. Valencia, 1993, Ann. Rev. Nucl. Part. Sci. 43, 729.

Littenberg, L., 1989a, Phys. Rev. D39, 3322.

Littenberg, L. S., 1989b, in Proceedings of the Workshop on CP Violation at a Kaon Factory, editor J.N. Ng, TRIUMF, Vancouver, Canada (1989), p. 19.

Lu, M. and M. Wise, 1994, Phys. Lett. B324, 461.

Lu, M., M. Wise, and M. Savage, 1992, Phys. Rev. D46, 5026.

Luke, M. and M. Savage, 1994, Phys. Lett. B321, 88.

Luke, M., M. Savage, and M. B. Wise, 1995, Phys. Lett. B343, 329.

Lusignoli, M., 1989, Nucl. Phys. B325, 33.

Mannel, T., W. Roberts, and Z. Ryzak, 1992, Nucl. Phys. B368, 204.

Mannel, T., 1993, in QCD - 20 Years Later, editors P.M. Zerwas and H.A. Kastrup, World Scientific, Singapore, (1993), p. 634.

Mannel, T., 1994, Nucl. Phys. B413, 396.

Manohar, A. and M. Wise, 1994, Phys. Rev. D49, 1310.

Marciano, W. and Z. Parsa, 1995, preprint, DOE/ER/40561-224-INT95-17-09.

Misiak, M. and M. Münz, 1995, Phys. Lett. B344, 308.

Misiak, M., 1991, Phys. Lett. B269, 161.

Misiak, M., 1993, Nucl. Phys. B393, 23.

Misiak, M., 1994, Phys. Lett. B321, 113.

Misiak, M., 1995, Nucl. Phys. B439, 461E.

Narison, S., 1994, Phys. Lett. B322, 247.

Neubert, M. and B. Stech, 1991, Phys. Rev. D44, 775.

Neubert, M., 1992, Phys. Rev. D45, 2451.

Neubert, M., 1994a, Phys. Lett. B338, 84.

Neubert, M., 1994b, Phys. Rev. D49, 4623.

Neubert, M., 1994c, Phys. Rept. 245, 259.

Nierste, U., 1995, Ph.D. dissertation, Munich Technical University hep-ph/9510323.

Nir, Y., 1989, Phys. Lett. B221, 184.

Nir, Y., 1992, in Proceedings of the the 20th Annual SLAC Summer Institute on Particle Physics, Stanford, CA, 13-24 July 1992, p. 81.

Novikov, V. A., A. I. Vainshtein, V. I. Zakharov, and M. A. Shifman, 1977, Phys. Rev. D16, 223.

O’Donnell, P. J. and H. K. K. Tung, 1991, Phys. Rev. D43, R2067.

Ohl, K. E. et al., 1990, Phys. Rev. Lett. 64, 2755.

Ovsyannikov, L. V., 1956, Dokl. Acad. Nauk SSSR 109, 1112.

Palmer, W. and B. Stech, 1993, Phys. Rev. D48, 4174.

Papadimitriou, V. et al., 1991, Phys. Rev. D44, 573. 
Particle Data Group, 1994, Phys. Rev. D50.

Patterson, R., 1995, proceedings of the XXVII. International Conference on High Energy Physics, Glasgow, 1994, editors P.J. Bussey and I.G. Knowles, IOP Publications Ltd., Bristol, $1995, \mathrm{pi} 49$.

Peccei, R. D. and K. Wang, 1995, Phys. Lett. B349, 220.

Pich, A. and E. de Rafael, 1985, Phys. Lett. B158, 477.

Pich, A. and E. de Rafael, 1991, Nucl. Phys. B358, 311.

Politzer, H. and M. Wise, 1988a, Phys. Lett. B206, 681.

Politzer, H. and M. Wise, 1988b, Phys. Lett. B208, 504.

Ponce, W., 1981, Phys. Rev. D23, 1134.

Pott, N., 1995, Technical University Munich preprint TUM-T31-93/95.

Prades, J., C. Dominguez, J. Penarrocha, A. Pich, and E. de Rafael, 1991, Z. Phys. C51, 287.

Rein, D. and L. M. Sehgal, 1989, Phys. Rev. D39, 3325.

Ritchie, J. L. and S. G. Wojcicki, 1993, Rev. Mod. Phys. 65, 1149.

Rosner, J. L., 1992, in B-Decays, editor S. L. Stone, World Scientific, Singapore, (1992).

Sachrajda, C. T., 1992, in Heavy Flavours, editors A. J. Buras and M. Lindner, World Scientific, Singapore, (1992), p. 415.

Sachrajda, C., 1994, in B-Decays (2nd Edition), editor S. L. Stone, World Scientific, Singapore, (1994), p. 602.

Savage, M. and M. Wise, 1990, Phys. Lett. B250, 151.

Schmidtler, M. and K. R. Schubert, 1992, Z.Phys. C 53, 347.

Sharpe, S. R., 1991, Nucl. Phys. B (Proc. Suppl.) 20, 429.

Sharpe, S. R., 1994, Nucl. Phys. B (Proc. Suppl.) 34, 403.

Shifman, M., N. G. Uraltsev, and A. I. Vainshtein, 1994, Int. J. Mod. Phys. A9, 2467.

Shifman, M., N. G. Uraltsev, and A. I. Vainshtein, 1995, Phys. Rev. D51, 2217.

Soni, A., 1995, preprint, hep-lat 9510036.

Stueckelberg, E. C. G. and A. Petermann, 1953, Helv. Phys. Acta 26, 499.

Symanzik, K., 1970, Comm. Math. Phys. 18, 227.

't Hooft, G. and M. Veltman, 1972a, Nucl. Phys. B50, 318.

't Hooft, G. and M. Veltman, 1972b, Nucl. Phys. B44, 189.

't Hooft, G., 1973, Nucl. Phys. B61, 455.

Tanimoto, M., 1992, Phys. Lett. B274, 463.

Thorndike, E., 1995, talk presented at the EPS-HEP Conference, Brussels, Belgium, July 1995.

Vainshtein, A. I., V. I. Zakharov, V. A. Novikov, and M. A. Shifman, 1976, Sov. J. Nucl. Phys. 23, 540 .

Vainshtein, A. I., V. I. Zakharov, and M. A. Shifman, 1977, JEPT 45, 670.

Voloshin, M. and M. Shifman, 1987, Sov. J. Nucl. Phys. 45, 292.

Voloshin, M., 1995, Phys. Rev. D51, 3948.

Vysotskij, M. I., 1980, Sov. J. Nucl. Phys. 31, 797.

Weaver, M. et al., 1994, Phys. Rev. Lett. 72, 3758.

Webber, B. R., 1994, talk presented at the International Conference on High Energy Physics,

Glasgow, Scotland, July 20-27.

Weinberg, S., 1973, Phys. Rev. D8, 3497.

Wilson, K. G. and W. Zimmermann, 1972, Comm. Math. Phys. 24, 87.

Winstein, B. and L. Wolfenstein, 1993, Rev. Mod. Phys. 65, 1113. 
Witten, E., 1977, Nucl. Phys. B122, 109.

Wolfenstein, L., 1964, Phys. Rev. Lett. 13, 562.

Wolfenstein, L., 1983, Phys. Rev. Lett. 51, 1841. 